\title{
Adiabatic hydrodynamics: the eightfold way to dissipation
}

\author{
Felix M. Haehl, ${ }^{a}$ R. Loganayagam ${ }^{b}$ and Mukund Rangamani ${ }^{a}$ \\ ${ }^{a}$ Centre for Particle Theory $\&$ Department of Mathematical Sciences, \\ Durham University, South Road, Durham DH1 3LE, U.K. \\ ${ }^{b}$ Institute for Advanced Study, \\ Einstein Drive, Princeton, NJ 08540, U.S.A. \\ E-mail: f.m.haehl@gmail.com, nayagam@gmail.com, \\ mukund.rangamani@durham.ac.uk
}

ABSTRACT: Hydrodynamics is the low-energy effective field theory of any interacting quantum theory, capturing the long-wavelength fluctuations of an equilibrium Gibbs density matrix. Conventionally, one views the effective dynamics in terms of the conserved currents, which should be expressed via the constitutive relations in terms of the fluid velocity and the intensive parameters such as the temperature, chemical potential, etc... However, not all constitutive relations are acceptable; one has to ensure that the second law of thermodynamics is satisfied on all physical configurations. In this paper, we provide a complete solution to hydrodynamic transport at all orders in the gradient expansion compatible with the second law constraint.

The key new ingredient we introduce is the notion of adiabaticity, which allows us to take hydrodynamics off-shell. Adiabatic fluids are such that off-shell dynamics of the fluid compensates for entropy production. The space of adiabatic fluids is quite rich, and admits a decomposition into seven distinct classes. Together with the dissipative class this establishes the eightfold way of hydrodynamic transport. Furthermore, recent results guarantee that dissipative terms beyond leading order in the gradient expansion are agnostic of the second law. While this completes a transport taxonomy, we go on to argue for a new symmetry principle, an Abelian gauge invariance that guarantees adiabaticity in hydrodynamics. We suggest that this symmetry is the macroscopic manifestation of the microscopic KMS invariance. We demonstrate its utility by explicitly constructing effective actions for adiabatic transport. The theory of adiabatic fluids, we speculate, provides a useful starting point for a new framework to describe non-equilibrium dynamics, wherein dissipative effects arise by Higgsing the Abelian symmetry.

KEYwORDS: Holography and quark-gluon plasmas, Effective field theories, Anomalies in Field and String Theories, Quantum Dissipative Systems

ARXIV EPRINT: 1502.00636 


\section{Contents}

I An invitation to adiabatic hydrodynamics 2

1 Introduction $\quad 2$

2 Adiabatic hydrodynamics $\quad 12$

$\begin{array}{ll}2.1 \text { The adiabaticity equation } & 12\end{array}$

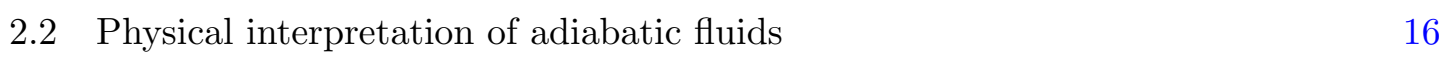

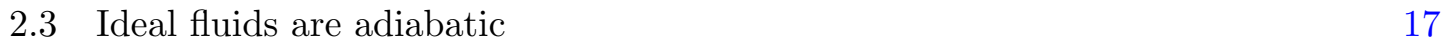

$\begin{array}{lll}2.4 & \text { The adiabatic free energy current } & 18\end{array}$

3 Classification of adiabatic transport $\quad 19$

4 Class H: hydrostatics from adiabaticity $\quad 25$

4.1 Hydrostatic partition functions 26

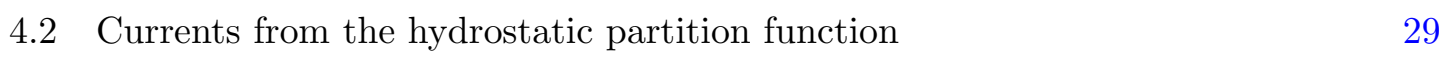

5 Class D: dissipative terms $\quad 32$

5.1 Constraints on dissipative transport 32

$\begin{array}{lll}5.2 & \text { Differential operators for dissipation } & 35\end{array}$

5.3 Examples: low order Class D differential operators 38

II The classification of adiabatic constitutive relations 40

6 Class L: Lagrangian solutions to adiabaticity equation 40

6.1 Bianchi identities in Class L 42

6.2 Noether current in Class L 44

6.3 Hydrostatic partition function for Class L 45

7 Hydrodynamic Ward identities in Class L 47

$\begin{array}{lll}7.1 & \text { A constrained variational principle for hydrodynamics } & 47\end{array}$

$\begin{array}{lll}7.2 & \text { Reference fields and conservation equations } & 48\end{array}$

$\begin{array}{lll}7.3 & \text { Gauge redundancy of reference fields } & 51\end{array}$

$\begin{array}{ll}7.4 & \text { Variational principle on reference manifold } \\ 7.5 & 52\end{array}$

7.5 Static gauge on the reference manifold \& hydrodynamic fields 53

$\begin{array}{lll}7.6 & \text { Field redefinitions in Class L } & 56\end{array}$ 
8 Applications of adiabatic fluids $\quad \mathbf{5 8}$

$\begin{array}{lll}8.1 & \text { Neutral fluids up to second order in gradients } & 58\end{array}$

$\begin{array}{lll}\text { 8.1.1 Zeroth order in gradients } & 59\end{array}$

$\begin{array}{lll}8.1 .2 & \text { First order in gradients } & 59\end{array}$

8.1.3 Second order in gradients $\quad 61$

$\begin{array}{lll}8.2 & \text { Parity-odd fluids in } 3 \text { dimensions } & 65\end{array}$

8.2.1 Zeroth order in gradients $\quad 65$

$\begin{array}{lll}\text { 8.2.2 First order in gradients } & 65\end{array}$

9 Class B: Berry-like transport $\quad 68$

$\begin{array}{lll}9.1 & \text { The Berry constitutive relations } & 69\end{array}$

$\begin{array}{lll}9.2 & \text { Examples of Class B transport } & 72\end{array}$

9.3 Embedding Class B in Class L? 74

10 Class C: conserved entropy $\quad 75$

11 The vector classes: transverse free energy currents $\quad 78$

$\begin{array}{ll}11.1 \text { The hydrostatic Class } \mathrm{H}_{V} & 78\end{array}$

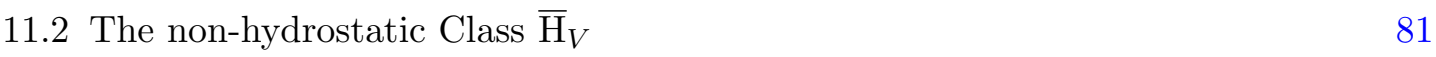

11.2.1 General construction of Class $\overline{\mathrm{H}}_{V} \quad 81$

11.2.2 Example: second order charged fluid $\quad 83$

12 Class A: lagrangian solution to anomalous adiabaticity equation $\quad \mathbf{8 5}$

$\begin{array}{lll}12.1 & \text { Flavour anomalies } & 86\end{array}$

$\begin{array}{lr}12.2 \text { Mixed anomalies } & 90\end{array}$

$\begin{array}{ll}12.3 \text { Variational calculus for mixed anomalies } & 92\end{array}$

12.4 On-shell dynamics of anomalous adiabatic fluids 95

13 Schwinger-Keldysh formalism for Class L and application to Class A $\quad 97$

$\begin{array}{lc}\text { 13.1 Schwinger-Keldysh fields on the reference manifold } & 97\end{array}$

$\begin{array}{ll}13.2 \text { Hydrodynamic currents in common/difference basis } & 101\end{array}$

13.3 Anomalous Ward identities in the Schwinger-Keldysh formalism 103

$\begin{array}{ll}13.4 \text { Effective actions for Class D? } & 106\end{array}$

III The eightfold way to dissipation and its lagrangian unification 108

14 The eightfold way 108

$\begin{array}{ll}14.1 \text { The route to classification } & 108\end{array}$

14.2 Completeness of the adiabatic taxonomy 113

$\begin{array}{ll}14.3 \text { Example I: charged parity-even fluids } & 116\end{array}$

$\begin{array}{ll}\text { 14.4 Example II: Weyl invariant fluid dynamics } & 120\end{array}$ 
14.5 Adiabatic fluids in holography and kinetic theory

14.5.1 Holographic fluids and adiabaticity

14.5.2 Kinetic theory and adiabatic fluids

15 Class $\mathrm{L}_{\mathrm{T}}$ : eightfold Lagrangian

15.1 Introducing $U(1)_{\mathrm{T}}$ invariance

15.2 The fields and their transformation properties

15.3 Bianchi identities in Class $\mathrm{L}_{\mathrm{T}}$

15.4 The adiabatic Lagrangian $\mathcal{L}_{\mathrm{T}}$

16 Hydrodynamic Ward identities and the second law in Class $\mathrm{L}_{\mathrm{T}}$

$\begin{array}{lr}\text { A Adiabaticity equation for consistent currents } & \mathbf{1 5 6}\end{array}$

$\begin{array}{ll}\text { A.1 Bardeen-Zumino currents } & 156\end{array}$

$\begin{array}{lll}\text { A.2 The consistent Gibbs current } & 157\end{array}$

$\begin{array}{lll}\text { A.3 Noether construction for free-energy current } & 158\end{array}$

B Class ND: from adiabatic to non-dissipative fluids $\quad 160$

$\begin{array}{ll}\text { B.1 Legendre transformation to an entropic description } & 160\end{array}$

$\begin{array}{lll}\text { B.2 Gauge fixing and the non-dissipative effective action } & 162\end{array}$

B.3 Static gauge: symmetries of non-dissipative effective actions 164 
C Topological currents in odd spacetime dimensions $\quad 165$

$\begin{array}{ll}\text { C.1 Generalized Euler current } & 166\end{array}$

$\begin{array}{lll}\text { C.2 Chern current } & 167\end{array}$

$\begin{array}{lr}\text { D Adiabatic hydrodynamics with Weyl invariance } & 167\end{array}$

$\begin{array}{ll}\text { D.1 Weyl transformation } & 168\end{array}$

$\begin{array}{ll}\text { D.2 Weyl connection } & 168\end{array}$

$\begin{array}{lll}\text { D.3 Covariant form of Weyl transformations } & 170\end{array}$

$\begin{array}{ll}\text { D.4 Weyl covariance and conservation equations } & 171\end{array}$

$\begin{array}{ll}\text { D.5 Velocity compatible Weyl connection } & 172\end{array}$

$\begin{array}{lll}\text { D.6 Class L for Weyl covariant fluids } & 173\end{array}$

$\begin{array}{ll}\text { VI Computational details } & 175\end{array}$

$\begin{array}{lr}\text { E Useful variational formulae } & \mathbf{1 7 5}\end{array}$

$\begin{array}{lll}\text { E.1 Mapping variations of hydrodynamic fields } & 175\end{array}$

E.2 Relating variations of hydrodynamic fields to reference parameterization 175

$\begin{array}{lll}\text { E.3 Variational rules for anomalous hydrodynamics } & 177\end{array}$

$\begin{array}{ll}\text { F Details of the neutral fluid computation at second order } & 177\end{array}$

$\begin{array}{lll}\text { F.1 Variational calculus for the second order scalars } & 178\end{array}$

$\begin{array}{lll}\text { F.2 Transport coefficients for neutral fluids } & 182\end{array}$

$\begin{array}{lll}\text { F.3 } & \text { Entropy current for the neutral fluid } & 185\end{array}$

$\begin{array}{lr}\text { G The hydrostatic entropy current } & 187\end{array}$

$\begin{array}{ll}\text { G.1 The entropy analysis of Bhattacharyya } & 187\end{array}$

$\begin{array}{lll}\text { G.2 Comparison with the charged fluid analysis of Bhattacharyya } & 188\end{array}$

$\begin{array}{lr}\text { H Bianchi identities for anomalous hydrodynamics } & 189\end{array}$

$\begin{array}{lll}\text { H.1 Bianchi identities from anomalous part of effective action } & 189\end{array}$

$\begin{array}{lll}\text { H.2 On-shell constraints from the full Lagrangian } & 193\end{array}$

H.3 Bianchi identities of anomalous Schwinger-Keldysh action 194

$\begin{array}{llr}\text { I Class } \mathrm{L}_{\mathrm{T}} \text { details } & \mathbf{1 9 6}\end{array}$

$\begin{array}{lll}\text { I.1 Consistency of } U(1)_{\mathrm{T}} \text { transformations } & 196\end{array}$

$\begin{array}{lll}\text { I.2 Deriving Class } \mathrm{L}_{\mathrm{T}} \text { Bianchi identities } & 198\end{array}$

$\begin{array}{ll}\text { J Notation and conventions } & 201\end{array}$ 


\section{Part I}

\section{An invitation to adiabatic hydrodynamics}

\section{Introduction}

Hydrodynamics, as is well known, is the universal long-wavelength effective description of near-equilibrium dynamics of interacting quantum systems. Given its wide range of applicability and the simplicity of its dynamical content, it behooves us to understand the derivation of classical hydrodynamic equations from first principles. While many attempts have been made to distill the essentials of the theory and derive the low energy dynamics following rules of effective field theory, it is perhaps fair to say that to date a completely autonomous theory of hydrodynamics remains in absentia.

The traditional approach to hydrodynamics involves identifying the conserved currents such as energy-momentum and charge currents. Firstly, one invokes an appropriate Gibbsian ensemble to describe equilibrium thermodynamics. The Gibbs free energy, as a function of temperature $T$ and chemical potentials $\mu_{i}$, determines the equilibrium data: pressures, internal energies, charge densities, etc., which constitute the components of the currents in the inertial frame chosen by the equilibrium configuration denoted by a unit timelike vector $u^{\mu}$. This explicitly constructs the ideal fluid currents which satisfy apposite conservation equations. One then allows arbitrary long-wavelength (infra-red) fluctuations of the intensive variables $\left(T, \mu_{i}\right)$ and the local thermal frame $u^{\mu}$. The fluctuations of the Gibbs density matrix in a current algebra language translate into higher derivative operators correcting the ideal fluid constitutive relations. As in usual effective field theory one allows these operators (respecting requisite symmetries) with arbitrary coefficients. In the case at hand we should admit local functions of the intensive thermodynamic parameters $\left(T, \mu_{i}\right)$; these are the transport coefficients of hydrodynamics. Note that the dynamics is still enforced by the conservation of the currents.

Thus far the construction of the low energy hydrodynamic theory seems analogous to any other effective field theory albeit in a current algebra language. The main novelty of hydrodynamics is that it has a constraint: one expects that the hydrodynamic evolution locally respects the second law of thermodynamics. More abstractly, in addition to the conserved currents which capture the dynamical information, one posits the existence of an entropy current, which is constrained to have non-negative divergence. This approach to hydrodynamics, which we dub as the current algebra approach, is the canonical method to determining both the constitutive relations and the constraints on the transport coefficients. This viewpoint has been well appreciated for many decades now, cf., [1] for a clear discussion.

Since the second law of thermodynamics is stated as an inequality one usually finds that it imposes sign-definiteness constraints on transport data. For instance, one learns at the first non-trivial order in the gradient expansion that viscosities and conductivities are 
positive definite to ensure entropy production. What is perhaps less familiar is the recent discovery that one also encounters explicit constraints fixing some transport coefficients in terms of others [2]. An example of this is the Gibbs-Duhem and Euler relations obeyed at zeroth order in derivative expansion. Said differently, by a careful analysis one can show that the allowed class of operators respecting the second law is smaller than one might a-priori have imagined. In all known examples studied so far, the transport coefficients thus constrained can be obtained from analyzing general hydrostatic equilibria - they are determined in terms of the so called hydrostatic or thermodynamic response parameters. In particular, these constraints can be understood in terms of subjecting the fluid to arbitrary stationary sources (background metric, gauge fields) and obtaining the desired relations by writing down the generating function for the current correlators, or equivalently the equilibrium partition function $[3,4]$.

The class of hydrostatic transport coefficients is quite rich. Not only does it comprise of novel constraints on higher order hydrodynamic data, but it also importantly includes the class of anomalous transport coefficients which provide an interesting insight into the underlying quantum dynamics in thermodynamic systems. While the presence of anomalous transport coefficients was first clearly encountered in fluid/gravity computations of $[5,6]$, they were soon understood as being necessary from the canonical current algebra framework of fluid dynamics in [7]. More recently, starting from the work of [8], it has been appreciated that the anomalous contribution to transport belongs to the hydrostatic class. We now have clear picture of how to derive the constraints on them using the equilibrium partition function [3, 9-13] (for related work on anomaly induced transport we refer the reader to [14-35]). We will have occasion to describe these results in due course, but for now we simply record the fact that the equilibrium partition function provides a powerful way to study the constraints on hydrodynamic transport.

Recently, building on the hydrostatic analysis, Sayantani Bhattacharyya [36, 37] derived a remarkable theorem about hydrodynamic transport. She proved that:

- All the dangerous terms in hydrodynamics which could potentially lead to entropy destruction, are constrained to vanish from the hydrostatic analysis and there are no further equality constraints beyond hydrostatics.

- Of the entropy producing dissipative terms, only the ones at leading order in the gradient expansion are constrained to be sign-definite.

Essentially the upshot of this analysis is the following: once one understands the leading deviations from a perfect fluid and is able to analyze hydrostatic configurations, one has completed the task of constructing the hydrodynamic effective field theory (at least as a current algebra).

While this result captures the essence of the second law constraints, it still leaves unanswered questions about the structure of hydrodynamics. One may think of the situation in the following vein: a-priori in the current algebra approach, constructing tensor valued operators which correct the ideal fluid conserved currents is a question in representation theory. Given the intensive parameters $\left\{T, \mu_{i}\right\}$ and the hydrodynamic velocity field $u^{\mu}$ 
(which is a unit timelike vector), we simply have to build tensors with suitable symmetries to appear in the energy-momentum tensor $T^{\mu \nu}$ and charge currents $J_{i}^{\mu}$. The number of such tensors can be inferred from a straightforward counting exercise at any desired order. Complications start to arise when we impose the constraints of the second law since this poses a non-linear constraint, potentially mixing terms across derivative orders. Indeed the proof of the statements quoted above in [36] relies on a careful unpacking of such mixing (see $[2,37]$ ). It would be ideal if we could understand the second law constraints in a more straightforward fashion. Ideally, one would like to have a complete classification of hydrodynamic transport, both dissipative and non-dissipative, which respects the democratic ordering of the gradient expansion. ${ }^{1}$

This compels us to further our understanding of hydrodynamics, a task we will undertake in this work. Our primary result will indeed be a complete classification of hydrodynamic transport at all orders in the gradient expansion. In the process we will also be able to identify the origins of various curious results that have been uncovered in recent analysis of hydrodynamic transport, both from studies in kinetic theory and in holography. The analysis of transport in holographic fluids, which began with the pioneering work of [40] and was extended by the fluid/gravity correspondence to the non-linear level [41], provides a rather fertile laboratory for testing non-linear hydrodynamics.

To explain more precisely the rationale behind our analysis, we start with the following observation. In order to ascertain the structure of hydrodynamics, it would be ideal if we could formulate the effective field theory, not in terms of the currents as described above, but rather directly in terms of a Wilsonian effective action. The question then becomes: what are the natural variables for such an action and how does one incorporate the fact that the theory is intrinsically dissipative?

As a toy problem, one can focus on the structure of hydrodynamic effective actions in the absence of dissipation. At the very least this attempt can help us learn about the constraints resulting from demanding an off-shell (by definition), off-equilibrium, effective action for hydrodynamics. Happily, such a formalism exists. It was invented in the distant past to formulate the dynamics of ideal fluids coupled to gravitational degrees of freedom $[42,43]$. In recent years this effective action formalism has been revived starting from the work of $[44,45]$. These works formulate the story in terms of the Goldstone degrees of freedom associated with individual fluid elements. A systematic exploration for neutral fluids was undertaken in [46] wherein a detailed comparison with the conventional current algebra approach to hydrodynamics was made. ${ }^{2}$ In particular, it was noticed that demanding the presence of an effective action appears to pose stronger constraints than what would be encountered by the existence of an entropy current with desired properties. $^{3}$ Said differently, there were relations amongst transport coefficients (the functions

\footnotetext{
${ }^{1}$ In many circumstances one also demands that the Onsager relations [38, 39] are upheld, by invoking the microscopic time-reversal symmetry. We will for the most part be agnostic about these relations, and relegate comments regarding them to section 18.

${ }^{2}$ This approach has also been used to study parity-odd transport in 3 dimensions [47-49].

${ }^{3}$ For non-dissipative fluids one imposes a strong constraint on the entropy current: we demand it to be divergence free off-shell. So the theory actually has an additional conserved current due to entropy non-production.
} 
of intensive parameters multiplying higher order tensor structures in the currents) which remained inexplicable. Curiously, some of these relations are also manifested in the class of holographic fluids, which prompted us to examine the situation further.

A crucial check of this effective action formalism was provided in [30] where it was shown that one can recover the anomalous transport data for non-abelian flavour anomalies in arbitrary even dimensions (see [20] for abelian anomalies in 2 dimensions). This analysis highlighted two important facts about the effective action approach: (i) the symmetries of the theory (ii) the necessity of doubling the degrees of freedom, a la Schwinger-Keldysh along with non-trivial cross terms (a.k.a. influence functionals of Feynman-Vernon [50]) in the effective action. We will postpone a discussion of the doubling to later, but the symmetries are worth examining at present.

The effective action for $d$-dimensional hydrodynamics is constructed in terms of $d-1$ scalar fields $\phi^{I}$ which capture energy-momentum transport along with a set of fields c which transform in a bifundamental representation of the flavour symmetry. One allows arbitrary volume preserving field reparametrizations of the fields, $\phi^{I} \mapsto f^{I}(\phi)$ and $\mathrm{c} \mapsto \mathrm{c} g(\phi)$. This implies that the effective action is invariant under a generalized volume-preserving diffeomorphism group; this guarantees entropy non-production. More specifically the conservation of configuration space volume $d \phi^{1} \wedge \cdots \wedge d \phi^{d-1}$ is interpreted as the statement of entropy current conservation. That is one identifies $\star \boldsymbol{J}_{S}=\star(s \boldsymbol{u})=d \phi^{1} \wedge \cdots \wedge d \phi^{d-1}$ as the 1-form entropy current, with $s$ being the entropy density and $\mathbf{u}$ the fluid velocity. Note that the formalism forces the entropy current to take its ideal fluid form at all orders in the hydrodynamic gradient expansion. The resulting constitutive relation may then said to be presented in the entropy frame. ${ }^{4}$

While one can tie in the entropy conservation with the presence of the enlarged symmetry, one would as such like to understand the rationale behind its existence and check the consistency of employing it to define a conserved entropy current. Moreover, if we were to extend the effective action approach to physically relevant dissipative fluids, we need to understand how to allow for entropy production. In fact, empirically the failure point of the formalism appears to be at an even simpler situation. An attempt to extend the considerations of anomalous transport to mixed flavour-Lorentz anomalies suggests that modifications to the entropy current from the form $\boldsymbol{J}_{S}=s \boldsymbol{u}$ are imperative. ${ }^{5}$ This being impossible in the framework described above one is led to look elsewhere.

Let us therefore step back and ask the following: what is the canonical choice of variables for a hydrodynamic effective action? A natural set of hydrodynamic variables in terms of which an effective action ought to be written, one would guess, are simply the intensive thermodynamic variables that characterize the Gibbs ensemble $T, \mu_{i}$ etc., and the fluid velocity $u^{\mu}$. In the non-dissipative effective action temperature is viewed as a

\footnotetext{
${ }^{4}$ Frame choices in hydrodynamic current algebra are a reflection of field redefinitions. For example, there being no a-priori canonical choice for the velocity field in a relativistic fluid one can choose to define it conveniently. The above choice is just as natural as the often made choice of Landau frame, wherein the velocity field is taken to be the unit-timelike eigenvector of the energy-momentum current.

${ }^{5}$ We do not have a complete proof of this statement, but the ease with which we are able to recover all the results in the formalism described below suggests to us that this is the correct intuition.
} 
function of its thermodynamic conjugate entropy density, while the velocity and chemical potential (and entropy itself) are indirectly defined in terms of the fields $\phi^{I}$ and c. As such the on-shell action computes not quite the thermodynamic Gibbs potential, but rather, its Legendre transform with respect to the entropy density. If one were interested in allowing deformation of the entropy current, working with Gibbs potential is more natural. ${ }^{6}$ However, in the hydrodynamic gradient expansion computing the Legendre transformation is non-trivial. ${ }^{7}$ Inspired by the structural aspects of the formal Legendre transformation we establish now a new formalism that naturally incorporates the hydrodynamic variables as the basic fields and provides a framework to describe what we call adiabatic fluids, which are a generalization of the non-dissipative fluids discussed hitherto.

To motivate the study of adiabatic fluids, let us ask the following question: "what is the most convenient way to implement the second law of thermodynamics, which apriori is stated as an inequality, in practice?" As we discussed before the conventional current-algebraic approach is to work on-shell by classifying independent tensors, but this is limiting from the point of view of constructing an action principle. A useful trick for implementing inequality constraints is in fact to go off-shell using a suitable set of Lagrange multipliers, which sometimes is referred to as the Liu procedure [51]. The basic idea can be explained as follows: suppose we want to constrain the solutions of a set of linear equations with an inequality constraint. We add to the inequality of interest a suitable linear combination of the dynamical equations with Lagrange multipliers. While the new quantity defined thus, also satisfies the same inequality, it has the distinct advantage that we are no longer on-shell. Said differently, incorporating the dynamical equations of motion into the inequalities, we can uplift the constraints off-shell and analyze them without having to solve for the dynamically independent set of data.

Specifically, we take the on-shell statement of non-negative entropy production $\nabla_{\mu} J_{S}^{\mu} \geq 0$ and upgrade it to an off-shell statement which reads:

$$
\begin{aligned}
\nabla_{\mu} J_{S}^{\mu}+\boldsymbol{\beta}_{\mu}\left(\nabla_{\nu} T^{\mu \nu}-J_{\nu} \cdot F^{\mu \nu}-\mathrm{T}_{H}^{\mu \perp}\right) \\
+\left(\Lambda_{\boldsymbol{\beta}}+\boldsymbol{\beta}^{\lambda} A_{\lambda}\right) \cdot\left(D_{\nu} J^{\nu}-\mathrm{J}_{H}^{\perp}\right) \equiv \Delta \geq 0 .
\end{aligned}
$$

Here $\boldsymbol{\beta}_{\mu}$ is the Lagrange multiplier for the energy-momentum conservation equation involving the energy-momentum tensor $T^{\mu \nu}$ and the charge current $J^{\mu}$, and $\left(\Lambda_{\boldsymbol{\beta}}+\boldsymbol{\beta}^{\lambda} A_{\lambda}\right)$ is the Lagrange multiplier for the charge conservation equation. We have denoted the background metric and flavour gauge fields by $\left\{g_{\mu \nu}, A_{\mu}\right\}$ respectively and $F_{\mu \nu}$ denotes the field-strength associated with $A_{\mu}$. Note that we have written the conservation equations for a general situation including contributions from sources and anomalies (which are captured by the

\footnotetext{
${ }^{6}$ Hence the common use of Landau-Ginzburg free-energy funtionals to describe condensed matter systems.

${ }^{7}$ This has the insalubrious effect of making comparisons between the effective actions and the equilibrium partition functions rather complex and involved. In special cases such as the anomalous effective action one can carry out the Legendre transformation trivially owing to independence of such terms from the entropy density, cf., [30].
} 
Hall currents $J_{H}^{\perp}$ and $\left.T_{H}^{\mu \perp}\right){ }^{8}$ The notation will become clear when we set-up the problem in greater detail in due course.

It is convenient to take these Lagrange multiplier fields to be the basic hydrodynamic fields. At zeroth order in derivative expansion, thermodynamics demands that $\boldsymbol{\beta}^{\mu}=u^{\mu} / T$ be the velocity field rescaled by the temperature and $\Lambda_{\boldsymbol{\beta}}+\boldsymbol{\beta}^{\lambda} A_{\lambda}=\mu / T$ be the chemical potential measured in thermal units. We will use a part of the field redefinition freedom to assume that these simple relations hold to arbitrary orders in derivative expansion. In fact, these variables naturally encompass all of the hydrodynamic degrees of freedom; by rescaling the normalized velocity field by the temperature we have ensured that $\boldsymbol{\beta}^{\mu}$ is an unconstrained vector field. We refer to these fields as the thermal vector and the thermal twist respectively; they are the physical degrees of freedom in local equilibrium.

The off-shell rewriting of the second law of thermodynamics in (1.1) turns out to provide sufficient control to classify all hydrodynamic transport. A-priori, we can distinguish between two kinds of transport coefficients: on the one hand those that contribute to offshell entropy production, i.e., lead to positive definite $\Delta \neq 0$ - these are the dissipative (Class D) parts of transport. On the other hand we have those which comprise the marginal case of no entropy production, i.e., those where the production of entropy is compensated for by the flow of energy-momentum and charge. The latter form the boundary of the domain of physically admissible constitutive relations and have $\Delta=0$. Understanding this marginal case turns out to be the crucial step that allows us to complete our classification. This therefore motivates for us the study of adiabatic hydrodynamics, defined as the constitutive relations which solve (1.1) with $\Delta=0$, which we will refer to as the adiabaticity equation. ${ }^{9}$ The class of adiabatic constitutive relations subsumes (but is not identical to) the pre-existing discussions of non-dissipative fluids.

A large part of our work will be devoted to identifying and classifying all constitutive relations $\left\{T^{\mu \nu}, J^{\mu}, J_{S}^{\mu}\right\}$ that solve the adiabaticity equation (1.1) with $\Delta=0$. At a broad brush level there are two types of adiabatic transport: hydrostatic (Class $\mathrm{H}$ ) and nonhydrostatic or hydrodynamic (Class $\overline{\mathrm{H}})$. The former can be inferred from the dynamics of the fluid in hydrostatic equilibrium described earlier by subjecting it to time-independent spatially varying external sources. The latter are more diverse; some can be obtained using a simple Lagrangian formalism involving the hydrodynamic fields $\left\{\boldsymbol{\beta}^{\mu}, \Lambda_{\boldsymbol{\beta}}\right\}$, but there are others which evade such a simple description. We have found it convenient to isolate the solutions of the adiabaticity equation into seven classes based on their origins. Together with the dissipative Class D, we are led to the eightfold way of hydrodynamic transport, as illustrated in figure $1 .^{10}$

We emphasize that we classify (off-shell inequivalent) constitutive relations i.e., give combinations of currents that satisfy the adiabaticity equation. However, it is convenient

\footnotetext{
${ }^{8}$ If the underlying quantum system of interest is anomaly-free then we can set the Hall currents to zero; we refer to the corresponding version of (1.1) (with $\Delta=0$ ) as the non-anomalous adiabaticity equation for definiteness.

${ }^{9}$ The adiabaticity equation we study in some detail below was first introduced in [8] to aid the analysis of anomaly induced hydrodynamic transport using the standard current algebra approach to hydrodynamics.

${ }^{10}$ We give a preview of this classification in some detail in section 3.
} 


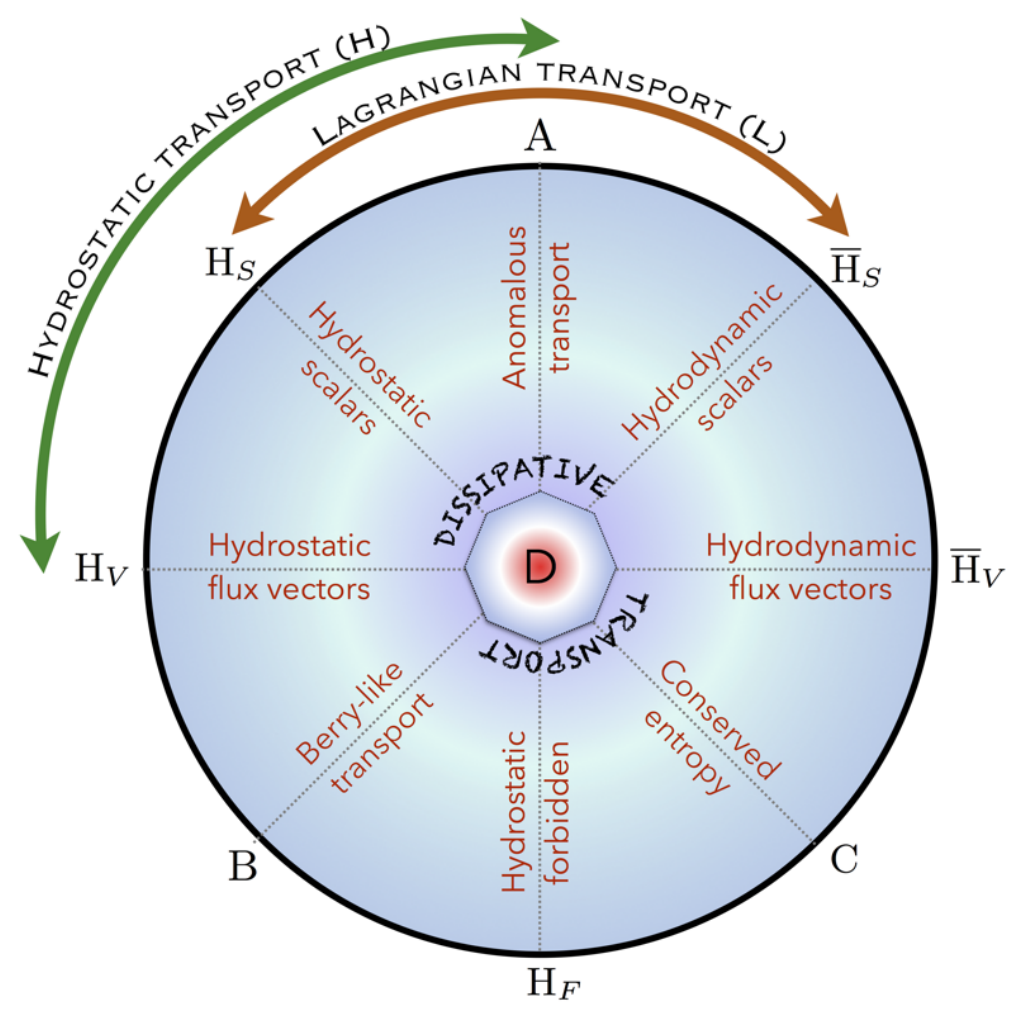

Figure 1. The eightfold way of hydrodynamic transport.

for purposes of taxonomy to refer to some more primitive object that generates such constitutive relations. The classification turns out to be canonically motivated by the choice of the grand canonical free energy current (obtained by Legendre transforming the entropy current) which is a spacetime vector. This vector can be either longitudinal (aligned to the thermal vector) or transverse. Since longitudinal vectors are characterized by their magnitude, we can refer to it as the scalar component. Hence all the classes in figure 1 refer to either scalar or vector structures. For example, $\mathrm{H}_{S}$ and $\overline{\mathrm{H}}_{S}$ refer to hydrostatic and hydrodynamic terms that can be encoded in a scalar Lagrangian. Similarly, $\mathrm{H}_{V}$ and $\overline{\mathrm{H}}_{V}$ are classes of transport that transform as transverse vectors. Whether at the end of the day such terms show up as tensors, vectors or scalars in the constitutive relations, is largely a matter of convention and frame choice.

We also provide evidence that certain well studied hydrodynamic systems respect the adiabatic classification. In particular, strongly coupled conformal plasmas which can be described holographically via the fluid/gravity correspondence [41, 52], as well as existing results in kinetic theory [53] manifestly exhibit the eightfold path. Furthermore, as noted in our short companion paper [54], second order transport for a neutral holographic fluid, is encapsulated in a simple effective action (built out of the sources and the thermal vector and twist)! We take this as striking evidence of physical fluid systems being cognizant of the adiabaticity equation and the sevenfold constitutive relations which solve it.

To demonstrate that our classification is exhaustive, we argue that all solutions to the adiabaticity equation can be obtained from a master effective action. This eightfold effective 
action clearly is a functional of the hydrodynamic fields $\left\{\boldsymbol{\beta}^{\mu}, \Lambda_{\boldsymbol{\beta}}\right\}$ and the background sources $\left\{g_{\mu \nu}, A_{\mu}\right\}$. Rather surprisingly, a complete picture emerges only upon including a second set of background sources, $\left\{\tilde{g}_{\mu \nu}, \tilde{A}_{\mu}\right\}$, which morally speaking appear to be a proxy for the Schwinger-Keldysh partners of the basic sources. Furthermore, this doubling of sources comes with an interesting new Abelian gauge symmetry with an associated gauge field $\mathrm{A}^{(\mathrm{T})}{ }_{\mu} !^{11}$

In the thermofield construction one has sources for the left $(\mathrm{L})$ and right $(\mathrm{R})$ degrees of freedom; these are specific linear combinations of the sources $\left\{g_{\mu \nu}, A_{\mu}\right\}$ and $\left\{\tilde{g}_{\mu \nu}, \tilde{A}_{\mu}\right\}$. The necessity to double of the degrees of freedom, whilst curious for adiabatic transport, has already been encountered previously in attempts to construct effective actions for anomalous hydrodynamic transport, which forms a special case, in [30]. What is really intriguing is the gauge field $\mathrm{A}^{(\mathrm{T})}{ }_{\mu}$ and its associated gauge invariance $U(1)_{\mathrm{T}}$, which along with the diffeomorphism and gauge invariance forms the symmetries of the effective action. ${ }^{12}$ The latter act canonically on the fields above, but the $U(1)_{\mathrm{T}}$ gauge symmetry acts non-trivially. All fields carry $U(1)_{\mathrm{T}}$ charges, with the gauge transformation acting as a diffeomorphism or flavour gauge transformation in the direction of $\boldsymbol{\beta}^{\mu}, \Lambda_{\boldsymbol{\beta}}$. In addition, $\tilde{g}_{\mu \nu}$ and $\tilde{A}$ further undergo transformations depending on the physical fields $\left\{\boldsymbol{\beta}^{\mu}, \Lambda_{\boldsymbol{\beta}}, g_{\mu \nu}, A_{\mu}\right\}$. The Bianchi identity associated with $U(1)_{\mathrm{T}}$ gauge invariance immediately leads to the adiabaticity equation. In fact, armed with this enlarged space of fields one can immediately write down a diffeomorphism, flavour gauge and $U(1)_{\mathrm{T}}$ invariant effective action which captures all of the adiabatic transport.

We have chosen to structure the paper into six parts owing to the complexities of the results we uncover. To help orient readers through the maze of results we also provide a flowchart in figure 2 to indicate the inter-relations between various sections.

Part I: we begin in section 2 with a definition of the basic statement of the adiabatic fluids and explain some of the general properties of such fluids directly from the study of the adiabaticity equation. In section 3 we present a quick overview of the diverse classes of adiabatic constitutive relations; this provides a short synopsis of part II. We then turn in section 4 to connecting our construction with the hydrostatic analysis of $[3,4]$. We go on to argue that the adiabaticity equation we introduce can be thought of as an off-equilibrium off-shell extension of the hydrostatic constitutive relations. In section 5 we discuss dissipative constitutive relations, reviewing the results of $[36,37]$ in a language adapted to our analysis. This allows us to give an alternate proof of the theorem, classifying dissipative transport coefficients into those constrained by the second law, and those that are agnostic to entropy production.

Part II: this is the central part of the paper where we study adiabatic constitutive relations. For the most part we focus on the non-anomalous adiabaticity equation as

\footnotetext{
${ }^{11}$ It is tempting for various reasons to christen this $U(1)_{T}$ symmetry as KMS-gauge invariance. We will however refrain from doing so since we won't fully justify the connections with the Schwinger-Keldysh framework in the present work and leave a full exposition for the future [55].

${ }^{12} \mathrm{~A}$ clue to the existence of such a structure is provided by the analysis of hydrostatic partition functions satisfying the Euclidean consistency condition in the presence of gravitational anomalies [13].
} 


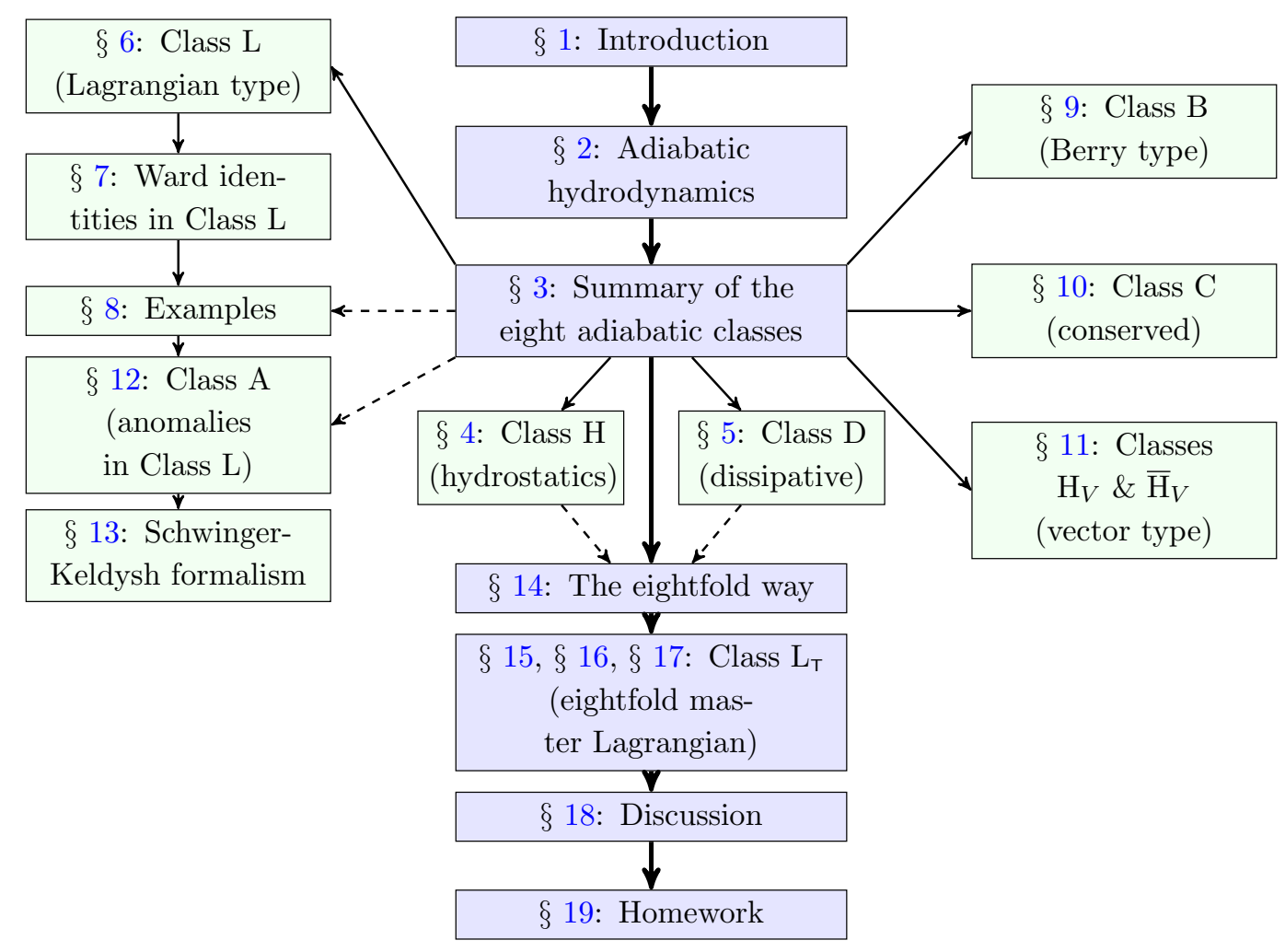

Figure 2. Flowchart giving the structure of this paper. Light blue sections in the middle column form the main thread of our analysis. The sections in light green contain detailed constructions of the various classes and could be skipped on a first read. The sections in the left column are concerned with various Lagrangian descriptions. The classes in the right column are those that do not fit into a simple Lagrangian framework (without $U(1)_{\mathrm{T}}$ ).

inclusion of anomalies ends up providing a specific particular solution. We first show in section 6 how to construct Lagrangian solutions to the adiabaticity equation in the absence of anomalies. Any diffeomorphism and gauge invariant Lagrangian has a set of Bianchi identities which together with the canonical definition of the entropy current leads to the adiabaticity equation. Having established that the background symmetries guarantee adiabaticity we turn to describing how to obtain hydrodynamic Ward identities in section 7 in terms of a constrained variational principle. To illustrate the efficacy of our formalism, we quickly review some basic examples discussed in the context of non-dissipative fluids in section 8 .

In section 9-section 11 we turn to the set of adiabatic constitutive relations which do not belong to Class L, i.e., they don't admit a Lagrangian description. Our first example is the Berry-like class of adiabatic transport section 9 - these are non-hydrostatic, nondissipative parts of transport which include well studied examples of transport such as Hall viscosity and Hall conductivity, but also others which have been encountered in holographic fluids. In section 10 we describe the class of conserved entropy currents which are agnostic to physical current transport. We argue in particular that such constitutive relations 
will be encountered in the presence of topological ground state degeneracy. In section 11 constitutive relations determined by transverse vector currents are described; there are some new ingredients here for such transport appears to have never been studied in the literature (outside hydrostatics).

In section 12 we turn to the problem of finding Lagrangian solutions to the anomalous hydrodynamic transport. We also demonstrate that a complete story for obtaining anomalous Ward identities requires working in a Schwinger-Keldysh doubled theory. We review the thermofield doubled Schwinger-Keldysh construction for hydrodynamics in section 13, taking the opportunity to highlight certain obvious tension with adiabaticity and the role of influence functionals. We also explain why the terms we introduce in the anomalies both in the current and in our previous discussion [30] are necessary and sensible.

Part III: we discuss how we can use adiabatic hydrodynamics to provide a complete classification of hydrodynamic transport in section 14 . Not only do we provide an algorithm for the eightfold classification, amplifying on the results presented in [54], but we also finally prove that the classification is exhaustive. To exemplify our construction, we provide evidence that various hydrodynamic systems are cognizant of the eightfold classification (section 14.5) and give a concise summary of a variety of fluid systems (up to second order in gradients) in section 14.6. We also outline the basic construction of an effective action which encompasses all of the adiabatic constitutive relations in section 15 and section 16, though we leave a fuller exposition of the construction to a later publication [55]. In section 17 we show how the eightfold way of adiabatic transport is captured by this effective action.

Part IV: we end the main thread of the paper with a discussion in section 18 and highlight some open questions which we think can be addressed with existing technology in section 19 .

Part V: there are several extensions to our general analysis of adiabatic constitutive relations which are interesting to explore. We have chosen to present some of these outside the main line of development so as to keep the flow of the paper more straightforward. In appendix A we provide a translation from the covariant to the consistent form of anomalous adiabaticity equation. In appendix B we argue that the Lagrangians we introduce are canonically related via a Legendre transformation to those described earlier in the literature on non-dissipative fluids. Our presentation clarifies the origins of various symmetries encountered in the previous discussion. Appendix $\mathrm{C}$ provides a construction of topological currents which play a role in constructing Class $\mathrm{C}$ adiabatic constitutive relations. Finally in appendix D we give a complete characterization of Weyl invariant adiabatic fluids relevant in the study of holographic fluids, generalizing the discussion of [56].

Part VI: in these appendices we provide some technical details which are omitted from the main text. Appendix E provides useful technical details for implementing the variational calculus in Class L theories. Appendix $\mathrm{F}$ gives a detailed discussion of second order neutral fluid hydrodynamics. In appendix $G$ we compare our construction with that of $[36,37]$. Appendix $\mathrm{H}$ contains details of the derivation of Bianchi identities in the presence of anomalies. Appendix I verifies that the symmetries of the eightfold Lagrangian are 
consistent and provides some details for deriving the various Bianchi identities quoted in the text.

Appendix J provides a quick reference of the various symbols we introduced through the course of or discussion.

\section{Adiabatic hydrodynamics}

We would like to construct a hydrodynamic effective field theory which not only incorporates the fundamental symmetries present in the underlying microscopic quantum system, but also and in addition is cognizant of the basic constraint of such effective theories, viz., the second law of thermodynamics. As described in section 1 the complications of the inequality constraints imposed by the second law can be tackled by taking the constraints off-shell using a suitable combination of the dynamical equations of motion [51]. While this procedure allows exploration of the off-shell constraints, it is actually more efficacious to first undertake a full classification in the marginal situation where the second law is imposed as a statement of entropy conservation (as opposed to entropy production). This split is guided by the fact that hydrodynamic transport can a-priori be categorized as being either adiabatic ${ }^{13}$ or dissipative.

Aided by this intuition we therefore propose to study a class of hydrodynamic theories which we call adiabatic fluids. These are fluids where entropy production is compensated for off-shell by the dynamics of the theory. Having an off-shell formalism allows much insight into how one might construct hydrodynamic effective actions. In fact it will turn out that much of the constraints of the second law can be gleaned from an analysis of adiabatic transport; explicitly dissipative terms will turn out to be quite tractable in the sequel.

\subsection{The adiabaticity equation}

Consider a fluid characterized by normalized velocity field $u^{\mu}$ (with $u^{\mu} u_{\mu}=-1$ ), temperature $T$ and chemical potential $\mu$ moving in a background geometry $\mathcal{M}$ with metric $g_{\mu \nu}$ and a background flavour gauge field $A_{\mu}$ which generically will be taken to be non-abelian. ${ }^{14}$ We will work in $d$ spacetime dimensions and will assume that the hydrodynamic fields $\left\{u^{\mu}, T, \mu\right\}$ as well as the background sources $\left\{g_{\mu \nu}, A_{\mu}\right\}$ are slowly varying on this spacetime manifold throughout our discussion.

While we could choose to work with the hydrodynamic fields defined above it is in fact convenient to repackage them into an unnormalized vector field and a scalar field. By a simple redefinition we therefore introduce the hydrodynamic fields (denoted collectively by $\mathcal{B}$ )

$$
\mathcal{B} \equiv\left\{\boldsymbol{\beta}, \Lambda_{\boldsymbol{\beta}}\right\}, \quad \boldsymbol{\beta}^{\mu} \equiv \frac{u^{\mu}}{T}, \quad \Lambda_{\boldsymbol{\beta}} \equiv \frac{\mu}{T}-\frac{u^{\sigma}}{T} A_{\sigma} .
$$

The fields $\left\{\boldsymbol{\beta}^{\mu}, \Lambda_{\boldsymbol{\beta}}\right\}$ which we refer to as the thermal vector and thermal twist, encode the same hydrodynamic data as the fields $\left\{u^{\mu}, T, \mu\right\}$. We can explicitly invert the above

\footnotetext{
${ }^{13}$ We use the word adiabatic in a precise technical sense defined below. Reference [36] uses this word synonymously with hydrostatics, which we prefer not to do. There is more to adiabaticity than equilbirum, as we shall unearth in the course of our discussion.

${ }^{14}$ Generalizations to arbitrary number of flavour symmetries is straightforward.
} 
relations to get

$$
u^{\mu}=\frac{\boldsymbol{\beta}^{\mu}}{\sqrt{-g_{\alpha \lambda} \boldsymbol{\beta}^{\alpha} \boldsymbol{\beta}^{\lambda}}}, \quad T=\frac{1}{\sqrt{-g_{\alpha \lambda} \boldsymbol{\beta}^{\alpha} \boldsymbol{\beta}^{\lambda}}}, \quad \mu=\frac{\Lambda_{\boldsymbol{\beta}}+\boldsymbol{\beta}^{\sigma} A_{\sigma}}{\sqrt{-g_{\alpha \lambda} \boldsymbol{\beta}^{\alpha} \boldsymbol{\beta}^{\lambda}}} .
$$

Thus for the rest of the discussion, the dynamical content of hydrodynamics is captured by the $d+1$ degrees of freedom in the vector field $\boldsymbol{\beta}^{\mu}$ and scalar field $\Lambda_{\boldsymbol{\beta}}$.

A general hydrodynamic system as reviewed in section 1 is characterized by a set of currents: we have the energy-momentum tensor $T^{\mu \nu}$ and a charge current $J^{\mu}$ which should be considered dynamical. In addition we have an entropy current $J_{S}^{\mu}$ which enforces the constraint of the second law. It is also useful to include the free energy current $\mathcal{G}^{\mu}$, which is a particular linear combination of the above, which we will encounter shortly, cf., (2.18). To simplify notation, we will collect the various currents we have introduced into a single set by introducing a collection of tensor fields $\mathcal{C}_{\mathcal{H}}$

$$
\mathcal{C}_{\mathcal{H}} \equiv\left\{T^{\mu \nu}, J^{\mu}, J_{S}^{\mu}\right\},
$$

where instead of $J_{S}^{\mu}$ we often equivalently consider the Gibbs free energy current $\mathcal{G}^{\mu}$ to be defined in due course.

These currents should all be thought of as given by local covariant functionals of the background and hydrodynamical fields which we also collectively denote as $\mathbf{\Psi}$

$$
\boldsymbol{\Psi} \equiv\left\{g_{\mu \nu}, A_{\mu}, \boldsymbol{\beta}^{\mu}, \Lambda_{\boldsymbol{\beta}}\right\}
$$

Then we can write for our currents $\mathcal{C}_{\mathcal{H}}=\mathcal{C}_{\mathcal{H}}[\Psi]$ or more explicitly, for the fundamental currents we have

$$
\begin{aligned}
T^{\mu \nu} & =T^{\mu \nu}[\mathbf{\Psi}]=T^{\mu \nu}\left[g_{\alpha \beta}, A_{\alpha}, \boldsymbol{\beta}^{\alpha}, \Lambda_{\boldsymbol{\beta}}\right] \\
J^{\mu} & =J^{\mu}[\mathbf{\Psi}]=J^{\mu}\left[g_{\alpha \beta}, A_{\alpha}, \boldsymbol{\beta}^{\alpha}, \Lambda_{\boldsymbol{\beta}}\right] \\
J_{S}^{\mu} & =J_{S}^{\mu}[\mathbf{\Psi}]=J_{S}^{\mu}\left[g_{\alpha \beta}, A_{\alpha}, \boldsymbol{\beta}^{\alpha}, \Lambda_{\boldsymbol{\beta}}\right] .
\end{aligned}
$$

These relations are termed constitutive relations.

The dynamical information of hydrodynamics comprises simply of the statement of conservation modulo source terms (which do work on the system) and anomalies. In general we can write the conservation equations for a microscopic quantum theory with flavour and Lorentz anomalies in the presence of background sources as:

$$
\nabla_{\nu} T^{\mu \nu}=J_{\nu} \cdot F^{\mu \nu}+\mathrm{T}_{H}^{\mu \perp} \quad D_{\nu} J^{\nu}=\mathrm{J}_{H}^{\perp} .
$$

Here, $F_{\mu \nu}$ and $D_{\mu}$ denote the field-strength and gauge-covariant derivative associated with $A_{\mu}$ while $\left\{\mathrm{T}_{H}^{\mu \perp}, \mathrm{J}_{H}^{\perp}\right\}$ are the covariant Lorentz and flavour anomalies respectively. ${ }^{15}$ The

\footnotetext{
${ }^{15}$ If $\mathcal{P}[\boldsymbol{F}, \boldsymbol{R}]$ is the anomaly polynomial, then the covariant anomalies are determined using the following equations:

$$
\mathrm{J}_{H}^{\perp}{ }^{\star} \mathbf{1} \equiv \frac{\partial \mathcal{P}}{\partial \boldsymbol{F}}, \quad \Sigma_{H}^{\perp \nu}{ }^{\star}{ }^{\star} \mathbf{1} \equiv 2 \frac{\partial \mathcal{P}}{\partial \boldsymbol{R}^{\mu}{ }_{\nu}}, \quad \mathrm{T}_{H}^{\mu \perp} \equiv \frac{1}{2} \nabla_{\nu} \Sigma_{H}^{\perp \mu \nu} .
$$

Here $\Sigma_{H}^{\perp \mu \nu}$ is the torque on the system due to Lorentz anomaly. We adopt a bold-face notation for differential forms. In general our notation follows that of $[12,13,30]$ where the reader will find further details on the conventions used herein. We will be more explicit when we solve the anomalous adiabaticity equation in section 12. Some further useful details are collected in appendices A and J.
} 
center-dot "." is reserved for gauge index contraction which we will never write explicitly. The gauge-covariant derivative acts on tensors $X_{\rho \cdots \sigma}^{\mu \cdots \nu}$ in a familiar fashion, viz.,

$$
D_{\alpha} X_{\rho \cdots \sigma}^{\mu \cdots \nu}=\nabla_{\alpha} X_{\rho \cdots \sigma}^{\mu \cdots \nu}+\left[A_{\alpha}, X_{\rho \cdots \sigma}^{\mu \cdots \nu}\right]
$$

with

$$
\begin{aligned}
\nabla_{\alpha} X_{\rho \cdots \sigma}^{\mu \cdots \nu}=\partial_{\alpha} X^{\mu \cdots \nu}{ }_{\rho \cdots \sigma} & +\Gamma_{\lambda \alpha}^{\mu} X_{\rho{ }^{\lambda \cdots \sigma}}^{\lambda \cdots \nu}+\ldots+\Gamma_{\lambda \alpha}^{\nu} X^{\mu \cdots \lambda}{ }_{\rho \cdots \sigma} \\
& -\Gamma_{\rho \alpha}^{\lambda} X_{\lambda{ }^{\mu \cdots \sigma}}^{\mu \cdots \nu}-\ldots-\Gamma_{\sigma \alpha}^{\lambda} X_{\rho \cdots \lambda}^{\mu \cdots \nu} .
\end{aligned}
$$

Here [, ] represents the appropriate adjoint action of the flavour algebra. The equations (2.6), which we term as the hydrodynamic Ward identities, together with $\nabla_{\mu} J_{S}^{\mu} \geq 0$ capturing the essence of the second law, complete the specification of the hydrodynamic effective field theory in the current algebra language.

The task of a hydrodynamicist is to provide these constitutive relations, order by order in gradients of the fields $\boldsymbol{\Psi}$, subject to symmetry and second law requirements, cf., [1] for the classic treatment. We will refer the reader to the vast literature on hydrodynamic constitutive relations which have been computed (in certain cases up to the second order in the gradient expansion); see $[52,57]$ for a partial summary of certain results in the past few years. ${ }^{16}$

While most analyses of the second law constraints are done by classifying first on-shell independent data, as explained in section 1 it is useful to work off-shell. To this end we want to extend the statement of the second law, viz.,

$$
\exists J_{S}^{\mu}[\Psi]: \quad \nabla_{\mu} J_{S}^{\mu} \geq 0,
$$

to a more amenable one which is agnostic of dynamics. The simplest way to proceed is to use the fact that linear combinations of the equations of motion can be added to (2.10) without affecting the inequality [51]. All we need is appropriate Lagrange multipliers to ensure that the vectorial energy conservation and the scalar charge conservation equations can be combined with the gradient of the entropy current. The canonical choice is simply to take the Lagrange multipliers to be the hydrodynamic fields $\mathcal{B}=\left\{\boldsymbol{\beta}^{\mu}, \Lambda_{\boldsymbol{\beta}}\right\}$ themselves. One way to motivate this choice is to exploit the field redefinition freedom inherent in fluid dynamics, to align the Lagrange multiplier fields to the velocity (rescaled by the temperature) and chemical potential.

This then leads us to the following statement of the second law of thermodynamics:

$$
\begin{aligned}
\nabla_{\mu} J_{S}^{\mu}+\boldsymbol{\beta}_{\mu}\left(\nabla_{\nu} T^{\mu \nu}-J_{\nu} \cdot F^{\mu \nu}-\mathrm{T}_{H}^{\mu \perp}\right) \\
+\left(\Lambda_{\boldsymbol{\beta}}+\boldsymbol{\beta}^{\lambda} A_{\lambda}\right) \cdot\left(D_{\nu} J^{\nu}-\mathrm{J}_{H}^{\perp}\right)=\Delta \geq 0 .
\end{aligned}
$$

We have introduced $\Delta$ as the placeholder for the entropy production resulting from the dissipative constitutive relations.

\footnotetext{
${ }^{16}$ These computations are typically done by fixing a fluid frame (e.g., in the Landau frame one demands that the non-ideal parts of $T^{\mu \nu}$ and $J^{\mu}$ are transverse to velocity). We will a-priori make no such assumptions though at various stages of our analysis we will present results by making certain frame choices.
} 
Often when confronted with solving constraints given as inequalities, it is simplest to examine the boundary of the acceptable domain. In the present case this amounts to switching off dissipation by setting $\Delta=0$. The part of the constitutive relation which does not contribute to $\Delta$ will be termed adiabatic.

This canonical split allows us to motivate the adiabaticity equation. By definition it captures the marginal situation where dissipation is turned off, i.e., $\Delta=0$ :

$$
\begin{aligned}
\nabla_{\mu} J_{S}^{\mu}+\boldsymbol{\beta}_{\mu}\left(\nabla_{\nu} T^{\mu \nu}-J_{\nu} \cdot F^{\mu \nu}-\mathrm{T}_{H}^{\mu \perp}\right) \\
+\left(\Lambda_{\boldsymbol{\beta}}+\boldsymbol{\beta}^{\lambda} A_{\lambda}\right) \cdot\left(D_{\nu} J^{\nu}-\mathrm{J}_{H}^{\perp}\right)=0 .
\end{aligned}
$$

The constitutive relations which satisfy the adiabaticity equation are called adiabatic constitutive relations. ${ }^{17}$ Note that this relation is being imposed off-shell on the hydrodynamical system of interest, a fact that will be of crucial import in our discussion. For most of this paper we will be concerned with the adiabatic case. However, we will, at some early stage of the discussion (cf., section 5), describe the dissipative part of hydrodynamics building on the results of $[36,37]$ using the lessons learned from our adiabatic analysis.

It is worthwhile recording here a version of the adiabaticity equation that holds when we consider non-anomalous fluids. Since the quantum anomaly manifests itself through the Hall current terms $T_{H}^{\mu \perp}$ and $J_{H}^{\perp}$ setting them to zero allows us to capture the desired equation for non-anomalous adiabatic fluids, viz.,

$$
\nabla_{\mu} J_{S}^{\mu}+\boldsymbol{\beta}_{\mu}\left(\nabla_{\nu} T^{\mu \nu}-J_{\nu} \cdot F^{\mu \nu}\right)+\left(\Lambda_{\boldsymbol{\beta}}+\boldsymbol{\beta}^{\lambda} A_{\lambda}\right) \cdot D_{\nu} J^{\nu}=0
$$

In the initial part of our discussion we will find it convenient to work with the nonanomalous case first, and then build up to include the presence of anomalies. There is in fact a useful perspective that helps to segregate the anomalous contribution from the rest. Apart from anomalies appearing via the Hall currents, the adiabaticity equation is linear in the constitutive relations and relates terms of the same derivative order in the constitutive relations. This means that we can treat anomalous terms in (2.12) as "inhomogeneous source terms". Thus they can be removed by picking a suitable particular solution of adiabaticity equation. As a result we will for the most part assume that such anomalous terms have been appropriately dealt with and focus on the non-anomalous adiabaticity equation by setting them to zero, i.e., work with the homogeneous equation (2.13). In section 12 we will describe how the particular solutions to incorporate anomalous effects can be obtained.

It is important to appreciate the following fact: when we refer here and in the sequel to finding solutions to (2.12) we mean that we would like to find a set of hydrodynamic currents $\mathcal{C}_{\mathcal{H}}[\boldsymbol{\Psi}]$ which satisfy this equation off-shell. Thus we would like to determine families of constitutive relations parameterized by the transport coefficients that are adiabatic. As in any structural analysis of hydrodynamic transport we will not be interested in fixing values of transport coefficients. That can only be accomplished once we have an understanding

\footnotetext{
${ }^{17}$ We provide a translation of the adiabaticity equation in terms of the consistent currents which are sometimes more natural when working with effective actions in appendix A.
} 
of the microscopic quantum system whose hydrodynamic description we seek. ${ }^{18}$ With this understanding we will continue to speak of solving the adiabaticity equation, hopefully without causing any confusion.

\subsection{Physical interpretation of adiabatic fluids}

Let us physically understand the nature of the fluid systems that satisfy (2.12), by qualifying the adjective 'adiabatic'. Suppose we restrict ourselves to fluid configurations $\left\{\boldsymbol{\beta}^{\mu}, \Lambda_{\boldsymbol{\beta}}\right\}$ which satisfy the hydrodynamic equations of motion (2.6). Let us re-characterize them for the present discussion as

$$
\begin{aligned}
\nabla_{\nu} T^{\mu \nu} & \simeq J_{\nu} \cdot F^{\mu \nu}+\mathrm{T}_{H}^{\mu \perp} \\
D_{\nu} J^{\nu} & \simeq \mathrm{J}_{H}^{\perp}
\end{aligned}
$$

with the symbol $\simeq$ referring to the fact that these equations hold only in this restricted sense (i.e., on-shell). We can then assign a conserved entropy current to this restricted set of fluid configurations, i.e., $\nabla_{\mu} J_{S}^{\mu} \simeq 0$. Thus, the constitutive relations which solve adiabaticity equation describe entropy-conserving (i.e., adiabatic) transport once hydrodynamic equations are imposed. In this sense the adiabatic fluids are on-shell equivalent to the non-dissipative fluids as defined in [46]. One way to interpret the adiabaticity equation is to take the view that we have taken entropy conservation off-shell using the hydrodynamic fields as Lagrange multipliers, along the lines espoused in [51].

Alternately, the adiabaticity equation is actually a stronger assertion than just entropy conservation. Say, instead of taking hydrodynamics on-shell via (2.14), we impose

$$
\begin{aligned}
\nabla_{\nu} T^{\mu \nu} & \simeq J_{\nu} \cdot F^{\mu \nu}+\mathrm{T}_{H}^{\mu \perp}+f_{e x t}^{\mu} \\
D_{\nu} J^{\nu} & \simeq \mathrm{J}_{H}^{\perp}+Q_{e x t}
\end{aligned}
$$

where $f_{e x t}^{\mu}$ is the force per unit volume due to an external system and $Q_{e x t}$ is the charge injected per unit time per unit volume by the external system. Let us assume that this injection of energy-momentum and charge happens adiabatically and the entropy injected into the fluid is $\nabla_{\mu} J_{S}^{\mu} \simeq S_{\text {ext }}$. The adiabaticity equation is the statement that all these cannot be together true for arbitrary $\left\{f_{\text {ext }}^{\mu}, Q_{\text {ext }}, S_{\text {ext }}\right\}$. In fact this transfer can be adiabatic if and only if $T S_{\text {ext }}+u_{\nu} f_{\text {ext }}^{\nu}+\mu \cdot Q_{e x t} \simeq 0$, i.e., if and only if external system satisfies adiabaticity equation. Thus, any two systems which satisfy adiabaticity equation can be combined to a bigger system which satisfies adiabaticity equation, in a way reminiscent of the classical discussions on thermodynamics by Carnot and others.

Thus the adiabaticity hypothesis brings in a sense of linearity into hydrodynamics, much like the superposition principle of quantum mechanics. This allows us to focus the discussion on isolated systems, with the potential downside that we do not have access to the dissipative part of hydrodynamics.

\footnotetext{
${ }^{18}$ In the interest of full disclosure, we should add that certain constitutive relations which are forbidden by demanding existence of hydrostatic equilibrium can be viewed as fixing certain transport coefficients to being functions of others (which are the only physical ones).
} 
The main motivation for considering adiabatic hydrodynamics is the observation that non-dissipative parts of many actual hydrodynamic theories coincide with what one finds in adiabatic hydrodynamics. We note that not all solutions of adiabaticity equation might arise in a given microscopic QFT. For example one might want to impose additional constraints (like Euclidean consistency $[11,13]$ ) and identify on-shell equivalent or fluid frameequivalent expressions to eliminate potentially unphysical solutions. Thus, we generally expect the solutions of adiabaticity equation to furnish a super-set of physically admissible non-dissipative constitutive relations up to field redefinitions.

\subsection{Ideal fluids are adiabatic}

Having presented the basic equation of interest, we now turn to asking how one might characterize the solutions to the adiabaticity equation. After all we are interested in using these as the first step in understanding more realistic fluid systems (including dissipation). To this end we need to show that we have a non-empty solution set to (2.12).

It is natural to study the non-anomalous adiabatic constitutive relations order by order in derivative expansion. Let us illustrate how this works at zeroth order in derivative expansion. The most general constitutive relation with zero derivatives of the hydrodynamic data is ${ }^{19}$

$$
J_{S}^{\mu}=s u^{\mu}, \quad T^{\mu \nu}=\epsilon u^{\mu} u^{\nu}+p P^{\mu \nu}, \quad J^{\mu}=q u^{\mu} .
$$

where the entropy density $s$, energy density $\epsilon$, pressure $p$ and charge density $q$ are scalar functions of $T$ and $\mu$. The tensor $P_{\mu \nu}=g_{\mu \nu}+u_{\mu} u_{\nu}$ is the projector transverse to the velocity.

The adiabaticity condition (2.13) can then be written quite simply as

$$
u^{\alpha}\left(T \nabla_{\alpha} s+\mu \cdot \nabla_{\alpha} q-\nabla_{\alpha} \epsilon\right)+(T s+\mu \cdot q-\epsilon-p) \Theta=0,
$$

where $\Theta \equiv \nabla_{\mu} u^{\mu}$ is the fluid expansion. If we insist that this hold for an arbitrary fluid configuration, then the combination in each of the parentheses should individually vanish. This then implies that the fluid should satisfy the first law

$$
\delta \epsilon=T \delta s+\mu \cdot \delta q,
$$

and the Euler relation

$$
\epsilon+p=T s+\mu \cdot q
$$

Thus, we recover standard constitutive relations describing thermodynamics from the formalism of adiabatic hydrodynamics.

We will soon see that the family of adiabatic fluids is far richer as evidenced by our eightfold classification illustrated in figure 1 . We will shortly provide a short synopsis of the different classes in section 3. The reader impatient to see some more examples is invited to consult section 8 where we study neutral fluids and parity-odd charged fluids at higher orders.

\footnotetext{
${ }^{19}$ We have reverted to $\left\{u^{\mu}, T, \mu\right\}$ so as to write the constitutive relations in their familiar form.
} 


\subsection{The adiabatic free energy current}

We have phrased our discussion of the adiabaticity equation in terms of the entropy current. However, since we are describing via the hydrodynamic expansion the fluctuations in the Gibbsian density matrix, it makes more sense to ask about the behaviour of the free energy current itself. This involves using the standard definition of the grand canonical free energy current. In terms of the other hydrodynamic currents introduced hitherto: ${ }^{20}$

$$
\begin{aligned}
\mathcal{G}^{\sigma} & =-T \mathrm{~N}^{\sigma}, \\
& \equiv-T\left[J_{S}^{\sigma}+\boldsymbol{\beta}_{\nu} T^{\nu \sigma}+\left(\Lambda_{\boldsymbol{\beta}}+\boldsymbol{\beta}^{\nu} A_{\nu}\right) \cdot J^{\sigma}\right] .
\end{aligned}
$$

Assuming we know the free energy current we can solve for the entropy current by inverting the above relation

$$
\begin{aligned}
J_{S}^{\sigma} & =-\left[\boldsymbol{\beta}_{\nu} T^{\nu \sigma}+\left(\Lambda_{\boldsymbol{\beta}}+\boldsymbol{\beta}^{\nu} A_{\nu}\right) \cdot J^{\sigma}+\frac{\mathcal{G}^{\sigma}}{T}\right] \\
& \equiv\left(J_{S}^{\sigma}\right)_{\mathrm{can}}-\frac{\mathcal{G}^{\sigma}}{T}
\end{aligned}
$$

This expression is useful in that it segregates the various contributions to the entropy current. The terms $-\left[\boldsymbol{\beta}_{\nu} T^{\nu \sigma}+\left(\Lambda_{\boldsymbol{\beta}}+\boldsymbol{\beta}^{\nu} A_{\nu}\right) \cdot J^{\sigma}\right]$ are usually interpreted as the canonical part of the entropy current $\left(J_{S}^{\mu}\right)_{\text {can }}$. On the other hand the vector $-\mathcal{G}^{\sigma} / T$ is called the non-canonical part of the entropy current. Thus, passing to grand canonical ensemble can be thought of as focusing our attention on the part of entropy flow which is not simply related to energy and charge flow. We can think of free energy (up to a factor of $T$ ) as just the name given to this part of entropy.

While in the present discussion the grand canonical free energy current appears as a convenient book keeping device for the non-canonical part of the entropy current, it will soon transpire when we consider hydrostatics that it has a natural interpretation in terms of a partition function.

The notion of the free energy current is quite useful in the context of anomalous hydrodynamics. While the presence of a quantum anomaly does not necessarily introduce entropy into the fluid, ${ }^{21}$ the charge and energy-momentum injection is inevitably accompanied by a free energy injection. The free energy per unit time per unit volume injected by anomalies is

$$
\begin{aligned}
\mathcal{G}_{H}^{\perp} & \equiv-T\left[\boldsymbol{\beta}_{\nu} \mathrm{T}_{H}^{\nu \perp}+\left(\Lambda_{\boldsymbol{\beta}}+\boldsymbol{\beta}^{\nu} A_{\nu}\right) \cdot \mathrm{J}_{H}^{\perp}\right] \\
& =-\left[u_{\nu} \mathrm{T}_{H}^{\nu \perp}+\mu \cdot \mathrm{J}_{H}^{\perp}\right] .
\end{aligned}
$$

\footnotetext{
${ }^{20}$ While the physical free energy current is $\mathcal{G}^{\sigma}$, it is often convenient to write expression for $\mathrm{N}^{\sigma}$ (which is the free energy rescaled by $-T^{-1}$ ). This quantity naturally appears as a Noether charge in our effective action constructions. As a result we will use both quantities interchangeably for much of our discussion.

${ }^{21}$ The anomalous contribution to the entropy current can typically be chosen to vanish for flavour anomalies. The story for Lorentz anomalies is a bit more involved and is discussed in section 12 .
} 
Using this definition, we can now write the grand canonical version of the adiabaticity equation (2.12) as (we include $\Delta$ for completeness)

$$
\begin{aligned}
-\left[\nabla_{\sigma}\left(\frac{\mathcal{G}^{\sigma}}{T}\right)-\frac{\mathcal{G}_{H}^{\perp}}{T}\right] & =\frac{1}{2} T^{\mu \nu} \delta_{\mathfrak{B}} g_{\mu \nu}+J^{\mu} \cdot \delta_{\mathfrak{B}} A_{\mu}+\Delta \\
& =T^{\mu \nu} \nabla_{\mu}\left(\frac{u_{\nu}}{T}\right)+J^{\sigma} \cdot\left[D_{\sigma}\left(\frac{\mu}{T}\right)-\frac{E_{\sigma}}{T}\right]+\Delta .
\end{aligned}
$$

Here $E^{\mu}=F^{\mu \nu} u_{\nu}$ is the electric field and $\delta_{\mathcal{B}}$ represents the Lie derivatives using the diffeomorphism and flavour transformations generated by $\left\{\boldsymbol{\beta}^{\mu}, \Lambda_{\boldsymbol{\beta}}\right\}$ :

$$
\begin{aligned}
\delta_{\mathfrak{B}} g_{\mu \nu} & \equiv £_{\boldsymbol{\beta}} g_{\mu \nu}=\nabla_{\mu} \boldsymbol{\beta}_{\nu}+\nabla_{\nu} \boldsymbol{\beta}_{\mu}, \\
\delta_{\mathfrak{B}} A_{\mu} & \equiv £_{\boldsymbol{\beta}} A_{\mu}+\partial_{\mu} \Lambda_{\boldsymbol{\beta}}+\left[A_{\mu}, \Lambda_{\boldsymbol{\beta}}\right]=D_{\mu}\left(\Lambda_{\boldsymbol{\beta}}+\boldsymbol{\beta}^{\nu} A_{\nu}\right)+\boldsymbol{\beta}^{\nu} F_{\nu \mu} .
\end{aligned}
$$

In this expression, we used $£_{\boldsymbol{\beta}}$ to denotes the Lie derivative along the vector field $\boldsymbol{\beta}^{\mu}$.

It is useful to record the expression for the Lie derivative in terms of the more familiar hydrodynamic decomposition. A quick evaluation leads to

$$
\begin{aligned}
& \delta_{\mathcal{B}} g_{\mu \nu}=2 \nabla_{(\mu} \boldsymbol{\beta}_{\nu)}=\frac{2}{T}\left[\sigma_{\mu \nu}+P_{\mu \nu} \frac{\Theta}{d-1}-\left(\mathfrak{a}_{(\mu}+\nabla_{(\mu} \log T\right) u_{\nu)}\right] \\
& \delta_{\mathcal{B}} A_{\mu}=D_{\mu}\left(\Lambda_{\boldsymbol{\beta}}+\boldsymbol{\beta}^{\nu} A_{\nu}\right)+\boldsymbol{\beta}^{\nu} F_{\nu \mu}=u^{\alpha} D_{\alpha}\left(\frac{\mu}{T}\right) u_{\mu}-\frac{1}{T} \mathfrak{v}_{\mu} .
\end{aligned}
$$

We use the standard decomposition of the gradient of the velocity field into the transverse traceless shear-strain rate $\sigma_{\mu \nu}$, the anti-symmetric vorticity $\omega_{\mu \nu}$, the vectorial acceleration $\mathfrak{a}_{\nu}$ and scalar expansion $\Theta$ respectively, viz.,

$$
\nabla_{\mu} u_{\nu}=\sigma_{(\mu \nu)}+\omega_{[\mu \nu]}-u_{\mu} \mathfrak{a}_{\nu}+P_{\mu \nu} \frac{\Theta}{d-1}
$$

and the flavour fields decompose as

$$
\mathfrak{v}^{\mu}=E^{\mu}-T P^{\mu \nu} \nabla_{\nu}\left(\frac{\mu}{T}\right), \quad E^{\mu}=F^{\mu \nu} u_{\nu} .
$$

An alternate form of (2.21) can be given by using the fluid acceleration $\mathfrak{a}^{\alpha} \equiv u^{\mu} \nabla_{\mu} u^{\alpha}$ to eliminate the thermal gradients:

$$
\begin{aligned}
-\left[\left(\nabla_{\sigma}+\mathfrak{a}_{\sigma}\right) \mathcal{G}^{\sigma}-\mathcal{G}_{H}^{\perp}\right]=J_{S}^{\sigma}\left(\nabla_{\sigma}+\mathfrak{a}_{\sigma}\right) T & \\
& +T^{\mu \nu}\left(\nabla_{\nu}+\mathfrak{a}_{\nu}\right) u_{\mu}+J^{\sigma} \cdot\left[D_{\sigma} \mu+\mathfrak{a}_{\sigma} \mu-E_{\sigma}\right]+T \Delta .
\end{aligned}
$$

This form of the equation is quite useful in making comparisons with traditional hydrodynamic analysis. ${ }^{22}$

\section{Classification of adiabatic transport}

We now provide a telegraphic summary of the different solutions (i.e., constitutive relations) to the adiabaticity equation (2.12) we will encounter in the course of our discussion.

\footnotetext{
${ }^{22}$ Recall that in the current algebra approach one typically chooses to eliminate thermal gradients in favour of velocity derivatives. Some useful formulae and commonly used notation are collected in the tables of appendix J.
} 
- Class H (hydrostatic constitutive relations) section 4: consider placing a fluid on a stationary background wherein there exists a timelike Killing vector and a Killing gauge transformation, $\left\{K^{\mu}, \Lambda_{K}\right\}$, that leave the background sources $g_{\mu \nu}$ and $A_{\mu}$ invariant. In section 4 we explain our reasoning for taking it as an axiom that the obvious hydrostatic fluid configuration given by $\left\{\boldsymbol{\beta}^{\mu}, \Lambda_{\boldsymbol{\beta}}\right\}=\left\{K^{\mu}, \Lambda_{K}\right\}$ immediately gives solutions to the adiabaticity equation. We dub this premise as the hydrostatic principle. This gives us the hydrostatic (i.e., time-independent) configurations of a generic fluid dynamical system. These configurations lead to hydrostatic constitutive relations which capture the thermodynamic response parameters that are encoded in terms of an equilibrium partition function.

We emphasize here that we want to classify adiabatic constitutive relations, i.e., currents $\mathcal{C}_{\mathcal{H}}[\boldsymbol{\Psi}]$ solving (2.12). In some cases the solutions can be efficiently encoded in a generating function that may be parametrized in terms of other tensor structures. The physical currents should thus be treated as functionals of these auxiliary (solution generating) tensor structures. With this clarification we record here that the hydrostatic class can be further sub-divided into two:

- Class $\mathrm{H}_{S}$ : hydrostatic partition functions transforming as thermal scalars, ${ }^{23}$ which comprise the class of non-anomalous equilibrium generating functions discussed in $[3,4]$.

- Class $\mathrm{H}_{V}$ : hydrostatic partition functions transforming as thermal vectors such as those relevant for understanding transcendental anomaly induced transport which generalizes the parity-odd part of Cardy formula to higher dimensions [13]. Further details are provided in section 11.

- Class $\mathbf{H}_{\boldsymbol{F}}$ (hydrostatic forbidden) section 4: at a given order in derivatives, Classes $\mathrm{H}_{S}$ and $\mathrm{H}_{V}$ are exhausted after classifying all independent scalars and vectors that survive the hydrostatic limit. Together they parameterize the (Euclidean) equilibrium partition function. However, this counting generically does not match the classification of all possible tensor structures in the equilibrium constitutive relations at this order. The mismatch is due to a number of hydrostatic relations $\mathrm{H}_{F}$ which arise as constraints from the existence of an equilibrium configuration.

As the simplest example, consider a neutral fluid at zeroth order in derivative expansion. A-priori there are two possible tensor structures in the stress tensor constitutive relations: $u^{\mu} u^{\nu}$ and $P^{\mu \nu}$. The pressure which multiplies $P^{\mu \nu}$ parameterizes hydrostatic partition function. Moreover, the existence of hydrostatic equilibrium at this level is equivalent to the Euler relation, $\epsilon+p=T s$, which enforces that the coefficient of $u^{\mu} u^{\nu}$ (energy density) is not independent, but rather is determined in terms of pressure (this is the hydrostatic version of the analysis in section 2.3). This statement can be viewed as saying that there is one relation between two a-priori independent pieces of transport data, a feature that characterizes Class $\mathrm{H}_{F}$ more generally.

\footnotetext{
${ }^{23}$ The transformation property here refers to behaviour under Kaluza-Klein gauge transformations where we treat the Euclidean thermal circle relevant in hydrostatic as the compact direction we reduce on.
} 
- Class L (Lagrangian solutions) section 6, section 7: if we are on-shell the content of (2.12) with $\Delta=0$ is simply that of entropy conservation, but we have now allowed ourselves to take this off-shell. By doing so we have gained the distinct advantage of being able to ask the following question: "What are the Lagrangian theories that respect the adiabaticity equation?" This turns out to be surprisingly easy to answer. Consider any local diffeomorphic and gauge invariant Lagrangian density $\mathcal{L}\left[g_{\mu \nu}, A_{\mu}, \boldsymbol{\beta}^{\mu}, \Lambda_{\boldsymbol{\beta}}\right]$ viewed as a functional of the background metric $g^{\mu \nu}$, background gauge potential $A_{\mu}$ and the hydrodynamic variables $\left\{\boldsymbol{\beta}^{\mu}, \Lambda_{\boldsymbol{\beta}}\right\}$. $\boldsymbol{\beta}^{\mu}$ is a vector field under diffeomorphisms and $\Lambda_{\boldsymbol{\beta}}$ transforms as appropriate for a gauge parameter. We then have a set of Bianchi identities arising from these background symmetries. These identities then simply imply the adiabaticity equation with the entropy current $J_{S}^{\mu}=s u^{\mu}$ where $s$ is taken to be the Euler-Lagrange derivative of the Lagrangian with respect to the temperature, keeping fixed the chemical potentials and the background sources. In other words the adiabaticity equation follows quite trivially in a wide class of Lagrangian hydrodynamic theories. ${ }^{24}$

Given a Lagrangian theory of hydrodynamics, we not only want to show that the adiabaticity equation is satisfied, but also obtain the correct dynamical equations, which as we have emphasized, are simply conservation equations. Unfortunately, the simple unconstrained variational principle with respect to hydrodynamic variables $\left\{\boldsymbol{\beta}^{\mu}, \Lambda_{\boldsymbol{\beta}}\right\}$ does not result in the desired energy-momentum and charge conservation Ward identities. So the non-trivial part of the construction involves demonstrating the existence of a novel constrained variational principle that obeys the desired dynamics. In fact, such a principle is easy to state: fixing $\left\{g_{\mu \nu}, A_{\mu}\right\}$, and extremizing $\int_{\mathcal{M}} \sqrt{-g} \mathcal{L}[\Psi]$ under constrained variations that stay on Lie orbits of $\left\{\boldsymbol{\beta}^{\mu}, \Lambda_{\boldsymbol{\beta}}\right\}$ gives on-shell hydrodynamic configurations.

There is an equivalent but more convenient way of parameterizing such variations. Start by fixing a reference hydrodynamic configuration $\left\{\beta^{a}, \Lambda_{\beta}\right\}$ on a (fiducial) reference manifold $\mathbb{M}$ which is diffeomorphic to the physical spacetime $\mathcal{M}$. Then consider a diffeomorphism field $\varphi^{a}(x)$ and a gauge transformation $c(x)$ which relate $\mathcal{M}$ and $\mathbb{M}$. Said differently, the true physical configuration $\left\{\boldsymbol{\beta}^{\mu}, \Lambda_{\boldsymbol{\beta}}\right\}$ on $\mathcal{M}$ is given by the pullback of the reference configuration $\left\{\beta^{a}, \Lambda_{\beta}\right\}$ along $\varphi^{a}(x), c(x)$ :

$$
\begin{aligned}
\boldsymbol{\beta}^{\mu}(x) & =\frac{\partial x^{\mu}}{\partial \varphi^{a}} \beta^{a}[\varphi(x)] \\
\Lambda_{\boldsymbol{\beta}}(x) & =c(x) \Lambda_{\beta}[\varphi(x)] c^{-1}(x)+\boldsymbol{\beta}^{\sigma}(x) \partial_{\sigma} c(x) c^{-1}(x)
\end{aligned}
$$

The fields $\varphi^{a}(x)$ and $c(x)$ are just a convenient parametrization of a given Lie orbit of $\left\{\boldsymbol{\beta}^{\mu}, \Lambda_{\boldsymbol{\beta}}\right\}$. So instead of doing a constrained variation of $\left\{\boldsymbol{\beta}^{\mu}, \Lambda_{\boldsymbol{\beta}}\right\}$ along Lie orbits, we can equivalently do an unconstrained variation of the fields $\left\{\varphi^{a}(x), c(x)\right\}$ holding fixed the hydrodynamic reference configuration. We demonstrate that this prescription leads to the desired Ward identities.

In addition, as suggested by choice of notation for the fields, we have a wonderful bonus - we can derive and explain the symmetries of the non-dissipative effective action

\footnotetext{
${ }^{24}$ For the moment, we are ignoring situations with anomaly induced transport. We will later explain how to find Lagrangian solutions to anomalous hydrodynamics, which is indeed possible, albeit with some interesting technical complications.
} 
in a simple and effective manner, see appendix B. This analysis will be tantamount to saying that all transport described by the non-dissipative action formalism (Class ND) is contained within the Class L constitutive relations. Furthermore, we will demonstrate that Class $\mathrm{H}_{S} \subset$ Class $\mathrm{L}$ by showing that the equilibrium limit of the Lagrangians we construct, reproduces the (scalar part of) hydrostatic partition function. However, the scalar Lagrangian density also has hydrodynamic scalar densities $\left(\right.$ Class $\overline{\mathrm{H}}_{S}$ ) (which vanish in equilibrium); strictly speaking $\mathrm{L}=\mathrm{H}_{S} \cup \overline{\mathrm{H}}_{S}$ with the hydrostatic scalars forming the coset $\mathrm{H}_{S}=\mathrm{L} / \overline{\mathrm{H}}_{S}$.

The simplest non-trivial example of non-dissipative fluids is a neutral fluid at second order in gradient expansion. This system has been studied from an effective action perspective in [46]. We revisit this example in section 8 (see also appendix F) where we describe how we can exploit our new Lagrangian formalism to understand features of the transport. The neutral fluid Lagrangian is parameterized by five scalar functions which determine 15 transport coefficients. This implies 10 linear differential relation amongst transport coefficients. 5 of these are hydrostatic and were obtained earlier in [3] - they make up Class $\mathrm{H}_{F}$. The remaining 5 relations are adiabatic combinations of transport that do not lead to any dissipation. Curiously as reported in [54] all second order transport for strongly coupled Weyl invariant holographic fluids is obtained from Class L.

- Class B (Berry-like transport) section 9: these comprise of the class of solutions to the adiabaticity equation which are non-hydrostatic but explicitly non-dissipative. They turn out to satisfy the adiabaticity equation trivially and as a result such transport coefficients are completely unconstrained in the current algebra approach. The nomenclature owes its origin to the fact that these can be viewed as Berry curvature induced transport. Heuristically these arise when we adiabatically traverse a closed loop in the fluid configuration space [58]. To wit, consider the following constitutive relations:

$$
\begin{aligned}
\left(T^{\mu \nu}\right)_{\mathrm{B}} & \equiv-\frac{1}{4}\left(\mathcal{N}^{(\mu \nu)(\alpha \beta)}-\mathcal{N}^{(\alpha \beta)(\mu \nu)}\right) \delta_{\mathcal{B}} g_{\alpha \beta}+\mathcal{X}^{(\mu \nu) \alpha} \cdot \delta_{\mathcal{B}} A_{\alpha} \\
\left(J^{\alpha}\right)_{\mathrm{B}} & \equiv-\frac{1}{2} \mathcal{X}^{(\mu \nu) \alpha} \delta_{\mathcal{B}} g_{\mu \nu}-\mathcal{S}^{[\alpha \beta]} \cdot \delta_{\mathcal{B}} A_{\beta} \\
\left(J_{S}^{\alpha}\right)_{\mathrm{B}} & \equiv-\frac{u_{\sigma}}{T}\left(T^{\alpha \sigma}\right)_{B}-\frac{\mu}{T} \cdot\left(J^{\alpha}\right)_{B}
\end{aligned}
$$

where $\left\{\mathcal{N}^{\mu \nu \alpha \beta}, \mathcal{X}^{\mu \nu \alpha}, \mathcal{S}^{\alpha \beta}\right\}$ are arbitrary tensors and $\delta_{\mathcal{B}}$ denotes the Lie derivative associated to the diffeomorphism and gauge transformation generated by $\left\{\boldsymbol{\beta}^{\mu}, \Lambda_{\boldsymbol{\beta}}\right\}$. A prime example for structures of the type (3.2) are the parity-odd shear tensor in 3 dimensions which contributes to Hall viscosity. There also turn out to be some novel combinations in parity-even neutral fluids; the (shear) $\times$ (vorticity) term which is present in conformal fluids is of this type (as is a combination seen in the analysis of [46]).

- Class C (conserved entropy) section 10: this is the simplest class of adiabatic transport, comprising of identically conserved entropy current, with vanishing physical currents. Since we want non-trivial entropy current, we classify such terms up to exact gradients, i.e., cohomologically. Wen-Zee currents [59] and their generalizations discussed by $[60,61]$ which we explore (and extend) in section $\mathrm{C}$ provide examples of such terms. 
One can view this as capturing the transport of topological states of the hydrodynamical system. These are finite since they arise as solutions to a cohomology problem.

- Class $\overline{\mathbf{H}}_{V}$ (Hydrodynamic vectors) section 11: just as Class $\mathrm{H}_{V}$ contains hydrostatic vectors that give rise to transverse free energies, Class $\overline{\mathrm{H}}_{V}$ does the same for hydrodynamic vectors which are vanishing in equilibrium. ${ }^{25}$ In particular, consider the following constitutive relations:

$$
\begin{aligned}
&\left(T^{\mu \nu}\right)_{\overline{\mathrm{H}}_{V}} \equiv \frac{1}{2}\left[D_{\lambda} \mathfrak{C}_{\mathcal{N}}^{\lambda(\mu \nu)(\alpha \beta)}\right.\left.\delta_{\mathcal{B}} g_{\alpha \beta}+2 \mathfrak{C}_{\mathcal{N}}^{\lambda(\mu \nu)(\alpha \beta)} D_{\lambda} \delta_{\mathcal{B}} g_{\alpha \beta}\right] \\
&+D_{\lambda} \mathfrak{C}_{\mathcal{X}}^{\lambda(\mu \nu) \alpha} \cdot \delta_{\mathcal{B}} A_{\alpha}+2 \mathfrak{C}_{\mathcal{X}}^{\lambda(\mu \nu) \alpha} \cdot D_{\lambda} \delta_{\mathcal{B}} A_{\alpha} \\
&\left(J^{\alpha}\right)_{\overline{\mathrm{H}}_{V}} \equiv \frac{1}{2}\left[D_{\lambda} \mathfrak{C}_{\mathcal{X}}^{\lambda(\mu \nu) \alpha} \delta_{\mathfrak{B}} g_{\mu \nu}+2 \mathfrak{C}_{\mathcal{X}}^{\lambda(\mu \nu) \alpha} D_{\lambda} \delta_{\mathcal{B}} g_{\mu \nu}\right] \\
&+D_{\lambda} \mathfrak{C}_{\mathcal{S}}^{\lambda(\alpha \beta)} \cdot \delta_{\mathcal{B}} A_{\beta}+2 \mathfrak{C}_{\mathcal{S}}^{\lambda(\alpha \beta)} \cdot D_{\lambda} \delta_{\mathcal{B}} A_{\beta}
\end{aligned}
$$

where $\mathfrak{C}_{\mathcal{N}}^{\lambda(\mu \nu)(\alpha \beta)}=\mathfrak{C}_{\mathcal{N}}^{\lambda(\alpha \beta)(\mu \nu)}$ and $\left\{\mathfrak{C}_{\mathcal{N}}^{\lambda \mu \nu \alpha \beta}, \mathfrak{C}_{\mathcal{X}}^{\lambda \mu \nu \alpha}, \mathfrak{C}_{\mathcal{S}}^{\lambda \alpha \beta}\right\}$ are transverse in their first index and are otherwise arbitrary local functionals of $\boldsymbol{\Psi}$. This trivially solves the adiabaticity equation with a particular choice of transverse free energy current. The simplest examples of such transport can be found at second order charged fluid dynamics. The constitutive relations are messy, cf., (11.22) and (11.24), but the free energy current is simpler and given by the transverse vectors $\sigma^{\mu \nu} \mathfrak{v}_{\nu}$ and $\Theta \mathfrak{v}^{\mu}$.

- Class A (anomalous transport terms) section 12, section 13: anomalous transport is hydrostatic [9-13, 23]. It is however instructive to extract anomalous transport from an off-shell, off-equilibrium approach, as it provides non-trivial checks on our formalism. By generalizing the arguments of [30] for flavour anomalies we construct an off-shell effective action for mixed flavour and gravitational anomalies in Class $\mathrm{H}_{S}$. This procedure however does not capture the $\mathrm{H}_{V}$ terms that are necessary for ensuring Euclidean consistency (the transcendental terms of [11]).

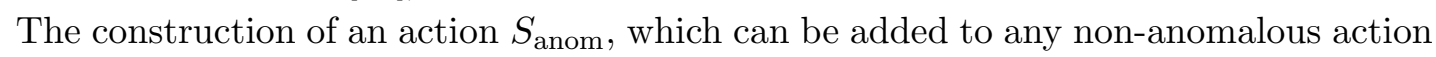
from Class L to account for the presence of mixed anomalies, proceeds in two steps. Firstly, we need to use the anomaly inflow mechanism which extends the physical spacetime $\mathcal{M}$ to a $(d+1)$-dimensional bulk spacetime $\mathcal{M}_{d+1}$ with $\partial \mathcal{M}_{d+1}=\mathcal{M}$. The gauge and gravitational connections of the latter are such that their boundary inflow compensates for the gauge noninvariance of the physical theory on $\mathcal{M}$. In section 12 we consider the following bulk action:

$$
S_{\mathrm{anom}}=\int_{\mathcal{M}_{d+1}} \boldsymbol{V}_{\mathcal{P}}[\boldsymbol{A}, \boldsymbol{\Gamma}, \hat{\boldsymbol{A}}, \hat{\boldsymbol{\Gamma}}]
$$

where $\boldsymbol{V}_{\mathcal{P}}$ is the transgression form that interpolates between the gauge and gravitational connections $\boldsymbol{A}, \boldsymbol{\Gamma}^{\mu}{ }_{\nu}$ and their "shadows" $\hat{\boldsymbol{A}}=\boldsymbol{A}+\mu \boldsymbol{u}$ and $\hat{\boldsymbol{\Gamma}}^{\mu}{ }_{\nu}=\boldsymbol{\Gamma}^{\mu}{ }_{\nu}+\Omega^{\mu}{ }_{\nu} \boldsymbol{u}$. Here $\Omega^{\mu}{ }_{\nu}$ is the out-of-equilibrium spin chemical potential extending the definition of [12]. The transgression form $\boldsymbol{V}_{\mathcal{P}}$ is itself given in terms of the anomaly polynomial.

\footnotetext{
${ }^{25}$ Given that these constitutive relations are primarily determined by the free energy current, it might be more natural to call this the Gibbsian vector class.
} 
As expected, the stress tensor and charge current derived from $S_{\text {anom }}$ are nonequilibrium generalizations of the anomalous currents found in $[8,12]$. In the presence of gravitational anomalies the entropy current is modified by an anomalous contribution (which vanishes in equilibrium) from its canonical form $J_{S}^{\mu}=s u^{\mu}{ }^{26}$

Secondly, an anomalous effective action should also lead to the correct hydrodynamic Ward identities; this however fails if we just consider (3.4). As discussed by us in the context of flavour anomalies [30], getting the Ward identities to work requires a SchwingerKeldysh doubled theory. We describe the general framework of such doubled constructions in section 13 and show how to obtain the correct anomalous constitutive relations and Ward identities using two copies ( $\mathrm{R}$ and $\mathrm{L}$ ) of the transgression form (3.4) together with a third transgression form that acts as an influence functional connecting the two copies,

$$
S_{\text {tot,anom }}=\int_{\mathbb{M}_{d+1}}\left(\boldsymbol{V}_{\mathcal{P}}\left[\mathbb{A}_{R}, \mathbb{T}_{R}, \hat{\mathbb{A}}_{R}, \hat{\mathbb{T}}_{R}\right]-\boldsymbol{V}_{\mathcal{P}}\left[\mathbb{A}_{\mathrm{L}}, \mathbb{T}_{\mathrm{L}}, \hat{\mathbb{A}}_{\mathrm{L}}, \hat{\mathbb{T}}_{\mathrm{L}}\right]+\boldsymbol{V}_{\mathcal{P}}\left[\hat{\mathbb{A}}_{\mathrm{R}}, \hat{\mathbb{T}}_{\mathrm{R}} ; \hat{\mathbb{A}}_{\mathrm{L}}, \hat{\mathbb{T}}_{\mathrm{L}}\right]\right)
$$

A rather pleasant consequence of viewing hydrodynamic fields $\left\{\varphi^{a}, c\right\}$ as maps from a fiducial reference spacetime to a physical configuration is that one has a clear picture of the doubled gauge symmetries (especially diffeomorphisms) encountered in the SchwingerKeldysh constructions. While there are indeed two sets of gravitational sources, they are both obtained by pushing-forward data from a single reference manifold $\mathbb{M}_{d+1}$.

* The eightfold Lagrangian (Class $\mathrm{L}_{\mathrm{T}}$ ) section 15: the classification of adiabatic constitutive relations described above can be motivated on physical grounds. Furthermore, one can show that the 7 classes above (Classes $\mathrm{H}_{S}, \mathrm{H}_{V}, \overline{\mathrm{H}}_{S}, \overline{\mathrm{H}}_{V}, \mathrm{~B}, \mathrm{C}, \mathrm{A}$ ) exhaust all adiabatic transport. One can in fact obtain all of these solutions from a single master action. This effective action is parameterized by the hydrodynamic fields $\left\{\boldsymbol{\beta}^{\mu}, \Lambda_{\boldsymbol{\beta}}\right\}$, the background sources $\left\{g_{\mu \nu}, A_{\mu}\right\}$, their Schwinger-Keldysh like doubles $\left\{\tilde{g}_{\mu \nu}, \tilde{A}_{\mu}\right\}$, and a new Abelian gauge field $A^{(T)}{ }_{\mu}$. While the doubling of the sources, whilst perhaps unanticipated, could be reconciled with the fact that non-equilibrium dynamics is incomplete without such a construction, the new gauge principle hints at some hidden underlying structure. We describe why this is necessary and point out some of its physical implications below. For now we shall simply record that a set of hydrodynamic currents $\mathcal{C}_{\mathcal{H}}[\boldsymbol{\Psi}]$ satisfying the adiabaticity equation can be derived rather straightforwardly from the effective action

$$
S_{\mathrm{T}}=\int d^{d} x \sqrt{-g}\left(\frac{1}{2} T^{\mu \nu} \tilde{g}_{\mu \nu}+J^{\mu} \tilde{A}_{\mu}-\frac{1}{T} \mathcal{G}^{\mu} A^{(\mathrm{T})} \mu\right)
$$

Before proceeding to a detailed discussion of the specific classes of solutions to the adiabaticity equation, let us remark on how this construction is related to the earlier work on non-dissipative fluids and how the knowledge of adiabatic transport enables us to complete the classification of hydrodynamic transport.

\footnotetext{
${ }^{26}$ The modification of the entropy current was one of the main reasons for us to upgrade from the nondissipative effective action formalism used to analyze flavour anomalies in [20, 30].
} 
o Class ND (non-dissipative fluids) (appendix B): this class encompasses nondissipative fluids as described in the formalism of [45] with the fundamental degrees of freedom expressed in terms of fluid element Goldstone fields as reviewed in section 1. We explain in appendix B how this family of fluids can be embedded into Class L. We show this by starting with the Lagrangian written in terms of the hydrodynamic fields and implementing a Legendre transformation with respect to temperature. The fundamental variable is then the entropy current itself in the new parameterization. By passing to the reference manifold and examining the redundancies in the map from the fiducial spacetime $\mathbb{M}$ to the physical spacetime $\mathcal{M}$ implemented by the fields $\left\{\phi^{I}, c\right\}$ mentioned in section 1 , we unearth the origins of the volume-preserving diffeomorphism and chemical shift symmetries postulated in the effective action constructions of [45, 46].

- Class D (dissipative transport) section 5: having classified all terms that solve the adiabaticity equation (2.12) with $\Delta=0$, we are left with some terms that are genuinely dissipative, i.e., terms for which no choice of entropy current exists that would remove them. These dissipative terms can be further subdivided: on the one hand, we have transport coefficients whose sign is constrained by the requirement of $\nabla_{\mu} J_{S}^{\mu} \geq 0$ for arbitrary fluid flows $\left(\right.$ Class $\left.\mathrm{D}_{v}\right)$. Such terms only show up at the leading order in derivative expansion. On the other hand, there are sub-dissipative terms which are completely unconstrained (Class $\mathrm{D}_{s}$ ). It transpires, using a result established in [36] that $\mathrm{D}_{v}$ terms appear only at the first order in gradient expansion. We describe this argument in our language, providing at the same time a simple characterization of dissipative constitutive relations.

\section{Class H: hydrostatics from adiabaticity}

We have defined adiabatic fluids to be the set of hydrodynamic constitutive relations that satisfy (2.12). While in section 2 we have argued that this set comprises of the obvious example of ideal fluids, we would like to ascertain and classify other solutions to the adiabaticity equation. We will proceed to establish the existence of various classes of solutions to (2.12) in the reminder. To keep the logical flow of the arguments simple we will start with statements that hold in great generality and subsequently specialize to more special cases.

Our first case of interest is what we called Class $\mathrm{H}$ in section 3. We specialize to timeindependent configurations in hydrodynamics (i.e., we limit ourselves to hydrostatics). In order to ascertain non-trivial constraints on fluids from this hydrostatic restriction we need to turn on external sources, e.g., background metric and gauge fields, which themselves are time-independent to begin with. Therefore let us assume that there exists a Killing vector and Killing gauge transformation collectively denoted by $\mathcal{K} \equiv\left\{K^{\mu}, \Lambda_{K}\right\}$ such that $\delta_{\mathfrak{K}} g_{\mu \nu}=0$ and $\delta_{\mathcal{K}} A_{\mu}=0$. We will further assume that $K^{\mu}$ is timelike everywhere on the manifold the fluid propagates on. ${ }^{27}$ To wit, a stationary background source configuration

\footnotetext{
${ }^{27}$ In particular, we demand by virtue of $K$ being globally timelike on $\mathcal{M}$ that the background the fluid propagates on is free of ergosurfaces. This is necessary in order for the fluid configuration to have a stationary solution aligned with the Killing field.
} 
is encoded as

$$
\mathcal{K} \equiv\left\{K^{\mu}, \Lambda_{K}\right\}, \quad g_{\mu \nu} K^{\mu} K^{\nu} \leq 0 \longrightarrow \delta_{\mathcal{K}} g_{\mu \nu}=\delta_{\mathcal{K}} A_{\mu}=0
$$

There is a natural hydrostatic configuration associated with this background given by $\left\{\boldsymbol{\beta}^{\mu}, \Lambda_{\boldsymbol{\beta}}\right\}=\left\{K^{\mu}, \Lambda_{K}\right\}$. This configuration is time-independent since $\delta_{\mathcal{K}} \boldsymbol{\beta}^{\mu}=\delta_{\mathcal{K}} K^{\mu}=0$ and $\delta_{\mathcal{K}} \Lambda_{\beta}=\delta_{\mathcal{K}} \Lambda_{K}=0$. It therefore follows that for any functional $\mathcal{Z}[\boldsymbol{\Psi}]$ of the fluid dynamical variables we have

$$
\delta_{\mathcal{B}} \mathcal{Z}\left[g_{\alpha \beta}, A_{\alpha}, \boldsymbol{\beta}^{\alpha}, \Lambda_{\boldsymbol{\beta}}\right]=\delta_{\mathcal{K}} \mathcal{Z}\left[g_{\alpha \beta}, A_{\alpha}, K^{\alpha}, \Lambda_{K}\right]=0 .
$$

The hydrostatic principle: we now formulate an important non-trivial statement about the configurations we have just described. A-priori by aligning the fluid velocity and the gauge parameter to the background Killing structure only results in an off-shell configuration of the system. The hydrostatic principle asserts that these off-shell hydrostatic configurations constructed above are also automatically on-shell, i.e., they automatically solve the hydrodynamic equations (2.6). We will see that this holds true for all adiabatic constitutive relations that we will consider in the sequel.

Let us now define the hydrostatic limit of various currents we have defined in the previous sections by just substituting $\left\{\boldsymbol{\beta}^{\mu}, \Lambda_{\boldsymbol{\beta}}\right\}=\left\{K^{\mu}, \Lambda_{K}\right\}$. The hydrodstatic currents are then simply obtained as

$$
\left(\mathcal{C}_{\mathcal{H}}\right)_{\text {Hydrostatic }}=\left.\mathcal{C}_{\mathcal{H}}\right|_{\left\{\boldsymbol{\beta}^{\mu}, \Lambda_{\boldsymbol{\beta}}\right\}=\left\{K^{\mu}, \Lambda_{K}\right\}} \cdot
$$

The utility of these currents is that they allow us to write down an expression for the hydrostatic partition function, the generating function for correlators of the currents $\mathcal{C}_{\mathcal{H}}$.

\subsection{Hydrostatic partition functions}

Consider a fluid on a background manifold $\mathcal{M}$ with metric $g_{\mu \nu}$ and gauge field $A_{\mu}$. When the background sources satisfy (4.1) we can construct a Wick-rotated manifold over which a partition function can be defined. We begin by identifying every point $p \in \mathcal{M}$, with the point $p^{\prime}$ in its future separated from it by a unit affine distance along the vector $K^{\mu}$. More precisely, we identify the points $p$ and $p^{\prime}$ if there is a curve $x^{\mu}(\tau)$ such that

$$
x^{\mu}(\tau=0)=x^{\mu}(p), \quad x^{\mu}(\tau=1)=x^{\mu}\left(p^{\prime}\right), \quad \frac{d x^{\mu}}{d \tau}=K^{\mu} .
$$

We will also assume that $\left\{g_{\mu \nu}, A_{\mu}\right\}$ are sufficiently slowly varying (spatially) so that there are no caustics within a unit affine distance. This identification then converts the original spacetime $\mathcal{M}$ into a fibre bundle with a timelike circle fibred over a spacelike base space $\Sigma_{\mathcal{M}}{ }^{28}$ For definiteness, we will also choose an embedding of the base space into the original spacetime as a spatial hypersurface (this is equivalent to fixing a gauge for the

\footnotetext{
${ }^{28}$ In order to do this without any ambiguity, one needs to prescribe how the flavour fibres at $p$ and $p^{\prime}$ should be identified - we will identify these flavour fibres with a flavour transformation given by $\Lambda_{K}$, i.e., we take the local gauge choice at $p$ and $p^{\prime}$ to be related by the gauge transformation generated by $\Lambda_{K}$. This then gives fibre bundles charged under the flavour group over the spatial base space.
} 
Kaluza-Klein (KK) gauge field which arises when we reduce along the timelike circle). For consistency, we will require that our final results should not depend on this (arbitrary) choice of embedding.

Since $K^{\mu}$ is a Killing vector field, we can Wick rotate the background $\mathcal{M} \mapsto \mathcal{M}_{E}$ by a suitable analytic continuation of its orbits. Likewise we also Wick-rotate all the hydrostatic currents to obtain suitable Euclidean currents ${ }^{29}$

$$
\left(\mathcal{C}_{\mathcal{H}}\right)_{\text {Hydrostatic }} \mapsto\left(\mathcal{C}_{\mathcal{H}}\right)_{\mathrm{E}}
$$

With these manipulations we are now in a position to define the grand canonical partition function $e^{i W_{E}}$ following [3, 4]. It is given as the path integral over the manifold $\mathcal{M}_{E}$ constructed above with thermal boundary condition along the $\tau$-circle. This path integral is dominated by the hydrostatic saddle point we have just described so it is trivial to write down the final answer. Let us distinguish situations with and without anomalies since the final answer for the generating function depends on the microscopics of the theory.

(i). Non-anomalous hydrostatic partition functions: in the absence of anomalies, $W_{E}$ is just the Wick rotation of the total hydrostatic free energy, viz.,

$$
W_{E}=-\int_{\Sigma_{E}}\left(\frac{\mathcal{G}_{E}^{\mu}}{T}\right) d^{d-1} S_{\mu}
$$

where $d^{d-1} S_{\sigma}$ is the area form on the base space $\Sigma_{E}$ which is defined using the choice of base space embedding we described above (thus $\Sigma_{E}=\Sigma_{\mathcal{M}}$ ).

One can easily check that this answer for $W_{E}$ is embedding independent. Setting $\left\{\boldsymbol{\beta}^{\mu}, \Lambda_{\boldsymbol{\beta}}\right\}=\left\{K^{\mu}, \Lambda_{K}\right\}$ in (2.21) and using (4.1) we get

$$
\nabla_{\mu}\left(\frac{\mathcal{G}^{\mu}}{T}\right)_{\text {Hydrostatic }}=0 .
$$

This means that its Wick-rotated counterpart $\frac{\mathcal{G}_{E}^{\mu}}{T}$ is also divergenceless (i.e., it is conserved). This then implies that $W_{E}$ is embedding independent. As advertised earlier, knowledge of the Euclidean Gibbs current is sufficient information to recover the generating function of current correlators.

(ii). Anomalous hydrostatic partition functions: the above argument can be extended to situations where we have quantum anomalies with some minor modifications. As evident from (2.21), the covariant free energy current is no longer conserved in the adiabatic limit because of the free energy injection due to anomalies. This issue can be solved however if we choose to work with consistent free energy current instead. ${ }^{30}$

\footnotetext{
${ }^{29}$ In this section we will use the subscript $E$ uniformly to denote the Wick rotated fields of interest.

${ }^{30}$ The distinction between the covariant and the consistent currents is that the latter is naturally obtained from a (anomalous) quantum effective action by varying with respect to sources and is so named because it satisfies the Wess-Zumino consistency conditions. We provide a quick review of the translation between the covariant and consistent currents in appendix A. A detailed account of the issues can also be found in $[3,10]$ and in standard references such as $[62,63]$.
} 
For the present discussion we simply assume that the consistent current is obtained from the covariant one by a well-defined prescription. Once this is done, the adiabaticity equation can be written directly in terms of the consistent currents, see (A.11). Given the consistent currents, using (4.1) in (A.11) we get

$$
\nabla_{\mu}\left(\frac{\mathcal{G}_{\text {cons }}^{\mu}}{T}\right)_{\text {Hydrostatic }}=0,
$$

provided we choose to work in hydrostatic gauge defined via

$$
\text { Hydrostatic Gauge: } \quad \partial_{\nu} K^{\mu}=0, \quad \Lambda_{K}=0 .
$$

As in the preceding discussion this then suffices to define a generating functional, since we can consider the integral of $\frac{\mathcal{G}_{\text {cons, }, E}^{\mu}}{T}$ over the base space $\Sigma_{E}$ in analogy with (4.6). Thus modulo a restricted gauge choice, the consistent free energy current leads to a Euclidean partition function that is well-defined, and independent of the choice of base-space embedding, even in the presence of anomalies.

It is convenient to perform an inverse Wick-rotation of the Euclidean partition function $W_{E}$ so that we may use the metric with Lorentzian signature. This amounts to

$$
W_{\text {Hydrostatic }}=-\left[\int_{\Sigma_{E}}\left(\frac{\mathcal{G}_{\text {cons }}^{\mu}}{T}\right) d^{d-1} S_{\mu}\right]_{\text {Hydrostatic }}
$$

As we will see shortly, this hydrostatic partition function is a very powerful way to characterize a large subset of adiabatic constitutive relations.

A classification of hydrostatic partition functions: the basic lesson from the above discussion is that the choice of hydrostatic partition functions is characterized by the (consistent) free energy current $\mathcal{G}_{\text {cons }}^{\mu}$. Being a vector in spacetime, it can naturally be decomposed into a longitudinal part along $\boldsymbol{\beta}^{\mu}$ and transverse part, i.e., $\mathcal{G}^{\mu}=\mathfrak{S} \boldsymbol{\beta}^{\mu}+\mathfrak{V}^{\mu}$ with $\mathfrak{V}_{\mu} \boldsymbol{\beta}^{\mu}=0$. Accounting for the presence of anomalies as well, this prompts a further subdivision of the hydrostatic partition functions into three sub-classes.

1. Class $\mathrm{H}_{S}$ : these are hydrostatic partition functions that lift to spacetime scalars. In this case we can extend the definition (4.9) to write the final answer as a complete spacetime integral (as opposed to a spatial integral) over $\mathcal{M}=\Sigma_{E} \times I_{K}$ where $I_{K}$ is an interval of unit affine length along the Killing direction $K$.

$$
W_{\text {Hydrostatic }}=\int_{\Sigma_{E}} P_{S}\left[\mathbf{\Psi}_{\mathcal{K}}\right] K^{\sigma} d^{d-1} S_{\sigma}=\int_{\Sigma_{E} \times I_{K}} d^{d} x \sqrt{-g} P_{S}\left[\mathbf{\Psi}_{\mathcal{K}}\right],
$$

where $\boldsymbol{\Psi}_{\mathcal{K}}=\left\{g_{\mu \nu}, A_{\mu}, K^{\mu}, \Lambda_{K}\right\}$. This is the canonical form in which we expect to see generating functions for correlators of currents.

2. Class $\mathrm{H}_{V}$ : these are terms in the hydrostatic partition function which lift to transverse spacetime vectors as suggested by (4.9). More explicitly, they are allowed contributions of the form

$$
W_{\text {Hydrostatic }}=\int_{\Sigma_{E}}\left(P_{V}^{\sigma}\left[\mathbf{\Psi}_{\mathcal{K}}\right]\right)_{\text {Hydrostatic }} d^{d-1} S_{\sigma},
$$


with

$$
\left(\boldsymbol{\beta}_{\sigma} P_{V}^{\sigma}\left[\mathbf{\Psi}_{\mathcal{K}}\right]\right)_{\text {Hydrostatic }}=K_{\sigma}\left(P_{V}^{\sigma}\left[\mathbf{\Psi}_{\mathcal{K}}\right]\right)_{\text {Hydrostatic }}=0 .
$$

Note that in order for this to be independent of embedding, we will also require that the vector field $P_{V}^{\sigma}$ be conserved, $\left(\nabla_{\sigma} P_{V}^{\sigma}\right)_{\text {Hydrostatic }}=0$. We will discuss these constitutive relations further in section 11.1.

3. Class A: these are non-gauge invariant, non-diffeomorphism invariant terms that are added to the hydrostatic partition function to reproduce flavour and gravitational anomalies. We will explain how to obtain the anomalous constitutive relations in an off-shell Lagrangian formalism extending the analysis of [30] in section 12.

One of the main outcomes of demanding the existence of equilibrium on arbitrary time-independent backgrounds is that it serves to delineate a set of constraints on the transport due to the second law. Certain terms if present in the constitutive relations will give rise to sign-indefinite contributions to $\nabla_{\mu} J_{S}^{\mu}$ - these have to be forbidden if we want to ensure that the divergence of the entropy current is positive definite. This leads us to an important class of terms which are the hydrostatic forbidden terms $\left(\mathrm{H}_{F}\right)$. To wit,

Class $\mathbf{H}_{\boldsymbol{F}}$ : transport coefficients in Classes $\mathrm{H}_{S}$ and $\mathrm{H}_{V}$ can be enumerated by listing all off-shell independent hydrostatic scalars and vectors at a given order in derivative expansion. The existence of equilibrium imposes that all other hydrostatic tensor structures that can appear in the constitutive relations be constrained to occur as linear combinations of the unconstrained ones. This consistency of the equilibrium partition function gives a number of hydrostatic relations that we call as $\mathrm{H}_{F}$ (for hydrostatic forbidden). ${ }^{31}$

We will have more to say about these various categories in the course of our discussion.

\subsection{Currents from the hydrostatic partition function}

Given a hydrostatic partition function the constitutive relations can be derived from a straightforward variational principle. One can effectively think of the partition function as a generating function, from which one derives the currents by varying with respect to the sources [3]. One can also obtain the entropy current using the boundary terms of the variational calculus. We review how this can be done as described in the recent discussion of $[36,37]$. We will recast this in a language that will be at once familiar, and at the same time set the stage for further discussions in section 6 .

First let us see how one can recover the hydrostatic energy momentum tensor and currents from (4.9). Consider the variation of the partition function under a small change of sources $\left\{g_{\mu \nu}, A_{\mu}\right\}$. It is useful to think of this variation as arising from a very slow time-dependence of the sources. Indeed any deviation from equilibrium can be measured by the temporal changes - we will make extensive use of the fact that $\delta_{\mathfrak{B}} g_{\mu \nu}$ and $\delta_{\mathfrak{B}} A_{\mu}$ will capture the linear time dependence away from equilibrium in what follows.

\footnotetext{
${ }^{31}$ Empirically, terms in Class $\mathrm{H}_{F}$ appear to account for about a third of the total number of transport coefficients at a given order in the gradient expansion (beyond leading order). See tables 7 and 8 for a summary of the counting in a variety of examples.
} 
We want to calculate the change in $W_{\text {Hydrostatic }}$ between two time slices separated by an infinitesimal displacement $\boldsymbol{\beta}^{\alpha} \delta t$. This can be done by using Gauss law:

$$
\begin{aligned}
\delta W_{\text {Hydrostatic }}= & -\delta\left[\int_{\Sigma_{E}}\left(\frac{\mathcal{G}_{\text {cons }}^{\mu}}{T}\right) d^{d-1} S_{\mu}\right]_{\text {Hydrostatic }} \\
= & \delta t\left[\int_{\Sigma_{E}}\left[-\nabla_{\sigma}\left(\frac{\mathcal{G}_{\text {cons }}^{\sigma}}{T}\right)\right] \boldsymbol{\beta}^{\alpha} d^{d-1} S_{\alpha}\right]_{\text {Hydrostatic }} \\
& +\delta t\left[\int_{\partial \Sigma_{E}}\left(\frac{\mathcal{G}_{\text {cons }}^{j}}{T}\right) d^{d-2} S_{j}\right]_{\text {Hydrostatic }}
\end{aligned}
$$

The bulk piece can then be simplified using the adiabaticity equation for the consistent free energy current (A.11). Restricting to the hydrostatic gauge we obtain with a single variation $^{32}$

$$
\begin{aligned}
\delta W_{\text {Hydrostatic }}= & {\left[\int_{\Sigma_{E}}\left(\frac{1}{2} T_{\text {cons }}^{\mu \nu} \delta g_{\mu \nu}+J_{\text {cons }}^{\mu} \cdot \delta A_{\mu}\right) \boldsymbol{\beta}^{\alpha} d^{d-1} S_{\alpha}\right]_{\text {Hydrostatic }} } \\
& +\delta t\left[\int_{\partial \Sigma_{E}}\left(\frac{\mathcal{G}_{\text {cons }}^{j}}{T}\right) d^{d-2} S_{j}\right]_{\text {Hydrostatic }}
\end{aligned}
$$

where $\delta g_{\mu \nu}=\delta t \delta_{\mathfrak{B}} g_{\mu \nu}$ and $\delta A_{\mu}=\delta t \delta_{\mathfrak{B}} A_{\mu}$. Further, we have chosen our time slices such that there is no linear time dependence in $\left\{\boldsymbol{\beta}^{\alpha}, \Lambda_{\boldsymbol{\beta}}\right\}$, i.e., $\delta \boldsymbol{\beta}^{\alpha}=\delta t \delta_{\mathfrak{B}} \boldsymbol{\beta}^{\alpha}=0$ and $\delta \Lambda_{\boldsymbol{\beta}}=\delta t \delta_{\mathfrak{B}} \Lambda_{\boldsymbol{\beta}}=0$.

Since any variation can be mimicked by a slow time-dependence, we conclude that, in general

$$
\begin{aligned}
\delta W_{\text {Hydrostatic }}= & {\left[\int_{\Sigma_{E}}\left(\frac{1}{2} T_{\text {cons }}^{\mu \nu} \delta g_{\mu \nu}+J_{\text {cons }}^{\mu} \cdot \delta A_{\mu}\right) \boldsymbol{\beta}^{\alpha} d^{d-1} S_{\alpha}\right]_{\text {Hydrostatic }} } \\
& +\left[\int_{\partial \Sigma_{E}}\left(\not \Theta_{\mathrm{PS}}\right)^{j} d^{d-2} S_{j}\right]_{\text {Hydrostatic }}
\end{aligned}
$$

where $\left(\phi \Theta_{\mathrm{PS}}\right)^{j}$ is a boundary term linear in variations of fields, arising out of integration by parts.

For the particular kind of slow time dependence under consideration, we can write $\left(\not \Theta_{\mathrm{PS}}\right)^{j}=\delta t\left(\phi_{\mathcal{B}} \Theta_{\mathrm{PS}}\right)^{j}$ where $\phi_{\mathcal{B}} \Theta_{\mathrm{PS}}$ is obtained by changing all the variations $\delta(\ldots)$ into Lie-derivative $\delta_{\mathcal{B}}(\ldots)$. A comparison of (4.15) against (4.14) then yields

$$
\left(\phi_{\mathcal{B}} \Theta_{\mathrm{PS}}\right)^{j}=\frac{\mathcal{G}_{\text {cons }}^{j}}{T}
$$

Thus, when we vary the sources in the hydrostatic partition function, we get a bulk variation which allows us to figure out the consistent currents and a boundary variation which gives us the information about the spatial component of free energy current. Since the temporal component of free energy current (i.e., free energy density) is already captured by the

\footnotetext{
${ }^{32}$ If we are dealing with non-anomalous systems, the subscript cons may be freely omitted.
} 
partition function before variation, we can then reconstruct the entire free energy current. By using the free energy current thus obtained as the non-canonical part of the entropy current, we can finally compute the entropy current associated with the partition function.

A clear algorithmic procedure for doing this which is inspired by our Class L discussion, can be phrased as follows (cf., also appendix G):

1. From $W_{\text {Hydrostatic }}$ determine $\mathcal{G}_{\text {cons }}^{0}$. By varying it, determine the currents $\left\{T^{\mu \nu}, J^{\mu}\right\}$ and the boundary term gives $\mathcal{G}_{\text {cons }}^{j}$. When covariantized, the latter is just the presymplectic potential $\left(\Varangle \Theta_{\mathrm{PS}}\right)^{\mu}$ which arises as the surface term when varying the hydrostatic partition function.

2. Having obtained the hydrostatic currents, one then takes them off-shell by giving them linear time dependence. To do so, one adds in non-hydrostatic terms in $\left\{T^{\mu \nu}, J^{\mu}\right\}$ by unlinking $\mathcal{B}$ from $\mathcal{K}$. Effectively what this amounts to is that the linear variation of the background fields, in the direction of $\left\{\boldsymbol{\beta}^{\mu}, \Lambda_{\boldsymbol{\beta}}\right\}$, i.e., $\delta_{\mathfrak{B}}$ defined in (2.22) plays the role of time derivative.

3. One similarly upgrades the boundary term from $\left(\Varangle \Theta_{\mathrm{PS}}\right)^{\mu}$ to $\left(\oiint_{\mathcal{B}} \Theta_{\mathrm{PS}}\right)^{\mu}$ to obtain the linear time dependence in the spatial component of free energy current.

4. In Class $\mathrm{H}_{S}$ the non-canonical part of the entropy current is simply obtained by combining the temporal and the spatial components of the free energy current:

$$
\left(J_{S}^{\mu}\right)_{n o n-c a n}=\boldsymbol{\beta}^{\mu} P_{S}[\Psi]-\left(\oiint_{\mathcal{B}} \Theta_{\mathrm{PS}}\right)^{\mu} .
$$

We note in passing that we can add a total derivative term $\nabla_{\nu} \mathcal{K}^{[\mu \nu]}$ (Komar terms) to the above whilst still retaining a conserved entropy current, cf., section 6.3 .

5. In Class $\mathrm{H}_{V}$ the vector in the partition function can be just covariantised to give the non-canonical part of the entropy current [10].

6. By adding this non-canonical part to the canonical part of entropy current $\left(J_{S}^{\mu}\right)_{\text {can }}=$ $-\boldsymbol{\beta}_{\nu} T^{\mu \nu}-\frac{\mu}{T} J^{\mu}$, we thus reproduce the prescription for computing the hydrostatic entropy current given in [37].

With this, we have thus given a direct, covariant and off-shell rederivation of the entire theory of hydrostatic partition function and its associated entropy current developed in references [3, 37]. Before moving away from hydrostatics, we remark on two crucial ideas we have used above to considerably simplify the existing derivations. First is the relation between consistent free energy current and hydrostatic partition function first proposed in [10]. The second idea is the off-shell adiabaticity equation (2.12) (first introduced in [8]) which will continue to play a crucial role in what follows.

To summarize, given a hydrostatic partition function which is a functional of the background (time-independent) sources we can recover on-shell currents $\mathcal{C}_{\mathcal{H}}$ which satisfy the adiabaticity equations (2.12). This provides us with our first class of examples of non-trivial adiabatic fluids, though soon we will be enlarging our repertoire. 


\section{Class D: dissipative terms}

In our discussion thus far we have motivated focusing on the solutions to the adiabaticity equation (2.12) which switches off dissipation, viz., $\Delta=0$. While we will explore a rather intricate structure of adiabatic constitutive relations in part II, one would imagine that this would be a small part hydrodynamic transport. After all, most of the phenomena we intuitively grasp in hydrodynamics have to do with dissipation. Strangely enough, this turns out not to be true. Nevertheless, any complete classification of transport has to tackle the constraints on such dissipative terms as well. We will now argue that this is relatively straightforward and, using a key result proved in [36, 37], make a case for a complete transport classification, once the adiabatic story is complete.

\subsection{Constraints on dissipative transport}

The second law of thermodynamics requires $\Delta \geq 0$, so let us recall what is known about dissipative transport. The simplest dissipative terms are the shear and bulk viscosities and charge conductivity, usually denoted as $\{\eta, \zeta, \sigma\}$ respectively and enter at first order in gradients. Recall that at leading order in the gradient expansion, the currents are (see [57]):

$$
\begin{aligned}
T^{\mu \nu} & =\epsilon u^{\mu} u^{\nu}+p P^{\mu \nu}-2 \eta \sigma^{\mu \nu}-\zeta \Theta P^{\mu \nu}, \\
J^{\mu} & =q u^{\mu}+\sigma_{\mathrm{Ohm}} \mathfrak{v}^{\mu}, \quad J_{S}^{\mu}=s u^{\mu}-\frac{\mu}{T} \sigma_{\mathrm{Ohm}} \mathfrak{v}^{\mu} .
\end{aligned}
$$

which leads to entropy generation

$$
\Delta=\frac{1}{T}\left(2 \eta \sigma_{\mu \nu} \sigma^{\mu \nu}+\zeta \Theta^{2}+\sigma_{\mathrm{Ohm}} \mathfrak{v}^{2}\right) .
$$

This is consistent with the second law and positive definite for

$$
\eta \geq 0, \quad \zeta \geq 0, \quad \sigma_{\mathrm{Ohm}} \geq 0 .
$$

The fact that there are no adiabatic terms at first order, as well as absence of (noncanonical) corrections to the entropy current, are well known. These statements can be easily derived from the standard current algebra perspective. However, one may intuitively expect the higher order story to be much more complex, making it unclear how to proceed. This intuition turns out to be wrong.

For the moment assume that the adiabatic part of the constitutive relations has been dealt with. First, let us use the positivity of viscosities and conductivities to define a subclass $\mathrm{D}_{v} \subset \mathrm{D}$ which contains genuinely dissipative/viscous transport. These are terms that are constrained to be sign-definite by the second law. Terms which are not of this form will be called sub-dissipative $\left(\mathrm{D}_{s} \subset \mathrm{D}\right)$; these will be allowed to take on any value without causing trouble for the second law. Much of the material that follows in this subsection was first explained in $[36,37]$. We are mostly going to paraphrase the results first, before unveiling a more abstract proof inspired by our analysis of adiabaticity in part II.

Let us understand why the split $\mathrm{D}=\mathrm{D}_{v} \cup \mathrm{D}_{s}$ exists. In the hydrodynamic gradient expansion the divergence of the entropy current captured by $\Delta$ itself admits a gradient 
expansion (starting at second order as ideal fluids are non-dissipative):

$$
\Delta=\Delta_{2}+\Delta_{3}+\cdots
$$

where $\Delta_{2}$ is the quantity given in the r.h.s. of (5.2) (or generalizations thereof). The higher order $\Delta_{k}$ arise from gradient corrections to both the entropy current and the constitutive relations. When we compute $\Delta_{k}$ we can assemble this into a linear combination of scalar operators with exactly $k$ derivatives $\left(\partial^{k}\right)$. There is a basis of such operators; in the current algebraic approach one usually works with a basis of on-shell independent scalars at a given derivative order, but this is not necessary (as we shall see). The statement about the splitting of Class D is equivalent to the statement that the scalar operators admit such a decomposition.

Scalars of interest at $\mathcal{O}\left(\partial^{k}\right)$ are composite operators and can either be (i) 'descendants' of operators constrained at lower orders or (ii) simple 'product-composites' of lower order operators. The descendant operators are obtained by acting with derivatives on lower order tensor structures, while product-composites are simply obtained by contraction. Examples of the former are operators such as $\left\{\Theta u^{\mu} \nabla_{\mu} \Theta, \sigma^{\mu \nu} u^{\alpha} \nabla_{\alpha} \sigma_{\mu \nu}\right\}$, while $\left\{\Theta^{3}, \sigma^{\alpha \beta} \sigma_{\beta \gamma} \sigma_{\alpha}^{\gamma}, \Theta \sigma_{\mu \nu} \sigma^{\mu \nu}\right\}$ exemplify the latter at $\mathcal{O}\left(\partial^{3}\right) .{ }^{33}$

The product-composites are simple; since they are invariants built out of terms that are already constrained, their coefficients can be arbitrary whilst still respecting the second law (in the gradient expansion). For example, taking the viscosities and conductivities to be positive, we ensure $\Delta_{2} \geq 0$ and thence the contribution to $\Delta_{3}$ from such product-composite form, is simply sub-dominant and poses no obstruction to the second law. To wit,

$$
\begin{gathered}
2 \eta \sigma_{\mu \nu} \sigma^{\mu \nu}+\zeta \Theta^{2}+\gamma_{1} \Theta^{3}+\gamma_{2} \sigma^{\alpha \beta} \sigma_{\beta \gamma} \sigma_{\alpha}{ }_{\alpha}+\gamma_{3} \Theta \sigma_{\mu \nu} \sigma^{\mu \nu} \geq 0 \\
\Longrightarrow \quad \eta, \zeta \geq 0, \text { and }\left\{\gamma_{1}, \gamma_{2}, \gamma_{3}\right\} \text { unconstrained. }
\end{gathered}
$$

The descendants are a-priori trickier to handle; at any given derivative order they give rise to new scalar invariants which have not been encountered at lower orders. Their contribution to $\Delta$ cannot be subsumed into lower order terms. One way to argue for their importance is to note that one can find fluid configurations where the lower order gradients are locally made to vanish, making the descendants important in some domain. Since we want the second law to hold in all possible scenarios, one must therefore control the descendants. The rather non-trivial fact is that these are also easy to handle beyond the leading order.

Let us understand this a bit more carefully following the impressively clear and complete analysis of dissipative transport of $[37]$ (see $[2,36]$ for earlier results). As explained there, scalar operators contributing to $\Delta$ are of three types: ${ }^{34}$

\footnotetext{
${ }^{33}$ It is useful at this stage to infer from our hydrostatic discussion that the operator $\delta_{\mathcal{B}}$ serves the role of capturing time derivatives about equilibrium configuration. All the aforementioned operators can be written directly in terms of $\delta_{\mathcal{B}} g_{\mu \nu}$ (likewise occurrences of $\mathfrak{v}_{\mu}$ can be expressed in terms of $\delta_{\mathcal{B}} A_{\mu}$ ).

${ }^{34}$ In [37] a fourth type was introduced called $\Delta_{n o n-d i s s}$ - these will be accounted for in our adiabatic story as they end up having net zero contribution to entropy production. Note that we will not include them explicitly in our counting of Class $\mathrm{C}$ constitutive relations as these terms are exact differentials and thus trivial in cohomology.
} 
- Terms that contribute to $\Delta_{2}$ at leading order which need to be controlled to ensure $\Delta \geq 0$. They belong to $\mathrm{D}_{v}$ and impose constraints on transport (such terms were called $\Delta_{2 n d-o r d e r}$ in [37]). Note that we can write them effectively in terms of $\left(\delta_{\mathfrak{B}} g\right)^{2}$ and $\left(\delta_{\mathfrak{B}} A\right)^{2}$ for they appear only at quadratic order.

- Descendant terms at any given order which are composite scalars built from a $(k-1)^{\text {st }}$ order independent operator and a first order operator, i.e., of the form $\delta_{\mathfrak{B}} g D \mathcal{O}_{k-2}$ where $\mathcal{O}_{k-2}$ could be a composite-product. Such terms were denoted as $\Delta_{\text {diss-imp }}$ in [37].

- Composite-product terms which simply take the form $\left(\delta_{\mathfrak{B}} g\right)^{k}$ and $\left(\delta_{\mathfrak{B}} A\right)^{k}$. Terms of this type were called $\Delta_{\text {diss-product }}$ in [37].

Given this decomposition, we have schematically

$$
\begin{aligned}
\Delta & =\alpha_{2 g}\left(\delta_{\mathfrak{B}} g\right)^{2}+\alpha_{2 A}\left(\delta_{\mathcal{B}} A\right)^{2}+\sum_{k=3}^{\infty}\left[\kappa_{k g} \delta_{\mathfrak{B}} g D \mathcal{O}_{k-2}+\gamma_{k g}\left(\delta_{\mathfrak{B}} g\right)^{k}+\cdots\right] \\
& \sim \alpha_{2 g}\left[\delta_{\mathfrak{B}} g+\sum_{k=3}^{\infty} \frac{\kappa_{k g}}{2 \alpha_{2 g}} D \mathcal{O}_{k-2}\right]^{2}+\sum_{k=3}^{\infty} \gamma_{k g}\left(\delta_{\mathfrak{B}} g\right)^{k}+\cdots
\end{aligned}
$$

where we have only written out the composite higher order terms explicitly for the metric variation and elided over writing the gauge variations. In the second line we have indicated a merger of the descendant and leading order terms into a quadratic form which plays a role in the argument below. With this parameterization

- $\alpha_{2 g}, \alpha_{2 A} \geq 0$ for the second law to hold.

- $\gamma_{k}$ are unconstrained since they multiply terms which are parametrically smaller than the leading order contributions. These are clearly in the sub-dissipative Class $\mathrm{D}_{s}$.

- By completing squares, one can take care of the descendant terms as well (they are effectively in Class $\mathrm{D}_{s}$ despite appearances). Indeed, as written, positivity of the second line of (5.6) is ensured once we demand $\alpha_{2 g}, \alpha_{2 A}$ etc., to be positive definite. One further needs to ensure that the cross-terms obtained in the process can be assembled into positive-definite quadratic form.

The remarkable statement of [37] is that this can always be done, recursively order by order in the gradient expansion! More specifically, the aforementioned reference used the specific example of (parity-even) charged fluid at third order in gradients to illustrate the general picture proposed in [36]. But the general structure emerging from that analysis makes it clear that the construction can be extended to higher orders. The argument in [37] can be worded as follows: given a hydrostatic entropy current, there exists a hydrodynamic correction $J_{\text {extra }}^{\mu}$ which serves to absorb the contributions from the descendant contribution $\Delta_{\text {diss-imp }}$ to convert them into a positive-definite quadratic form. Inspired by our construction of solutions to the adiabaticity equation we give a more abstract discussion 
below in section 5.2 using a class of tensor-valued differential operators that subsume the above statements efficiently.

The upshot of this discussion is that Class $\mathrm{D}=\mathrm{D}_{v} \cup \mathrm{D}_{s}$ with $\mathrm{D}_{v}$ contributions which are sensitive to the second law making their appearance only at the leading order in the gradient expansion. So while there are many potential contributions to Class D, most of them are agnostic to the constraints and thus can be treated democratically.

\subsection{Differential operators for dissipation}

We will now give a succinct summary of the results of [36, 37], using a compact notation that is inspired by our construction of adiabatic constitutive relations. The impatient reader may choose to skip this section at first reading.

We begin our discussion by constructing a set of tensor structures that provide Class D constitutive relations. We will see in the sequel that these will be quite useful in demarcating the second law constraints quite effectively for they provide us with a simple way to assemble both the product-composite and descendant operators into a positive definite form.

Let us begin with the non-anomalous grand canonical adiabaticity equation (2.21)

$$
-\nabla_{\sigma}\left(\frac{\mathcal{G}^{\sigma}}{T}\right)=\frac{1}{2} T^{\mu \nu} \delta_{\mathfrak{B}} g_{\mu \nu}+J^{\mu} \cdot \delta_{\mathcal{B}} A_{\mu}+\Delta
$$

where we have included $\Delta$ denoting entropy production and dissipation. Second law of thermodynamics demands that $\Delta \geq 0$. We will now arrange our conserved currents in an appropriate form where this condition can be imposed readily.

Consider a differential operator $\Upsilon$ which is tensor valued, i.e., it is a map from the space of tensors to themselves involving some derivations. For the moment we will be rather abstract about the precise form of this operator, but we have here in mind operators constructed from $\Psi$ and quantities such as $\delta_{\mathcal{B}} g_{\mu \nu}$ and $\delta_{\mathcal{B}} A_{\mu}$. Since in the course of manipulations we will have to reshuffle derivatives around, we also denote the corresponding adjoint differential operator obtained via integration by parts as $\Upsilon^{\dagger}$.

Let us examine the following constitutive relations, which we suggestively refer to as Class D constitutive relations:

$$
\begin{aligned}
\left(T^{\mu \nu}\right)_{\mathrm{D}} \equiv-\frac{1}{2}\left[\Upsilon_{\boldsymbol{\eta}_{g}}^{\dagger} \boldsymbol{\eta} \Upsilon_{\boldsymbol{\eta}_{g}}\right. & \left.+\Upsilon_{\boldsymbol{\sigma}_{g}}^{\dagger} \boldsymbol{\sigma} \Upsilon_{\boldsymbol{\sigma}_{g}}\right]^{(\mu \nu)(\alpha \beta)} \delta_{\mathcal{B}} g_{\alpha \beta} \\
& -\left[\Upsilon_{\boldsymbol{\eta}_{g}}^{\dagger} \boldsymbol{\eta} \Upsilon_{\boldsymbol{\eta}_{A}}+\Upsilon_{\boldsymbol{\sigma}_{g}}^{\dagger} \boldsymbol{\sigma} \Upsilon_{\boldsymbol{\sigma}_{A}}\right]^{(\mu \nu) \alpha} \cdot \delta_{\mathcal{B}} A_{\alpha} \\
\left(J^{\alpha}\right)_{\mathrm{D}} \equiv-\frac{1}{2}\left[\Upsilon_{\boldsymbol{\eta}_{A}}^{\dagger} \boldsymbol{\eta} \Upsilon_{\boldsymbol{\eta}_{g}}\right. & \left.+\Upsilon_{\boldsymbol{\sigma}_{A}}^{\dagger} \boldsymbol{\sigma} \Upsilon_{\boldsymbol{\sigma}_{g}}\right]^{\alpha(\mu \nu)} \delta_{\mathcal{B}_{B}} g_{\mu \nu} \\
& -\left[\Upsilon_{\boldsymbol{\eta}_{A}}^{\dagger} \boldsymbol{\eta} \Upsilon_{\boldsymbol{\eta}_{A}}+\Upsilon_{\boldsymbol{\sigma}_{A}}^{\dagger} \boldsymbol{\sigma} \Upsilon_{\boldsymbol{\sigma}_{A}}\right]^{\alpha \beta} \cdot \delta_{\mathcal{B}} A_{\beta}
\end{aligned}
$$

By construction these currents vanish in hydrostatics, so they potentially contribute to $\Delta$. Furthermore, by exploiting the structure of (5.8) we have ensured that the r.h.s. of (5.7) is at least quadratic in $\delta_{\mathfrak{B}} g$ and $\delta_{\mathfrak{B}} A$ as expected. 
We need to now specify the representation content of the operators $\Upsilon$ that appear above. We denote the vector representation as Vect and the two-index symmetric tensor representation by $\mathrm{Sym}_{2}$. Clearly, $A_{\mu} \in$ Vect and $g_{\mu \nu} \in \mathrm{Sym}_{2}$, where we shall use the isomorphism provided by metric on $\mathcal{M}$ to raise and lower indices when necessary. With this understanding

- $\left\{\Upsilon_{\boldsymbol{\eta}_{g}}, \Upsilon_{\boldsymbol{\sigma}_{g}}\right\}$ are tensor-valued differential operators which take two-indexed symmetric tensor fields $\left(\mathrm{Sym}_{2}\right)$ to diffeomorphism representations denoted by Tens $\boldsymbol{\eta}$ and $\mathrm{Tens}_{\boldsymbol{\sigma}}$ respectively, where the latter are those which are direct sums of tensor representations, viz.,

$$
\Upsilon_{\boldsymbol{\eta}_{g}}: \mathrm{Sym}_{2} \rightarrow \text { Tens }_{\boldsymbol{\eta}}, \quad \Upsilon_{\boldsymbol{\sigma}_{g}}: \mathrm{Sym}_{2} \rightarrow \mathrm{Tens}_{\boldsymbol{\sigma}}
$$

- Similarly, $\left\{\Upsilon_{\boldsymbol{\eta}_{A}}, \Upsilon_{\boldsymbol{\sigma}_{A}}\right\}$ are tensor-valued differential operators which take vector fields (Vect) to diffeomorphism representations Tens $\boldsymbol{\eta}$ and Tens $\boldsymbol{\sigma}$ respectively.

$$
\Upsilon_{\boldsymbol{\eta}_{A}}: \text { Vect } \rightarrow \text { Tens }_{\boldsymbol{\eta}}, \quad \Upsilon_{\boldsymbol{\sigma}_{A}}: \text { Vect } \rightarrow \text { Tens }_{\boldsymbol{\sigma}}
$$

- The adjoints of these differential operators act in the opposite direction, mapping direct sums of tensor representations back to $\mathrm{Sym}_{2}$ and Vect representations respectively.

$$
\begin{aligned}
\Upsilon_{\boldsymbol{\eta}_{g}}^{\dagger}: \text { Tens }_{\boldsymbol{\eta}} \rightarrow \text { Sym }_{2}, & \Upsilon_{\boldsymbol{\sigma}_{g}}^{\dagger}: \text { Tens }_{\boldsymbol{\sigma}} \rightarrow \text { Sym }_{2}, \\
\Upsilon_{\boldsymbol{\eta}_{A}}^{\dagger}: \text { Tens }_{\boldsymbol{\eta}} \rightarrow \text { Vect }, & \Upsilon_{\boldsymbol{\sigma}_{A}}^{\dagger}: \text { Tens }_{\boldsymbol{\sigma}} \rightarrow \text { Vect }
\end{aligned}
$$

- Finally, the symbols $\{\boldsymbol{\eta}, \boldsymbol{\sigma}\}$ denote arbitrary tensor fields in the product representations

$$
\left\{\text { Tens }_{\boldsymbol{\eta}} \otimes \text { Tens }_{\boldsymbol{\eta}}, \text { Tens }_{\boldsymbol{\sigma}} \otimes \text { Tens }_{\boldsymbol{\sigma}}\right\}
$$

which can be thought of as intertwiners to ensure that the net operator acting on either $\mathrm{Sym}_{2}$ or Vect is in the representations indicated by the index structure in (5.8).

It is useful to note that we can write the Class $\mathrm{D}$ constitutive relations also in a matrix form

$$
\left(\begin{array}{c}
T^{\mu \nu} \\
J^{\alpha}
\end{array}\right)_{\mathrm{D}}=-\left(\begin{array}{ll}
\Upsilon_{\boldsymbol{\eta}_{g}}^{\dagger} & \Upsilon_{\boldsymbol{\sigma}_{g}}^{\dagger} \\
\Upsilon_{\boldsymbol{\eta}_{A}}^{\dagger} & \Upsilon_{\boldsymbol{\sigma}_{A}}^{\dagger}
\end{array}\right)\left(\begin{array}{cc}
\boldsymbol{\eta} & 0 \\
0 & \boldsymbol{\sigma}
\end{array}\right)\left(\begin{array}{ll}
\Upsilon_{\boldsymbol{\eta}_{g}} & \Upsilon_{\boldsymbol{\eta}_{A}} \\
\Upsilon_{\boldsymbol{\sigma}_{g}} & \Upsilon_{\boldsymbol{\sigma}_{A}}
\end{array}\right)\left(\begin{array}{c}
\frac{1}{2} \delta_{\mathcal{B}} g \\
\delta_{\mathcal{B}} A
\end{array}\right)
$$

which better exhibits the algebraic structure of this class of constitutive relations.

Substituting the above expression back into the right hand side of grand canonical adiabaticity equation and removing the adjoint operators via integration by parts, we 
obtain

$$
\begin{aligned}
& \frac{1}{2}\left(T^{\mu \nu}\right)_{\mathrm{D}} \delta_{\mathcal{B}} g_{\mu \nu}+\left(J^{\alpha}\right)_{\mathrm{D}} \cdot \delta_{\mathcal{B}} A_{\alpha} \\
&=-\frac{1}{2} \delta_{\mathcal{B}} g_{\mu \nu}\left[\Upsilon_{\boldsymbol{\eta}_{g}}^{\dagger} \boldsymbol{\eta} \Upsilon_{\boldsymbol{\eta}_{g}}+\Upsilon_{\boldsymbol{\sigma}_{g}}^{\dagger} \boldsymbol{\sigma} \Upsilon_{\boldsymbol{\sigma}_{g}}\right]^{(\mu \nu)(\alpha \beta)} \frac{1}{2} \delta_{\mathcal{B}} g_{\alpha \beta} \\
&-\frac{1}{2} \delta_{\mathcal{B}} g_{\mu \nu}\left[\Upsilon_{\boldsymbol{\eta}_{g}}^{\dagger} \boldsymbol{\eta} \Upsilon_{\boldsymbol{\eta}_{A}}+\Upsilon_{\boldsymbol{\sigma}_{g}}^{\dagger} \boldsymbol{\sigma} \Upsilon_{\boldsymbol{\sigma}_{A}}\right]^{(\mu \nu) \alpha} \cdot \delta_{\mathcal{B}} A_{\alpha} \\
&-\frac{1}{2} \delta_{\mathcal{B}} A_{\alpha} \cdot\left[\Upsilon_{\boldsymbol{\eta}_{A}}^{\dagger} \boldsymbol{\eta} \Upsilon_{\boldsymbol{\eta}_{g}}+\Upsilon_{\boldsymbol{\sigma}_{A}}^{\dagger} \boldsymbol{\sigma} \Upsilon_{\boldsymbol{\sigma}_{g}}\right]^{\alpha(\mu \nu)} \delta_{\mathcal{B}} g_{\mu \nu} \\
&-\delta_{\mathcal{B}} A_{\alpha} \cdot\left[\Upsilon_{\boldsymbol{\eta}_{A}}^{\dagger} \boldsymbol{\eta} \Upsilon_{\boldsymbol{\eta}_{A}}+\Upsilon_{\boldsymbol{\sigma}_{A}}^{\dagger} \boldsymbol{\sigma} \Upsilon_{\boldsymbol{\sigma}_{A}}\right]^{\alpha \beta} \cdot \delta_{\mathcal{B}} A_{\beta} \\
&=- {\left[\frac{1}{2} \Upsilon_{\boldsymbol{\eta}_{g}} \delta_{\mathcal{B}} g+\Upsilon_{\boldsymbol{\eta}_{A}} \delta_{\mathcal{B}} A\right] \boldsymbol{\eta}\left[\frac{1}{2} \Upsilon_{\boldsymbol{\eta}_{g}} \delta_{\mathcal{B}} g+\Upsilon_{\boldsymbol{\eta}_{A}} \delta_{\mathcal{B}} A\right] } \\
&-\left[\frac{1}{2} \Upsilon_{\boldsymbol{\sigma}_{g}} \delta_{\mathfrak{B}} g+\Upsilon_{\boldsymbol{\sigma}_{A}} \delta_{\mathcal{B}} A\right] \boldsymbol{\sigma}\left[\frac{1}{2} \Upsilon_{\boldsymbol{\sigma}_{g}} \delta_{\mathfrak{B}} g+\Upsilon_{\boldsymbol{\sigma}_{A}} \delta_{\mathcal{B}} A\right]+\nabla_{\alpha}\left(\mathrm{N}^{\alpha}\right)_{\mathrm{D}}
\end{aligned}
$$

where we have kept the total derivative term from integration by parts.

Let us take $\left(\mathcal{G}^{\alpha}\right)_{\mathrm{D}}=-T\left(\mathrm{~N}^{\alpha}\right)_{\mathrm{D}}$, which in the micro-canonical ensemble is equivalent to taking the entropy current to be

$$
\left(J_{S}^{\alpha}\right)_{\mathrm{D}} \equiv-\frac{u_{\beta}}{T}\left(T^{\alpha \beta}\right)_{\mathrm{D}}-\frac{\mu}{T} \cdot\left(J^{\alpha}\right)_{\mathrm{D}}+\left(\mathrm{N}^{\alpha}\right)_{\mathrm{D}} \cdot
$$

With this choice we have a simple expression for the entropy production within this class of constitutive relations (using (5.7)):

$$
\begin{aligned}
\Delta=\left[\frac{1}{2} \Upsilon_{\boldsymbol{\eta}_{g}} \delta_{\mathfrak{B}} g\right. & \left.+\Upsilon_{\boldsymbol{\eta}_{A}} \delta_{\mathcal{B}} A\right] \boldsymbol{\eta}\left[\frac{1}{2} \Upsilon_{\boldsymbol{\eta}_{g}} \delta_{\mathcal{B}} g+\Upsilon_{\boldsymbol{\eta}_{A}} \delta_{\mathcal{B}} A\right] \\
& +\left[\frac{1}{2} \Upsilon_{\boldsymbol{\sigma}_{g}} \delta_{\mathcal{B}} g+\Upsilon_{\boldsymbol{\sigma}_{A}} \delta_{\mathcal{B}} A\right] \boldsymbol{\sigma}\left[\frac{1}{2} \Upsilon_{\boldsymbol{\sigma}_{g}} \delta_{\mathcal{B}} g+\Upsilon_{\boldsymbol{\sigma}_{A}} \delta_{\mathcal{B}} A\right]
\end{aligned}
$$

which is completely parameterized by the differential operators $\Upsilon$ and the intertwiners between different representations $\{\boldsymbol{\eta}, \boldsymbol{\sigma}\}$. We want to ensure that $\Delta \geq 0$ for the second law, which is easy to insist for (5.15) has the structure of a quadratic form with the intertwiners playing the role of the metric. Then demanding that $\{\boldsymbol{\eta}, \boldsymbol{\sigma}\}$ transform in appropriately symmetric representations to provide a positive definite quadratic form, viz.,

$$
\boldsymbol{\eta} \in \operatorname{Sym}_{+}\left(\text {Tens }_{\boldsymbol{\eta}} \otimes \text { Tens }_{\boldsymbol{\eta}}\right), \quad \boldsymbol{\sigma} \in \mathrm{Sym}_{+}\left(\mathrm{Tens}_{\boldsymbol{\sigma}} \otimes \mathrm{Tens}_{\boldsymbol{\sigma}}\right)
$$

with the subscript + denoting that the eigenvalues are non-negative definite. This gives us a solution to $\Delta \geq 0$.

Should we consider intertwiners not transforming in the symmetric tensor product representation we will find that they would correspond to adiabatic or hydrostatic forbidden constitutive relations. For instance taking the anti-symmetric representation will lead to Class B adiabatic constitutive relations as we shall discuss in section 9.

To summarize the above construction, by suitably picking tensor structures we are in a position to engineer constitutive relations that are guaranteed to satisfy the second law of thermodynamics. 


\subsection{Examples: low order Class D differential operators}

Let us now examine the results of our earlier discussion in section 5.1 in light of this renewed understanding. The easiest way to proceed is to start with the leading order in gradients. Since (5.15) already has two factors of $\delta_{\mathfrak{B}}$ on the r.h.s., a contribution to $\Delta_{2}$ necessarily requires $\Upsilon$ to be a tensor operator involving no derivatives. For instance, picking

$$
\Upsilon_{\boldsymbol{\eta}_{g}}=\Upsilon_{\sigma_{A}}=\mathrm{Id}, \quad \Upsilon_{\boldsymbol{\eta}_{A}}=\Upsilon_{\sigma_{g}}=0,
$$

and $\boldsymbol{\eta} \in \mathrm{Sym}_{2} \otimes \mathrm{Sym}_{2}$ and $\boldsymbol{\sigma} \in$ Vect $\otimes$ Vect to be also zero derivative tensors built from the background metric $g_{\mu \nu}$ and the hydrodynamic field $\boldsymbol{\beta}^{\mu}$ we can recover (5.2). To be specific, (5.2) is reproduced by taking (5.17) combined with

$$
\boldsymbol{\eta}_{(0)}^{\mu \nu \rho \sigma}=T \zeta P^{\mu \nu} P^{\rho \sigma}+2 T \eta P^{\rho<\mu} P^{\nu>\sigma}, \quad \boldsymbol{\sigma}_{(0)}^{\alpha \beta}=T \sigma_{\mathrm{Ohm}} P^{\alpha \beta},
$$

where the subscript (0) means that these intertwiners are the contributions at lowest order in a derivative expansion (the corresponding constitutive relations are first order, of course) and $<\cdot>$ denotes the symmetric transverse traceless projection. ${ }^{35}$

At higher order in derivative expansion an interesting subset of Class D constitutive relations can be obtained by restricting to $\Upsilon$-operators which do not act as derivatives. For instance, in the case of second order constitutive relations, we can consider arbitrary first order transverse tensors $\mathcal{X}^{(\mu \nu) \alpha}$ and the following correction to (5.17):

$$
\begin{aligned}
& \Upsilon_{\boldsymbol{\eta}_{g}}=\Upsilon_{\boldsymbol{\sigma}_{A}}=\mathrm{Id}, \\
& \Upsilon_{\boldsymbol{\sigma}_{g}}=0, \\
&\left(\Upsilon_{\left.\boldsymbol{\eta}_{A}\right)_{\rho \sigma}}{ }^{\alpha}=-\frac{1}{T \zeta} \frac{1}{(d-1)^{2}} P_{\rho \sigma} \mathcal{X}_{\lambda}^{\lambda \alpha}-\frac{1}{2 T \eta} \mathcal{X}_{<\rho \sigma>}{ }^{\alpha},\right.
\end{aligned}
$$

together with the following first order correction to the intertwiners (5.18):

$$
\eta_{(1)}^{\mu \nu \rho \sigma}=\frac{1}{2}\left(\mathcal{N}^{(\mu \nu)(\rho \sigma)}+\mathcal{N}^{(\rho \sigma)(\mu \nu)}\right), \quad \sigma_{(1)}^{\alpha \beta}=\mathcal{S}^{(\alpha \beta)},
$$

where $\left\{\mathcal{N}^{\mu \nu \alpha \beta}, \mathcal{S}^{\alpha \beta}\right\}$ are arbitrary tensors involving one derivative. From (5.19) and intertwiners $\left\{\left(\boldsymbol{\eta}_{(0)}+\boldsymbol{\eta}_{(1)}\right)^{\mu \nu \rho \sigma},\left(\boldsymbol{\sigma}_{(0)}+\boldsymbol{\sigma}_{(1)}\right)^{\alpha \beta}\right\}$ we get a large set of Class D constitutive relations at second order:

$$
\begin{aligned}
\left(T^{\mu \nu}\right)_{\mathrm{D}} & \equiv\left(-2 \eta \sigma^{\mu \nu}-\zeta \Theta P^{\mu \nu}\right)-\frac{1}{4}\left(\mathcal{N}^{(\mu \nu)(\alpha \beta)}+\mathcal{N}^{(\alpha \beta)(\mu \nu)}\right) \delta_{\mathfrak{B}} g_{\alpha \beta}+\mathcal{X}^{(\mu \nu) \alpha} \cdot \delta_{\mathcal{B}} A_{\alpha}+\mathcal{O}\left(\partial^{3}\right) \\
\left(J^{\alpha}\right)_{\mathrm{D}} & \equiv\left(\sigma_{\mathrm{Ohm}} \mathfrak{v}^{\alpha}\right)+\frac{1}{2} \mathcal{X}^{(\mu \nu) \alpha} \delta_{\mathcal{B}} g_{\mu \nu}-\mathcal{S}^{(\alpha \beta)} \cdot \delta_{\mathcal{B}} A_{\beta}+\mathcal{O}\left(\partial^{3}\right) \\
\left(\mathcal{G}^{\sigma}\right)_{\mathrm{D}} & =0
\end{aligned}
$$

As we will see in section 14.3, classifying first order transverse tensors $\left\{\mathcal{N}^{\mu \nu \alpha \beta}, \mathcal{X}^{\mu \nu \alpha}, \mathcal{S}^{\alpha \beta}\right\}$ captures all Class D constitutive relations of the parity-even charged fluids at second order via (5.21).

\footnotetext{
${ }^{35}$ Explicitly, the projector is given by $X^{<\alpha \beta>}=\left(P_{\mu}^{\alpha} P_{\nu}^{\beta}-\frac{1}{d-1} P^{\alpha \beta} P_{\mu \nu}\right) X^{\mu \nu}$.
} 
At any given order in derivatives we can construct further constitutive relations by considering non-trivial $\Upsilon$-tensors in such a way that the entropy production (5.15) is manifestly a quadratic form. For example, a simple subset of Class D constitutive relations at any order is obtained by the parameterization (5.21) with arbitrary tensor structures $\left\{\mathcal{N}^{\mu \nu \rho \sigma}, \mathcal{S}^{\alpha \beta}\right\}$ for the entropy production of these terms automatically assembles itself in a quadratic form. Note that the contribution of the object $\mathcal{X}^{\mu \nu \alpha}$ is more complicated and it only takes the form of (5.21) at second order. At higher orders its mixing with other terms in (5.21) must be taken into account. With non-trivial choice of $\Upsilon$ we can easily obtain the descendant terms while composite-products are obtained by making judicious choices for the intertwiners $\{\boldsymbol{\eta}, \boldsymbol{\sigma}\}$ themselves. We discuss more examples where Class D transport shows up in section 14.3.

Before we conclude our discussion of dissipative terms, it is worth highlighting the following point. In most of the preceding analyses of hydrodynamics (such as the discussion of second order Weyl invariant neutral fluids in [64, 65], generic neutral fluids in [2], or charged fluids in [37]) the modus operandi has always been to down the constitutive relations for the conserved currents at a given order in the gradient expansion. One then ascertains the form of the entropy current which ensures that the second law of thermodynamics is upheld. Usually the entropy currents are determined modulo certain ambiguities (see below). However, to ensure that the entropy production is sign-definite $\Delta \geq 0$, say by completing the contributions into a positive definite quadratic form, one needs to understand contributions to the conserved currents at higher orders as well. This is most clearly illustrated in the neutral fluid analysis of [2]. One of the advantages of working with the operators $\Upsilon$ and the intertwiners $\{\boldsymbol{\eta}, \boldsymbol{\sigma}\}$ is that we can in one fell swoop ascertain the combination of transport that ensures $\Delta \geq 0$. This makes the analysis at higher orders much more straightforward.

We note that when we shift the entropy current by

$$
J_{S}^{\mu} \mapsto J_{S}^{\mu}+\frac{1}{\sqrt{-g}} £_{V}\left[\sqrt{-g} J_{S}^{\mu}\right]=J_{S}^{\mu}+V^{\mu} D_{\sigma} J_{S}^{\sigma}+D_{\nu}\left(V^{\nu} J_{S}^{\mu}-J_{S}^{\nu} V^{\mu}\right)
$$

the amount of entropy produced $\Delta$ shifts by a Lie derivative $\Delta \mapsto \Delta+\frac{1}{\sqrt{-g}} £_{V}[\sqrt{-g} \Delta]$ which preserves the condition $\Delta \geq 0$. Thus such a shift moves us within the space of admissible Class D constitutive relations. In the holographic context this is reflected in the pullback ambiguity in the construction of the entropy current [64].

The explicit construction of Class D constitutive relations in section 5.2 establishes the main statement we made at the end of section 5.1; all of the product-composite and descendant scalar operators can be assembled into a form where the only operative restriction from the second law of thermodynamics applies at $\Delta_{2}$ order. Thus as claimed in $[36,37$ the only inequality constraints of interest arising from the second law operate at the leading order in the gradient expansion. All higher order terms can be subsumed via the derivative operators into the sub-dissipative Class $\mathrm{D}_{s}$.

The reader interested solely in the constraints arising from the second law can stop here. As described in [36] once we figured out the terms in Classes $\mathrm{H}_{F}$ and $\mathrm{D}_{v}$, one is done. There is no more information from the second law. However, those intrepid souls willing to brave the winding pathways of the eightfold way are encouraged to continue onto part II. 


\section{Part II}

\section{The classification of adiabatic constitutive relations}

\section{Class L: Lagrangian solutions to adiabaticity equation}

Following the analysis of section 4 we have seen that the set of adiabatic fluids is much larger than just the ideal fluid family. In principle, we could continue on from our analysis at zero derivative level as in section 2.3, and solve the adiabaticity equation at higher derivative orders, to find new families of adiabatic constitutive relations. In practice, however, the number of terms proliferates very fast and the analysis becomes complicated. Hence, one therefore would like to seek more practical ways of solving adiabaticity equation or writing down adiabatic constitutive relations. The most elegant solution would be to mimic our discussion in hydrostatics (Class $\mathrm{H}$ ) and construct the generating function for the adiabatic constitutive relations, consistent with our desire of being off-shell and off-equilibrium.

In this section, we will describe a method to generate a large class of adiabatic constitutive relations in the absence of anomalies. Though this does not give all possible solutions, at any given derivative order, many solutions seem to fall into this class. We will call this class of adiabatic constitutive relations as Class L (where L stands for Lagrangianderivable) as one can find a local Lagrangian or Landau-Ginzburg free energy functional which succinctly encodes the constitutive relations. As presaged this will be quite close to the Euclidean partition function in hydrostatics. In particular, observe that in Class $\mathrm{H}_{S}$, the generating functional was given by the longitudinal part of the free energy current. That is, the natural decomposition of any covariant free energy current,

$$
\mathcal{G}^{\mu}=\mathfrak{S} \boldsymbol{\beta}^{\mu}+\mathfrak{V}^{\mu}, \quad \mathfrak{V}^{\mu} \boldsymbol{\beta}_{\mu}=0
$$

gives a natural scalar object $\mathfrak{S}$ which in hydrostatics took the role of the partition function scalar density $P_{S}\left[\mathbf{\Psi}_{\mathcal{K}}\right]$ in (4.10). If we consider the full set of scalar invariants (up to field redefinitions), including ones that vanish in equilibrium, then we can write down an off-shell Lagrangian density $\mathcal{L} \sim f_{\mathfrak{S}} \mathfrak{S}$ which parameterizes Class L. The non-hydrostatic part of this construction comprises of those scalars which identically vanish in equilibrium. All in all such Lagrangian densities will completely parameterize the longitudinal part of the free energy current in (6.1).

Let us now carry out this construction in detail. Constitutive relations in Class L are parametrized by a Lagrangian density $\mathcal{L}\left[g_{\mu \nu}, A_{\mu}, \boldsymbol{\beta}^{\mu}, \Lambda_{\boldsymbol{\beta}}\right]$ which we will assume to be a local scalar functional of its arguments, i.e., under gauge transformations and diffeomorphisms $\mathcal{L}$ transforms like a scalar field. Intuitively, $\mathcal{L}$ can be thought of as some sort of a generalized 
pressure functional for the adiabatic fluid. ${ }^{36}$ We may write

$$
S_{\text {hydro }}=\int d^{d} x \sqrt{-g} \mathcal{L}[\mathbf{\Psi}] .
$$

Consider now a variation of this Lagrangian functional which, after sufficient number of integration by parts, can be brought to the form

$$
\begin{aligned}
\frac{1}{\sqrt{-g}} \delta & (\sqrt{-g} \mathcal{L})-\nabla_{\mu}\left(\Varangle \Theta_{\mathrm{PS}}\right)^{\mu} \\
& =\frac{1}{2} T^{\mu \nu} \delta g_{\mu \nu}+J^{\mu} \cdot \delta A_{\mu}+T \mathfrak{h}_{\sigma} \delta \boldsymbol{\beta}^{\sigma}+T \mathfrak{n} \cdot\left(\delta \Lambda_{\boldsymbol{\beta}}+A_{\sigma} \delta \boldsymbol{\beta}^{\sigma}\right)
\end{aligned}
$$

Here $\left(\$ \Theta_{\mathrm{PS}}\right)^{\mu}$ denotes the surface terms generated due to integration by parts and is related to the pre-symplectic potential. The symbol $\phi$ denotes that it is linear in variations of fields. The reader may simply take (6.3) as the defining statement of the variational principle.

So far $\mathfrak{h}_{\sigma}$ and $\mathfrak{n}$ which multiply variations of the hydrodynamic fields (and are thus conjugate to them) are simply defined by the above variational principle; they will have a role to play in the sequel. We will refer to them as the adiabatic heat current and adiabatic charge density respectively.

The variation of the Lagrangian makes it easy to obtain the currents $\mathcal{C}_{\mathcal{H}}$. For instance we read off $\left\{T^{\mu \nu}, J^{\mu}\right\}$ from the above variation and take $J_{S}^{\mu}=s u^{\mu}$ with

$$
\left.s \equiv\left(\frac{1}{\sqrt{-g}} \frac{\delta}{\delta T} \int \sqrt{-g} \mathcal{L}[\mathbf{\Psi}]\right)\right|_{\left\{u^{\sigma}, \mu, g_{\alpha \beta}, A_{\alpha}\right\}=\text { fixed }}
$$

Here $\frac{\delta}{\delta T}$ is the variational (i.e., Euler-Lagrange) derivative. The free energy current can be obtained using (2.18). It is convenient to rewrite this expression in terms of the adiabatic currents $\left\{\mathfrak{h}_{\sigma}, \mathfrak{n}\right\}$ for simplification of future computations. Note that

$$
\begin{aligned}
T \mathfrak{h}_{\sigma} \delta \boldsymbol{\beta}^{\sigma}+T \mathfrak{n} \cdot\left(\delta \Lambda_{\boldsymbol{\beta}}+A_{\sigma} \delta \boldsymbol{\beta}^{\sigma}\right)= & \left(\mathfrak{h}_{\sigma}+\mathfrak{n} \cdot A_{\sigma}\right) \delta u^{\sigma}+\mathfrak{n} \cdot \delta\left(\mu-u^{\sigma} A_{\sigma}\right) \\
& -\left[\mathfrak{h}_{\sigma} \boldsymbol{\beta}^{\sigma}+\mathfrak{n} \cdot\left(\Lambda_{\boldsymbol{\beta}}+A_{\sigma} \boldsymbol{\beta}^{\sigma}\right)\right] \delta T
\end{aligned}
$$

which in turn implies that

$$
\begin{gathered}
s=-\left[\mathfrak{h}_{\sigma} \boldsymbol{\beta}^{\sigma}+\mathfrak{n} \cdot\left(\Lambda_{\boldsymbol{\beta}}+A_{\sigma} \boldsymbol{\beta}^{\sigma}\right)\right]=-\frac{1}{T}\left[\mathfrak{h}_{\sigma} u^{\sigma}+\mathfrak{n} \cdot \mu\right] \\
\Longrightarrow \quad T s+\mu \cdot \mathfrak{n}+u^{\sigma} \mathfrak{h}_{\sigma}=0 .
\end{gathered}
$$

In the above and in what follows, we will often want to convert general variations of hydrodynamic fields $\left\{u^{\sigma}, T, \mu\right\}$ in terms of variations of $\left\{\boldsymbol{\beta}^{\mu}, \Lambda_{\boldsymbol{\beta}}\right\}$ and vice versa. This can readily be done by using the defining equation (2.1) and explicit expressions can be found in (E.1) for convenience. In sum from $\mathcal{L}$ we have access to both the physical and adiabatic currents respectively, with the latter determining the entropy current.

\footnotetext{
${ }^{36}$ We will later see that upon restricting to hydrostatic configurations, $\mathcal{L}$ reduces to the hydrostatic partition function $W_{\text {Hydrostatic }}$ which suggests this intuition.
} 


\subsection{Bianchi identities in Class $\mathrm{L}$}

The invariance of $S_{\text {hydro }}$ under gauge/diffeomorphisms implies certain identities obeyed by $\left\{T_{\mu \nu}, J_{\mu}, \mathfrak{h}_{\sigma}, \mathfrak{n}\right\} .{ }^{37}$ Interpreting these identities in a particular manner will prove conducive to showing that having a Lagrangian $\mathcal{L}[\boldsymbol{\Psi}]=\mathcal{L}\left[g_{\mu \nu}, A_{\mu}, \boldsymbol{\beta}^{\mu}, \Lambda_{\boldsymbol{\beta}}\right]$ leads to currents which solve the adiabaticity equation.

To see this, consider the diffeomorphism and gauge variations induced by a pair of arbitrary vector field and scalar collectively denoted as $X \equiv\left\{\xi^{\mu}, \Lambda\right\}$ on the basic hydrodynamic fields:

$$
\begin{aligned}
& \delta_{x} g_{\mu \nu} \equiv £_{\xi} g_{\mu \nu}=\nabla_{\mu} \xi_{\nu}+\nabla_{\nu} \xi_{\mu} \\
& \delta_{x} A_{\mu} \equiv £_{\xi} A_{\mu}+\left[A_{\mu}, \Lambda\right]+\partial_{\mu} \Lambda=D_{\mu}\left[\Lambda+\xi^{\nu} A_{\nu}\right]+\xi^{\nu} F_{\nu \mu} \\
& \delta_{x} \boldsymbol{\beta}^{\mu} \equiv £_{\xi} \boldsymbol{\beta}^{\mu}=\xi^{\nu} \nabla_{\nu} \boldsymbol{\beta}^{\mu}-\boldsymbol{\beta}^{\nu} \nabla_{\nu} \xi^{\mu} \\
& \delta_{x} \Lambda_{\boldsymbol{\beta}}+A_{\nu} \delta_{x} \boldsymbol{\beta}^{\nu} \equiv \xi^{\mu} D_{\mu}\left[\Lambda_{\boldsymbol{\beta}}+\boldsymbol{\beta}^{\nu} A_{\nu}\right]-\boldsymbol{\beta}^{\mu} D_{\mu}\left[\Lambda+\xi^{\nu} A_{\nu}\right] \\
&-\xi^{\mu} \boldsymbol{\beta}^{\nu} F_{\mu \nu}+\left[\Lambda_{\boldsymbol{\beta}}+\boldsymbol{\beta}^{\nu} A_{\nu}, \Lambda+\xi^{\lambda} A_{\lambda}\right]
\end{aligned}
$$

where the symbol $£_{\xi}$ denotes the Lie derivative along the vector field $\xi^{\mu}$.

Plugging this variation into the expression appearing on the r.h.s. of (6.3) followed by a straightforward integration by parts gives

$$
\begin{aligned}
& \frac{1}{2} T^{\mu \nu} \delta_{x} g_{\mu \nu}+J^{\mu} \cdot \delta_{x} A_{\mu}+T \mathfrak{h}_{\mu} \delta_{x} \boldsymbol{\beta}^{\mu}+T \mathfrak{n} \cdot\left(\delta_{x} \Lambda_{\boldsymbol{\beta}}+A_{\mu} \delta_{x} \boldsymbol{\beta}^{\mu}\right) \\
& =\nabla_{\mu} \mathrm{N}^{\mu}[X]+\xi_{\mu}\left[-\nabla_{\nu} T^{\mu \nu}+J_{\nu} \cdot F^{\mu \nu}+\frac{g^{\mu \nu}}{\sqrt{-g}} \delta_{\mathcal{B}}\left(\sqrt{-g} T \mathfrak{h}_{\nu}\right)+g^{\mu \nu} T \mathfrak{n} \cdot \delta_{\mathcal{B}} A_{\nu}\right] \\
& \quad+\left(\Lambda+\xi^{\lambda} A_{\lambda}\right) \cdot\left[-D_{\nu} J^{\nu}+\frac{1}{\sqrt{-g}} \delta_{\mathcal{B}}(\sqrt{-g} T \mathfrak{n})\right]
\end{aligned}
$$

with $\mathrm{N}^{\mu}[X]$ defined as

$$
\mathrm{N}^{\mu}[X] \equiv \xi_{\nu} T^{\mu \nu}+\left(\Lambda+\xi^{\lambda} A_{\lambda}\right) \cdot J^{\mu}-T \boldsymbol{\beta}^{\mu}\left[\xi^{\nu} \mathfrak{h}_{\nu}+\left(\Lambda+\xi^{\lambda} A_{\lambda}\right) \cdot \mathfrak{n}\right]
$$

We have denoted the diffeomorphism and gauge variation induced by $\left\{\boldsymbol{\beta}^{\mu}, \Lambda_{\boldsymbol{\beta}}\right\}$ as $\delta_{\mathcal{B}}$ which is defined in (2.22). The Noether current $\mathrm{N}^{\mu}[\mathcal{B}]$ will play a role in constructing the noncanonical part of the entropy current or equivalently the free energy current. ${ }^{38}$

\footnotetext{
${ }^{37}$ The material in this subsection was worked out in collaboration with Kristan Jensen.

${ }^{38}$ In the process of deriving various variational expressions we find the following identities quite useful to collect terms:

$$
\frac{1}{\sqrt{-g}} \delta_{\mathcal{B}}(\sqrt{-g} S)=\nabla_{\alpha}\left(\boldsymbol{\beta}^{\alpha} S\right), \quad \frac{1}{\sqrt{-g}} \delta_{\mathcal{B}}\left(\sqrt{-g} V_{\sigma}\right)=£_{\boldsymbol{\beta}} V_{\sigma}+V_{\sigma} \nabla_{\alpha} \boldsymbol{\beta}^{\alpha}
$$

where $S$ and $V_{\alpha}$ are arbitrary scalar and one-form fields, respectively. We have made use of the latter in derving (6.8) and will use the former in, e.g., (6.13).
} 
We can now plug the expression (6.8) into (6.3) and integrate over the background geometry to obtain a statement for the total variation

$$
\begin{aligned}
\delta_{x} \int \sqrt{-g} \mathcal{L}[\Psi]= & \int \sqrt{-g} \xi_{\mu}\left[-\nabla_{\nu} T^{\mu \nu}+J_{\nu} \cdot F^{\mu \nu}+\frac{g^{\mu \nu}}{\sqrt{-g}} \delta_{\mathcal{B}}\left(\sqrt{-g} T \mathfrak{h}_{\nu}\right)+g^{\mu \nu} T \mathfrak{n} \cdot \delta_{\mathcal{B}} A_{\nu}\right] \\
& +\int \sqrt{-g}\left(\Lambda+\xi^{\lambda} A_{\lambda}\right) \cdot\left[-D_{\nu} J^{\nu}+\frac{1}{\sqrt{-g}} \delta_{\mathcal{B}}(\sqrt{-g} T \mathfrak{n})\right] \\
& + \text { Boundary terms. }
\end{aligned}
$$

Since $\mathcal{L}$ is a scalar under the background diffeomorphism and gauge transformation, the integral on the l.h.s. has to vanish, $\delta_{x} S_{\text {hydro }}=0$, up to boundary terms. This immediately implies for arbitrary $\left\{\xi^{\mu}, \Lambda\right\}$ one has the diffeomorphism and gauge Bianchi identities:

$$
\begin{aligned}
\nabla_{\nu} T^{\mu \nu} & =J_{\nu} \cdot F^{\mu \nu}+\frac{g^{\mu \nu}}{\sqrt{-g}} \delta_{\mathcal{B}}\left(\sqrt{-g} T \mathfrak{h}_{\nu}\right)+g^{\mu \nu} T \mathfrak{n} \cdot \delta_{\mathcal{B}} A_{\nu} \\
D_{\sigma} J^{\sigma} & =\frac{1}{\sqrt{-g}} \delta_{\mathcal{B}}(\sqrt{-g} T \mathfrak{n})
\end{aligned}
$$

These are the Bianchi identities we are after and per se they hold off-shell. If we think of $\left\{T^{\mu \nu}, J^{\mu}, \mathfrak{h}_{\sigma}, \mathfrak{n}\right\}$ as tensor-valued functionals of $\boldsymbol{\Psi}$, obtained from the variational principle (6.3), then these equations hold identically for the currents for all configurations.

We can supplement (6.12) with another identity which follows from our definition of the entropy current (6.4)

$$
\nabla_{\sigma} J_{S}^{\sigma}=\nabla_{\sigma}\left(T s \boldsymbol{\beta}^{\sigma}\right)=\frac{1}{\sqrt{-g}} \delta_{\mathcal{B}}(\sqrt{-g} T s)
$$

which is again valid off-shell.

We can now easily check that (6.12) and (6.13) together imply the adiabaticity equation (2.13) in the absence of anomalies, for

$$
\begin{aligned}
\nabla_{\mu} J_{S}^{\mu}+\boldsymbol{\beta}_{\mu}\left(\nabla_{\nu} T^{\mu \nu}-J_{\nu} \cdot F^{\mu \nu}\right)+\left(\Lambda_{\boldsymbol{\beta}}+\boldsymbol{\beta}^{\lambda} A_{\lambda}\right) \cdot D_{\nu} J^{\nu} \\
=\frac{1}{\sqrt{-g}}\left[\delta_{\mathcal{B}}(\sqrt{-g} T s)+\boldsymbol{\beta}^{\sigma} \delta_{\mathcal{B}}\left(\sqrt{-g} T \mathfrak{h}_{\sigma}\right)\right. \\
\left.\quad+\sqrt{-g} T \boldsymbol{\beta}^{\sigma} \mathfrak{n} \cdot \delta_{\mathcal{B}} A_{\sigma}+\frac{\mu}{T} \cdot \delta_{\mathcal{B}}(\sqrt{-g} T \mathfrak{n})\right] \\
=\frac{1}{\sqrt{-g}} \delta_{\mathcal{B}}\left(\sqrt{-g}\left[T s+u^{\sigma} \mathfrak{h}_{\sigma}+\mu \cdot \mathfrak{n}\right]\right) \\
=0 .
\end{aligned}
$$

We have used the basic definitions (2.1) and the relation (6.6) derived earlier. We should emphasize that by virtue of the Bianchi identities (6.12) holding off-shell we have demonstrated that the Lagrangian system defined by $\mathcal{L}[\mathbf{\Psi}]$ satisfies the non-anomalous adiabaticity equation (2.13) off-shell. We will postpone a more detailed discussion of the anomalous situation until section 12; suffice it to say for now that there is a Lagrangian construction that gives a particular solution to (2.12). 
Sometimes it is convenient to write the combinations that occur above in a conventional hydrodynamic expansion. Upon explicit evaluation one finds

$$
\begin{aligned}
\frac{1}{\sqrt{-g}} & \delta_{\mathcal{B}}\left(\sqrt{-g} T \mathfrak{h}_{\sigma}\right)+T \mathfrak{n} \cdot \delta_{\mathcal{B}} A_{\sigma} \\
& =\nabla_{\lambda}\left(\mathfrak{h}_{\sigma} u^{\lambda}\right)+\mathfrak{h}_{\lambda}\left(\nabla_{\sigma}+\mathfrak{a}_{\sigma}\right) u^{\lambda}+s\left(\nabla_{\sigma}+\mathfrak{a}_{\sigma}\right) T-\mathfrak{n} \cdot\left[E_{\sigma}-D_{\sigma} \mu-\mathfrak{a}_{\sigma} \mu\right]
\end{aligned}
$$

and

$$
\frac{1}{\sqrt{-g}} \delta_{\mathcal{B}}(\sqrt{-g} T \mathfrak{n})=D_{\sigma}\left(\mathfrak{n} u^{\sigma}\right)+[\mathfrak{n}, \mu]
$$

In the above expressions we encounter the fluid acceleration vector $\mathfrak{a}_{\sigma}$ and the rest frame electric field $E_{\sigma}=F_{\sigma \lambda} u^{\lambda}$ introduced earlier.

\subsection{Noether current in Class L}

Having seen that Lagrangian systems of hydrodynamics as formulated above satisfy adiabaticity equation off-shell, we now proceed to extract some more basic lessons. Most of these follow from the basic variational principle and are encoded in the Noether current for the Class L constitutive relations which is related to the free energy current of the system.

We proceed by first deriving the Noether theorem for our Lagrangian system. By substituting (6.12) into (6.8), we get

$$
\nabla_{\mu} \mathrm{N}^{\mu}[X]=\frac{1}{2} T^{\mu \nu} \delta_{x} g_{\mu \nu}+J^{\mu} \cdot \delta_{x} A_{\mu}+T \mathfrak{h}_{\mu} \delta_{x} \boldsymbol{\beta}^{\mu}+T \mathfrak{n} \cdot\left(\delta_{x} \Lambda_{\boldsymbol{\beta}}+A_{\mu} \delta_{x} \boldsymbol{\beta}^{\mu}\right)
$$

with $\mathrm{N}^{\mu}[X]$ as given in (6.9). The primary content of Noether theorem is that a current $\mathrm{N}^{\mu}[X]$ satisfying the above equation exists.

It is easy to see that every Noether current satisfying (6.17) gives a free energy current satisfying the adiabaticity equation $(2.21)$ with $\mathcal{G}_{H}^{\perp}=0$ (for non-anomalous fluids). In particular, we see that we solve (2.21) by identifying $\left\{\xi^{\mu}, \Lambda\right\}=\left\{\boldsymbol{\beta}^{\mu}, \Lambda_{\boldsymbol{\beta}}\right\}$ (but we will still keep $\left\{g_{\mu \nu}, A_{\mu}\right\}$ general) and take

$$
\begin{aligned}
\mathcal{G}^{\sigma} & =-T \mathrm{~N}^{\sigma}[\mathcal{B}] \\
& =-T\left(\boldsymbol{\beta}_{\nu} T^{\sigma \nu}+\left(\Lambda_{\boldsymbol{\beta}}+\boldsymbol{\beta}^{\lambda} A_{\lambda}\right) \cdot J^{\sigma}-T \boldsymbol{\beta}^{\sigma}\left[\boldsymbol{\beta}^{\nu} \mathfrak{h}_{\nu}+\left(\Lambda_{\boldsymbol{\beta}}+\boldsymbol{\beta}^{\lambda} A_{\lambda}\right) \cdot \mathfrak{n}\right]\right) \\
\mathcal{G}_{H}^{\perp} & =0 .
\end{aligned}
$$

Thus we see that the free energy current coincides (up to a factor of $T$ ) with the Noether current (or the non-canonical part of the entropy current) $\mathrm{N}^{\sigma}[\mathcal{B}]$, cf., (2.18).

The corresponding entropy current is also easily constructed: we remind the reader that the non-canonical part of the entropy current is $-\mathcal{G}^{\sigma} / T=\mathrm{N}^{\sigma}[\mathcal{B}]$ so that the total entropy current is given by

$$
\begin{aligned}
J_{S}^{\sigma} & =\mathrm{N}^{\sigma}[\mathcal{B}]-\boldsymbol{\beta}_{\lambda} T^{\sigma \lambda}-\left(\Lambda_{\boldsymbol{\beta}}+\boldsymbol{\beta}^{\lambda} A_{\lambda}\right) \cdot J^{\sigma} \\
& =\mathrm{N}^{\sigma}[\mathcal{B}]-\frac{u_{\lambda}}{T} T^{\sigma \lambda}-\frac{\mu}{T} \cdot J^{\sigma} \\
& =-T \boldsymbol{\beta}^{\sigma}\left[\boldsymbol{\beta}^{\nu} \mathfrak{h}_{\nu}+\left(\Lambda_{\boldsymbol{\beta}}+\boldsymbol{\beta}^{\lambda} A_{\lambda}\right) \cdot \mathfrak{n}\right] .
\end{aligned}
$$


Thus, the choice of free energy/entropy currents is in one to one correspondence with the choice of the Noether current, consistent with our identification in (6.6).

Let us now try to get an alternate expression for $\mathrm{N}^{\mu}[X]$ which will be useful later on. We have from (6.3) and (6.17) the simple identity

$$
\begin{aligned}
\nabla_{\mu} \mathrm{N}^{\mu}[X] & =\frac{1}{\sqrt{-g}} \delta_{x}(\sqrt{-g} \mathcal{L})-\nabla_{\mu}\left(\phi_{x} \Theta_{\mathrm{PS}}\right)^{\mu} \\
& =\nabla_{\mu}\left[\xi^{\mu} \mathcal{L}-\left(\phi_{x} \Theta_{\mathrm{PS}}\right)^{\mu}\right]
\end{aligned}
$$

where we have assumed that $\mathcal{L}$ transforms as a scalar. This shows that the vector $\xi^{\mu} \mathcal{L}-$ $\left(\oiint_{x} \Theta_{\mathrm{PS}}\right)^{\mu}$ (which is often called the canonical Noether current) has the same divergence as $\mathrm{N}^{\mu}[X]$. Assuming there are no cohomological obstructions, we can then write

$$
\mathrm{N}^{\mu}[X]=\xi^{\mu} \mathcal{L}-\left(\phi_{X} \Theta_{\mathrm{PS}}\right)^{\mu}+\nabla_{\nu} \mathcal{K}^{\mu \nu}[X]
$$

where $\mathcal{K}^{\mu \nu}[X]=-\mathcal{K}^{\nu \mu}[X]$ is called the Komar charge of the system. We will call this decomposition of $\mathrm{N}^{\mu}[X]$ as Komar decomposition. This gives an alternate expression for free energy current as

$$
\mathcal{G}^{\sigma}=-T \mathrm{~N}^{\sigma}[\mathcal{B}]=-T\left(\boldsymbol{\beta}^{\sigma} \mathcal{L}-\left(\oiint_{\mathcal{B}} \Theta_{\mathrm{PS}}\right)^{\sigma}+\nabla_{\nu} \mathcal{K}^{\sigma \nu}[\mathcal{B}]\right)
$$

and

$$
\begin{aligned}
J_{S}^{\mu} & =s u^{\mu} \\
& =-\boldsymbol{\beta}_{\nu} T^{\mu \nu}-\left(\Lambda_{\boldsymbol{\beta}}+\boldsymbol{\beta}^{\lambda} A_{\lambda}\right) \cdot J^{\mu}+\mathrm{N}^{\mu}[\mathcal{B}] \\
& =-\boldsymbol{\beta}_{\nu} T^{\mu \nu}-\left(\Lambda_{\boldsymbol{\beta}}+\boldsymbol{\beta}^{\lambda} A_{\lambda}\right) \cdot J^{\mu}+\boldsymbol{\beta}^{\mu} \mathcal{L}-\left(\phi_{\mathcal{B}} \Theta_{\mathrm{PS}}\right)^{\mu}+\nabla_{\nu} \mathcal{K}^{\mu \nu}[\mathcal{B}]
\end{aligned}
$$

Note that the pre-symplectic potential $\left(\phi_{\mathrm{PS}}\right)^{\mu}$ appears in these expressions only through $\left(\phi_{\mathcal{B}} \Theta_{\mathrm{PS}}\right)^{\mu}$. Since $\delta_{\mathfrak{B}} \boldsymbol{\beta}^{\mu}=0$ and $\delta_{\mathfrak{B}} \Lambda_{\boldsymbol{\beta}}=0$, this means to get the free energy current or the entropy current, we need not actually get the contributions to $\left(\phi_{\mathcal{B}} \Theta_{\mathrm{PS}}\right)^{\mu}$ from varying $\left\{\boldsymbol{\beta}^{\mu}, \Lambda_{\boldsymbol{\beta}}\right\}$. The contribution to the pre-symplectic potential can be obtained by just varying $\left\{g_{\mu \nu}, A_{\mu}\right\}$ and then see what we obtain when we integrate by parts.

\subsection{Hydrostatic partition function for Class $\mathrm{L}$}

Our discussion of the Class L solutions to the adiabaticity equation has so far been unconstrained, in that we have only assumed that the currents can be derived from a Lagrangian $\mathcal{L}[\boldsymbol{\Psi}]$. We now relate this story to the analysis of section 4 where we also derived the currents from a generating function. In order to ascertain the connection we now specialize to hydrostatics. Since we have an explicit expression for the free energy current in Class L, we can invoke the arguments that led to (4.6) to come up with a hydrostatic partition function for theories in Class L.

We will now argue that Class L provides an off-shell generalization for hydrostatics. Note however, that Class $\mathrm{L}$ can at best incorporate Class $\mathrm{H}_{S}$ as we are required to write the integral of a spacetime scalar density for the effective action $S_{\text {hydro }}$. 
First we constrain the sources to support a Killing field configuration $\mathcal{K} \equiv\left\{K^{\mu}, \Lambda_{K}\right\}$ which we will momentarily identify with $\mathcal{B}=\left\{\boldsymbol{\beta}^{\mu}, \Lambda_{\boldsymbol{\beta}}\right\}$. Further using (6.22) we can write on the base space $\Sigma_{E}$

$$
\begin{aligned}
W_{\text {Hydrostatic }} & =-\left[\int_{\Sigma_{E}} \frac{\mathcal{G}^{\sigma}}{T} d^{d-1} S_{\sigma}\right]_{\text {Hydrostatic }}=\left[\int_{\Sigma_{E}} \mathrm{~N}^{\sigma}[\mathcal{B}] d^{d-1} S_{\sigma}\right]_{\text {Hydrostatic }} \\
& =\left[\int_{\Sigma_{E}}\left(\boldsymbol{\beta}^{\sigma} \mathcal{L}-\left(\oiint_{\mathcal{B}} \Theta_{\mathrm{PS}}\right)^{\sigma}+\nabla_{\nu} \mathcal{K}^{\sigma \nu}[\mathcal{B}]\right) d^{d-1} S_{\sigma}\right]_{\text {Hydrostatic }} \\
& =\int_{\Sigma_{E}} \mathcal{L}_{\text {Hydrostatic }} K^{\sigma} d^{d-1} S_{\sigma}+\left[\int_{\partial \Sigma_{E}} \frac{1}{2} \mathcal{K}^{\sigma \nu}[\mathcal{B}] d^{d-2} S_{\sigma \nu}\right]_{\text {Hydrostatic }}
\end{aligned}
$$

where we have used the fact that $\delta_{\mathfrak{B}}$ annihilates functions in hydrostatics (4.2) to drop the $\left(\phi_{\mathcal{B}} \Theta_{\mathrm{PS}}\right)^{\sigma}$ contribution. Here, $\mathcal{L}_{\text {Hydrostatic }}$ denotes $\mathcal{L}[\boldsymbol{\Psi}]$ with $\left\{\boldsymbol{\beta}^{\mu}, \Lambda_{\boldsymbol{\beta}}\right\}$ replaced by $\left\{K^{\mu}, \Lambda_{K}\right\}$. We finally obtain

$$
W_{\text {Hydrostatic }}=\int_{\Sigma_{E} \times I_{K}} d^{d} x \sqrt{-g} \mathcal{L}_{\text {Hydrostatic }}+\text { Boundary contributions },
$$

where the integral is performed over the manifold $\Sigma_{E} \times I_{K}$ where $I_{K}$ is an interval of unit affine length along $K^{\mu}$. So in the end we get the simple result that the hydrostatic partition function is just the integral over the Lagrangian after taking the hydrostatic limit of $\mathcal{L}[\Psi]$.

Lest the reader be misled into thinking that we recover the complete set of hydrostatic partition functions (Class $\mathrm{H}$ ) from the Class L family of adiabatic fluids we hasten to add an important caveat alluded to earlier. It should be clear from (6.25) that we obtain from $\mathcal{L}[\Psi]$ only those hydrostatic partition functions that can be written as spacetime scalars, since we have an integral over the entire manifold $\mathcal{M}=\Sigma_{E} \times I_{K}$. This is what we called $\mathrm{H}_{S}$ in our discussion in section 4 . From the categorization explained there, there are two other classes of terms in the partition function which do not obviously arise from Class L Lagrangians: the Class $\mathrm{H}_{V}$ terms involving integrals over transverse vectors and the Class A terms which play a role in anomalous hydrodynamic transport. ${ }^{39}$ Thus, apart from these terms (which seem to be a finite set of terms in any spacetime dimension) we recover most of the theory of partition functions and the adiabatic constitutive relations that they lead to.

Given the connections to hydrostatics, it is useful therefore to decompose Class L into explicit contributions from hydrostatics $\mathrm{H}_{S}$ and genuine hydrodynamic scalars $\overline{\mathrm{H}}_{S}$. To wit, one has $\mathrm{L}=\mathrm{H}_{S} \cup \overline{\mathrm{H}}_{S}$. The hydrodynamic scalars, which necessarily involve one $\delta_{\mathfrak{B}}$ insertion (by definition), are identically vanishing in equilibrium - they require us to turn on time dependence to contribute. Moreover, as a result the values of the hydrostatic scalars can be freely changed by contributions proportional to the Class $\overline{\mathrm{H}}_{S}$ terms. Hence, hydrostatic scalars $\mathrm{H}_{S} \subset \mathrm{L}$ take values in a coset: $\mathrm{H}_{S}=\mathrm{L} / \overline{\mathrm{H}}_{S}$.

\footnotetext{
${ }^{39}$ Since we have focused on Lagrangian solutions to non-anomalous adiabaticity equation (2.13) it is not surprising that we have not yet encountered Class A. We will encounter Class A terms when we turn to a detailed discussion of anomalies in section 12 .
} 
Finally, we can make a precise connection between the Noether current construction outlined in section 6.2 and the entropy current constrained by hydrostatics. As explained in section 4 the hydrostatic entropy current has been studied in some detail in [36, 37]. As we now understand, varying (6.25) with respect to the metric and gauge field (we do not vary $\left\{\boldsymbol{\beta}^{\mu}, \Lambda_{\boldsymbol{\beta}}\right\}$ since they are fixed to $\left\{K^{\mu}, \Lambda_{K}\right\}$ in the hydrostatic limit) we obtain

$$
\begin{aligned}
\delta W_{\text {Hydrostatic }}= & \int d^{d} x \sqrt{-g}\left[\frac{1}{2} T^{\mu \nu} \delta g_{\mu \nu}+J^{\sigma} \cdot \delta A_{\sigma}\right]_{\text {Hydrostatic }} \\
& + \text { Boundary contributions }
\end{aligned}
$$

which agrees with the rule given in [3]. Further, if we just keep the first order deviations from hydrostatics in the equation for the non-canonical part of the entropy current $\mathrm{N}^{\sigma}[\mathcal{B}]=$ $\boldsymbol{\beta}^{\sigma} \mathcal{L}-\left(\phi_{\mathcal{B}} \Theta_{\mathrm{PS}}\right)^{\sigma}+\nabla_{\nu} \mathcal{K}^{\sigma \nu}[\mathcal{B}]$, we get the prescription given in [37]. This was explained in detail in section 4.2 and we elaborate on the connections further in appendix G.

\section{Hydrodynamic Ward identities in Class L}

Up to this point our discussion of Class L has been quite abstract. We have only exploited the diffeomorphism and gauge symmetry to extract the Bianchi identities (6.12), which in turn led to the adiabaticity equation. As such we have in fact been treating the hydrodynamic fields $\left\{\boldsymbol{\beta}^{\mu}, \Lambda_{\boldsymbol{\beta}}\right\}$ effectively as non-dynamical fields, thus working off-shell as far as the hydrodynamic fields are concerned. The only exception is the hydrostatic limit studied in section 6.3 , where we went on-shell by simply setting $\left\{\boldsymbol{\beta}^{\mu}, \Lambda_{\boldsymbol{\beta}}\right\}=\left\{K^{\mu}, \Lambda_{K}\right\}$ and invoking the hydrostatic principle.

This is clearly unsatisfactory; the utility of a Lagrangian is that it not only allows us to construct an off-shell action, but that it also comes equipped with a variational principle that captures the on-shell dynamics by giving us the equations of motion. We will now proceed to address this lacunae and give a variational procedure to go on-shell. Our goal is to simply to give the hydrodynamic fields $\left\{\boldsymbol{\beta}^{\mu}, \Lambda_{\boldsymbol{\beta}}\right\}$ appropriate dynamics which enforces the conservation equations in (2.6) (with $T_{H}^{\mu \perp}=J_{H}^{\perp}=0$ in the absence of anomalies).

\subsection{A constrained variational principle for hydrodynamics}

Let us go back to the derivation of the Bianchi type identities in section 6.1. Inspection of the Bianchi identities (6.12) which are obeyed by all Class L constitutive relations suggests that on-shell equations of non-anomalous hydrodynamics (2.6) would be satisfied (with anomaly terms set to zero) if the fields $\left\{\boldsymbol{\beta}^{\mu}, \Lambda_{\boldsymbol{\beta}}\right\}$ obeyed the following equations:

$$
\begin{aligned}
\frac{1}{\sqrt{-g}} \delta_{\mathcal{B}}\left(\sqrt{-g} T \mathfrak{h}_{\mu}\right)+T \mathfrak{n} \cdot \delta_{\mathcal{B}} A_{\mu} & \simeq 0, \\
\frac{1}{\sqrt{-g}} \delta_{\mathcal{B}}(\sqrt{-g} T \mathfrak{n}) & \simeq 0 .
\end{aligned}
$$

These equations have to arise for consistency of our formalism as the dynamical equations of motion obtained by varying the fields $\left\{\boldsymbol{\beta}^{\mu}, \Lambda_{\boldsymbol{\beta}}\right\}$. It is clear a-priori that this is 
not going to happen naturally; the basic variational equation (6.3) if interpreted naïvely would lead to $\mathfrak{h}_{\sigma}+\mathfrak{n} \cdot A_{\sigma}=0$ and $\mathfrak{n}=0$ (assuming $T \neq 0$ ), which is certainly not what we would like to have. The key point we have to understand is the following: given that the dynamical degrees of freedom comprise of a vector $\boldsymbol{\beta}^{\mu}$ and a scalar $\Lambda_{\boldsymbol{\beta}}$, we have to decide what variations of these fields to admit as being physical. Our argument above shows that an unconstrained variation of these fields is inconsistent with the dynamics we seek, so the question is whether a suitable constrained variational principle exists.

We would like to claim now that such a constrained variation of $\left\{\boldsymbol{\beta}^{\mu}, \Lambda_{\boldsymbol{\beta}}\right\}$ exists and it naturally leads to the correct hydrodynamic Ward identities upon using the Bianchi identities (6.12). To see how the desired equations can be obtained from a variational principle, consider the following: fix the metric and gauge field and extremize $S_{\text {hydro }}[\boldsymbol{\Psi}]$ among a family of $\mathcal{B}=\left\{\boldsymbol{\beta}^{\mu}, \Lambda_{\boldsymbol{\beta}}\right\}$ which are related to each other via Lie transport. We will denote this class of variations by $\delta$ to distinguish it from the variation we have considered hitherto without the Lie transport constraint.

To wit, given an arbitrary $X=\left\{\xi^{\mu}, \Lambda\right\}$ we define this constrained variation as:

$$
\delta: \quad \delta \boldsymbol{\beta}^{\mu}=\delta_{x} \boldsymbol{\beta}^{\mu}, \quad \delta \Lambda_{\boldsymbol{\beta}}=\delta_{x} \Lambda_{\boldsymbol{\beta}}, \quad \delta g_{\mu \nu}=\delta A_{\mu}=0 .
$$

Our claim is that the on-shell hydrodynamic configurations are precisely those $\left\{\boldsymbol{\beta}^{\mu}, \Lambda_{\boldsymbol{\beta}}\right\}$ which satisfy $\delta S_{\text {hydro }}[\mathbf{\Psi}]=0$ up to boundary contributions.

To show this, we use the definition of $\delta$, (7.2), in (6.3) to write

$$
\begin{aligned}
\frac{1}{\sqrt{-g}} \delta(\sqrt{-g} \mathcal{L})-\nabla_{\mu}\left(\phi_{\mathrm{PS}}\right)^{\mu} & =T \mathfrak{h}_{\sigma} \delta_{x} \boldsymbol{\beta}^{\sigma}+T \mathfrak{n} \cdot\left(\delta_{x} \Lambda_{\boldsymbol{\beta}}+A_{\sigma} \delta_{x} \boldsymbol{\beta}^{\sigma}\right) \\
& =-T \mathfrak{h}_{\sigma} \delta_{\mathfrak{B}} \xi^{\sigma}-T \mathfrak{n} \cdot\left(\delta_{\mathfrak{B}} \Lambda+A_{\sigma} \delta_{\mathfrak{B}} \xi^{\sigma}\right),
\end{aligned}
$$

where we have used $\delta_{x} \boldsymbol{\beta}=£_{\xi} \boldsymbol{\beta}=-£_{\boldsymbol{\beta}} \xi=-\delta_{\mathcal{B}} \xi$. Integrating the above equation by parts to move the derivatives over from the variational parameters $\left\{\xi^{\mu}, \Lambda\right\}$ results in

$$
\begin{aligned}
\frac{1}{\sqrt{-g}} \delta(\sqrt{-g} \mathcal{L}[\Psi])= & \left(\frac{1}{\sqrt{-g}} \delta_{\mathcal{B}}\left(\sqrt{-g} T \mathfrak{h}_{\sigma}\right)+T \mathfrak{n} \cdot \delta_{\mathfrak{B}} A_{\sigma}\right) \xi^{\sigma} \\
& +\frac{1}{\sqrt{-g}} \delta_{\mathcal{B}}[\sqrt{-g} T \mathfrak{n}] \cdot\left(\Lambda+A_{\sigma} \xi^{\sigma}\right) \\
& +\nabla_{\mu}\left\{\left(\phi \Theta_{\mathrm{PS}}\right)^{\mu}-T \boldsymbol{\beta}^{\mu}\left[\mathfrak{h}_{\sigma} \xi^{\sigma}+\mathfrak{n} \cdot\left(\Lambda+A_{\sigma} \xi^{\sigma}\right)\right]\right\} .
\end{aligned}
$$

Insisting that this be zero for arbitrary $X=\left\{\xi^{\mu}, \Lambda\right\}$ then directly leads to the required equations (7.1).

Thus as advertised Lagrangian theories of hydrodynamic fields equipped with a suitable variational principle, give rise to the correct hydrodynamic equations of motion and simultaneously provide an off-shell solution to the adiabaticity equation.

\subsection{Reference fields and conservation equations}

In section 7.1 we gave a constrained variational principle which enabled us to derive the hydrodynamic equations from the Lagrangian $\mathcal{L}[\Psi]$. While prescriptive, it is cumbersome 


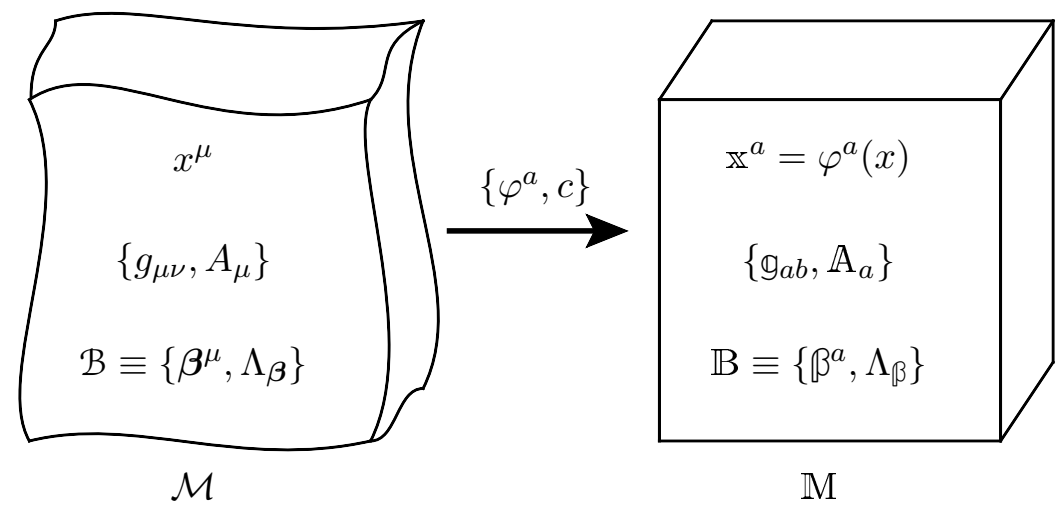

Figure 3. Illustration of the connection between the physical and reference fields for Class L adiabatic fluids. The fields on the physical spacetime manifold $\mathcal{M}$ are related to those on the reference manifold $\mathbb{M}$ by a pull-back using the dynamical fields $\left\{\varphi^{a}, c\right\}$. The constrained variation on $\mathcal{M}$ which gives the correct equations of motion corresponds to varying $\left\{\varphi^{a}, c\right\}$ while holding $\left\{\beta^{a}, \Lambda_{\beta}\right\}$ fixed.

to work in the space of constrained variations to derive dynamics. It is somewhat more satisfactory to shift to a description where these constraints are automatically implemented by an action, rather than being imposed by hand.

To do this, we need to decompose the variations of $\left\{\boldsymbol{\beta}^{\mu}, \Lambda_{\boldsymbol{\beta}}\right\}$ into those allowed by the constraint, and those in the orthogonal space of variations (which are forbidden by the constraint). The former lie in the Lie orbit of an admissible configuration. We can exploit this characterization in decomposing the degrees of freedom into the truly dynamical ones and the ones held rigid under the variation. To ascertain the physical space of variations, we pick a reference configuration $\left\{\beta^{\mu}, \Lambda_{\beta}\right\}$ in each Lie orbit and then express the actual $\left\{\boldsymbol{\beta}^{\mu}, \Lambda_{\boldsymbol{\beta}}\right\}$ by Lie dragging this reference configuration by a gauge transformation and diffeomorphism. We thus seek to decompose the hydrodynamic fields into

(i) A heavy component which is the reference configuration that one does not vary when extremizing (denoted by the blackboard bold font characters).

(ii) A light component which is given by the Lie drag modes that one varies when extremizing.

We begin by systematically first establishing a reference configuration. It is convenient to imagine that these reference configurations live on some other spacetime $\mathbb{M}$ which is gauge equivalent and diffeomorphic to the original spacetime. We will use the (first half of the) lowercase Latin alphabet to denote the spacetime indices on $\mathbb{M}$ to distinguish them from lowercase Greek indices used for the original spacetime $\mathcal{M}$.

Let $x^{\mu}$ be coordinates on $\mathcal{M}$ and $\mathbb{x}^{a}$ be coordinates on $\mathbb{M} . \mathbb{B} \equiv\left\{\beta^{a}, \Lambda_{\beta}\right\}$ be the reference hydrodynamic fields living on $\mathbb{M}$. The actual $\left\{\boldsymbol{\beta}^{\mu}, \Lambda_{\boldsymbol{\beta}}\right\}$ are obtained by introducing a diffeomorphism field $\varphi^{a}(x)$ and a gauge transformation field $c(x)$ from physical spacetime $\mathcal{M}$ to $\mathbb{M}$ and then using them to pull-back $\left\{\beta^{a}, \Lambda_{\beta}\right\}$. In order to do this, let us introduce the matrix $\partial_{\mu} \varphi^{a} \equiv \frac{\partial \varphi^{a}}{\partial x^{\mu}}$ and its inverse $e_{a}^{\mu} \equiv \frac{\partial x^{\mu}}{\partial \varphi^{a}}$ that can be used to pull-back tensor 
indices. For definiteness, let us think of these matrices as functions of $x$, viz., living on the actual spacetime $\mathcal{M}$. They satisfy

$$
e_{a}^{\mu} \partial_{\nu} \varphi^{a}=\delta_{\nu}^{\mu}, \quad e_{a}^{\mu} \partial_{\mu} \varphi^{b}=\delta_{b}^{a} .
$$

With this definition the pull-back of the reference configuration is given by

$$
\begin{aligned}
\boldsymbol{\beta}^{\mu} & =e_{a}^{\mu}(x) \mathbb{\beta}^{a}[\varphi(x)] \\
\Lambda_{\boldsymbol{\beta}} & =c(x) \Lambda_{\beta}[\varphi(x)] c^{-1}(x)+\boldsymbol{\beta}^{\sigma}(x) \partial_{\sigma} c(x) c^{-1}(x) .
\end{aligned}
$$

Note that $\Lambda_{\boldsymbol{\beta}}$ transforms with the correct inhomogeneous piece so that $\Lambda_{\boldsymbol{\beta}}+A_{\sigma} \boldsymbol{\beta}^{\sigma}$ transforms covariantly. More precisely, consider a flavour transformation $A_{\sigma} \mapsto g^{-1} A_{\sigma} g+$ $g^{-1} \partial_{\sigma} g$ and $\left(\Lambda_{\boldsymbol{\beta}}+A_{\sigma} \boldsymbol{\beta}^{\sigma}\right) \mapsto g^{-1}\left(\Lambda_{\boldsymbol{\beta}}+A_{\sigma} \boldsymbol{\beta}^{\sigma}\right) g$. It follows from the above expressions that this corresponds to a left transformation of $c$ given by $c \mapsto g^{-1} c$ with $\Lambda_{\beta}$ kept fixed.

The decomposition given in (7.6) means that changing $\left\{\varphi^{a}, c\right\}$ takes $\left\{\boldsymbol{\beta}^{\mu}, \Lambda_{\boldsymbol{\beta}}\right\}$ along a Lie orbit whereas changing the functional form of $\left\{\beta^{a}, \Lambda_{\beta}\right\}$ takes $\left\{\boldsymbol{\beta}^{\mu}, \Lambda_{\boldsymbol{\beta}}\right\}$ from one Lie orbit to another Lie orbit. So, in order to get the hydrodynamic equations, we should extremize $S_{\text {hydro }}[\boldsymbol{\Psi}]$ with respect to variations of the $\left\{\varphi^{a}, c\right\}$ fields keeping the functional form of $\left\{\beta^{a}, \Lambda_{\beta}\right\}$ fixed. See figure 3 for an illustration of the situation.

It is easy to intuit how this has to work in principle. We simply have to consider the variation of the relation (7.6) between the physical fields and the reference parameterization introduced above. We would then plug this into (6.3) to ascertain the variations of the Lagrangian in the physically admissible directions. To see how this actually works in practice, let us begin by recording out the variation of $\boldsymbol{\beta}^{\mu}$ and $\Lambda_{\boldsymbol{\beta}}$ in terms of the reference and physical fields. Relegating the details of the actual computation to appendix E we quote here the final result of import:

$$
\begin{aligned}
& \delta \boldsymbol{\beta}^{\mu}=e_{a}^{\mu} \delta \beta^{a}-\delta_{\mathcal{B}}\left(e_{a}^{\mu} \delta \varphi^{a}\right) \\
& \delta \Lambda_{\boldsymbol{\beta}}=c \delta \Lambda_{\beta} c^{-1}+e_{a}^{\sigma} \delta \beta^{a}\left(\partial_{\sigma} c\right) c^{-1}+\delta_{\mathcal{B}}\left[\delta c c^{-1}-e_{a}^{\sigma} \delta \varphi^{a} \partial_{\sigma} c c^{-1}\right],
\end{aligned}
$$

where the variations of $\left\{\varphi^{a}, c\right\}$ appear as variations along Lie orbit as we wanted.

We can now substitute the above equation into (6.3). Once again we have to do an integration by parts to isolate the terms proportional to the dynamical degrees of freedom. After a bit of algebra we get

$$
\begin{aligned}
\frac{1}{\sqrt{-g}} \delta & (\sqrt{-g} \mathcal{L})-\nabla_{\mu}\left\{\left(\not \Theta_{\mathrm{PS}}\right)^{\mu}+u^{\mu} \mathfrak{n} \cdot \delta c c^{-1}-u^{\mu} e_{a}^{\sigma} \delta \varphi^{a}\left[\mathfrak{h}_{\sigma}+\mathfrak{n} \cdot\left(\partial_{\sigma} c c^{-1}+A_{\sigma}\right)\right]\right\} \\
= & \frac{1}{2} T^{\mu \nu} \delta g_{\mu \nu}+J^{\mu} \cdot \delta A_{\mu}+e_{a}^{\sigma}\left[\mathfrak{h}_{\sigma}+\mathfrak{n} \cdot\left(\partial_{\sigma} c c^{-1}+A_{\sigma}\right)\right] T \delta \beta^{a}+c^{-1} \mathfrak{n} c \cdot T \delta \Lambda_{\beta} \\
& -\frac{1}{\sqrt{-g}} \delta_{\mathcal{B}}(\sqrt{-g} T \mathfrak{n}) \cdot\left\{\delta c c^{-1}-e_{a}^{\sigma} \delta \varphi^{a}\left(\partial_{\sigma} c c^{-1}+A_{\sigma}\right)\right\} \\
& +e_{a}^{\sigma} \delta \varphi^{a}\left[\frac{1}{\sqrt{-g}} \delta_{\mathcal{B}}\left(\sqrt{-g} T \mathfrak{h}_{\sigma}\right)+T \mathfrak{n} \cdot \delta_{\mathcal{B}} A_{\sigma}\right] .
\end{aligned}
$$

The dynamical equations of motion can be now read off from the terms proportional to $\delta \varphi^{a}$ and $\delta c$ respectively. Inspection of (7.8) make it clear immediately that isolating these terms we end up with the equations of motion (7.1). 
Thus, extremizing $S_{\text {hydro }}[\Psi]$ with respect to $\left\{\varphi^{a}, c\right\}$ variations gives the correct energymomentum and charge conservation equations as required. As such we have transplanted a constrained variational problem into one where the variation is unconstrained for the physical fields $\left\{\varphi^{a}, c\right\}$ but the price one pays is to introduce a set of reference fields which are held rigid in the process.

\subsection{Gauge redundancy of reference fields}

In section 7.2 we decomposed the hydrodynamic fields $\left\{\boldsymbol{\beta}^{\mu}, \Lambda_{\boldsymbol{\beta}}\right\}$ into fixed reference fields $\left\{\beta^{a}, \Lambda_{\beta}\right\}$ and dynamical fields $\left\{\varphi^{a}, c\right\}$ parameterizing the Lie orbits of this reference configuration. Let us now scrutinize the decomposition (7.6) more closely to ascertain the symmetries of our new parameterization.

The decomposition (7.6) introduces a new redundancy in description. We can always do a gauge transformation or diffeomorphism on the reference configurations and compensate for it, say, by changing $\left\{\varphi^{a}, c\right\}$, so that we end up getting the same physical hydrodynamic configuration. For example, consider the following gauge transformation for the charged fields:

$$
\begin{aligned}
\Lambda_{\beta} & \mapsto f^{-1} \Lambda_{\beta} f-f^{-1} \beta^{\sigma} \partial_{\sigma} f \\
c & \mapsto c f,
\end{aligned}
$$

where $f$ is any flavour gauge transformation on $\mathbb{M}$. It is then simple to see that this transformation leaves $\Lambda_{\beta}$ unaffected. Hence, these transformations should be thought of as a gauge redundancy in our description which forces us to identify

$$
\left\{\Lambda_{\beta}, c\right\} \sim\left\{f^{-1} \Lambda_{\beta} f-f^{-1} \boldsymbol{\beta}^{\sigma} \partial_{\sigma} f, c f\right\} .
$$

as they give the same hydrodynamic field $\Lambda_{\boldsymbol{\beta}}$.

There is a similar redundancy in diffeomorphisms on the reference manifold $\mathbb{M}$ given by

$$
\begin{aligned}
\varphi^{a} & \mapsto f^{a}(\varphi) \\
\Lambda_{\beta}\left[\varphi^{a}\right] & \mapsto \Lambda_{\beta}\left[f^{a}(\varphi)\right] \\
\mathbb{\beta}^{a}\left[\varphi^{b}\right] & \mapsto \frac{\partial f^{a}}{\partial \varphi^{c}} \mathbb{\beta}^{c}\left[f^{b}(\varphi)\right]
\end{aligned}
$$

where $\varphi^{a}(x)$ are understood to be the coordinates on $\mathbb{M}$ obtained by pushing forward $x$ coordinates. Hence, any two configurations which differ by such $\varphi$-diffeomorphisms should also be thought of as the same fluid configuration as they lead to identical hydrodynamic fields $\left\{\boldsymbol{\beta}^{\mu}, \Lambda_{\boldsymbol{\beta}}\right\}$.

As with any gauge redundancy, it is convenient to pass to a formalism which is covariant with respect to this set of transformations. Moreover, since the redundancy is the gauge and diffeomorphism properties of our fields, we can just introduce a metric and flavour gauge fields on $\mathbb{M}$ to properly account for it. Endowing $\mathbb{M}$ with these structures would allow us to covariantize all the transformations. We will do this by first pushing forward 
the metric and flavour gauge fields on the actual spacetime $\mathcal{M}$ to $\mathbb{M}$ using $\left\{\varphi^{a}, c\right\}$ i.e., we define

$$
\begin{aligned}
& \mathscr{g}_{a b}[\varphi] \equiv e_{a}^{\mu} e_{b}^{\nu} g_{\mu \nu} \\
& \mathbb{A}_{a}[\varphi] \equiv e_{a}^{\mu}\left[c^{-1} A_{\mu} c+c^{-1} \partial_{\mu} c\right] .
\end{aligned}
$$

Given the push-forward relation for the metric, the Christoffel connection transforms in a related fashion:

$$
\mathbb{\square}_{b c}^{a}[\varphi] \equiv e_{c}^{\lambda} \partial_{\mu} \varphi^{a}\left[\Gamma_{\nu \lambda}^{\mu} e_{b}^{\nu}+\partial_{\lambda} e_{b}^{\mu}\right] .
$$

We can use the connections $\mathbb{A}, \mathbb{\llbracket}$ to define covariant derivatives on $\mathbb{M}$ which can then be used to construct invariants of $\varphi$-diffeomorphisms and $c$ gauge transformations. One can also easily check that the connections on $\mathcal{M}$ and $\mathbb{M}$ are consistent with Lie transport. That is, for the pushforward of a general tensor field, ${ }^{40}$

$$
\mathbb{T}^{a_{1} a_{2} \cdots}{ }_{b_{1} b_{2} \cdots[}[\varphi] \equiv\left(\partial_{\mu_{1}} \varphi^{a_{1}}\right)\left(\partial_{\mu_{2}} \varphi^{a_{2}}\right) \cdots e_{b_{1}}^{\nu_{1}} e_{b_{2}}^{\nu_{2}} \cdots \cdots T_{\nu_{1} \nu_{2} \cdots}^{\mu_{1} \mu_{2} \cdots}
$$

we have

$$
\left(\partial_{\mu_{1}} \varphi^{a_{1}}\right)\left(\partial_{\mu_{2}} \varphi^{a_{2}}\right) \cdots e_{b_{1}}^{\nu_{1}} e_{b_{2}}^{\nu_{2}} \cdots\left[£_{\xi} T^{\mu_{1} \mu_{2} \cdots}{ }_{\nu_{1} \nu_{2} \cdots}\right]=£_{(\xi . \partial) \varphi} \mathbb{T}^{a_{1} a_{2} \cdots}{ }_{b_{1} b_{2} \cdots} \ldots
$$

\subsection{Variational principle on reference manifold}

We can now reformulate the variational principle that gives rise to the hydrodynamic equations in terms of $\left\{\mathfrak{g}_{a b}, \mathbb{A}_{a}\right\}$. We begin by observing that invariance of $S_{\text {hydro }}[\Psi]$ under diffeomorphisms and gauge transformations means that

$$
S_{\text {hydro }}[\Psi]=\int_{\mathcal{M}} \sqrt{-g} \mathcal{L}[\Psi]=\int_{\mathbb{M}} \sqrt{-g} \mathcal{L}\left[\mathfrak{g}, \mathbb{A}, \mathbb{\beta}, \Lambda_{\beta}\right] \equiv \int_{\mathbb{M}} \sqrt{-g} \mathbb{L}[\Psi]
$$

where we use the condensed notation $\Downarrow$ to denote the collection of "hydrodynamic" fields on the reference manifold.

It follows then that we can get hydrodynamic equations by extremising $\int_{\mathbb{M}} \sqrt{-g} \mathbb{L}[\mathbb{\Psi}]$ by varying $\left\{\varphi^{a}, c\right\}$ inside $\{g, \mathbb{A}\}$ keeping $\left\{\mathbb{B}, \Lambda_{\beta}\right\}$ fixed. To see how this works, we will begin by decomposing the variations of $\{g, \mathbb{A}\}$ into (i) variation of reference sources and (ii) variation of the dynamical fields $\left\{\varphi^{a}, c\right\}$. Using (7.12), we obtain

$$
\begin{aligned}
& \delta \mathbb{g}_{a b}[\varphi]=\delta\left(\mathbb{g}_{a b}[\varphi]\right)-\delta \varphi^{c} \frac{\partial}{\partial \varphi^{c}} \mathfrak{g}_{a b}[\varphi]=e_{a}^{\mu} e_{b}^{\nu} \delta g_{\mu \nu}-\delta_{\varphi} \mathbb{g}_{a b} \\
& \delta \mathbb{A}_{a}[\varphi]=\delta\left(\mathbb{A}_{a}[\varphi]\right)-\delta \varphi^{c} \frac{\partial}{\partial \varphi^{c}} \mathbb{A}_{a}[\varphi]=e_{a}^{\mu} c^{-1}\left(\delta A_{\mu}\right) c-\delta_{\varphi} \mathbb{A}_{a}
\end{aligned}
$$

where $\delta_{\varphi}$ is the Lie drag on $\mathbb{M}$ along $\left\{\delta \varphi^{a},-c^{-1} \delta c\right\}$, viz.,

$$
\begin{aligned}
\delta_{\varphi} \mathbb{G}_{a b} & \equiv \mathbb{D}_{a} \delta \varphi_{b}+\mathbb{D}_{b} \delta \varphi_{a} \\
\delta_{\varphi} \mathbb{A}_{a} & \equiv \mathbb{D}_{a}\left(-c^{-1} \delta c+\mathbb{A}_{b} \delta \varphi^{b}\right)+\delta \varphi^{b} \mathbb{F}_{b a} .
\end{aligned}
$$

\footnotetext{
${ }^{40}$ We have written here the expression for an uncharged tensor field transforming solely under diffeomorphisms; including gauge transformations is straightforward which we leave as an exercise.
} 
In the above expressions we have introduced the covariant derivatives $\mathbb{D}$ and field strength $\mathbb{F}$ which are defined with the reference connections $\mathbb{A}, \mathbb{\square}$ respectively in the usual fashion.

With these definitions in place, let us then translate the variational calculus onto the reference manifold $\mathbb{M}$. First, when we vary $\int_{\mathbb{M}} \sqrt{-g} \mathbb{L}[\mathbb{\Psi}]$ with respect to $\left\{\mathfrak{g}_{a b}, \mathbb{A}_{a}\right\}$, we get the currents:

$$
\delta \int_{\mathbb{M}} \sqrt{-\mathfrak{g}} \mathbb{L}[\Psi]=\int_{\mathbb{M}} \sqrt{-\mathfrak{g}}\left(\frac{1}{2} \mathbb{T}^{a b} \delta \mathfrak{g}_{a b}+\mathbb{J}^{a} \cdot \delta \mathbb{A}_{a}+\mathbb{T} \mathfrak{h}_{a} \delta \beta^{a}+\mathbb{T} \mathfrak{n} \cdot\left(\delta \Lambda_{\beta}+\mathbb{A}_{b} \delta \mathbb{\beta}^{b}\right)\right),
$$

where all boldface currents are understood as the pushforwards of physical currents on $\mathcal{M}$ to the reference manifold. If we further extremize $\int_{\mathbb{M}} \sqrt{-\mathfrak{g}} \mathbb{L}$ with respect to just the $\varphi$ part of $\left\{\mathfrak{g}_{a b}, \mathbb{A}_{a}\right\}$ (holding fixed the functional form of $\left\{\beta^{a}, \Lambda_{\beta}\right\}$ ), we are led to conservation equations for the energy-momentum tensor and similarly for the charge current. To obtain these we have to perform the constrained variation $\delta$ which amounts to Lie drags of the sources along $\left\{\delta \varphi^{a},-c^{-1} \delta c\right\}$. Using (7.17) we learn that one should employ the variations

$$
\delta \mathbb{g}_{a b}=-\delta_{\varphi} \mathbb{I}_{a b}, \quad \delta \mathbb{A}_{a}=-\delta_{\varphi} \mathbb{A}_{a}, \quad \delta \beta^{a}=0, \quad \delta \Lambda_{\beta}=0
$$

Applying this variation to (7.19) leads to

$$
\delta \int_{\mathbb{M}} \sqrt{-\mathfrak{g}} \mathbb{L}[\Psi]=\int_{\mathbb{M}} \sqrt{-\mathfrak{g}}\left\{\delta \varphi^{c}\left[\mathbb{D}_{a} \mathbb{T}_{c}^{a}-\mathbb{J}^{a} \cdot \mathbb{F}_{c a}\right]+\left(-c^{-1} \delta c+\mathbb{A}_{a} \delta \varphi^{a}\right) \cdot \mathbb{D}_{c} \mathbb{J}^{c}\right\}
$$

We can now read off the hydrodynamic conservation equations on the reference manifold:

$$
\mathbb{D}_{a} \mathbb{T}_{c}^{a}-\mathbb{J}^{a} \cdot \mathbb{F}_{c a}=0, \quad \mathbb{D}_{c} \mathbb{J}^{c}=0 .
$$

This makes the variational principle on the reference manifold very simple in practice because it allows us to skip the computation of Bianchi identities ${ }^{41}$ and obtain the correct hydrodynamic Ward identities directly. The covariance of our reference manifold formalism makes it very easy to translate these results back to equations of motion on the physical spacetime $\mathcal{M}$ : one just replaces Latin indices by Greek indices and switches bold-face letters back to normal font. This prescription is thus equivalent to the one given in section 7.2.

\subsection{Static gauge on the reference manifold \& hydrodynamic fields}

Given a covariant form of an action with some redundancies it is sometimes convenient to pass to a gauge fixed version and focus on the physical degrees of freedom. To this end we can partially fix the gauge symmetries in the reference fields. Ignoring any possible Gribov type topological ambiguities, let us use the gauge transformation and diffeomorphism freedom on $\mathbb{M}$ to set

$$
\Lambda_{\beta}=0, \quad \mathbb{\beta}^{a=0}=1 \quad \text { and } \quad \mathbb{\beta}^{a=I}=0 \quad \text { for } I \in\{1, \ldots, d-1\} .
$$

\footnotetext{
${ }^{41}$ While we have not explicitly indicated how to get the Bianchi identities, these follow in the same manner as on the physical spacetime $\mathcal{M}$. We simply vary $\int_{\mathbb{M}} \sqrt{-g} \mathbb{L}$ with respect to $\Downarrow$ and follow the same set of arguments as in section 6.1. Modulo a font and index change, the algebra remains identical.
} 
In what follows, we will refer to this as the static reference gauge. As is clear from above, we will henceforth use uppercase Latin alphabets to denote spatial indices on $\mathbb{M}$.

Let us now examine the residual gauge redundancy that is left unfixed in the static reference gauge. The following set of $\varphi$-diffeomorphisms and $c$ gauge transformations survive the static reference gauge fixing of (7.23)

$$
\begin{aligned}
\varphi^{J} & \mapsto h^{J}\left(\varphi^{I}\right), \quad \operatorname{det}\left(\frac{\partial h^{J}}{\partial \varphi^{I}}\right) \neq 0, \\
\varphi^{0} & \mapsto \varphi^{0}+g\left(\varphi^{I}\right), \\
c & \mapsto c f\left(\varphi^{I}\right) .
\end{aligned}
$$

We will refer to the transformations in first line as transverse diffeomorphisms in $\varphi$-space. Further, we will call the shift symmetry in $\varphi^{0}$ in the second line as the thermal shift and the (right) spatial gauge transformations of $c$ in the third line as the chemical shift. ${ }^{42}$

It is useful to compare the redundancies in the parameterization using the reference manifold variables with those present in our discussion of the hydrostatic state in section 4 . One can in fact check that the redundancies in the two cases are precisely the same. For example, in static reference gauge, the metric and gauge field on $\mathbb{M}$ assume a 'Kaluza-Klein' form familiar from hydrostatics:

$$
\begin{aligned}
\mathfrak{g}_{a b} d \varphi^{a} d \varphi^{b} & =-\frac{1}{\mathbb{T}^{2}}\left(d \varphi^{0}+\mathbb{a}_{I} d \varphi^{I}\right)^{2}+\mathbb{p}_{I J} d \varphi^{I} d \varphi^{J} \\
\mathfrak{g}^{a b} \frac{\partial}{\partial \varphi^{a}} \otimes \frac{\partial}{\partial \varphi^{b}} & =-\mathbb{T}^{2} \frac{\partial}{\partial \varphi^{0}} \otimes \frac{\partial}{\partial \varphi^{0}}+\mathbb{P}^{I J}\left(\frac{\partial}{\partial \varphi^{I}}-\mathbb{a}_{I} \frac{\partial}{\partial \varphi^{0}}\right) \otimes\left(\frac{\partial}{\partial \varphi^{J}}-\mathbb{a}_{J} \frac{\partial}{\partial \varphi^{0}}\right) \\
\mathbb{A}_{a} d \varphi^{a} & =c^{-1}\left(\frac{\mu}{\mathbb{T}}\right) c\left(d \varphi^{0}+\mathbb{a}_{I} d \varphi^{I}\right)+\mathfrak{a}_{I} d \varphi^{I} \\
\mathfrak{u}^{a} \frac{\partial}{\partial \varphi^{a}} & =\mathbb{T} \frac{\partial}{\partial \varphi^{0}}, \quad \cup_{a} d \varphi^{a}=-\frac{1}{\mathbb{T}}\left(d \varphi^{0}+\mathbb{a}_{I} d \varphi^{I}\right)
\end{aligned}
$$

where $\mathbb{a}_{I}$ is a gauge field for the thermal shift, whereas $\mathrm{a}_{I}$ is the spatial component of the reference gauge field. As in hydrostatics, we have the useful rule of thumb that the tensor components with only 0's in down (covariant) indices and only Is in up (contravariant) indices are invariant under thermal shift. This follows from the fact that the thermal shift invariant basis on tangent and cotangent bundles of $\mathbb{M}$ are given by

$$
\left\{d \varphi^{0}+\mathbb{a}_{I} d \varphi^{I}, d \varphi^{I}\right\}, \quad \text { and } \quad\left\{\frac{\partial}{\partial \varphi^{0}}, \frac{\partial}{\partial \varphi^{I}}-\mathbb{a}_{I} \frac{\partial}{\partial \varphi^{0}}\right\},
$$

respectively. It is thus convenient to replace $\left\{e_{I}^{\sigma}\right\}$ which transforms under thermal shift with a set of thermal shift invariant vectors $\left\{P_{I}^{\sigma}\right\}$ instead. Let us define the hybrid hydrodynamic projectors

$$
P_{I}^{\sigma} \equiv \frac{\partial x^{\sigma}}{\partial \varphi^{I}}-\mathbb{a}_{I} \frac{\partial x^{\sigma}}{\partial \varphi^{0}}
$$

\footnotetext{
${ }^{42}$ The rationale for this terminology, which is inspired from the formalism of non-dissipative fluids [45], will be come clear in appendix B. We will demonstrate there how the class of adiabatic fluids encompasses the non-dissipative fluid framework explicitly.
} 
They satisfy

$$
\begin{array}{rlrl}
P_{I}^{\alpha} \partial_{\beta} \varphi^{I} & =P_{\beta}^{\alpha}=\delta_{\beta}^{\alpha}+u^{\alpha} u_{\beta}, & \partial_{\alpha} \varphi^{I} P_{J}^{\alpha}=\delta_{J}^{I}, \\
-T u_{\sigma} P_{I}^{\sigma} & =\left(\partial_{\sigma} \varphi^{0}+\mathbb{a}_{J} \partial_{\sigma} \varphi^{J}\right) P_{I}^{\sigma}=P_{I}^{\sigma} \partial_{\sigma} \varphi^{0}+\mathbb{a}_{I}=0, \\
g_{\alpha \beta} P_{I}^{\alpha} P_{J}^{\beta} & =\mathbb{P}_{I J}, & \mathbb{P}^{I J} P_{I}^{\alpha} P_{J}^{\beta}=P^{\alpha \beta}, \\
g_{\alpha \beta} P_{I}^{\beta} & =\mathbb{P}^{I}{ }_{J} \partial_{\alpha} \varphi^{J}, & \mathbb{P}^{I J} P_{J}^{\alpha}=g^{\alpha \beta} \partial_{\beta} \varphi^{I}
\end{array}
$$

Using these thermal shift invariant vectors, we can write

$$
e_{a}^{\sigma} \delta \varphi^{a}=P_{I}^{\sigma} \delta \varphi^{I}+\boldsymbol{\beta}^{\sigma}\left[\delta \varphi^{0}+\mathbb{a}_{I} \delta \varphi^{I}\right] .
$$

This thermal shift invariant form is often useful in explicit computations. For example, when varying $\sqrt{-g} \mathbb{L}[\Psi]$, we get (7.8) where $\delta \varphi^{a}$ always occur in this combination.

The static reference gauge has the merit that once we adopt it, only dynamical fields show up in the description; all gauge redundancies are eliminated. Hydrodynamics in static reference gauge is then completely described by the following set of degrees of freedom:

- $(d-1)$ spatial $\varphi^{I}$ S which satisfy $u^{\sigma} \partial_{\sigma} \varphi^{I}=0$,

- a field $\varphi^{0}$ such that $T=u^{\alpha} \partial_{\alpha} \varphi^{0}$ and

- a field $c$ such that $\mu=u^{\alpha}\left(\partial_{\alpha} c\right) c^{-1}+u^{\alpha} A_{\alpha}$.

Further, we can solve for $u^{\sigma}$ itself directly in terms of these dynamical fields. We get

$$
u^{\sigma}=\frac{1}{\sqrt{\operatorname{det}_{d-1}\left(g^{\mu \nu} \partial_{\mu} \varphi^{I} \partial_{\nu} \varphi^{J}\right)}} \varepsilon^{\sigma \alpha_{1} \ldots \alpha_{d-1}} \partial_{\alpha_{1}} \varphi^{1} \cdots \partial_{\alpha_{d-1}} \varphi^{d-1} .
$$

This follows from the fact that $u^{\sigma}$ is orthogonal to the $(d-1)$ vector fields $\partial_{\sigma} \varphi^{I}$ and is hence parallel to $\varepsilon^{\sigma \alpha_{1} \ldots \alpha_{d-1}} \partial_{\alpha_{1}} \varphi^{1} \cdots \partial_{\alpha_{d-1}} \varphi^{d-1}$. The square-root pre-factor then ensures the correct normalization appropriate for a $d$-velocity.

A more elegant way of writing the above expressions is to introduce a spatial volume form on the space of $\varphi^{I} \mathrm{~S}$ using the spatial part of the pushforward co-metric $\mathrm{g}^{a b}$, i.e., we define $\mathbb{P}^{I J} \equiv g^{\mu \nu} \partial_{\mu} \varphi^{I} \partial_{\nu} \varphi^{J}$ whose inverse then defines a thermal shift invariant spatial metric $\mathfrak{p}_{I J}$ introduced in (7.25). Using this expression one can work out the parameterization of hydrodynamic fields $\left\{\boldsymbol{\beta}^{\mu}, \Lambda_{\boldsymbol{\beta}}\right\}$ in terms of $\left\{\varphi^{a}, c\right\}$. For completeness let us record these expressions which read:

$$
\begin{aligned}
u^{\sigma} & =\frac{1}{(d-1) !} \varepsilon^{\sigma \alpha_{1} \ldots \alpha_{d-1}} \varepsilon_{I_{1} \cdots I_{d-1}}^{(\mathfrak{p})} \prod_{i=1}^{d-1} \partial_{\alpha_{i}} \varphi^{I_{i}} \\
T & =\frac{1}{(d-1) !} \varepsilon^{\sigma \alpha_{1} \ldots \alpha_{d-1}} \varepsilon_{I_{1} \cdots I_{d-1}}^{(\mathfrak{p})} \partial_{\sigma} \varphi^{0} \prod_{i=1}^{d-1} \partial_{\alpha_{i}} \varphi^{I_{i}} \\
& =\frac{1}{(d-1) !} \varepsilon^{\sigma \alpha_{1} \ldots \alpha_{d-1}} \varepsilon_{I_{1} \cdots I_{d-1}}^{(\mathfrak{p})}\left(\partial_{\sigma} \varphi^{0}+\mathbb{a}_{I} \partial_{\sigma} \varphi^{I}\right) \prod_{i=1}^{d-1} \partial_{\alpha_{i}} \varphi^{I_{i}} \\
\mu & =\frac{1}{(d-1) !} \varepsilon^{\sigma \alpha_{1} \ldots \alpha_{d-1}} \varepsilon_{I_{1} \cdots I_{d-1}}^{(\mathfrak{p})}\left(\left(\partial_{\sigma} c\right) c^{-1}+A_{\sigma}\right) \prod_{i=1}^{d-1} \partial_{\alpha_{i}} \varphi^{I_{i}}
\end{aligned}
$$


where $\varepsilon_{I_{1} \cdots I_{d-1}}^{(\mathbb{P})}$ is the spatial volume form associated with $\mathbb{P}_{I J}$. We can also give a similar expression for thermal shift invariant vectors $\left\{P_{I}^{\sigma}\right\}$ defined in (7.27):

$$
P_{I}^{\sigma}=\frac{1}{(d-2) !} \varepsilon^{\sigma \alpha \beta_{1} \ldots \beta_{d-2}} \varepsilon_{I J_{1} \cdots J d-2}^{(\mathrm{p})} u_{\alpha} \prod_{i=1}^{d-2} \partial_{\beta_{i}} \varphi^{J_{i}}
$$

More generally one can express a $k$-fold anti-symmetric tensor product of the projection tensors $P_{I}^{\alpha}$ in terms of the $\varphi^{I}$ fields as

$$
(k+1) ! u^{[\sigma} P_{\left[I_{1}\right.}^{\alpha_{1}} \ldots P_{\left.I_{k}\right]}^{\left.\alpha_{k}\right]}=\frac{1}{(d-1-k) !} \varepsilon^{\sigma \alpha_{1} \ldots \alpha_{k} \beta_{1} \ldots \beta_{d-1-k}} \varepsilon_{I_{1} \cdots I_{k} J_{1} \cdots J}^{(\mathrm{p})} \prod_{d-1-k} \prod_{i=1}^{d-1-k} \partial_{\beta_{i}} \varphi^{J}{ } .
$$

The expressions derived above, express the hydrodynamic fields as gauge-invariant composite fields formed out of the basic dynamic fields $\left\{\varphi^{0}, \varphi^{I}, c\right\}$. While this is the most economical presentation in terms of the dynamical degrees of freedom, the gauge fixing introduces a degree of non-linearity in the mapping between the physical fields and the hydrodynamical variables. Nevertheless, there is a certain simplicity to the parameterization: the hydrodynamic equations can then be obtained by writing $\int \sqrt{-g} \mathcal{L}[\Psi]$ or $\int_{\mathbb{M}} \sqrt{-g} \mathbb{L}[\Psi]$ as a functional of $\left\{g_{\mu \nu}, A_{\alpha}, \varphi^{0}, \varphi^{I}, c\right\}$ and extremising with respect to the dynamical fields $\left\{\varphi^{0}, \varphi^{I}, c\right\}$. Moreover, the hydrostatic limit in the static reference gauge can be obtained by setting

$$
c=\mathbb{1}, \quad \partial_{\sigma} \varphi^{0}=T_{0} \delta_{\sigma}^{0}, \quad \partial_{\sigma} \varphi^{I}=\delta_{\sigma}^{I},
$$

i.e., we can just pull-back the reference configuration through what is essentially an identity map between the spacetime and $\mathbb{M}$. This is to be expected given the close analogy between the two formalisms noted around (7.25). Then the formalism we just described reduces to the hydrostatic formalism described in $[3,4]$ as expected. One can easily derive explicit expressions for the partition function by recasting the results of section 6.3 in the static reference gauge.

The reader familiar with the discussion of non-dissipative fluids $[45,46]$ will undoubtedly see some similarities with the variables used in that context. However, there are some subtle (but important) distinctions; we are not yet within the remit of that framework. ${ }^{43}$ The connection between the formalism outlined herein and that used in the aforementioned references is explained in appendix $\mathrm{B}$, where we demonstrate that non-dissipative fluids (Class ND) are a subclass of adiabatic fluids.

\subsection{Field redefinitions in Class $L$}

In this subsection, we will examine the field redefinitions of the hydrodynamic fields $\left\{u^{\sigma}, T, \mu\right\}$ which leaves the on-shell physics invariant. While we are allowed to do a general redefinition of the hydrodynamic fields, this does not translate into a general redefinition of

\footnotetext{
${ }^{43}$ We draw the attention of the reader to the fact that $T$ and $\mu$ are treated symmetrically in this description, with $e^{\varphi^{0}}$ playing an analogous role to $c$. This is similar to a model considered in [66].
} 
the Lagrange multiplier fields $\left\{\boldsymbol{\beta}^{\mu}, \Lambda_{\boldsymbol{\beta}}\right\}$. We remind the reader that we have already used up a subset of field redefinitions so as to have a simple relationship between the Lagrange multiplier fields $\left\{\boldsymbol{\beta}^{\mu}, \Lambda_{\boldsymbol{\beta}}\right\}$ and the original hydrodynamic fields $\left\{u^{\sigma}, T, \mu\right\}$, see section 2.1. In this subsection, we will examine the residual redefinitions which are allowed for the Lagrange multiplier fields $\left\{\boldsymbol{\beta}^{\mu}, \Lambda_{\boldsymbol{\beta}}\right\}$.

One of the advantages of shifting to $\left\{\boldsymbol{\beta}^{\mu}, \Lambda_{\boldsymbol{\beta}}\right\}$ was that the hydrostatic configurations can simply be described by aligning $\left\{\boldsymbol{\beta}^{\mu}, \Lambda_{\boldsymbol{\beta}}\right\}$ to the Killing fields $\left\{K^{\mu}, \Lambda_{K}\right\}$. An admissible field redefinition should preserve this feature. This then suggests that we consider redefinitions of the form

$$
\begin{aligned}
& \boldsymbol{\beta}^{\mu} \mapsto \boldsymbol{\beta}^{\mu}-\delta_{\mathcal{B}} V^{\mu}=\boldsymbol{\beta}^{\mu}+£_{V} \boldsymbol{\beta}^{\mu}, \\
& \Lambda_{\boldsymbol{\beta}} \mapsto \Lambda_{\boldsymbol{\beta}}-\delta_{\mathcal{B}} \Lambda_{V}=\Lambda_{\boldsymbol{\beta}}+£_{V} \Lambda_{\boldsymbol{\beta}}+\left[\Lambda_{\boldsymbol{\beta}}, \Lambda_{V}\right]-\boldsymbol{\beta}^{\sigma} \partial_{\sigma} \Lambda_{V}
\end{aligned}
$$

for some general diffeomorphism and flavour parameter $\left\{V^{\mu}, \Lambda_{V}\right\}$. The presence of $\delta_{\mathcal{B}}$ ensures that the nice features of hydrostatics survive these redefinitions. This is the most general class of redefinitions that are admissible for $\left\{\boldsymbol{\beta}^{\mu}, \Lambda_{\boldsymbol{\beta}}\right\}$.

In Class L, there is a more concrete way of seeing why two fluids related by (7.35) should be considered on-shell equivalent. Using (7.4) we can write down the change in Lagrangian induced by field redefinitions in (7.35):

$$
\begin{aligned}
\mathcal{L}[\Psi] \mapsto \mathcal{L}[\Psi]+\left(\frac{1}{\sqrt{-g}} \delta_{\mathcal{B}}\left(\sqrt{-g} T \mathfrak{h}_{\sigma}\right)+T \mathfrak{n} \cdot \delta_{\mathcal{B}} A_{\sigma}\right) V^{\sigma} & \\
& +\frac{1}{\sqrt{-g}} \delta_{\mathcal{B}}[\sqrt{-g} T \mathfrak{n}] \cdot\left(\Lambda_{V}+A_{\sigma} V^{\sigma}\right)+\nabla_{\mu}(\ldots),
\end{aligned}
$$

viz., the Lagrangian is shifted by terms proportional to the equations of motion and a boundary term. In a field theory, this is the most general redefinition admissible in the Lagrangian density. What this means in practice is that we can effectively focus on the basis of on-shell independent scalars parameterizing $\mathcal{L}[\Psi]$, which greatly simplifies the computation (see for example appendix F).

An alternate way to get at the same result is to shift to the description based on reference manifolds and pullback fields. So consider then replacing $\mathcal{L}[\Psi]$ by the functional on the reference manifold $\mathbb{L}[\Psi]$. Variation of this functional under arbitrary variation of fields $\left\{\varphi^{a}, c\right\}$ leads to terms proportional to the equations of motion $\nabla_{\nu} T^{\mu \nu}=J_{\nu} \cdot F^{\mu \nu}$ and $D_{\mu} J^{\mu}=0$, which effectively means, using the notation introduced in (7.2),

$$
\delta \mathcal{L}_{k}[\mathbf{\Psi}]=\left(\nabla_{\alpha} T_{(k)}^{\alpha \mu}-J_{(k)}^{\alpha} \cdot F^{\alpha \mu}\right) e_{\mu a} \delta \varphi^{a}+D_{\alpha} J_{(k)}^{\alpha} \cdot\left\{-\delta c c^{-1}+e_{a}^{\sigma} \delta \varphi^{a}\left(\partial_{\sigma} c c^{-1}+A_{\sigma}\right)\right\} .
$$

Here we are working order by order in the gradient expansion, which as explained earlier is completely kosher in the absence of anomalies. $\mathcal{L}_{k}$ denotes the scalar contribution at $k^{\text {th }}$ order in gradients. Using (7.7), we can relate these field redefinitions of the pull-back fields to the redefinitions of $\left\{\boldsymbol{\beta}^{\mu}, \Lambda_{\boldsymbol{\beta}}\right\}$ in (7.35):

$$
V^{\mu}=e_{a}^{\mu} \delta \varphi^{a}, \quad \Lambda_{V}=-\delta c c^{-1}+e_{a}^{\sigma} \delta \varphi^{a} \partial_{\sigma} c c^{-1}
$$


In practice we can use this redefinition freedom as follows: say we are interested in contributions to the currents at $k^{\text {th }}$ order in gradients. We can implement a shift of $\Downarrow$ in all the terms up to the $(k-1)^{\text {st }}$ order, so that we pick up a contribution to the Lagrangian proportional to the conservation equation to one lower order than we are interested in. In other words

$$
\begin{aligned}
\sum_{l=1}^{k} \delta \mathcal{L}_{l}[\mathbf{\Psi}]=\sum_{l=1}^{k-1}\left(\nabla_{\alpha} T_{(l)}^{\alpha \mu}-J_{(l)}^{\alpha} F^{\alpha \mu}\right) e_{\mu a} \delta \varphi^{a} & \\
& +\sum_{l=1}^{k-1} \nabla_{\alpha} J_{(l)}^{\alpha} \cdot\left\{-\delta c c^{-1}+e_{a}^{\sigma} \delta \varphi^{a}\left(\partial_{\sigma} c c^{-1}+A_{\sigma}\right)\right\}+\cdots
\end{aligned}
$$

where we have only retained terms up to $k^{\text {th }}$ order in the gradients. By a suitable choice of $\delta \varphi^{a}$ and $\delta c$ we can eliminate some of the terms in $\mathcal{L}_{k}[\Psi]$.

The upshot of this discussion is that we can always choose to parameterize $\mathcal{L}[\mathbf{\Psi}]$ solely in terms of the on-shell independent scalars at a given order in the gradient expansion. This has a significant effect in simplifying the computations. An explicit verification of this statement at the level of neutral fluids at second order in gradients can be found in appendix $\mathrm{F}^{4}{ }^{44}$

\section{Applications of adiabatic fluids}

Having in the previous sections given a rather abstract discussion of the Class L adiabatic fluids, we now turn to some specific examples. We first describe how neutral fluids can be understood in this language and derive the constraints arising from demanding adiabaticity on such fluids up to the second order in hydrodynamic gradient expansion. We also comment briefly on the case of charged parity-odd fluids in 3 dimensions working to first order in the gradient expansion. We choose these specific examples for their simplicity and also because they have been previously analyzed in the framework of non-dissipative fluids (Class ND) in [46] and [48] respectively. Later on in section 14 we will also have occasion to describe charged fluids, when we illustrate the general classification scheme we develop.

\subsection{Neutral fluids up to second order in gradients}

Consider a neutral non-anomalous fluid for which we wish to find the constraints imposed by adiabaticity. Since there are no charges we ignore the field $\Lambda_{\boldsymbol{\beta}}$ and the corresponding gauge field source $A_{\mu}$; thus our Lagrangian is going to be a function only of the hydrodynamic field $\boldsymbol{\beta}^{\mu}$ and the background metric source $g_{\mu \nu}$. Our strategy will be to follow intuition from hydrodynamics and write down a Lagrangian density order by order in the gradients of these fields. So we have

$$
\mathcal{L}\left[\boldsymbol{\beta}^{\mu}, g_{\mu \nu}\right]=\mathcal{L}_{0}\left[\boldsymbol{\beta}^{\mu}, g_{\mu \nu}\right]+\mathcal{L}_{1}\left[\boldsymbol{\beta}^{\mu}, g_{\mu \nu}\right]+\mathcal{L}_{2}\left[\boldsymbol{\beta}^{\mu}, g_{\mu \nu}\right]+\cdots
$$

where $\mathcal{L}_{k}$ involves terms with exactly $k$ derivatives acting on the fields. We will now proceed to construct the first three terms in the above gradient expansion and derive the corresponding hydrodynamic constitutive relations.

\footnotetext{
${ }^{44}$ Field redefinitions affecting the entropy current have been described previously in section 5.3.
} 


\subsubsection{Zeroth order in gradients}

At leading order in the gradient expansion, we want a scalar function built out of $g_{\mu \nu}$ and $\boldsymbol{\beta}^{\mu}$. Clearly, there is only one such scalar which is the norm of $\boldsymbol{\beta}^{\mu}$, which we can trade for the temperature $T$ from (2.2). So we can write our leading Lagrangian as

$$
\mathcal{L}_{0}\left[\boldsymbol{\beta}^{\mu}, g_{\mu \nu}\right]=p(T), \quad T=\frac{1}{\sqrt{-g_{\mu \nu} \boldsymbol{\beta}^{\mu} \boldsymbol{\beta}^{\nu}}} .
$$

Now we can apply the variational calculus of section 6 to this Lagrangian and extract the currents. We already know the Bianchi identities and the dynamical equations they are supposed to satisfy on general grounds. Thus all we need is the analog of (6.3) for our specific choice of $\mathcal{L}_{0}$. A simple calculation using (E.1) leads to ${ }^{45}$

$$
\frac{1}{\sqrt{-g}} \delta\left(\sqrt{-g} \mathcal{L}_{0}\right)=\frac{1}{2}\left[\left(T p^{\prime}-p\right) u^{\mu} u^{\nu}+p P^{\mu \nu}\right] \delta g_{\mu \nu}+T^{3} p^{\prime} \boldsymbol{\beta}_{\sigma} \delta \boldsymbol{\beta}^{\sigma}
$$

There are no boundary terms, and the currents are just what we expect for an ideal fluid

$$
\begin{aligned}
T_{(0)}^{\mu \nu} & =\left(T p^{\prime}-p\right) u^{\mu} u^{\nu}+p P^{\mu \nu}, \quad J_{S,(0)}^{\mu}=p^{\prime} u^{\mu} \\
\mathfrak{h}_{(0)}^{\sigma} & =p^{\prime}(T) T^{2} \boldsymbol{\beta}^{\sigma}
\end{aligned}
$$

where we identify $\epsilon(T)=T p^{\prime}(T)-p(T)$ with $p(T)$ being the pressure (or negative of the free energy). We have already verified that the ideal fluid satisfies the adiabaticity equation directly in section 2.3 , but it of course now also follows from the variational calculus.

\subsubsection{First order in gradients}

Moving to the next order in gradients, we find that there are two one derivative scalars that we can write down $\boldsymbol{\beta}^{\sigma} \nabla_{\sigma} T$ and $\nabla_{\mu} \boldsymbol{\beta}^{\mu}$, both of which can be multiplied by an arbitrary function of the scalar $T$. However, these two scalars are not independent as Lagrangian entries. They are equivalent up to a total derivative term owing to the identity: $f(T) \nabla_{\mu} \boldsymbol{\beta}^{\mu}=\nabla_{\mu}\left(f(T) \boldsymbol{\beta}^{\mu}\right)-f^{\prime}(T) \boldsymbol{\beta}^{\mu} \nabla_{\mu} T$. We will therefore only pick one of them to include in $\mathcal{L}_{1}$. Since it is simpler to vary the gradient of the temperature, we parameterize the first order Lagrangian as

$$
\mathcal{L}_{1}\left[\boldsymbol{\beta}^{\mu}, g_{\mu \nu}\right]=\boldsymbol{\beta}^{\sigma} \nabla_{\sigma} f_{1}(T)
$$

whose variation again leads to

$$
\begin{aligned}
\frac{1}{\sqrt{-g}} \delta\left(\sqrt{-g} \mathcal{L}_{1}\right)= & \frac{f_{1}^{\prime}}{2}\left(\boldsymbol{\beta}^{\sigma} \nabla_{\sigma} T g^{\mu \nu}-T \nabla_{\sigma} \boldsymbol{\beta}^{\sigma} u^{\mu} u^{\nu}\right) \delta g_{\mu \nu}+\nabla_{\sigma}\left(f_{1}^{\prime} \boldsymbol{\beta}^{\sigma} \delta T\right) \\
& +f_{1}^{\prime}\left(\nabla_{\mu} T-T^{3}\left(\nabla_{\sigma} \boldsymbol{\beta}^{\sigma}\right) \boldsymbol{\beta}_{\mu}\right) \delta \boldsymbol{\beta}^{\mu}
\end{aligned}
$$

The stress tensor arising from adding $\mathcal{L}_{1}$ is again of the perfect fluid form, except that the definitions of the energy density and pressure are shifted by terms involving $\boldsymbol{\beta}^{\sigma} \nabla_{\sigma} T$ and $\nabla_{\sigma} \boldsymbol{\beta}^{\sigma}$. The final expressions for the transport data is then:

$$
\begin{aligned}
T_{(1)}^{\mu \nu} & =-f_{1}^{\prime}\left(T \nabla_{\sigma} \boldsymbol{\beta}^{\sigma}+\boldsymbol{\beta}^{\sigma} \nabla_{\sigma} T\right) u^{\mu} u^{\nu}+f_{1}^{\prime} \boldsymbol{\beta}^{\sigma} \nabla_{\sigma} T P^{\mu \nu} \\
J_{S,(1)}^{\mu} & =-\frac{1}{T} f_{1}^{\prime}\left(u^{\sigma} \nabla_{\sigma} \log T+T \nabla_{\sigma} \boldsymbol{\beta}^{\sigma}\right)
\end{aligned}
$$

\footnotetext{
${ }^{45}$ Derivatives with respect to temperature are denoted by a prime, viz., $f^{\prime}(T)=\frac{d f}{d T}$.
} 
In addition we have the adiabatic heat and pre-symplectic currents given by

$$
\begin{aligned}
\mathfrak{h}_{(1)}^{\mu} & =f_{1}^{\prime}\left(\nabla^{\mu} \log T-T^{2}\left(\nabla_{\sigma} \boldsymbol{\beta}^{\sigma}\right) \boldsymbol{\beta}^{\mu}\right) \\
\left(\not \Theta_{\mathrm{PS}}\right)^{\mu} & =f_{1}^{\prime}\left(T^{2} u_{\alpha} \delta \boldsymbol{\beta}^{\alpha}+\frac{1}{2} T u^{\alpha} u^{\beta} \delta g_{\alpha \beta}\right) \boldsymbol{\beta}^{\mu}
\end{aligned}
$$

The stress tensor appears to be in the ideal fluid form, but given that there are various gradient terms lurking around, we would like to ascertain whether there are genuine viscous contributions. The hydrodynamic stress tensor at first order is expected to contain terms involving the shear and expansion of the fluid whose coefficients are the shear and bulk viscosities, cf., the Landau frame expression given in (F.12) (see also our discussion in section 5). To compare with conventional expressions in hydrodynamics it is useful to write the answer for the stress tensor (8.7) in a more familiar form.

Usually hydrodynamic stress tensors are given in terms of basis of independent tensors which are identified by invoking on-shell relations at one lower order, cf., [41] for a nice review of the procedure. For neutral fluids derivatives of the temperature are typically eliminated in favour of velocity gradients; using the conservation of the ideal fluid we obtain

$$
\nabla_{\mu} T \simeq \frac{\epsilon+p}{\epsilon^{\prime}(T)} \Theta u_{\mu}-\frac{\epsilon+p}{p^{\prime}(T)} \mathfrak{a}_{\mu}=T v_{s}^{2} \Theta u_{\mu}-T \mathfrak{a}_{\mu} .
$$

We have introduced the speed of sound $v_{s}^{2}(T)$ to simplify future expressions:

$$
v_{s}^{2} \equiv \frac{d p}{d \epsilon}
$$

If we eliminate the temperature gradients using the above, we find for the gradients of $\boldsymbol{\beta}^{\mu}$ the following expressions:

$$
\begin{aligned}
\nabla_{\mu} \boldsymbol{\beta}_{\nu} & \simeq \frac{1}{T}\left(\sigma_{\mu \nu}+\omega_{\mu \nu}+\frac{\Theta}{d-1} P_{\mu \nu}\right)-\frac{v_{s}^{2}}{T} \Theta u_{\mu} u_{\nu}, \\
\nabla_{\sigma} \boldsymbol{\beta}^{\sigma} & \simeq \frac{\Theta}{T}\left(1+v_{s}^{2}\right) .
\end{aligned}
$$

Armed with this information we can then rewrite the hydrodynamic currents in (8.7) as

$$
\begin{aligned}
T_{(1)}^{\mu \nu} & \stackrel{\text { ideal }}{\simeq}-f_{1}^{\prime} \Theta\left[u^{\mu} u^{\nu}+v_{s}^{2} P^{\mu \nu}\right], \\
J_{S,(1)}^{\mu} & \stackrel{\text { ideal }}{\simeq}-\frac{1}{T} f_{1}^{\prime} \Theta u^{\mu},
\end{aligned}
$$

where we have made clear with the notation $\stackrel{\text { ideal }}{\simeq}$ that we are only taking the ideal part of the fluid on-shell. We also can check that the free energy current vanishes $\mathcal{G}_{(1)}^{\sigma} \simeq 0$ using (2.18). Further, using that we have the pre-symplectic potential in (8.8), one can obtain the Komar charge using (6.23). Noting as described in section 6.2 that we only need the variation of the background metric under Lie transport by $\boldsymbol{\beta}^{\mu}$ i.e., $\delta_{\mathfrak{B}} g_{\mu \nu}=2 \nabla_{(\mu} \boldsymbol{\beta}_{\nu)}$ we have

$$
\mathcal{K}^{\mu \nu}[\mathcal{B}] \stackrel{\text { ideal }}{\simeq} 0
$$


We are now in a position to discuss some physical aspects of the first order Class L term (8.5). The first peculiar feature to note is that while the adiabaticity equation (2.13) is clearly satisfied, the form of entropy current is counterintuitive. It is well known that a neutral fluid has no correction to the ideal fluid entropy current at first order. In fact, by using the standard current algebra logic in hydrodynamics, one can show that an entropy current with non-negative divergence demands vanishing of the coefficient of $\Theta u^{\mu}$ at first order (similarly for the other a-priori allowed vector $\mathfrak{a}^{\mu}$ ) [2].

Clearly, in the present case what is happening is that the entropy production is being compensated for by the energy-momentum tensor off-shell (note that we have not imposed the first order conservation equations as yet). However, we can make a somewhat more clean statement by examining the stress tensor itself. Since we are free to make a certain amount of field redefinitions as discussed in section 7.6, which amount in hydrodynamics language to choice of fluid frame, in comparing the stress tensor we should account for this. At leading order in the gradient expansion the simplest way to proceed is to project the stress tensor (8.12) onto frame invariant (i.e., field redefinition independent) tensor structures. This is implemented by employing the tensor and scalar projectors $C_{T}^{\mu \nu}$ and $C_{S}$ respectively [3]:

$$
\begin{aligned}
C_{T}^{\mu \nu} & =P^{\mu \alpha} P^{\nu \beta} T_{\alpha \beta}^{(1)}-\frac{1}{d-1} P^{\mu \nu} P^{\alpha \beta} T_{\alpha \beta}^{(1)}, \\
C_{S} & =\frac{1}{d-1} P^{\mu \nu} T_{\mu \nu}^{(1)}-v_{s}^{2} u^{\mu} u^{\nu} T_{\mu \nu}^{(1)} .
\end{aligned}
$$

Acting with these operators on (8.5), we find the results to vanish identically. In other words, there is no frame independent on-shell information in the stress tensor. More importantly, the term in (8.5) which resembles the bulk viscosity term $\Theta P^{\mu \nu}$ should not be interpreted as such; it is not a genuine contribution to the dissipative transport.

For the first order Lagrangian (8.5) we saw that the process of taking the ideal fluid part on-shell led to a stress tensor with no physical information. We claim that the field redefinitions described in section 7.6 can be used to remove (8.5) by setting $f_{1}=$ constant, leading to the same conclusion as above.

Let us see how this can be used at first order for the Lagrangian term (8.5). We start with the ideal fluid contribution and write

$$
\delta \mathcal{L}_{0}[\boldsymbol{\beta}]+\mathcal{L}_{1}[\boldsymbol{\beta}]=p^{\prime}(T)\left(\nabla_{\mu} \log T-v_{s}^{2} \Theta u_{\mu}+\mathfrak{a}_{\mu}\right) e_{a}^{\mu} \delta \varphi^{a}+f_{1}^{\prime}(T) u^{\sigma} \nabla_{\sigma} \log T+\cdots
$$

Writing $v_{s}^{2} p^{\prime} \Theta=\left(v_{s}^{2} p^{\prime}-T\left(v_{s}^{2} p^{\prime}\right)^{\prime}\right) u^{\mu} \nabla_{\mu} \log T$ up to a total derivative we see that picking

$$
e_{a}^{\mu} \delta \varphi^{a}=-\frac{f_{1}^{\prime}}{p^{\prime}\left(1+v_{s}^{2}\right)-T\left(v_{s}^{2} p^{\prime}\right)^{\prime}} u^{\mu}
$$

we can eliminate the $\mathcal{L}_{1}$ completely as required.

\subsubsection{Second order in gradients}

At the second order in gradient expansion we have many new scalar functions built from the background metric and hydrodynamic fields. One can use the standard fluid dynamical 
parameterization and write the terms as: ${ }^{46}$

$$
\begin{array}{llll}
\sigma^{2} \equiv \sigma_{\mu \nu} \sigma^{\mu \nu}, & \omega^{2} \equiv \omega_{\mu \nu} \omega^{\nu \mu}, & \mathfrak{a}^{2} \equiv \mathfrak{a}_{\mu} \mathfrak{a}^{\mu}, & \Theta^{2}, \quad R \\
\nabla_{\mu} T \nabla^{\mu} T, & \Theta u^{\mu} \nabla_{\mu} T, & \mathfrak{a}^{\mu} \nabla_{\mu} T, & u^{\mu} \nabla_{\mu} T u^{\nu} \nabla_{\nu} T, \\
R_{00} \equiv R_{\mu \nu} u^{\mu} u^{\nu}, & u^{\mu} \nabla_{\mu} \Theta, & \nabla^{2} T, & u^{\mu} u^{\nu} \nabla_{\mu} \nabla_{\nu} T,
\end{array}
$$

where we have introduced the shear tensor $\sigma_{\mu \nu}$ and the vorticity tensor $\omega_{\mu \nu}$; cf., (2.24) and table 11 for their definition. A-priori we have thirteen independent functions of temperature multiplying these scalars and making for a rather formidable computation. ${ }^{47}$ However, there are some simplifications which we can exploit:

(i) the four terms in the third line can be related to others up to total derivatives

(ii) the four terms in the second line can be related to those in the first by a first order field redefinition (one chooses $e_{a}^{\mu} \delta \varphi^{a}$ to be aligned along either $\mathfrak{a}^{\mu}$ or $\Theta u^{\mu}$ ).

All in all we have five independent terms to consider which still makes for a somewhat complex computation. The end result is that the adiabatic part of 15 independent transport coefficients for a neutral fluid at second order [2] is determined in terms of five functions of temperature, pretty much along the lines of the non-dissipative effective action computation of [46]. We postpone a full discussion of how this works in full detail (including explicit verification of our field redefinitions) to appendix $\mathrm{F}$.

For now we will restrict ourselves to Weyl invariant neutral fluids which are much easier to describe. For one there are only 5 independent transport coefficients [41, 67]. Furthermore, since a pre-requisite for Weyl invariance is that the Lagrangian must be invariant under Weyl rescalings of the background metric $g_{\mu \nu}$, we also have a reduction in the number of terms which enter the Lagrangian. The Weyl transformation properties of various fields are well known. We further develop a Weyl covariant formulation of adiabatic hydrodynamics to deal with fluids arising from conformal field theories, extending [56], in appendix D, where the reader can find some of the necessary details for the computations below.

The Weyl covariant second order scalars at our disposal are (each with Weyl weight ${ }^{48}$ $w=+2)$

$$
\sigma^{2}, \quad \omega^{2}, \quad\left({ }^{\mathcal{W}} R\right), \quad g^{\mu \nu}\left(\mathcal{D}_{\mu}^{\mathcal{W}} \log T\right)\left(\mathcal{D}_{\nu}^{\mathcal{W}} \log T\right) .
$$

\footnotetext{
${ }^{46}$ The advantage of this parameterization is that it is easier to read off the energy-momentum tensor upon variation. It is straightforward to use (2.1) to rewrite these in terms of $\boldsymbol{\beta}^{\mu}$ and its derivatives if necessary.

${ }^{47}$ A classification of independent second order scalars was done in [2]. Specifically, off-shell genuine second order scalars were shown to be five in total, which are the last four scalars in (8.17) along with the Ricci scalar $R$. The others are products of one-derivative objects. While [2] took these to be the first four scalars of (8.17) after using (8.9), we have a-priori included the terms in the second line since we choose to remain off-shell.

${ }^{48} \mathrm{~A}$ tensor is said to have Weyl weight $w$ if under Weyl rescalings of the background metric $g_{\mu \nu} \rightarrow e^{2 \phi} \tilde{g}_{\mu \nu}$ it transforms homogeneously with a rescaling $e^{-w \phi}$. The metric itself has Weyl weight $w=-2$ with these conventions. For more details please see appendix D.
} 
Using the identification (D.31), the Weyl covariant derivative is defined in (D.6) and the associated Ricci scalar in (D.15). Since the temperature $T$ has Weyl weight $w=+1$ it follows that the Lagrangian which is invariant under Weyl transformations has to take the form

$$
\begin{aligned}
\mathcal{L}_{2}^{\mathcal{W}}= & k_{\sigma} T^{d-2} \sigma^{2}+k_{\omega} T^{d-2} \omega^{2} \\
& +k_{R} T^{d-2}\left[R-(d-2)(d-1) \mathfrak{a}^{2}+\frac{d-2}{d-1} \Theta^{2}-2(d-2)(d-1) \mathfrak{a}^{\alpha} \nabla^{\alpha} \log T\right. \\
& \left.+2(d-2) \Theta u^{\alpha} \nabla_{\alpha} \log T\right] \\
& +k_{T} T^{d-2}\left(\left(\nabla_{\mu} \log T\right)^{2}+2 \mathfrak{a}^{\mu} \nabla_{\mu} \log T-\frac{2 \Theta}{d-1} u^{\mu} \nabla_{\mu} \log T+\mathfrak{a}^{2}-\frac{\Theta^{2}}{(d-1)^{2}}\right)
\end{aligned}
$$

where $k_{\sigma}, k_{\omega}, k_{R}$ and $k_{T}$ are constants. All the dependence on the thermal vector is implicit in (8.19); if necessary we can convert all the terms to appropriate combinations of $\boldsymbol{\beta}^{\mu}$ and its derivatives. The field redefinition freedom discussed in section 7.6 allows us to set $k_{T}=0$ which we shall do forthwith (cf., appendix $\mathrm{F}$ for further details).

The variation of the various terms in the Lagrangian can be computed using the rules given in (E.1) in a straightforward (albeit tedious) manner. One of the advantages of using the standard parameterization in terms of the velocity and temperature instead of $\boldsymbol{\beta}^{\mu}$ is that simplifications at intermediate steps using hydrodynamic identities are transparent. We present the variation of the full neutral fluid in appendix F; see (F.5a)-(F.5e) from which the relevant details for the Weyl invariant fluid can be extracted. Let us therefore pass directly to a discussion of the stress tensor.

The Weyl covariant stress tensor for conformal fluid is expressed in a succinct manner in the following basis of five independent tensors [56] (see also [57]) as: ${ }^{49}$

$$
\begin{aligned}
T_{(2), \mathcal{W}}^{\mu \nu}=\tau u^{\alpha} & \mathcal{D}_{\alpha}^{\mathcal{W}} \sigma^{\mu \nu}+\kappa C^{\mu \alpha \nu \beta} u_{\alpha} u_{\beta} \\
& +\lambda_{1} \sigma^{\langle\mu \alpha} \sigma_{\alpha}{ }^{\nu\rangle}+\lambda_{2} \sigma^{\langle\mu \alpha} \omega_{\alpha}{ }^{\nu\rangle}+\lambda_{3} \omega^{\langle\mu \alpha} \omega_{\alpha}{ }^{\nu\rangle},
\end{aligned}
$$

where the longitudinal Weyl covariant derivative evaluates to

$$
u^{\alpha} \mathcal{D}_{\alpha}^{\mathcal{W}} \sigma^{\mu \nu}=P_{\rho}^{\mu} P_{\sigma}^{\nu} u^{\alpha} \nabla_{\alpha} \sigma^{\rho \sigma}+\frac{\Theta}{d-1} \sigma^{\mu \nu} .
$$

This expression is written in the so called Landau frame where the corrections to the ideal fluid stress tensor are demanded to be perpendicular to the velocity field, i.e., $T^{\mu \nu}=$ $T_{(0)}^{\mu \nu}+\sum_{k \geq 1} T_{(k)}^{\mu \nu}$ with $u_{\mu} T_{(k)}^{\mu \nu}=0$. We can equivalently write (8.20) in a basis adapted to our classification scheme:

$$
\begin{aligned}
T_{(2), \mathcal{W}}^{\mu \nu}= & \left(\lambda_{1}-\kappa\right) \sigma^{<\mu \alpha} \sigma_{\alpha}^{\nu>}+\left(\lambda_{2}+2 \tau-2 \kappa\right) \sigma^{<\mu \alpha} \omega_{\alpha}^{\nu>} \\
& +\tau\left(u^{\alpha} \mathcal{D}_{\alpha}^{\mathcal{W}} \sigma^{\mu \nu}-2 \sigma^{<\mu \alpha} \omega_{\alpha}^{\nu>}\right)+\lambda_{3} \omega^{<\mu \alpha} \omega_{\alpha}^{\nu>} \\
& +\kappa\left(C^{\mu \alpha \nu \beta} u_{\alpha} u_{\beta}+\sigma^{<\mu \alpha} \sigma_{\alpha}^{\nu>}+2 \sigma^{<\mu \alpha} \omega_{\alpha}^{\nu>}\right) .
\end{aligned}
$$

\footnotetext{
${ }^{49}$ When comparing with the expressions in these papers we warn the reader that there are some convention differences (mostly involving factors of two and the sign in the definition of $\omega_{\mu \nu}$ and some index contractions). See footnote 91 for a mapping between conventions of various papers.
} 
As we will see shortly, this adiabaticity adapted basis is more natural for it does not mix the different classes in the eightfold way; each term will turn out to be at home in a unique class. The first two terms will turn out to be forced to vanish in Class L, while the remaining three will be unconstrained.

The raw expressions obtained from the variation in appendix $\mathrm{F}$ are somewhat unilluminating written as they are in a non-standard basis of tensors. As before we have to use the on-shell equations of motion for the ideal fluid (8.9) to eliminate the thermal gradient terms. A somewhat more tricky proposition is the fact that the stress tensor which solves the adiabaticity equation is not necessarily in the Landau frame. Since the solution to the adiabaticity equation (2.13) in Class L for non-anomalous fluids has $J_{S}^{\mu}=s u^{\mu}$ one may in fact view the result as naturally being cast in the entropy frame (see also [46, 48]). To compare the results with the Landau frame presentation, we first switch off the first order terms (since they carry no physical information). We then project the stress tensor computed by the variational principle onto the frame invariant tensor and scalar parts. This is a relatively trivial exercise and one can then read off the coefficients of the independent tensors used in (F.12). The projectors in question are given explicitly in (8.14). Carrying out the aforementioned computation we find the following set of transport coefficients for a Weyl invariant neutral fluid $[65]^{50}$

$$
\begin{aligned}
\eta & =\zeta=0, \\
\tau & =-\left(2(d-2) k_{R}+2 k_{\sigma}\right) T^{d-2}, \\
\kappa & =-2(d-2) k_{R} T^{d-2}, \\
\lambda_{1} & =-2(d-2) k_{R} T^{d-2}, \\
\lambda_{2} & =4 k_{\sigma} T^{d-2}, \\
\lambda_{3} & =-2\left((d-2) k_{R}-2 k_{\omega}\right) T^{d-2} .
\end{aligned}
$$

The scaling with temperature can of course be determined on dimensional grounds. Equivalently, the Weyl covariant stress tensor in Class L is forced to take the form

$$
\begin{aligned}
T_{(2), \mathcal{W}}^{\mu \nu}=\tau\left(u^{\alpha} \mathcal{D}_{\alpha}^{\mathcal{W}} \sigma^{\mu \nu}-2 \sigma^{<\mu \alpha} \omega_{\alpha}^{\nu>}\right)+\lambda_{3} \omega^{<\mu \alpha} \omega_{\alpha}^{\nu>} \\
+\kappa\left(C^{\mu \alpha \nu \beta} u_{\alpha} u_{\beta}+\sigma^{<\mu \alpha} \sigma_{\alpha}^{\nu>}+2 \sigma^{<\mu \alpha} \omega_{\alpha}^{\nu>}\right)
\end{aligned}
$$

which is written in the basis of (8.22) and can be derived from a two-derivative Lagrangian density

$$
\mathcal{L}_{2}^{\mathcal{W}}=\frac{1}{4}\left[-\frac{2 \kappa}{(d-2)}\left({ }^{\mathcal{W}} R\right)+2(\kappa-\tau) \sigma^{2}+\left(\lambda_{3}-\kappa\right) \omega^{2}\right] .
$$

What is interesting about the result (8.23) is the following: given that there are a-priori three parameters allowed in our Lagrangian, $\left\{k_{\sigma}, k_{\omega}, k_{R}\right\}$, after exploiting field redefinition freedom, we expect two relations between the five transport coefficients. These can be ascertained by inspection of (8.24) to be the simple linear relations:

$$
\lambda_{1}=\kappa, \quad \lambda_{2}=2(\kappa-\tau) .
$$

\footnotetext{
${ }^{50}$ The first derivation of the second order transport coefficients was carried out explicitly in $d=4$ by $[41,67]$.
} 
These relations are actually quite fascinating; we have an infinite class of hydrodynamic constitutive relations for which they hold thanks to the holographic fluid/gravity correspondence, cf., [52]. We will return to a complete discussion of holographic fluids and its relation to the adiabatic eightfold way in section 14 .

\subsection{Parity-odd fluids in 3 dimensions}

Our second example concerns the class of parity-odd charged fluids in 3 spacetime dimensions. This system has been described in the non-dissipative effective action framework originally [47] and was revisited more recently in [48]. The investigations of the latter reference revealed that there is some tension in incorporating aspects of Hall transport in this framework (see also [46]). This has been addressed in an intriguing recent development [49], wherein the Hall viscosity coefficient was captured in terms of a non-local term in the effective action. For the present we will focus on local Lagrangians, but will comment on the non-local terms at the end. Given our discussion of the neutral fluid in section 8.1 we will be a bit brief in the following, indicating just the salient results.

\subsubsection{Zeroth order in gradients}

Since we are dealing with charged fluids, we now have the full set of hydrodynamic fields $\boldsymbol{\Psi}$ to consider. At leading order in the gradient expansion, we need a scalar function of these fields. A moment's thought suffices to note that the only function of relevance is a scalar function of temperature and chemical potential (which will be the Gibbs free energy for the system). To wit, we have

$$
\mathcal{L}_{0}[\Psi]=p(T, \mu)
$$

Applying the variational calculus of section 6 using (E.1) leads to ${ }^{51}$

$$
\begin{aligned}
\frac{1}{\sqrt{-g}} \delta\left(\sqrt{-g} \mathcal{L}_{0}\right)=\frac{1}{2}\left(\left(T p^{\prime}+\right.\right. & \left.\mu \dot{p}-p) u^{\mu} u^{\nu}+p P^{\mu \nu}\right) \delta g_{\mu \nu}+\dot{p} u^{\sigma} \delta A_{\sigma} \\
& +\left(T p^{\prime}+\mu \dot{p}\right) T^{2} \boldsymbol{\beta}_{\sigma} \delta \boldsymbol{\beta}^{\sigma}+T \dot{p}\left(\delta \Lambda_{\boldsymbol{\beta}}+A_{\sigma} \delta \boldsymbol{\beta}^{\sigma}\right)
\end{aligned}
$$

There are no boundary terms, and we have the currents for an ideal charged fluid

$$
\begin{array}{ll}
T_{(0)}^{\mu \nu}=\left(T p^{\prime}+\mu \dot{p}-p\right) u^{\mu} u^{\nu}+p P^{\mu \nu}, & J_{(0)}^{\mu}=\dot{p} u^{\mu}, \quad J_{S,(0)}^{\mu}=p^{\prime} u^{\mu} \\
\mathfrak{h}_{(0)}^{\sigma}=\left(T p^{\prime}+\mu \dot{p}\right) T \boldsymbol{\beta}^{\sigma}, & \mathfrak{n}_{(0)}=\dot{p}
\end{array}
$$

In the present instance, $p$ is the pressure of the system and the charge density is given by the thermodynamics to be $q=\dot{p}$ and $\epsilon=p^{\prime}+\mu \dot{p}-p$.

\subsubsection{First order in gradients}

Moving to the next order in gradients, we find that there are no interesting parity-even one derivative scalars build from $\boldsymbol{\Psi}$. The argument for this follows along similar lines as

\footnotetext{
${ }^{51}$ We will continue to denote temperature derivatives with a prime, while derivatives with respect to chemical potential are denoted with an over-dot.
} 
that presented in section 8.1, so we will refrain from repeating it again here. Physically, of course, this is easily understood by noting that there are no non-trivial solutions to the adiabaticity equation at first order in gradients.

However, if we have a system that is parity-odd, then in 3 spacetime dimensions we can write down two scalars which, following [48], we parameterize as

$$
\mathcal{L}_{1}[\Psi]=\tilde{\mathfrak{w}}(T, \mu) \varepsilon^{\rho \sigma \lambda} u_{\rho} \nabla_{\sigma} u_{\lambda}+\tilde{\mathfrak{b}}(T, \mu) \varepsilon^{\rho \sigma \lambda} u_{\rho} \nabla_{\sigma} A_{\lambda} .
$$

In fact these are the two terms which are allowed in the hydrostatic partition function [68]. We have set to zero the parity-even first order terms such as the charged analog of the term discussed in (8.5). They do not contribute to physical transport data. As a result, one can essentially view $\mathcal{L}_{1}[\Psi]$ as the off-shell extension of the equilibrium partition function.

Varying the Lagrangian density we find for the hydrodynamic currents

$$
\begin{aligned}
T_{(1)}^{\alpha \beta}=2 \varepsilon^{(\alpha \rho \sigma} u^{\beta)}\left(2 \tilde{\mathfrak{w}} \nabla_{\rho} u_{\sigma}+\tilde{\mathfrak{b}} \nabla_{\rho} A_{\sigma}-u_{\rho}\left[\tilde{\mathfrak{w}}^{\prime} \nabla_{\sigma} T+\dot{\tilde{\mathfrak{w}}} \nabla_{\sigma} \mu\right]\right) \\
+u^{\alpha} u^{\beta}\left(\left[T \tilde{\mathfrak{w}}^{\prime}+\mu \dot{\tilde{\mathfrak{w}}}+2 \tilde{\mathfrak{w}}\right] \Omega+\left[T \tilde{\mathfrak{b}}^{\prime}+\mu \dot{\tilde{\mathfrak{b}}}+\tilde{\mathfrak{b}}\right] \mathfrak{B}\right) \\
J_{(1)}^{\alpha}=\varepsilon^{\alpha \rho \sigma} \tilde{\mathfrak{b}} \nabla_{\rho} u_{\sigma}-\varepsilon^{\alpha \rho \sigma} u_{\rho}\left(\tilde{\mathfrak{b}}^{\prime} \nabla_{\sigma} T+\dot{\tilde{\mathfrak{b}}} \nabla_{\sigma} \mu\right)+[\dot{\tilde{\mathfrak{w}}} \Omega+\dot{\tilde{\mathfrak{b}}} \mathfrak{B}] u^{\alpha}
\end{aligned}
$$

with the pre-symplectic potential

$$
\left(\not \Theta_{\mathrm{PS}}\right)_{(1)}^{\sigma}=\tilde{\mathfrak{w}}(T, \mu) \varepsilon^{\rho \sigma \lambda} u_{\rho} \delta u_{\lambda}+\tilde{\mathfrak{b}}(T, \mu) \varepsilon^{\rho \sigma \lambda} u_{\rho} \delta A_{\lambda}
$$

and the adiabatic heat current and adiabatic charge density entering the Bianchi identities

$$
\begin{aligned}
& \mathfrak{h}_{(1)}^{\alpha}=\left(\left[T \tilde{\mathfrak{w}}^{\prime}+\mu \dot{\tilde{\mathfrak{w}}}+2 \tilde{\mathfrak{w}}\right] \Omega+\left[T \tilde{\mathfrak{b}}^{\prime}+\mu \dot{\tilde{\mathfrak{b}}}+\tilde{\mathfrak{b}}\right] \mathfrak{B}\right) u^{\alpha} \\
& \quad+\varepsilon^{\alpha \rho \sigma}\left(2 \tilde{\mathfrak{w}} \nabla_{\rho} u_{\sigma}-\dot{\tilde{\mathfrak{w}}} u_{\rho} \nabla_{\sigma} \mu-\tilde{\mathfrak{w}}^{\prime} u_{\rho} \nabla_{\sigma} T+\tilde{\mathfrak{b}} \nabla_{\rho} A_{\sigma}\right) \\
& \mathfrak{n}_{(1)}=\dot{\tilde{\mathfrak{w}}} \Omega+\dot{\tilde{\mathfrak{b}}} \mathfrak{B}
\end{aligned}
$$

where we defined the parity-odd scalars

$$
\Omega \equiv \varepsilon^{\rho \sigma \lambda} u_{\rho} \nabla_{\sigma} u_{\lambda}, \quad \mathfrak{B} \equiv \frac{1}{2} \varepsilon^{\rho \sigma \lambda} u_{\rho} B_{\sigma \lambda}=\varepsilon^{\rho \sigma \lambda} u_{\rho} \nabla_{\sigma} A_{\lambda} .
$$

The entropy current derived from (8.33) and (8.34) reads

$$
J_{S,(1)}^{\mu}=\left(\tilde{\mathfrak{w}}^{\prime} \Omega+\tilde{\mathfrak{b}}^{\prime} \mathfrak{B}\right) u^{\mu} .
$$

These expressions are reasonably similar to the ones derived in the non-dissipative effective action formalism by [48]. One can pursue their algorithm to extract the transport coefficients as we summarize below.

Firstly, the most general parity-odd first order stress tensor and charge current are given by the following Landau frame expressions $[68]^{52}$

$$
\begin{aligned}
T_{(1)}^{\alpha \beta} & =\left(-\zeta \Theta+\tilde{\chi}_{B} \mathfrak{B}+\tilde{\chi}_{\Omega} \Omega\right) P^{\alpha \beta}-2 \eta \sigma^{\alpha \beta}-\tilde{\eta}_{H} \varepsilon^{\mu \nu(\alpha} u_{\mu} \sigma_{\nu}^{\beta)}, \\
J_{(1)}^{\alpha} & =\sigma_{\mathrm{Ohm}} \mathfrak{v}^{\alpha}+\tilde{\chi}_{T} \varepsilon^{\alpha \mu \nu} u_{\mu} \nabla_{\nu} T+\tilde{\chi}_{E} \varepsilon^{\alpha \mu \nu} u_{\mu} E_{\nu}+\tilde{\sigma}_{H} \varepsilon^{\alpha \mu \nu} u_{\mu} \mathfrak{v}_{\nu} .
\end{aligned}
$$

\footnotetext{
${ }^{52}$ The analysis of [68] also a-priori allows the two further parity-even vectors contributions in the charge current, viz., $J_{(1)}^{\alpha}=-\chi_{T} T \mathfrak{a}^{\alpha}+\chi_{E} E^{\alpha}$. We have used the fact that the only parity-even contribution compatible with the second law is the conductivity term exhibited in (8.38) and thus set $\chi_{T}=\chi_{E}=0$.
} 
where we use the parity-even vector introduced in (2.25). The coefficient $\tilde{\eta}_{H}$ is called the Hall viscosity and $\tilde{\sigma}_{H}$ is the (hydrodynamic) Hall conductivity. We will refer to $\tilde{\chi}_{T}$ as the odd Ohm conductivity and $\tilde{\chi}_{T}$ as the odd thermal conductivity.

To compare this expression with (8.31) we have to project once again onto frame invariant combinations. Doing so we find the following relations (setting $\tilde{\mu} \equiv \frac{\epsilon+p}{q}=\mu+\frac{s T}{q}$ and $v_{s}^{2} \equiv\left[\frac{\partial p}{\partial \epsilon}\right]_{q}$ with $q=\dot{p}$ for brevity):

$$
\begin{aligned}
\tilde{\chi}_{\Omega} & =v_{s}^{2}\left(2 \tilde{\mathfrak{w}}-T \frac{\partial \tilde{\mathfrak{w}}}{\partial T}-\mu \frac{\partial \tilde{\mathfrak{w}}}{\partial \mu}\right)+\left[\frac{\partial p}{\partial q}\right]_{\epsilon}\left(\tilde{\mathfrak{b}}-\frac{\partial \tilde{\mathfrak{w}}}{\partial \mu}\right), \\
\tilde{\chi}_{B} & =v_{s}^{2}\left(\tilde{\mathfrak{b}}-T \frac{\partial \tilde{\mathfrak{b}}}{\partial T}-\mu \frac{\partial \tilde{\mathfrak{b}}}{\partial \mu}\right)-\left[\frac{\partial p}{\partial q}\right]_{\epsilon} \frac{\partial \tilde{\mathfrak{b}}}{\partial \mu}, \\
T \tilde{\chi}_{T} & =\left(\tilde{\mathfrak{b}}-T \frac{\partial \tilde{\mathfrak{b}}}{\partial T}-\mu \frac{\partial \tilde{\mathfrak{b}}}{\partial \mu}\right)-\frac{1}{\tilde{\mu}}\left(2 \tilde{\mathfrak{w}}-T \frac{\partial \tilde{\mathfrak{w}}}{\partial T}-\mu \frac{\partial \tilde{\mathfrak{w}}}{\partial \mu}\right), \\
\tilde{\chi}_{E} & =-\frac{\partial \tilde{\mathfrak{b}}}{\partial \mu}+\frac{1}{\tilde{\mu}}\left(\frac{\partial \tilde{\mathfrak{w}}}{\partial \mu}-\tilde{\mathfrak{b}}\right), \\
\tilde{\sigma}_{H}+\tilde{\chi}_{E} & =-\frac{2}{\tilde{\mu}}\left(\tilde{\mathfrak{b}}-\frac{1}{\tilde{\mu}} \tilde{\mathfrak{w}}\right), \\
\sigma_{\mathrm{Ohm}} & =\eta=\zeta=\tilde{\eta}_{H}=0 .
\end{aligned}
$$

We see from (8.39) that the stress tensor does not have any frame-invariant tensor data and thus the Hall viscosity $\tilde{\eta}_{H}$ predicted by (8.30) vanishes, consistent with $[46,48]$. Examining the charge current, one finds that the Hall conductivity $\tilde{\sigma}_{H}$ and $\tilde{\chi}_{E}$ can be taken to be the independent transport coefficients:

$$
\begin{aligned}
& \tilde{\mathfrak{w}}=\frac{\tilde{\mu}^{2}}{2}\left[\frac{\tilde{\mu} \frac{\partial}{\partial \mu}\left(\tilde{\chi}_{E}+\tilde{\sigma}_{H}\right)+2 \tilde{\chi}_{E}}{\frac{\partial}{\partial \mu}\left(\frac{s T}{q}\right)}\right]+\frac{\tilde{\mu}^{2}}{2}\left(\tilde{\chi}_{E}+\tilde{\sigma}_{H}\right), \\
& \tilde{\mathfrak{b}}=\frac{\tilde{\mu}}{2}\left[\frac{\tilde{\mu} \frac{\partial}{\partial \mu}\left(\tilde{\chi}_{E}+\tilde{\sigma}_{H}\right)+2 \tilde{\chi}_{E}}{\frac{\partial}{\partial \mu}\left(\frac{s T}{q}\right)}\right] .
\end{aligned}
$$

It is a-priori tempting, based on the linear relation involving the odd Ohm and Hall conductivities, to speculate that one can fix $\tilde{\sigma}_{H}$ in terms of the other transport coefficients.

We expect in general three relations amongst the set $\left\{\tilde{\chi}_{\Omega}, \tilde{\chi}_{B}, \tilde{\chi}_{T}, \tilde{\chi}_{E}, \tilde{\sigma}_{H}\right\}$. One of these is the expression in the penultimate line of (8.39). Another which can be ascertained by eliminating the Lagrangian scalars from the first four lines of (8.39) is

$$
\tilde{\chi}_{B}-\frac{1}{\tilde{\mu}} \tilde{\chi}_{\Omega}-v_{s}^{2} T \tilde{\chi}_{T}=\left[\frac{\partial p}{\partial q}\right]_{\epsilon} \tilde{\chi}_{E}
$$

These results of course agree with those derived earlier in [68] (see also [3]). The final relation can be written using (8.40) eliminating the Lagrangian scalars $\tilde{\mathfrak{w}}, \tilde{\mathfrak{b}}$. We have not been able to derive a simple closed form answer, since we seem to need to employ thermodynamic identities in an involved fashion. 
Passing to a simpler context of Weyl invariant fluids, reveals an unnecessary nuance. Now, $\zeta=\tilde{\chi}_{B}=\tilde{\chi}_{\Omega}=0$ by the tracelessness of the stress tensor and $\tilde{\chi}_{T}=0$ by virtue of $\nabla_{\nu} T$ not being homogeneous under Weyl rescaling (see appendix $\mathrm{D}$ ). Then the only non-vanishing vector transport are the conductivities and the parity-odd ones have to be determined in terms of the Lagrangian functions. ${ }^{53}$ One linear combination of $\tilde{\chi}_{E}$ and $\tilde{\sigma}_{H}$ gives a linear combination of the Lagrangian scalars, but this allows both of them to be independent. ${ }^{54}$

Despite these complications we suspect that Class L does not allow for the most general form of the Hall conductivity $\tilde{\sigma}_{H}$. At the same time, a curious fact of the Class L effective action is the vanishing of the Hall viscosity as has been noted earlier in [48]. In summary we see that in Class L

$$
\tilde{\eta}_{H}=0, \quad \tilde{\sigma}_{H}=\text { fixed } .
$$

As we shall see later in section 9 these relations are akin to the relation between $\left\{\tau, \lambda_{1}, \lambda_{2}\right\}$ for the neutral fluid (8.26). Since $\tilde{\sigma}_{H}$ and $\tilde{\eta}_{H}$ are completely unconstrained by the second law [68] one should find that any value for the Hall viscosity is acceptable in hydrodynamics. We defer comments on non-local Lagrangians and the recent construction of [49] to section 9, since understanding deviations from relations such as (8.26) and (8.42) is part of a more general endeavour of constructing actions for Berry-like transport. Once we understand that they are Berry-like terms (Class B), we will be able to find an effective action which allows arbitrary non-vanishing value of $\tilde{\eta}_{H}$ and $\tilde{\sigma}_{H}$ in our extended formalism (see section 17).

\section{Class B: Berry-like transport}

We have explored for the most part of our discussion, solutions to the adiabaticity equation classified by a Lagrangian $\mathcal{L}[\mathbf{\Psi}]$, which we termed as Class L adiabatic fluids. This raises an interesting question: "Are all solutions to (2.12) obtained from a suitable Lagrangian?" The answer surprisingly turns out to be no! There are several classes of adiabatic transport that do not appear to fit into a simple Lagrangian description. We have already hinted that anomalous transport (Class A) requires more structure. In particular, in [30] we argued that an anomalous hydrodynamic effective action necessarily involves a Schwinger-Keldysh doubling of fields in order to satisfy the correct Ward identities. One might argue that such transport comprising typically of finite set of terms (those governed by the anomaly) is special.

However, strangely enough, this doubling trick by itself does not appear to suffice in general. We find three additional classes of transport, of which two (Class B and Class $\overline{\mathrm{H}}_{V}$ ) are non-finite classes (the third Class $\mathrm{C}$ is finite). To complete our classification scheme and to understand the nature of adiabatic transport, we have to indeed analyze what such

\footnotetext{
${ }^{53}$ One can confirm that (8.33) trivializes in a Weyl invariant fluid.

${ }^{54}$ The one exception to this argument is the special case of an equation of state where we have a temperature independence, e.g., $p(T, \mu)=C \mu^{3}$ for some constant $C$ as one encounters for an extremal black hole (in the holographic context), since this implies that $\frac{\partial}{\partial \mu}\left(\frac{s T}{q}\right)=0$.
} 
constitutive relations mean. Therefore, before trying to enlarge the set of Lagrangian Class L transport by incorporating Schwinger-Keldysh doubling and influence functionals (c.f., section 13), let us now explicitly construct the parts of adiabatic transport which do not seem to fit into Class L.

We start with adiabatic transport that we call Class B (for Berry curvature inspired constitutive relations). These Berry terms actually arise in familiar contexts of hydrodynamic transport, and have indeed been encountered hitherto, without the general structure however being appreciated. The simplest examples of these arise in parity-odd fluids in $2+1$ dimensions, where the Hall transport falls in this class, cf., section 8.2. We first introduce the basic tensor structures and constitutive relations in section 9.1 and then exhibit some examples to illustrate the construction. ${ }^{55}$

\subsection{The Berry constitutive relations}

We now describe a large set of solutions to the non-anomalous adiabaticity equation based purely on exploiting some tensor structures which conspire effectively. The logic is going to be similar to our discussion in section 5 . We will start with the grand canonical adiabaticity equation (2.21) reproduced here for convenience:

$$
-\nabla_{\sigma}\left(\frac{\mathcal{G}^{\sigma}}{T}\right)=\frac{1}{2} T^{\mu \nu} \delta_{\mathfrak{B}} g_{\mu \nu}+J^{\mu} \cdot \delta_{\mathfrak{B}} A_{\mu}
$$

We are going to use the fact that the r.h.s. involves an explicit contribution from the variation of the background fields along $\mathcal{B}$ via the $\left\{\delta_{\mathfrak{B}} g_{\mu \nu}, \delta_{\mathfrak{B}} A_{\mu}\right\}$ insertions. Imagine picking an ansatz for the conserved currents which also contains an explicit insertion of these operators, i.e., schematically consider $T^{\mu \nu} \propto \delta_{\mathfrak{B}} g_{\alpha \beta}$ and $J^{\alpha} \propto \delta_{\mathcal{B}} A_{\beta}$. If the intertwining tensors that complete the map above have the appropriate symmetries, then it is plausible that upon further contraction with $\delta_{\mathfrak{B}} g_{\mu \nu}$ or $\delta_{\mathcal{B}} A_{\mu}$ we ensure that the divergence of the free energy current vanishes. This means that we can solve the adiabaticity equation with the no free energy current; the conserved currents themselves conspire to ensure lack of dissipation.

Inspired by the above argument, consider the following constitutive relations: ${ }^{56}$

$$
\begin{aligned}
\left(T^{\mu \nu}\right)_{\mathrm{B}} & \equiv-\frac{1}{4}\left(\mathcal{N}^{(\mu \nu)(\alpha \beta)}-\mathcal{N}^{(\alpha \beta)(\mu \nu)}\right) \delta_{\mathcal{B}} g_{\alpha \beta}+\mathcal{X}^{(\mu \nu) \alpha} \cdot \delta_{\mathcal{B}} A_{\alpha} \\
\left(J^{\alpha}\right)_{\mathrm{B}} & \equiv-\frac{1}{2} \mathcal{X}^{(\mu \nu) \alpha} \delta_{\mathcal{B}} g_{\mu \nu}-\mathcal{S}^{[\alpha \beta]} \cdot \delta_{\mathcal{B}} A_{\beta}
\end{aligned}
$$

where $\left\{\mathcal{N}^{\mu \nu \alpha \beta}, \mathcal{X}^{\mu \nu \alpha}, \mathcal{S}^{\alpha \beta}\right\}$ are arbitrary tensors (modulo field redefinitions). Here $(\alpha \beta)$ and $[\alpha \beta]$ indicates the usual projection to the symmetric and anti-symmetric parts respectively.

\footnotetext{
${ }^{55}$ Using the differential operators introduced in section 5.2 one can in a single swoop construct all Class B constitutive relations. This discussion being somewhat abstract is better appreciated once the basic story is laid out.

${ }^{56}$ The conflation of the notation with the tensor structures used for describing Class D constitutive relations in section 5.3 is intentional. It will allow us later to talk about a single tensor structure whose symmetric part contributes to Class D and anti-symmetric part to Class B.
} 
Substituting the above constitutive relations into the adiabaticity equation in the grand canonical ensemble, we get

$$
\begin{aligned}
\frac{1}{2}\left(T^{\mu \nu}\right)_{\mathrm{B}} & \delta_{\mathcal{B}} g_{\mu \nu}+\left(J^{\alpha}\right)_{\mathrm{B}} \cdot \delta_{\mathcal{B}} A_{\alpha} \\
= & -\frac{1}{8}\left(\mathcal{N}^{(\mu \nu)(\alpha \beta)}-\mathcal{N}^{(\alpha \beta)(\mu \nu)}\right) \delta_{\mathfrak{B}} g_{\mu \nu} \delta_{\mathfrak{B}} g_{\alpha \beta} \\
& +\frac{1}{2} \mathcal{X}^{(\mu \nu) \alpha} \cdot\left(\delta_{\mathcal{B}} g_{\mu \nu} \delta_{\mathcal{B}} A_{\alpha}-\delta_{\mathcal{B}} A_{\alpha} \delta_{\mathfrak{B}} g_{\mu \nu}\right)-\delta_{\mathcal{B}} A_{\alpha} \cdot \mathcal{S}^{[\alpha \beta]} \cdot \delta_{\mathcal{B}} A_{\beta} \\
= & 0
\end{aligned}
$$

we see that we solve the adiabaticity equation (9.1) if we simply take $\left(\mathcal{G}^{\sigma}\right)_{\mathrm{B}}=0$.

In the micro-canonical ensemble, this is equivalent to taking the entropy current to have a purely canonical contribution, viz.,

$$
\begin{aligned}
\left(J_{S}^{\alpha}\right)_{\mathrm{B}} \equiv & -\boldsymbol{\beta}_{\beta}\left(T^{\alpha \beta}\right)_{\mathrm{B}}-\left(\Lambda_{\boldsymbol{\beta}}+\boldsymbol{\beta}^{\beta} A_{\beta}\right) \cdot\left(J^{\alpha}\right)_{\mathrm{B}} \\
= & \frac{1}{T}\left\{\frac{1}{4}\left(\mathcal{N}^{(\alpha \beta)(\mu \nu)}-\mathcal{N}^{(\mu \nu)(\alpha \beta)}\right) u_{\beta}+\frac{1}{2} \mu \cdot \mathcal{X}^{(\mu \nu) \alpha}\right\} \delta_{\mathcal{B}} g_{\mu \nu} \\
& -\frac{1}{T}\left\{\mathcal{X}^{(\alpha \beta) \nu} u_{\beta}-\mu \cdot \mathcal{S}^{[\alpha \nu]}\right\} \cdot \delta_{\mathcal{B}} A_{\nu}
\end{aligned}
$$

Thus, equations (9.2) and (9.4) give a large set of adiabatic constitutive relations. The set of constitutive relations parameterized by these expressions is what we term to be Class B. ${ }^{57}$

Before we proceed further with our analysis, let us pause to motivate our terminology. The tensors which multiply $\delta_{\mathfrak{B}} g_{\mu \nu}$ and $\delta_{\mathfrak{B}} A_{\mu}$ are anti-symmetric for the most part (the only symmetric tensor is the compensator $\mathcal{X}$ which mixes the two sources). Such anti-symmetric tensors may be viewed as curvatures in the phase space of hydrodynamic fields $\boldsymbol{\Psi}$; indeed, they have the correct symmetries to be interpreted as such. Phase space connections and associated curvatures typically contribute to the Berry phase picked up by the system when it is made to traverse a closed loop in configuration space. While we have not quite justified why $\left\{\mathcal{N}^{[(\mu \nu) \mid(\alpha \beta)]}, \mathcal{S}^{[\mu \nu]}\right\}$ should be thought of as configuration space curvatures, supporting evidence for this interpretation can be advanced by examining the physics of Hall viscosity [58]. As we will see below the Hall viscosity term is the simplest example of Class B transport, and the fact that it is associated with the dynamics of quantum states in the phase space makes it plausible to forward a rationale for our terminology.

All the Class B constitutive relations trivially satisfy hydrostatic principle because they vanish in hydrostatic equilibrium. They thus drop out of the hydrodynamic equations in the hydrostatic limit. These are thus examples of non-hydrostatic but non-dissipative constitutive relations. In fact, some aspects of these as we shall see have been encountered in previous analyses but were not identified to belong to this general class. For instance in the analysis of [68] it was noticed that the Hall transport coefficients are unconstrained by any form of the second law, while [46] noticed a similar feature for a particular combination

\footnotetext{
${ }^{57}$ See, however, the slight generalization below.
} 
of second order transport coefficients for a neutral fluid. We will now show how these arise within the general construction above.

While a general Class B term can be characterized by the tensors $\{\mathcal{N}, \mathcal{X}, \mathcal{S}\}$ with the indicated symmetry properties, which can be classified by working in the gradient expansion, a slight generalization allows us to write a complete solution to Berry transport. Recall our discussion of tensor valued derivative operators and the set of intertwining tensors used to describe dissipative Class D transport in section 5.2. This construction can be exploited to give non-trivial Class B relations as well. This is not quite useful for the purposes of classification, but does provide an alternative perspective on the Berry-like transport whilst simultaneously indicating some degenerate situations where naïve Class D terms are actually adiabatic. Furthermore, it substantiates our earlier statement in section 5 regarding the adiabatic nature of non-symmetric intertwiners.

Firstly let us ask when $\Delta$ obtained in (5.15) vanishes. As the interwiners $\{\boldsymbol{\eta}, \boldsymbol{\sigma}\}$ connect two identical representations, this will happen whenever they transform in an anti-symmetric representation, i.e.,

$$
\Delta=0 \Longrightarrow \boldsymbol{\eta} \in \operatorname{Asym}\left(\operatorname{Tens}_{\boldsymbol{\eta}} \otimes \text { Tens }_{\boldsymbol{\eta}}\right), \quad \boldsymbol{\sigma} \in \operatorname{Asym}\left(\operatorname{Tens}_{\boldsymbol{\sigma}} \otimes \text { Tens }_{\boldsymbol{\sigma}}\right) .
$$

So we clearly have an adiabatic constitutive relation. Thus, equations (5.8) and (5.14) along with the conditions (9.5) give a large set of Class B solutions. One can get even more by generalizing the intertwiner matrix in (5.12) to contain off-diagonal mixed intertwiners $\boldsymbol{\kappa} \in\left(\mathrm{Tens}_{\boldsymbol{\eta}} \otimes \mathrm{Tens}_{\boldsymbol{\sigma}}\right)$ as follows:

$$
\left(\begin{array}{c}
T^{\mu \nu} \\
J^{\alpha}
\end{array}\right)_{\mathrm{B}}=-\left(\begin{array}{ll}
\Upsilon_{\boldsymbol{\eta}_{g}}^{\dagger} & \Upsilon_{\boldsymbol{\sigma}_{g}}^{\dagger} \\
\Upsilon_{\boldsymbol{\eta}_{A}}^{\dagger} & \Upsilon_{\boldsymbol{\sigma}_{A}}^{\dagger}
\end{array}\right)\left(\begin{array}{cc}
\boldsymbol{\eta} & \boldsymbol{\kappa} \\
-\boldsymbol{\kappa} & \boldsymbol{\sigma}
\end{array}\right)\left(\begin{array}{ll}
\Upsilon_{\boldsymbol{\eta}_{g}} & \Upsilon_{\boldsymbol{\eta}_{A}} \\
\Upsilon_{\boldsymbol{\sigma}_{g}} & \Upsilon_{\boldsymbol{\sigma}_{A}}
\end{array}\right)\left(\begin{array}{c}
\frac{1}{2} \delta_{\mathcal{B}} g \\
\delta_{\mathcal{B}} A
\end{array}\right)
$$

The additional $\boldsymbol{\kappa}$-intertwiners with opposite sign drop out of the entropy production (5.13), so they contribute to Class B.

While the description in terms of the tensor valued differential operators $\Upsilon$ and intertwiners $\{\boldsymbol{\eta}, \boldsymbol{\kappa}, \boldsymbol{\sigma}\}$ provides a complete description, there is one difference between this picture and the simpler parameterization introduced in (9.2), which should be borne in mind. This concerns the free energy flux. When the tensor operators $\Upsilon$ in (5.8) are just tensors (i.e., they do not involve derivative operators), they reproduce the simple parametrization (9.2). For in this case no integration by parts is necessary to obtain (5.14), so the Noether current is just zero (which is consistent with (9.4)). However, in the more general case that the constitutive relations (5.8) contain genuine derivative operators, one has to do an integration by parts in (5.13), leaving behind some total derivative terms that give a non-canonical contribution to $\left(J_{S}^{\mu}\right)_{\mathrm{B}}$.

Further, this construction makes it also clear that there are certain constitutive relations which we would want to think of as dissipative, which end up nevertheless in Class B. These are situations wherein the intertwiners $\{\boldsymbol{\eta}, \boldsymbol{\kappa}, \boldsymbol{\sigma}\}$ themselves are built from higher order mixed symmetry representations. Consider for example, the following tensor repre- 
sentations

$$
\begin{gathered}
\square \equiv \operatorname{Sym}_{2}, \\
\mathcal{N}_{\mathrm{B}}^{[(\alpha \beta)(\mu \nu)]}=\square \square^{(\alpha \beta)(\mu \nu)(\rho \lambda)} \delta_{\mathcal{B}} g_{\rho \lambda}, \quad \mathcal{N}_{\mathrm{D}}^{((\alpha \beta)(\rho \lambda))}=\square \square \\
\square \quad \square_{\mathcal{B}}^{(\alpha \beta)(\mu \nu)(\rho \lambda)} \delta_{\mu \nu}
\end{gathered}
$$

i.e., we obtain the intertwiners from the same underlying representation $\square$ by contracting different sets of indices. However, when we compute $\Delta$ we find

$$
\begin{aligned}
\Delta & =\delta_{\mathfrak{B}} g_{\alpha \beta} \mathcal{N}_{\mathrm{D}}^{((\alpha \beta)(\rho \lambda))} \delta_{\mathfrak{B}} g_{\rho \lambda} \\
& =\delta_{\mathfrak{B}} g_{\alpha \beta} \square{ }^{(\alpha \beta)(\mu \nu)(\rho \lambda)} \delta_{\mathfrak{B}} g_{\rho \lambda} \delta_{\mathfrak{B}} g_{\mu \nu} \\
& =\delta_{\mathfrak{B}} g_{\alpha \beta} \mathcal{N}_{\mathrm{B}}^{[(\alpha \beta)(\mu \nu)]} \delta_{\mathfrak{B}} g_{\mu \nu} \\
& =0
\end{aligned}
$$

A similar analysis can be carried out for the flavour charges, by working with the representation Vect instead. In fact, we can make the general statement by choosing to work with the representations $\mathrm{Tens}_{\boldsymbol{\eta}}$ and Tens $\boldsymbol{\sigma}$ respectively. Let

$$
\boldsymbol{\eta} \equiv \text { Tens }_{\boldsymbol{\eta}}, \quad \boldsymbol{\sigma} \equiv \text { Tens }_{\boldsymbol{\sigma}},
$$

and define

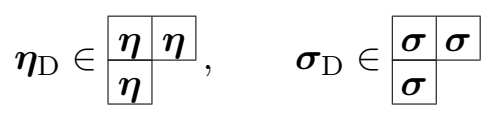

will provide examples of naïve Class D terms which secretly belong to Class B, since they do not produce any entropy.

Before proceeding with explicit examples of Class B terms, we should point out that the constitutive relations above (9.2) are subject to field redefinition ambiguities. For instance we can redefine the thermal vector and twist as in (7.35) which would then affect the intertwiners. We leave it as an exercise for the reader to work out what the changes induced are, noting that they involve rather messy algebra. We will however have a bit more to say about this as we develop the eightfold effective action in section 17 .

\subsection{Examples of Class B transport}

Let us now consider some examples of Berry transport. By construction, Class B constitutive relations have at least one derivative (since $\delta_{\mathfrak{B}} g_{\mu \nu}$ and $\delta_{\mathcal{B}} A_{\mu}$ is linear in the gradients of $\left.\left\{\boldsymbol{\beta}^{\mu}, \Lambda_{\boldsymbol{\beta}}\right\}\right)$. Thus, there are no examples in zero derivative order.

Hall transport in 3 dimensions: at one derivative order, in 3-dimensional parity violating fluids, there is an adiabatic constitutive relation that can be obtained by setting 
$\mathcal{N}^{\mu \nu \alpha \beta}=-\tilde{\eta}_{H} T u_{\rho} \varepsilon^{\rho \mu \alpha} P^{\nu \beta}$ along with $\mathcal{X}^{\mu \nu \alpha}=0$ and $\mathcal{S}^{\alpha \beta}=-\tilde{\sigma}_{H} T u_{\rho} \varepsilon^{\rho \alpha \beta}$. We obtain then for the currents

$$
\begin{aligned}
\left(T^{\mu \nu}\right)_{\mathrm{B}} & =-\tilde{\eta}_{H} \varepsilon^{\alpha \beta(\mu} u_{\alpha} \sigma_{\beta}^{\nu)} \\
\left(J^{\alpha}\right)_{\mathrm{B}} & =\tilde{\sigma}_{H} \cdot \varepsilon^{\alpha \rho \sigma} u_{\rho} \mathfrak{v}_{\sigma} \\
\left(J_{S}^{\alpha}\right)_{\mathrm{B}} & =\frac{\mu}{T} \cdot \tilde{\sigma}_{H} \cdot \varepsilon^{\alpha \rho \sigma} u_{\rho} \mathfrak{v}_{\sigma} .
\end{aligned}
$$

We recognize the transport coefficients $\tilde{\sigma}_{H}$ and $\tilde{\eta}_{H}$ as the Hall conductivity and Hall viscosity respectively, from our discussion in section 8.2. As mentioned earlier the fact that the Hall transport terms on-shell lead to an exactly conserved entropy current (from the adiabaticity equation) was the reason that [68] found in the current algebra approach no constraint on them from the second law. Since the tensor structures vanish in hydrostatics, so we have no information regarding these terms from the equilibrium partition function.

Berry terms in neutral fluids: our second example for Class B constitutive relations is perhaps in the simplest hydrodynamic system imaginable, a neutral fluid! While there is no adiabatic transport at first order, we have seen that there are adiabatic parts to each of the 15 transport coefficients of a neutral fluid, cf., appendix F. Amongst these lurks a term of the form (9.2). Since $\delta_{\mathfrak{B}} g_{\mu \nu}=2 \nabla_{(\mu} \boldsymbol{\beta}_{\nu)}$ can be written using (8.11) in terms of the shear etc., and is clearly a first order term, we pick for the tensor $\mathcal{N}^{\mu \nu \alpha \beta}$ another first order contribution. The symmetries we require fix this tensor uniquely to be

$$
\mathcal{N}^{\mu \nu \alpha \beta}=2 T\left(\lambda_{\sigma} \sigma^{\mu \nu} P^{\alpha \beta}+\lambda_{\omega} \omega^{\mu \alpha} P^{\nu \beta}\right)
$$

Using the decomposition of the gradient of $\boldsymbol{\beta}^{\mu}$ we can express the stress tensor in a simple form:

$$
\left(T^{\mu \nu}\right)_{\mathrm{B}}=-\lambda_{\sigma}\left(\Theta \sigma^{\mu \nu}-\sigma^{2} P^{\mu \nu}\right)-\lambda_{\omega}\left(\omega^{\mu \alpha} \sigma_{\alpha}^{\nu}+\omega^{\nu \alpha} \sigma_{\alpha}^{\mu}\right)
$$

Let us compare this with the parametrization of the second order Landau frame stress tensor given in (F.12). Using two simple identities

$$
\begin{aligned}
\lambda_{0} \Theta \sigma_{\mu \nu}+\xi_{2} P_{\mu \nu} \sigma^{2} & =\frac{\lambda_{0}+\xi_{2}}{2}\left(\Theta \sigma_{\mu \nu}+P_{\mu \nu} \sigma^{2}\right)+\frac{\lambda_{0}-\xi_{2}}{2}\left(\Theta \sigma_{\mu \nu}-P_{\mu \nu} \sigma^{2}\right) \\
\sigma_{\langle\mu}{ }^{\alpha} \omega_{\alpha \nu\rangle} & =-\frac{1}{2}\left(\omega^{\mu \alpha} \sigma_{\alpha}^{\nu}+\omega^{\nu \alpha} \sigma_{\alpha}^{\mu}\right)
\end{aligned}
$$

we identify the two coefficients $\lambda_{\sigma}$ and $\lambda_{\omega}$ as determining linear combinations of the transport coefficients, viz.,

$$
\lambda_{\sigma}=\frac{\xi_{2}-\lambda_{0}}{2}, \quad \lambda_{2}=2 \lambda_{\omega}
$$

The fact that the two tensor structures appearing in (9.13) are non-dissipative was in fact was noticed in the analysis of [46], but again it was not appreciated then that these were part of a larger set of adiabatic transport data in hydrodynamics. 


\subsection{Embedding Class B in Class L?}

Given a couple of examples at our disposal let us take stock of whether we can identify a way to embed Class B into Class L. Each of our two examples has been explored in the non-dissipative effective action framework. So we can make some informed statements about whether or not this is possible. Since the details seem to be a-priori distinct in the two cases we will address them in turn.

Hall transport: the analysis of [48], building on earlier work of [47] and [46], argued that there is no local effective action that captures Hall viscosity. Furthermore, it was found in that construction that the Hall conductivity was not an independent transport coefficient, but rather a linear combination of it and the coefficient $\tilde{\chi}_{E}$ introduced in [68] was fixed by the effective action. More specifically, the tensor structures involved are the ones displayed in (9.11).

We find a very similar relation in the Class L construction outlined in section 8.2. In particular, in (8.39) we have derived the parity-odd transport coefficients in terms of the Lagrangian scalars $\{\tilde{\mathfrak{b}}, \tilde{\mathfrak{w}}\}$. As there are six parity-odd transport coefficients and only two scalar densities, we expect four relations amongst the transport. Two of these are hydrostatic relations which eliminate two combinations of of $\left\{\tilde{\chi}_{\Omega}, \tilde{\chi}_{B}, \tilde{\chi}_{T}\right\}$. One such relation is easy to find algebraically and is given in (8.41); the other appears to be complicated to obtain in closed form. The third relation, which is not hydrodynamic, appears to fix Hall conductivity $\tilde{\sigma}_{H}$, which is invisible in hydrostatics, in terms of a hydrostatic response parameter $\tilde{\chi}_{E},[48,68]$. The final relation is the one that sets the Hall viscosity $\tilde{\eta}_{H}=0$.

A-priori, given that the Hall conductivity and viscosity are adiabatic, any value for these hydrodynamic transport coefficient is acceptable. So it is in fact somewhat curious that the Class L theory fixes their value so specifically. The results obtained herein are consistent with the effective action analysis of [48] (which involves a Legendre transformation - see appendix B). It should be noted that recently [49] have argued that a suitable non-local term allows one to at least obtain non-vanishing Hall viscosity. The construction involved constructing a Wess-Zumino term in the configuration space of fluids (using the Lagrangian scalar variables of the non-dissipative effective action formalism of [45]). ${ }^{58}$ While this construction does indeed give a non-vanishing Hall viscosity, it however constrains it to be of the specific functional form $\tilde{\eta}_{H}=s f(q / s)$, as opposed to a general function of $s$ and $q$ (or $T$ and $\mu$ after Legendre transformation). We believe this is significant and points to a different resolution of the puzzle of Class B Hall transport terms. Indeed, we will later exhibit a Lagrangian system in section 15 which will give us the most general form of Hall transport.

Neutral fluids: the situation in the neutral fluid case is similar. $\lambda_{2}$ is fixed in Class $\mathrm{L}$, and is constrained to obeying the relations (8.26) and (F.18), in situations with and without Weyl invariance respectively. Furthermore, these relations appear to be upheld in two extreme corners: for Weyl invariant strongly coupled holographic plasmas as well as

\footnotetext{
${ }^{58}$ A general construction of Wess-Zumino terms for a wide class of physical systems with various choices of internal symmetries was described in [69].
} 
in kinetic theory. ${ }^{59}$ Once again we do not know of a simple modification to incorporate these terms in Class L, but we will make a case for an extended Lagrangian which allows arbitrary values for Class B transport coefficients in due course.

\section{Class C: conserved entropy}

In hydrodynamics, the conserved currents $\left\{T^{\mu \nu}, J^{\mu}\right\}$ are canonically defined, but the entropy current $J_{S}^{\mu}$ is a more abstruse object. It has no microscopic counterpart, arising as it does due to coarse-graining inherent in the statistical description of the quantum system of interest. Per se one only requires an entropy current satisfying $\nabla_{\mu} J_{S}^{\mu} \geq 0$ or (2.11) to exist, with no implication of uniqueness. The ambiguities in entropy current have been well appreciated in various discussions, cf., $[2,37,64]$ for a sampling of recent literature where this issue is clearly spelt out.

We will now argue that there is one more type of adiabatic constitutive relation solving (2.12) which relies potential ambiguities in the entropy current. Recall that in Class L one can always add arbitrary Komar terms as in (6.21) to any entropy current determined by the Noether construction (in the absence of interesting cohomology). Such ambiguities in the entropy current are physically uninteresting and we won't discuss them further. However, there may actually be other entropy current contributions which are cohomologically non-trivial but still identically conserved without producing energy-momentum or charge transport. These terms are not accounted for in our previous analysis because all adiabatic classes so far led to non-trivial energy-momentum or charge currents, even taking into account field redefinitions.

Let us therefore examine as our next class of non-anomalous adiabatic constitutive relation a very simple set of currents. At any order in the gradient expansion one can consider a family of exactly conserved vectors $\mathrm{J}^{\mu}, \nabla_{\mu} \mathrm{J}^{\mu}=0$. Since we are only interested in solutions to $(2.12)$ we can simply set

$$
\left(J_{S}^{\mu}\right)_{\mathrm{C}}=J^{\mu}, \quad\left(T^{\mu \nu}\right)_{\mathrm{C}}=0, \quad\left(J^{\mu}\right)_{\mathrm{C}}=0
$$

and achieve this desired outcome! As long as we have conserved vector fields $\boldsymbol{J}^{\mu}[\boldsymbol{\Psi}]$ we have achieved a trivial adiabatic constitutive relation.

For reasons described earlier, not all conserved vector fields $\mathrm{J}^{\mu}$, or equivalently their dual current $(d-1)$-forms $\mathbf{j}$, are physically interesting. A trivial class of conserved currents can be obtained by taking $\mathrm{J}^{\mu}=\nabla_{\nu} \mathrm{X}^{[\mu \nu]}$ for some anti-symmetric tensor $\mathrm{X}^{\mu \nu}$; in other words the entropy current $(d-1)$ form is exact $\star \mathbf{j}=d(\star \mathbf{x}) \Longrightarrow d(\star \mathbf{j})=0$. As in any physical application, we are interested in cohomologically non-trivial conserved currents. These are similar to the Komar terms encountered in Class L which are uninteresting as long as there are no boundaries. We shall later see that in the extended Lagrangian theory these will correspond to total derivative boundary terms. We will henceforth quotient the

\footnotetext{
${ }^{59}$ In the holographic context the relations are only valid in two derivative Einstein-Hilbert theory. Higher derivative corrections appear to spoil the Class L relation fixing $\lambda_{2}$, see [70-72] and our discussion in section 14.5.
} 
space of conserved currents by such exactly conserved currents and Class C will comprise of cohomologically non-trivial currents.

Since here we have no energy-momentum or charge transport, but solely entropy flux along the chosen vector field, one has a macroscopic manifestation of entropy without any physical effect. While one might a-priori think that even non-trivial elements of the cohomology, i.e., non-exact $(d-1)$-current forms are uninteresting, there are certain choices of $\mathrm{J}^{\mu}$ which are worth exploring closely.

To do so, let us consider some examples, starting as usual with parity-even charged fluids. For vectors built out of $\boldsymbol{\Psi}$ and their gradients, it is clear that there is no conserved vector at first order in gradients; the three parity-even vectors $\mathfrak{a}^{\mu}, \Theta u^{\mu}$ and $\mathfrak{v}^{\mu}$ are generically non-conserved. At higher orders it is possible to find conserved vectors, but most of these are exact differentials of the form $\nabla_{\nu} \mathrm{X}^{[\mu \nu]}$. For instance, we have five such vectors at second order in gradients, since we have a plethora of first order anti-symmetric tensors [37],

$$
\mathrm{X}^{\mu \nu} \in\left\{u^{[\mu} \mathfrak{a}^{\nu]}, \omega^{\mu \nu}, u^{[\mu} \mathfrak{v}^{\nu]}, u^{[\mu} \nabla^{\nu]}\left(\frac{\mu}{T}\right), P^{\mu \alpha} P^{\nu \beta} F_{\alpha \beta}\right\},
$$

which give an exactly conserved entropy current at second order. These we discard for being trivial cohomological elements.

One however has a non-trivial conserved current in odd spacetime dimensions owing to topological considerations. The simplest example is in three-dimensional parity-even neutral fluids where, inspired by Wen-Zee shift current [59] which appears in Hall transport, we have the following second order conserved vector:

$$
\mathrm{J}_{\text {Euler }}^{\sigma}=\frac{1}{2} c_{\text {Euler }} \varepsilon^{\sigma \alpha \beta} \varepsilon^{\mu \nu \lambda} u_{\mu}\left(\nabla_{\alpha} u_{\nu} \nabla_{\beta} u_{\lambda}-\frac{1}{2} R_{\nu \lambda \alpha \beta}\right),
$$

where $R_{\alpha \beta \gamma \delta}$ is the Riemann tensor and $c_{\text {Euler }}$ is an arbitrary constant. The nomenclature is motivated by the fact that the conserved topological charge associated with this current is the Euler characteristic of the codimension-one spatial slice normal to $u^{\mu}[61]$. It is easy to check conservation directly, though the analysis is greatly facilitated by writing the associated current 2-form. We give a simple derivation of this fact and the generalization to arbitrary odd $d=2 n+1$ dimensions in appendix C. ${ }^{60}$ From there we find that in general

$$
\begin{aligned}
\mathrm{J}_{\text {Euler }}^{\sigma}= & -\frac{1}{2^{n}} c_{\text {Euler }} \varepsilon^{\sigma \alpha_{1} \alpha_{2} \ldots \alpha_{2 n-1} \alpha_{2 n}} u_{\mu} \varepsilon^{\mu \nu_{1} \nu_{2} \ldots \nu_{2 n-1} \nu_{2 n}} \\
& \times\left(\frac{1}{2} R_{\nu_{1} \nu_{2} \alpha_{1} \alpha_{2}}-\nabla_{\alpha_{1}} u_{\nu_{1}} \nabla_{\alpha_{2}} u_{\nu_{2}}\right) \\
& \times \ldots\left(\frac{1}{2} R_{\nu_{2 n-1} \nu_{2 n} \alpha_{2 n-1} \alpha_{2 n}}-\nabla_{\alpha_{2 n-1}} u_{\nu_{2 n-1}} \nabla_{\alpha_{2 n}} u_{\nu_{2 n}}\right)
\end{aligned}
$$

is a conserved current present at the $(2 n)^{\text {th }}$ derivative order and gives a Class $\mathrm{C}$ solution to (2.12).

\footnotetext{
${ }^{60}$ These currents were recently revisited in the context of parity-odd Hall fluids in [60] and [61]. The latter work independently generalized the construction to arbitrary odd dimensions. Our discussion in appendix $\mathrm{C}$ provides a complementary perspective.
} 
Let us understand the physical consequence of the Euler current contribution to entropy current in $d=3$. The Euler current reduces to the Euler character of the spatial two manifold on which we place our fluid. Let us for simplicity take $\mathcal{M}_{3}=\mathbb{R} \times \Sigma_{2}$ where $\Sigma_{2}$ is a compact two manifold. Then $\mathrm{J}_{\text {Euler }}^{\mu} u_{\mu}$ is a measure of the topology of $\Sigma_{2}$, and in particular its integral gives the Euler character (and hence the genus) of this two-manifold. Since there is no a-priori reason to restrict attention to spherical or planar topology, we can consider fluids on negatively curved Riemann surfaces and extract a contribution from $\mathrm{J}_{\text {Euler }}^{\mu}$. The topological contribution will compute a degeneracy in terms of the Euler character $s=c_{\text {Euler }} \chi$.

This situation can be realized holographically. A three-dimensional CFT such as the M2-brane worldvolume (ABJM) theory can be placed on $\mathcal{M}_{3}=\mathbb{R} \times \Sigma_{2} \cdot{ }^{61}$ While the vacuum dynamics of this theory is ill-behaved owning to the conformal coupling of the massless scalars (transforming in the $\mathbf{8}_{v}$ of $\mathrm{SO}(8)_{R}$ ), it is plausible that the thermal corrections stabilize the theory. In the strong coupling limit the gravity dual is given by supergravity on $\mathrm{AdS}_{4} \times \mathbf{S}^{7}$. The four-dimensional Gauss-Bonnet term is the leading correction to the two derivative Einstein-Hilbert dynamics. This term is however topological and integrates to a pure boundary term and thus does not affect dynamics. It does however change the degeneracy of the thermal density matrix. In particular, in the presence of this GaussBonnet term, a black hole in $\mathrm{AdS}_{4}$ picks up a contribution from the Wald functional [73] proportional to the Euler character of the spatial two-manifold which is the bifurcation surface. For the CFT on $\mathbb{R} \times \Sigma_{2}$ the black hole horizon is such that its spatial crosssections and especially the bifurcation surface have the same topology as $\Sigma_{2}$. Then the Wald entropy does get a contribution proportional to $\chi\left(\Sigma_{2}\right)$ which is indeed what we see purely from a field theory analysis. One can furthermore check that the pull-back of the Wald functional on the horizon onto the boundary, to define a boundary entropy current as in [64] will indeed give a contribution of the form (10.3). Similar remarks apply to higher (odd) dimensional CFTs on topologically non-trivial backgrounds. Indeed (10.4) entropy current is obtained from a particular Lovelock term in $\mathrm{AdS}_{d+1}$.

The above discussion can be extended to charged fluids. For example we can consider the Chern current in even spacetime dimensions

$$
\mathrm{J}_{\text {Chern }}^{\sigma}=\frac{1}{2^{n}} c_{\text {Chern }} \varepsilon^{\sigma \alpha_{1} \alpha_{2} \cdots \alpha_{2 n-1} \alpha_{2 n}} F_{\alpha_{1} \alpha_{2}} \cdots F_{\alpha_{2 n-1} \alpha_{2 n}},
$$

which despite being exact does contribute to the degeneracy of states and thus the entropy. One easy way to intuit this is to look at the a three-dimensional field theory again $(n=1)$. Now we have a background magnetic field in the spatial manifold which is well known to contribute to ground state degeneracy (e.g., classic Landau level physics). In higher dimensions we would be picking up contributions when the topology of the gauge bundle is non-trivial (e.g., instanton bundle in $d=5$ ). It would be interesting to investigate other such contributions from combinations of the background sources and realizations of such effects in physical fluids.

\footnotetext{
${ }^{61}$ These considerations extend to other three-dimensional QFTs; we simply choose the ABJM theory for illustrative purposes.
} 


\section{The vector classes: transverse free energy currents}

We now turn to another family of solutions to (2.12) which are not captured by Class L Lagrangians. The transport terms constructed in this section rely on the presence of a set of vector fields. These fields could be hydrostatic whence the transport will be characterized by the Class $\mathrm{H}_{V}$ terms encountered in section 4 . It also transpires that we can have nonhydrostatic vector fields which give solutions to the adiabaticity equation; we will name the set of constitutive relations thus determined as belonging to Class $\overline{\mathrm{H}}_{V}$ (in analogy with our distinction in the scalar case).

\subsection{The hydrostatic Class $\mathrm{H}_{V}$}

In section 4.1 we have already mentioned the fact that hydrostatic partition functions are either classified by scalar densities or by conserved transverse vectors $P_{V}^{\sigma}$ which satisfy

$$
K_{\sigma}\left(P_{V}^{\sigma}\right)_{\text {Hydrostatic }}=\nabla_{\sigma}\left(P_{V}^{\sigma}\right)_{\text {Hydrostatic }}=0 .
$$

Their contribution to the equilibrium partition function is as indicated in (4.11). We note that such terms have been studied in the context of Cardy-like formulae in higher dimensions [11, 13, 18, 74]. These terms first showed up as 'integration constants' of the anomaly induced transport [8,22]; that they contribute as vectors to the partition function was first realized in [3]. These terms are sometimes termed transcendental anomaly induced transport terms in order to distinguish them from the 'rational' anomaly induced terms in Class A. These names emphasizes the fact that Cardy-like formulae for Class $\mathrm{H}_{V}$ transport always involve extra transcendental factors of $2 \pi$ unlike the Class A transport. ${ }^{62}$ The supersymmetric cousins of $\mathrm{H}_{V}$ play a crucial role in the recent proposals for Cardylike formulae applicable to supersymmetric partition functions [76]. We will now review the structure of these terms as discussed in these references mainly to give a complete representation of all the classes of adiabatic transport.

Let us begin with a simple example in two-dimensional fluids $(d=2)$. Consider the following adiabatic constitutive relations

$$
\left(T^{\alpha \beta}\right)_{\mathrm{H}_{V}}=-2 \tilde{c}_{g} T^{2} \varepsilon^{(\alpha \gamma} u_{\gamma} u^{\beta)}, \quad\left(J^{\alpha}\right)_{\mathrm{H}_{V}}=0, \quad\left(J_{S}^{\alpha}\right)_{\mathrm{H}_{V}}=-2 \tilde{c}_{g} T \varepsilon^{\alpha \gamma} u_{\gamma} .
$$

This corresponds to a transverse free energy current

$$
\left(\mathcal{G}^{\alpha}\right)_{\mathrm{H}_{V}}=\tilde{c}_{g} T^{2} \varepsilon^{\sigma \gamma} u_{\gamma}
$$

These constitutive relations can be derived from a hydrostatic partition function

$$
W_{\text {Hydrostatic }}=-\int_{\Sigma_{E}}\left(\frac{1}{T}\left(\mathcal{G}^{\sigma}\right)_{\mathrm{H}_{V}}\left[\boldsymbol{\Psi}_{\mathcal{K}}\right]\right)_{\text {Hydrostatic }} d^{d-1} S_{\sigma} .
$$

It is easily checked that these constitutive relations solve adiabaticity equation (2.21) for an arbitrary number $\tilde{c}_{g}$. It was argued in [11] that for a general field theory, we have $\tilde{c}_{g}=$

\footnotetext{
${ }^{62}$ Such contributions are also well understood in the holographic context and we refer the reader to [75] and references therein for a detailed discussion.
} 
$2(2 \pi)^{2} c_{g}$ where $c_{g}$ is the Lorentz anomaly of the underlying two-dimensional theory. For 2d CFTs, this is just the (parity-odd part of) Cardy formula. Thus, the parity-odd Cardy formula relates the coefficients that appear in the Class $\mathrm{H}_{V}$ with the anomaly coefficients that appear in Class A constitutive relations (which we will encounter in section 12).

The above construction (and the corresponding parity-odd Cardy-like formulae) can be generalized to arbitrary even dimensions following [13]. We will give a description of the construction below for completeness, but the reader may find the discussion below more comprehensible after reading through our Class A section, section 12.

Given the close relation between the Class $\mathrm{H}_{V}$ and Class A constitutive relations that Cardy formula implies, it is useful to set them in a common formalism. To this end, it is convenient to introduce a new gauge field $\mathrm{A}^{(\mathrm{T})}{ }_{\mu}$ and an associated chemical potential $\boldsymbol{\mu}^{(\mathrm{T})}$. It turns out to be natural to treat the temperature as the chemical potential (by thinking of it as the twist in the thermal circle), so $\boldsymbol{\mu}^{(\mathrm{T})}=T$. This is equivalent to introducing $\Lambda_{\boldsymbol{\beta}}^{(\mathrm{T})}$ such that $\Lambda_{\boldsymbol{\beta}}^{(\mathrm{T})}+\boldsymbol{\beta}^{\sigma} \mathrm{A}_{\sigma}^{(\mathrm{T})}=1$. We will take the field-strength $\mathrm{F}^{(\mathrm{T})}{ }_{\mu \nu}$ corresponding to this new gauge field to zero. ${ }^{63}$

With this gauge field, Class $\mathrm{H}_{V}$ constitutive relations take the same form as Class A constitutive relations (but now involving $\boldsymbol{\mu}^{(\mathrm{T})}$ in addition) and the coefficients in Class $\mathrm{H}_{V}$ correspond to pure and mixed anomalies involving the new gauge field $A^{(T)}{ }_{\mu}$. The higher dimensional analogues of Cardy formula can then be phrased as fixing the new anomaly coefficients in terms of the usual anomaly coefficients. In $d=2 n$ dimensions, Class $\mathrm{H}_{V}$ constitutive relations are characterized by the exact forms encoding these new anomalies. Thus, consider an anomaly polynomial relevant to Class $\mathrm{H}_{V}$ of the form

$$
\mathcal{P}_{\mathrm{H}_{V}} \equiv \sum_{j=1}^{\left\lfloor\frac{n+1}{2}\right\rfloor}\left(\mathrm{F}^{(\mathrm{T})}\right)^{2 j} \wedge \mathcal{P}_{\left(\mathrm{H}_{V}, j\right)}[\boldsymbol{F}, \boldsymbol{R}]
$$

where $\mathcal{P}_{\left(\mathrm{H}_{V}, j\right)}[\boldsymbol{F}, \boldsymbol{R}]$ denotes an exact $2(n+1-2 j)$-form made by wedging the flavour field strength 2-form $\boldsymbol{F}$ and the Riemann curvature 2-form $\boldsymbol{R}^{\mu}{ }_{\nu}$. For a given set of $\left\lfloor\frac{n+1}{2}\right\rfloor$ exact forms $\left\{\mathcal{P}_{\left(\mathrm{H}_{V}, j\right)}[\boldsymbol{F}, \boldsymbol{R}]\right\}_{j=1,2, \ldots,\left\lfloor\frac{n+1}{2}\right\rfloor}$, we can then construct a Class $\mathrm{H}_{V}$ constitutive relation. ${ }^{64}$ We note that $\mathrm{CPT}$ invariance only allows $\mathcal{P}_{\mathrm{H}_{V}}$ which are even in $\mathrm{F}^{(\mathrm{T})}[10,13]$. This also ensures that once $\mathrm{F}^{(\mathrm{T})}$ is set to zero, all the new anomalies introduced via $\mathcal{P}_{\mathrm{H}_{V}}$ vanishes as they should.

As mentioned above, the detailed form of Class A constitutive relations and how they are derived starting from an anomaly polynomial are described in section 12 and appendix A. It is a straightforward exercise to repeat the derivation with a new gauge field $A^{(\mathrm{T})}{ }_{\mu}$ and the anomaly polynomial $\mathcal{P}_{\mathrm{H}_{V}}$ followed by setting $\mathrm{F}^{(\mathrm{T})}{ }_{\mu \nu}=0$ at the end. We will present here the result of this exercise and refer the reader to our sections on

\footnotetext{
${ }^{63}$ In fact, a lot of this formalism will play an important role in the construction of an extended Lagrangian theory for adiabatic hydrodynamics in part III. We defer physical statements till section 15; for now the reader may simply take the introduction of $A^{(T)}$ and associated quantities a convenient way to encode Class $\mathrm{H}_{V}$ constitutive relations as was done in [13].

${ }^{64}$ The anomaly polynomial $\mathcal{P}_{\mathrm{H}_{V}}$ was called $\mathcal{P}_{\text {trans }}$ in [13]. We pretty much follow their notation for differential forms etc., and further notational conventions are as explained in section 12 (see also appendix J).
} 
Class A for more details. We will need the definition of the spin chemical potential from equation (12.21)

$$
{\Omega^{\mu}}_{\nu}=\frac{1}{2} T\left(D_{\nu} \boldsymbol{\beta}^{\mu}-D^{\mu} \boldsymbol{\beta}_{\nu}\right)
$$

along with the definition of "hydrodynamical shadow" gauge field and spin connection from (12.2) and (12.19) respectively. We have

$$
\begin{aligned}
\hat{\boldsymbol{A}} & =\boldsymbol{A}+\mu \boldsymbol{u}, \\
\hat{\boldsymbol{\Gamma}}^{\mu}{ }_{\nu} & =\boldsymbol{\Gamma}^{\mu}{ }_{\nu}+\Omega^{\mu}{ }_{\nu} \boldsymbol{u} .
\end{aligned}
$$

In analogy with (12.23), let us also define the bulk Hall currents for the sequence of anomaly polynomials $\left\{\boldsymbol{P}_{\left(\mathrm{H}_{V}, j\right)}[\boldsymbol{F}, \boldsymbol{R}]\right\}_{j=1,2, \ldots,\left\lfloor\frac{n+1}{2}\right\rfloor}$ as

$$
\star_{2 n+1}\left(\mathbf{J}_{H}\right)_{\left(\mathrm{H}_{V}, j\right)}=\frac{\partial \mathcal{P}_{\left(\mathrm{H}_{V}, j\right)}}{\partial \boldsymbol{F}}, \quad \star_{2 n+1}\left(\boldsymbol{\Sigma}_{H}\right)_{\left(\mathrm{H}_{V}, j\right)}{ }^{b}{ }_{a}=2 \frac{\partial \boldsymbol{\mathcal { P }}_{\left(\mathrm{H}_{V}, j\right)}}{\partial \boldsymbol{R}^{a}{ }_{b}} .
$$

We will denote by hats the corresponding objects evaluated for the shadow connections.

We are now ready to present the general form of Class $\mathrm{H}_{V}$ constitutive relations. Using (12.25) for the new anomaly polynomial and setting $\mathrm{F}^{(\mathrm{T})}{ }_{\mu \nu}=0$, we get for the heat current $\boldsymbol{q}_{\mathrm{H}_{V}}$, the spin current $\boldsymbol{\Sigma}_{\mathrm{H}_{V}}$, the charge current $\boldsymbol{J}_{\mathrm{H}_{V}}$, and a contribution to the entropy current $\left(\boldsymbol{J}_{S, \mathrm{H}_{V}}^{\prime}\right)$ the following expressions in differential form notation:

$$
\begin{aligned}
\star \boldsymbol{J}_{\mathrm{H}_{V}} & =-\sum_{j=1}^{\left\lfloor\frac{n+1}{2}\right\rfloor} T^{2 j} \boldsymbol{u} \wedge(2 \boldsymbol{\omega})^{2 j-1} \wedge\left(\hat{\mathbf{J}}_{H}\right)_{\left(\mathrm{H}_{V}, j\right)}, \\
\star \boldsymbol{\Sigma}_{\mathrm{H}_{V}}{ }^{\beta}{ }_{\alpha} & =-\sum_{j=1}^{\left\lfloor\frac{n+1}{2}\right\rfloor} T^{2 j} \boldsymbol{u} \wedge(2 \boldsymbol{\omega})^{2 j-1} \wedge\left(\hat{\boldsymbol{\Sigma}}_{H}\right)_{\left(\mathrm{H}_{V}, j\right)}{ }^{\beta} \alpha, \\
\star \boldsymbol{q}_{\mathrm{H}_{V}} & =-\sum_{j=1}^{\left\lfloor\frac{n+1}{2}\right\rfloor}(2 j-1) T^{2 j} \boldsymbol{u} \wedge(2 \boldsymbol{\omega})^{2 j-2} \wedge \widehat{\mathcal{P}}_{\left(\mathrm{H}_{V}, j\right)}, \\
\star \boldsymbol{J}_{S, \mathrm{H}_{V}}^{\prime} & =-\sum_{j=1}^{\left\lfloor\frac{n+1}{2}\right\rfloor} 2 j T^{2 j-1} \boldsymbol{u} \wedge(2 \boldsymbol{\omega})^{2 j-1} \wedge\left(\hat{\mathbf{J}}_{H}\right)_{\left(\mathrm{H}_{V}, j\right)} .
\end{aligned}
$$

This in turn gives (see eqs. (12.28) and (12.30)) an energy momentum tensor and an entropy current of the form

$$
\begin{aligned}
\left(T^{\alpha \beta}\right)_{\mathrm{H}_{V}} & =q_{\mathrm{H}_{V}}^{\alpha} u^{\beta}+q_{\mathrm{H}_{V}}^{\beta} u^{\alpha}+\frac{1}{2} D_{\rho}\left(\Sigma_{\mathrm{H}_{V}}^{\alpha[\beta \rho]}+\Sigma_{\mathrm{H}_{V}}^{\beta[\alpha \rho]}-\Sigma_{\mathrm{H}_{V}}^{\rho(\alpha \beta)}\right), \\
\left(J_{S}^{\alpha}\right)_{\mathrm{H}_{V}} & =\left(J_{S}^{\prime}\right)_{\mathrm{H}_{V}}^{\alpha}-\frac{1}{2} \boldsymbol{\beta}_{\sigma}\left(\hat{\Sigma}_{H}\right)_{\mathrm{H}_{V}}^{\perp[\alpha \sigma]}
\end{aligned}
$$

along with a charge current given by $\left(J^{\alpha}\right)_{\mathrm{H}_{V}}$. Here, $\left(\hat{\Sigma}_{H}\right)_{\mathrm{H}_{V}}^{\perp[\alpha \sigma]}$ is defined via

$$
\star\left(\hat{\boldsymbol{\Sigma}}_{H}\right)_{\mathrm{H}_{V}}{ }^{\beta}{ }_{\alpha}=\sum_{j=1}^{\left\lfloor\frac{n+1}{2}\right\rfloor}[d(T \boldsymbol{u})]^{2 j-1} \wedge\left(\hat{\boldsymbol{\Sigma}}_{H}\right)_{\left(\mathrm{H}_{V}, j\right)}{ }^{\beta} \alpha .
$$


These constitutive relations then solve the adiabaticity equation (2.12) without the anomalies (since the limit $\mathrm{F}^{(\mathrm{T})}{ }_{\mu \nu}=0$ sets all the new anomalies to zero).

The corresponding free energy current is given by $\left(\mathcal{G}^{\sigma}\right)_{\mathrm{H}_{V}}=\left(\mathcal{G}^{\prime \sigma}\right)_{\mathrm{H}_{V}}+\frac{1}{2} u_{\alpha}\left(\hat{\Sigma}_{H}\right)_{\mathrm{H}_{V}}^{\perp[\sigma \alpha]}$ with

$$
\star \mathcal{G}_{\mathrm{H}_{V}}^{\prime}=\sum_{j=1}^{\left\lfloor\frac{n+1}{2}\right\rfloor} T^{2 j} \boldsymbol{u} \wedge(2 \boldsymbol{\omega})^{2 j-2} \wedge \widehat{\mathcal{P}}_{\left(\mathrm{H}_{V}, j\right)} .
$$

In the hydrostatic limit $u_{\alpha}\left(\hat{\Sigma}_{H}\right)_{\mathrm{H}_{V}}^{\perp[\sigma \alpha]}=0$ and only the $\left(\mathcal{G}^{\prime \sigma}\right)_{\mathrm{H}_{V}}$ part of the free-energy current contributes to the hydrostatic partition function. Thus,

$$
\begin{aligned}
W_{\text {Hydrostatic }} & =-\int_{\Sigma_{E}}\left(\frac{1}{T}\left(\mathcal{G}^{\sigma}\right)_{\mathrm{H}_{V}}\left[\mathbf{\Psi}_{\mathcal{K}}\right]\right)_{\text {Hydrostatic }} d^{d-1} S_{\sigma} . \\
& =-\int_{\Sigma_{E}} \sum_{j=1}^{\left\lfloor\frac{n+1}{2}\right\rfloor} T^{2 j-1} \boldsymbol{u} \wedge(2 \boldsymbol{\omega})^{2 j-2} \wedge \widehat{\mathcal{P}}_{\left(\mathrm{H}_{V}, j\right)} .
\end{aligned}
$$

We note that this free-energy current is transverse, thus justifying our nomenclature in calling these class of terms as Class $\mathrm{H}_{V}$.

In any even space-time dimensions, given a set of exact forms $\left\{\mathcal{P}_{\left(\mathrm{H}_{V}, j\right)}[\boldsymbol{F}, \boldsymbol{R}]\right\}_{j=1,2, \ldots,\left\lfloor\frac{n+1}{2}\right\rfloor}$ of appropriate degree, we can construct an adiabatic constitutive relations using the formulae above. Additional physical considerations over and above second law such as Euclidean consistency, cf., [11, 13], can be used to fix these exact forms in terms of the original anomaly polynomial of the theory. These 'parity-odd Cardy formula' or 'replacement rule' thus fix $\mathcal{P}_{\mathrm{H}_{V}}$ that appears in the Class $\mathrm{H}_{V}$ in terms of the anomaly polynomial $\mathcal{P}[\boldsymbol{F}, \boldsymbol{R}]$ that controls Class A constitutive relations. Following $[11,13]$, this relation takes the form

$$
\mathcal{P}_{\mathrm{H}_{V}}\left[\boldsymbol{F}, \boldsymbol{R}, \mathrm{F}^{(\mathrm{T})}\right]=\mathcal{P}\left[\boldsymbol{F}, \operatorname{tr} \boldsymbol{R}^{2 k} \mapsto \operatorname{tr} \boldsymbol{R}^{2 k}+2\left(2 \pi \mathrm{F}^{(\mathrm{T})}\right)^{2 k}\right]-\mathcal{P}\left[\boldsymbol{F}, \operatorname{tr} \boldsymbol{R}^{2 k}\right] .
$$

For example, if the anomaly polynomial that controls Class $\mathrm{A}$ is taken to be $\mathcal{P}=$ $c_{g}\left(\operatorname{tr} \boldsymbol{R}^{2}\right)^{2}$, then the $\mathcal{P}_{\mathrm{H}_{V}}$ controlling Class $\mathrm{H}_{V}$ is fixed by the Cardy formula to be $\mathcal{P}_{\mathrm{H}_{V}}=4 c_{g}\left(2 \pi \mathrm{F}^{(\mathrm{T})}\right)^{2} \wedge \operatorname{tr} \boldsymbol{R}^{2}+4 c_{g}\left(2 \pi \mathrm{F}^{(\mathrm{T})}\right)^{4}$.

This completes the discussion of hydrostatic transverse free energy currents which give rise to adiabatic constitutive relations.

\subsection{The non-hydrostatic Class $\overline{\mathbf{H}}_{V}$}

Let us now turn to another set of solutions to the adiabaticity equations involving vectorial degrees of freedom. We should be focusing now on vectorial contributions that vanish in hydrostatic equilibrium (should they not do so, we would be able to include them in our discussion of Class $\mathrm{H}_{V}$ ).

\subsubsection{General construction of Class $\overline{\mathbf{H}}_{V}$}

For the simplest way to motivate the construction, it is convenient to start with the nonanomalous adiabaticity equation in the grand canonical ensemble which we derived in (2.21) 
and requoted in (9.1) during the Class B discussion of section 9. The free energy current decomposes into longitudinal and transverse vectors as in (6.1). Longitudinal vectors can all be obtained directly from Class L; so we only need to focus now on transverse vectors to find the remaining solutions to (9.1).

The r.h.s. of (9.1) involves at least one factor of $\delta_{\mathfrak{B}} g_{\mu \nu}$ or $\delta_{\mathfrak{B}} A_{\mu}$, which vanish in hydrostatics since $\delta_{\mathcal{K}} g_{\mu \nu}=\delta_{\mathcal{K}} A_{\mu}=0$. In other words, if we take the hydrostatic configurations off-shell by unlinking $\mathcal{B} \neq \mathcal{K}$ then the Gibbs free energy flux is produced at $\mathcal{O}\left(\delta_{\mathfrak{B}}\right)$. The statement of hydrostatic principle is simply at the this order we have compensating energymomentum and charge flow to ensure adiabaticity.

However, now consider the situation where the Gibbs free energy flux is itself quadratic in departures from equilibrium, i.e., $\mathcal{G}^{\lambda} \sim \mathcal{O}\left(\delta_{\mathfrak{B}}^{2}\right)$. This would be invisible from an hydrostatic analysis. Taking divergence of such a term we should expect then that the r.h.s. of (9.1) would have contributions at $\mathcal{O}\left(\delta_{\mathfrak{B}}^{2}\right)$ (when the derivative hits the tensor structure multiplying the $\delta_{\mathcal{B}}$ terms), as well as terms which behave as $\delta_{\mathfrak{B}} D_{\mu} \delta_{\mathfrak{B}}$. Since the r.h.s. itself involves one $\delta_{\mathfrak{B}}$ insertion, it follows that the terms of interest should have the currents containing combinations of $\delta_{\mathfrak{B}}$ and $D \delta_{\mathfrak{B}}$ terms.

This simple reasoning then leads to the following ansatz for the energy-momentum and charge currents:

$$
\begin{aligned}
&\left(T^{\mu \nu}\right)_{\overline{\mathrm{H}}_{V}} \equiv \frac{1}{2}\left[D_{\rho} \mathfrak{C}_{\mathcal{N}}^{\rho(\mu \nu)(\alpha \beta)} \delta_{\mathfrak{B}} g_{\alpha \beta}+2 \mathfrak{C}_{\mathcal{N}}^{\rho(\mu \nu)(\alpha \beta)} D_{\rho} \delta_{\mathcal{B}} g_{\alpha \beta}\right] \\
&+D_{\rho} \mathfrak{C}_{\mathcal{X}}^{\rho(\mu \nu) \alpha} \cdot \delta_{\mathcal{B}} A_{\alpha}+2 \mathfrak{C}_{\mathcal{X}}^{\rho(\mu \nu) \alpha} \cdot D_{\rho} \delta_{\mathcal{B}} A_{\alpha} \\
&\left(J^{\alpha}\right)_{\overline{\mathrm{H}}_{V}} \equiv \frac{1}{2}\left[D_{\rho} \mathfrak{C}_{\mathcal{X}}^{\rho(\mu \nu) \alpha} \delta_{\mathcal{B}} g_{\mu \nu}+2 \mathfrak{C}_{\mathcal{X}}^{\rho(\mu \nu) \alpha} D_{\rho} \delta_{\mathfrak{B}} g_{\mu \nu}\right] \\
&+D_{\rho} \mathfrak{C}_{\mathcal{S}}^{\rho(\alpha \beta)} \cdot \delta_{\mathcal{B}} A_{\beta}+2 \mathfrak{C}_{\mathcal{S}}^{\rho(\alpha \beta)} \cdot D_{\rho} \delta_{\mathcal{B}} A_{\beta}
\end{aligned}
$$

where $\mathfrak{C}_{\mathcal{N}}^{\rho(\mu \nu)(\alpha \beta)}=\mathfrak{C}_{\mathcal{N}}^{\rho(\alpha \beta)(\mu \nu)}$. These tensor fields are local functions of $\boldsymbol{\Psi}$ and their gradients.

This solves adiabaticty equation with the free energy current

$$
\begin{aligned}
\left(\mathrm{N}^{\rho}\right)_{\overline{\mathrm{H}}_{V}} & \equiv-\left(\frac{\mathcal{G}^{\rho}}{T}\right)_{\overline{\mathrm{H}}_{V}} \\
& =\frac{1}{4} \delta_{\mathcal{B}} g_{\mu \nu} \mathfrak{C}_{\mathcal{N}}^{\rho(\mu \nu)(\alpha \beta)} \delta_{\mathfrak{B}} g_{\alpha \beta}+\delta_{\mathfrak{B}} g_{\mu \nu} \mathfrak{C}_{\mathcal{X}}^{\rho(\mu \nu) \alpha} \cdot \delta_{\mathcal{B}} A_{\alpha}+\delta_{\mathcal{B}} A_{\alpha} \cdot \mathfrak{C}_{\mathcal{S}}^{\rho(\alpha \beta)} \cdot \delta_{\mathcal{B}} A_{\beta}
\end{aligned}
$$

As should be clear from the construction, the tensors $\left\{\mathfrak{C}_{\mathcal{N}}, \mathfrak{C}_{\mathcal{X}}, \mathfrak{C}_{\mathcal{S}}\right\}$ are a-priori completely arbitrary with the indicated symmetry structure (modulo field redefinitions - see below). Moreover, it is clear, that we will only obtain genuinely Class $\overline{\mathrm{H}}_{V}$ constitutive relations if we make sure that the free energy current will be transverse, i.e., we demand that the first index of the tensors be transverse:

$$
\mathfrak{C}_{\mathcal{N}}^{\rho(\mu \nu)(\alpha \beta)} u_{\rho}=\mathfrak{C}_{\mathcal{X}}^{\rho(\mu \nu) \alpha} u_{\rho}=\mathfrak{C}_{\mathcal{S}}^{\rho(\alpha \beta)} u_{\rho}=0
$$

The solution to (9.1) characterized by the constitutive relations (11.15) and the free energy current (11.16) is the Class $\overline{\mathrm{H}}_{V}$ of Gibbsian vectors. The astute reader might wonder 
why we choose to call this Class $\overline{\mathrm{H}}_{V}$ as opposed to $\mathrm{G}_{V}$ to indicate the Gibbsian structure employed in the construction. Our choice will be rationalized when we argue that these nonhydrostatic vectors can be obtained from a generalized Lagrangian density (with enhanced symmetry) in section 15 .

From the construction it is clear that $\overline{\mathrm{H}}_{V}$ terms contribute to the constitutive relations only from the second order in gradients; the presence of an explicit derivative and a single $\delta_{\mathfrak{B}}$ in (11.15) ensures that we cannot get a first order contribution (even in the parity-odd case). In the context of proving the completeness of our classification (cf., Theorem 1 in section 14), we will demonstrate that the parameterization (11.15), (11.16) is complete, i.e., every non-hydrostatic adiabatic constitutive relation with transverse free energy current is of this form.

\subsubsection{Example: second order charged fluid}

Let us look at an example to illustrate the construction. Unfortunately the simplest setting where we encounter this class happens to be for a charged fluid at second order in gradients, owing to the observation above. We want a second order contribution to the free energy which implies that the tensors $\left\{\mathfrak{C}_{\mathcal{N}}, \mathfrak{C}_{\mathcal{X}}, \mathfrak{C}_{\mathcal{S}}\right\}$ should be zero-derivative objects from (11.15). A-priori we have the following inequivalent tensor structures at our disposal:

$$
\begin{aligned}
\mathfrak{C}_{\mathcal{N}}^{\rho(\mu \nu)(\alpha \beta)} & \in\left\{P^{\rho(\mu} \boldsymbol{\beta}^{\nu)} P^{\alpha \beta}, P^{\rho(\mu} \boldsymbol{\beta}^{\nu)} \boldsymbol{\beta}^{\alpha} \boldsymbol{\beta}^{\beta}, P^{\rho(\mu} P^{\nu)(\alpha} \boldsymbol{\beta}^{\beta)}\right\}, \\
\mathfrak{C}_{\mathcal{X}}^{\rho(\mu \nu) \alpha} & \in\left\{P^{\rho(\mu} P^{\nu) \alpha}, P^{\mu \nu} P^{\rho \alpha}\right\}, \\
\mathfrak{C}_{\mathcal{S}}^{\rho(\alpha \beta)} & \in\left\{P^{\rho(\alpha} \boldsymbol{\beta}^{\beta)}\right\},
\end{aligned}
$$

and permutations thereof. These tensor structures have to be contracted with $\delta_{\mathfrak{B}} g_{\mu \nu}$ and $\delta_{\mathfrak{B}} A_{\alpha}$ in the free energy current (11.16). These linear variations can be expressed in terms of hydrodynamical objects using (2.23) which we reproduce here for convenience:

$$
\begin{aligned}
\delta_{\mathfrak{B}} g_{\mu \nu} & =2 \nabla_{(\mu} \boldsymbol{\beta}_{\nu)}=\frac{2}{T}\left[\sigma_{\mu \nu}+P_{\mu \nu} \frac{\Theta}{d-1}-\left(\mathfrak{a}_{(\mu}+\nabla_{(\mu} \log T\right) u_{\nu)}\right] \\
\delta_{\mathcal{B}} A_{\mu} & =D_{\mu}\left(\Lambda_{\boldsymbol{\beta}}+\boldsymbol{\beta}^{\nu} A_{\nu}\right)+\boldsymbol{\beta}^{\nu} F_{\nu \mu}=u^{\alpha} D_{\alpha}\left(\frac{\mu}{T}\right) u_{\mu}-\frac{1}{T} \mathfrak{v}_{\mu}
\end{aligned}
$$

with the vector $\mathfrak{v}^{\mu}$ defined in (2.25). However, upon evaluating these variations on-shell as in (E.2), we find that the tensors in (11.18) give only two different non-zero contributions to constitutive relations (others are linear combinations of these two). Without loss of generality, we choose to parameterize the two non-trivial choices leading to inequivalent transverse vector contributions to the free energy current at this order as

$$
\mathfrak{C}_{\mathcal{N}}^{\rho(\mu \nu)(\alpha \beta)}=\mathfrak{C}_{\mathcal{S}}^{\rho(\alpha \beta)}=0, \quad \mathfrak{C}_{\mathcal{X}}^{\rho(\mu \nu) \alpha}=T C_{1}(T, \mu) P^{\rho<\mu} P^{\nu>\alpha}+T C_{2}(T, \mu) P^{\mu \nu} P^{\rho \alpha} .
$$

This gives the following transverse vector contributions to the free energy:

$$
\left(\mathcal{G}^{\rho}\right)_{\overline{\mathrm{H}}_{V}}=2 C_{1} \sigma^{\rho \alpha} \mathfrak{v}_{\alpha}+2 C_{2} \Theta \mathfrak{v}^{\rho} .
$$

The contributions to the energy-momentum tensor and charge current are rather cumbersome, so we give separately the terms that correspond to the $C_{1}(T, \mu)$ term and to the 
$C_{2}(T, \mu)$ term, respectively. From $C_{1}$ we get

$$
\begin{aligned}
\left(T^{\mu \nu}\right)_{\overline{\mathrm{H}}_{V}}= & -C_{1} \frac{d p}{d q} \Theta \sigma^{\mu \nu}-2 C_{1} D^{<\mu} \mathfrak{v}^{\nu>}+\left(\mathfrak{D} C_{1}-2 C_{1}\right) \mathfrak{v}^{<\mu} \mathfrak{a}^{\nu>}-\dot{C}_{1} \mathfrak{v}^{<\mu} E^{\nu>} \\
& -\left[\frac{q}{\varepsilon+p}\left(\mathfrak{D} C_{1}-C_{1}\right)-\dot{C}_{1}\right] \mathfrak{v}^{<\mu} \mathfrak{v}^{\nu>}+\frac{(3-d) C_{1}}{(d-1)}\left[\sigma^{(\mu \rho} \mathfrak{v}_{\rho} u^{\nu)}+\frac{1}{d-1} \Theta \mathfrak{v}^{(\mu} u^{\nu)}\right] \\
& -\frac{(d+1) C_{1}}{(d-1)} \omega^{(\mu \rho} \mathfrak{v}_{\rho} u^{\nu)}-C_{1} \Theta \mathfrak{v}^{(\mu} u^{\nu)}, \\
\left(J^{\alpha}\right)_{\overline{\mathrm{H}}_{V}}= & {\left[-C_{1} \sigma^{2}\right] u^{\alpha}+\left[\frac{q}{\varepsilon+p}\left(\mathfrak{D} C_{1}-C_{1}\right)-\dot{C}_{1}\right] \sigma^{\alpha \rho} \mathfrak{v}_{\rho}-\left(\mathfrak{D C} C_{1}\right) \sigma^{\alpha \rho} \mathfrak{a}_{\rho} } \\
& +\dot{C}_{1} \sigma^{\alpha \rho} E_{\rho}+2 C_{1} D_{\rho} \sigma^{\rho \alpha}+\frac{(3-d) C_{1}}{2(d-1)} \frac{q}{\varepsilon+p}\left[\sigma^{\rho \alpha} \mathfrak{v}_{\rho}+\frac{1}{d-1} \Theta \mathfrak{v}^{\alpha}\right] \\
& -\frac{(d+1) C_{1}}{2(d-1)} \frac{q}{\varepsilon+p} \omega^{\rho \alpha} \mathfrak{v}_{\rho}-\frac{C_{1}}{2} \frac{q}{\varepsilon+p} \Theta \mathfrak{v}^{\alpha},
\end{aligned}
$$

which is built from the following fluid frame invariant scalar, vector and tensor contributions: ${ }^{65}$

$$
\begin{aligned}
C_{S}= & \frac{d p}{d q} C_{1} \sigma^{2} \\
C_{V}^{\alpha}= & {\left[\frac{q}{\varepsilon+p}\left(\mathfrak{D} C_{1}-C_{1}\right)-\dot{C}_{1}\right] \sigma^{\alpha \rho} \mathfrak{v}_{\rho}+\frac{(d+1) C_{1}}{(d-1)} \frac{q}{\varepsilon+p} \omega^{\alpha \rho} \mathfrak{v}_{\rho}-\left(\mathfrak{D} C_{1}\right) \sigma^{\alpha \rho} \mathfrak{a}_{\rho} } \\
& +\dot{C}_{1} \sigma^{\alpha \rho} E_{\rho}+2 C_{1} D_{\rho} \sigma^{\rho \alpha}, \\
C_{T}^{\mu \nu}= & -C_{1} \frac{d p}{d q} \Theta \sigma^{\mu \nu}-2 C_{1} D^{<\mu} \mathfrak{v}^{\nu>}+\left(\mathfrak{D} C_{1}-2 C_{1}\right) \mathfrak{v}^{<\mu} \mathfrak{a}^{\nu>}-\dot{C}_{1} \mathfrak{v}^{<\mu} E^{\nu>} \\
& -\left[\frac{q}{\varepsilon+p}\left(\mathfrak{D} C_{1}-C_{1}\right)-\dot{C}_{1}\right] \mathfrak{v}^{<\mu} \mathfrak{v}^{\nu>} .
\end{aligned}
$$

In the above expressions we use the abbreviation $\mathfrak{D} C_{1} \equiv T C_{1}^{\prime}+\mu \dot{C}_{1}$, where primes denote $T$-derivatives and over-dots denote $\mu$-derivatives. The $C_{2}$ tensor structure on the other hand gives the following transport:

$$
\begin{aligned}
\left(T^{\mu \nu}\right)_{\overline{\mathrm{H}}_{V}}= & \left\{\left(\mathfrak{D} C_{2}\right) \mathfrak{a}^{\alpha} \mathfrak{v}_{\alpha}-\left[\frac{q}{\varepsilon+p}\left(\mathfrak{D} C_{2}-C_{2}\right)-\dot{C}_{2}\right] \mathfrak{v}^{2}-\dot{C}_{2}\left(\mathfrak{v}^{\alpha} \cdot E_{\alpha}\right)-2 C_{2} D_{\alpha} \mathfrak{v}^{\alpha}\right. \\
& \left.-C_{2} \frac{d p}{d q} \Theta^{2}\right\} P^{\mu \nu}-2 C_{2}\left[\sigma^{(\mu \rho} \mathfrak{v}_{\rho} u^{\nu)}-\omega^{(\mu \rho} \mathfrak{v}_{\rho} u^{\nu)}+\frac{1}{d-1} \Theta \mathfrak{v}^{(\mu} u^{\nu)}\right], \\
\left(J^{\alpha}\right)_{\overline{\mathrm{H}}_{V}}= & {\left[C_{2} \Theta^{2}\right] u^{\alpha}+\left[\frac{q}{\varepsilon+p}\left(\mathfrak{D} C_{2}-C_{2}\right)-\dot{C}_{2}\right] \Theta \mathfrak{v}^{\alpha}-\left(\mathfrak{D} C_{2}-2 C_{2}\right) \Theta \mathfrak{a}^{\alpha} } \\
& +\dot{C}_{2} \Theta E^{\alpha}+2 C_{2} P^{\alpha \rho} \nabla_{\rho} \Theta-\frac{q}{\varepsilon+p} C_{2}\left[\sigma^{\rho \alpha} \mathfrak{v}_{\rho}-\omega^{\rho \alpha} \mathfrak{v}_{\rho}+\frac{1}{d-1} \Theta \mathfrak{v}^{\alpha}\right],
\end{aligned}
$$

\footnotetext{
${ }^{65}$ For the form of frame-invariant data we use the conventions of $[3,48]$ as described in $(8.14)$.
} 
with frame-invariant data as follows:

$$
\begin{aligned}
C_{S} & =\left(\mathfrak{D} C_{2}\right) \mathfrak{a}^{\alpha} \mathfrak{v}_{\alpha}-\left[\frac{q}{\varepsilon+p}\left(\mathfrak{D} C_{2}-C_{2}\right)-\dot{C}_{2}\right] \mathfrak{v}^{2}-\dot{C}_{2}\left(\mathfrak{v}^{\alpha} \cdot E_{\alpha}\right)-2 C_{2} D_{\alpha} \mathfrak{v}^{\alpha}, \\
C_{V}^{\alpha} & =\left[\frac{q}{\varepsilon+p}\left(\mathfrak{D} C_{2}-C_{2}\right)-\dot{C}_{2}\right] \Theta \mathfrak{v}^{\alpha}-\left(\mathfrak{D} C_{2}-2 C_{2}\right) \Theta \mathfrak{a}^{\alpha}+\dot{C}_{2} \Theta E^{\alpha}+2 C_{2} P^{\alpha \rho} \nabla_{\rho} \Theta, \\
C_{T}^{\mu \nu} & =0 .
\end{aligned}
$$

All the above expressions are written in the basis of independent scalars, vectors and tensors as listed in table 2. From that table and the list in table 7, it is evident that most of the above terms are fixed in terms of Class B and Class D parameters. The only terms that are not obvious to classify are the combinations $\left(C_{V}^{\alpha}, C_{T}^{\mu \nu}\right)=\left(D_{\rho} \sigma^{\rho \alpha}, D^{<\mu_{\mathfrak{v}} \nu>}\right)$ and $\left(C_{S}, C_{V}^{\alpha}\right)=\left(D_{\alpha} \mathfrak{v}^{\alpha},-P^{\alpha \rho} \nabla_{\rho} \Theta\right)$. It would be interesting to study the second order charged fluid in more detail and see if these combinations get fixed in Class $\mathrm{L}$.

From these calculations, we can now also see why there are no Class $\overline{\mathrm{H}}_{V}$ terms in neutral fluids at second order. In neutral fluids, only the tensor structure $\mathfrak{C}_{\mathcal{N}}$ would be relevant. Hence we would take the tensors in the first line of (11.18) and would compute the associated constitutive relations. To this end we would perform contractions with the on-shell expression of $\delta_{\mathfrak{B}} g_{\mu \nu}$. However, as can be seen from (E.2), for uncharged fluids $\delta_{\mathcal{B}} g_{\mu \nu}$ only has pieces that are either completely transverse or completely longitudinal. A quick glance at the structure of the possible $\mathfrak{C}_{\mathcal{N}}$ terms shows that in this way we could not get a transverse free energy current.

We find it rather curious that Class $\overline{\mathrm{H}}_{V}$ constitutive relations are sparse (at least at low orders in the gradient expansion). There is no reason for the number of transverse vectors to be limited at a given order in gradient expansion, but it appears that many such contributions are subsumed in other Classes. It would be useful to have a clear intuition for why this is the case. One hopes that by studying such constitutive relations in various hydrodynamic systems would help reveal some rationale for the paucity of Class $\overline{\mathrm{H}}_{V}$.

\section{Class A: lagrangian solution to anomalous adiabaticity equation}

The framework of adiabatic fluids whilst sufficiently general to allow us to discuss anomalous hydrodynamics and being formulated as such to incorporate these effects, we have so far refrained from analyzing such systems explicitly. The main novelty with the anomalous constitutive relations is that one has to account for contributions which account for a modification of the equations of motion due to the presence of flavour and gravitational anomalies. Indeed it was in attempting to understand these constitutive relations that the adiabaticity equation was first proposed in [8].

Thus far the only exposure to anomalies we have had has been in the context of hydrostatics. It has been well known for a while that Class $\mathrm{H}$ contains Class A, see for example the analyses of [3, 10-13]. However, we have established that Class $\mathrm{H}_{S} \subset$ Class L, which begs the question whether we can understand the anomalous transport in terms of an effective action. Indeed, one could take the philosophy that for the adiabatic 
fluid framework achieve its stated goal of enabling us understand how hydrodynamics can be derived from an action principle, we would need to demonstrate that the anomalous transport can be captured by a Lagrangian, thus establishing that Class A $\subset$ Class L.

There is reason for optimism on this front, since [30] have demonstrated that purely flavour anomalies can be captured by non-dissipative effective actions (Class ND); cf., appendix B. So it would seem that by suitably reverse engineering the construction of [30] and implementing the Legendre transformation we should be able to solve for anomalous contributions to the adiabatic hydrodynamics. Indeed this is all that needs to be done in the case of flavour anomalies. The mixed flavour and gravitational anomaly story however turns out to be a bit more intricate. In fact as mentioned earlier, it provides us with a strong rationale to switch from the formalism of non-dissipative fluid effective actions to the framework proposed herein.

In this section we will show that a specific class of anomalous terms is a subset of Class L, i.e., they can be formulated in terms of a Lagrangian. For the case of flavour anomalies, this is a simple modification of [30] which we will use as a guiding template. We will extend that analysis to the case of general mixed anomalies in what follows. We will focus on constructing particular solutions to the anomalous adiabaticity equation (2.12). This will be sufficient to capture all the flavour and mixed contributions which are neither gauge nor diffeomorphism invariant, but will be insufficient to capture the gauge and diffeomorphism terms that are necessary for the consistency of the Euclidean partition function. The terms we are unable to include are the $\mathrm{H}_{V}$ terms discussed in section 11, which comprise of the transcendental anomaly contributions [13]. In the present section our main aim will be on finding solutions to the off-shell adiabaticity equation; only in section 13.3 will we worry about the on-shell conditions and the anomalous Ward identities for these require Schwinger-Keldysh doubling of the degrees of freedom. Subsequently in section 15 we will give a prescription that does appear to capture all anomaly induced transport in an extended theory of adiabatic hydrodynamics.

\subsection{Flavour anomalies}

Let us begin our discussion by recalling some salient facts from the analysis of [30] in the context of effective actions for flavour anomalies. In the framework of Class ND effective actions [30] showed that an effective action given as a transgression form provides a solution to (2.12) with $\mathrm{T}_{H}^{\mu \perp}=0$. More specifically, it was shown that for a hydrodynamic system in $d=2 n$ dimensions living on a spacetime manifold $\mathcal{M}$ one has a local effective action in one higher dimension. ${ }^{6}$ We have an effective action that can be succinctly written on an extended spacetime $\mathcal{M}_{d+1}$ with $\partial \mathcal{M}_{d+1}=\mathcal{M}$ being the physical spacetime where the fluid propagates. The effective action takes the beguilingly simple form

$$
S_{\text {anom }}=\int_{\mathcal{M}_{d+1}} \sqrt{-g_{d+1}} \mathcal{L}_{\text {anom }}=\int_{\mathcal{M}_{d+1}} \boldsymbol{V}_{\mathcal{P}}[\boldsymbol{A}, \hat{\boldsymbol{A}}]=\int_{\mathcal{M}_{d+1}} \frac{\boldsymbol{u}}{2 \boldsymbol{\omega}} \wedge(\mathcal{P}[\boldsymbol{F}]-\widehat{\mathcal{P}}[\hat{\boldsymbol{F}}])
$$

\footnotetext{
${ }^{66}$ This follows from the fact that we can use the anomaly inflow mechanism [77] to construct a local effective action by coupling our anomalous quantum system to a topological theory in the higher dimension.
} 
In the equation above, we have also provided an explicit expression for the transgression form $\boldsymbol{V}_{\mathcal{P}}[\boldsymbol{A}, \hat{\boldsymbol{A}}]$ in terms of in terms of the hydrodynamic velocity 1 -form $\boldsymbol{u}$, the vorticity 2form $\boldsymbol{\omega}$ and the anomaly polynomial $\mathcal{P}[\boldsymbol{F}]$ which is a $d+2=2 n+2$ form built from the gauge invariant field strengths. ${ }^{67}$ Note that $d \boldsymbol{u}=2 \boldsymbol{\omega}-\boldsymbol{u} \wedge \boldsymbol{a}$ where $\boldsymbol{a}$ is the acceleration 1-form.

The transgression form denoted herein as $\boldsymbol{V}_{\mathcal{P}}[\boldsymbol{A}, \hat{\boldsymbol{A}}]$ is a functional of two gauge connections $\boldsymbol{A}$ and $\hat{\boldsymbol{A}}$. The former is simply the background gauge field source in differential form notation, while the latter is what was called in [30] as the "hydrodynamical shadow gauge field". It is a linear combination of the background source and the hydrodynamic velocity field defined as

$$
\hat{\boldsymbol{A}}=\boldsymbol{A}+\mu \boldsymbol{u}
$$

or more directly in terms of the hydrodynamic fields $\mathcal{B}$ we have for its components

$$
\hat{A}_{\mu}=A_{\mu}+T^{2} \boldsymbol{\beta}_{\mu}\left(\Lambda_{\boldsymbol{\beta}}+\boldsymbol{\beta}^{\sigma} A_{\sigma}\right) .
$$

The symbol $\widehat{\mathcal{P}}$ denotes the anomaly polynomial evaluated over the shadow gauge field. This shadow field appears pretty much universally in all attempts to understand anomalous transport in hydrodynamics; it was first encountered during an attempt to solve the anomalous adiabaticity equation in [8] and plays a significant role in the anomalous hydrostatic partition function (for reasons that will be transparent soon) [10-13].

As written the anomalous effective action is simply a functional of the background sources $\left\{g_{\mu \nu}, A_{\mu}\right\}$ and the hydrodynamic fields $\mathcal{B}=\left\{\boldsymbol{\beta}^{\mu}, \Lambda_{\boldsymbol{\beta}}\right\}$. The gauge field dependence is manifest, while the velocity field $u^{\mu}$ can be expressed in terms of $\mathcal{B}$ using (2.1). What is perhaps less clear is the dependence on the background metric, but owing to the presence of the shadow field in the transgression form, one has a non-trivial metric dependence. To be sure we are extending our sources and hydrodynamic fields to live on $\mathcal{M}_{d+1}$. We will use the same symbols to denote the bulk hydrodynamic fields only differentiating the components by the indices when necessary. Lowercase Latin indices from the later half of the alphabet will denote bulk indices, with $\perp$ being used to denote the direction normal to the physical spacetime $\mathcal{M}$. To wit,

$$
\boldsymbol{\Psi}_{d+1}=\left\{g_{m n}, A_{m}, \boldsymbol{\beta}^{m}, \Lambda_{\boldsymbol{\beta}}\right\}, \quad \boldsymbol{\beta}^{\perp}=0 .
$$

Thus, despite its origins within the framework of non-dissipative effective actions in [30], (12.1) should be viewed as a particular element of Class L for our purposes with $\mathcal{L}=\boldsymbol{V}_{\mathcal{P}}[\boldsymbol{A}, \hat{\boldsymbol{A}}]$. Strictly speaking we are now extending our definition of Class $\mathrm{L}$ to include local Lagrangians in one higher dimension, as we must, if we insist on dealing with anomalous symmetries.

Generically transgressions are defined on a space of interpolating connections. For instance, given two connections say $\boldsymbol{A}_{1}$ and $\boldsymbol{A}_{2}$ respectively, the transgression form denoted

\footnotetext{
${ }^{67}$ We will follow the notational conventions of $[8,11-13,30]$ using bold-face letters to indicate differential forms etc. . Furthermore, to retain compact expressions we perform some formal manipulations with differential forms as in the aforementioned references. Divisions by a differential form implicitly indicates that the numerator when expanded out always has a factor which cancels the form we divide by; the expression in (12.1) is a $2 n+1$ form written as if it were a ratio of a $2 n+3$ form and a 2 form. We refer the reader to the above references where these concepts are explained in greater detail.
} 
more generally as $\mathcal{T}\left[\boldsymbol{A}_{1}, \boldsymbol{A}_{2}\right]$ can be viewed as a functional of a continuous set of connections $\boldsymbol{A}_{t}$ with $t \in[0,1]$ interpolating between $\boldsymbol{A}_{t=0}=\boldsymbol{A}_{1}$ and $\boldsymbol{A}_{t=1}=\boldsymbol{A}_{2}$. One can write this quite succinctly for gauge connections as

$$
\mathcal{T}\left[\boldsymbol{A}_{1}, \boldsymbol{A}_{2}\right]=\int_{0}^{1} d t\left[\frac{d \boldsymbol{A}_{t}}{d t} \cdot\left(\frac{\partial \mathcal{P}}{\partial \boldsymbol{F}}\right)_{t}\right]
$$

with

$$
\boldsymbol{A}_{t}=t \boldsymbol{A}_{t=1}+(1-t) \boldsymbol{A}_{t=0} .
$$

Having this explicit expression is useful for carrying out the variational calculus we need to do to check that the functional $S_{\text {anom }}$ does indeed provide a solution to the anomalous adiabaticity equation (2.12) with $\mathrm{T}_{H}^{\mu \perp}=0$.

For the particular choice of connections $\boldsymbol{A}_{t=0}=\hat{\boldsymbol{A}}$ and $\boldsymbol{A}_{t=1}=\boldsymbol{A}$ we define an interpolation from the hydrodynamic shadow field to the physical gauge field source via $\boldsymbol{A}_{t}=\boldsymbol{A}+(1-t) \mu \boldsymbol{u}$. The corresponding field-strengths are given by

$$
\begin{aligned}
& \boldsymbol{F}=d \boldsymbol{A}+\boldsymbol{A}^{2}=\boldsymbol{B}+\boldsymbol{u} \wedge \boldsymbol{E}, \\
& \hat{\boldsymbol{F}}=d \hat{\boldsymbol{A}}+\hat{\boldsymbol{A}}^{2}=\hat{\boldsymbol{B}}+\boldsymbol{u} \wedge \hat{\boldsymbol{E}}=\boldsymbol{B}+2 \boldsymbol{\omega} \mu+\boldsymbol{u} \wedge(\boldsymbol{E}-D \mu-\boldsymbol{a} \mu) .
\end{aligned}
$$

$\boldsymbol{a}$ and $\boldsymbol{\omega}$ are the acceleration and vorticity defined after (12.1), while $\boldsymbol{B}$ and $\boldsymbol{E}$ are the rest frame magnetic 2-form and electric 1-form respectively. The interpolating field-strength is $\boldsymbol{F}_{t}=t \boldsymbol{F}+(1-t) \hat{\boldsymbol{F}}$ since $(\Delta \boldsymbol{A})^{2}=0$. One can, of course, check explicitly that

$$
\begin{aligned}
\boldsymbol{V}_{\mathcal{P}}[\boldsymbol{A}, \hat{\boldsymbol{A}}] & \equiv \int_{0}^{1} d t\left[\frac{d \boldsymbol{A}_{t}}{d t} \cdot\left(\frac{\partial \mathcal{P}}{\partial \boldsymbol{F}}\right)_{t}\right]=\frac{\boldsymbol{u}}{2 \boldsymbol{\omega}} \wedge \int_{0}^{1} d t\left[\frac{d \boldsymbol{F}_{t}}{d t} \cdot\left(\frac{\partial \mathcal{P}}{\partial \boldsymbol{F}}\right)_{t}\right] \\
& =\frac{\boldsymbol{u}}{2 \boldsymbol{\omega}} \wedge(\mathcal{P}-\widehat{\mathcal{P}}),
\end{aligned}
$$

as indicated above.

To compute the variation of these transgression forms, we need to evaluate $\delta \boldsymbol{V}_{\mathcal{P}}[\boldsymbol{A}, \hat{\boldsymbol{A}}]$. The explicit computation is described in appendix D of [30] and we quote the final result:

$$
\delta \boldsymbol{V}_{\mathcal{P}}[\boldsymbol{A}, \hat{\boldsymbol{A}}]=\delta \boldsymbol{A} \cdot \star_{2 n+1} \mathbf{J}_{H}-\delta \hat{\boldsymbol{A}} \cdot \star_{2 n+1} \hat{\mathbf{J}}_{H}+d\left\{\delta \boldsymbol{A} \cdot \star \boldsymbol{J}_{\mathcal{P}}+\delta \boldsymbol{u} \wedge \star \boldsymbol{q}_{\mathcal{P}}\right\} .
$$

Here $\mathbf{J}_{H}$ is the Hall current defined directly in terms of the variation of the anomaly polynomial:

$$
\star_{2 n+1} \mathbf{J}_{H}=\frac{\partial \mathcal{P}}{\partial \boldsymbol{F}},
$$

with a similar expression for the shadow Hall current $\hat{\mathbf{J}}_{H}$. The two other currents appearing in (12.9) are defined in terms of the boundary terms arising from the variation

$$
\int_{0}^{1} d t\left[\delta \boldsymbol{A}_{t} \cdot\left(\frac{\partial^{2} \mathcal{P}}{\partial \boldsymbol{F} \partial \boldsymbol{F}}\right)_{t} \cdot \frac{d \boldsymbol{A}_{t}}{d t}\right]=\delta \boldsymbol{A} \cdot \star \boldsymbol{J}_{\mathcal{P}}+\delta \boldsymbol{u} \wedge \star \boldsymbol{q}_{\mathcal{P}}
$$

where we have used $\boldsymbol{u} \wedge \frac{d \boldsymbol{A}_{t}}{d t}=0$ and parameterized the terms involved in the variation in terms of gauge potential variation and the velocity field variation. These quantities $\boldsymbol{J}_{\mathcal{P}}$ 
and $\boldsymbol{q}_{\mathcal{P}}$ are determined directly from the variational calculus to be

$$
\begin{aligned}
\star \boldsymbol{J}_{\mathcal{P}} & \equiv \int_{0}^{1} d t\left[\left(\frac{\partial^{2} \mathcal{P}}{\partial \boldsymbol{F} \partial \boldsymbol{F}}\right)_{t} \cdot \frac{d \boldsymbol{A}_{t}}{d t}\right] \\
& =\frac{\boldsymbol{u}}{2 \boldsymbol{\omega}} \wedge\left\{\frac{\partial \mathcal{P}}{\partial \boldsymbol{F}}-\frac{\partial \widehat{\mathcal{P}}}{\partial \hat{\boldsymbol{F}}}\right\},
\end{aligned}
$$

and

$$
\begin{aligned}
\star \boldsymbol{q}_{\mathcal{P}} & =\int_{0}^{1} d s \int_{0}^{s} d t\left[\mu \cdot\left(\frac{\partial^{2} \mathcal{P}}{\partial \boldsymbol{F} \partial \boldsymbol{F}}\right)_{t} \cdot \frac{d \boldsymbol{A}_{t}}{d t}\right] \\
& =-\frac{\boldsymbol{u}}{(2 \boldsymbol{\omega})^{2}} \wedge\left\{\mathcal{P}-\widehat{\mathcal{P}}-(\boldsymbol{F}-\hat{\boldsymbol{F}}) \cdot \frac{\partial \widehat{\mathcal{P}}}{\partial \hat{\boldsymbol{F}}}\right\} .
\end{aligned}
$$

So far the variational calculus did not call for any details of how we are parameterizing the hydrodynamic fields. While we have indeed written the final expression in terms of $\delta \boldsymbol{u}$, the variation of the velocity field, it is easy to convert this to the hydrodynamic field variations using (E.1). Explicitly one can evaluate variation of the action in terms of the sources and the hydrodynamic fields ${ }^{68}$ to obtain an explicit answer for the variation as

$$
\begin{aligned}
\delta \int_{\mathcal{M}_{d+1}} \boldsymbol{V}_{\mathcal{P}}[\boldsymbol{A}, \hat{\boldsymbol{A}}]=f_{\mathcal{M}}\left\{\left(\mathrm{J}_{H}^{m}-P_{n}^{m} \hat{\mathrm{J}}_{H}^{n}\right) \cdot \delta A_{m}\right. & \quad-\mu \cdot \hat{\mathrm{J}}_{H}^{q}\left[P_{q}^{(m} u^{n)} \delta g_{m n}+\left(P_{q m}+u_{q} u_{m}\right) T \delta \boldsymbol{\beta}^{m}\right] \\
& \left.\quad-T u_{q} \hat{\mathrm{J}}_{H}^{q} \cdot\left(\delta \Lambda_{\boldsymbol{\beta}}+A_{m} \delta \boldsymbol{\beta}^{m}\right)\right\} \\
& +\int_{\mathcal{M}} \sqrt{-g}\left[J_{\mathcal{P}}^{\alpha} \cdot \delta A_{\alpha}+q_{\mathcal{P}}^{(\alpha} u^{\beta)} \delta g_{\alpha \beta}\right] .
\end{aligned}
$$

In deriving the above we have used (E.10) to write the variation of $\hat{\boldsymbol{A}}$ in terms of the physical fields and their variations. For notational simplicity we have also abbreviated

$$
f_{\mathcal{M}} \equiv \int_{\mathcal{M}_{d+1}} \sqrt{-g_{d+1}}
$$

so as to avoid cluttering up the equations.

As we see there are two types of contributions to the variation of our Lagrangian $\boldsymbol{V}_{\mathcal{P}}[\boldsymbol{A}, \hat{\boldsymbol{A}}]$. On the one hand, we have some bulk variations (the first two lines in the r.h.s. of (12.14)) which define the bulk currents living on $\mathcal{M}_{d+1}$. To wit,

$$
\begin{array}{ll}
T_{(d+1)}^{m n}=-\mu \cdot \hat{\mathrm{J}}_{H}^{q}\left(P_{q}^{m} u^{n}+P_{q}^{n} u^{m}\right), & J_{(d+1)}^{m}=\mathrm{J}_{H}^{m}-P_{n}^{m} \hat{\mathrm{J}}_{H}^{n}, \\
\mathfrak{h}_{m}^{(d+1)}=-\mu \cdot \hat{\mathrm{J}}_{H}^{q}\left(P_{q m}+u_{q} u_{m}\right) T, & \mathfrak{n}_{(d+1)}=T u_{q} \hat{\mathrm{J}}_{H}^{q} .
\end{array}
$$

\footnotetext{
${ }^{68}$ One can also convert this variation to one involving the reference fields introduced in section 7.2. These should also be viewed as living on the reference bulk spacetime since the hydrodynamic fields which they are a proxy for are defined in terms of maps from there to the physical spacetime. We will shortly come back to this viewpoint to facilitate some parts of the analysis.
} 
These have to to satisfy the analog of the bulk adiabaticity equation. This can be shown directly by running our argument for the Bianchi identity in the bulk theory; cf., the discussion around (H.12) for an explicit proof of this statement.

More interesting for us are the boundary terms in the last line of (12.14) - these are the contributions that arise from the inflow mechanism. In particular, they capture the constitutive relations for anomalous hydrodynamics. Since we have the terms explicitly in terms of the source variations we can directly read off from here using (6.3) the anomalous currents to be

$$
\left(T^{\alpha \beta}\right)_{\mathrm{A}}=q_{\mathcal{P}}^{\alpha} u^{\beta}+q_{\mathcal{P}}^{\beta} u^{\alpha}, \quad\left(J^{\alpha}\right)_{\mathrm{A}}=J_{\mathcal{P}}^{\alpha}, \quad\left(J_{S}^{\alpha}\right)_{\mathrm{A}}=0
$$

These currents satisfy the anomalous adiabaticity equation (2.12) on the boundary manifold $\mathcal{M}$. This was first established in [8] and follows immediately from the previous analysis of [30]. ${ }^{69}$ As described there, by isolating the anomalous contributions and solving the adiabaticity equation to give the above particular solution (12.17), one has accounted for all flavour anomalies. One can then couple the anomalous Lagrangian $\mathcal{L}_{\text {anom }}$ to any non-anomalous adiabatic fluid Lagrangian system and continue to satisfy adiabaticity.

Finally, let us make a remark on the construction above which will be useful for generalizations. The anomalous Lagrangian density $\sqrt{g_{d+1}} \mathcal{L}_{\text {anom }}$ is a scalar density on the bulk spacetime manifold. Per se, in keeping with our general philosophy this is an off-shell quantity, since we have nowhere insisted in our construction above that the fields be onshell. However, restricting to hydrostatics by enforcing $\mathcal{B}=\mathcal{K}$ one ends up with an on-shell construction which as we now appreciate is related to the hydrostatic partition function $W_{\text {Hydrostatic }}(6.25)$.

In general the relation between the hydrostatic partition function and the nondissipative fluid formalism is complicated by a non-linear Legendre transform (see section B). However, for the flavour anomalies the fact that $\mathcal{L}_{\text {anom }}(12.1)$ is independent of the entropy density makes the Legendre transformation trivial. ${ }^{70}$ This also to some extent underscores the rationale for introduction of the shadow gauge field $\hat{A}$; the shadow field plays a crucial role in ensuring the correct properties of the hydrostatic partition function as has been described in earlier works.

\subsection{Mixed anomalies}

We would now like to generalize anomalous adiabatic fluids to the case where we have gravitational or mixed anomalies. One of the motivations for reviewing in some detail the flavour case in the previous subsection, was that it provides a hint of how one should generalize the construction to incorporate gravitational effects. To a large extent a specific solution to the anomalous adiabaticity equation in the presence of mixed anomalies can be obtained by treating the gravitational field as a non-abelian flavour field. This is roughly

\footnotetext{
${ }^{69}$ We will give a more detailed derivation for the mixed anomalies in section 12.2. Setting the gravitational terms in that analysis to zero will demonstrate the claim herein explicitly.

${ }^{70}$ This was the reason why the direct comparison of the non-dissipative effective action with the hydrostatic partition function worked quite seamlessly in the consistency checks carried out in [30].
} 
the correct intuition, though as we will see in the course of a more thorough analysis below there are some subtleties we need to deal with. In particular, we will see that the entropy current is modified in the presence of gravitational effects, no longer vanishing as in (12.17).

We are going to start our discussion for the mixed anomaly by mimicking the discussion for the flavour case. Specifically, since there is a close connection between the off-shell Lagrangian and the on-shell hydrostatic partition function we are going to take inspiration from the latter which is by now well understood for mixed anomalies [12]. Once again we imagine that the set of anomalies of our underlying quantum system is encoded in an anomaly polynomial $\mathcal{P}[\boldsymbol{A}, \boldsymbol{\Gamma}]$ with $\boldsymbol{A}$ being the gauge connection and $\boldsymbol{\Gamma}$ the spin connection for the background geometry. ${ }^{71}$ We are also unabashedly going to work in the bulk geometry $\mathcal{M}_{d+1}$ with the physical spacetime $\mathcal{M}=\partial \mathcal{M}_{d+1}$ as before. The general set of conditions we impose on the geometry is similar to that encountered in section 12.1, though we will have to add some new ingredients as we proceed.

In analogy with the flavour story, let us consider modifying Class L by adding to the Lagrangian a higher dimensional term of the form

$$
\int_{\mathcal{M}_{d+1}} \sqrt{-g_{d+1}} \mathcal{L}_{\text {anom }}=\int_{\mathcal{M}_{d+1}} \boldsymbol{V}_{\mathcal{P}}[\boldsymbol{A}, \boldsymbol{\Gamma}, \hat{\boldsymbol{A}}, \hat{\boldsymbol{\Gamma}}]=\int_{\mathcal{M}_{d+1}} \frac{\boldsymbol{u}}{2 \boldsymbol{\omega}} \wedge(\mathcal{P}[\boldsymbol{F}, \boldsymbol{R}]-\widehat{\mathcal{P}}[\hat{\boldsymbol{F}}, \hat{\boldsymbol{R}}])
$$

The specific choice of the Lagrangian is motivated by the fact that $\boldsymbol{V}_{\mathcal{P}}[\boldsymbol{A}, \boldsymbol{\Gamma}, \hat{\boldsymbol{A}}, \hat{\boldsymbol{\Gamma}}]$ is the canonical form for the scalar part of the hydrostatic anomalous partition function (Class $\mathrm{H}_{S}$ ). The key difference from (12.1) is the dependence on the background geometry; the anomaly polynomial now is a functional both of the background field strength and the background curvature $\boldsymbol{R}$.

Let us examine the dependence of the potential anomalous Lagrangian introduced above: apart from the backgound sources $\left\{g_{m n}, A_{m}\right\}$ we have also the shadow fields appearing in $\mathcal{L}_{\text {anom. }}$ The shadow flavour gauge connection $\hat{\boldsymbol{A}}$ is the same as before being given by (12.2). The shadow spin connection however is new and requires to be defined. A natural course of action is to follow the partition function analysis of [12] and demand that this be given in terms of the background spin connection, the velocity field, and a spin chemical potential $\Omega^{\mu}{ }_{\nu}$ as

$$
\hat{\boldsymbol{\Gamma}}^{\mu}{ }_{\nu}=\boldsymbol{\Gamma}^{\mu}{ }_{\nu}+{\Omega^{\mu}}_{\nu} \boldsymbol{u} .
$$

Modulo defining the spin chemical potential we are now equipped with a putative anomalous Lagrangian.

The spin chemical potential should couple to the background metric structure since its origins are in the Lorentzian structure of the local tangent space geometry on $\mathcal{M}$ (and hence by inflow on $\mathcal{M}_{d+1}$ ). In hydrostatic equilibrium the analysis of [12] shows that it is related to the gradient of the Killing vector $K^{\mu}$ which extends trivially to a Killing field on $\mathcal{M}_{d+1}$. The relation in the hydrodynamic variables living on the physical spacetime $\mathcal{M}$

\footnotetext{
${ }^{71}$ We will mostly work with the one-form spin connection since it is most conducive for the purposes of carrying out the formal manipulations. Translating this to the Christoffel connection is reasonably straightforward. We will in fact do so when we write out some explicit components.
} 
is just

$$
\left(\Omega^{\mu}{ }_{\nu}\right)_{\text {Hydrostatic }}=T D_{\nu} K^{\mu}
$$

with $T$ being the equilibrium temperature. We could take this expression off-equilibrium and off-shell by simply replacing $K^{\mu} \mapsto \boldsymbol{\beta}^{\mu}$. However, we need to be careful with the symmetries: for a Killing vector field $D_{(\mu} K_{\nu)}=0$ by virtue of Killing's equation. Hence only the antis-symmetric part of the tensor $D_{\mu} K^{\nu}$ is non-zero. Away from equilibrium when we consider $D_{\mu} \boldsymbol{\beta}^{\nu}$ we are likely to encounter both the symmetric and anti-symmetric contributions. The naïve generalization $K^{\mu} \mapsto \boldsymbol{\beta}^{\mu}$ would retain both, but we claim that the correct off-shell extension of (12.20) should only keep the anti-symmetric part. ${ }^{72}$ With this motivation we define:

$$
{\Omega^{\mu}}_{\nu}=\frac{1}{2} T\left(D_{\nu} \boldsymbol{\beta}^{\mu}-D^{\mu} \boldsymbol{\beta}_{\nu}\right) \equiv T \mathfrak{Q}_{\nu \beta}^{\mu \alpha} D_{\alpha} \boldsymbol{\beta}^{\beta},
$$

where we have introduced the antisymmetrizer $\mathfrak{Q}_{\nu \beta}^{\mu \alpha}=\frac{1}{2}\left(\delta_{\beta}^{\mu} \delta_{\nu}^{\alpha}-g^{\mu \alpha} g_{\nu \beta}\right)$ for future convenience. By construction we ensure that in hydrostatic equilibrium we recover the spin chemical potential of [12].

The main claim we wish to make is that the Lagrangian density $\boldsymbol{V}_{\mathcal{P}}[\boldsymbol{A}, \boldsymbol{\Gamma}, \hat{\boldsymbol{A}}, \hat{\boldsymbol{\Gamma}}]$ provides a solution to the adiabaticity equation (2.12). Furthermore, the currents derived from this Lagrangian are consistent with those derived earlier in [12] in hydrostatic equilibrium. We will establish this by a straightforward computation.

\subsection{Variational calculus for mixed anomalies}

The anomalous Lagrangian density $\boldsymbol{V}_{\mathcal{P}}[\boldsymbol{A}, \boldsymbol{\Gamma}, \hat{\boldsymbol{A}}, \hat{\boldsymbol{\Gamma}}](12.18)$ is once again a transgression form. Let us therefore focus on the transgression formula between the pairs of gauge and spin connections $\left\{\boldsymbol{A}_{t=1}, \boldsymbol{\Gamma}_{t=1}\right\}=\{\boldsymbol{A}, \boldsymbol{\Gamma}\}$ and $\left\{\boldsymbol{A}_{t=0}, \boldsymbol{\Gamma}_{t=0}\right\}=\{\hat{\boldsymbol{A}}, \hat{\boldsymbol{\Gamma}}\}$ respectively. Since the gravitational connection $\boldsymbol{\Gamma}$ behaves exactly like a non-abelian gauge connection, the calculation is a straightforward generalization of what we had to in the case of the flavour anomaly.

We begin by considering the first variation of the transgression form, which is given directly by the analog of (12.14), except that now we have terms coming from the spin connection. This can be written in a reasonably compact form by introducing bulk Hall currents and boundary anomalous currents as:

$$
\begin{aligned}
& \delta \boldsymbol{V}_{\mathcal{P}}[\boldsymbol{A}, \boldsymbol{\Gamma}, \hat{\boldsymbol{A}}, \hat{\boldsymbol{\Gamma}}]=\delta \boldsymbol{A} \wedge \cdot^{\star 2 n+1} \mathbf{J}_{H}-\delta \hat{\boldsymbol{A}} \wedge \cdot{ }^{\star 2 n+1} \hat{\mathbf{J}}_{H} \\
& +\frac{1}{2} \delta \boldsymbol{\Gamma}_{b}^{a} \wedge^{\star 2 n+1} \boldsymbol{\Sigma}_{H}{ }^{b}{ }_{a}-\frac{1}{2} \delta \hat{\boldsymbol{\Gamma}}^{a}{ }_{b} \wedge^{\star_{2 n+1}} \hat{\boldsymbol{\Sigma}}_{H}{ }^{b}{ }_{a} \\
& +d\left\{\delta \boldsymbol{A} \wedge \cdot \star \boldsymbol{J}_{\mathcal{P}}+\frac{1}{2} \delta \boldsymbol{\Gamma}^{\alpha}{ }_{\beta} \wedge \star \boldsymbol{\Sigma}_{\mathcal{P}}{ }^{\beta}{ }_{\alpha}+\delta \boldsymbol{u} \wedge \star \boldsymbol{q}_{\mathcal{P}}\right\},
\end{aligned}
$$

\footnotetext{
${ }^{72}$ We do not have an a-priori reason to motivate this particular choice; what we can see is a post-facto argument. Choosing the spin chemical potential to contain the symmetric part of the gradient results in a tension with the off-equilibrium adiabaticity equation; see the discussion around (12.30) for what goes wrong. We note that choosing the anti-symmetric part makes the shadow connection metric compatible (but not torsion free). This property however does not uniquely characterize our choice.
} 
The bulk Hall currents are themselves given in terms of the derivatives of the anomaly polynomial with respect to the field strengths and are given by

$$
{ }^{{ }_{2 n+1}} \mathbf{J}_{H}=\frac{\partial \mathcal{P}}{\partial \boldsymbol{F}}, \quad{ }^{{ }_{2 n+1}} \boldsymbol{\Sigma}_{H}{ }^{b}{ }_{a}=2 \frac{\partial \mathcal{P}}{\partial \boldsymbol{R}^{a} b},
$$

These currents will play a role as before in determining the amount of anomaly inflow into the boundary theory. The anomaly induced boundary currents can also be determined explicitly and the only change due to the spin connection is a slight generalization of (12.13) for the current $\boldsymbol{q}_{\mathcal{P}}$ to include appropriate gravitational contributions. The flavour anomaly induced current $\boldsymbol{J}_{\mathcal{P}}$ being blind to the spin connection remains unchanged as in (12.13). In addition we have a spin anomaly current $\boldsymbol{\Sigma}_{\mathcal{P}}$. To write down the expressions for the currents, it is useful to introduce an electromagnetic splitting of the field strength and curvature:

$$
\begin{aligned}
\boldsymbol{B} & =\boldsymbol{F}-\boldsymbol{u} \wedge \boldsymbol{E}, & \boldsymbol{E} & =-\boldsymbol{i}_{u} \boldsymbol{F}, \\
\left(\boldsymbol{B}_{R}\right)^{\alpha}{ }_{\beta} & =\boldsymbol{R}^{\alpha}{ }_{\beta}-\boldsymbol{u} \wedge \boldsymbol{E}_{R}{ }_{\beta}{ }_{\beta}, & \boldsymbol{E}_{R}^{\alpha}{ }_{\beta} & =-\boldsymbol{i}_{u} \boldsymbol{R}_{\beta}^{\alpha},
\end{aligned}
$$

where $\boldsymbol{i}_{u}$ denotes contractions with $u^{\mu}$. With this information we can now write down all three currents, as we do below for completeness: ${ }^{73}$

$$
\begin{aligned}
& \star \boldsymbol{J}_{\mathcal{P}}=\int_{0}^{1} d t\left[\left(\frac{\partial^{2} \mathcal{P}}{\partial \boldsymbol{F} \partial \boldsymbol{F}}\right)_{t} \cdot \frac{d \boldsymbol{A}_{t}}{d t}+\left(\frac{\partial^{2} \mathcal{P}}{\partial \boldsymbol{F} \partial \boldsymbol{R}^{\alpha}{ }_{\beta}}\right) \frac{d\left(\boldsymbol{\Gamma}_{\beta}^{\alpha}\right)_{t}}{d t}\right] \\
& =\frac{\boldsymbol{u}}{2 \boldsymbol{\omega}} \wedge\left\{\frac{\partial \mathcal{P}}{\partial \boldsymbol{F}}-\frac{\partial \widehat{\mathcal{P}}}{\partial \hat{\boldsymbol{F}}}\right\}=\frac{\partial \boldsymbol{V}_{\mathcal{P}}}{\partial \boldsymbol{B}}, \\
& \star \boldsymbol{\Sigma}_{\mathcal{P}}{ }^{\beta}{ }_{\alpha}=2 \int_{0}^{1} d t\left[\left(\frac{\partial^{2} \mathcal{P}}{\partial \boldsymbol{R}^{\alpha}{ }_{\beta} \partial \boldsymbol{R}^{\gamma}{ }_{\delta}}\right)_{t} \frac{d\left(\boldsymbol{\Gamma}^{\gamma}\right)_{t}}{d t}+\left(\frac{\partial^{2} \mathcal{P}}{\partial \boldsymbol{R}^{\alpha}{ }_{\beta} \partial \boldsymbol{F}}\right) \cdot \frac{d \boldsymbol{A}_{t}}{d t}\right] \\
& =2 \frac{\boldsymbol{u}}{2 \boldsymbol{\omega}} \wedge\left\{\frac{\partial \mathcal{P}}{\partial \boldsymbol{R}^{\alpha}{ }_{\beta}}-\frac{\partial \widehat{\mathcal{P}}}{\partial \hat{\boldsymbol{R}}^{\alpha}{ }_{\beta}}\right\}=2 \frac{\partial \boldsymbol{V}_{\mathcal{P}}}{\partial\left(\boldsymbol{B}_{R}\right)^{\alpha}{ }_{\beta}}, \\
& \star \boldsymbol{q}_{\mathcal{P}}=\int_{0}^{1} d t(1-t)\left[\mu \cdot\left(\frac{\partial^{2} \mathcal{P}}{\partial \boldsymbol{F} \partial \boldsymbol{F}} \cdot \frac{d \boldsymbol{A}_{t}}{d t}+\frac{\partial^{2} \mathcal{P}}{\partial \boldsymbol{F} \partial \boldsymbol{R}^{\alpha}{ }_{\beta}} \frac{d\left(\boldsymbol{\Gamma}_{\beta}^{\alpha}\right)_{t}}{d t}\right)\right. \\
& \left.+\Omega^{\alpha}{ }_{\beta}\left(\frac{\partial^{2} \mathcal{P}}{\partial \boldsymbol{R}^{\alpha}{ }_{\beta} \partial \boldsymbol{R}^{\gamma} \delta} \frac{d\left(\boldsymbol{\Gamma}^{\gamma}\right)_{t}}{d t}+\frac{\partial^{2} \mathcal{P}}{\partial \boldsymbol{R}^{\alpha}{ }_{\beta} \partial \boldsymbol{F}} \cdot \frac{d \boldsymbol{A}_{t}}{d t}\right)\right] \\
& =-\frac{\boldsymbol{u}}{(2 \boldsymbol{\omega})^{2}} \wedge\left[\boldsymbol{\mathcal { P }}-\widehat{\mathcal{P}}-(\boldsymbol{F}-\hat{\boldsymbol{F}}) \cdot \frac{\partial \widehat{\mathcal{P}}}{\partial \hat{\boldsymbol{F}}}-\left(\boldsymbol{R}_{\beta}^{\alpha}-\hat{\boldsymbol{R}}^{\alpha}{ }_{\beta}\right) \frac{\partial \widehat{\mathcal{P}}}{\partial \hat{\boldsymbol{R}}^{\alpha}{ }_{\beta}}\right]=\frac{\partial \boldsymbol{V}_{\mathcal{P}}}{\partial(2 \boldsymbol{\omega})},
\end{aligned}
$$

In order to obtain bulk and boundary Bianchi identities from the basic variation (12.22), we need to follow the same logic as in the case of flavour anomalies. We perform the detailed computation in appendix $\mathrm{H}$ and only quote the result for the Bianchi identities of the (physical) boundary theory:

$$
\begin{aligned}
D_{\beta}\left(T^{\alpha \beta}\right)_{\mathrm{A}}=\left(J^{\beta}\right)_{\mathrm{A}} \cdot & F^{\alpha}{ }_{\beta}+\frac{g^{\alpha \sigma}}{\sqrt{-g}} \delta_{\mathcal{B}}\left[\sqrt{-g} T\left(q_{\mathcal{P}}\right)_{\sigma}\right] \\
& +\frac{1}{2} D_{\gamma}\left(\Sigma_{H}^{\perp[\alpha \gamma]}-\hat{\Sigma}_{H}^{\perp[\alpha \gamma]}\right)-\left(\mu \cdot \hat{\mathrm{J}}_{H}^{\perp}+\frac{1}{2} \Omega^{\nu}{ }_{\mu} \hat{\Sigma}_{H}{ }^{\perp \mu}{ }_{\nu}\right) u^{\alpha},
\end{aligned}
$$

\footnotetext{
${ }^{73}$ For further details we refer the reader to [12].
} 
and

$$
D_{\alpha}\left(J^{\alpha}\right)_{\mathrm{A}}=\mathrm{J}_{H}^{\perp}-\hat{\mathrm{J}}_{H}^{\perp},
$$

which are satisfied by the anomalous currents

$$
\begin{aligned}
\left(T^{\alpha \beta}\right)_{\mathrm{A}} & =\left.\frac{2}{\sqrt{-g}} \frac{\delta S_{\text {anom }}}{\delta g_{\alpha \beta}}\right|_{\text {boundary }}=q_{\mathcal{P}}^{\alpha} u^{\beta}+q_{\mathcal{P}}^{\beta} u^{\alpha}+\frac{1}{2} D_{\rho}\left(\Sigma_{\mathcal{P}}^{\alpha[\beta \rho]}+\Sigma_{\mathcal{P}}^{\beta[\alpha \rho]}-\Sigma_{\mathcal{P}}^{\rho(\alpha \beta)}\right) \\
\left(J^{\alpha}\right)_{\mathrm{A}} & =\left.\frac{1}{\sqrt{-g}} \frac{\delta S_{\text {anom }}}{\delta A_{\alpha}}\right|_{\text {boundary }}=J_{\mathcal{P}}^{\alpha}
\end{aligned}
$$

We now want convert these Bianchi identities into an adiabaticity equation and check that (2.12) is satisfied with an appropriate choice of currents. Since the energy-momentum and charge currents are defined by varying the Lagrangian with respect to the sources, these currents are already manifest in the above expressions. Plugging these in and demanding that the following adiabaticity equation be upheld

$$
\begin{aligned}
D_{\alpha}\left(J_{S}^{\alpha}\right)_{\mathrm{A}}+\boldsymbol{\beta}_{\alpha}\left[D_{\sigma}\left(T^{\alpha \sigma}\right)_{\mathrm{A}}\right. & \left.-\left(J^{\sigma}\right)_{\mathrm{A}} \cdot F_{\sigma}^{\alpha}-\frac{1}{2} D_{\gamma} \Sigma_{H}^{\perp[\alpha \gamma]}\right] \\
& +\left(\Lambda_{\boldsymbol{\beta}}+\boldsymbol{\beta}^{\alpha} A_{\alpha}\right) \cdot\left(D_{\sigma}\left(J^{\sigma}\right)_{\mathrm{A}}-\mathrm{J}_{H}^{\perp}\right)=0
\end{aligned}
$$

results in a non-trivial solution for $\left(J_{S}^{\alpha}\right)_{\mathrm{A}}$ ! More precisely, we find that in addition to (12.28), we need to define the following entropy current in order to get a solution to $(12.29)$ :

$$
\left(J_{S}^{\alpha}\right)_{\mathrm{A}}=-\frac{1}{2} \boldsymbol{\beta}_{\sigma} \hat{\Sigma}_{H}^{\perp[\alpha \sigma]} .
$$

The expressions (12.28) and (12.30) define a required particular solution to (2.12) that can be used to remove the anomaly terms (both flavour and Lorentz anomalies). What is curious in our construction is the fact that we have necessarily had to modify the entropy current in order to achieve this. Specifically, the entropy current $\left(J_{S}^{\alpha}\right)_{A}$ does not quite satisfy (6.4) anymore. We conclude that the solution to the anomalous adiabaticity equation requires modifying the entropy current apart from noting that given the variational principles, it is the only current that we are free to manipulate.

While the reader might consider the above set of statements somewhat ad-hoc, we should add that the structure of the terms is rather tightly constrained. We have not been able to find any other Lagrangian solution to the anomalous adiabaticity equation. Moreover, small modifications such as allowing the spin chemical potential to be defined directly in terms of the gradient of the velocity field (i.e., without the anti-symmetrization introduced in (12.21)) ends up destroying the structure. One intuition we can offer is the following: in contrast to the flavour anomaly discussion of section 12.1 the new element we have to account for is the background metric variation. Since the diffeomorphism symmetry enters more universally any slight modification of the structures results in inconsistencies. We believe that this is indicative of some underlying structure that can be used to formulate our arguments more robustly — we will pursue this line of thought in the future (see however section 15 for some preliminary ideas on this front). 
It is worth recording that in hydrostatic equilibrium $\hat{\Sigma}_{H}^{\perp[\alpha \beta]}=0$ and we reproduce the result of [12]. Furthermore, we also see that the anomalous stress-tensor and charge current in (12.28) take precisely the form that is expected by naïvely generalizing hydrostatic results. We take these to be important consistency checks of our construction. Attempting to solve the adiabaticity equation directly to obtain off-shell currents, leads to somewhat different constitutive relations. While this will be discussed elsewhere [78], it is worth noting that this result uses a different spin chemical potential (in particular they take the gradient of the thermal vector eschewing the projection to the anti-symmetric part as in $(12.21))$.

More generally, it is worth keeping in mind that the Class A constitutive relations are particular solutions to the inhomogeneous adiabaticity equation. As always these are ambiguous to shifts by homogeneous solutions. In terms of the current discussion, we have the freedom to add into Class $\mathrm{A}$ any other adiabatic constitutive relation, whilst maintaining adiabaticity. So two a-priori different looking solutions to the anomalous adiabaticity equation should be demonstrably related by adding in a linear combination of terms from the other six classes. More formally, Class A constitutive relations take values in the coset (Adiabatic constitutive relations)/(Class $\{\mathrm{L}, \mathrm{B}, \mathrm{C}, \mathrm{V}\}$ ) with $\mathrm{V}=\mathrm{H}_{V} \cup \overline{\mathrm{H}}_{V}$ denoting the vector classes.

\subsection{On-shell dynamics of anomalous adiabatic fluids}

Given that we have off-shell adiabatic constitutive relations (12.28), (12.30) we can ask whether our anomalous effective action (12.18) satisfies the correct on-shell constraints. A-priori we expect based on our knowledge of the flavour anomaly discussion of [30], that the on-shell Ward identities are not going to be obeyed by our Lagrangian system. We will show in section 13.3 that a thermofield doubled construction can fix this problem. For now we are simply going to use the construction of the previous subsections to show that the on-shell equations we obtain from Class L anomalous hydrodynamics are incorrect.

To get started, let us assume that $\int_{\mathcal{M}_{d+1}} \boldsymbol{V}_{\mathcal{P}}[\boldsymbol{A}, \boldsymbol{\Gamma}, \hat{\boldsymbol{A}}, \hat{\boldsymbol{\Gamma}}]$ provides for us a particular solution to the anomalous adiabaticity equation. The complete hydrodynamical system as we have discussed hitherto is then a combination of a non-anomalous part and the anomalous terms we have just taken care of. So we can write an effective action for our system as a sum of two contributions

$$
S_{\mathrm{eff}}[\boldsymbol{\Psi}]=\int_{\mathcal{M}} \sqrt{-g} \mathcal{L}_{\mathrm{n}-\mathrm{a}}[\boldsymbol{\Psi}]+\int_{\mathcal{M}_{d+1}} \boldsymbol{V}_{\mathcal{P}}[\boldsymbol{A}, \boldsymbol{\Gamma}, \hat{\boldsymbol{A}}, \hat{\boldsymbol{\Gamma}}]
$$

and treat the entire bulk + boundary dynamics as part of an extended Class L system. In what follows we will denote the contribution from the non-anomalous terms in Class $\mathrm{L}$ arising from $\mathcal{L}_{\text {n-a }}$ by the subscript ' $\mathrm{n}$-a' so as to keep track of them explicitly. These terms then are required to satisfy the non-anomalous Bianchi identities from (6.12):

$$
\begin{aligned}
& D_{\nu} T_{\mathrm{n}-\mathrm{a}}^{\mu \nu}=\left(J_{\nu}\right)_{\mathrm{n}-\mathrm{a}} \cdot F^{\mu \nu}+\frac{g^{\mu \nu}}{\sqrt{-g}} \delta_{\mathcal{B}}\left(\sqrt{-g} T\left(\mathfrak{h}_{\nu}\right)_{\mathrm{n}-\mathrm{a}}\right)+g^{\mu \nu} T \mathfrak{n}_{\mathrm{n}-\mathrm{a}} \cdot \delta_{\mathcal{B}} A_{\nu}, \\
& D_{\sigma} J_{\mathrm{n}-\mathrm{a}}^{\sigma}=\frac{1}{\sqrt{-g}} \delta_{\mathcal{B}}\left(\sqrt{-g} T \mathfrak{n}_{\mathrm{n}-\mathrm{a}}\right) .
\end{aligned}
$$


We note that we are not adding any bulk non-anomalous terms since the presumption is that the physical fluid lives on $\mathcal{M}$ with the bulk fields on $\mathcal{M}_{d+1}$ simply providing us with an efficient way to keep track of the inflow and Hall currents.

Since we are interested in the on-shell dynamics, let us introduce the reference fields $\left\{\beta^{a}, \Lambda_{\beta}\right\}$ and their related pullback fields $\left\{\varphi^{a}, c\right\} .{ }^{74}$ The dynamical information of the theory is obtained by extremizing the effective action $S_{\text {eff }}$ with respect to the pullback fields.

Performing the required manipulations, we firstly find sensible equations of motion for bulk quantities which we quote in section H.2. Similarly, for the boundary degrees of freedom we find that the extremization in the Lie orbit of the reference sources $\left\{\mathfrak{g}_{a b}, \mathbb{A}_{a}\right\}$ leads to

$$
\begin{aligned}
\frac{g^{\mu \nu}}{\sqrt{-g}} \delta_{\mathcal{B}}\left(\sqrt{-g} T\left[\left(\mathfrak{h}_{\nu}\right)_{\mathrm{n}-\mathrm{a}}+\left(q_{\mathcal{P}}\right)_{\nu}\right]\right)+g^{\mu \nu} T \mathfrak{n}_{\mathrm{n}-\mathrm{a}} \cdot \delta_{\mathfrak{B}} A_{\nu} & \simeq 0, \\
\frac{1}{\sqrt{-g}} \delta_{\mathcal{B}}\left(\sqrt{-g} T \mathfrak{n}_{\mathrm{n}-\mathrm{a}}\right) & \simeq 0 .
\end{aligned}
$$

Note that the anomalous part of the action only contributes a single term proportional $\left(q_{\mathcal{P}}\right)_{\mu}$. This can be seen from (H.6) where all the boundary terms except the very last one give vanishing contribution when we restricted to the constrained variation in the Lie orbit of the reference sources.

Using then the Bianchi identities (12.26), (12.27) together with the on-shell dynamical equations (12.33) we find that the on-shell fluid configurations on the boundary $\mathcal{M}$ obey

$$
\begin{aligned}
D_{\beta}\left(T_{\mathrm{n}-\mathrm{a}}^{\alpha \beta}+\left(T^{\alpha \beta}\right)_{\mathrm{A}}\right) \simeq & \left(J_{\mathrm{n}-\mathrm{a}}^{\sigma}+\left(J^{\sigma}\right)_{\mathrm{A}}\right) \cdot F^{\alpha}{ }_{\sigma}+\frac{1}{2} D_{\gamma}\left(\Sigma_{H}^{\perp[\alpha \gamma]}-\hat{\Sigma}_{H}^{\perp[\alpha \gamma]}\right) \\
& -\left(\mu \cdot \hat{\mathrm{J}}_{H}^{\perp}+\frac{1}{2} \Omega^{\nu}{ }_{\mu} \hat{\Sigma}_{H}{ }^{\perp \mu}{ }_{\nu}\right) u^{\alpha}, \\
D_{\sigma}\left(J_{\mathrm{n}-\mathrm{a}}^{\sigma}+\left(J^{\sigma}\right)_{\mathrm{A}}\right) \simeq & \mathrm{J}_{H}^{\perp}-\hat{\mathrm{J}}_{H}^{\perp} .
\end{aligned}
$$

We note that these are not quite the correct hydrodynamic equations. The anomalous Ward identities should rather be

$$
\begin{aligned}
D_{\beta}\left(T_{\mathrm{n}-\mathrm{a}}^{\alpha \beta}+\left(T^{\alpha \beta}\right)_{\mathrm{A}}\right) & \simeq\left(J_{\mathrm{n}-\mathrm{a}}^{\sigma}+\left(J^{\sigma}\right)_{\mathrm{A}}\right) \cdot F^{\alpha}{ }_{\sigma}+\frac{1}{2} D_{\gamma} \Sigma_{H}^{\perp[\alpha \gamma]}, \\
D_{\sigma}\left(J_{\mathrm{n}-\mathrm{a}}^{\sigma}+\left(J^{\sigma}\right)_{\mathrm{A}}\right) & \simeq \mathrm{J}_{H}^{\perp} .
\end{aligned}
$$

The troublesome terms are the shadow (hatted) currents on the r.h.s. of (12.34).

Thus, we conclude that further modification is required in how one formulates the field theory of the pullback fields for it to match with the usual hydrodynamics. This will be the focus of section 13.3 where we will follow the analysis of [30] to show that the correct Ward identities which require removing the shadow terms from the r.h.s. of (12.34), can be obtained by working with a doubled set of degrees of freedom. Before applying the SchwingerKeldysh technology to Class A, let us now set up an appropriate general formalism.

\footnotetext{
${ }^{74}$ For the discussion of anomalous fluids the reference fields and the reference manifold are taken to be $(d+1)$-dimensional. However, apart from using different indices as summarized in table 13 we will refrain from introducing a new notation for the bulk reference quantities; hopefully it will be clear from the context whether we are discussing the bulk or the boundary reference data.
} 


\section{Schwinger-Keldysh formalism for Class L and application to Class A}

Thus far we have tried to formulate hydrodynamics in terms of response to a single set of background fields $\left\{g_{\mu \nu}, A_{\mu}\right\}$. However, since hydrodynamics is ultimately a statistical system, we should allow for statistical fluctuations. By the fluctuation-dissipation theorem these statistical fluctuations are closely tied to allowing statistical dissipation. The correct framework for dealing with this is the Schwinger-Keldysh formalism [79, 80] whereby the dynamical fields (and the background sources) are doubled.

Now the astute reader would wonder why we bring up the issue of doubling the fields since the basic premise of the adiabatic fluid formalism is that it is conservative; on-shell the adiabaticity equation ensures that no entropy is produced thus allowing no dissipation. Nevertheless since we are interested in classifying hydrodynamic transport in general, it is worthwhile analyzing the situation in the adiabatic case which provides a useful context for the general discussion. Furthermore, our discussion of anomalous transport in section 12 confronts us with the issue of getting unwanted shadow contributions to the Ward identities. As we will show, this is an artifact of working with a single copy theory and doubling the degrees of freedom recovers for us the correct Ward identities, despite falling within the purview of adiabatic transport (Class A) as in [30].

There are two issues we wish to highlight in the hydrodynamic Schwinger-Keldysh functionals that we will construct below. The first is the doubling of degrees of freedom and the associated symmetries. The second is the fact that such functionals a-priori allow interactions between the two sets of degrees of freedom. These terms are are sometimes referred to as influence functionals following [50]. In fact, our previous discussion of Lagrangian constructions of anomalous hydrodynamic effective actions in [30] already exemplified the occurrence of such interaction terms. We will however take this opportunity to rephrase the construction in a more canonical fashion. Along the way we will see some advantages of the Class L reference field formalism for the Schwinger-Keldysh functionals. While this appears to hold useful clues in understanding how to apply the Schwinger-Keldysh construction in generic non-equilibrium dynamics, we will also find a certain tension with the adiabaticity equation. The following discussion should be viewed as a first step in setting up the general construction and we will in particular highlight some of the missing ingredients. A fuller exposition of these ideas will however be postponed to a future publication [55].

\subsection{Schwinger-Keldysh fields on the reference manifold}

With these facts in mind, let us now consider uplifting our construction from a single copy of fields $\Psi$ to the Schwinger-Keldysh doubled system. We start by doubling the fields to a pair of left and right fields indexed by sub/superscripts $L$ and $R$ respectively, $\left\{\boldsymbol{\Psi}_{\mathrm{L}}, \boldsymbol{\Psi}_{\mathrm{R}}\right\}$. In particular we not only double the background sources to $\left\{g_{\mu \nu}^{\mathrm{L}}, A_{\mu}^{\mathrm{L}}\right\}$ and $\left\{g_{\mu \nu}^{\mathrm{R}}, A_{\mu}^{\mathrm{R}}\right\}$ but we also double the dynamical fields to $\left\{\varphi_{\mathrm{L}}, c_{\mathrm{L}}\right\}$ and $\left\{\varphi_{\mathrm{R}}, c_{\mathrm{R}}\right\}$. We will want to couple both sets of these physical fields to the background fields.

This has one important implication: since there are now two sets of pull-back fields which we can apply on the reference fields $\left\{\beta^{a}, \Lambda_{\beta}\right\}$ living on $\mathbb{M}$, we also have two sets of hydrodynamic fields $\left\{\boldsymbol{\beta}^{\mu}, \Lambda_{\boldsymbol{\beta}}\right\}_{\mathrm{L}}$ and $\left\{\boldsymbol{\beta}^{\mu}, \Lambda_{\boldsymbol{\beta}}\right\}_{\mathrm{R}}$ coupling to the corresponding backgrounds. 


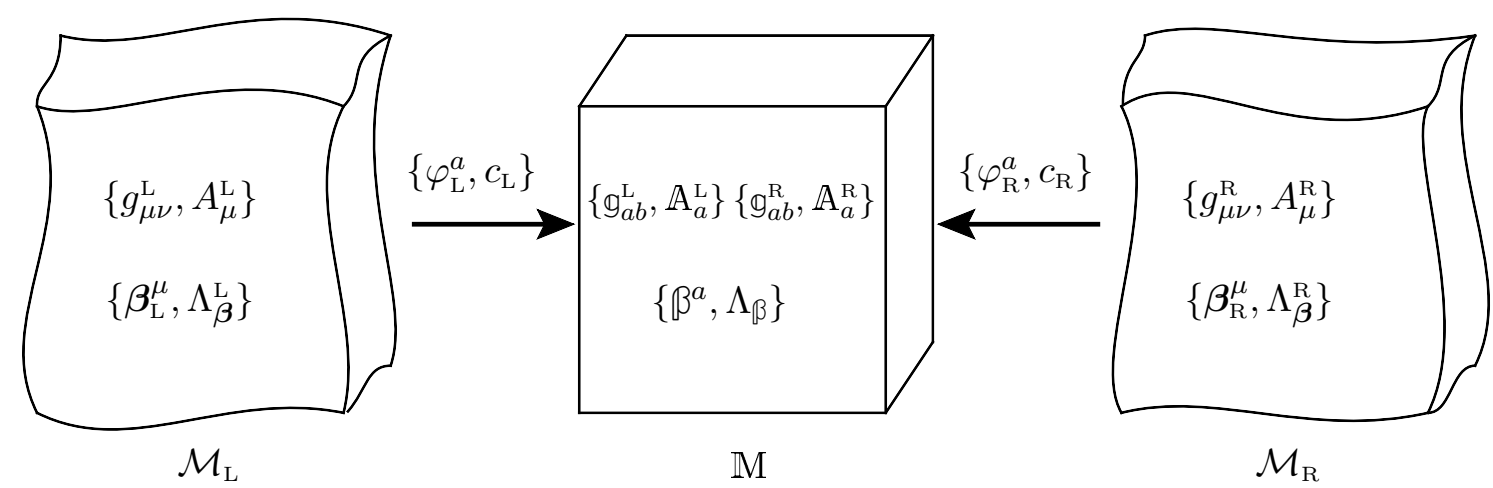

Figure 4. Illustration of the Schwinger-Keldysh setup. The physical spacetime manifold $\mathcal{M}$ has been doubled. However, the two copies are not entirely independent as they are both related to the same reference configuration on $\mathbb{M}$ via pull-backs using the dynamical fields $\{\varphi, c\}_{\mathrm{L}, \mathrm{R}}$. Despite the presence of two copies of source fields on $\mathbb{M}$ there is only one diffeomorphism and gauge redundancy involved; invariance under this symmetry implies Schwinger-Keldysh Bianchi identities.

Indeed this is to be expected since we literally wanted to double the numbers of degrees of freedom of our system. Furthermore, it is clearly possible to derive both copies of the theory on the physical spacetime $\mathcal{M}$ from the same reference configuration on $\mathbb{M}$. To wit, the R-fields are related to the reference fields via

$$
\begin{aligned}
& \boldsymbol{\beta}_{\mathrm{R}}^{\mu}\left(x_{\mathrm{R}}\right)=\left(e_{\mathrm{R}}\right)_{a}^{\mu} \beta^{a}\left[\varphi_{\mathrm{R}}\left(x_{\mathrm{R}}\right)\right], \\
& \Lambda_{\boldsymbol{\beta}}^{\mathrm{R}}\left(x_{\mathrm{R}}\right)=c_{\mathrm{R}}\left(x_{\mathrm{R}}\right) \Lambda_{\mathbb{\beta}}\left[\varphi_{\mathrm{R}}\left(x_{\mathrm{R}}\right)\right] c_{\mathrm{R}}^{-1}\left(x_{\mathrm{R}}\right)+\boldsymbol{\beta}^{\sigma}\left(x_{\mathrm{R}}\right) \partial_{\sigma} c_{\mathrm{R}}\left(x_{\mathrm{R}}\right) c_{\mathrm{R}}^{-1}\left(x_{\mathrm{R}}\right),
\end{aligned}
$$

and similarly for the L-fields. On the other hand, if we push-forward the sources from the physical manifold $\mathcal{M}$ onto the reference manifold $\mathbb{M}$, we get two copies of reference sources:

$$
\begin{aligned}
g_{\mu \nu}^{\mathrm{R}}\left(x_{\mathrm{R}}\right) & =\partial_{\mu} \varphi_{\mathrm{R}}^{a} \partial_{\nu} \varphi_{\mathrm{R}}^{b} \mathrm{~g}_{a b}^{\mathrm{R}}\left[\varphi_{\mathrm{R}}\left(x_{\mathrm{R}}\right)\right], \\
A_{\mu}^{\mathrm{R}}\left(x_{\mathrm{R}}\right) & =\partial_{\mu} \varphi_{\mathrm{R}}^{a}\left[c_{\mathrm{R}}\left(x_{\mathrm{R}}\right) \mathbb{A}_{a}^{\mathrm{R}}\left[\varphi_{\mathrm{R}}\left(x_{\mathrm{R}}\right)\right] c_{\mathrm{R}}^{-1}\left(x_{\mathrm{R}}\right)\right]-\partial_{\mu} c_{\mathrm{R}}\left(x_{\mathrm{R}}\right) c_{\mathrm{R}}^{-1}\left(x_{\mathrm{R}}\right),
\end{aligned}
$$

and similarly for the L-sources. See figure 4 for an illustration.

We now see a major advantage of introducing the reference configuration. In traditional treatments of the Schwinger-Keldysh formalism while one is fine with doubling the physical degrees of freedom, doubling of the background sources, especially the metric, poses an important puzzle. If we have a physical set of degrees of freedom living on a spacetime manifold $\mathcal{M}$ are we working with a pair of diffeomorphic manifolds $\mathcal{M}_{\mathrm{R}}, \mathcal{M}_{\mathrm{L}}$ in the doubled theory? If so, since points on two distinct manifolds may only be related up to an overall diffeomorphism, how does one map degrees of freedom on the right to those on the left? The pay-off of introducing a reference manifold is that it provides a common ground for comparing the two different degrees of freedom. Since $\mathcal{M}_{\mathrm{L}} \hookleftarrow \mathbb{M} \mapsto \mathcal{M}_{\mathrm{R}}$, we can bring all the physical fields onto the reference manifold using the pullback fields, perform all manipulations on the reference manifold and thence push forward to the physical spacetime. We believe this is the correct way to understand the symmetries of the Schwinger-Keldysh formalism. 
Having identified the relevant degrees of freedom we can write the Schwinger-Keldysh action generically as

$$
\begin{aligned}
S_{\mathrm{SK}}\left[\boldsymbol{\Psi}_{\mathrm{R}}, \boldsymbol{\Psi}_{\mathrm{L}}\right] & =\int_{\mathcal{M}_{\mathrm{R}}} \sqrt{-g_{\mathrm{R}}} \mathcal{L}\left[\boldsymbol{\Psi}_{\mathrm{R}}\right]-\int_{\mathcal{M}_{\mathrm{L}}} \sqrt{-g_{\mathrm{L}}} \mathcal{L}\left[\boldsymbol{\Psi}_{\mathrm{L}}\right]+S_{I F}\left[\boldsymbol{\Psi}_{\mathrm{R}}, \mathbf{\Psi}_{\mathrm{L}}\right], \\
& =\int_{\mathbb{M}}\left(\sqrt{-\mathfrak{g}_{\mathrm{R}}} \mathbb{L}\left[\Psi_{\mathrm{R}}\right]-\sqrt{-\mathfrak{g}_{\mathrm{L}}} \mathbb{L}\left[\Psi_{\mathrm{R}}\right]+\sqrt{-\mathfrak{g}_{\mathrm{R}}} \mathbb{L}_{I F}\left[\Psi_{\mathrm{R}}, \Psi_{\mathrm{L}}\right]\right) .
\end{aligned}
$$

We have allowed here for the possibility of a non-vanishing influence functional that couples the two sets of degrees of freedom. In the process we have arbitrarily chosen to write $\mathbb{L}_{I F}$ as a scalar field with the metric on $\mathbb{M}$ taken to be $\mathscr{g}_{\mathrm{R}} \cdot{ }^{75}$

For the situations we have discussed so far we do not need to include such terms, but anomalous (and also dissipative) parts of the constitutive relations will turn out to involve non-trivial influence functionals. At the moment we will not impose any constraints on $\mathcal{L}_{I F}$ (apart from the obvious symmetry requirements).

We now want to describe the variational principle on the reference manifold for Schwinger-Keldysh actions of the form (13.3). Varying $S_{\mathrm{SK}}$ with respect to the various fields and sources yields a-priori two sets of currents associated with the two sets of degrees of freedom:

$$
\begin{aligned}
\delta S_{\mathrm{SK}}\left[\boldsymbol{\Psi}_{\mathrm{R}}, \boldsymbol{\Psi}_{\mathrm{L}}\right]=\int_{\mathbb{M}} \sqrt{-\mathfrak{g}_{\mathrm{R}}}\{ & \frac{1}{2} \mathbb{T}_{\mathrm{R}}^{a b} \delta \mathfrak{g}_{a b}^{\mathrm{R}}-\frac{1}{2} \mathfrak{s} \mathbb{T}_{\mathrm{L}}^{a b} \delta \mathfrak{g}_{a b}^{\mathrm{L}}+\mathbb{J}_{\mathrm{R}}^{a} \cdot \delta \mathbb{A}_{a}^{\mathrm{R}}-\mathfrak{s} \mathbb{J}_{\mathrm{L}}^{a} \cdot \delta \mathbb{A}_{a}^{\mathrm{L}} \\
& +\left(\mathbb{T}_{\mathrm{R}} \mathbb{h}_{a}^{\mathrm{R}}-\mathfrak{s} \mathbb{T}_{\mathrm{L}} \mathbb{h}_{a}^{\mathrm{L}}\right) \delta \beta^{a}+\mathbb{T}_{\mathrm{R}} \mathrm{m}_{\mathrm{R}} \cdot\left(\delta \Lambda_{\beta}+\mathbb{A}_{a}^{\mathrm{R}} \delta \beta^{a}\right) \\
& \left.-\mathbb{T}_{\mathrm{L}} \mathfrak{s} \mathrm{m}_{\mathrm{L}} \cdot\left(\delta \Lambda_{\beta}+\mathbb{A}_{a}^{\mathrm{L}} \delta \beta^{a}\right)\right\}
\end{aligned}
$$

where we abbreviated $\mathfrak{s}=\sqrt{-g_{\mathrm{L}}} / \sqrt{-\mathfrak{g}_{\mathrm{R}}} .76$ While this defines the general SchwingerKeldysh constitutive relations, the equations of motion are obtained by extremizing $S_{\mathrm{SK}}$ with respect to $\left\{\varphi^{a}, c\right\}_{\mathrm{R}, \mathrm{L}}$ inside $\left\{\mathscr{G}_{a b}[\varphi], \mathbb{A}_{a}[\varphi]\right\}_{\mathrm{R}, \mathrm{L}}$, holding fixed $\left\{\beta^{a}, \Lambda_{\beta}\right\}$. Since there are now two copies of pullback fields $\left\{\varphi^{a}, c\right\}_{\mathrm{R}, \mathrm{L}}$, there are a-priori two copies of diffeomorphism and gauge symmetries. For example, we can obtain equations of motion for $\left\{\mathbb{T}_{\mathrm{R}}^{a b}, \mathbb{J}_{\mathrm{R}}^{a}\right\}$ by infinitesimally varying the right pullback fields. To wit, consider a variation $\left\{\delta \varphi_{\mathrm{R}}^{a},-c_{\mathrm{R}}^{-1} \delta c_{\mathrm{R}}\right\}$ inside the R-fields:

$$
\begin{array}{ll}
\delta \mathfrak{g}_{a b}^{\mathrm{R}}=-\delta_{\varphi} \mathbb{g}_{a b}^{\mathrm{R}}, & \delta \mathbb{A}_{a}^{\mathrm{R}}=-\delta_{\varphi} \mathbb{A}_{a}^{\mathrm{R}}, \\
\delta \mathfrak{g}_{a b}^{\mathrm{L}}=0, & \delta \mathbb{A}_{a}^{\mathrm{L}}=0, \\
\delta \mathbb{\beta}^{a}=0, & \delta \Lambda_{\beta}=0 .
\end{array}
$$

\footnotetext{
${ }^{75}$ Of course, the choice of the influence functional Lagrangian density being given in terms of the $\mathrm{R}$ metric is simply a matter of convenience. The choice matters in practical terms; when we have to define index contractions, covariant derivatives etc., which will be done with $g_{\mu \nu}^{\mathrm{R}}$ with the above convention.

${ }^{76}$ Note that temperature and chemical potential come as $\mathrm{R}$ and $\mathrm{L}$ versions since they depend on the metric
} and gauge field, respectively:

$$
\mathbb{T}_{\mathrm{R} / \mathrm{L}}=\frac{1}{\sqrt{-\mathfrak{g}_{a b}^{\mathrm{R} / \mathrm{L}} \mathbb{\beta}^{a} \beta^{b}}}, \quad \frac{\mu_{\mathrm{R} / \mathrm{L}}}{\mathbb{T}_{\mathrm{R} / \mathrm{L}}}=\Lambda_{\beta}+\beta^{c} \mathbb{A}_{c}^{\mathrm{R} / \mathrm{L}}
$$


Applying this variation to (13.4), we obtain

$$
\delta S_{\mathrm{SK}}\left[\boldsymbol{\Psi}_{\mathrm{R}}, \boldsymbol{\Psi}_{\mathrm{L}}\right]=\int_{\mathbb{M}} \sqrt{-\mathfrak{g}_{\mathrm{R}}}\left\{\delta \varphi_{\mathrm{R}}^{c}\left[\mathbb{D}_{a}\left(\mathbb{T}_{\mathrm{R}}\right)_{c}^{a}-\mathbb{J}_{\mathrm{R}}^{a} \cdot \mathbb{F}_{c a}^{\mathrm{R}}\right]+\left(-c_{\mathrm{R}}^{-1} \delta c_{\mathrm{R}}+\mathbb{A}_{a}^{\mathrm{R}} \delta \varphi_{\mathrm{R}}^{a}\right) \cdot \mathbb{D}_{c} \mathbb{J}_{\mathrm{R}}^{c}\right\}
$$

From this we can read off the usual equations of motion for $\left\{\mathbb{T}_{\mathrm{R}}^{a b}, \mathbb{J}_{\mathrm{R}}^{a}\right\}$. Equations of motion for the left copy can be derived in an analogous fashion.

However, note that it is not obvious how the two copies should be coupled. In order to make this deficiency of a naïve Schwinger-Keldysh formalism more apparent, let us briefly demonstrate how it fails to give a sensible adiabaticity equation. To this end, let us consider Bianchi identities on the reference manifold as obtained from a common diffeomorphism and gauge transformation along $\left\{\oiint^{a}, \wedge\right\}$ :

$$
\begin{aligned}
& \delta_{x} \mathfrak{G}_{a b}^{\mathrm{R} / \mathrm{L}}=£_{\S} \mathfrak{g}_{a b}^{\mathrm{R} / \mathrm{L}}, \quad \delta_{x} \beta^{a}=£_{\S} \beta^{a}, \\
& \delta_{x} \mathbb{A}_{a}^{\mathrm{R} / \mathrm{L}}=£_{\S} \mathbb{A}_{a}^{\mathrm{R} / \mathrm{L}}+\left[\mathbb{A}_{a}^{\mathrm{R} / \mathrm{L}}, \Lambda\right]+\partial_{a} \Lambda, \\
& \delta_{x} \Lambda_{\beta}+\mathbb{A}_{a}^{\mathrm{R} / \mathrm{L}} \delta_{x} \beta^{a}=\oint^{a} \mathbb{D}_{a}\left[\Lambda_{\beta}+\beta^{b} \mathbb{A}_{b}^{\mathrm{R} / \mathrm{L}}\right]-\beta^{a} \mathbb{D}_{a}\left[\Lambda+\S^{b} \mathbb{A}_{b}^{\mathrm{R} / \mathrm{L}}\right] \\
& -\oiint^{a} \beta^{b} \mathbb{F}_{a b}^{\mathrm{R} / \mathrm{L}}+\left[\Lambda_{\beta}+\beta^{a} \mathbb{A}_{a}^{\mathrm{R} / \mathrm{L}}, \Lambda+\oiint^{b} \mathbb{A}_{b}^{\mathrm{R} / \mathrm{L}}\right] .
\end{aligned}
$$

Inserting this in (13.4) we can do exactly the same integration by parts as in section 6.1 to obtain:

$$
\begin{aligned}
& \delta_{x} S_{\mathrm{SK}}\left[\boldsymbol{\Psi}_{\mathrm{R}}, \boldsymbol{\Psi}_{\mathrm{L}}\right]=\int_{\mathbb{M}} \sqrt{-\mathfrak{g}_{\mathrm{R}}} \S_{a}\left[-\mathbb{D}_{b}\left(\mathbb{T}_{\mathrm{R}}^{a b}-\mathfrak{s} \mathbb{T}_{\mathrm{L}}^{a b}\right)+\left(\mathbb{J}_{b}^{\mathrm{R}} \cdot \mathbb{F}_{\mathrm{R}}^{a b}-\mathfrak{s} \mathbb{J}_{b}^{\mathrm{L}} \cdot \mathbb{F}_{\mathrm{L}}^{a b}\right)\right. \\
& +\frac{\mathfrak{g}_{\mathrm{R}}^{a b}}{\sqrt{-\mathfrak{g}_{\mathrm{R}}}} \delta_{\mathbb{B}}\left(\sqrt{-\mathfrak{g}_{\mathrm{R}}}\left[\mathbb{T}_{\mathrm{R}} \mathbb{h}_{b}^{\mathrm{R}}-\mathbb{T}_{\mathrm{L}} \mathfrak{s} \mathbb{h}_{b}^{\mathrm{L}}\right]\right) \\
& \left.+\left(\mathfrak{g}_{\mathrm{R}}^{a b} \mathbb{T}_{\mathrm{R}} \mathrm{m}_{\mathrm{R}} \cdot \delta_{\mathbb{B}} \mathbb{A}_{b}^{\mathrm{R}}-\mathfrak{g}_{\mathrm{R}}^{a b} \mathbb{T}_{\mathrm{L}} \mathfrak{s} \mathrm{m}_{\mathrm{L}} \cdot \delta_{\mathbb{B}} \mathbb{A}_{b}^{\mathrm{L}}\right)\right] \\
& +\int_{\mathbb{M}} \sqrt{-\mathfrak{g}_{\mathrm{R}}}\left(\Lambda+\mathscr{g}^{c} \mathbb{A}_{c}^{\mathrm{R}}\right) \cdot\left[-\mathbb{D}_{c} \mathbb{J}_{\mathrm{R}}^{c}+\frac{1}{\sqrt{-\mathfrak{g}_{\mathrm{R}}}} \delta_{\mathbb{B}}\left(\sqrt{-\mathfrak{g}_{R}} \mathbb{T}_{\mathrm{R}} \mathrm{m}_{\mathrm{R}}\right)\right] \\
& -\int_{\mathbb{M}} \sqrt{-g_{\mathrm{R}}}\left(\Lambda+\mathscr{f}^{c} \mathbb{A}_{c}^{\mathrm{L}}\right) \cdot\left[-\mathbb{D}_{c}\left(\mathfrak{s} \mathbb{J}_{\mathrm{L}}^{c}\right)+\frac{1}{\sqrt{-g_{\mathrm{R}}}} \delta_{\mathbb{B}}\left(\sqrt{-g_{R}} \mathbb{T}_{\mathrm{L}} \mathfrak{s} \mathrm{m}_{\mathrm{L}}\right)\right] \text {. }
\end{aligned}
$$

where $\delta_{\mathbb{B}}$ denotes Lie transport along $\mathbb{B}=\left\{\beta^{a}, \Lambda_{\beta}\right\}$. We can directly read off Bianchi identities from this variation. We define the reference manifold entropy current in the same way as on physical spacetime $\mathcal{M}$, i.e. $\mathbb{J}_{S}^{a}=\left(\mathbb{T}_{\mathrm{R}} \mathbb{S}_{\mathrm{R}}-\mathfrak{s} \mathbb{T}_{\mathrm{L}} \mathbb{S}_{\mathrm{L}}\right) \mathbb{\beta}^{a}$ with $\mathbb{\Phi}_{\mathrm{R} / \mathrm{L}}$ defined as the functional derivative of $S_{\mathrm{SK}}$ with respect to $\mathbb{T}_{\mathrm{R} / \mathrm{L}}$ exactly as in (6.4). Using the Bianchi identities from (13.8) it is then straightforward to derive the following adiabaticity 
equation in complete analogy to (6.14):

$$
\begin{aligned}
& \mathbb{D}_{a} \mathbb{J}_{S}^{a}+\beta_{a}\left[\mathbb{D}_{b}\left(\mathbb{T}_{\mathrm{R}}^{a b}-\mathfrak{s} \mathbb{T}_{\mathrm{L}}^{a b}\right)-\left(\mathbb{J}_{b}^{\mathrm{R}} \cdot \mathbb{F}_{\mathrm{R}}^{a b}-\mathfrak{s} \mathbb{J}_{b}^{\mathrm{L}} \cdot \mathbb{F}_{\mathrm{L}}^{a b}\right)\right] \\
& +\left[\left(\Lambda_{\beta}+\beta^{c} \mathbb{A}_{c}^{\mathrm{R}}\right) \cdot \mathbb{D}_{a} \mathbb{J}_{\mathrm{R}}^{a}-\left(\Lambda_{\beta}+\beta^{c} \mathbb{A}_{c}^{\mathrm{L}}\right) \cdot \mathbb{D}_{a}\left(\mathfrak{s} \mathbb{J}_{\mathrm{L}}^{a}\right)\right] \\
& =\frac{1}{\sqrt{-g_{\mathrm{R}}}} \delta_{\mathbb{B}}\left(\sqrt { - \mathfrak { g } _ { \mathrm { R } } } \left[\left(\mathbb{T}_{\mathrm{R}} \mathbb{S}_{\mathrm{R}}-\mathfrak{s} \mathbb{T}_{\mathrm{L}} \mathbb{S}_{\mathrm{L}}\right)+\mathfrak{u}^{a}\left(\mathbb{h}_{a}^{\mathrm{R}}-\mathfrak{s} \mathbb{h}_{a}^{\mathrm{L}}\right)\right.\right. \\
& \left.\left.+\left(\mathbb{T}_{\mathrm{R}} \mu_{\mathrm{R}} \cdot \mathrm{m}_{\mathrm{R}}-\mathfrak{s} \mathbb{T}_{\mathrm{L}} \mu_{\mathrm{L}} \cdot \mathrm{m}_{\mathrm{L}}\right)\right]\right)=0 .
\end{aligned}
$$

Clearly this form of a Schwinger-Keldysh adiabaticity equation is not satisfactory: in the hydrodynamic limit where right and left quantities coincide, the equation collapses to something trivial. This indicates that a proper Schwinger-Keldysh formalism must contain a rule to connect the right and left contours. Note that in the absence of influence functionals we can separately derive the Bianchi identities on the left and right and check that they are upheld. While this in itself is not ideal, at least in the absence of influence functionals adiabaticity continues to hold in the doubled theory. The issues alluded to above, start arising only when the two sides start talking to each other.

In section 15 we will construct a doubled formalism where an additional symmetry ensures a sensible adiabaticity equation. For the moment, we leave it with the observation that a naïve Schwinger-Keldysh formalism as developed in the present section suffers from various deficiencies.

\subsection{Hydrodynamic currents in common/difference basis}

Regardless of the problems pointed out in the previous subsection, from working on the reference manifold we nevertheless gained a distinct advantage: we have a way of defining currents in a basis of common and difference fields. Let us now briefly outline how this allows to compute the hydrodynamic currents of interest in a rather simple way. By taking linear combinations of the sources we can consider the average source and the difference source which will be useful in our discussion. Generalizing the notion to also include the hydrodynamic fields, we define the following average and difference fields on the reference manifold:

$$
\bar{\Downarrow} \equiv \Downarrow_{\mathrm{R}}-\Downarrow_{\mathrm{L}}, \quad \breve{\Downarrow}=\frac{1}{2}\left(\Psi_{\mathrm{R}}+\Downarrow_{\mathrm{L}}\right) .
$$

For completeness we record the explicit definition of the difference hydrodynamic fields which will play an important role in what follows:

$$
\begin{aligned}
\overline{\mathfrak{g}}_{a b}(\mathbb{x}) & =\mathfrak{g}_{a b}^{\mathrm{R}}(\mathbb{x})-\mathfrak{g}_{a b}^{\mathrm{L}}(\mathbb{x}), \\
\overline{\mathbb{A}}_{a}(\mathbb{x}) & =\mathbb{A}_{a}^{\mathrm{R}}(\mathbb{x})-\mathbb{A}_{a}^{\mathrm{L}}(\mathbb{x}),
\end{aligned}
$$

where $\mathbb{x}^{a}$ are some coordinates on $\mathbb{M}$; for instance, one could consider the above difference fields as functionals of the diffeomorphism fields by setting $\mathbb{x}^{a}=\varphi_{\mathrm{R}}^{a}(x)$.

In any system out of equilibrium, we are interested in analyzing the causal response of sources. This amounts to considering linear combinations of correlation functions with operator insertions in both the $\mathrm{R}$ and $\mathrm{L}$ copies of the theory. The causal correlation 
functions involve a single variation with respect to the difference source [81]. It is therefore useful to remember that the Schwinger-Keldysh construction (13.3) couples the difference source to the average current and the average source to the difference current:

$$
\begin{aligned}
\delta S_{\mathrm{SK}}\left[\boldsymbol{\Psi}_{\mathrm{R}}, \mathbf{\Psi}_{\mathrm{L}}\right]=\int_{\mathbb{M}} \sqrt{-\breve{\mathfrak{g}}}\{ & \frac{1}{2}\left(\mathfrak{s}_{\mathrm{R}} \mathbb{T}_{\mathrm{R}}^{a b}-\mathfrak{s}_{\mathrm{L}} \mathbb{T}_{\mathrm{L}}^{a b}\right) \delta \breve{g}_{a b}+\frac{1}{4}\left(\mathfrak{s}_{\mathrm{R}} \mathbb{T}_{\mathrm{R}}^{a b}+\mathfrak{s}_{\mathrm{L}} \mathbb{T}_{\mathrm{L}}^{a b}\right) \delta \overline{\mathfrak{g}}_{a b} \\
& +\left(\mathfrak{s}_{\mathrm{R}} \mathbb{J}_{\mathrm{R}}^{a}-\mathfrak{s}_{\mathrm{L}} \mathbb{J}_{\mathrm{L}}^{a}\right) \cdot \delta \breve{\mathbb{A}}_{a}+\frac{1}{2}\left(\mathfrak{s}_{\mathrm{R}} \mathbb{J}_{\mathrm{R}}^{a}+\mathfrak{s}_{\mathrm{L}} \mathbb{J}_{\mathrm{L}}^{a}\right) \cdot \delta \overline{\mathbb{A}}_{a} \\
& +\left(\mathbb{T}_{\mathrm{R}} \mathfrak{s}_{\mathrm{R}} \mathbb{h}_{a}^{\mathrm{R}}-\mathbb{T}_{\mathrm{L}} \mathfrak{s}_{\mathrm{L}} \mathbb{h}_{a}^{\mathrm{L}}\right) \delta \beta^{a} \\
& +\left(\mathbb{T}_{\mathrm{R}} \mathfrak{s}_{\mathrm{R}} \mathrm{m}_{\mathrm{R}}-\mathbb{T}_{\mathrm{L}} \mathfrak{s}_{\mathrm{L}} \mathrm{m}_{\mathrm{L}}\right) \cdot\left(\delta \Lambda_{\beta}+\breve{\mathbb{A}}_{a} \delta \beta^{a}\right) \\
& \left.+\frac{1}{2}\left(\mathbb{T}_{\mathrm{R}} \mathfrak{s}_{\mathrm{R}} \mathrm{m}_{\mathrm{R}}+\mathbb{T}_{\mathrm{L}} \mathfrak{s}_{\mathrm{L}} \mathrm{m}_{\mathrm{L}}\right) \cdot\left(\overline{\mathbb{A}}_{a} \delta \mathbb{\beta}^{a}\right)\right\}
\end{aligned}
$$

where $\mathfrak{s}_{\mathrm{R}, \mathrm{L}} \equiv \sqrt{-\mathfrak{g}_{\mathrm{R}, \mathrm{L}}} / \sqrt{-\breve{\mathfrak{g}}}$.

While the above discussion remains valid for generic non-equilibrium dynamics, our interest is in the hydrodynamic limit where we only allow small (long-wavelength) departures from thermodynamic equilibrium. One useful consequence is that we can obtain the hydrodynamic currents by working to linear order in deviations from the equilibrium configuration wherein the left and right degrees of freedom are identified. Said differently, we consider linear deviations of the diffeomorphism and gauge transformation fields about a common equilibrium configuration: $\varphi_{\mathrm{R}}^{a}(x)=\varphi_{\mathrm{L}}^{a}(x) \equiv \varphi^{a}(x)$ and $c_{\mathrm{R}}(x)=c_{\mathrm{L}}(x) \equiv c(x)$. From the action (13.3) one obtains the hydrodynamic constitutive relations by varying with respect to the reference sources $\{g, \mathbb{A}\}_{R, L}$ and taking the hydrodynamic limit:

$$
\begin{aligned}
& \mathbb{T}_{\text {hydro }}^{a b}=\left.\frac{2}{\sqrt{-g_{\mathrm{R}}}}\left(\frac{\delta S_{\mathrm{SK}}}{\delta \mathbb{g}_{a b}^{\mathrm{R}}\left[\varphi_{\mathrm{R}}\right]}-\frac{\delta S_{\mathrm{SK}}}{\delta \mathbb{g}_{a b}^{\mathrm{L}}\left[\varphi_{\mathrm{L}}\right]}\right)\right|_{\begin{array}{l}
\varphi_{\mathrm{R}}^{a}(x)=\varphi_{\mathrm{L}}^{a}(x) \equiv \varphi^{a}(x) \\
c_{\mathrm{R}}(x)=c_{\mathrm{L}}(x) \equiv c(x)
\end{array}} \\
& \mathbb{J}_{\text {hydro }}^{a}=\left.\frac{1}{\sqrt{-g_{\mathrm{R}}}}\left(\frac{\delta S_{\mathrm{SK}}}{\delta \mathbb{A}_{a}^{\mathrm{R}}\left[\varphi_{\mathrm{R}}\right]}-\frac{\delta S_{\mathrm{SK}}}{\delta \mathbb{A}_{a}^{\mathrm{L}}\left[\varphi_{\mathrm{L}}\right]}\right)\right|_{\begin{array}{l}
\varphi_{\mathrm{R}}^{a}(x)=\varphi_{\mathrm{L}}^{a}(x) \equiv \varphi^{a}(x) \\
c_{\mathrm{R}}(x)=c_{\mathrm{L}}(x) \equiv c(x)
\end{array}}
\end{aligned}
$$

where $\varphi^{a}=\frac{1}{2}\left(\varphi_{\mathrm{R}}^{a}+\varphi_{\mathrm{L}}^{a}\right)$ is the common part of $\varphi_{\mathrm{R}}$ and $\varphi_{\mathrm{L}}$ which coincides with $\varphi_{\mathrm{R}}, \varphi_{\mathrm{L}}$ in the hydrodynamic limit. From (13.11) it transpires that the hydrodynamic currents (13.12) are the common currents which can equivalently be obtained by varying $S_{\mathrm{SK}}$ with respect to $\left\{\overline{\mathfrak{g}}_{a b}, \overline{\mathrm{A}}_{a}\right\}$ and then taking the hydrodynamic coincidence limit. Note however, that such a variation yields the same expressions as the right hand side of (13.12) only in the hydrodynamic limit (i.e., to linear order in difference fields). Beyond linear order, the natural Schwinger-Keldysh currents are defined by (13.4).

Similarly, the desired equations of motion in the hydrodynamic limit can be obtained making use of this simplified variational principle: in analogy to the discussion of section 7.4 we can do a variation of $\left\{\varphi^{a}, c\right\}$ inside the difference sources, holding the reference configuration of $\left\{\beta^{a}, \Lambda_{\beta}\right\}$ fixed:

$$
\begin{array}{rlrl}
\delta_{x} \overline{\mathfrak{I}}_{a b} & =-\delta_{\varphi} \overline{\mathfrak{I}}_{a b}, & & \delta_{x} \overline{\mathbb{A}}_{a}=-\delta_{\varphi} \overline{\mathbb{A}}_{a}, \\
\delta_{x} \beta^{a}=0, & \delta_{x} \Lambda_{\beta}=0 .
\end{array}
$$


This variational principle applied to (13.11) directly yields equations of motion for the hydrodynamic common currents. Its relation to the variational principle of the previous subsection should be seen as being consistent to linear order in difference fields (which is good enough if we take the hydrodynamic limit at the end of the day).

While it is clear that the above Schwinger-Keldysh formalism can achieve some things, it is certainly not entirely satisfying. By postulating a reference manifold configuration underlying both copies of the physical theory together with a way the symmetries act there, we are able to obtain equations of motion for the hydrodynamic currents. However, we have no obvious way of defining an entropy current on the reference manifold from first principles. In order to obtain an adiabaticity equation for the currents obtained in this subsection, we need to construct the reference manifold entropy current by hand such that adiabaticity is satisfied. This is the strategy that we will follow in the construction of Class A constitutive relations (see section 13.3 below).

\subsection{Anomalous Ward identities in the Schwinger-Keldysh formalism}

The analysis of section 12 underscores the fact that, while the anomalous adiabaticity equation can be solved within the framework of Class L adiabatic fluids, one fails to recover the desired on-shell Ward identities. The reason for this failure can be traced to the fact that the amount of anomaly inflow into a single copy theory, respecting the adiabatic principle, is a bit too much [30]. As one can see from the transgression form characterization of the anomalous Lagrangian (12.18), the inflow to the boundary manifold $\mathcal{M}$ from the bulk topological theory comprises not only of the anomaly in the physical background sources $\{\boldsymbol{A}, \boldsymbol{\Gamma}\}$ but also involves an extra bit of inflow from the shadow fields $\{\hat{\boldsymbol{A}}, \hat{\boldsymbol{\Gamma}}\}$. The latter have to be removed from the system in order to obtain the correct physical Ward identities. ${ }^{77}$

Fortunately we now know a cure for this problem; as discussed in [30] and reviewed in the previous subsections the Schwinger-Keldysh formalism provides a natural framework to understand the Ward identities. In particular, we will find it quite useful in the following discussion to be able to have non-trivial influence functional contributions. The symmetries of the Schwinger-Keldysh construction pick out a unique influence functional which in turn implies the desired anomalous Ward identities (12.35).

Let us hark back to the discussion of section 12.4 where we took our anomalous effective action in the single copy theory to be $S_{\text {eff }}[\boldsymbol{\Psi}]$, cf., (12.31). From that discussion, it is clear that we need to add to the total action $S_{\text {eff }}$ another term which fixes the dynamics by ensuring that we have the correct amount of inflow. In the double-field context, we are thus looking for a total action of the form

$$
\begin{aligned}
S_{\text {tot }} & \equiv S_{\text {tot }}\left[\mathbf{\Psi}_{\mathrm{L}}, \mathbf{\Psi}_{\mathrm{R}}\right] \\
& =S_{\text {eff }}\left[\boldsymbol{\Psi}_{\mathrm{R}}\right]-S_{\text {eff }}\left[\mathbf{\Psi}_{\mathrm{L}}\right]+S_{I F}\left[\mathbf{\Psi}_{\mathrm{R}}, \mathbf{\Psi}_{\mathrm{L}}\right],
\end{aligned}
$$

with $S_{\text {eff }}[\mathbf{\Psi}]$ being given in (12.31) and $S_{I F}$ is a cross-contour term that involves fields from both copies of the theory.

\footnotetext{
${ }^{77}$ We recall that the extra terms in (12.34) all involve the shadow fields in the r.h.s. of the physical conservation equations.
} 
It proves convenient for reasons mentioned above to write the action $S_{\text {tot }}$ on the reference manifold directly. This can always be achieved using (13.1). As explained in section 13.1 one then has a single background geometry where all the currents live. The resulting action will depend on $\Downarrow_{\mathrm{R}}=\left\{\mathscr{g}_{a b}^{\mathrm{R}}\left[\varphi_{\mathrm{R}}\right], \mathbb{A}_{a}^{\mathrm{R}}\left[\varphi_{\mathrm{R}}\right], \mathbb{\beta}^{a}\left[\varphi_{\mathrm{R}}\right], \Lambda_{\beta}\left[\varphi_{\mathrm{R}}\right]\right\}$ and similarly for $\Downarrow_{\mathrm{L}}$, i.e., we effectively view it as an effective action for two copies of sources while keeping one copy of hydrodynamic degrees of freedom $\left\{\beta^{a}, \Lambda_{\beta}\right\}$. We thus write

$$
\begin{aligned}
S_{\text {tot }} \equiv S_{\text {tot }}\left[\varphi_{\mathrm{R}}, c_{\mathrm{R}}, \varphi_{\mathrm{L}}, c_{\mathrm{L}}\right]=\left(S_{\mathrm{n}-\mathrm{a}}\left[\mathbb{\Psi}_{\mathrm{R}}\right]\right. & \left.+\int_{\mathbb{M}_{d+1}} \boldsymbol{V}_{\mathcal{P}}\left[\Psi_{\mathrm{R}}\right]\right) \\
& -\left(S_{\mathrm{n}-\mathrm{a}}\left[\Psi_{\mathrm{L}}\right]+\int_{\mathbb{M}_{d+1}} \boldsymbol{V}_{\mathcal{P}}\left[\Psi_{\mathrm{L}}\right]\right)+S_{I F}\left[\Psi_{\mathrm{R}}, \Psi_{\mathrm{L}}\right]
\end{aligned}
$$

As remarked earlier, by working in terms of the reference manifold $\mathbb{M}$ (and its bulk extension which we denote as $\mathbb{M}_{d+1}$ ), we circumvent potential confusions about the presence of two copies of the spacetime manifold with two metrics and two gauge and diffeomorphism symmetries. Despite the fact that there are still two copies of source fields living on $\mathbb{M}$, there is only one physical gauge and diffeomorphism invariance involved in (13.15). Equations of motion in the hydrodynamic limit can then be obtained from a simple variational principle as described in section 13.2.

Since the contributions from $S_{\text {eff }}[\Psi]$ have already been computed in section 12.4 , we now turn to an explicit description of the contributions that come from $S_{I F}$. Using the same arguments as [30], we can infer what the form of $S_{I F}\left[\Psi_{\mathrm{R}}, \Downarrow_{\mathrm{L}}\right]$ ought to be. Its form is pretty much dictated by ensuring that we have the correct amount of inflow: it needs to be a transgression form between the two sets of shadow fields. We therefore claim that the precise term to add as our anomalous influence functional is the transgression from hatted fields on the right towards hatted fields on the left contour, i.e.,

$$
\begin{aligned}
S_{I F} & =\int_{\mathbb{M}_{d+1}} \boldsymbol{V}_{\mathcal{P}}\left[\hat{\mathbb{A}}_{\mathrm{R}}, \hat{\mathbb{T}}_{\mathrm{R}} ; \hat{\mathbb{A}}_{\mathrm{L}}, \hat{\mathbb{T}}_{\mathrm{L}}\right] \\
& \equiv \int_{\mathbb{M}_{d+1}} \boldsymbol{V}_{\mathcal{P}}\left(\hat{\boldsymbol{A}}\left[\Psi_{\mathrm{R}}\right], \hat{\boldsymbol{\Gamma}}\left[\Psi_{\mathrm{R}}\right] ; \hat{\boldsymbol{A}}\left[\Psi_{\mathrm{L}}\right], \hat{\boldsymbol{\Gamma}}\left[\Psi_{\mathrm{L}}\right]\right),
\end{aligned}
$$

The main thing we need to check is that the above cross-term influence functional provides the right correction terms necessary to fix the anomalous hydrodynamic Ward identities (12.34) without influencing the physical currents (12.30). We can use the same kind of manipulations as in section $\mathrm{H}$ to verify this. As we pointed out in the general discussion of Class L Schwinger-Keldysh formalism, section 13.1, a non-linear treatment requires carefully separating $\mathrm{R}$ and $\mathrm{L}$ degrees of freedom. However, we are eventually interested in the hydrodynamic limit of the currents, i.e., the coincidence limit where difference fields are set to zero. Therefore we can employ the simpler formalism where we vary the Schwinger-Keldysh action directly with respect to difference sources, disregarding any current contributions that contain hydrodynamically vanishing quantities, c.f., section 13.2. After explicit computation (see section H.3), we obtain for the variation of the 
entire anomaly part of the action in the hydrodynamic coincidence limit ${ }^{78}$

$$
\begin{aligned}
\left.\delta S_{\text {anom }}\right|_{\text {hydro }} \equiv & \left.\delta\left(S_{I F}+\int_{\mathbb{M}_{d+1}}\left(\boldsymbol{V}_{\mathcal{P}}\left[\Psi_{\mathrm{R}}\right]-\boldsymbol{V}_{\mathcal{P}}\left[\Psi_{\mathrm{L}}\right]\right)\right)\right|_{\text {hydro }} \\
= & f_{\mathbb{M}_{d+1}}\left[\frac{1}{2} \mathbb{D}_{P}\left(\mathbb{\mathbb { V }}_{H}^{M[N P]}+\mathbb{\mathbb { Z }}_{H}^{N[M P]}-\mathbb{Z}_{H}^{P(M N)}\right) \frac{1}{2} \delta \overline{\mathfrak{g}}_{M N}+\mathbb{J}_{H}^{M} \cdot \delta \overline{\mathbb{A}}_{M}\right] \\
& +\int_{\mathbb{M}} \sqrt{-\breve{g}}\left\{\left[\frac{1}{2} \mathbb{D}_{c}\left(\mathbb{\Sigma}_{\mathcal{P}}^{a[b c]}+\mathbb{\Sigma}_{\mathcal{P}}^{b[a c]}-\mathbb{\Sigma}_{\mathcal{P}}^{c(a b)}\right)+2{\mathbb{q}_{\mathcal{P}}^{(a 1}}^{(a)}\right] \frac{1}{2} \delta \overline{\mathfrak{g}}_{a b}+\mathbb{J}_{\mathcal{P}}^{a} \cdot \delta \overline{\mathbb{A}}_{a}\right\} .
\end{aligned}
$$

where hydro denotes the limit where all the expressions that are not variations of difference fields are evaluated at $\varphi_{\mathrm{R}}^{a}(x)=\varphi_{\mathrm{L}}^{a}(x) \equiv \varphi^{a}(x)$ and $c_{\mathrm{R}}(x)=c_{\mathrm{L}}(x) \equiv c(x)$. Further, $\left\{\overline{\mathfrak{g}}_{a b}, \overline{\mathbb{A}}_{a}\right\}$ denote the difference sources as introduced in section 13.2. Equations of motion are now obtained by varying $\left\{\varphi^{a}, c\right\}$ inside the difference sources on $\mathbb{M}$, i.e. (13.13). Following that logic, we immediately obtain the bulk on-shell equation of motion, which we now write for the reference fields

$$
\frac{1}{2} \mathbb{D}_{N} \mathbb{D}_{P}\left(\mathbb{Z}_{H}^{M[N P]}+\mathbb{\Sigma}_{H}^{N[M P]}-\mathbb{Z}_{H}^{P(N M)}\right) \simeq \mathbb{F}_{M P} \cdot \mathbb{J}_{H}^{P}, \quad \mathbb{D}_{P} \mathbb{J}_{H}^{P} \simeq 0 .
$$

The boundary equations of motion from $S_{\text {anom }}$ then take the form

$$
\mathbb{D}_{b}\left[\frac{1}{2} \mathbb{D}_{c}\left(\mathbb{\Sigma}_{\mathcal{P}}^{a[b c]}+\mathbb{Z}_{\mathcal{P}}^{b[a c]}-\mathbb{Z}_{\mathcal{P}}^{c(a b)}\right)+2{q_{\mathcal{P}}}^{\left(a u^{b}\right.}{ }^{b)}\right] \simeq \mathbb{F}_{c}^{a} \cdot \mathbb{J}_{\mathcal{P}}^{c}+\frac{1}{2} \mathbb{D}_{c} \mathbb{Z}_{H}^{\perp[a c]}, \quad \mathbb{D}_{a} \mathbb{J}_{\mathcal{P}}^{a} \simeq \mathbb{J}_{H}^{\perp}
$$

where all fields are in the hydrodynamic limit, i.e., the R- and L-fields have been identified. After combining the R- and L-pieces, the additional non-anomalous contributions to the boundary equations of motion are the same as in (12.32) with (12.33). Putting all of this together we get the hydrodynamic equations of motion for the action (13.15):

$$
\begin{aligned}
& \mathbb{D}_{b}\left[\mathbb{T}_{\mathrm{n}-\mathrm{a}}^{a b}+\frac{1}{2} \mathbb{D}_{c}\left(\mathbb{\Sigma}_{\mathcal{P}}^{a[b c]}+\mathbb{Z}_{\mathcal{P}}^{b[a c]}-\mathbb{\Sigma}_{\mathcal{P}}^{c(a b)}\right)+2 \mathbb{q}_{\mathcal{P}}^{(a} u^{b)}\right] \simeq \mathbb{F}_{c}^{a} \cdot\left(\mathbb{J}_{\mathrm{n}-\mathrm{a}}^{c}+\mathbb{J}_{\mathcal{P}}^{c}\right)+\frac{1}{2} \mathbb{D}_{c} \mathbb{\Sigma}_{H}^{\perp[a c]}, \\
& \mathbb{D}_{a}\left[\mathbb{J}_{\mathrm{n}-\mathrm{a}}^{a}+\mathbb{J}_{\mathcal{P}}^{a}\right] \simeq \mathbb{J}_{H}^{\perp} .
\end{aligned}
$$

When written in terms of quantities on the physical spacetime $\mathcal{M}$, these are the usual equations of motion (12.35) for hydrodynamics with mixed flavour and gravitational anomaly. ${ }^{79}$

This completes for us the derivation of the equations of motion for the full SchwingerKeldysh action. We conclude that a Schwinger-Keldysh formulation with suitable Feynman-Vernon term is capable of imposing the correct dynamics on our theory. Working in a formalism with doubled set of degrees of freedom is inevitable if we want to circumvent having various Ward identities contaminated by unwanted hatted anomaly contributions.

\footnotetext{
${ }^{78}$ We adhere to our index conventions stated earlier:

- Boundary physical manifold $\mathcal{M}$ : indices from the Greek alphabet.

- Bulk physical manifold $\mathcal{M}_{d+1}$ : indices from the second half of the lowercase Latin alphabet.

- Boundary reference manifold $\mathbb{M}$ : indices from the first half of the lowercase Latin alphabet.

- Bulk reference manifold $\mathbb{M}_{d+1}$ : indices from the second half of the uppercase Latin alphabet.

${ }^{79}$ The translation between the physical and reference manifolds is simple: we replace $\Downarrow \mapsto \Psi$ and change indices back to Greek.
} 


\subsection{Effective actions for Class D?}

In section 5 we have seen that dissipative transport is well under control. The positivity of the leading order transport coefficients and lack of further constraints is reminiscent of general structures of effective field theories, wherein unitarity imposes positivity controls on kinetic terms but leaves typically higher order interactions arbitrary. ${ }^{80}$

Ideally, it would be great if we can give a complete picture for dissipative transport by constructing an effective action; once this is understood we would be able to make a clear analysis from a microscopic perspective. It has long been understood that such an effective action has to be described using the Schwinger-Keldysh construction. We would like to offer some critical thoughts at this juncture, why this construction requires further bolstering, paving the way for a general picture in section 15 .

To incorporate dissipation in the Schwinger-Keldysh doubled formalism, it is necessary to incorporate interactions between the left and right fields via the Feynman-Vernon influence functionals [50]. Then, upon integrating out one set of degrees of freedom that we take to be the difference fields $\left(\boldsymbol{\Psi}_{\mathrm{L}}-\boldsymbol{\Psi}_{\mathrm{R}}\right)$, one obtains an effective action for the common fields $\boldsymbol{\Psi}_{\mathrm{L}}+\boldsymbol{\Psi}_{\mathrm{R}}$ with dissipative interactions. What we are after then is a constraint on the influence functionals ensuring that such interactions are compatible with the second law. We should emphasize that this is a very physical requirement since the second law of thermodynamics is a macroscopic manifestation of microscopic unitarity.

If we naïvely construct influence functionals without any constraint, then we have seen that the adiabaticity equation fails. One should then worry about terms that violate the second law. In particular, using generic influence functionals one can construct effective actions which allow non-vanishing Class $\mathrm{H}_{F}$ terms, which as we have seen are forbidden by adiabaticity (in fact hydrostatics). One potential issue is that unconstrained influence functions violate the fluctuation-dissipation relations which typically are encoded by the Kubo-Martin-Schwinger (KMS) condition in non-equilibrium dynamics. While there is some understanding of how these relations are to be imposed in the Schwinger-Keldysh formalism we are as yet unaware of a complete treatment in the hydrodynamic context (see for instance [83-85] for some progress in this direction).

Ideally, we would like some element in the Schwinger-Keldysh construction which forbids influence functionals that lead to disallowed constitutive relations (such as Class $\mathrm{H}_{F}$ ). Given our previous discussions and in particular section 13.3, let us take a step back and see what we can learn from the adiabatic effective actions. For Class L constitutive relations, the Schwinger-Keldysh construction is rather straightforward. We simply have (13.3) with $\mathbb{L}_{I F}=0$ since there is no need for any interaction terms in either Class $\mathrm{H}_{S}$ or Class $\overline{\mathrm{H}}_{S}$. So this does not provide us with much guidance on how to proceed.

Anomalous transport of Class A is however more interesting, as we need a non-trivial influence functional (13.16), required in order to satisfy the Ward identities. While our construction was be guided by the symmetries and the rigidity of anomalies, it should be

\footnotetext{
${ }^{80}$ This analogy comes with caveats. In relativistic quantum field theories there are sign-definiteness constraints on the leading corrections to the positive definite quadratic kinetic terms arising from causality as discussed for instance in [82].
} 
borne in mind that the final answer in (13.15) is not derived from first principles. While we have presumably fixed the non-covariant part of the influence functional correctly by demanding the Ward identities, it is plausible that there are additional pieces which ensure that the term we propose satisfies the KMS condition.

In any event the story about influence functionals for adiabatic transport is incomplete - we have not been able thus far to incorporate the vector classes $\mathrm{H}_{V}, \overline{\mathrm{H}}_{V}$ and C into an effective action framework, nor the Berry-like transport terms of Class B. It appears intuitive that writing an effective action for such transport does require some form of SchwingerKeldysh doubling; indeed we will see glimpses of such a structure in section 15-section 17. We will argue there for a new symmetry principle which circumvents all these problems. The set of influence functionals will be constrained in precisely the right way by the presence of a new symmetry to ensure adiabaticity. Furthermore, we will see a more natural variational principle deriving the Ward identities of hydrodynamics. We however forewarn the reader that whilst this structure is tantalizing, we postpone a complete discussion of the implications vis a vis the Schwinger-Keldysh constructions to a future publication [55]. 


\section{Part III \\ The eightfold way to dissipation and its lagrangian unification}

\section{The eightfold way}

We are now in a position to outline the complete classification of hydrodynamic transport at arbitrary orders in the gradient expansion building on the results derived in section 4section 13. We will give the algorithm for the intrepid hydrodynamicist to implement the construction at any desired order.

\subsection{The route to classification}

We will work off-shell in the most general fluid frame. We first compile a list of all tensor structures that can appear in constitutive relations $\mathcal{C}_{\mathcal{H}}[\Psi]$. We will sequentially eliminate elements of this collection by assigning them to distinct classes suggested by the eightfold way. The algorithm for understanding the transport classification can be implemented in the following sequence:

- The first step of our analysis is to remove the particular solutions of Class A by picking the correct particular solution leading to the anomalous constitutive relations, i.e., $\left\{\left(\mathcal{G}^{\sigma}\right)_{\mathrm{A}},\left(T^{\mu \nu}\right)_{\mathrm{A}},\left(J^{\mu}\right)_{\mathrm{A}}\right\}$ given in terms of the anomaly polynomial $\mathcal{P}[\boldsymbol{F}, \boldsymbol{R}]$. These anomalous currents take the form

$$
\begin{aligned}
\left(J_{S}^{\alpha}\right)_{\mathrm{A}} & =-\frac{1}{2} \boldsymbol{\beta}_{\sigma} \hat{\Sigma}_{H}^{\perp[\alpha \sigma]} \\
\left(T^{\alpha \beta}\right)_{\mathrm{A}} & =q_{\mathcal{P}}^{\alpha} u^{\beta}+q_{\mathcal{P}}^{\beta} u^{\alpha}+\frac{1}{2} D_{\rho}\left(\Sigma_{\mathcal{P}}^{\alpha[\beta \rho]}+\Sigma_{\mathcal{P}}^{\beta[\alpha \rho]}-\Sigma_{\mathcal{P}}^{\rho(\alpha \beta)}\right), \\
\left(J^{\alpha}\right)_{\mathrm{A}} & =J_{\mathcal{P}}^{\alpha},
\end{aligned}
$$

where the various pieces in these currents are given in terms of the transgression form $\boldsymbol{V}_{\mathcal{P}} \equiv \frac{\boldsymbol{u}}{2 \boldsymbol{\omega}} \wedge(\mathcal{P}[\boldsymbol{F}, \boldsymbol{R}]-\widehat{\mathcal{P}}[\hat{\boldsymbol{F}}, \hat{\boldsymbol{R}}])$ as

$$
\begin{aligned}
\star \boldsymbol{q}_{\mathcal{P}} & =\frac{\partial \boldsymbol{V}_{\mathcal{P}}}{\partial(2 \boldsymbol{\omega})}, & \star \boldsymbol{\Sigma}_{\mathcal{P}}{ }^{\beta}{ }_{\alpha} & =2 \frac{\partial \boldsymbol{V}_{\mathcal{P}}}{\partial\left(\boldsymbol{B}_{R}\right)^{\alpha}{ }_{\beta}}, \\
\star \boldsymbol{J}_{\mathcal{P}} & =\frac{\partial \boldsymbol{V}_{\mathcal{P}}}{\partial \boldsymbol{B}}, & { }^{2 n+1} \boldsymbol{\Sigma}_{H^{b}}{ }^{b}{ }_{a} & =2 \frac{\partial \mathcal{P}}{\partial \boldsymbol{R}^{a}{ }_{b}} .
\end{aligned}
$$

- We then remove the terms that are forbidden by the hydrostatic analysis. This involves discarding Class $\mathrm{H}_{F}$ terms $\left\{\left(\mathcal{G}^{\sigma}\right)_{\mathrm{H}_{F}},\left(T^{\mu \nu}\right)_{\mathrm{H}_{F}},\left(J^{\mu}\right)_{\mathrm{H}_{F}}\right\}$ from the constitutive relations. These terms are tensor structures allowed by symmetry, but forbidden by the second law. 
- There are combinations which can never be removed irrespective of choice of entropy current. These irreducibly dissipative combinations belong to Class D . All other combinations solve non-anomalous adiabaticity equation which is homogeneous in derivative order. We will henceforth proceed derivative order by derivative order with no mixing of adiabatic constitutive relations at different orders. The dissipative constitutive relations take the form (5.8):

$$
\begin{aligned}
&\left(T^{\mu \nu}\right)_{\mathrm{D}} \equiv-\frac{1}{2}\left[\Upsilon_{\boldsymbol{\eta}_{g}}^{\dagger} \boldsymbol{\eta} \Upsilon_{\boldsymbol{\eta}_{g}}\right.\left.+\Upsilon_{\boldsymbol{\sigma}_{g}}^{\dagger} \boldsymbol{\sigma} \Upsilon_{\boldsymbol{\sigma}_{g}}\right]^{(\mu \nu)(\alpha \beta)} \delta_{\mathcal{B}_{\mathcal{B}}} g_{\alpha \beta} \\
&- {\left[\Upsilon_{\boldsymbol{\eta}_{g}}^{\dagger} \boldsymbol{\eta} \Upsilon_{\boldsymbol{\eta}_{A}}+\Upsilon_{\boldsymbol{\sigma}_{g}}^{\dagger} \boldsymbol{\sigma} \Upsilon_{\boldsymbol{\sigma}_{A}}\right]^{(\mu \nu) \alpha} \cdot \delta_{\mathcal{B}} A_{\alpha} } \\
&\left(J^{\alpha}\right)_{\mathrm{D}} \equiv-\frac{1}{2}\left[\Upsilon_{\boldsymbol{\eta}_{A}}^{\dagger} \boldsymbol{\eta} \Upsilon_{\boldsymbol{\eta}_{g}}+\Upsilon_{\boldsymbol{\sigma}_{A}}^{\dagger} \boldsymbol{\sigma} \Upsilon_{\boldsymbol{\sigma}_{g}}\right]^{\alpha(\mu \nu)} \delta_{\mathcal{B}} g_{\mu \nu} \\
&-\left[\Upsilon_{\boldsymbol{\eta}_{A}}^{\dagger} \boldsymbol{\eta} \Upsilon_{\boldsymbol{\eta}_{A}}+\Upsilon_{\boldsymbol{\sigma}_{A}}^{\dagger} \boldsymbol{\sigma} \Upsilon_{\boldsymbol{\sigma}_{A}}\right]^{\alpha \beta} \cdot \delta_{\mathcal{B}} A_{\beta} .
\end{aligned}
$$

where the dissipative Noether current $\left(\mathrm{N}^{\sigma}\right)_{\mathrm{D}}$ is determined by integration by parts as in (5.13). As shown in section 5.3, a large subset of Class D constitutive relations up to the second order in derivative expansion can be obtained instead by the simpler task of classifying transverse tensor structures $\left\{\mathcal{N}^{\mu \nu \rho \sigma}, \mathcal{X}^{\mu \nu \alpha}, \mathcal{S}^{\alpha \beta}\right\}$ and plugging them into

$$
\begin{aligned}
\left(T^{\mu \nu}\right)_{\mathrm{D}, 2 \partial} & \equiv\left(-2 \eta \sigma^{\mu \nu}-\zeta \Theta P^{\mu \nu}\right)-\frac{1}{4}\left(\mathcal{N}^{(\mu \nu)(\alpha \beta)}+\mathcal{N}^{(\alpha \beta)(\mu \nu)}\right) \delta_{\mathfrak{B}} g_{\alpha \beta}+\mathcal{X}^{(\mu \nu) \alpha} \cdot \delta_{\mathfrak{B}} A_{\alpha} \\
\left(J^{\alpha}\right)_{\mathrm{D}, 2 \partial} & \equiv\left(\sigma_{\mathrm{Ohm}} \mathfrak{v}^{\alpha}\right)+\frac{1}{2} \mathcal{X}^{(\mu \nu) \alpha} \delta_{\mathfrak{B}} g_{\mu \nu}-\mathcal{S}^{(\alpha \beta)} \cdot \delta_{\mathcal{B}} A_{\beta} \\
\left(\mathcal{G}^{\sigma}\right)_{\mathrm{D}, 2 \partial} & =0 .
\end{aligned}
$$

- We then remove the Class $\mathrm{C}$ constitutive relations by eliminating the non-trivial conserved vectors that can serve to provide contributions to the entropy current:

$$
\left(T^{\mu \nu}\right)_{\mathrm{C}}=0, \quad\left(J^{\mu}\right)_{\mathrm{C}}=0, \quad\left(\mathcal{G}^{\sigma}\right)_{\mathrm{C}}=-T \mathrm{~J}^{\sigma},
$$

where $\mathrm{J}^{\sigma}$ are identically conserved topological currents.

- At the next step, we will remove Class B by looking at all combinations in that derivative order that solve adiabaticity equation with zero free energy current. In order to adhere to the derivative counting one should simple classify the intertwining tensors $\{\mathcal{N}, \mathcal{X}, \mathcal{S}\}$ without the derivative operators $\Upsilon$ as the latter mixes derivative orders: ${ }^{81}$

$$
\begin{aligned}
\left(T^{\mu \nu}\right)_{\mathrm{B}} & \equiv-\frac{1}{4}\left(\mathcal{N}^{(\mu \nu)(\alpha \beta)}-\mathcal{N}^{(\alpha \beta)(\mu \nu)}\right) \delta_{\mathfrak{B}} g_{\alpha \beta}+\mathcal{X}^{(\mu \nu) \alpha} \cdot \delta_{\mathcal{B}} A_{\alpha} \\
\left(J^{\alpha}\right)_{\mathrm{B}} & \equiv-\frac{1}{2} \mathcal{X}^{(\mu \nu) \alpha} \delta_{\mathcal{B}} g_{\mu \nu}-\mathcal{S}^{[\alpha \beta]} \cdot \delta_{\mathcal{B}} A_{\beta} \\
\left(\mathcal{G}^{\sigma}\right)_{\mathrm{B}} & =0
\end{aligned}
$$

\footnotetext{
${ }^{81}$ Note that this argument seems to indicate that $\Upsilon$ construction while useful in constructing solutions for Class B, serves little purpose in the classification program, where all we care about is the set of solutions at a given derivative order.
} 
At a given derivative order, say $k^{\text {th }}$, let there be $N_{\text {tot }}$ functions which parameterize constitutive relations solving the non-anomalous adiabaticity equation and have non-trivial free energy current. We will examine the grand canonical adiabaticity equation (2.21) and focus on the expression for the most general adiabatic free energy current. This is in fact easier to deal with than the stress-tensor and charge currents owing to the fact that we only have to classify vectors and not symmetric tensors. As we have already taken the effort to remove Class B terms in the preceding steps, the most general adiabatic free energy current will then be written in terms of just these $N_{\text {tot }}$ functions we enumerate.

Let us decompose the adiabatic free energy current into a longitudinal scalar and vector part by the ansatz:

$$
-\frac{\mathcal{G}^{\mu}}{T}=\mathcal{L} \boldsymbol{\beta}^{\mu}-P_{\sigma}^{\mu} \frac{\mathcal{G}^{\sigma}}{T}
$$

using the hydrodynamic field and the transverse spatial projector $P_{\mu \nu}$. Further, let $N_{\mathcal{L}}$ be the number of functional combinations that appear in the scalar part $\mathcal{L}$. Without loss of generality, let us assume our parametrization is such that we can then divide the $N_{\text {tot }}$ number of functions in $\mathcal{G}^{\sigma}$ into $N_{\mathcal{L}}$ functions that appear in $\mathcal{L}$ and the reminder (that do not appear in $\mathcal{L}$ ). Now, set the $N_{\text {tot }}-N_{\mathcal{L}}$ number of functions that do not appear in $\mathcal{L}$ to zero. We are then left with a $N_{\mathcal{L}}$ functions worth of solution of adiabaticity equation which we will denote by $\left\{\mathcal{G}_{S c}^{\sigma}, T_{S c}^{\mu \nu}, J_{S c}^{\mu}\right\}$. After subtracting this solution, we have $N_{\text {tot }}-N_{\mathcal{L}}$ solutions with purely transverse free energy current denoted by $\left\{\mathcal{G}_{V}^{\sigma}, T_{V}^{\mu \nu}, J_{V}^{\mu}\right\}$.

Let us now focus on $\left\{\mathcal{G}_{S c}^{\sigma}, T_{S c}^{\mu \nu}, J_{S c}^{\mu}\right\}$. The decomposition for these solutions (14.7) then reduces to

$$
-\frac{\mathcal{G}_{S c}^{\mu}}{T}=\mathcal{L} \beta^{\mu}-P_{\sigma}^{\mu} \frac{\mathcal{G}_{S c}^{\sigma}}{T}
$$

where the data is now parameterized by scalar functions.

In the next step, let us use $\mathcal{L}$ as the Lagrangian and then construct $N_{\mathcal{L}}$ functions worth of Class L constitutive relations which are of the form

$$
\begin{aligned}
\mathcal{G}_{\mathcal{L}}^{\sigma} & =-T\left(\boldsymbol{\beta}^{\sigma} \mathcal{L}-\left(\phi_{\mathcal{B}} \Theta_{\mathrm{PS}}\right)^{\sigma}+\nabla_{\nu} \mathcal{K}^{\sigma \nu}[\mathcal{B}]\right), \\
T_{\mathcal{L}}^{\mu \nu} & =\frac{2}{\sqrt{-g}} \frac{\delta(\sqrt{-g} \mathcal{L})}{\delta g_{\mu \nu}} \\
J_{\mathcal{L}}^{\mu} & =\frac{1}{\sqrt{-g}} \frac{\delta(\sqrt{-g} \mathcal{L})}{\delta A_{\mu}}
\end{aligned}
$$

Since this accounts for all the $N_{\mathcal{L}}$ solutions, the difference $\left\{\mathcal{G}_{S c}^{\sigma}-\mathcal{G}_{\mathcal{L}}^{\sigma}, T_{S c}^{\mu \nu}-T_{\mathcal{L}}^{\mu \nu}, J_{S c}^{\mu}-J_{\mathcal{L}}^{\mu}\right\}$ can only contain repetitions, trivial solutions or a mix of other classes, such as Class B or even $\overline{\mathrm{H}}_{V}$ type solutions. ${ }^{82}$ All that matters for our discussion is that the currents are derivable from some form of a generating function (as in, e.g., Class L). In that case we can set the controlling functions to be some functionals of the hydrostatic and hydrodynamic

\footnotetext{
${ }^{82}$ It is useful to know that even though we are generating solutions from a scalar Lagrangian density we can indeed get some transverse vector components in the free energy current.
} 
parameters in $\mathcal{L}$. We can discard them without loss of generality, and choose a basis of solutions such that

$$
\left\{\mathcal{G}_{S c}^{\sigma}, T_{S c}^{\mu \nu}, J_{S c}^{\mu}\right\}=\left\{\mathcal{G}_{\mathcal{L}}^{\sigma}, T_{\mathcal{L}}^{\mu \nu}, J_{\mathcal{L}}^{\mu}\right\}
$$

- The above discussion takes care of all Class L (which divides further into $\mathrm{H}_{S}$ and $\overline{\mathrm{H}}_{S}$ ) constitutive relations. At this point, we have accounted for five adiabatic classes $\left\{\mathrm{H}_{S}, \overline{\mathrm{H}}_{S}, \mathrm{~A}, \mathrm{~B}, \mathrm{C}\right\}$ in addition to the Class $\mathrm{D}$ terms (and we have eliminated $\mathrm{H}_{F}$ terms).

- We are now left with the remaining constitutive relations with transverse free energies, $\left\{\mathcal{G}_{V}^{\sigma}, T_{V}^{\mu \nu}, J_{V}^{\mu}\right\}=\left\{\left(\mathcal{G}^{\sigma}\right)_{\mathrm{H}_{V}}+\left(\mathcal{G}^{\sigma}\right)_{\overline{\mathrm{H}}_{V}},\left(T^{\mu \nu}\right)_{\mathrm{H}_{V}}+\left(T^{\mu \nu}\right)_{\overline{\mathrm{H}}_{V}},\left(J^{\mu}\right)_{\mathrm{H}_{V}}+\left(J^{\mu}\right)_{\overline{\mathrm{H}}_{V}}\right\}$.

- The Class $\mathrm{H}_{V}$ terms can be eliminated by invoking the replacement rule arising from Euclidean consistency and thus employing a similar trick as in the Class A discussion earlier. We define a modified anomaly polynomial $\mathcal{P}$ via

$$
\mathcal{P}_{\mathrm{H}_{V}}\left[\boldsymbol{F}, \boldsymbol{R}, \mathrm{F}^{(\mathrm{T})}\right]=\mathcal{P}\left[\boldsymbol{F}, \operatorname{tr} \boldsymbol{R}^{2 k} \mapsto \operatorname{tr} \boldsymbol{R}^{2 k}+2\left(2 \pi \mathrm{F}^{(\mathrm{T})}\right)^{2 k}\right]-\mathcal{P}\left[\boldsymbol{F}, \operatorname{tr} \boldsymbol{R}^{2 k}\right]
$$

and find the constitutive relations

$$
\begin{aligned}
\left(J_{S}^{\alpha}\right)_{\mathrm{H}_{V}} & =\left(J_{S}^{\prime}\right)_{\mathrm{H}_{V}}^{\alpha}-\frac{1}{2} \boldsymbol{\beta}_{\sigma}\left(\hat{\Sigma}_{H}\right)_{\mathrm{H}_{V}}^{\perp[\alpha \sigma]} \\
\left(T^{\alpha \beta}\right)_{\mathrm{H}_{V}} & =q_{\mathrm{H}_{V}}^{\alpha} u^{\beta}+q_{\mathrm{H}_{V}}^{\beta} u^{\alpha}+\frac{1}{2} D_{\rho}\left(\Sigma_{\mathrm{H}_{V}}^{\alpha[\beta \rho]}+\Sigma_{\mathrm{H}_{V}}^{\beta[\alpha \rho]}-\Sigma_{\mathrm{H}_{V}}^{\rho(\alpha \beta)}\right), \\
\left(J^{\alpha}\right)_{\mathrm{H}_{V}} & =J_{\mathrm{H}_{V}}^{\alpha}
\end{aligned}
$$

where

$$
\begin{array}{rlrl}
\star \boldsymbol{q}_{\mathrm{H}_{V}} & =\frac{\partial \boldsymbol{V}_{\mathcal{P}_{\mathrm{H}_{V}}}}{\partial(2 \boldsymbol{\omega})}, & \star \boldsymbol{\Sigma}_{\mathrm{H}_{V}}{ }^{\beta}{ }^{\alpha}=2 \frac{\partial \boldsymbol{V}_{\mathcal{P}_{\mathrm{H}_{V}}}}{\partial\left(\boldsymbol{B}_{R}\right)^{\alpha}{ }_{\beta}}, \\
\star \boldsymbol{J}_{\mathrm{H}_{V}}=\frac{\partial \boldsymbol{V}_{\mathcal{P}_{\mathrm{H}_{V}}}}{\partial \boldsymbol{B}}, & \star_{2 n+1}\left(\boldsymbol{\Sigma}_{H}\right)_{\mathrm{H}_{V}}{ }^{b}{ }_{a}=2 \frac{\partial \boldsymbol{P}_{\mathrm{H}_{V}}}{\partial \boldsymbol{R}^{a}{ }_{b}} .
\end{array}
$$

One main difference from the Class A constitutive relations (apart from the presence of $\mathrm{A}^{(\mathrm{T})}$ in $\mathcal{P}_{\mathrm{H}_{V}}$ ) is the fact that the entropy current (and thus the free energy current) gets a non-trivial contribution in this class. The additional contribution to the entropy current $\left(J_{S}^{\prime}\right)_{\mathrm{H}_{V}}^{\alpha}$ is defined in terms of the $\mathrm{H}_{V}$ transgression form:

$$
\star \boldsymbol{J}_{S, \mathrm{H}_{V}}^{\prime}=\frac{\partial\left(\boldsymbol{V}_{\mathcal{P}_{\mathrm{H}_{V}}}\right)}{\partial \mathrm{B}^{(\mathrm{T})}} .
$$

where $\mathrm{B}^{(\mathrm{T})}$ is the two-form magnetic field for $\mathrm{A}^{(\mathrm{T})}, \mathrm{B}^{(\mathrm{T})}=\mathrm{F}^{(\mathrm{T})}-\boldsymbol{u} \wedge i_{\boldsymbol{u}} \mathrm{F}^{(\mathrm{T})}$. 
- Having dealt with the other classes the rest will go into Class $\overline{\mathrm{H}}_{V}$ and takes the general form

$$
\begin{aligned}
&\left(\mathcal{G}^{\rho}\right)_{\overline{\mathrm{H}}_{V}}=-T\left[\frac{1}{4} \delta_{\mathcal{B}} g_{\mu \nu} \mathfrak{C}_{\mathcal{N}}^{\rho((\mu \nu) \mid(\alpha \beta))} \delta_{\mathcal{B}} g_{\alpha \beta}\right. \\
&\left.+\delta_{\mathcal{B}} g_{\mu \nu} \mathfrak{C}_{\mathcal{X}}^{\rho(\mu \nu) \alpha} \cdot \delta_{\mathcal{B}} A_{\alpha}+\delta_{\mathcal{B}} A_{\alpha} \cdot \mathfrak{C}_{\mathcal{S}}^{\rho(\alpha \beta)} \cdot \delta_{\mathcal{B}} A_{\beta}\right] \\
&\left(T^{\mu \nu}\right)_{\overline{\mathrm{H}}_{V}}=\frac{1}{2}\left[D_{\rho} \mathfrak{C}_{\mathcal{N}}^{\rho((\mu \nu) \mid(\alpha \beta))} \delta_{\mathfrak{B}} g_{\alpha \beta}+2 \mathfrak{C}_{\mathcal{N}}^{\rho((\mu \nu) \mid(\alpha \beta))} D_{\rho} \delta_{\mathcal{B}} g_{\alpha \beta}\right] \\
& \quad+D_{\rho} \mathfrak{C}_{\mathcal{X}}^{\rho(\mu \nu) \alpha} \cdot \delta_{\mathcal{B}} A_{\alpha}+2 \mathfrak{C}_{\mathcal{X}}^{\rho(\mu \nu) \alpha} \cdot D_{\rho} \delta_{\mathcal{B}} A_{\alpha} \\
&\left(J^{\alpha}\right)_{\overline{\mathrm{H}}_{V}}=\frac{1}{2}\left[D_{\rho} \mathfrak{C}_{\mathcal{X}}^{\rho(\mu \nu) \alpha} \delta_{\mathcal{B}} g_{\mu \nu}+2 \mathfrak{C}_{\mathcal{X}}^{\rho(\mu \nu) \alpha} D_{\rho} \delta_{\mathcal{B}} g_{\mu \nu}\right] \\
&+D_{\rho} \mathfrak{C}_{\mathcal{S}}^{\rho(\alpha \beta)} \cdot \delta_{\mathcal{B}} A_{\beta}+2 \mathfrak{C}_{\mathcal{S}}^{\rho(\alpha \beta)} \cdot D_{\rho} \delta_{\mathcal{B}} A_{\beta} .
\end{aligned}
$$

- Once we have accounted for these terms we have exhausted all possible hydrodynamic constitutive relations; the eightfold path is complete and the most general constitutive relations allowed by symmetries at a given order in derivatives can be written as

$$
\begin{aligned}
\mathcal{G}^{\sigma} & =\left(\mathcal{G}^{\sigma}\right)_{\mathrm{A}}+\left(\mathcal{G}^{\sigma}\right)_{\mathrm{H}_{F}}+\left(\mathcal{G}^{\sigma}\right)_{\mathrm{D}}+\left(\mathcal{G}^{\sigma}\right)_{\mathrm{C}}+\left(\mathcal{G}^{\sigma}\right)_{\mathrm{B}}+\mathcal{G}_{\mathcal{L}}^{\sigma}+\mathcal{G}_{V}^{\sigma}, \\
T^{\mu \nu} & =\left(T^{\mu \nu}\right)_{\mathrm{A}}+\left(T^{\mu \nu}\right)_{\mathrm{H}_{F}}+\left(T^{\mu \nu}\right)_{\mathrm{D}}+\left(T^{\mu \nu}\right)_{\mathrm{C}}+\left(T^{\mu \nu}\right)_{\mathrm{B}}+T_{\mathcal{L}}^{\mu \nu}+T_{V}^{\mu \nu}, \\
J^{\mu} & =\left(J^{\mu}\right)_{\mathrm{A}}+\left(J^{\mu}\right)_{\mathrm{H}_{F}}+\left(J^{\mu}\right)_{\mathrm{D}}+\left(J^{\mu}\right)_{\mathrm{C}}+\left(J^{\mu}\right)_{\mathrm{B}}+J_{\mathcal{L}}^{\mu}+J_{V}^{\mu} .
\end{aligned}
$$

Theorem 1. All hydrodynamic transport is exhaustively classified by one of the aforementioned seven adiabatic classes, viz., $\left\{\mathrm{H}_{S}, \overline{\mathrm{H}}_{S}, \mathrm{~A}, \mathrm{~B}, \mathrm{C}, \mathrm{H}_{V}, \overline{\mathrm{H}}_{V}\right\}$ and the forbidden constitutive relations of Class $\mathrm{H}_{F}$, in addition to the dissipative Class $\mathrm{D}=\mathrm{D}_{v} \cup \mathrm{D}_{s}$.

The constructive algorithm described above outlines the general structure of the proof we would like to present. However, in order to complete the proof, we need a precise argument stating that our constructions exhaust the terms in the non-Lagrangian classes $\left\{\overline{\mathrm{H}}_{V}, \mathrm{~B}, \mathrm{D}\right\}$ completely. We will give such an argument in the following subsection, section 14.2. However, before we give an independent proof, let us anticipate the result of section 15-section 17 which makes it clear that there exists a master effective field theory which will encompass precisely the adiabatic classes and thus provides a much more direct proof of the completeness of our classification:

Theorem 2. The sevenfold classes of adiabatic hydrodynamic transport can be obtained from a scalar Lagrangian density $\mathcal{L}_{\mathrm{T}}\left[\boldsymbol{\beta}^{\mu}, \Lambda_{\boldsymbol{\beta}}, g_{\mu \nu}, A_{\mu}, \tilde{g}_{\mu \nu}, \tilde{A}_{\mu}, \mathrm{A}^{(\mathrm{T})}{ }_{\mu}\right]$ :

$$
\mathcal{L}_{\mathrm{T}}=\frac{1}{2} T^{\mu \nu} \tilde{g}_{\mu \nu}+J^{\mu} \cdot \tilde{A}_{\mu}+\left(J_{S}^{\sigma}+\boldsymbol{\beta}_{\nu} T^{\nu \sigma}+\left(\Lambda_{\boldsymbol{\beta}}+\boldsymbol{\beta}^{\nu} A_{\nu}\right) \cdot J^{\sigma}\right) \mathrm{A}_{\sigma}^{(\mathrm{T})}
$$

As indicated the Lagrangian density depends not only on the hydrodynamic fields and the background sources, but also the 'Schwinger-Keldysh' partners of the sources $\left\{\tilde{g}_{\mu \nu}, \tilde{A}_{\mu}\right\}$ and a new KMS-gauge gauge field $\mathrm{A}^{(\mathrm{T})}{ }_{\mu}$. This Lagrangian is invariant under diffeomorphisms 
and gauge transformations ${ }^{83}$ and under $U(1)_{\mathrm{T}}$ which acts only on the sources as a thermal diffeomorphism or gauge transformation along $\mathcal{B}$. The $U(1)_{\mathrm{T}}$ gauge invariance implies a Bianchi identity, which is nothing but the adiabaticity equation (2.12). Furthermore, a constrained variational principle for the fields $\left\{\boldsymbol{\beta}^{\mu}, \Lambda_{\boldsymbol{\beta}}\right\}$ ensures that the dynamics of the theory is simply given by conservation.

Given the Lagrangian $\mathcal{L}_{\mathrm{T}}$ we are essentially done, since all we need to do is to show that by picking appropriate scalar densities in the extended space of fields gives rise to a solution in one of the aforementioned eight classes. This is relatively straightforward as we shall see in section 17. What is less apparent at first sight is the rationale for the existence of the extended set of degrees of freedom and the extra $U(1)_{T}$ symmetry. The reader might take these as part of our construction for the present, though we believe that the Class $\mathrm{L}_{\mathrm{T}}$ story we are about to present hints at some fundamental truisms that ought to be valid in non-equilibrium dynamics of QFTs (and potentially fixing some of the problems described in section 13).

Before presenting the detailed construction of $\mathcal{L}_{\mathrm{T}}$ in section 15 -section 17 , we shall now give an independent proof of the completeness claimed in Theorem 1 and then illustrate our eightfold classification for various fluid systems.

\subsection{Completeness of the adiabatic taxonomy}

In the lead up to the statement of Theorem 1 we have already covered a reasonable amount of ground vis a vis a proof of the statement. We will in the following complete some of the open issues which that discussion left out and argue that out eightfold classification is complete. The proof per se will be phrased in a physical language; it can be made mathematically rigorous as necessary but we prefer to illustrate the basic statements in a fashion that makes them more intuitive.

The key component of the proof is to realize that one needs to control the free energy current $\mathcal{G}^{\sigma}$ in order to ascertain the behaviour of transport. In much of our discussion, including section 14.1, we have emphasized the fact that the free energy current is a spacetime vector simplifies the classification scheme. Accounting for all possible vectors that can appear in $\mathcal{G}^{\sigma}$ would suffice for our purposes of demonstrating completeness.

Let us first examine adiabatic constitutive relations. We invoke the decomposition (14.7) of the free energy current. Using the argument following this equation in section 14.1, it is clear that the entire contribution to the longitudinal part of the free energy current is captured by the Class $\mathrm{L}\left(=\mathrm{H}_{S} \cup \overline{\mathrm{H}}_{S}\right)$ by picking a set of generating scalars which are either hydrostatic $\left(\mathrm{H}_{S}\right)$ of hydrodynamic $\left(\overline{\mathrm{H}}_{S}\right)$. Furthermore, anomalies are dealt with using the particular solutions of the adiabaticity equation, leaving us then with situations of traverse vector free energy current and some situations where the free energy is vanishing (Class B) or identically conserved (Class C). In addition we have the dissipative constitutive relations. Of these Class $\mathrm{C}$ terms are easy to handle and like with the anomalies one quickly can exhaust the space of cohomologically non-trivial conserved currents.

\footnotetext{
${ }^{83}$ Anomalies if present are dealt with using the inflow mechanism [77]. $\mathcal{L}_{\mathrm{T}}$ then includes a topological theory in $d+1$ dimensions coupled to the physical $d$-dimensional QFT (at the boundary/edge).
} 
Thus for a full proof of Theorem 1 we need to ascertain that the parameterizations we gave for Classes $\left\{\mathrm{H}_{V}, \overline{\mathrm{H}}_{V}, \mathrm{~B}, \mathrm{D}\right\}$ are exhaustive. Let us make a couple of remarks:

- For Class D, the argument is clear since we can always recast the most general positive definite form using a set of tensor valued differential operators $\Upsilon$ and suitably chosen intertwiners $\{\boldsymbol{\eta}, \boldsymbol{\sigma}\}$ as discussed in section 5.2.

- For Class B (without $\Upsilon$ operators), one can argue that in the vector space of terms, all the combinations orthogonal to $\left\{\delta_{\mathfrak{B}} g_{\mu \nu}, \delta_{\mathfrak{B}} A_{\mu}\right\}$ necessarily take the form given in eqs. (9.2) and (9.4).

- The hydrostatic transverse vector free energy currents are likewise easy to tackle by focusing on a limited set of terms that survive equilibrium.

- The only unresolved problem is how to argue that our construction for $\overline{\mathrm{H}}_{V}$ does not miss any terms. The issue here is that there are too few of them in our explicit examples to see how they work in general. We will present an argument in favour of the completeness of our classification below.

To prove the completeness of our parametrization (14.14) of Class $\overline{\mathrm{H}}_{V}$, we need to consider the set of all possible transverse free energy currents $\left(\mathcal{G}^{\sigma}\right)_{\overline{\mathrm{H}}_{V}}$. Since we are only interested in non-hydrostatic currents (otherwise we could describe them in Class $\mathrm{H}_{V}$ ), they need to contain at least one factor of $\delta_{\mathfrak{B}} g_{\mu \nu}{ }^{84}$ Let us parameterize such currents as

$$
\left(\mathrm{N}^{\sigma}\right)_{\overline{\mathrm{H}}_{V}} \equiv-\frac{1}{T}\left(\mathcal{G}^{\sigma}\right)_{\overline{\mathrm{H}}_{V}}=\mathfrak{C}^{\sigma \mu \nu} \delta_{\mathfrak{B}} g_{\mu \nu} \quad \text { with } \quad u_{\sigma} \mathfrak{C}^{\sigma \mu \nu}=0
$$

where $\mathfrak{C}^{\sigma \mu \nu}$ is some tensor. Now consider the divergence of this current:

$$
\nabla_{\sigma}\left(\mathrm{N}^{\sigma}\right)_{\overline{\mathrm{H}}_{V}}=\left(\nabla_{\sigma} \mathfrak{C}^{\sigma \mu \nu}\right) \delta_{\mathfrak{B}} g_{\mu \nu}+\mathfrak{C}^{\sigma \mu \nu} \nabla_{\sigma}\left(\delta_{\mathfrak{B}} g_{\mu \nu}\right)
$$

From the structure of the adiabaticity equation (5.7), it is clear that this free energy current is only adiabatic if the divergence (14.18) is proportional to $\delta_{\mathfrak{B}} g_{\mu \nu}$. This is clear by examining the adiabaticity equation (2.21). In (14.18) first term in the r.h.s. has an explicit $\delta_{\mathfrak{B}} g_{\mu \nu}$ which would serve. The second term however contains descendant operators (using the terminology from section 5). These are independent tensors and do not contain a free $\delta_{\mathfrak{B}} g_{\mu \nu}$. Alternately, one simply notes that there are fluid configurations which are hydrostatic locally (i.e., $\delta_{\mathfrak{B}} g_{\mu \nu}=0$ at some point on $\mathcal{M}$ ) but such that $\nabla_{\sigma}\left(\delta_{\mathfrak{B}} g_{\mu \nu}\right) \neq 0$, the above divergence can only be consistent with adiabaticity if every term contains at least one undifferentiated factor of $\delta_{\mathfrak{B}} g_{\mu \nu}$. Either way we require $\mathfrak{C}^{\sigma \mu \nu}$ to contain a factor of $\delta_{\mathfrak{B}} g_{\mu \nu}$ in general. However, this requirement implies that $\left(\mathcal{G}^{\sigma}\right)_{\overline{\mathrm{H}}_{V}}$ contains two factors of $\delta_{\mathfrak{B}} g_{\mu \nu}$ and hence is precisely captured by our Class $\overline{\mathrm{H}}_{V}$ parametrization (14.14).

\footnotetext{
${ }^{84}$ In this subsection, we will content ourselves with the discussion of neutral fluids. In charged fluids, one could also use $\delta_{\mathcal{B}} A_{\mu}$ instead of $\delta_{\mathcal{B}} g_{\mu \nu}$ to describe deviations from equilibrium, which leads to a completely analogous discussion.
} 
Let us now consider the slightly more general situation where the object $\mathfrak{C}$ in (14.17) is not a tensor, but a tensor-valued derivative operator. W.l.o.g. we can parameterize this situation as

$$
\left(\mathrm{N}^{\sigma}\right)_{\overline{\mathrm{H}}_{V}}=\mathfrak{C}^{\sigma \lambda(\mu \nu)} \nabla_{\lambda}\left(\delta_{\mathfrak{B}} g_{\mu \nu}\right) \quad \text { with } \quad u_{\sigma} \mathfrak{C}^{\sigma \lambda \mu \nu}=0,
$$

where $\mathfrak{C}^{\sigma \lambda \mu \nu}$ is some tensor. Let us consider separately the cases where $\mathfrak{C}^{\sigma \lambda(\mu \nu)}$ is symmetric and anti-symmetric in its first two indices, respectively:

- Anti-symmetric case $\left(\mathfrak{C}^{\sigma \lambda(\mu \nu)}=\mathfrak{C}^{[\sigma \lambda](\mu \nu)}\right)$ : One can easily see that the anti-symmetric component does not provide anything new as it can always be canceled by a combination of terms of other classes and an uninteresting Komar piece; to see this, observe that $\left(\mathrm{N}^{\sigma}\right)_{\overline{\mathrm{H}}_{V}}$ can be decomposed as follows:

$$
\begin{aligned}
\left(\mathrm{N}^{\sigma}\right)_{\overline{\mathrm{H}}_{V}}= & \mathfrak{C}^{[\sigma \lambda](\mu \nu)} \nabla_{\lambda}\left(\delta_{\mathfrak{B}} g_{\mu \nu}\right) \\
= & \nabla_{\lambda}\left(\mathfrak{C}^{[\sigma \lambda](\mu \nu)} \delta_{\mathcal{B}} g_{\mu \nu}\right)-\left(\nabla_{\lambda} \mathfrak{C}^{[\sigma \lambda](\mu \nu)}\right) \delta_{\mathfrak{B}} g_{\mu \nu} \\
= & \nabla_{\lambda}\left(\mathfrak{C}^{[\sigma \lambda](\mu \nu)} \delta_{\mathfrak{B}} g_{\mu \nu}\right)-P_{\rho}^{\sigma}\left(\nabla_{\lambda} \mathfrak{C}^{[\rho \lambda](\mu \nu)}\right) \delta_{\mathcal{B}} g_{\mu \nu} \\
& +u^{\sigma}\left(u_{\rho} \nabla_{\lambda} \mathfrak{C}^{[\rho \lambda](\mu \nu)}\right) \delta_{\mathfrak{B}} g_{\mu \nu}
\end{aligned}
$$

In the last line the first term is Komar (hence uninteresting), the second is captured by $\overline{\mathrm{H}}_{V}$ (it's of the form (14.17)) and the last one is longitudinal (and thus in Class $\mathrm{L})$. We conclude that this does not lead to any transport not captured by either $\overline{\mathrm{H}}_{V}$ or in one of the other Classes we have already accounted for.

- Symmetric case $\left(\mathfrak{C}^{\sigma \lambda(\mu \nu)}=\mathfrak{C}^{(\sigma \lambda)(\mu \nu)}\right)$ : in the symmetric case, consider the divergence of the free energy current (14.19):

$$
\nabla_{\sigma}\left(\mathrm{N}^{\sigma}\right)_{\overline{\mathrm{H}}_{V}}=\left(\nabla_{\sigma} \mathfrak{C}^{(\sigma \lambda)(\mu \nu)}\right) \nabla_{\lambda}\left(\delta_{\mathfrak{B}} g_{\mu \nu}\right)+\mathfrak{C}^{(\sigma \lambda)(\mu \nu)} \nabla_{\sigma} \nabla_{\lambda}\left(\delta_{\mathfrak{B}} g_{\mu \nu}\right) .
$$

W.l.o.g. we can assume that $\mathfrak{C}^{\sigma \lambda \mu \nu}$ does not contain any undifferentiated $\delta_{\mathfrak{B}} g_{\mu \nu}$ (if it did, we would be back in the case parameterized by (14.17)). A-priori the second term in (14.21) does then not contain any undifferentiated $\delta_{\mathfrak{B}} g_{\mu \nu}$. Hence the only way that this situation can be compatible with adiabaticity, is that a cancellation between the two terms happens such that their sum is proportional to $\delta_{\mathfrak{B}} g_{\mu \nu}$.

Let us see under what conditions it could happen that the second term is canceled by the first. Note that $\nabla_{(\sigma} \nabla_{\lambda)}\left(\delta_{\mathfrak{B}} g_{\mu \nu}\right)$ is a genuine $3^{\text {rd }}$ order descendant object which is not a product of lower order tensors. Since the first term in (14.21) contains a $2^{\text {nd }}$ order descendant factor, a cancellation between terms can only happen if $\mathfrak{C}^{\sigma \lambda \mu \nu}$ itself contains a factor of $\nabla_{\kappa}\left(\delta_{\mathcal{B}} g_{\alpha \beta}\right)$. If the two terms were to cancel, we would hence require that

$$
\mathfrak{C}^{(\sigma \lambda)(\mu \nu)}=\overline{\mathfrak{C}}^{(\sigma \lambda \kappa)[(\mu \nu) \mid(\alpha \beta)]} \nabla_{\kappa}\left(\delta_{\mathfrak{B}} g_{\alpha \beta}\right)
$$

such that

$$
\nabla_{\sigma}\left(\mathrm{N}^{\sigma}\right)_{\overline{\mathrm{H}}_{V}}=\left(\nabla_{\sigma} \overline{\mathfrak{C}}^{(\sigma \lambda \kappa)[(\mu \nu) \mid(\alpha \beta)]}\right) \nabla_{\lambda}\left(\delta_{\mathcal{B}} g_{\mu \nu}\right) \nabla_{\kappa}\left(\delta_{\mathcal{B}} g_{\alpha \beta}\right)
$$


This divergence could now in principle be proportional to $\delta_{\mathfrak{B}} g_{\sigma \tau}$. But again this does not provide anything new, for we recognize the free energy current in this case as a special Class B term (with non-trivial $\Upsilon$-operators acting as derivative operators such that the free energy current is non-zero). The easiest way to see this is by recognizing that the divergence (14.23) is of the form of a Class B divergence (5.13) with

$$
\left(\Upsilon_{\boldsymbol{\eta}_{g}}\right)_{\lambda}=2 \nabla_{\lambda}, \quad \boldsymbol{\eta}^{(\lambda \kappa)[(\mu \nu) \mid(\alpha \beta)]}=-\nabla_{\sigma} \overline{\mathfrak{C}}^{(\sigma \lambda \kappa)[(\mu \nu) \mid(\alpha \beta)]} .
$$

From the symmetry structure of $\boldsymbol{\eta}$ it is clear that we are dealing with a Class B term as constructed in section 9.1.

These considerations show that an ansatz of the form (14.19) does not lead to any constitutive relations that are not captured by the ones we already have.

Similarly, one can proceed and consider ansätze where $\left(\mathcal{G}^{\sigma}\right)_{\overline{\mathrm{H}}_{V}}$ contains higher derivatives of $\delta_{\mathfrak{B}} g_{\mu \nu}$, but analogous arguments as the one presented above would show that this is only consistent with adiabaticity if $\left(\mathcal{G}^{\sigma}\right)_{\overline{\mathrm{H}}_{V}}$ is secretly a combination of Class $\overline{\mathrm{H}}_{V}$, $\mathrm{B}$ and Komar terms as we parameterized them in section 14.1. Together with our earlier comments, this completes our proof that the parametrization (11.15), (11.16) exhausts all possible non-hydrostatic, adiabatic transverse free energy currents.

\subsection{Example I: charged parity-even fluids}

To exemplify our general story we turn to an example that has been discussed in some detail in [37], viz., a charged parity-even fluid. Neutral fluids are clearly a subset obtained by setting the chemical potential and charge density to zero. We will describe first outline the classification in general and then indicate in section 14.4 how to specialize to Weyl invariant case (which has the advantage of being able to be tested holographically).

We begin by counting the total number of transport coefficients: there is one frame invariant scalar (for definiteness, let us take it to be in $P^{\mu \nu}$ part of the energy momentum tensor), one frame invariant transverse vector (for definiteness, let us take it to be in the charge current) and one frame invariant transverse traceless tensor occurring as a part of the energy momentum tensor. The final result of this counting is summarized in tables 1 and 2 .

The first order constitutive relations are a-priori parameterized by one scalar $\Theta$, three transverse vectors $\left\{\mathfrak{a}^{\mu}, E^{\mu}, \mathfrak{v}^{\mu}\right\}$, and one transverse traceless tensor $\sigma^{\mu \nu}$. The constitutive relations

$$
\begin{aligned}
T^{\mu \nu} & =\epsilon u^{\mu} u^{\nu}+p P^{\mu \nu}-2 \eta \sigma^{\mu \nu}-\zeta \Theta P^{\mu \nu} \\
J^{\mu} & =q u^{\mu}+\sigma_{\mathrm{Ohm}} \mathfrak{v}^{\mu}+\chi_{E} E^{\mu}-\chi_{T} T \mathfrak{a}^{\mu} \\
J_{S}^{\mu} & =s u^{\mu}+\alpha_{1} \mathfrak{a}^{\mu}+\alpha_{2} \Theta u^{\mu}+\alpha_{3} \mathfrak{v}^{\mu}+\alpha_{4} E^{\mu}
\end{aligned}
$$

satisfy the second law of thermodynamics provided the following relations hold:

- The coefficient of sign-indefinite terms in $\Delta$ vanish. These are the $\mathrm{H}_{F}$ constraints and at this order they can be shown to imply:

$$
\epsilon+p=T s+q \mu, \quad d \epsilon=T d s+\mu d q .
$$




\begin{tabular}{|c|c|c|}
\hline \multicolumn{3}{|c|}{$2^{\text {nd }}$ order charged hydrostatic response } \\
\hline Scalars & Vectors & Tensors \\
\hline$\omega^{2}$ & $\omega^{\mu \nu} \mathfrak{a}_{\nu}$ & $\omega^{\alpha<\mu} \omega^{\nu>}{ }_{\alpha}$ \\
$\omega_{\alpha \beta} B^{\alpha \beta}$ & $\omega^{\mu \nu} E_{\nu}$ & $\omega^{\alpha<\mu} B^{\nu>}{ }_{\alpha}$ \\
$B^{2}$ & $B^{\mu \nu} E_{\nu}$ & $B^{\alpha<\mu} B^{\nu>}{ }_{\alpha}$ \\
$\mathfrak{a}_{\alpha} E^{\alpha}$ & $B^{\mu \nu} \mathfrak{a}_{\nu}$ & $\mathfrak{a}^{<\mu} E^{\nu>}$ \\
$E^{2}$ & & $E^{<\mu} E^{\nu>}$ \\
$\mathfrak{a}^{2}$ & & $\mathfrak{a}^{<\mu} \mathfrak{a}^{\nu>}$ \\
$R$ & & $R^{<\mu>}$ \\
\hline$R_{\alpha \beta} u^{\alpha} u^{\beta}$ & $P_{\nu}^{\mu} D_{\lambda} \omega^{\nu \lambda}$ & $F_{R}^{<\mu \nu>} \equiv u^{\alpha} u^{\beta} R^{<\mu}{ }_{\alpha}{ }^{\nu>}$ \\
$D_{\mu} E^{\mu}$ & $P_{\nu}^{\mu} D_{\lambda} B^{\nu \lambda}$ & $D^{<\mu} E^{\nu>}$ \\
\hline $9 S+6 V+9 T=\mathbf{2 4}=17 \mathrm{H}_{F}+7 \mathrm{H}_{S}+0 \mathrm{H}_{V}+0 \mathrm{~A}$ \\
\hline
\end{tabular}

Table 1. The 24 hydrostatic response terms for parity-even charged fluids at $2^{\text {nd }}$ order in derivative expansion. Among them, $\mathrm{H}_{F}=17$ combinations are forbidden by hydrostatic principle whereas the remaining $\mathrm{H}_{S}=7$ combinations are generated by using the first 7 scalars in the Lagrangian.

If these conditions which are familiar from thermodynamics (as the Euler relation and first law) are not satisfied then there is an obstruction to the existence of a hydrostatic partition function.

- In addition one finds that (see [37] or earlier works such as [68] for a derivation)

$$
\alpha_{1}=\alpha_{2}=\alpha_{3}=\alpha_{4}=0, \quad \chi_{E}=\chi_{E}=0
$$

- The coefficient of sign-definite terms contributing to $\Delta$ must be correct. Evaluating the relevant terms we obtain (5.2) as expected and learn that viscosities and conductivities are positive definite. In section 5.2 we have already given the result that these terms can be obtained from the Class D parametrization (14.3) by choosing $\Upsilon_{\boldsymbol{\eta}_{g}}=\Upsilon_{\boldsymbol{\sigma}_{A}}=\mathrm{Id}$ and $\Upsilon_{\boldsymbol{\sigma}_{g}}=\Upsilon_{\boldsymbol{\eta}_{A}}=0$ along with the Class $\mathrm{D}_{v}$ tensor structures

$$
\eta^{\mu \nu \alpha \beta}=T \zeta P^{\mu \nu} P^{\alpha \beta}+2 T \eta P^{\alpha<\mu} P^{\nu>\beta}, \quad \sigma^{\alpha \beta}=T \sigma_{\mathrm{Ohm}} P^{\alpha \beta} .
$$

Having dispensed with the leading order, let us move to the second order constitutive relations. There are in total 51 parity-even 2-derivative terms that can appear in the charged fluid constitutive relations [3, 37] among which 9 scalars, 6 transverse vectors and 9 transverse traceless tensors (i.e., a total of 24 terms as in table 1) enter the hydrostatic description. The existence of hydrostatics imposes a series of constraints on these 24 terms. The most straightforward way to derive these constraints is to write down the most general hydrostatic partition function using the first 7 hydrostatic scalars multiplied by arbitrary functions of $T, \mu$. Note that we have discarded the last 2 hydrostatic scalars which can be removed by an integration by parts in the partition function. Hence, we conclude $\mathrm{H}_{S}=7$. 


\begin{tabular}{|c|c|c|}
\hline \multicolumn{2}{|c|}{$2^{\text {nd }}$ order charged non-hydrostatic transport } \\
\hline Scalars & Vectors & Tensors \\
\hline$\Theta^{2}$ & & $\sigma^{\alpha<\mu} \sigma^{\nu>}{ }_{\alpha}$ \\
$\sigma^{2}$ & & $\Theta \sigma^{\mu \nu}$ \\
$\mathfrak{v}_{\alpha} \mathfrak{a}^{\alpha}$ & $\Theta \mathfrak{a}^{\mu}$ & \\
$\mathfrak{v}_{\alpha} E^{\alpha}$ & $\Theta E^{\mu}$ & \\
$\mathfrak{v}^{2}$ & $\Theta \mathfrak{v}^{\mu}$ & \\
& $\sigma^{\mu \nu} \mathfrak{v}_{\nu}$ & $\mathfrak{v}^{<\mu} \mathfrak{v}^{\nu>}$ \\
& $\sigma^{\mu \nu} \mathfrak{a}_{\nu}$ & $\mathfrak{v}^{<\mu} \mathfrak{a}^{\nu>}$ \\
& $\sigma^{\mu \nu} E_{\nu}$ & $\mathfrak{v}^{<\mu} E^{\nu>}$ \\
& $\omega^{\mu \nu} \mathfrak{v}_{\nu}$ & $\sigma^{\alpha<\mu} \omega^{\nu>}{ }_{\alpha}$ \\
& $B^{\mu \nu} \mathfrak{v}_{\nu}$ & $\sigma^{\alpha<\mu} B^{\nu>}{ }_{\alpha}$ \\
\hline$\left(u^{\alpha} D_{\alpha}\right) \Theta$ & $P_{\nu}^{\mu}\left(u^{\alpha} D_{\alpha}\right) E^{\nu}$ & $\left(u^{\alpha} D_{\alpha}\right) \sigma^{\mu \nu}$ \\
$D_{\mu} \mathfrak{v}^{\mu}$ & $P^{\mu \nu} D_{\nu} \Theta$ & $D^{<\mu} \mathfrak{v}^{\nu>}$ \\
& $P_{\nu}^{\mu} D_{\lambda} \sigma^{\nu \lambda}$ & \\
\hline $7 S+11 V+9 T=27=5 \overline{\mathrm{H}}_{S}+2 \overline{\mathrm{H}}_{V}+11 \mathrm{~B}+9 \mathrm{D}$ \\
\hline
\end{tabular}

Table 2. The 27 non-hydrostatic transport terms for parity-even charged fluid at $2^{\text {nd }}$ order in derivative expansion. Among these, $\overline{\mathrm{H}}_{S}=5$ combinations are generated by inserting the first 5 non-hydrostatic scalars into the Lagrangian. Two combinations are generated by inserting terms proportional to the non-hydrostatic vectors $\left\{\sigma^{\mu \nu} \mathfrak{v}_{\nu}, \Theta \mathfrak{v}^{\mu}\right\}$ in the free energy current, thus $\overline{\mathrm{H}}_{V}=2$. Among the rest, there are 11 combinations in Class B and 9 combinations in Class D. Explicit expressions for these 20 combinations are given in table 7 .

For this system, there are no hydrostatically conserved vectors $\left(\mathrm{H}_{V}=0\right)$ and no possible anomalies $(\mathrm{A}=0)$.

By varying this partition function, we then get the most general hydrostatic energy momentum tensor consistent with hydrostatic principle. This procedure then fixes the 24 response coefficients in terms of 7 functions that appear in the partition function. Eliminating these 7 functions, we get $24-7=17$ relations thus giving the number of hydrostatically forbidden combinations as $\mathrm{H}_{F}=17$.

We now turn to the non-hydrostatic transport parameters listed in table 2 . There are 7 scalars, 11 transverse vectors and 9 transverse traceless tensors which add up to 27 nonhydrostatic transport coefficients. Of these, 5 combinations can be obtained from including the first 5 non-hydrostatic scalars into a Lagrangian (the last 2 scalars can be discarded via integration by parts). These 5 combinations give $\overline{\mathrm{H}}_{S}=5$.

The remainder of the analysis involves figuring out which of the residual $27-5=22$ terms can be obtained in the three non-Lagrangian classes $\left\{\overline{\mathrm{H}}_{V}, \mathrm{~B}, \mathrm{D}\right\}$. These are classified by the set of admissible tensor structures which were described in the preceding. We have already shown in section 11.2 that $\overline{\mathrm{H}}_{V}=2$ by explicit construction; this involved 
considering all zeroth order tensor structures $\left\{\mathfrak{C}_{\mathcal{N}}^{\rho((\mu \nu) \mid(\alpha \beta))}, \mathfrak{C}_{\mathcal{X}}^{\rho(\mu \nu) \alpha}, \mathfrak{C}_{\mathcal{S}}^{\rho(\alpha \beta)}\right\}$ and plugging them into (14.14). Independent data was obtained from the two combinations

$$
\begin{aligned}
\mathfrak{C}_{\mathcal{X}}^{\rho(\mu \nu) \alpha} & =T C_{1}(T, \mu) P^{\rho<\mu} P^{\nu>\alpha}+T C_{2}(T, \mu) P^{\mu \nu} P^{\rho \alpha} \\
\Rightarrow \quad \mathcal{G}^{\rho} & =2 C_{1} \sigma^{\rho \alpha} \mathfrak{v}_{\alpha}+2 C_{2} \Theta \mathfrak{v}^{\rho} .
\end{aligned}
$$

Let us now turn to Classes B and D. All independent Class B and D constitutive relations are shown in table 7 . The $11+9$ combinations presented there can be constructed by classifying all possible first order transverse tensor structures $\left\{\mathcal{N}^{\mu \nu \alpha \beta}, \mathcal{X}^{\mu \nu \alpha}, \mathcal{S}^{\alpha \beta}\right\}$ in the constitutive relations (14.6) and (14.4), respectively. All inequivalent Class B terms can be obtained (up to numerical factors) by plugging the following 11 tensor structures into (14.6): ${ }^{85}$

$$
\begin{aligned}
\mathcal{N}^{\mu \nu \alpha \beta} & \in T\left\{\sigma^{\mu \nu} P^{\alpha \beta}, \omega^{\mu \alpha} P^{\nu \beta}, B^{\mu \alpha} P^{\nu \beta}\right\}, \\
\mathcal{X}^{\mu \nu \alpha} & \in T\left\{P^{\mu \nu} \mathfrak{v}^{\alpha}, P^{\mu \nu} E^{\alpha}, P^{\mu \nu} \mathfrak{a}^{\alpha}, \mathfrak{v}^{<\mu} P^{\nu>\alpha}, E^{<\mu} P^{\nu>\alpha}, \mathfrak{a}^{<\mu} P^{\nu>\alpha}\right\}, \\
\mathcal{S}^{\alpha \beta} & \in T\left\{\omega^{\alpha \beta}, B^{\alpha \beta}\right\} .
\end{aligned}
$$

The 9 Class $\mathrm{D}$ terms listed in table 7 can be obtained by plugging the following tensor structures into (14.4):

$$
\begin{aligned}
\mathcal{N}^{\mu \nu \alpha \beta} & \in T\left\{\sigma^{\mu \nu} P^{\alpha \beta}, \sigma^{\mu \alpha} P^{\nu \beta}, \Theta P^{\mu \nu} P^{\alpha \beta}\right\}, \\
\mathcal{X}^{\mu \nu \alpha} & \in T\left\{P^{\mu \nu} \mathfrak{v}^{\alpha}, P^{\mu \nu} E^{\alpha}, P^{\mu \nu} \mathfrak{a}^{\alpha}, \mathfrak{v}^{<\mu} P^{\nu>\alpha}, E^{<\mu} P^{\nu>\alpha}, \mathfrak{a}^{<\mu} P^{\nu>\alpha}\right\}, \\
S^{\alpha \beta} & =0 .
\end{aligned}
$$

These lists for Classes B and D are exhaustive in the sense that any other tensor structure leads to Class B and D constitutive relations with frame invariant data being the same as of those already obtained (or linear combinations thereof). ${ }^{86}$ Note that we are not guaranteed that the parametrization (14.4) is exhaustive - we only verify this a-posteriori by observing that we have found a total of 51 inequivalent terms in all the classes of transport which matches the total number of inequivalent tensor structures that are possible for the second order charged fluids.

We can generalize the above discussion to parity-odd transport using the results of [86]. There are 2 additional parity-odd pseudo-vectors at first order in $d=4$ (the magnetic field vector $B^{\mu}$ and the vorticity vector $\left.\ell^{\mu}=\varepsilon^{\mu \alpha \rho \sigma} u_{\alpha} \nabla_{\rho} u_{\sigma}\right) .27$ parity-odd terms at second order $(27=6 S+9 V+12 T)$. Out of these second order terms $12=4 S+2 V+6 T$ are hydrostatic and can be obtained from an equilibrium partition function parameterized by two scalars: hence $\mathrm{H}_{S}=2$ and $\mathrm{H}_{F}=10$ (which includes A). Additionally one can see from their table 2 that $\overline{\mathrm{H}}_{S}=2$. This leaves us with 13 further terms $(7 \mathrm{~V}+6 \mathrm{~T})$ which should belong to the other classes. We leave it as an exercise for the reader to complete the classification for this case.

\footnotetext{
${ }^{85}$ Note that each of the tensor structures appears with an arbitrary function of $T, \mu$ multiplying it. The transport coefficients themselves are determined by suitable (differential) linear combinations of these functions.

${ }^{86}$ For instance, an obvious structure one might want to add to Class D parameterization is $\mathcal{S}^{\alpha \beta}=\sigma^{\alpha \beta}$. However, this gives constitutive relations which are a linear combination of the Class B terms and the Class D terms originating from $\mathcal{X}^{\mu \nu \alpha}=P^{\mu \nu} \mathfrak{v}^{\alpha}$.
} 


\begin{tabular}{|c|c|c|}
\hline \multicolumn{2}{|c|}{$2^{\text {nd }}$ order Weyl invariant neutral fluids: hydrostatic response } \\
\hline Scalars & Vectors & Tensors \\
\hline$\omega^{2}$ & & $\omega^{\alpha<\mu} \omega^{\nu>}{ }_{\alpha}$ \\
${ }^{\mathcal{W}} R$ & & $C^{<\mu \alpha \nu>\beta} u_{\alpha} u_{\beta}$ \\
\hline & $P_{\nu}^{\mu} \mathcal{D}_{\lambda}^{\mathcal{W}} \omega^{\nu \lambda}$ & \\
\hline \multicolumn{3}{|c|}{$2 T=\mathbf{2}=0 \mathrm{H}_{F}+2 \mathrm{H}_{S}+0 \mathrm{H}_{V}+0 \mathrm{~A}$} \\
\hline
\end{tabular}

Table 3. The 2 hydrostatic response terms for Weyl invariant parity-even neutral fluid at $2^{\text {nd }}$ order in derivative expansion. We have listed the scalars and vectors though they do not contribute to frame-invariant transport data. Both the relevant symmetric tensors can be obtained by using the 2 scalars in the Lagrangian, see section 8.1 for details.

\begin{tabular}{|c|c|c|}
\hline \multicolumn{2}{|c|}{$2^{\text {nd }}$ order Weyl invariant neutral fluids: non-hydrostatic transport } \\
\hline Scalars & Vectors & Tensors \\
\hline$\sigma^{2}$ & & $\sigma^{\alpha<\mu} \sigma^{\nu>}{ }_{\alpha}$ \\
& & $\sigma^{\alpha<\mu} \omega^{\nu>}{ }_{\alpha}$ \\
\hline & $P_{\nu}^{\mu} \mathcal{D}_{\lambda}^{\mathcal{W}} \sigma^{\nu \lambda}$ & $\left(u^{\alpha} \mathcal{D}_{\alpha}^{\mathcal{W}}\right) \sigma^{\mu \nu}$ \\
\hline \multicolumn{3}{|c|}{$3 T=\mathbf{3}=1 \overline{\mathrm{H}}_{S}+0 \overline{\mathrm{H}}_{V}+1 \mathrm{~B}+1 \mathrm{D}$} \\
\hline
\end{tabular}

Table 4. The 3 non-hydrostatic transport terms for Weyl invariant parity-even neutral fluid at $2^{\text {nd }}$ order in derivative expansion. We have listed the scalars and vectors though they do not contribute to frame-invariant transport data. Among the symmetric tensors, $\overline{\mathrm{H}}_{S}=1$ combination is generated by inserting $\sigma^{2}$ into the Lagrangian. We have $\overline{\mathrm{H}}_{V}=0$ since $\sigma^{\alpha<\mu} \omega^{\nu>}{ }_{\alpha}$ is in Class B and $\sigma^{\alpha<\mu} \sigma^{\nu>}{ }_{\alpha}$ is in Class D.

\subsection{Example II: Weyl invariant fluid dynamics}

While the second order charged fluid allows us to illustrate the complete set of constitutive relations within the eightfold way, it is useful to record some examples which can be tested at least theoretically using the fluid/gravity correspondence [41, 52]. We therefore give a summary of how various terms for Weyl invariant fluids (both neutral and charged) fit into our classification scheme. In section 14.5 we will provide explicit evidence of the eightfold classification in holography.

Let us now consider the case of second order fluid dynamics with Weyl invariance. The case of uncharged fluids is summarized in tables 3 and 4 .

There is a total of 5 Weyl invariant second order symmetric tensors that can enter the most general symmetry allowed constitutive relations, c.f. (8.20). Our Lagrangian analysis in section 8.1.3 showed explicitly that the 2 combinations $\omega^{\alpha<\mu} \omega^{\nu>}{ }_{\alpha}$ and $C^{<\mu \alpha \nu>\beta} u_{\alpha} u_{\beta}$ are in the hydrostatic Class $\mathrm{H}_{S}$ and a third term $\left(u^{\alpha} \mathcal{D}_{\alpha}^{\mathcal{W}}\right) \sigma^{\mu \nu}$ is Class $\overline{\mathrm{H}}_{S}$. These 3 terms can be obtained from a Lagrangian constructed out of the 3 Weyl invariant scalars. 2 more tensor structures in table $4, \sigma^{\alpha<\mu} \omega^{\nu>}{ }_{\alpha}$ and $\sigma^{\alpha<\mu} \sigma^{\nu>}{ }_{\alpha}$, are non-Lagrangian terms in Class B and Class D, respectively. Both these combinations are surprisingly absent in 


\begin{tabular}{|c|c|c|}
\hline \multicolumn{2}{|c|}{$2^{\text {nd }}$ order Weyl invariant charged fluids: hydrostatic response } \\
\hline Scalars & Vectors & Tensors \\
\hline$\omega^{2}$ & & $\omega^{\alpha<\mu} \omega^{\nu>}{ }_{\alpha}$ \\
$\omega_{\alpha \beta} B^{\alpha \beta}$ & $\omega^{\mu \nu} E_{\nu}$ & $\omega^{\alpha<\mu} B^{\nu>}{ }_{\alpha}$ \\
$B^{2}$ & $B^{\mu \nu} E_{\nu}$ & $B^{\alpha<\mu} B^{\nu>}{ }_{\alpha}$ \\
$E^{2}$ & & $E^{<\mu} E^{\nu>}$ \\
${ }^{\mathcal{W}} R$ & & $C^{\mu \alpha \nu \beta} u_{\alpha} u_{\beta}$ \\
\hline $\mathcal{D}_{\mu}^{\mathcal{W}} E^{\mu}$ & $P_{\nu}^{\mu} \mathcal{D}_{\lambda}^{\mathcal{W}} B^{\nu \lambda}$ & $\mathcal{D}_{<\mu}^{\mathcal{W}} E_{\nu>}$ \\
& $P_{\nu}^{\mu} \mathcal{D}_{\lambda}^{\mathcal{W}} \omega^{\nu \lambda}$ \\
\hline \multicolumn{2}{|c|}{$4 V+6 T=\mathbf{1 0}=5 \mathrm{H}_{F}+5 \mathrm{H}_{S}+0 \mathrm{H}_{V}+0 \mathrm{~A}$} \\
\hline
\end{tabular}

Table 5. The 10 hydrostatic response terms for Weyl invariant, parity-even, charged fluid at $2^{\text {nd }}$ order in derivative expansion. We have also enumerated the scalars despite the fact that they do not appear in the constitutive relations of a Weyl invariant fluid. Among the constitutive relations generated by the vectors and symmetric tensors, $\mathrm{H}_{F}=5$ combinations are forbidden by hydrostatic principle whereas the remaining $\mathrm{H}_{S}=5$ combinations are generated by using the first 5 scalars in the Lagrangian.

fluids dual to Einstein gravity - we only generate the particular linear combination of the 3 non-hydrostatic terms that is in $\overline{\mathrm{H}}_{S}$, cf., section 8.1 for a discussion.

We now turn to the case of second order charged fluids with Weyl invariance. All symmetry allowed tensor structures are summarized in tables 5 and 6 .

The hydrostatic case is easy to intuit. We have 10 hydrostatic vectors and symmetric tensors which generate constitutive relations. $\mathrm{H}_{S}=5$ of these terms can be obtained from a Lagrangian that contains the 5 hydrostatic Weyl invariant scalars. The remaining $\mathrm{H}_{F}=5$ are forbidden by the second law constraint. Beyond hydrostatics, there are another 13 vectors and tensors, $\overline{\mathrm{H}}_{S}=3$ of which can be generated by Lagrangians. The remaining 10 non-Lagrangian terms split into $\left\{\overline{\mathrm{H}}_{V}, \mathrm{~B}, \mathrm{D}\right\}=\{1,6,3\}$. Explicit expressions for the 10 combinations are given in table 7 . Their corresponding constitutive relations as listed in table 7 are precisely those that are obtained in the general constructions (14.29), (14.30) and (14.31), but restricting to Weyl invariant tensor structures. Practically, this means deleting all those constitutive relations that were obtained in the non-Lagrangian classes before, which contain non-Weyl invariant objects such as $\Theta$ and $\mathfrak{a}^{\mu}$.

\subsection{Adiabatic fluids in holography and kinetic theory}

Given the relations obtained for adiabatic Weyl invariant fluids in Class L up to second order in gradients, we have now some structural understanding of the class of hydrodynamic systems we are dealing with. We can for instance ask if there are physical theories which are aware of the adiabatic eightfold classification. The answer turns out to be a resounding confirmation of using adiabaticity to classify hydrodynamic transport. We will explain this statement, by examining to distinct hydrodynamic systems that are physically motivated 


\begin{tabular}{|c|c|c|}
\hline \multicolumn{2}{|c|}{$2^{\text {nd }}$ order Weyl invariant charged fluids: non-hydrostatic transport } \\
\hline Scalars & Vectors & Tensors \\
\hline$\sigma^{2}$ & & $\sigma^{\alpha<\mu} \sigma^{\nu>}{ }_{\alpha}$ \\
$\mathfrak{v}_{\alpha} E^{\alpha}$ & & \\
$\mathfrak{v}^{2}$ & & \\
& $\sigma^{\mu \nu} \mathfrak{v}_{\nu}$ & $\mathfrak{v}^{<\mu} \mathfrak{v}^{\nu>}$ \\
& $\sigma^{\mu \nu} E_{\nu}$ & $\mathfrak{v}^{<\mu} E^{\nu>}$ \\
& $\omega^{\mu \nu} \mathfrak{v}_{\nu}$ & $\sigma^{\alpha<\mu} \omega^{\nu>}{ }_{\alpha}$ \\
& $B^{\mu \nu} \mathfrak{v}_{\nu}$ & $\sigma^{\alpha<\mu} B^{\nu>}{ }_{\alpha}$ \\
\hline \multirow{3}{*}{$\mathcal{D}_{\mu}^{\mathcal{W}} \mathfrak{v}^{\mu}$} & $P_{\nu}^{\mu}\left(u^{\alpha} \mathcal{D}_{\alpha}^{\mathcal{W}}\right) E^{\nu}$ & $\left(u^{\alpha} \mathcal{D}_{\alpha}^{\mathcal{W}}\right) \sigma^{\mu \nu}$ \\
\hline \multicolumn{2}{|c|}{$P_{\nu}^{\mu} \mathcal{D}_{\lambda}^{\mathcal{W}} \sigma^{\nu \lambda}$} & $\mathcal{D}_{<\mu}^{\mathcal{W}} \mathfrak{v}_{\nu>}$ \\
\hline \multicolumn{2}{|c|}{$6 V+7 T=\mathbf{1 3}=3 \overline{\mathrm{H}}_{S}+1 \overline{\mathrm{H}}_{V}+6 \mathrm{~B}+3 \mathrm{D}$} \\
\hline
\end{tabular}

Table 6. The 13 non-hydrostatic transport terms for Weyl invariant, parity-even charged fluid at $2^{\text {nd }}$ order in derivative expansion. Among these, $\overline{\mathrm{H}}_{S}=3$ combinations are generated by inserting the first 3 non-hydrostatic scalars into the Lagrangian. One combination is generated by inserting a term proportional to the non-hydrostatic vector $\sigma^{\mu \nu} \mathfrak{v}_{\nu}$ in the free energy current, thus $\overline{\mathrm{H}}_{V}=1$. Explicit expressions for the 6 combinations in Class B and 3 combinations in Class D can be found in table 7.

and for which we have data to compare transport coefficients. First, we look at holographic fluids arising in the fluid/gravity correspondence [41, 52] which arise from strongly coupled gauge theory plasmas. Subsequently we examine the fluids arising from weakly coupled quantum field theories which have been understood using kinetic theory. We will focus exclusively on second order transport since first order transport is entirely in Class $\mathrm{D}_{v}$.

\subsubsection{Holographic fluids and adiabaticity}

We begin by examining the transport properties of holographic fluids using the AdS/CFT correspondence. As is well known the class of fluids dual to two derivative Einstein-Hilbert gravity saturate the KSS bound [87] for shear viscosity $\eta / s=\frac{1}{4 \pi}-$ at first order. Since $\eta \sigma^{\mu \nu}$ is a Class $\mathrm{D}_{v}$ term, we have no information to gain from an adiabatic analysis. It is nevertheless interesting to note that entropy production encoded in $\Delta=2 \eta \sigma_{\mu \nu} \sigma^{\mu \nu}$ is minimized (assuming the KSS bound $\eta / s \geq \frac{1}{4 \pi}$ ).

At second order in the gradient expansion we have more to say, since there are definitely adiabatic parts to transport as we have discovered above. Let us start by understanding the relations (8.26) in the eightfold way. The transport coefficient $\left(\lambda_{1}-\kappa\right)$ is dissipative (Class $\left.\mathrm{D}_{s}\right)$; indeed, this term contributes to entropy production as $\nabla_{\mu} J_{S}^{\mu} \sim\left(\lambda_{1}-\kappa\right) \sigma_{\alpha \nu} \sigma^{\nu \beta} \sigma^{\alpha}{ }_{\beta}$ which is sub-dominant to the leading order $\eta \sigma^{\mu \nu} \sigma_{\mu \nu}$ entropy production. A-priori this ought not to be visible in an effective action (either Class $\mathrm{L}$ or $\mathrm{L}_{\mathrm{T}}$ ). On the other hand, $\left(\lambda_{2}+2 \tau-2 \kappa\right)$ is a Class B term, for which we ought to be writing a Class $\mathrm{L}_{\mathrm{T}}$ effective action. The three remaining terms in (8.22) encode the adiabatic part of the second or- 
der transport and are in one-to-one correspondence with the three free functions in the Lagrangian (8.25) parameterizing the Class L Landau-Ginzburg free energy. Correspondingly they are unconstrained by any hydrodynamic analysis. This is the general expectation from our classification scheme. Let us now turn to seeing what has been computed in the literature so far.

Firstly, we find a relation between the transport coefficients $\lambda_{1}$ and $\kappa$ :

$$
\lambda_{1}-\kappa=0 .
$$

This constraint follows directly in all dimensions for any large central charge quantum field theory whose holographic dual is given by Einstein-Hilbert gravity. This can be ascertained from the analysis of [65], see their eq. (4.6). It also holds in the case of charged fluids in $d=4$ (we believe it probably holds in all dimensions) as can be verified from eqs. (4.14) and (5.2) of [88]. Its validity in more general theories has not been checked as far as we are aware; it would be interesting to examine this relation in more general theories of gravity. As demonstrated in [46] it is a necessary consequence of non-dissipation; one can show that the on-shell entropy current is conserved iff $\kappa=\lambda_{1}$ as we expect from the adiabaticity perspective. The relation we note is not visible in hydrostatics since $\sigma_{\mu \nu}$ vanishes in equilibrium and one therefore is unable to fix the value of $\lambda_{1} ; \kappa$ on the other hand is part of thermodynamic response, cf., [3]. On the other hand we have seen that our Class L Landau-Ginzburg free energy lands us precisely on this subspace of allowed constitutive relations, cf., (8.26).

Curiously, we also find a second relation which fixes $\lambda_{2}$ despite it being a Class B term. In Class L we find this captured by the second relation (8.26). In fact, using (14.32) we can express this after eliminating $\kappa$ as a relation between $\tau, \lambda_{1}, \lambda_{2}$ when it is even more fascinating and easily recognized in holography:

$$
\tau=\lambda_{1}-\frac{1}{2} \lambda_{2}
$$

This is precisely the universal relation between second order transport shown to hold in a very broad class of theories by [89]. They derived the relation in two derivative theories of gravity coupled to arbitrary matter fields (scalars and gauge fields). ${ }^{87}$ This relation also holds naturally in the non-dissipative effective action approach, but is not demanded per se from entropy conservation [46]; the latter analysis leaves $\lambda_{2}$ unconstrained. From our modern perspective of adiabaticity, this makes sense as the term is part of Class B transport. It is rather surprising that not only the Class L theories fix the value of $\lambda_{2}$ but they also do so in a manner consistent with holography! ${ }^{88}$

Going beyond the two derivative gravity theories, we can ask if the relations (8.26) or (14.33) hold once we include higher order corrections to the gravitational Lagrangian.

\footnotetext{
${ }^{87}$ The relation given in eq. (9) of [89] uses different conventions and also has a small typo. The authors define the shear tensor with an extra factor of 2 relative to our definition. Furthermore, the sign in the definition of $\omega_{\mu \nu}$ should be flipped; this affects the sign of $\lambda_{2}$. We thank Michael Haack and Amos Yarom for double checking these results carefully and for discussions on implications and generalizations of this statement.

${ }^{88}$ The relation holds without imposing Weyl invariance in Class L as can be seen explicitly in appendix F.
} 
This would correspond to the finite coupling corrections to the strong coupling limit of the holographic plasma. So far it has been checked that (14.33) holds perturbatively in Gauss-Bonnet theories to leading order in the higher-derivative coupling [70], though not to next to leading order $[71,72]$. Curiously enough, higher derivative corrections that arise in string theory (from Type IIB flux compactification on $\mathbf{S}^{5}$ ) uphold this relation to one additional order [72] (to $\mathcal{O}\left(\lambda^{-3 / 2}\right)$ in the strong coupling perturbation expansion for the $\mathcal{N}=4 \mathrm{SYM}$ plasma). However, the original relations as stated in (8.26) are satisfied only to leading order in the higher derivative correction to gravity. From the adiabatic fluid perspective, (8.26) is a bit more fundamental since $\kappa-\lambda_{1}$ provides a measure for entropy production.

Viewing these relations as fixing a Class D and Class B term respectively is itself an interesting statement, independent of the precise values. While any physical fluid would of course have specific values of transport coefficients, one generically expects that the second order Weyl transport is a point in the five-dimensional space of parameters. Having extra constraints fixing two parameters in terms of the others is an interesting statement which deserves to be understood better. Moreover, the value chosen for $\lambda_{1}$ is such that no entropy is produced. This is rather remarkable hinting that holographic fluids are even more perfect than hitherto believed to be.

Finally, for completeness let us record the values of $\left\{k_{\sigma}, k_{\omega}, k_{R}\right\}$ that are suggested by holography. Translating the results of [65] we have ${ }^{89}$

$$
\begin{aligned}
k_{R} & =-\frac{c_{\mathrm{eff}}}{d-2}\left(\frac{4 \pi}{d}\right)^{d-2}, \\
k_{\omega} & =\frac{d-2}{2} k_{R}, \\
k_{\sigma} & =\frac{c_{\mathrm{eff}}}{2 d}\left(\frac{4 \pi}{d}\right)^{d-2} \text { Harmonic }\left(\frac{2}{d}-1\right),
\end{aligned}
$$

where Harmonic $(x)=\gamma_{e}+\frac{\Gamma^{\prime}(x)}{\Gamma(x)}$ is the Harmonic number function ( $\gamma_{e}$ is Euler's constant). Thus, the fluid-gravity result for second order neutral fluid transport can be determined explicitly from a Lagrangian density

$$
\mathcal{L}^{\mathcal{W}}=c_{\mathrm{eff}}\left(\frac{4 \pi T}{d}\right)^{d}-c_{\mathrm{eff}}\left(\frac{4 \pi T}{d}\right)^{d-2}\left[\frac{{ }^{\mathcal{w}} R}{(d-2)}+\frac{1}{2} \omega^{2}+\frac{1}{d} \text { Harmonic }\left(\frac{2}{d}-1\right) \sigma^{2}\right]
$$

where we have included also the zero derivative pressure term.

It is really amazing that the simple effective action (14.35) captures all the non-trivial results about the thermodynamics of a strongly coupled plasma along with the non-linear part of transport. Only the value of the first order Class D term, shear viscosity, is undetermined and indeed modulo this contribution (which is of course important), holographic plasmas are effectively adiabatic. Coupled with the low value of shear viscosity [87], it follows that flows of these plasmas tend to minimize the amount of dissipation. The nearly

\footnotetext{
${ }^{89}$ We use $c_{\text {eff }}$ to denote the effective central charge of the field theory; $c_{\mathrm{eff}}=\frac{\ell_{\mathrm{AdS}}}{16 \pi G_{N}}$. For $\mathcal{N}=4 \mathrm{SYM}$ in $d=4$ with gauge group $\mathrm{SU}(N)$ this is $\frac{1}{8 \pi^{2}} N^{2}$.
} 
perfect fluid picture, persists even more strongly perhaps at second order in gradients. We argue in section 18 that this suggests a minimum entropy production conjecture, which would be fascinating to understand in greater detail than explored herein.

It is an interesting challenge in fluid-gravity correspondence to give a gravity prescription to directly derive this expression. We advocate this as a sharp test for the proposals on how to think about AdS/CFT effective actions in the presence of horizons (see, for example [90]).

\subsubsection{Kinetic theory and adiabatic fluids}

We will now turn to examine the existing results in weakly coupled field theories in the light of our eightfold classification. Computations in weak coupling are surprisingly more difficult than the AdS/CFT computations in the previous subsection.

The transport coefficients in the hydrostatic Classes $\mathrm{H}_{S}$ and $\mathrm{H}_{V}$ are computable via straightforward Euclidean methods without any subtle issues regarding analytic continuation. At weak coupling, the leading contributions to these coefficients are generically given by free-theory results which can be then systematically corrected via perturbative expansion. These coefficients are also more amenable to numerical methods in lattice. The most common example is the computation of pressure as a function of temperature and chemical potential in various weakly coupled field theories.

The above state is to be contrasted with the non-hydrostatic classes which require realtime (Schwinger-Keldysh) techniques for their computation. Further, the leading contributions to these non-hydrostatic coefficients behave generically as inverse powers of coupling (and inverse powers of the logarithm of couplings) and often require careful resummations to deal with infrared issues. A common simplification in this context is to pass to an effective kinetic description. A paradigmatic example in this regard is the computation of viscosities and conductivities for weakly coupled theories [91, 92].

Computations of higher order transport coefficients are less common. Let us specialize to the case of $(3+1)$-dimensional parity-even, Weyl invariant neutral fluid transport coefficients at second order in derivative expansion. The hydrostatic transport coefficients in this case were computed by Moore and Sohrabi [93] whose result can be stated in our notations as

$$
-\lambda_{3}=3 \kappa=\frac{T^{2}}{48}\left(-4 N_{S}+2 N_{W F}+16 N_{V}\right)+O(g),
$$

where $N_{S}$ is the number of real scalars, $N_{W F}$ is the number of Weyl fermions and $N_{V}$ is the number of massless vectors in the theory. Here, $O(g)$ represents sub-leading corrections due to interactions..$^{90}$

The non-hydrostatic transport coefficients are more difficult to compute. For the $(3+$ 1)-dimensional parity-even, Weyl invariant neutral fluid, the leading order answers have been computed using kinetic theory in [53] (see also [67]). These leading answers are proportional to inverse powers of coupling (and inverse powers of the logarithm of couplings)

\footnotetext{
${ }^{90}$ We will refer the reader to [94-96] for a generalization of these results to parity odd transport coefficients. There is an unresolved discrepancy in the value of $\lambda_{3}$ between the results of [93] and [96].
} 
as expected. Thus, in contrast to the hydrostatic coefficients in (14.36) which are known up to zeroth order in coupling, the non-hydrostatic coefficients are known less precisely. The results of [53] translated into our notation takes the form ${ }^{91}$

$$
\begin{aligned}
\tau_{1} & =\frac{2 \eta^{2}}{\epsilon+p} \times 5.9 \text { to } 5.0 \quad \text { (varies with coupling) }: 6.10517 \text { in } \phi^{4} \text { theory } \\
\lambda_{1} & =\frac{4 \eta^{2}}{\epsilon+p} \times 5.2 \text { to } 4.1 \quad \text { (varies with coupling) }: 6.13264 \text { in } \phi^{4} \text { theory } \\
\kappa & =0, \quad \lambda_{2}=2 \tau .
\end{aligned}
$$

These results are valid for Debye screening lengths of the order of temperature (see [53] for more detailed plots of these transport coefficients as functions of coupling and the approximations involved).

We will draw the readers attention to a relation obtained by combining the last two transport results in (14.39)

$$
\lambda_{2}-2(\tau-\kappa)=0 .
$$

The relation $\lambda_{2}-2 \tau=0$ is a universal prediction of kinetic theory which follows naturally from the Boltzmann equation [53] and is consistent with earlier derivations [67]. The fact that [67] could ascertain $\lambda_{2}$ without knowing the collision kernel in the Boltzmann equation tells us that this is indeed a non-dissipative part of transport. Along with the fact that $\kappa=0$ at this order in coupling leads to the relation we have quoted above. We have however chosen to highlight the color of the sign, since it differs from the holographic result in (8.26). Indeed had the sign been consistent with the holographic result, we would have concluded that even in kinetic theory $\lambda_{2}$ would have had a value determined by a Class L Landau-Ginzburg free energy. It might be useful to cross-check this result independently (despite two independent confirmations above) to demonstrate that $\lambda_{2}$ is not obtainable from an effective action in kinetic theory. ${ }^{92}$

\footnotetext{
following is a useful dictionary to aid the translation between the references cited:

$$
\begin{aligned}
& \omega=-\omega_{[41]}=\omega_{[65]}=-\omega_{[89]}=-\omega_{[57]}=\omega_{[67]}=\omega_{[53]}, \\
& \sigma=\sigma_{[41]}=\sigma_{[65]}=\frac{1}{2} \sigma_{[89]}=\sigma_{[57]}=\frac{1}{2} \sigma_{[67]}=\frac{1}{2} \sigma_{[53]}, \\
& C=C_{[65]}=C_{[57]}=-\frac{1}{d-2} C_{[67]}=-\frac{1}{d-2} C_{[53]},
\end{aligned}
$$
}

${ }^{91}$ Unfortunately the literature is littered with a multitude of conventions for various hydrodynamic tensors which affects the numerical values of transport coefficients. As far as we have been able to ascertain the

with the un-subscripted symbols corresponding to the ones used in this text and $C$ is the tensor structure governing the curvature coupling of the fluid (given by Weyl tensor term $C^{\mu \rho \nu \sigma} u_{\rho} u_{\sigma}$ in our conventions). We have also taken the liberty to correct the sign in the definition of [89] based on a private correspondence with the authors (their eq. (7) should have an extra sign in front). In addition to further complicate signs, we have $\lambda_{2}$ multiplying different contracting of $\sigma^{\mu \nu}$ and $\omega^{\mu \nu}$ giving an additional sign to keep track of:

$$
\begin{array}{llll}
\text { coefficient } \lambda_{2}: & \left(\sigma_{\alpha}^{<\mu} \omega^{\alpha \nu>}\right) & \\
& \left(\sigma_{\alpha}^{<\mu} \omega^{\nu>\alpha}\right)_{[41]}, & \left(\sigma_{\alpha}^{<\mu} \omega^{\nu>\alpha}\right)_{[65]}, & \left(\sigma_{\alpha}^{<\mu} \omega^{\alpha \nu>}\right)_{[89]} \\
& \left(\sigma_{\alpha}^{<\mu} \omega^{\nu>\alpha}\right)_{[67]}, & \left(\sigma_{\alpha}^{<\mu} \omega^{\nu>\alpha}\right)_{[53]}, & \left(\sigma_{\alpha}^{<\mu} \omega^{\nu>\alpha}\right)_{[57]}
\end{array}
$$

${ }^{92}$ We thank Andrei Starinets for useful discussions on this point. 
We see that this combination chosen by kinetic theory is exactly the combination that we have identified as the Class B transport coefficient (and it is also one of the combinations which are zero in the two derivative gravity). We can thus state this universal result from kinetic theory as the statement that the Class B term is absent in the constitutive relation derived from kinetic theory. We will take this as an evidence that our eightfold classification gives a natural framework from which the kinetic theory results could be understood. It would be an interesting exercise to try to see whether one could simplify these kinetic theory computations using various techniques introduced in this paper.

\subsection{Eightfold classification for various fluid systems}

In the preceding subsections we have seen evidence for the eightfold classification of transport in various physical systems and we have also outlined how to transcribe the eightfold path in certain examples. We now give a comprehensive summary of the results in a tabular form for future reference. Tables 7 and 8 provide a classification of the total number of vector and tensor structures that give constitutive relations allowed by symmetry in various fluid systems up to second order in the derivative expansion.

The terms listed for Classes $\mathrm{H}_{S}$ and $\overline{\mathrm{H}}_{S}$ are scalars that can be used in a Lagrangian to generate independent constitutive relations. Similarly, Classes $\mathrm{H}_{V}$ and $\overline{\mathrm{H}}_{V}$ provide partition function vectors, i.e., free energy currents. Classes B and D are non-Lagrangian, so we give directly the expressions for stress tensors (and charge currents) that can be generated using the rules given in section 9 and section 5, respectively. Similarly, Class A terms are given directly as constitutive relations of stress tensor and charge current. Further taking into account a number $\mathrm{H}_{F}$ of terms that are disallowed by the existence of an equilibrium configuration (or equivalently by the second law of thermodynamics), the terms listed in each row of the tables exhaust the number of independent transport data. ${ }^{93}$

\section{Class $\mathrm{L}_{\mathrm{T}}$ : eightfold Lagrangian}

Our discussion thus far has focused on generating adiabatic constitutive relations and demonstrating how these help us classify hydrodynamic transport at arbitrary orders in the gradient expansion. This discussion is encapsulated in the statement of Theorem 1 . We have argued in that context for the completeness of our classification based on the structure of the adiabaticity equation.

We would now like to justify the statement more directly and in the process explain the basic rationale for considering adiabatic transport which has played a starring role. As a result we now introduce a novel ingredient in our analysis, which involves constructing a master Lagrangian. The adiabaticity equation is not derived from this master equation as a Bianchi identity of the underlying diffeomorphism and gauge symmetries as in the Class L discussion of section 6 , but rather follows as the statement of invariance under a new abelian gauge symmetry. The corresponding gauge field $\mathrm{A}^{(\mathrm{T})}{ }_{\mu}$ and the associated gauge group $U(1)_{\mathrm{T}}$ will be motivated below and argued to ensure that the associated Gauss Law

\footnotetext{
${ }^{93}$ We refrain from listing Class $\mathrm{C}$ constitutive relations in the tables as they are dimension specific and depend on the topology of the background $\mathcal{M}$ on which the fluid lives.
} 


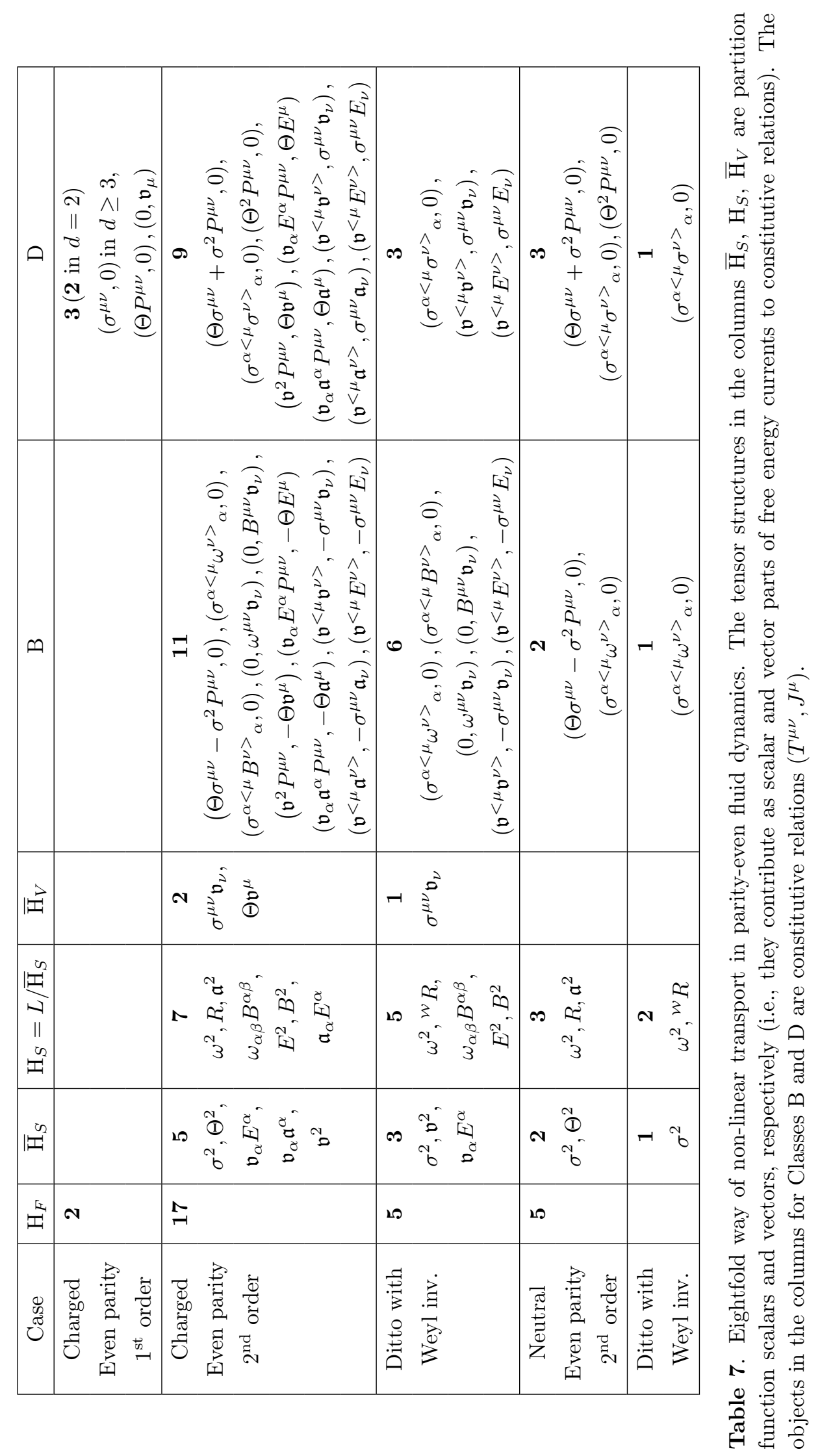




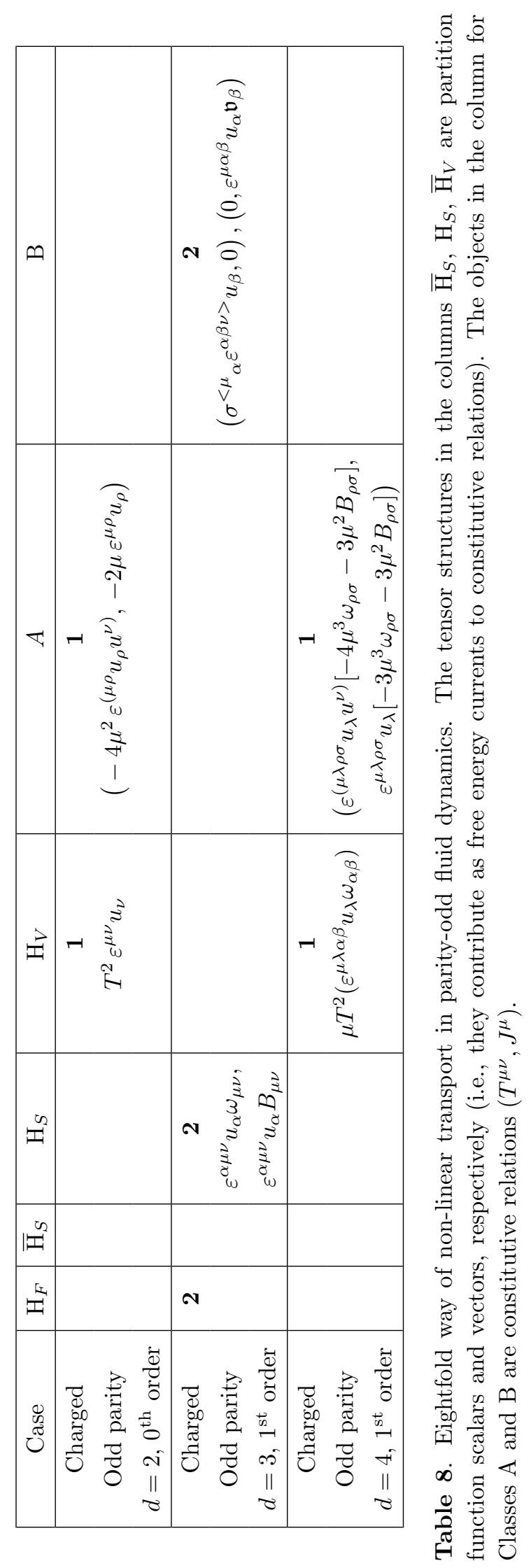


translates directly into the statement of adiabaticity. The main upshot of this construction is then to provide a constructive proof of Theorem 2 .

The framework which we christen Class $\mathrm{L}_{\mathrm{T}}$ (since it extends Class $\mathrm{L}$ to include the non-Lagrangian solutions to adiabaticity equation) involves not only a new symmetry, but also introduces some additional background fields. These are analogous to the SchwingerKeldysh counterparts of the metric and gauge field sources. The strategy we follow is to guess at a set of fields and invariances that are suggestive from our adiabatic analysis of part II. This allows us to postulate a master Lagrangian which generates precisely the constitutive relations consistent with adiabaticity equation, deriving for us the eightfold classification in the process. Furthermore, we will argue for the existence of an appropriate variational principle which yields exactly the hydrodynamic equations of motion. While satisfactory in terms of helping us complete our taxonomy, we don't provide here a fullfledged argument for why certain sources are doubled, nor do we give the actual relation between this construction and that of the Schwinger-Keldysh analysis of section $13 .{ }^{94} \mathrm{We}$ will just note here that from our preliminary analysis, the Class $\mathrm{L}_{\mathrm{T}}$ framework appears to capture some of the basic features necessary in the analysis of non-equilibrium transport. The lessons that we can glean from fleshing out this statement and the connections with the Schwinger-Keldysh construction will be deferred to a separate future publication [55].

\subsection{Introducing $U(1)_{\mathrm{T}}$ invariance}

We initially motivated the adiabaticity equation to capture the part of transport where off-shell entropy production was compensated by a flow of energy-momentum and charge. The latter currents are of course conserved as a consequence of diffeomorphism and gauge invariance of the underlying microscopic theory. In Class L we noticed further that working with a thermal density matrix parameterized by $\left\{\boldsymbol{\beta}^{\mu}, \Lambda_{\boldsymbol{\beta}}\right\}$ allowed us to extract a set of Bianchi identities which together imply the adiabaticity equation as a natural corollary, cf., section 6. We have however seen that Class L Lagrangians are not exhaustive in describing the space of adiabatic transport coefficients.

On the one hand this is to be expected because a genuine treatment of non-equilibrium field theory should require a Schwinger-Keldysh doubling of the degrees of freedom. From this viewpoint it is rather surprising that Class L Lagrangians already capture many aspects of adiabatic transport. On the other hand as we argued in section 13.4 there is something missing in a simple-minded construction of Schwinger-Keldysh doubled Lagrangian effective field theories. The problem is not so much in simply doubling the degrees of freedom and writing down independent Lagrangians for the left and right degrees of freedom, but rather in constraining the interactions between the two sets. The challenge is to keep the doubled degrees of freedom under control after coupling the two copies via Feynman-Vernon influence functionals. A-priori there would be two independent copies of diffeomorphism and flavour gauge symmetries that act on the system independently on the left and right. One might imagine breaking these down to the corresponding diagonal symmetries upon

\footnotetext{
${ }^{94}$ We will however note in the course of the discussion that the construction in Class $\mathrm{L}_{\mathrm{T}}$ appears to be consistent with the Schwinger-Keldysh doubling required for the anomalous hydrodynamic transport discussed in section 13 .
} 
introduction of the influence functionals. This however does not suffice to forbid terms that allow for violation of the second law of thermodynamics.

The principle of adiabaticity introduced in section 2 allows us to focus on the marginal case of zero entropy production. Based on this we would like to argue that we should understand first a basic principle that guarantees (2.12) as a statement of invariance. Since it asserts effectively that the entropy current is conserved on-shell (up to anomalous contributions), it is tempting to posit a gauge invariance whose associated current conservation leads directly to the adiabaticity equation. This we claim that will suffice to impose sufficient conditions on the Feynman-Vernon terms to ensure consistency with the second law of thermodynamics. More precisely, it ensures that such terms are consistent with the microscopic KMS condition.

Let us then record the ingredients we deem necessary to construct an effective Lagrangian for all the adiabatic constitutive relations. Firstly we have the low energy fluid degrees of freedom $\left\{\boldsymbol{\beta}, \Lambda_{\boldsymbol{\beta}}\right\}$ and the background sources $\left\{g_{\mu \nu}, A_{\mu}\right\}$. These can be viewed as functionals of the maps from some reference configuration, whence we can directly deal with the Goldstone bosons as described in section 13. We will in addition postulate the existence of a second set of sources, which we call $\left\{\tilde{g}_{\mu \nu}, \tilde{A}_{\mu}\right\}$.

The abelian symmetry which we will henceforth refer to as $U(1)_{\mathrm{T}}$ can be viewed as a KMS-gauge symmetry. ${ }^{95}$ This symmetry acts as a thermal diffeomorphism or flavour gauge transformation on the sources. As indicated above it corresponds to difference diffeomorphisms/gauge transformations which are aligned with the hydrodynamic fields $\left\{\boldsymbol{\beta}^{\mu}, \Lambda_{\boldsymbol{\beta}}\right\}$. The KMS-gauge gauge field corresponding to the $U(1)_{\mathrm{T}}$ symmetry will be denoted as $\mathrm{A}^{(\mathrm{T})}{ }_{\mu}$. We have in addition an associated holonomy field $\Lambda_{\boldsymbol{\beta}}^{(\mathrm{T})}$ and a $U(1)_{\mathrm{T}}$ chemical potential $\Lambda_{\boldsymbol{\beta}}^{(\mathrm{T})}+\boldsymbol{\beta}^{\mu} \mathrm{A}^{(\mathrm{T})} \mu$.

The diffeomorphism and flavour transformations on the fields in an obvious manner. On the contrary $U(1)_{\mathrm{T}}$ acts nonlinearly and mixes with flavour and diffeomorphism transformations:

- On all fields, $U(1)_{\mathrm{T}}$ acts as a longitudinal diffeomorphism and flavour gauge transformation along $\left\{\boldsymbol{\beta}^{\mu}, \Lambda_{\boldsymbol{\beta}}\right\}$.

- In addition, on $\left\{\tilde{g}_{\mu \nu}, \tilde{A}_{\mu}\right\}$, there is a further shift by $\left\{\delta_{\mathfrak{B}} g_{\mu \nu}, \delta_{\mathfrak{B}} A_{\mu}\right\}$.

- The field $\mathrm{A}^{(\mathrm{T})}{ }_{\mu}$ transforms as a connection for $U(1)_{\mathrm{T}}$ and $\Lambda_{\boldsymbol{\beta}}^{(\mathrm{T})}$ acts like a gauge transformation parameter, viz., $\Lambda_{\boldsymbol{\beta}}^{(\mathrm{T})}+\boldsymbol{\beta}^{\sigma} \mathrm{A}^{(\mathrm{T})}{ }_{\sigma}$ is invariant.

It is worth noting that from a Schwinger-Keldysh point of view, these transformation rules are not the most natural ones. It would have been more natural to retain the abelian part of the non-diagonal diffeomorphism and flavour gauge symmetries along $\mathcal{B}$. We anticipate that the difference is due to the fact that the natural basis of sources chosen here is

\footnotetext{
${ }^{95}$ The nomenclature is suggestive, but as we have explained in the text we will not justify this at present.
} 
not the canonical Schwinger-Keldysh choice. In fact it seems plausible to conjecture that

$$
\begin{aligned}
g_{\mu \nu}^{\mathrm{R}} & =g_{\mu \nu}, \\
A_{\mu}^{\mathrm{R}} & =A_{\mu} \\
g_{\mu \nu}^{\mathrm{L}} & =g_{\mu \nu}-\tilde{g}_{\mu \nu}-\boldsymbol{\beta}_{\mu} \mathrm{A}^{(\mathrm{T})}{ }_{\nu}-\boldsymbol{\beta}_{\nu} \mathrm{A}^{(\mathrm{T})}{ }_{\mu}, \\
A_{\mu}^{\mathrm{L}} & =A_{\mu}-\tilde{A}_{\mu}-\left(\Lambda_{\boldsymbol{\beta}}+\boldsymbol{\beta}^{\alpha} A_{\alpha}\right) \mathrm{A}^{(\mathrm{T})}{ }_{\mu}
\end{aligned}
$$

as the appropriate identifications for the right $(\mathrm{R})$ and left $(\mathrm{L})$ sources, respectively. We will however not flesh this out in great detail, since it (a) appears much cleaner in the formalism we introduce to write down $U(1)_{\mathrm{T}}$ invariant Lagrangians and (b) the connections with the Schwinger-Keldysh construction are being deferred to a separate publication [55] anyway. For the present the reader may therefore take our prescription merely as a technical tool to prove the completeness of our eightfold classification without worrying about the profound physical consequences.

\subsection{The fields and their transformation properties}

Let us start by writing down the extended set of fields and transformation properties based on the above discussion. We have the following fields which form the building blocks for the master Lagrangian:

1. the sources $\left\{g_{\mu \nu}, A_{\mu}\right\}$,

2. the fluid fields $\left\{\boldsymbol{\beta}^{\mu}, \Lambda_{\boldsymbol{\beta}}\right\}$,

3. partners for the sources $\left\{\tilde{g}_{\mu \nu}, \tilde{A}_{\mu}\right\}$ which are a symmetric tensor and a vector transforming in the adjoint representation of the flavour symmetry,

4. an additional $U(1)_{\mathrm{T}}$ gauge field $\mathrm{A}^{(\mathrm{T})}{ }_{\mu}$ and its holonomy field $\Lambda_{\boldsymbol{\beta}}^{(\mathrm{T})}$.

When necessary we will collectively refer to these fields as $\boldsymbol{\Psi}_{\mathrm{T}}$. The symmetries that any effective Lagrangian needs to preserve are diagonal diffeomorphisms/flavour gauge transformations (acting equally on sources and their partners) and in addition the abelian $U(1)_{T}$ thermal shift symmetry (which we claim enforces consistency of Feynman-Vernon terms).

Let us now record the transformation rules for the fields $\boldsymbol{\Psi}_{\mathrm{T}}$. We denote the transformation parameters of diffeomorphism, flavour, and $U(1)_{\mathrm{T}}$ transformations by $\left\{\xi, \Lambda, \Lambda^{(\mathrm{T})}\right\}$ respectively. In terms of these independent parameters, $U(1)_{\mathrm{T}}$ has a twisted action on the various fields. This is because fields transform non-linearly under it and part of the $U(1)_{\mathrm{T}}$ transformation involves diffeomorphisms and flavour gauge transformations. We will deal with the non-trivial mixing between diffeomorphism and flavour transformations on the one hand and $U(1)_{\mathrm{T}}$ on the other hand using the following trick: instead of using the original transformation parameters, we will move to a new basis of transformation parameters $\left\{\bar{\xi}^{\mu}, \bar{\Lambda}, \bar{\Lambda}^{(\mathrm{T})}\right\}$ which generate combinations of the original transformations which do not mix with each other. The original transformation parameters are related to these via

$$
\begin{aligned}
\xi^{\mu} & \equiv \bar{\xi}^{\mu}-\left(\bar{\Lambda}^{(\mathrm{T})}+\bar{\xi}^{\sigma} \mathrm{A}_{\sigma}^{(\mathrm{T})}\right) \boldsymbol{\beta}^{\mu}, & \bar{\xi}^{\mu} & \equiv \xi^{\mu}+\left(\Lambda^{(\mathrm{T})}+\xi^{\sigma} \mathrm{A}_{\sigma}^{(\mathrm{T})}\right) \boldsymbol{\beta}^{\mu}, \\
\Lambda & \equiv \bar{\Lambda}-\left(\bar{\Lambda}^{(\mathrm{T})}+\bar{\xi}^{\sigma} \mathrm{A}_{\sigma}^{(\mathrm{T})}{ }_{\sigma}\right) \Lambda_{\boldsymbol{\beta}}, & \bar{\Lambda} & \equiv \Lambda+\left(\Lambda^{(\mathrm{T})}+\xi^{\sigma} \mathrm{A}_{\sigma}^{(\mathrm{T})}{ }_{\sigma}\right) \Lambda_{\boldsymbol{\beta}}, \\
\Lambda^{(\mathrm{T})} & \equiv \bar{\Lambda}^{(\mathrm{T})}+\left(\bar{\Lambda}^{(\mathrm{T})}+\bar{\xi}^{\sigma} \mathrm{A}_{\sigma}^{(\mathrm{T})}\right) \boldsymbol{\beta}^{\nu} \mathrm{A}_{\nu}^{(\mathrm{T})}, & \bar{\Lambda}^{(\mathrm{T})} & \equiv \Lambda^{(\mathrm{T})}-\left(\Lambda^{(\mathrm{T})}+\xi^{\sigma} \mathrm{A}_{\sigma}^{(\mathrm{T})}\right) \boldsymbol{\beta}^{\nu} \mathrm{A}_{\nu}^{(\mathrm{T})} .
\end{aligned}
$$


We have given the translation between the two sets of gauge transformation parameters $\left\{\xi^{\mu}, \Lambda, \Lambda^{(\mathrm{T})}\right\}$ and $\left\{\bar{\xi}^{\mu}, \bar{\Lambda}, \bar{\Lambda}^{(\mathrm{T})}\right\}$ in both forward and reverse directions to facilitate translation between them in the future. A useful relation in converting between these parameters is

$$
\Lambda^{(\mathrm{T})}+\xi^{\sigma} \mathrm{A}_{\sigma}^{(\mathrm{T})}=\bar{\Lambda}^{(\mathrm{T})}+\bar{\xi}^{\sigma} \mathrm{A}_{\sigma}^{(\mathrm{T})} .
$$

The transformation rules: armed with this we are now in a position to write down the explicit transformations of various fields which takes a simple form in terms of the untwisted transformation parameters $\left\{\bar{\xi}^{\mu}, \bar{\Lambda}, \bar{\Lambda}^{(\mathrm{T})}\right\}: 96$

$$
\begin{aligned}
\delta_{x} g_{\mu \nu} & \equiv £_{\bar{\xi}} g_{\mu \nu}=D_{\mu} \bar{\xi}_{\nu}+D_{\nu} \bar{\xi}_{\mu}, \\
\delta_{x} A_{\mu} & \equiv £_{\bar{\xi}} A_{\mu}+\left[A_{\mu}, \bar{\Lambda}\right]+\partial_{\mu} \bar{\Lambda}=D_{\mu}\left(\bar{\Lambda}+\bar{\xi}^{\nu} A_{\nu}\right)+\bar{\xi}^{\nu} F_{\nu \mu}, \\
\delta_{x} \boldsymbol{\beta}^{\mu} & \equiv £_{\bar{\xi}} \boldsymbol{\beta}^{\mu}=\bar{\xi}^{\nu} D_{\nu} \boldsymbol{\beta}^{\mu}-\boldsymbol{\beta}^{\nu} D_{\nu} \bar{\xi}^{\mu} \\
\delta_{x} \Lambda_{\boldsymbol{\beta}}+A_{\nu} \delta_{x} \boldsymbol{\beta}^{\nu} & \equiv \bar{\xi}^{\mu} \delta_{\mathcal{B}} A_{\mu}-\boldsymbol{\beta}^{\mu} D_{\mu}\left(\bar{\Lambda}+\bar{\xi}^{\nu} A_{\nu}\right)+\left[\Lambda_{\boldsymbol{\beta}}+\boldsymbol{\beta}^{\lambda} A_{\lambda}, \bar{\Lambda}+\bar{\xi}^{\nu} A_{\nu}\right] .
\end{aligned}
$$

In terms of the original transformation parameters $\left\{\xi^{\mu}, \Lambda, \Lambda^{(\mathrm{T})}\right\}$, these transformations would mix diffeomorphism and flavour transformations with $U(1)_{\mathrm{T}}$. The advantage gained from working with $\left\{\bar{\xi}, \bar{\Lambda}, \bar{\Lambda}^{(\mathrm{T})}\right\}$ is an untwisting of $U(1)_{\mathrm{T}}$ such that $\left\{g_{\mu \nu}, A_{\mu}, \boldsymbol{\beta}^{\mu}, \Lambda_{\boldsymbol{\beta}}\right\}$ are blind to it. The partner sources $\left\{\tilde{g}_{\mu \nu}, \tilde{A}_{\mu}\right\}$ transform similarly, but in addition pick up an inhomogeneous piece which contains a source Lie-dragged along $\mathcal{B}$ under the $U(1)_{\mathrm{T}}$ action:

$$
\begin{aligned}
\delta_{x} \tilde{g}_{\mu \nu} & \equiv £_{\bar{\xi}} \tilde{g}_{\mu \nu}+\bar{\Lambda}^{(\mathrm{T})} \delta_{\mathcal{B}} g_{\mu \nu} \\
& =2 \tilde{g}_{\sigma(\mu} D_{\nu)} \bar{\xi}^{\sigma}+\bar{\xi}^{\sigma}\left(D_{\sigma} \tilde{g}_{\mu \nu}-\mathrm{A}_{\sigma}^{(\mathrm{T})} \delta_{\mathcal{B}} g_{\mu \nu}\right)+\left(\bar{\Lambda}^{(\mathrm{T})}+\bar{\xi}^{\sigma} \mathrm{A}_{\sigma}^{(\mathrm{T})}\right) \delta_{\mathcal{B}} g_{\mu \nu} \\
\delta_{x} \tilde{A}_{\mu} & \equiv £_{\bar{\xi}} \tilde{A}_{\mu}+\left[\tilde{A}_{\mu}, \bar{\Lambda}\right]+\bar{\Lambda}^{(\mathrm{T})} \delta_{\mathcal{B}} A_{\mu}
\end{aligned}
$$

Finally, the transformation of the $U(1)_{\mathrm{T}}$ connection and its holonomy are given by

$$
\begin{aligned}
\delta_{x} \mathrm{~A}^{(\mathrm{T})}{ }_{\mu} & \equiv £_{\bar{\xi}} \mathrm{A}^{(\mathrm{T})}{ }_{\mu}+\partial_{\mu} \bar{\Lambda}^{(\mathrm{T})}=D_{\mu}\left(\bar{\Lambda}^{(\mathrm{T})}+\bar{\xi}^{\nu} \mathrm{A}^{(\mathrm{T})}{ }_{\nu}\right)+\bar{\xi}^{\nu} \mathrm{F}^{(\mathrm{T})}{ }_{\nu \mu} \\
\delta_{x} \Lambda_{\boldsymbol{\beta}}^{(\mathrm{T})}+\mathrm{A}^{(\mathrm{T})}{ }_{\nu} \delta_{x} \boldsymbol{\beta}^{\nu} & \equiv \bar{\xi}^{\mu} \delta_{\mathcal{B}} \mathrm{A}^{(\mathrm{T})}{ }_{\mu}-\boldsymbol{\beta}^{\mu} D_{\mu}\left(\bar{\Lambda}^{(\mathrm{T})}+\bar{\xi}^{\nu} \mathrm{A}^{(\mathrm{T})}{ }_{\nu}\right) .
\end{aligned}
$$

The first line is just the usual transformation rule for the gauge field of an abelian symmetry; the second line is such that $\Lambda_{\boldsymbol{\beta}}^{(\mathrm{T})}+\boldsymbol{\beta}^{\nu} \mathrm{A}^{(\mathrm{T})}{ }_{\nu}$ is invariant. In fact, as we will see the present formalism is a natural extension of the Class $\mathrm{H}_{V}$ formalism of section 11.1. To obtain consistency between the two formalisms (see section 17.3), we are led to a natural choice for fixing the above invariant combination. We will choose

$$
\Lambda_{\boldsymbol{\beta}}^{(\mathrm{T})}+\boldsymbol{\beta}^{\sigma} \mathrm{A}_{\sigma}^{(\mathrm{T})}=1 .
$$

Given that the transformations rules have thus far been "pulled out of a hat", we demonstrate that they are consistent in appendix I. In particular, we will will check that they form an algebra such that the usual Wess-Zumino consistency conditions are satisfied. This allows us to proceed with confidence about these transformations.

\footnotetext{
${ }^{96}$ We denote the derivative operator which covariantly transforms under diffeomorphisms, flavour gauge, and $U(1)_{\top}$ transformations by $D_{\mu}$ in what follows. It is defined by appropriately extending (2.8) to incorporate $U(1)_{\mathrm{T}}$ transformations as well.
} 
Difference source combinations: while this completes the basic transformation rules from which all the subsequent expressions can be derived, it is convenient to consider a linear combination of the sources $\left\{g_{\mu \nu}, A_{\mu}\right\}$ and their partners $\left\{\tilde{g}_{\mu \nu}, \tilde{A}_{\mu}\right\}$ which is simplifies the expressions somewhat.

To appreciate this let us define the shifted partner sources

$$
\begin{gathered}
g_{\mu \nu}^{\prime} \equiv g_{\mu \nu}-\tilde{g}_{\mu \nu} \\
A_{\mu}^{\prime} \equiv A_{\mu}-\tilde{A}_{\mu}
\end{gathered}
$$

as well as the associated covariant derivative $D^{\prime}$ and field strength $F^{\prime}$ respectively. ${ }^{97}$ We define them as

$$
\begin{aligned}
D_{\alpha}^{\prime} X^{\mu \cdots \nu}{ }_{\rho \cdots \sigma} & =\nabla_{\alpha}^{\prime} X^{\mu \cdots \nu}{ }_{\rho \cdots \sigma}+\left[A_{\alpha}^{\prime}, X_{\rho \cdots \sigma}^{\mu \cdots \nu}\right], \\
F_{\mu \nu}^{\prime} & =\nabla_{\mu}^{\prime} A_{\nu}^{\prime}-\nabla_{\mu}^{\prime} A_{\nu}^{\prime}+\left[A_{\mu}^{\prime}, A_{\nu}^{\prime}\right]
\end{aligned}
$$

The primed covariant derivative acts on tensors as in (2.9).

Then it a simple exercise to see that we can rewrite (15.4) as

$$
\begin{aligned}
& \delta_{x} \tilde{g}_{\mu \nu}=\delta_{x} g_{\mu \nu}+\left(\bar{\Lambda}^{(\mathrm{T})}+\bar{\xi}^{\sigma} \mathrm{A}_{\sigma}^{(\mathrm{T})}\right) \delta_{\mathfrak{B}} g_{\mu \nu}-\left\{2 D_{(\mu}^{\prime}\left(g_{\nu) \rho}^{\prime} \bar{\xi}^{\rho}\right)+\bar{\xi}^{\sigma} \mathrm{A}_{\sigma}^{(\mathrm{T})} \delta_{\mathcal{B}} g_{\mu \nu}\right\}, \\
& \delta_{x} \tilde{A}_{\mu}=\delta_{x} A_{\mu}+\left(\bar{\Lambda}^{(\mathrm{T})}+\bar{\xi}^{\sigma} \mathrm{A}_{\sigma}^{(\mathrm{T})}{ }_{\sigma}\right) \delta_{\mathcal{B}} A_{\mu}-\left\{D_{\mu}^{\prime}\left(\bar{\Lambda}+\bar{\xi}^{\sigma} A_{\sigma}^{\prime}\right)+\bar{\xi}^{\sigma} F_{\sigma \mu}^{\prime}+\bar{\xi}^{\sigma} \mathrm{A}_{\sigma}^{(\mathrm{T})} \delta_{\mathcal{B}} A_{\mu}\right\} .
\end{aligned}
$$

In fact, we can more conveniently merge (15.3) and (15.10) into a transformation rule for the partner fields themselves. To wit,

$$
\begin{aligned}
& \delta_{x} g_{\mu \nu}^{\prime}=D_{\mu}^{\prime}\left(g_{\nu \rho}^{\prime} \bar{\xi}^{\rho}\right)+D_{\nu}^{\prime}\left(g_{\mu \rho}^{\prime} \bar{\xi}^{\rho}\right)+\bar{\xi}^{\sigma} \mathrm{A}_{\sigma}^{(\mathrm{T})}{ }_{\sigma} \delta_{\mathcal{B}} g_{\mu \nu}-\left(\bar{\Lambda}^{(\mathrm{T})}+\bar{\xi}^{\sigma} \mathrm{A}^{(\mathrm{T})}{ }_{\sigma}\right) \delta_{\mathfrak{B}} g_{\mu \nu}, \\
& \delta_{x} A_{\mu}^{\prime}=D_{\mu}^{\prime}\left(\bar{\Lambda}+\bar{\xi}^{\sigma} A_{\sigma}^{\prime}\right)+\bar{\xi}^{\sigma} F_{\sigma \mu}^{\prime}+\bar{\xi}^{\sigma} \mathrm{A}_{\sigma}^{(\mathrm{T})} \delta_{\mathcal{B}} A_{\mu}-\left(\bar{\Lambda}^{(\mathrm{T})}+\bar{\xi}^{\sigma} \mathrm{A}^{(\mathrm{T})}{ }_{\sigma}\right) \delta_{\mathcal{B}} A_{\mu} .
\end{aligned}
$$

Schwinger-Keldysh inspired combinations: above we have chosen to take the partner sources $\left\{g_{\mu \nu}^{\prime}, A_{\mu}^{\prime}\right\}$ without any potential contamination from $\mathrm{A}^{(\mathrm{T})}{ }_{\mu}$. However, attempts to reconcile the construction here with the Schwinger-Keldysh picture developed for Class A suggests that the combination that may be relevant is instead given as in (15.1). Taking this seriously let us consider the twisted partner sources (15.1) with suggestive names inspired by Schwinger-Keldysh construction. We can then rewrite (15.4) as

$$
\begin{aligned}
& \delta_{x} \tilde{g}_{\mu \nu}=\delta_{x} g_{\mu \nu}+\left(\bar{\Lambda}^{(\mathrm{T})}+\bar{\xi}^{\sigma} \mathrm{A}_{\sigma}^{(\mathrm{T})}\right) \delta_{\mathcal{B}} g_{\mu \nu}-\left\{2 D_{(\mu}^{\mathrm{L}}\left(g_{\nu) \rho}^{\mathrm{L}} \bar{\xi}^{\rho}\right)+\bar{\xi}^{\sigma} \mathrm{A}_{\sigma}^{(\mathrm{T})} \delta_{\mathcal{B}} g_{\mu \nu}\right\}, \\
& \delta_{x} \tilde{A}_{\mu}=\delta_{x} A_{\mu}+\left(\bar{\Lambda}^{(\mathrm{T})}+\bar{\xi}^{\sigma} \mathrm{A}_{\sigma}^{(\mathrm{T})}\right) \delta_{\mathcal{B}} A_{\mu}-\left\{D_{\mu}^{\mathrm{L}}\left(\bar{\Lambda}+\bar{\xi}^{\sigma} A_{\sigma}^{\mathrm{L}}\right)+\bar{\xi}^{\sigma} F_{\sigma \mu}^{\mathrm{L}}+\bar{\xi}^{\sigma} A_{\sigma}^{(\mathrm{T})} \delta_{\mathcal{B}} A_{\mu}\right\} .
\end{aligned}
$$

\footnotetext{
following:

$$
\begin{aligned}
D_{\mu}^{\prime}\left(g_{\nu \sigma}^{\prime} V^{\sigma}\right) & =D_{\mu}\left(g_{\nu \sigma}^{\prime} V^{\sigma}\right)+\frac{1}{2} V^{\sigma}\left(D_{\sigma} g_{\mu \nu}^{\prime}-D_{\mu} g_{\nu \sigma}^{\prime}-D_{\nu} g_{\mu \sigma}^{\prime}\right) \\
D_{\nu}^{\prime}\left(g_{\sigma \mu}^{\prime} T^{\mu \nu}\right) & =\frac{\sqrt{-g}}{\sqrt{-g^{\prime}}} D_{\nu}\left(\frac{\sqrt{-g^{\prime}}}{\sqrt{-g}} g_{\sigma \mu}^{\prime} T^{\mu \nu}\right)-\frac{1}{2} T^{\mu \nu} D_{\sigma} g_{\mu \nu}^{\prime}
\end{aligned}
$$
}

${ }^{97}$ Some useful identities for converting between covariant derivatives of two different metrics are the

where $V^{\sigma}$ and $T^{\mu \nu}$ are some general vector and a symmetric tensor respectively. 
and merge (15.3) and (15.12) into

$$
\begin{aligned}
\delta_{x} g_{\mu \nu}^{\mathrm{L}}= & 2 D_{(\mu}^{\mathrm{L}}\left(g_{\nu) \rho}^{\mathrm{L}} \bar{\xi}^{\rho}\right)+\bar{\xi}^{\sigma} \mathrm{A}_{\sigma}^{(\mathrm{T})} \delta_{\mathcal{B}} g_{\mu \nu}^{\mathrm{R}}-2 £_{\bar{\xi}}\left(\boldsymbol{\beta}_{(\mu} \mathrm{A}_{\nu)}^{(\mathrm{T})}\right)-\left(\bar{\Lambda}^{(\mathrm{T})}+\bar{\xi}^{\sigma} \mathrm{A}_{\sigma}^{(\mathrm{T})}\right) \delta_{\mathcal{B}} g_{\mu \nu}^{\mathrm{R}}, \\
\delta_{x} A_{\mu}^{\mathrm{L}}= & D_{\mu}^{\mathrm{L}}\left(\bar{\Lambda}+\bar{\xi}^{\sigma} A_{\sigma}^{\mathrm{L}}\right)+\bar{\xi}^{\sigma} F_{\sigma \mu}^{\mathrm{L}}+\bar{\xi}^{\sigma} \mathrm{A}^{(\mathrm{T})}{ }_{\sigma} \delta_{\mathcal{B}} A_{\mu}^{\mathrm{R}}-£_{\bar{\xi}}\left(\left(\Lambda_{\boldsymbol{\beta}}+\boldsymbol{\beta}^{\sigma} A_{\sigma}^{\mathrm{R}}\right) \mathrm{A}^{(\mathrm{T})}{ }_{\mu}\right) \\
& -\left[\Lambda_{\boldsymbol{\beta}}+\boldsymbol{\beta}^{\sigma} A_{\sigma}^{\mathrm{R}}, \bar{\Lambda}\right] \mathrm{A}^{(\mathrm{T})}{ }_{\mu}-\left(\bar{\Lambda}^{(\mathrm{T})}+\bar{\xi}^{\sigma} \mathrm{A}^{(\mathrm{T})}{ }_{\sigma}\right) \delta_{\mathcal{B}} A_{\mu}^{\mathrm{R}} .
\end{aligned}
$$

In much of our discussion we will only use the difference sources $\left\{g_{\mu \nu}^{\prime}, A_{\mu}^{\prime}\right\}$ and only briefly in the discussion involving anomalous hydrodynamics revert to the SchwingerKeldysh inspired $\left\{g_{\mu \nu}^{\mathrm{L}}, A_{\mu}^{\mathrm{L}}\right\}$. The translation between the two sets of languages being straightforward (the basic formulae are all given above), it should be simple to translate statements between the two if necessary.

\subsection{Bianchi identities in Class $\mathrm{L}_{\mathrm{T}}$}

We can now use the various fields introduced in the previous subsection to construct Lagrangians $\mathcal{L}_{\mathrm{T}}\left[\boldsymbol{\Psi}_{\mathrm{T}}\right]$ invariant under diffeomorphism, flavour, and $U(1)_{\mathrm{T}}$ transformations. This invariance yields Bianchi identities which we will now show imply the adiabaticity equation in the hydrodynamic limit (i.e., to linear order in the Schwinger-Keldysh difference fields). Let us parameterize the variation of $\mathcal{L}_{\mathrm{T}}$ by

$$
\begin{aligned}
\frac{1}{\sqrt{-g}} \delta & \left(\sqrt{-g} \mathcal{L}_{\mathrm{T}}\right)-\nabla_{\mu}\left(\not \Theta_{\mathrm{PS}}^{\mathrm{T}}\right)^{\mu} \\
= & \frac{1}{2} T_{\mathrm{L}}^{\mu \nu} \delta g_{\mu \nu}+J_{\mathrm{L}}^{\mu} \cdot \delta A_{\mu}+T \mathfrak{h}_{\sigma} \delta \boldsymbol{\beta}^{\sigma}+T \mathfrak{n} \cdot\left(\delta \Lambda_{\boldsymbol{\beta}}+A_{\sigma} \delta \boldsymbol{\beta}^{\sigma}\right) \\
& +\frac{1}{2} T_{\mathrm{L}^{c}}^{\mu \nu} \delta \tilde{g}_{\mu \nu}+J_{\mathrm{L}^{c}}^{\mu} \cdot \delta \tilde{A}_{\mu}+J_{\mathrm{T}}^{\sigma} \delta \mathrm{A}^{(\mathrm{T})}+T \mathfrak{n}_{\mathrm{T}}\left(\delta \Lambda_{\boldsymbol{\beta}}^{(\mathrm{T})}+\mathrm{A}^{(\mathrm{T})}{ }_{\sigma} \delta \boldsymbol{\beta}^{\sigma}\right) .
\end{aligned}
$$

The subscript $\mathrm{L}$ is supposed to indicate that these constitutive relations can be obtained from Class L as discussed in section 6. Similarly, the subscript $\mathrm{L}^{c}$ suggests that these will be all remaining adiabatic constitutive relations not obtainable from Class L.

The variation (15.14) defines the constitutive relations obtained from $\mathcal{L}_{\mathrm{T}}$. We can now explicitly perform the diffeomorphism, flavour gauge, and $U(1)_{\mathrm{T}}$ transformations using the explicit variations given in section 15.2. We simply replace $\delta$ in (15.14) by $\delta_{x}$ as defined by (15.3)-(15.5) and perform some necessary integration by parts to isolate the coefficients of the transformation parameters $\left\{\bar{\xi}^{\mu}, \bar{\Lambda}, \bar{\Lambda}^{(\mathrm{T})}\right\}$. As this exercise is a straightforward generalization of the analysis in Class L we simply quote the final answers for the Bianchi identities (some useful intermediate steps are given in appendix I).

- The diffeomorphism Bianchi identity is

$$
\begin{aligned}
D_{\mu}\left(T_{\mathrm{L}+\mathrm{L}^{c}}\right)_{\sigma}^{\mu}-J_{\mathrm{L}+\mathrm{L}^{c}}^{\nu} \cdot F_{\sigma \nu}-J_{\mathrm{T}}^{\nu} \cdot \mathrm{F}^{(\mathrm{T})}{ }_{\sigma \nu} \\
=\frac{1}{\sqrt{-g}} \delta_{\mathcal{B}}\left(\sqrt{-g} T \mathfrak{h}_{\sigma}\right)+T \mathfrak{n} \cdot \delta_{\mathcal{B}} A_{\sigma}+T \mathfrak{n}_{\mathrm{T}} \cdot \delta_{\mathcal{B}} \mathrm{A}_{\sigma}^{(\mathrm{T})} \\
\quad+D_{\nu}\left(g_{\sigma \mu}^{\prime} T_{\mathrm{L}^{c}}^{\mu \nu}\right)-\frac{1}{2} T_{\mathrm{L}^{c}}^{\mu \nu} D_{\sigma} g_{\mu \nu}^{\prime}-J_{\mathrm{L}^{c}}^{\nu} \cdot F_{\sigma \nu}^{\prime} \\
\quad-\mathrm{A}_{\sigma}^{(\mathrm{T})}\left(\frac{1}{2} T_{\mathrm{L}^{c}}^{\mu \nu} \delta_{\mathcal{B}} g_{\mu \nu}+J_{\mathrm{L}^{c}}^{\mu} \cdot \delta_{\mathcal{B}} A_{\mu}\right)-\tilde{A}_{\sigma} \cdot\left(D_{\mu} J_{\mathrm{L}^{c}}^{\mu}-\left[\tilde{A}_{\mu}, J_{\mathrm{L}^{c}}^{\mu}\right]\right) .
\end{aligned}
$$


- The flavour Bianchi identity is given by

$$
D_{\mu} J_{\mathrm{L}+\mathrm{L}^{c}}^{\mu}=\frac{1}{\sqrt{-g}} \delta_{\mathcal{B}}(\sqrt{-g} T \mathfrak{n})+\left(D_{\mu} J_{\mathrm{L}^{c}}^{\mu}-\left[\tilde{A}_{\mu}, J_{\mathrm{L}^{c}}^{\mu}\right]\right)
$$

- Finally, the $U(1)_{\mathrm{T}}$ Bianchi identity reads (after setting $\Lambda_{\boldsymbol{\beta}}^{(\mathrm{T})}+\boldsymbol{\beta}^{\sigma} \mathrm{A}^{(\mathrm{T})}{ }_{\sigma}=1$ ):

$$
\begin{aligned}
D_{\mu} \mathrm{N}_{\mathrm{T}}^{\mu}= & \frac{1}{2} T_{\mathrm{L}+\mathrm{L}^{c}}^{\mu \nu} \delta_{\mathcal{B}} g_{\mu \nu}+J_{\mathrm{L}+\mathrm{L}^{c}}^{\mu} \cdot \delta_{\mathcal{B}} A_{\mu}+\frac{1}{2} T_{\mathrm{L}^{c}}^{\mu \nu} \delta_{\mathcal{B}} \tilde{g}_{\mu \nu}+J_{\mathrm{L}^{c}}^{\mu} \cdot \delta_{\mathcal{B}} \tilde{A}_{\mu}+J_{\mathrm{T}}^{\mu} \delta_{\mathcal{B}} \mathrm{A}^{(\mathrm{T})}{ }_{\mu} \\
& +\left(D_{\mu} J_{\mathrm{T}}^{\mu}-\frac{1}{2} T_{\mathrm{L}^{c}}^{\mu \nu} \delta_{\mathcal{B}} g_{\mu \nu}-J_{\mathrm{L}^{c}}^{\mu} \cdot \delta_{\mathcal{B}} A_{\mu}-\frac{1}{\sqrt{-g}} \delta_{\mathcal{B}}\left(\sqrt{-g} T \mathfrak{n}_{\mathrm{T}}\right)\right)
\end{aligned}
$$

We have skipped several steps in the derivation of (15.17), which unlike the diffeomorphism and flavour Bianchi identities does require isolating the $U(1)_{\mathrm{T}}$ transformation explicitly. The intermediate steps can be found in appendix I. The essential steps involve reverting back to the original (twisted) transformation parameters $\left\{\xi^{\mu}, \Lambda, \Lambda^{(\mathrm{T})}\right\}$. It should hopefully be clear that this can be achieved without modifying the diffeomorphism and flavour Bianchi identities. After performing the required shift and defining

$$
\begin{aligned}
\mathrm{N}_{\mathrm{T}}^{\mu} \equiv-\frac{\mathcal{G}_{\mathrm{T}}^{\mu}}{T} \equiv & J_{\mathrm{T}}^{\mu}+\boldsymbol{\beta}_{\nu} T_{\mathrm{L}+\mathrm{L}^{c}}^{\mu \nu}+\left(\Lambda_{\boldsymbol{\beta}}+\boldsymbol{\beta}^{\nu} A_{\nu}\right) \cdot J_{\mathrm{L}+\mathrm{L}^{c}}^{\mu} \\
& -\left\{\mathfrak{h}_{\sigma} \boldsymbol{\beta}^{\sigma}+\mathfrak{n} \cdot\left(\Lambda_{\boldsymbol{\beta}}+\boldsymbol{\beta}^{\nu} A_{\nu}\right)+\mathfrak{n}_{\mathrm{T}}\right\} u^{\mu} \\
& -\boldsymbol{\beta}_{\nu} g_{\alpha \beta}^{\prime} g^{\mu \alpha} T_{\mathrm{L}^{c}}^{\beta \nu}-\left(\Lambda_{\boldsymbol{\beta}}+\boldsymbol{\beta}^{\nu} A_{\nu}^{\prime}\right) \cdot J_{\mathrm{L}^{c}}^{\mu} .
\end{aligned}
$$

we arrive at (15.17).

In fact, we can further simplify the above expressions, by noting a particularly interesting combination of the Bianchi identities that follows straightforwardly in the derivation (see appendix I). One finds that the grand canonical adiabaticity equation for $\left\{T_{\mathrm{L}^{c}}^{\mu \nu}, J_{\mathrm{L}^{c}}^{\mu}\right\}$ holds identically, i.e.,

$$
\begin{array}{r}
D_{\mu} J_{\mathrm{T}}^{\mu}=\frac{1}{2} T_{\mathrm{L}^{c}}^{\mu \nu} \delta_{\mathcal{B}} g_{\mu \nu}+J_{\mathrm{L}^{c}}^{\mu} \cdot \delta_{\mathcal{B}} A_{\mu}+\frac{1}{\sqrt{-g}} \delta_{\mathcal{B}}\left(\sqrt{-g} T \mathfrak{n}_{\mathrm{T}}\right) \\
\Longrightarrow \quad D_{\mu}\left(J_{\mathrm{T}}^{\mu}-\mathfrak{n}_{\mathrm{T}} u^{\mu}\right)=\frac{1}{2} T_{\mathrm{L}^{c}}^{\mu \nu} \delta_{\mathcal{B}} g_{\mu \nu}+J_{\mathrm{L}^{c}}^{\mu} \cdot \delta_{\mathcal{B}} A_{\mu} .
\end{array}
$$

Using this equation we then eliminate the last line of (15.17) to obtain

$$
D_{\mu} \mathrm{N}_{\mathrm{T}}^{\mu}=\frac{1}{2} T_{\mathrm{L}+\mathrm{L}^{c}}^{\mu \nu} \delta_{\mathcal{B}} g_{\mu \nu}+J_{\mathrm{L}+\mathrm{L}^{c}}^{\mu} \cdot \delta_{\mathcal{B}} A_{\mu}+\frac{1}{2} T_{\mathrm{L}^{c}}^{\mu \nu} \delta_{\mathcal{B}} \tilde{g}_{\mu \nu}+J_{\mathrm{L}^{c}}^{\mu} \cdot \delta_{\mathcal{B}} \tilde{A}_{\mu}+J_{\mathrm{T}}^{\mu} \delta_{\mathcal{B}} \mathrm{A}_{\mu}^{(\mathrm{T})}{ }_{\mu} .
$$

This is a useful expression in ascertaining $U(1)_{T}$ invariant Lagrangians. We have written both this and the more cumbersome expression (15.17) since the latter is more useful when we have anomalous terms, as it helps at various stages of the derivation to keep track of the origins of various contributions.

\subsection{The adiabatic Lagrangian $\mathcal{L}_{\mathrm{T}}$}

Given the Bianchi identities for the various symmetries in Class $\mathrm{L}_{\mathrm{T}}$ we can now examine the implications for the adiabaticity equation. In particular, we shall prove that a given 
constitutive relation furnishes a solution to the adiabaticity equation (2.12) if and only if it can be derived from a master Lagrangian $\mathcal{L}_{\mathrm{T}}$ that preserves diffeomorphism, flavour, and $U(1)_{\mathrm{T}}$ invariance.

Firstly, we should make contact with the adiabaticity equation. Unlike in Class L where we gave a prescription for the entropy density, we have not yet done so for $\mathcal{L}_{\mathrm{T}}\left[\boldsymbol{\Psi}_{\mathrm{T}}\right]$. Intuitively, we want to identify the $U(1)_{\mathrm{T}}$ invariance as being responsible for adiabaticity. However, it is clear that $J_{\mathrm{T}}^{\mu}$ should play some role in its definition since $U(1)_{\mathrm{T}}$ symmetry was introduced to ensure adiabaticity. In fact, the redefined counterpart $\mathrm{N}_{\mathrm{T}}^{\mu}$ is closely related to the grand canonical free energy current.

In fact we can make this a bit more precise by viewing the Bianchi identities inspired by our discussion about the Schwinger-Keldysh construction in section 13. Consider using the reference configuration in the Schwinger-Keldysh construction to pull-back the L fields onto the R-manifold. Then we have all the background sources living on the right manifold. Furthermore, working with the common hydrodynamic fields, we can attempt to derive a set of Bianchi-identities under the right diffeomorphism and flavour symmetries. This leads to an analog of (13.8), with the sole difference being that all the fields are on the R-manifold and we are working with the physical degrees of freedom. This set of identities in fact can be shown to be closely related to eqs. (15.15)-(15.16). To see this, note that the defining variation (15.14) can be rewritten as

$$
\begin{aligned}
\frac{1}{\sqrt{-g}} \delta & \left(\sqrt{-g} \mathcal{L}_{\mathrm{T}}\right)-\nabla_{\mu}\left(\phi \Theta_{\mathrm{PS}}^{\mathrm{T}}\right)^{\mu} \\
= & \frac{1}{2} T_{\mathrm{L}+\mathrm{L}^{c}}^{\mu \nu} \delta g_{\mu \nu}-\frac{1}{2} T_{\mathrm{L}^{c}}^{\mu \nu} \delta g_{\mu \nu}^{\prime}+J_{\mathrm{L}+\mathrm{L}^{c}}^{\mu} \cdot \delta A_{\mu}-J_{\mathrm{L}^{c}}^{\mu} \cdot \delta A_{\mu}^{\prime} \\
& +T \mathfrak{h}_{\sigma} \delta \boldsymbol{\beta}^{\sigma}+T \mathfrak{n} \cdot\left(\delta \Lambda_{\boldsymbol{\beta}}+A_{\sigma} \delta \boldsymbol{\beta}^{\sigma}\right)+J_{\mathrm{T}}^{\sigma} \delta \mathrm{A}^{(\mathrm{T})}{ }_{\sigma}+T \mathfrak{n}_{\mathrm{T}}\left(\delta \Lambda_{\boldsymbol{\beta}}^{(\mathrm{T})}+\mathrm{A}^{(\mathrm{T})} \delta \boldsymbol{\beta}^{\sigma}\right) .
\end{aligned}
$$

Apart from the last two terms (which are tied to the presence of the $U(1)_{\mathrm{T}}$ symmetry principle), this expression has the same structure as the Schwinger-Keldysh variation (13.4) (after pulling it to the R-manifold). Aided by this observation, we tentatively forward the following hypothesis: in the absence of anomalies the Schwinger-Keldysh sources can be identified with $\left\{g_{\mu \nu}^{\mathrm{R}}, A_{\mu}^{\mathrm{R}}\right\} \mapsto\left\{g_{\mu \nu}, A_{\mu}\right\}$ and $\left\{g_{\mu \nu}^{\mathrm{L}}, A_{\mu}^{\mathrm{L}}\right\} \mapsto\left\{g_{\mu \nu}^{\prime}, A_{\mu}^{\prime}\right\}$ respectively. ${ }^{98}$ Accordingly, (15.22) suggests that the currents map as $\left\{T_{\mathrm{R}}^{\mu \nu}, J_{\mathrm{R}}^{\mu}\right\} \mapsto\left\{T_{\mathrm{L}+\mathrm{L}^{c}}^{\mu \nu}, J_{\mathrm{L}+\mathrm{L}^{c}}^{\mu}\right\}$ and $\left\{T_{\mathrm{L}}^{\mu \nu}, J_{\mathrm{L}}^{\mu}\right\} \mapsto\left\{T_{\mathrm{L}^{c}}^{\mu \nu}, J_{\mathrm{L}^{c}}^{\mu}\right\}$. The constrained (Schwinger-Keldysh) variational principle (13.5), would then inspire us to put forward a constrained variational principle in Class $\mathrm{L}_{\mathrm{T}}$ which takes the following form: under diffeomorphisms and flavour gauge transformations only $\left\{\boldsymbol{\beta}^{\mu}, \Lambda_{\boldsymbol{\beta}}, g_{\mu \nu}^{\prime}, A_{\mu}^{\prime}\right\}$ transform while the R-sources $\left\{g_{\mu \nu}, A_{\mu}\right\}$ are held fixed. Modulo a sensible extension to the new fields $\left\{\mathrm{A}^{(\mathrm{T})}{ }_{\mu}, \Lambda_{\boldsymbol{\beta}}^{(\mathrm{T})}\right\}$ and to $U(1)_{\mathrm{T}}$ transformations, this is indeed what we will find in section 16.2 .

This close analogy to the Schwinger-Keldysh doubled formalism then suggests that in the hydrodynamic limit, where we want to consider only the fully retarded correlators, we should be working to leading order in the difference fields which are now $\left\{\tilde{g}_{\mu \nu}, \tilde{A}_{\mu}\right\}$ for

\footnotetext{
${ }^{98}$ In the present context we view this identification as heuristic. Studying anomalies as in section 17.3 shows that the identification of Schwinger-Keldysh fields should actually be twisted to involve the field $\mathrm{A}^{(\mathrm{T})}{ }_{\mu}$ as in (15.1).
} 
reasons explained in section 13 (see also [30]). For the present analysis it implies that once we are done with the various variations we should set $\tilde{g}_{\mu \nu} \rightarrow 0$ and $\tilde{A}_{\mu} \rightarrow 0$. In addition, as far as the new $U(1)_{\mathrm{T}}$ symmetry is concerned, the hydrodynamic limit corresponds to setting $A^{(\mathrm{T})}{ }_{\mu}=0$ and $\Lambda_{\boldsymbol{\beta}}^{(\mathrm{T})}=1$.

Once we set all the auxiliary fields $\left\{\tilde{g}_{\mu \nu}, \tilde{A}_{\mu}, \mathrm{A}^{(\mathrm{T})}{ }_{\mu}\right\}$ to zero and $\Lambda_{\beta}^{(\mathrm{T})}=1$, the $U(1)_{\mathrm{T}}$ Bianchi identity (15.17) takes the form:

$$
\begin{aligned}
& \left.D_{\mu} \mathrm{N}_{\mathrm{T}}^{\mu}\right|_{\Psi_{\mathrm{T}}^{\varnothing}}=\left.\frac{1}{2} T_{\mathrm{L}+\mathrm{L}^{c}}^{\mu \nu}\right|_{\Psi_{\mathrm{T}}^{\varnothing}} \delta_{\mathcal{B}} g_{\mu \nu}+\left.J_{\mathrm{L}+\mathrm{L}^{c}}^{\mu}\right|_{\Psi_{\mathrm{T}}^{\varnothing}} \cdot \delta_{\mathcal{B}} A_{\mu} \\
& \text { where } \quad \Psi_{\mathrm{T}}^{\varnothing}=\left\{\tilde{g}_{\mu \nu}=\tilde{A}_{\mu}=\mathrm{A}^{(\mathrm{T})}{ }_{\mu}=0, \quad \Lambda_{\boldsymbol{\beta}}^{(\mathrm{T})}=1, \& \boldsymbol{\Psi}=\text { arbitrary }\right\} .
\end{aligned}
$$

Thus $\left.\left\{\mathrm{N}_{\mathrm{T}}^{\mu}, T_{\mathrm{L}+\mathrm{L}^{c}}^{\mu \nu}, J_{\mathrm{L}+\mathrm{L}^{c}}^{\mu}\right\}\right|_{\Psi_{\mathrm{T}}^{\ominus}}$ is a constitutive relation that solves adiabaticity equation.

This shows that the constitutive relations derived from any diffeomorphism, flavour, and $U(1)_{\mathrm{T}}$ invariant Lagrangian $\mathcal{L}_{\mathrm{T}}$ are always guaranteed to be solutions of the adiabaticity equation. We will now argue that the converse is also true: every adiabatic constitutive relation can be obtained by this method.

Consider an arbitrary set of covariant constitutive relations $\left\{\mathrm{N}^{\mu}=-\mathcal{G}^{\mu} / T, T^{\mu \nu}, J^{\mu}\right\}$ such that they solve the grand canonical adiabaticity equation (2.21), i.e., they identically satisfy

$$
D_{\mu} \mathrm{N}^{\mu}=\frac{1}{2} T^{\mu \nu} \delta_{\mathcal{B}} g_{\mu \nu}+J^{\mu} \cdot \delta_{\mathcal{B}} A_{\mu}
$$

This implies that the combination $\mathrm{N}^{\mu} \mathrm{A}^{(\mathrm{T})}{ }_{\mu}+\frac{1}{2} T^{\mu \nu} \tilde{g}_{\mu \nu}+J^{\mu} \cdot \tilde{A}_{\mu}$ is invariant under flavour, diffeomorphism and $U(1)_{\mathrm{T}}$ transformations up to boundary terms. Hence, the following master Lagrangian provides an allowed effective description:

$$
\mathcal{L}_{\mathrm{T}}=\mathrm{N}^{\mu} \mathrm{A}^{(\mathrm{T})}{ }_{\mu}+\frac{1}{2} T^{\mu \nu} \tilde{g}_{\mu \nu}+J^{\mu} \cdot \tilde{A}_{\mu} .
$$

It is now easy to see that our procedure for generating adiabatic constitutive relation from $\mathcal{L}_{\mathrm{T}}\left[\boldsymbol{\Psi}_{\mathrm{T}}\right]$ exactly reproduces the original constitutive relations we started with, i.e.,

$$
\left.\left\{\mathrm{N}_{\mathrm{T}}^{\mu}, T_{\mathrm{L}+\mathrm{L}^{c}}^{\mu \nu}, J_{\mathrm{L}+\mathrm{L}^{c}}^{\mu}\right\}\right|_{\Psi_{\mathrm{T}}^{\varnothing}}=\left\{\mathrm{N}^{\mu}, T^{\mu \nu}, J^{\mu}\right\}
$$

Thus, we have shown any adiabatic constitutive relation can be obtained from some $\mathcal{L}_{\mathrm{T}}\left[\boldsymbol{\Psi}_{\mathrm{T}}\right]$. This establishes $\mathcal{L}_{\mathrm{T}}$ to be the generating function for all adiabatic constitutive relations and completes the proof of Theorem 2. In section 17 we will give a more detailed discussion of this fact and indeed show explicitly how the eightfold way is implemented in Class $\mathrm{L}_{\mathrm{T}}$. Before doing so let us however show how hydrodynamic equations of motion and entropy current conservation are obtained in Class $\mathrm{L}_{\mathrm{T}}$.

\section{Hydrodynamic Ward identities and the second law in Class $\mathrm{L}_{\mathrm{T}}$}

Having constructed the basic formalism for the construction of $U(1)_{\mathrm{T}}$ invariant Lagrangians in Class $\mathrm{L}_{\mathrm{T}}$, we now turn to demonstrating that the implied equations of motion are the 
usual conservation equations of hydrodynamics. We do this by giving a constrained variational principle that is completely analogous to the procedure in Class L, c.f., section 7 . The new features are, of course, that Class $\mathrm{L}_{\mathrm{T}}$ captures all of adiabatic transport and the presence of the additional $U(1)_{\mathrm{T}}$ symmetry whose associated Ward identity will turn out to be entropy current conservation.

\subsection{The Class $\mathrm{L}_{\mathrm{T}}$ variational principle}

Having derived a master Lagrangian $\mathcal{L}_{\mathrm{T}}$ that determines precisely those constitutive relations that satisfy adiabaticity equation, it remains to show that currents involved satisfy the correct hydrodynamical equations of motion. Our next goal is thus to define a constrained variational principle for $\mathcal{L}_{\mathrm{T}}\left[\boldsymbol{\Psi}_{\mathrm{T}}\right]$ which leads to the desired on-shell Ward identities.

Before we get into the technicalities, it should be clear that any variational principle must reduce to the Class L variational principle (7.2) when the auxiliary fields are absent. This means that the constrained variational principle described there must be extended in an $U(1)_{\mathrm{T}}$ invariant manner to the auxiliary fields $\left\{\tilde{g}_{\mu \nu}, \tilde{A}_{\mu}, \mathrm{A}^{(\mathrm{T})}{ }_{\mu}, \Lambda_{\boldsymbol{\beta}}^{(\mathrm{T})}\right\}$.

Consider a constrained variation $\delta$ of the following form: it acts on the single copy fields exactly as in Class L, i.e.,

$$
\delta: \quad \delta \boldsymbol{\beta}^{\mu}=\delta_{x} \boldsymbol{\beta}^{\mu}, \quad \delta \Lambda_{\boldsymbol{\beta}}=\delta_{x} \Lambda_{\boldsymbol{\beta}}, \quad \delta g_{\mu \nu}=\delta A_{\mu}=0,
$$

and it acts on the copy sources and $U(1)_{\mathrm{T}}$ fields in a similar way:

$$
\begin{aligned}
\delta: \quad & \delta \tilde{g}_{\mu \nu}=-\delta_{x} g_{\mu \nu}^{\prime} \Longrightarrow \delta g_{\mu \nu}^{\prime}=\delta_{x} g_{\mu \nu}^{\prime} \\
& \delta \tilde{A}_{\mu}=-\delta_{x} A_{\mu}^{\prime} \Longrightarrow \delta A_{\mu}^{\prime}=\delta_{x} A_{\mu}^{\prime}, \\
& \delta A^{(\mathrm{T})}=\delta_{x} A^{(\mathrm{T})}, \quad \delta \Lambda_{\boldsymbol{\beta}}^{(\mathrm{T})}=\delta_{x} \Lambda_{\boldsymbol{\beta}}^{(\mathrm{T})} .
\end{aligned}
$$

The choices for the variations of the auxiliary fields made in (16.2) involves treating them like the physical hydrodynamic fields. In other words the constrained variation $\delta$ consists of varying the fields $\left\{\boldsymbol{\beta}^{\mu}, \Lambda_{\boldsymbol{\beta}}, g_{\mu \nu}^{\prime}, A_{\mu}^{\prime}, \mathrm{A}^{(\mathrm{T})}{ }_{\mu}, \Lambda_{\boldsymbol{\beta}}^{(\mathrm{T})}\right\}$ along a Lie-orbit while keeping the sources $\left\{g_{\mu \nu}, A_{\mu}\right\}$ fixed. As alluded to in section 15.4, these transformation rules are very canonical if we consider our Class $\mathrm{L}_{\mathrm{T}}$ Lagrangian as a natural extension of the Schwinger-Keldysh formalism developed section 13.1 .

Given this constrained variational principle, we can plug the explicit variations into (15.14), integrate by parts where necessary and end up with the basic statement: ${ }^{99}$

$$
\begin{aligned}
\frac{1}{\sqrt{-g}} & \delta\left(\sqrt{-g} \mathcal{L}_{\mathrm{T}}\right)-\text { Boundary terms } \\
= & \left(\Lambda+\xi^{\nu} A_{\nu}\right) \cdot\left\{\frac{1}{\sqrt{-g}} \delta_{\mathcal{B}}(\sqrt{-g} T \mathfrak{n})+\left(D_{\mu} J_{\mathrm{L}^{c}}^{\mu}-\left[\tilde{A}_{\mu}, J_{\mathrm{L}^{c}}^{\mu}\right]\right)\right\} \\
& +\xi^{\sigma}\left\{\frac{1}{\sqrt{-g}} \delta_{\mathcal{B}}\left(\sqrt{-g} T \mathfrak{h}_{\sigma}\right)+T \mathfrak{n} \cdot \delta_{\mathcal{B}} A_{\sigma}+T \mathfrak{n}_{\mathrm{T}} \cdot \delta_{\mathcal{B}} \mathrm{A}_{\sigma}^{(\mathrm{T})}+J_{\mathrm{T}}^{\nu} \cdot \mathrm{F}^{(\mathrm{T})}{ }_{\sigma \nu}\right.
\end{aligned}
$$

\footnotetext{
${ }^{99}$ The quickest derivation of this expression involves starting from (I.23) which gives the unconstrained variation and setting the currents which arise from the variation of the physical sources, viz., $T_{\mathrm{L}+\mathrm{L}^{c}}^{\mu \nu}$ and $J_{\mathrm{L}+\mathrm{L}^{c}}^{\mu}$ to zero. The latter currents arise from the variations of the physical sources which are forbidden in (16.2).
} 


$$
\begin{aligned}
& +D_{\nu}\left(g_{\sigma \mu}^{\prime} T_{\mathrm{L}^{c}}^{\mu \nu}\right)-\frac{1}{2} T_{\mathrm{L}^{c}}^{\mu \nu} D_{\sigma} g_{\mu \nu}^{\prime}-J_{\mathrm{L}^{c}}^{\nu} \cdot F_{\sigma \nu}^{\prime} \\
& \left.-A_{\sigma}^{(T)}\left(\frac{1}{2} T_{\mathrm{L}^{c}}^{\mu \nu} \delta_{\mathfrak{B}} g_{\mu \nu}+J_{\mathrm{L}^{c}}^{\mu} \cdot \delta_{\mathfrak{B}} A_{\mu}\right)-\tilde{A}_{\sigma} \cdot\left(D_{\mu} J_{\mathrm{L}^{c}}^{\mu}-\left[\tilde{A}_{\mu}, J_{\mathrm{L}^{c}}^{\mu}\right]\right)\right\} \\
+\left(\Lambda^{(\mathrm{T})}+\right. & \left.\xi^{\sigma} \mathrm{A}_{\sigma}^{(\mathrm{T})}\right)\left\{-D_{\mu}\left(J_{S}\right)_{\mathrm{T}}^{\mu}+\frac{1}{2} T_{\mathrm{L}^{c}}^{\mu \nu} \delta_{\mathcal{B}} \tilde{g}_{\mu \nu}+J_{\mathrm{L}^{c}}^{\mu} \cdot \delta_{\mathcal{B}} \tilde{A}_{\mu}+J_{\mathrm{T}}^{\mu} \delta_{\mathcal{B}} \mathrm{A}^{(\mathrm{T})}{ }_{\mu}\right. \\
& \left.+\left(D_{\mu} J_{\mathrm{T}}^{\mu}-\frac{1}{2} T_{\mathrm{L}^{c}}^{\mu \nu} \delta_{\mathfrak{B}} g_{\mu \nu}-J_{\mathrm{L}^{c}}^{\mu} \cdot \delta_{\mathcal{B}} A_{\mu}-\frac{1}{\sqrt{-g}} \delta_{\mathcal{B}}\left(\sqrt{-g} T \mathfrak{n}_{\mathrm{T}}\right)\right)\right\}
\end{aligned}
$$

In deriving this expression we have to bear in mind the fact that the physical sources do not vary. As a result the contribution to the current $\mathrm{N}_{\mathrm{T}}^{\mu}$ from $T_{\mathrm{L}+\mathrm{L}^{c}}^{\mu \nu}$ and $J_{\mathrm{L}+\mathrm{L}^{c}}^{\mu}$ is missing. We have chosen to indicate this by defining the entropy current in Class $\mathrm{L}_{\mathrm{T}}$ directly via

$$
\left(J_{S}\right)_{\mathrm{T}}^{\mu}=\mathrm{N}_{\mathrm{T}}^{\mu}-\boldsymbol{\beta}_{\nu} T_{\mathrm{L}+\mathrm{L}^{c}}^{\mu \nu}-\left(\Lambda_{\boldsymbol{\beta}}+\boldsymbol{\beta}^{\nu} A_{\nu}\right) \cdot J_{\mathrm{L}+\mathrm{L}^{c}}^{\mu} .
$$

Thus extremizing $\mathcal{L}_{\mathrm{T}}$ for an arbitrary constrained variation (16.2) parameterized by $\left.\left\{\xi^{\sigma}, \Lambda\right\}\right)$ yields the equations:

$$
\begin{aligned}
& 0 \simeq \frac{1}{\sqrt{-g}} \delta_{\mathcal{B}}(\left.\sqrt{-g} T \mathfrak{h}_{\sigma}\right)+T \mathfrak{n} \cdot \delta_{\mathcal{B}} A_{\sigma}+T \mathfrak{n}_{\mathrm{T}} \cdot \delta_{\mathcal{B}} \mathrm{A}_{\sigma}^{(\mathrm{T})}+J_{\mathrm{T}}^{\nu} \cdot \mathrm{F}_{\sigma \nu}^{(\mathrm{T})} \\
&+D_{\nu}\left(g_{\sigma \mu}^{\prime} T_{\mathrm{L}^{c}}^{\mu \nu}\right)-\frac{1}{2} T_{\mathrm{L}^{c}}^{\mu \nu} D_{\sigma} g_{\mu \nu}^{\prime}-J_{\mathrm{L}^{c}}^{\nu} \cdot F_{\sigma \nu}^{\prime} \\
& \quad \mathrm{A}^{(\mathrm{T})}\left(\frac{1}{2} T_{\mathrm{L}^{c}}^{\mu \nu} \delta_{\mathcal{B}} g_{\mu \nu}+J_{\mathrm{L}^{c}}^{\mu} \cdot \delta_{\mathcal{B}} A_{\mu}\right)-\tilde{A}_{\sigma} \cdot\left(D_{\mu} J_{\mathrm{L}^{c}}^{\mu}-\left[\tilde{A}_{\mu}, J_{\mathrm{L}^{c}}^{\mu}\right]\right) \\
& 0 \simeq \frac{1}{\sqrt{-g}} \delta_{\mathcal{B}}(\sqrt{-g} T \mathfrak{n})+\left(D_{\mu} J_{\mathrm{L}^{c}}^{\mu}-\left[\tilde{A}_{\mu}, J_{\mathrm{L}^{c}}^{\mu}\right]\right) .
\end{aligned}
$$

Comparing this against the Bianchi identities (15.15) and (15.16), we get the hydrodynamic equations we expect, viz., the usual hydrodynamic equations of motion

$$
\begin{aligned}
D_{\mu}\left(T_{\mathrm{L}+\mathrm{L}^{c}}\right)_{\sigma}^{\mu} & \simeq J_{\mathrm{L}+\mathrm{L}^{c}}^{\nu} \cdot F_{\sigma \nu}, \\
D_{\mu} J_{\mathrm{L}+\mathrm{L}^{c}}^{\mu} & \simeq 0 .
\end{aligned}
$$

Since we have an additional symmetry $U(1)_{\mathrm{T}}$ we should also examine the coefficient of $\left(\Lambda^{(\mathrm{T})}+\xi^{\sigma} \mathrm{A}_{\sigma}^{(\mathrm{T})}\right)$ to see what further constraints are imposed on dynamics. We find now

$$
\begin{aligned}
D_{\mu}\left(J_{S}\right)_{\mathrm{T}}^{\mu} \simeq & \frac{1}{2} T_{\mathrm{L}^{c}}^{\mu \nu} \delta_{\mathcal{B}} \tilde{g}_{\mu \nu}+J_{\mathrm{L}^{c}}^{\mu} \cdot \delta_{\mathcal{B}} \tilde{A}_{\mu}+J_{\mathrm{T}}^{\mu} \delta_{\mathcal{B}} \mathrm{A}^{(\mathrm{T})}{ }_{\mu} \\
& +\left(D_{\mu} J_{\mathrm{T}}^{\mu}-\frac{1}{2} T_{\mathrm{L}^{c}}^{\mu \nu} \delta_{\mathcal{B}} g_{\mu \nu}-J_{\mathrm{L}^{c}}^{\mu} \cdot \delta_{\mathcal{B}} A_{\mu}-\frac{1}{\sqrt{-g}} \delta_{\mathcal{B}}\left(\sqrt{-g} T \mathfrak{n}_{\mathrm{T}}\right)\right)
\end{aligned}
$$

Comparing with the $U(1)_{\mathrm{T}}$ Bianchi identity (15.17) we can simplify the expression above to read:

$$
D_{\mu}\left[\mathrm{N}_{\mathrm{T}}^{\mu}-\left(J_{S}\right)_{\mathrm{T}}^{\mu}\right] \simeq \frac{1}{2} T_{\mathrm{L}+\mathrm{L}^{c}}^{\mu \nu} \delta_{\mathfrak{B}} g_{\mu \nu}+J_{\mathrm{L}+\mathrm{L}^{c}} \cdot \delta_{\mathcal{B}} A_{\mu}
$$

This is an additional dynamical equation of motion that should be satisfied by systems described by $\mathcal{L}_{\mathrm{T}}$. A-priori we seem to have too much, for in (16.7) we have all the equations 
of motion we actually want. When we however examine the hydrodynamic limit by setting the auxiliary fields to zero, $\boldsymbol{\Psi}_{\mathrm{T}}=\Psi_{\mathrm{T}}^{\varnothing}$, as described around (15.23), we can further eliminate terms from (16.9) to arrive at

$$
D_{\mu}\left(J_{S}\right)_{\mathrm{T}}^{\mu} \simeq 0,
$$

which is simply the statement of on-shell entropy conservation in Class $\mathrm{L}_{\mathrm{T}}$. As promised the $U(1)_{\mathrm{T}}$ transformations ensure adiabaticity off-shell, which in turn implies that the entropy current is conserved on-shell.

\subsection{Reference fields for Class $L_{T}$}

Let us now try to introduce new fields to convert this into an unconstrained variational problem. To do this, we will pass to a description whereby we factorise the dynamical fields into a rigid reference configuration and a pullback diffeomorphism and flavour transformation. The hydrodynamic equations will then be generated by the variations of the pull back fields keeping the reference fields fixed. Note that here we will refrain from giving a prescription for the precise form of $U(1)_{\mathrm{T}}$ action on the reference manifold. While it is clear that such a formalism must exist (in fact it is not very hard to write the analogue of (7.20) including the $U(1)_{\mathrm{T}}$ action), its detailed workings may depend on other issues which we defer to future work [55]. For the moment we just illustrate that the reference manifold variational principle of section 7.2 can be extended to the new field content, thus obtaining the hydrodynamic equations of motion.

We begin by imagining a copy of hydrodynamic fields $\left\{\beta^{a}, \Lambda_{\beta}\right\}$ living in a reference manifold M. In addition, we will posit a metric, a gauge field and a copy of $\left\{A^{(T)}{ }_{\sigma}, \Lambda_{\beta}^{(T)}\right\}$, viz., $\left\{\mathfrak{g}_{a b}^{\prime}, \mathbb{A}_{a}^{\prime}, \mathbb{A}^{(\mathrm{T})}, \Lambda_{\beta}^{(\mathrm{T})}\right\}$ on $\mathbb{M}$. The actual fields are obtained by introducing a diffeomorphism field $\varphi^{a}(x)$ and a gauge transformation field $c(x)$ from physical spacetime $\mathcal{M}$ to $\mathbb{M}$ and then using them to pull-back $\Psi_{\mathrm{T}} \equiv\left\{\mathbb{\beta}^{a}, \Lambda_{\beta}, \mathbb{I}_{a b}, \mathbb{A}_{a}, \mathscr{g}_{a b}^{\prime}, \mathbb{A}_{a}^{\prime}, \mathbb{A}_{a}^{(\mathrm{T})}, \Lambda_{\beta}^{(\mathrm{T})}\right\}$. Now consider constrained variations as defined by (16.1), (16.2). Their diffeomorphism and flavour parts (disregarding the $U(1)_{\mathrm{T}}$ action for now) are implemented on the reference manifold as follows:

$$
\begin{aligned}
& \delta \mathfrak{g}_{a b}=-\delta_{\varphi} \mathfrak{g}_{a b}, \quad \delta \mathbb{A}_{a}=-\delta_{\varphi} \mathbb{A}_{a}, \\
& \delta \mathbb{\beta}^{a}=0, \quad \delta \mathbb{g}_{a b}^{\prime}=0, \quad \delta \mathbb{A}_{a}^{(\mathrm{T})}=0 \\
& \delta \Lambda_{\beta}=0, \quad \delta \mathbb{A}_{a}^{\prime}=0, \quad \delta \Lambda_{\beta}^{(\mathrm{T})}=0,
\end{aligned}
$$

where $\delta_{\varphi}$ denotes Lie drag on $\mathbb{M}$ along $\left\{e_{a}^{\mu} \delta \varphi^{a},-(\delta c) c^{-1}\right\}$. Let us see how the variational principle works on $\mathbb{M}$ :

$$
\begin{aligned}
\frac{1}{\sqrt{-g}} \delta & \left(\sqrt{-g} \mathbb{L}_{\mathrm{T}}\right) \\
= & \frac{1}{2} \mathbb{T}_{\mathrm{L}+\mathrm{L}^{c}}^{a b} \delta g_{a b}-\frac{1}{2} \mathbb{T}_{\mathrm{L}^{c}}^{a b} \delta g_{a b}^{\prime}+\mathbb{J}_{\mathrm{L}+\mathrm{L}^{c}}^{a} \cdot \delta \mathbb{A}_{a}-\mathbb{J}_{\mathrm{L}^{c}}^{a} \cdot \delta \mathbb{A}_{a}^{\prime} \\
& +\mathbb{T} \mathbb{h}_{a} \delta \beta^{a}+\mathbb{T} \mathrm{m} \cdot\left(\delta \Lambda_{\beta}+\mathbb{A}_{c} \delta \beta^{c}\right)+\mathbb{J}_{\mathrm{T}}^{a} \delta \mathbb{A}_{a}^{(\mathrm{T})}+\mathbb{T} \mathrm{m}_{\mathrm{T}}\left(\delta \Lambda_{\beta}^{(\mathrm{T})}+\mathbb{A}_{c}^{(\mathrm{T})} \delta \beta^{c}\right) \\
= & -\frac{1}{2} \mathbb{T}_{\mathrm{L}+\mathrm{L}^{c}}^{a b}\left(\mathbb{D}_{a} \delta \varphi_{b}+\mathbb{D}_{b} \delta \varphi_{a}\right)-\mathbb{J}_{\mathrm{L}+\mathrm{L}^{c}}^{a} \cdot\left[\mathbb{D}_{a}\left(-c^{-1} \delta c+\mathbb{A}_{b} \delta \varphi^{b}\right)+\delta \varphi^{b} \mathbb{F}_{b a}\right] .
\end{aligned}
$$


As usual, we perform an integration by parts and obtain

$$
\begin{aligned}
& \frac{1}{\sqrt{-g}} \delta\left(\sqrt{-g} \mathbb{L}_{\mathrm{T}}\right)+\nabla_{a}(\cdots) \\
& \quad=\delta \varphi_{a}\left[\mathbb{D}_{b} \mathbb{T}_{\mathrm{L}+\mathrm{L}^{c}}^{a b}-\mathbb{J}_{\mathrm{L}+\mathrm{L}^{c}}^{b} \cdot \mathbb{F}_{b}^{a}\right]+\left(-c^{-1} \delta c+\mathbb{A}_{b} \delta \varphi^{b}\right) \cdot \mathbb{D}_{a} \mathbb{J}_{\mathrm{L}+\mathrm{L}^{c}}^{a}
\end{aligned}
$$

Demanding invariance under any such variation, we can read off the reference manifold version of the conservation equations (16.7) from (16.13).

\section{Eightfold adiabatic transport in Class $\mathrm{L}_{\mathrm{T}}$}

We have now all the ingredients in Class $\mathrm{L}_{\mathrm{T}}$ to demonstrate how all classes of adiabatic transport can be realized by a Lagrangian description. For this purpose, we will distinguish constitutive relations that are Lagrangian $\left(\mathcal{L}=\mathrm{H}_{S} \cup \overline{\mathrm{H}}_{S}\right)$, non-Lagrangian $\left(\mathcal{L}^{c}=\mathrm{B} \cup \mathrm{C} \cup\right.$ $\left.\overline{\mathrm{H}}_{V}\right)$, and anomaly induced $\left(\mathrm{A} \cup \mathrm{H}_{V}\right)$. The most general adiabatic constitutive relations thus split into ${ }^{100}$

$$
\begin{aligned}
\mathcal{G}^{\sigma} & =\mathcal{G}_{\mathcal{L}}^{\sigma}+\left(\mathcal{G}^{\sigma}\right)_{\mathcal{L}^{c}}+\left(\mathcal{G}^{\sigma}\right)_{\mathrm{A}, \mathrm{H}_{V}}, \\
T^{\mu \nu} & =T_{\mathcal{L}}^{\mu \nu}+\left(T^{\mu \nu}\right)_{\mathcal{L}^{c}}+\left(T^{\mu \nu}\right)_{\mathrm{A}, \mathrm{H}_{V}}, \\
J^{\mu} & =J_{\mathcal{L}}^{\mu}+\left(J^{\mu}\right)_{\mathcal{L}^{c}}+\left(J^{\mu}\right)_{\mathrm{A}, \mathrm{H}_{V}},
\end{aligned}
$$

where $\mathcal{G}^{\sigma} \equiv-T \mathrm{~N}^{\sigma}$. Comparing with (14.15), we see that Classes $\mathrm{D}$ and $\mathrm{H}_{F}$ have been discarded from the most general currents that would be allowed just by classifying off-shell inequivalent tensor structures. Since such terms are either dissipative or forbidden by the second law, we don't expect to see them in Class $\mathrm{L}_{\mathrm{T}}$ (which we claim captures precisely the second law-allowed adiabatic transport).

The fact that Class $\mathrm{D}$ and Class $\mathrm{H}_{F}$ cannot be realized in Class $\mathrm{L}_{\mathrm{T}}$ is easy to see: these constitutive relations explicitly break the $U(1)_{\mathrm{T}}$ invariance by virtue of not satisfying the adiabaticity equation. This is only to be expected, of course, for the Class $\mathrm{L}_{\mathrm{T}}$ formalism to achieve the desired goal of providing a description precisely of adiabatic transport.

In turn, we will now show how all the adiabatic constitutive relations which are allowed by second law can be obtained from a Class $\mathrm{L}_{\mathrm{T}}$ effective action.

\section{1 $\mathcal{L}_{\mathrm{T}}$ for Class $\mathrm{L}$ constitutive relations}

We start our discussion of the eightfold way in Class $\mathrm{L}_{\mathrm{T}}$ by reproducing Class $\mathrm{L}$ constitutive relations. As we will demonstrate momentarily, Class $\mathrm{L}_{\mathrm{T}}$ provides a natural extension of Class L, for it contains Class L as a rather trivial special case.

By definition Class L currents $\left\{\mathcal{G}_{\mathcal{L}}^{\sigma}, T_{\mathcal{L}}^{\mu \nu}, J_{\mathcal{L}}^{\mu}\right\}$ are precisely those which can be obtained from a scalar Lagrangian $\mathcal{L}[\Psi] \equiv \mathcal{L}\left[g_{\mu \nu}, A_{\mu}, \boldsymbol{\beta}^{\mu}, \Lambda_{\boldsymbol{\beta}}\right]$. In this case there is thus no need to build a master Lagrangian that involves the enhanced field content of Class $\mathrm{L}_{\mathrm{T}}$ as in (15.25); instead we can simply take

$$
\mathcal{L}_{\mathrm{T}}\left[\boldsymbol{\Psi}_{\mathrm{T}}\right]=\mathcal{L}[\boldsymbol{\Psi}]
$$

\footnotetext{
${ }^{100}$ For the purposes of giving simple expression, we have chosen here to group the adiabatic classes somewhat differently; in particular, we choose to group Class $\mathrm{H}_{V}$ with Class $\mathrm{A}$ as this is quite natural in the $U(1)_{\mathrm{T}}$ invariant formalism.
} 
This simplified prescription for Class L terms has the advantage that it is obvious how $\int \sqrt{-g} \mathcal{L}_{\mathrm{T}}$ reduces in hydrostatics to the equilibrium partition function. Indeed, precisely the same argument as for Class L (see section 6.3) proves that this consistency condition is met. Equivalently one can consider starting with $\mathcal{L}_{\mathrm{T}}$ and gauge fix the auxiliary fields $\left\{\tilde{g}_{\mu \nu}, \tilde{A}_{\mu}, \mathrm{A}^{(\mathrm{T})}{ }_{\mu}\right\}$ differently from $\Psi_{\mathrm{T}}^{\varnothing}$ in (15.23). For instance given that the Noether current is roughly determined by the Class L Lagrangian, $\mathrm{N}^{\mu}=\boldsymbol{\beta}^{\mu} \mathcal{L}-\left(\phi_{\mathcal{B}} \Theta_{\mathrm{PS}}\right)^{\sigma}+\nabla_{\nu} \mathcal{K}^{\sigma \nu}[\mathcal{B}]$, suggests that one might choose the following set of $\left\{\mathrm{A}^{(\mathrm{T})}{ }_{\mu}=-T^{2} \boldsymbol{\beta}_{\mu}, \Lambda_{\boldsymbol{\beta}}=0, \tilde{g}_{\mu \nu}=0, \tilde{A}_{\mu}=\right.$ $0\}$ to recover the Lagrangian density $\mathcal{L} .^{101}$

The constitutive relations obtained from the Class $\mathrm{L}_{\mathrm{T}}$ variational principle can be read off from (15.14) and (15.18):

$$
\begin{array}{lll}
T_{\mathrm{L}}^{\mu \nu}=T_{\mathcal{L}}^{\mu \nu}, & J_{\mathrm{L}}^{\mu}=J_{\mathcal{L}}^{\mu}, & \mathcal{G}_{\mathrm{T}}^{\sigma}=\mathcal{G}_{\mathcal{L}}^{\sigma}, \\
T_{\mathrm{L}^{c}}^{\mu \nu}=0, & J_{\mathrm{L}^{c}}^{\mu}=0, & J_{\mathrm{T}}^{\mu}=0 .
\end{array}
$$

From this it is clear that also the dynamics implied by the variational principle in section 16 reproduces everything we developed in Class L. Hence the choice (17.2) identically reproduces Class $\mathrm{L}$ with the nice additional feature that the adiabaticity equation (and entropy conservation as an equation of motion) is manifestly satisfied a-priori because the Lagrangian is trivially $U(1)_{\mathrm{T}}$ invariant.

\section{$17.2 \mathcal{L}_{\mathrm{T}}$ for non-Lagrangian constitutive relations (Classes $\mathrm{B}, \mathrm{C}$ and $\overline{\mathbf{H}}_{V}$ )}

Having reproduced Class $\mathrm{L}$ in Class $\mathrm{L}_{\mathrm{T}}$ in a rather trivial way, let us now turn to constitutive relations which were not captured by naïve Lagrangians of Class L. To wit, consider adiabatic constitutive relations $\left\{\left(\mathcal{G}^{\sigma}\right)_{\mathcal{L}^{c}},\left(T^{\mu \nu}\right)_{\mathcal{L}^{c}},\left(J^{\mu}\right)_{\mathcal{L}^{c}}\right\}$ which subsume terms of Classes $\mathrm{B}, \mathrm{C}$ and $\overline{\mathrm{H}}_{V}$ as constructed in (9.2), (10.1) and (11.15). While a Class L Lagrangian giving such terms does not exist, we can use our new machinery to construct the associated effective master Lagrangian as in (15.25):

$$
\mathcal{L}_{\mathrm{T}}\left[\mathbf{\Psi}_{\mathrm{T}}\right]=-\frac{\left(\mathcal{G}^{\mu}\right)_{\mathcal{L}^{c}}}{T} \mathrm{~A}^{(\mathrm{T})}{ }_{\mu}+\frac{1}{2}\left(T^{\mu \nu}\right)_{\mathcal{L}^{c}} \tilde{g}_{\mu \nu}+\left(J^{\mu}\right)_{\mathcal{L}^{c}} \cdot \tilde{A}_{\mu}
$$

Via (15.14) this defines some constitutive relations $\left\{\mathcal{G}_{\mathrm{T}}^{\sigma}, T_{\mathrm{L}+\mathrm{L}^{c}}^{\mu \nu}, J_{\mathrm{L}+\mathrm{L}^{c}}^{\mu}\right\}$ which mix the full field content $\left\{\boldsymbol{\beta}^{\mu}, \Lambda_{\boldsymbol{\beta}}, g_{\mu \nu}, A_{\mu}, \tilde{g}_{\mu \nu}, \tilde{A}_{\mu}, \mathrm{A}^{(\mathrm{T})}{ }_{\mu}\right\}$. The dynamical equations implied by the variational principle of section section 16.1 are precisely the hydrodynamic conservation equations and the conservation of the entropy current

$$
\left(J_{S}\right)_{\mathrm{T}}^{\mu}=-\frac{\mathcal{G}_{\mathrm{T}}^{\mu}}{T}-\boldsymbol{\beta}_{\nu} T_{\mathrm{L}+\mathrm{L}^{c}}^{\mu \nu}-\left(\Lambda_{\boldsymbol{\beta}}+\boldsymbol{\beta}^{\nu} A_{\nu}\right) \cdot J_{\mathrm{L}+\mathrm{L}^{c}}^{\mu} .
$$

As demonstrated in section 15.4, after setting the auxiliary fields to zero, these currents reduce to the desired ones:

$$
\left.\left\{\mathcal{G}_{\mathrm{T}}^{\sigma}, T_{\mathrm{L}+\mathrm{L}^{c}}^{\mu \nu}, J_{\mathrm{L}+\mathrm{L}^{c}}^{\mu}\right\}\right|_{\Psi_{\mathrm{T}}^{\varnothing}}=\left\{\left(\mathcal{G}^{\sigma}\right)_{\mathcal{L}^{c}},\left(T^{\mu \nu}\right)_{\mathcal{L}^{c}},\left(J^{\mu}\right)_{\mathcal{L}^{c}}\right\}
$$

\footnotetext{
${ }^{101}$ This statement should account for the contribution from the pre-symplectic current which is not always transverse. One might however be able to field redefine this contribution away, though we have not checked this statement in detail.
} 
and their Ward identities are still the standard hydrodynamic equations of motion together with conservation of the entropy current

$$
\left.\left(J_{S}\right)_{\mathrm{T}}^{\mu}\right|_{\Psi_{\mathrm{T}}^{\varnothing}}=-\frac{\left(\mathcal{G}^{\mu}\right)_{\mathcal{L}^{c}}}{T}-\boldsymbol{\beta}_{\nu}\left(T^{\mu \nu}\right)_{\mathcal{L}^{c}}-\left(\Lambda_{\boldsymbol{\beta}}+\boldsymbol{\beta}^{\nu} A_{\nu}\right) \cdot\left(J^{\mu}\right)_{\mathcal{L}^{c}}=\left(J_{S}^{\mu}\right)_{\mathcal{L}^{c}} .
$$

\section{$17.3 \mathcal{L}_{\mathrm{T}}$ for anomalies (Classes $\mathrm{A}$ and $\mathrm{H}_{V}$ )}

The construction of the master Lagrangian $\mathcal{L}_{\mathrm{T}}$ heavily relies on a doubling of the field content and demanding invariance under $U(1)_{\mathrm{T}}$. We already gave indications that this can be linked back to the Schwinger-Keldysh doubling that we employed previously in section 13.3 to describe anomalies. We would thus like to show that the master Lagrangian $\mathcal{L}_{\mathrm{T}}$ is capable of describing anomalies in an analogous (but not quite identical) fashion. In this section we will discuss Class A terms, but it is clear from section 11.1 that this immediately incorporates Class $\mathrm{H}_{V}$, as well. The reason for this is that, once terms associated to some anomaly polynomial $\mathcal{P}[\boldsymbol{F}, \boldsymbol{R}]$ are dealt with, the presence of $\mathrm{A}^{(\mathrm{T})}{ }_{\mu}$ and $\Lambda_{\boldsymbol{\beta}}^{(\mathrm{T})}$ allows us to perform the Class $\mathrm{H}_{V}$ generalization of $\mathcal{P}[\boldsymbol{F}, \boldsymbol{R}]$ directly by using the replacement rule (11.14). The fact that we are free to set $\Lambda_{\boldsymbol{\beta}}^{(\mathrm{T})}+\boldsymbol{\beta}^{\mu} \mathrm{A}^{(\mathrm{T})}{ }_{\mu}=1$ in the Class $\mathrm{L}_{\mathrm{T}}$ formalism ensures consistency with the Class $\mathrm{H}_{V}$ discussion of section 11.1.

Consider a particular constitutive relation $\left\{\left(\mathcal{G}^{\mu}\right)_{\mathrm{A}} \equiv-T\left(\mathrm{~N}^{\mu}\right)_{\mathrm{A}},\left(T^{\mu \nu}\right)_{\mathrm{A}},\left(J^{\mu}\right)_{\mathrm{A}}\right\}$ that satisfies the adiabaticity equation with covariant anomalies

$$
D_{\mu}\left(\mathrm{N}^{\mu}\right)_{\mathrm{A}}=\frac{1}{2}\left(T^{\mu \nu}\right)_{\mathrm{A}} \delta_{\mathcal{B}} g_{\mu \nu}+\left(J^{\mu}\right)_{\mathrm{A}} \cdot \delta_{\mathfrak{B}} A_{\mu}+\mathrm{N}_{H}^{\perp}
$$

with $\mathrm{N}_{H}^{\perp}=\boldsymbol{\beta}_{\sigma} \mathrm{T}_{H}^{\perp \sigma}+\left(\Lambda_{\boldsymbol{\beta}}+\boldsymbol{\beta}^{\nu} A_{\nu}\right) \cdot \mathrm{J}_{H}^{\perp}$. Then, the combination

$$
\mathcal{L}_{\mathrm{T}}\left[\boldsymbol{\Psi}_{\mathrm{T}}\right]=\left(\mathrm{N}^{\mu}\right)_{\mathrm{A}} \mathrm{A}^{(\mathrm{T})}{ }_{\mu}+\frac{1}{2}\left(T^{\mu \nu}\right)_{\mathrm{A}} \tilde{g}_{\mu \nu}+\left(J^{\mu}\right)_{\mathrm{A}} \cdot \tilde{A}_{\mu}
$$

is no more $U(1)_{\mathrm{T}}$ invariant. In fact, we get

$$
\delta_{x} \int \sqrt{-g} \mathcal{L}_{\mathrm{T}}=-\int \sqrt{-g} \bar{\Lambda}^{(\mathrm{T})} \mathrm{N}_{H}^{\perp}=-\int \sqrt{-g}\left[\bar{\Lambda}^{(\mathrm{T})} \boldsymbol{\beta}_{\sigma} \mathrm{T}_{H}^{\perp \sigma}+\bar{\Lambda}^{(\mathrm{T})}\left(\Lambda_{\boldsymbol{\beta}}+\boldsymbol{\beta}^{\nu} A_{\nu}\right) \cdot \mathrm{J}_{H}^{\perp}\right] .
$$

In order to account for this anomaly, we extend our natural construction of master Lagrangians $\mathcal{L}_{\mathrm{T}}$ to the bulk theory, using Hall currents as the bulk constitutive relations. We claim that the following Class $\mathrm{L}_{\mathrm{T}}$ action gives the correct anomalous boundary constitutive relations and adiabaticity equation as a result of bulk inflow:

$$
S_{\mathrm{T}}=\int_{\mathcal{M}} \sqrt{-g} \mathcal{L}_{\mathrm{T}}+f_{\mathcal{M}}\left(\mathrm{N}_{H}^{m} \mathrm{~A}^{(\mathrm{T})}{ }_{m}+\frac{1}{2} \mathrm{~T}_{H}^{m n} \tilde{g}_{m n}+\mathrm{J}_{H}^{m} \cdot \tilde{A}_{m}\right),
$$

where $\mathrm{N}_{H}^{m}=s u^{m}+\left(\boldsymbol{\beta}_{s} \mathrm{~T}_{H}^{m s}+\left(\Lambda_{\boldsymbol{\beta}}+\boldsymbol{\beta}^{n} A_{n}\right) \mathrm{J}_{H}^{m}\right)$ is the non-canonical part of bulk entropy current. It is easy to see that the combination (17.11) is $U(1)_{\mathrm{T}}$ invariant:

$$
\begin{aligned}
\delta_{x} S_{\mathrm{T}}= & -\int_{\mathcal{M}} \sqrt{-g} \bar{\Lambda}^{(\mathrm{T})} \mathrm{N}_{H}^{\perp}+f_{\mathcal{M}}\left[\mathrm{N}_{H}^{m} D_{m} \bar{\Lambda}^{(\mathrm{T})}+\bar{\Lambda}^{(\mathrm{T})}\left(\mathrm{T}_{H}^{m n} \delta_{\mathfrak{B}} g_{m n}+\mathrm{J}_{H}^{m} \delta_{\mathcal{B}} A_{m}\right)\right] \\
= & -\int_{\mathcal{M}} \sqrt{-g} \bar{\Lambda}^{(\mathrm{T})} \mathrm{N}_{H}^{\perp}+f_{\mathcal{M}} D_{m}\left[\bar{\Lambda}^{(\mathrm{T})}\left(s u^{m}+\boldsymbol{\beta}_{n} \mathrm{~T}_{H}^{m n}+\left(\Lambda_{\boldsymbol{\beta}}+\boldsymbol{\beta}^{p} A_{p}\right) \cdot \mathrm{J}_{H}^{m}\right)\right] \\
& -f_{\mathcal{M}} \bar{\Lambda}^{(\mathrm{T})}\left[D_{m}\left(s u^{m}\right)+\boldsymbol{\beta}^{m}\left(D_{n}\left(\mathrm{~T}_{H}\right)_{m}^{n}-\mathrm{J}_{H}^{n} \cdot F_{m n}\right)+\left(\Lambda_{\boldsymbol{\beta}}+\boldsymbol{\beta}^{p} A_{p}\right) \cdot D_{m} \mathrm{~J}_{H}^{m}\right] \\
= & 0
\end{aligned}
$$


where in the last step we evaluated the integral over the total derivative to cancel the boundary anomaly (using $u^{\perp}=0$ ). Furthermore, we used a bulk adiabaticity equation to kill the second bulk integral (equivalently we could have computed the bulk Bianchi identities explicitly to see that such a bulk adiabaticity equation is satisfied).

While the Lagrangian (17.11) is very natural and simple from the Class $\mathrm{L}_{\mathrm{T}}$ point of view, it is less clear how this can be made consistent with the Class A Lagrangian that we gave before in (13.15). In order to get a step closer towards the Class A SchwingerKeldysh Lagrangian, let us rotate to another basis of sources: it turns out that, while $\left\{g_{\mu \nu}, A_{\mu}, \tilde{g}_{\mu \nu}, \tilde{A}_{\mu}\right\}$ are convenient to construct the master Lagrangian, anomalies are easier to describe in a Schwinger-Keldysh inspired basis $\left\{g_{\mu \nu}^{\mathrm{R}}, A_{\mu}^{\mathrm{R}}, g_{\mu \nu}^{\mathrm{L}}, A_{\mu}^{\mathrm{L}}\right\}$ as we defined it in (15.1). The twisted difference sources $\left\{\left(g^{\mathrm{R}}-g^{\mathrm{L}}\right)_{\mu \nu},\left(A^{\mathrm{R}}-A^{\mathrm{L}}\right)_{\mu}\right\}$ then have a nice $U(1)_{\mathrm{T}}$ transformation:

$$
\delta_{x}\left(g^{\mathrm{R}}-g^{\mathrm{L}}\right)_{\mu \nu}=\delta_{\bar{\Lambda}^{(\mathrm{T}) \mathcal{B}}} g_{\mu \nu}, \quad \delta_{x}\left(A^{\mathrm{R}}-A^{\mathrm{L}}\right)_{\mu}=\delta_{\bar{\Lambda}^{(\mathrm{T}) \mathcal{B}}} A_{\mu},
$$

where $\delta_{\bar{\Lambda}^{(\top)} \mathcal{B}}$ denotes Lie drag along $\left\{\bar{\Lambda}^{(\mathrm{T})} \boldsymbol{\beta}^{\mu}, \bar{\Lambda}^{(\mathrm{T})} \Lambda_{\boldsymbol{\beta}}\right\}$.

We can account for the anomaly by viewing it as originating from inflow due to a bulk Chern-Simons theory. To this end, we need to switch from a description of covariant currents to one in terms of consistent currents, c.f., appendix A. The Bardeen-Zumino currents by which the two descriptions differ will be seen as part of the bulk inflow. To wit, the consistent Lagrangian is related to $\mathcal{L}_{\mathrm{T}}$ as follows:

$$
\begin{aligned}
\mathcal{L}_{\mathrm{T}}^{\text {cons }} \equiv & \left(\mathcal{L}_{\mathrm{T}}\right)_{\mathrm{A}}-\left(\mathcal{L}_{\mathrm{T}}\right)_{B Z} \\
= & \left.\left(\left(\mathrm{N}^{\mu}\right)_{\mathrm{A}}-\mathrm{N}_{B Z}^{\mu}\right) \mathrm{A}^{(\mathrm{T})}{ }_{\mu}+\frac{1}{2}\left(\left(T^{\mu \nu}\right)_{\mathrm{A}}-T_{B Z}^{\mu \nu}\right)\right) \tilde{g}_{\mu \nu}+\left(\left(J^{\mu}\right)_{\mathrm{A}}-J_{B Z}^{\mu}\right) \cdot \tilde{A}_{\mu} \\
= & \left(\left(J_{S}^{\mu}\right)_{\mathrm{A}}-J_{S, B Z}^{\mu}\right) \mathrm{A}^{(\mathrm{T})}{ }_{\mu}+\frac{1}{2}\left(\left(T^{\mu \nu}\right)_{\mathrm{A}}-T_{B Z}^{\mu \nu}\right)\left(g^{\mathrm{R}}-g^{\mathrm{L}}\right)_{\mu \nu} \\
& +\left(\left(J^{\mu}\right)_{\mathrm{A}}-J_{B Z}^{\mu}\right) \cdot\left(A^{\mathrm{R}}-A^{\mathrm{L}}\right)_{\mu},
\end{aligned}
$$

where $J_{S, B Z}^{\mu}=-\boldsymbol{\beta}^{\nu} \mathcal{T}_{\nu}^{\mu}$ as in appendix A. In order to show that the philosophy of section 13.3 is being upheld by the present construction, we want to demonstrate that the Bardeen-Zumino currents can be interpreted as an anomaly inflow due to the difference of two Chern-Simons terms. The bulk contribution of these Chern-Simons terms should give rise to the Hall currents. Indeed, we observe that the Bardeen-Zumino and Hall part of the action (17.11) can be written as

$$
\begin{aligned}
\int_{\mathcal{M}} \sqrt{-g} & \left(\mathcal{L}_{\mathrm{T}}\right)_{B Z}+f_{\mathcal{M}}\left(\mathrm{N}_{H}^{m} \mathrm{~A}^{(\mathrm{T})}{ }_{m}+\frac{1}{2} \mathrm{~T}_{H}^{m n} \tilde{g}_{m n}+\mathrm{J}_{H}^{m} \cdot \tilde{A}_{m}\right) \\
= & \int_{\mathcal{M}} \sqrt{-g}\left(J_{S, B Z}^{\mu} \mathrm{A}^{(\mathrm{T})}+\frac{1}{2} T_{B Z}^{\mu \nu}\left(g^{\mathrm{R}}-g^{\mathrm{L}}\right)_{\mu \nu}+J_{B Z}^{\mu} \cdot\left(A^{\mathrm{R}}-A^{\mathrm{L}}\right)_{\mu}\right) \\
& +f_{\mathcal{M}}\left(J_{S, H}^{m} \mathrm{~A}^{(\mathrm{T})}{ }_{m}+\frac{1}{2} \mathrm{~T}_{H}^{m n}\left(g^{\mathrm{R}}-g^{\mathrm{L}}\right)_{m n}+\mathrm{J}_{H}^{m} \cdot\left(A^{\mathrm{R}}-A^{\mathrm{L}}\right)_{m}\right) \\
= & f_{\mathcal{M}}\left(\boldsymbol{I}_{\mathrm{CS}}\left[\boldsymbol{A}_{\mathrm{R}}, \boldsymbol{\Gamma}_{\mathrm{R}}\right]-\boldsymbol{I}_{\mathrm{CS}}\left[\boldsymbol{A}_{\mathrm{L}}, \boldsymbol{\Gamma}_{\mathrm{L}}\right]\right) \\
& +\int_{\mathcal{M}} \sqrt{-g}\left(J_{S, B Z}^{\mu} \mathrm{A}^{(\mathrm{T})}\right)+f_{\mathcal{M}}\left(J_{S, H}^{m} \mathrm{~A}^{(\mathrm{T})}{ }_{m}\right)+\mathcal{O}\left(\left(\bar{\Psi}_{\mathrm{T}}\right)^{2}\right)
\end{aligned}
$$


where we used in the last step that the non-entropic currents in the second and third line are the linear terms in when we expand a difference of Chern-Simons terms. Since our answers are insensitive to terms that are quadratic in difference sources, we can perform the last step. Terms that are discarded since they are quadratic in difference fields are denoted as $\mathcal{O}\left(\left(\bar{\Phi}_{T}\right)^{2}\right)$.

As it stands (17.15) demonstrates consistency with the methods used in section 13.3 (Schwinger-Keldysh for Class A). What we see in the final expression of (17.15) is precisely the difference of Chern-Simons terms that could be converted into an appropriate transgression form. Since we are already in the doubled construction, the hatted Chern-Simons terms which would have been present appear to have cancelled out against the influence functional. However, we are forced in our $U(1)_{\mathrm{T}}$-invariant formalism to introduce some new pieces of data - the terms in the last line of (17.15) involving the Bardeen-Zumino current are not present in the Schwinger-Keldysh formalism. These terms determine the entropy current both in bulk and physical manifold uniquely in terms of the anomaly polynomial. Of course, these terms depend on the presence of $\mathrm{A}^{(\mathrm{T})} \mathrm{m}$ and could therefore not be seen in our previous naïve Schwinger-Keldysh treatment of Class A. Note also that in the Schwinger-Keldysh construction with the influence functionals one is unable to determine the precise form of the entropy current owing to our failure to derive the adiabaticity equation. As a result what we can compare naturally in the two formalisms is the constitutive relations and these agree as they must for consistency. We leave it as an open and interesting problem to finish delineating the connections between the two formalisms, at the very least to check how $U(1)_{\mathrm{T}}$ invariance helps restore adiabaticity explicitly. For now we simply take comfort in the fact that we have a Class $\mathrm{L}_{\mathrm{T}}$ effective action that gives us a consistent adiabatic solution for the anomalous constitutive relations.

\subsection{Field redefinitions}

An important consistency requirement on our eightfold classification is, of course, that it is (on-shell) invariant under field redefinitions. While we commented at various occasions on the most general allowed field redefinitions, the unified framework of Class $\mathrm{L}_{\mathrm{T}}$ simplifies the discussion considerably. ${ }^{102}$ Consider the most general field redefinitions that preserve the property of $\left\{\boldsymbol{\beta}^{\mu}, \Lambda_{\boldsymbol{\beta}}\right\}$ being in equilibrium aligned with the symmetry generators $\left\{K^{\mu}, \Lambda_{K}\right\}$ :

$$
\begin{aligned}
\boldsymbol{\beta}^{\mu} & \mapsto \boldsymbol{\beta}^{\mu}-\delta_{\mathcal{B}} \bar{V}^{\mu}=\boldsymbol{\beta}^{\mu}+£_{\bar{V}} \boldsymbol{\beta}^{\mu}, \\
\Lambda_{\boldsymbol{\beta}} & \mapsto \Lambda_{\boldsymbol{\beta}}-\delta_{\mathcal{B}} \Lambda_{\bar{V}}=\Lambda_{\boldsymbol{\beta}}+£_{\bar{V}} \Lambda_{\boldsymbol{\beta}}+\left[\Lambda_{\boldsymbol{\beta}}, \bar{\Lambda}_{V}\right]-\boldsymbol{\beta}^{\sigma} \partial_{\sigma} \bar{\Lambda}_{V}, \\
g_{\mu \nu}^{\prime} & \mapsto g_{\mu \nu}^{\prime}+£_{\bar{V}} g_{\mu \nu}^{\prime}-\bar{\Lambda}_{V}^{(\mathrm{T})} \delta_{\mathcal{B}} \tilde{g}_{\mu \nu}, \\
A_{\mu}^{\prime} & \mapsto A_{\mu}^{\prime}+£_{\bar{V}} A_{\mu}^{\prime}+\left[A_{\mu}^{\prime}, \bar{\Lambda}_{V}\right]-\bar{\Lambda}_{V}^{(\mathrm{T})} \delta_{\mathcal{B}} \tilde{A}_{\mu}, \\
\mathrm{A}^{(\mathrm{T})}{ }_{\mu} & \mapsto \mathrm{A}^{(\mathrm{T})}{ }_{\mu}+£_{\bar{V}} \mathrm{~A}^{(\mathrm{T})}{ }_{\mu}+\partial_{\mu} \bar{\Lambda}_{V}^{(\mathrm{T})}, \\
\Lambda_{\boldsymbol{\beta}}^{(\mathrm{T})} & \mapsto \Lambda_{\boldsymbol{\beta}}^{(\mathrm{T})}+£_{\bar{V}} \Lambda_{\boldsymbol{\beta}}^{(\mathrm{T})}-\boldsymbol{\beta}^{\sigma} \partial_{\sigma} \bar{\Lambda}_{V}^{(\mathrm{T})}, \\
g_{\mu \nu} & \mapsto g_{\mu \nu}, \quad A_{\mu} \mapsto A_{\mu} .
\end{aligned}
$$

\footnotetext{
${ }^{102}$ For example, an isolated proof for the field redefinition-invariance of Class B would be notoriously complicated.
} 
for some general diffeomorphism, flavour and $U(1)_{\mathrm{T}}$ parameters $\left\{V^{\mu}, \Lambda_{V}, \Lambda_{V}^{(\mathrm{T})}\right\}$ and their twisted counterparts $\left\{\bar{V}^{\mu}, \bar{\Lambda}_{V}, \bar{\Lambda}_{V}^{(\mathrm{T})}\right\}$. An argument completely analogous to the one presented for Class L (see section 7.6) shows that under these transformations the Lagrangian $\mathcal{L}_{\mathrm{T}}$ only changes by terms proportional to the equations of motion (16.6), (16.8). This can easily be seen from the fact that the above field redefinitions take the form of a constrained variation (16.1), (16.2).

Since the eightfold Lagrangian $\mathcal{L}_{\mathrm{T}}$ encompasses all seven classes of adiabatic transport and disallows Class $\mathrm{H}_{F}$, we see that the field redefinitions (17.16) preserve this structure. This provides a very general argument for our eightfold classification being on-shell field redefinition-invariant. 


\section{Part IV}

\section{Future prospects}

\section{Discussion}

We have described a framework for non-linear hydrodynamics that gives a complete classification of hydrodynamic transport, arguing in the process for an eightfold way of hydrodynamic dissipation. The key idea was to exploit the natural decomposition of transport in two primary categories: adiabatic and dissipative. While the notion of adiabaticity we introduce reduces on-shell to a more intuitive notion of non-dissipative (i.e., entropy conserving) dynamics, it more generally involves playing off entropy production against energy momentum and charge transport (off-shell). One of the remarkable outcomes of our analysis was demonstrating that much of transport beyond leading order is in fact adiabatic.

We have established that adiabatic transport is cleanly organized into seven distinct classes of constitutive relations. Some of these are easily understood such as the hydrostatic scalar Class $\mathrm{H}_{S}$ and anomaly induced Class A, which have been explored extensively in recent literature, whilst certain others are perhaps a bit more exotic and unfamiliar. Curiously, all but one of these classes $\left(\right.$ Class $\overline{\mathrm{H}}_{V}$ ) have been encountered in earlier investigations, though neither the structural aspects nor their import has been completely appreciated hitherto. Our aim in the sections above has been to clearly bring out these aspects for every one of these adiabatic classes.

Having figured out how to tackle adiabatic transport, we further argued that dissipative hydrodynamics is under much better control than one might have a-priori anticipated. The primary result in this context has already been derived in $[36,37]$. The adiabatic analysis allows for an alternative perspective which complements and bolsters the central point: dissipative transport is constrained by the second law of thermodynamics to obey sign-definiteness constraints only at the leading order in the gradient expansion.

From the point of view of the hydrodynamic effective field theory, the combination of adiabaticity and the fact that dissipative parts of higher order transport are unconstrained, restores a sense of democracy to hydrodynamics. Specifically, the standard current algebra description of hydrodynamic constitutive relations could have been a simple exercise in representation theory, were it not for the constraint imposed by the second law. As has been argued in the text and in the aforementioned references, the constraints at arbitrary orders in the gradient expansion are completely captured by demanding the existence of hydrostatic equilibrium. This class of forbidden constitutive relations, Class $\mathrm{H}_{F}$, is easily ascertained by writing down all symmetry allowed tensor structures for the basic currents (which survive the hydrostatic limit) and eliminating ones that do not arise from an effective action (the hydrostatic partition function). Thus, modulo the Class $\mathrm{H}_{F}$ constitutive relations, one finds the task of a hydrodynamicist is rather simple for every other constitutive relation is physically admissible and satisfies the second law (beyond leading order). 
While the major part of our construction of adiabatic constitutive relations was carried out straightforwardly using conventional techniques familiar in hydrodynamic analyses, we have also uncovered some new structures in our quest for constructing an effective action for hydrodynamics. We have argued for a new symmetry principle, an Abelian gauge symmetry $U(1)_{\mathrm{T}}$ arising as a consequence of the eightfold way. We have primarily used this symmetry to write down a generating functional for the adiabatic classes. Let us therefore take stock of the physical implications of this construction. The discussion below is meant to be heuristic and we hope to provide concrete evidence for it in the future [55].

One naively might imagine that a hydrodynamic effective action in the absence of dissipation should be rather simple, following the usual rules of effective field theory. This is indeed the case for the Class L constitutive relations where we have a straightforward construction of a Landau-Ginzburg functional (Lagrangian) in terms of the effective infrared degrees of freedom (the thermal vector and twist). However, as we have explicitly seen this is inadequate to capture all of adiabatic transport. Not only are the transverse vector based Classes $\mathrm{H}_{V}$ and $\overline{\mathrm{H}}_{V}$ outside the remit of such a Lagrangian density, but we have furthermore evidence that Berry-like (Class B) terms are unaccounted for. Moreover, an analysis of anomalous constitutive relations in Class A both herein and in our earlier work [30] has revealed the impossibility of satisfying both the Ward identities and the second law simultaneously, unless one is willing to enlarge the set of degrees of freedom.

This perspective is natural when one considers the passage to a complete theory of hydrodynamics including dissipation, or more generally views hydrodynamics as governing infra-red fluctuations of a density matrix. One naturally then anticipates invoking a real-time non-equilibrium formalism such as the Schwinger-Keldysh construction [79, 80], thereby motivating the exploration of implications of doubling the degrees of freedom inherent in this formalism. This doubling in the fluid dynamical context is often called Martin-Siggia-Rose or Janssen-deDominicis-Politi formalism (see [97]).

In the context of anomalies we have shown that this intuition can indeed be made to work, in part since the structure of allowed terms is tightly constrained by the underlying symmetry (flavour gauge and diffeomorphism). However, once we double the degrees of freedom and consider a pure, entangled state in the doubled theory as the starting point of our discussion, we are led naturally to inquire after the most general set of interactions allowed in the effective field theory. The key issue concerns the constraints on the Feynman-Vernon influence functionals [50] describing the interactions between the two sets of degrees of freedom. Obtaining the correct anomalous Ward identities without modifying the single copy constitutive relation forces upon us a particular set of anomaly induced influence functionals.

If we were to allow arbitrary influence functionals however, then it is easy to generate Class $\mathrm{H}_{F}$ constitutive relations at will, thus explicitly violating the second law. This is equivalent to stating that, given the usual symmetries, the emergence of entropy, dissipation and second law at long distances is not 'natural' in the 't Hooft-Wilson sense. Thus one needs an additional principle to forbid arbitrary influence functionals and solve this naturalness problem of second law. Our claim is that in the hydrodynamic effective field theory the $U(1)_{\mathrm{T}}$ symmetry by ensuring adiabaticity guarantees that the second law is upheld. 
Let us then sketch a scenario how this $U(1)_{\mathrm{T}}$ symmetry works in ensuring the second law in the hydrodynamic limit. We first recall that the hydrodynamic gradient expansion requires that the fluctuations about the underlying Gibbs density matrix are sufficiently long wavelength. Operationally this almost always implies that we are in the high temperature regime. In this limit Schwinger-Keldysh construction for the equilibrium density matrix is such that the common or average fields are macroscopic, but the difference fields are retained only to linear order (this is required to ensure that we extract the correct retarded correlators) [81]. Pictorially we can then imagine that while the two sets of degrees of freedom $\boldsymbol{\Psi}_{\mathrm{L}}$ and $\boldsymbol{\Psi}_{\mathrm{R}}$ live on different background spacetimes (or parts of the SchwingerKeldysh contour), the effective spacing between them is vanishingly small in the Euclidean time direction. Alternately, the difference fields encode the fluctuations or noise in the system and they are Avagadro-suppressed in a fluid.

We would like to view the fluid phase as the Higgs phase for $U(1)_{\mathrm{T}}$ with the difference fields acting as the Higgs fields which transform non-linearly under $U(1)_{\mathrm{T}}$. This ensures that both the $U(1)_{\mathrm{T}}$ gauge field and the difference fields are invisible at long distances. A crucial result in this paper is to show that, ignoring effects of $U(1)_{\mathrm{T}}$ ghost fields, one can posit a nonlinear transformations for difference fields (see eq. (15.4)) that ensures the removal of Class $\mathrm{H}_{F}$ and reproduces the seven adiabatic classes. It would be enormously satisfying to show that this success somehow extends to dissipative class when appropriate ghost fields are added and their effects are taken into account. Indeed we have noted in our Class $\mathrm{D}$ description that the intertwiners $\mathcal{N}$ and $\mathcal{S}$ are required to be positive definite (symmetric) quadratic forms in some tensor representation. It is worthwhile noting that these intertwiners typically take on the role of the kinetic terms of certain ghosts which naturally occur in the Martin-Siggia-Rose dissipative effective action [83]. Such a structure makes it natural to demand positivity for these quadratic forms which along with removal of Class $\mathrm{H}_{F}$ would then ensure second law. It would be interesting whether these set of statements can be made precise in order to provide a Wilsonian explanation for second law of thermodynamics.

We see that the well-behavedness of the hydrodynamic effective field theory appears from this perspective to be tied to the underlying presence of the $U(1)_{\mathrm{T}}$ symmetry. In some sense, this Abelian symmetry is the macroscopic manifestation of the Kubo-MartinSchwinger (KMS) condition which encodes the near-thermal correlations between two copies of Schwinger-Keldysh construction. Of course, it would be fascinating to develop these heuristic ideas and derive the consequences of the $U(1)_{T}$ symmetry not only in the hydrodynamic limit but more generally in non-equilibrium QFT. Based on this intuitive picture it is tempting to christen the symmetry as KMS-gauge invariance, but we presently refrain from doing so, hoping to provide more concrete evidence in its favour in the near future.

Having described some of the conceptual implications of our construction of the eightfold Lagrangian, we now turn to other aspects of our analysis. One useful perspective provided by the hydrodynamic effective actions in Classes $\mathrm{L}$ and $\mathrm{L}_{\mathrm{T}}$ relates to the entropy current. It is usually said that the notion of the entropy current is mysterious from a microscopic perspective. However, from our effective action viewpoint, the entropy current 
(or equivalently the free-energy current) is quite canonically derived as a Noether charge. This is a simple consequence of diffeomorphism invariance. It is well appreciated that in a dynamical theory of gravity where diffeomorphisms are gauged, there is a notion of entropy for spacetimes with horizons. This horizon entropy itself is constructed as a Noether charge [73]. More generally one may ascribe a gravitational entropy to any diffeomorphism invariant dynamics. This point was appreciated by the authors of [73] and was amplified in the context of ideal fluids by [98]. This then begs a related question: "Can we use the hydrodynamic entropy current to provide a Noether current for black holes out of equilibrium?" The answer to this question is clearly in the affirmative based on the direct isomorphism between the hydrodynamic entropy current and black hole entropy in the fluid/gravity context [64]. This then provides a possible avenue for ascertaining the non-equilibrium gravitational entropy current in time-dependent situations. ${ }^{103}$

We noted in section 1 that often in addition to the second law of thermodynamics, one requires hydrodynamic transport to satisfy the Onsager relations $[38,39]$. This requirement is based for the most part on the empirical observation that most physical fluids satisfy these relations. One can phrase the Onsager relations in our language as follows: "In systems with time-reversal symmetry there are no anti-symmetric contributions to transport." Paraphrasing this statement into our adiabatic classification, we learn that the Onsager relations forbid the presence of Class B terms in hydrodynamic transport. However, one must pay attention to the underlying assumptions about the dynamics; for example, the derivations in $[38,39]$ rely on either assuming that physical systems produce currents out of equilibrium only to extremize dissipation or (equivalently by the fluctuation-dissipation theorem) assume a Gaussian spectrum of fluctuations.

We are for the most part agnostic about these relations, though our framework is broad enough to allow exploration of such transport. For example, the relations we have found constraining Class B terms when deriving adiabatic constitutive relations from a Class L scalar Lagrangian density, such as the vanishing of Hall viscosity or the relation (14.33) in neutral fluids, can be viewed as higher-order versions of the Onsager relations. Indeed one might argue that demanding that all non-dissipative transport outside equilibrium be derivable from a Landau-Ginzburg free energy functional as in Class L (without Schwinger-Keldysh doubling) provides an alternate route to ensuring the Onsager relations are satisfied, even in time-reversal violating systems.

Perhaps more curiously, there appears to be an interesting constraint on Class B terms in holographic fluids. Firstly all known examples of such transport obey a membrane paradigm like formula. By this we mean that the precise value of the Class B transport coefficient is given in terms of some geometric quantity evaluated on the horizon of an asymptotically AdS black hole. For instance, eq. (32) of [99] expresses Hall viscosity as such, while eq. (6.24) of [68] provides an analogous expression for Hall conductivity. For parity-even fluids, the derivation of the universal Haack-Yarom relation between second order transport coefficients (14.33) also expresses the appropriate combination this way, cf., eq. (47) of [89]. In all these case the relevant membrane-paradigm quantity then vanishes

\footnotetext{
${ }^{103}$ We would like to thank Sayantani Bhattacharyya and Shiraz Minwalla for extensive discussions on this issue.
} 
at the horizon in the two-derivative theory because of some general feature of horizon geometry, forcing thence the vanishing of a Class B term. This seems rather generic in two derivative theories of gravity; inclusion of higher derivative interactions appears to allow non-vanishing Class B terms. For example, vis a vis the Haack-Yarom relation, one finds that it is upheld to linear order in Gauss-Bonnet corrections to gravity [70], but not beyond [71, 72]. Recent analysis by [72] in the physically more interesting situation of string theory induced derivative corrections to Type IIB supergravity appear to uphold this to one higher order. It would be interesting to analyze what feature of black hole horizons and gravitational dynamics plays a role in determining these aspects of transport.

Holographic fluids via the fluid/gravity correspondence [41, 52] provide an ideal environment to test various statements about fluid dynamics in general by allowing one to be able to compute explicit constitutive relations. We find it reassuring to see evidence for the eightfold way in the examples studied hitherto. We outline in section 19 a set of questions that should give us even better insight into how holography implements the classification. What is perhaps curious however, is certain specificity in these fluids. While there is no a-priori reason that every aspect of transport allowed by the second law should find a holographic manifestation, it is nevertheless amazing to find further evidence for the near-idealness of these systems. It has been known for a very long time, starting from the seminal work of [40], that holographic fluids tend to want to minimize the shear viscosity and saturate the famous bound $\frac{\eta}{s} \geq \frac{1}{4 \pi}[87] .{ }^{104}$ The low value of shear viscosity implies that these fluids minimize their entropy production for arbitrary flows. Fascinatingly, this statement also appears to be upheld at the next order in gradients.

For Weyl invariant holographic fluids we find that the subleading contribution (third order) to entropy production is forced to vanish if the Class D transport coefficient $\lambda_{1}-\kappa$ vanishes. This relation appears to hold in all two-derivative theories of gravity explored so far (but is violated upon inclusion of higher derivative interactions [72]). This then suggests that holographic fluids obtained in the long-wavelength limit of strongly interacting quantum dynamics, obey a principle of minimum dissipation. One might suspect that all such transport be derivable from a (Class L) Landau-Ginzburg free energy functional, which in turn should be obtainable from the bulk gravitational dynamics. The simplest test would be to derive (14.35) directly from the Einstein-Hilbert dynamics for gravity in asymptotically AdS spacetimes. It would be fascinating to develop this line of thought, for it should provide us with a geometric underpinning for deriving effective actions for generic non-equilibrium quantum dynamics. More generally the interplay of gravity with the extended framework of $U(1)_{\mathrm{T}}$ KMS-gauge invariant effective field theories could potentially provide important insights into formulating Wilsonian low energy dynamics for QFTs in mixed states.

\section{Homework problems}

We have at various stages of our discussion exemplified adiabatic fluids and the efficacy of the eightfold way using simple examples of hydrodynamic systems. While the structural

\footnotetext{
${ }^{104}$ We refer the reader to $[100,101]$ for critical discussions of this bound and survey of attempts to violate it in various systems.
} 
aspects of our discussion are completely clear in the abstract, it is remarkable and reassuring that physical fluid systems are aware of the eightfold classification. We gave some arguments in favour of this using the analysis of holographic fluids (particularly the neutral Weyl invariant fluid) as well as in kinetic theory in section 14.5.

Ideally, of course, we would have liked to give many more examples and furthermore argue that the classification scheme can be used as an efficient organizational principle vis a vis actual computations. While we do believe this to be true, making further progress requires data in other hydrodynamic systems, which we do have at hand at present. This provides us with an opportunity to outline a set of problems, which we think are solvable, some of which perhaps more straightforwardly than others. We therefore leave our readers who have made it this far with a few problems that we feel are worth exploring.

One natural venue for exploration is simply to obtain explicit results for constitutive relations using the fluid/gravity correspondence for holographic fluids. Weyl invariant neutral fluids are already covered in our analysis. An obvious next step would be to examine Weyl invariant charged fluid dynamics by explicitly studying the Einstein-Maxwell (for parity-even) or Einstein-Maxwell-Chern-Simons (for anomalous parity-odd) examples. We note that $[5,6,28,88]$ have studied specific aspects of transport in this particular set-up up to second order in gradients, but the full set of constitutive relations require turning on all possible background sources has yet to be done. Another direction to consider is neutral fluids without Weyl invariance, but since the simplest set-ups realizable holographically secretly enjoy higher dimensional conformal invariance [102] (see also [103, 104]) it is easy to intuit that these analyses won't provide more detailed insight (see however [105] for explicit conformal symmetry breaking by sources). One could perhaps also make use of higher derivative gravitational theories, though in that case care must be exercised to ensure that one is dealing with a unitary QFT dual. With this preamble let us state a few questions for each of our classes and some specific issues that can be understood.

- Class $\mathrm{H}_{F}$ : the hydrostatic analysis for charged fluids implies 17 hydrostatic forbidden constitutive relations at second order in gradients [3]. Find explicit expressions for these constraints and further specialize to the case with Weyl invariance. Use the set-up described above to demonstrate that fluid/gravity automatically incorporates the hydrostatic constraints. Furthermore, demonstrate that holographic fluids always respect the $\mathrm{H}_{F}$ constraints at any order in the gradient expansion.

- Class L: write down the $\mathrm{H}_{S} \cup \overline{\mathrm{H}}_{S}$ terms describing a fluid dual to Einstein-Maxwell system using the aforementioned holographic analysis, i.e., the analog of (8.25). More generally give a bulk prescription to compute the effective action for this system from the gravitational dynamics (perhaps building on the ideas in [90]).

- Class $\mathrm{H}_{V}$ : in the holographic context demonstrate how $\mathrm{H}_{V}$ terms arise from the extension of the Noether procedure of [73] by [106] to account for the presence of Chern-Simons interactions, i.e., extend the leading order argument in [75, 107] to arbitrary derivative order. 
- Class $\overline{\mathrm{H}}_{V}$ : this is the most interesting class of adiabatic constitutive relations. Thus far we have no data on such terms in any realistic hydrodynamic context. Do these terms arise in fluid-gravity correspondence at all? Or are they constrained to vanish as with certain Class B terms we have described in the text? Does there exist a simple membrane paradigmesque formula for them in terms of data on the horizon [108]?

- Class B: formulate a general membrane paradigm formula to compute all these terms in terms of horizon data, extending the results of [99]. Is there a role for an attractor like mechanism (for non-extremal black holes) which requires that these coefficients are in certain sense robust?

- Class C: explore the presence of exactly conserved topological currents in fluid/gravity. For example show how the Lovelock terms in the gravitational description of even-dimensional AdS spacetimes map to Euler currents in the boundary fluid entropy current using [64].

- Class D: derive a membrane paradigm formula for general Class D transport coefficients. Is there a role for the tensor valued differential operators $\Upsilon$ to show up in the bulk? Do holographic fluids always attempt to minimize the entropy production for a given fluid flow. Give evidence or disprove the minimum entropy production conjecture for two derivative theories of gravity. What is the corresponding statement in higher derivative theories of gravitational dynamics?

- Class A: derive the Feynman-Vernon terms for Class A described in [30] and section 12 from the fluid/gravity correspondence.

- Eightfold way: derive the eightfold way of transport from a general SchwingerKeldysh analysis [55]. Extend this eightfold classification to systems with spontaneously broken symmetries and other Goldstone modes such as superfluids (cf., [109] for a comprehensive treatment of superfluid dynamics and [110-112] for discussions about holographic superfluids). Is there a relation between the eightfold way and Hohenberg-Halperin classification [113] of dynamic critical phenomena?

\section{Acknowledgments}

It is a pleasure to thank Koushik Balasubramanian, Jyotirmoy Bhattacharya, Sayantani Bhattacharyya, Veronika Hubeny, Kristan Jensen, Hong Liu, Juan Maldacena, Shiraz Minwalla, Guy Moore, Dam Son, Andrei Starinets, and Amos Yarom for enjoyable discussions on various aspects of hydrodynamics. FH and MR would like to thank the Institute for Advanced Study, Princeton for hospitality during the course of this project. MR would in addition like to thank Yukawa Institute for Theoretical Physics, Kyoto, Univ. of Amsterdam, Aspen Center for Theoretical Physics and Tata Institute for Fundamental Research, Mumbai, for hospitality during the course of this project.

FH is supported by a Durham Doctoral Fellowship. RL gratefully acknowledges support from Institute for Advanced Study, Princeton. MR was supported in part by 
the Ambrose Monell foundation, by the STFC Consolidated Grants ST/J000426/1 and ST/L000407/1, by the NSF grant under Grant No. PHY-1066293, and by the European Research Council under the European Union's Seventh Framework Programme (FP7/20072013), ERC Consolidator Grant Agreement ERC-2013-CoG-615443: SPiN (Symmetry Principles in Nature). 


\section{Part V}

\section{Interconnections \& generalizations}

\section{A Adiabaticity equation for consistent currents}

We have elected to work with covariant currents and covariant anomalies in the bulk of the paper. But, for some applications, it is useful to work with consistent currents and consistent anomalies instead. In this appendix we describe various statements about how adiabaticity equation changes if one works with consistent currents. Some salient results (such as those necessary for hydrostatics) have already been quoted in the main text; the derivation of these results will be found herein.

\section{A.1 Bardeen-Zumino currents}

To begin with, we need to pass to one of the consistent anomalies from the covariant anomalies we have been working with earlier. To do this, we choose a Chern-Simons form $\boldsymbol{I}_{\mathrm{CS}}$ such that $d \boldsymbol{I}_{\mathrm{CS}}=\mathcal{P}$ where $\mathcal{P}$ is the anomaly polynomial of the system under consideration. The consistent anomalies are then given by

$$
\mathcal{J}^{\star} 1 \equiv \frac{\partial \boldsymbol{I}_{\mathrm{CS}}}{\partial \boldsymbol{A}}, \quad \mathcal{T}_{\mu}{ }^{{ }^{\star}} 1 \equiv \frac{\partial \boldsymbol{I}_{\mathrm{CS}}}{\partial \boldsymbol{\Gamma}^{\mu}{ }_{\nu}} .
$$

It is also useful to work with Bardeen-Zumino current and energy momentum tensor $\left\{J_{B Z}^{\mu}, T_{B Z}^{\mu \nu}\right\}$ which are local functions of sources $\left\{A_{\mu}, g_{\mu \nu}\right\}$ given by

$$
\begin{aligned}
J_{B Z}^{\mu}{ }^{\star} d x_{\mu} & \equiv \frac{\partial \boldsymbol{I}_{\mathrm{CS}}}{\partial \boldsymbol{F}}, \quad\left(\Sigma_{B Z}\right)^{\sigma \nu}{ }_{\mu}{ }^{\star} d x_{\sigma} \equiv 2 \frac{\partial \boldsymbol{I}_{\mathrm{CS}}}{\partial \boldsymbol{R}^{\mu}{ }_{\nu}}, \\
T_{B Z}^{\mu \nu} & \equiv \frac{1}{2} \nabla_{\sigma}\left[\left(\Sigma_{B Z}\right)^{\mu[\nu \sigma]}+\left(\Sigma_{B Z}\right)^{\nu[\mu \sigma]}-\left(\Sigma_{B Z}\right)^{\sigma(\nu \mu)}\right] .
\end{aligned}
$$

We will not need the detailed forms above for our analysis, though given any quantum system we can determine the anomaly polynomial and thence the currents if necessary. All we need for the moment is the following fact: the Bardeen-Zumino current and energy momentum tensor obey conservation type equations with a right hand side that has the difference between the covariant anomaly and the consistent anomaly, viz.,

$$
\begin{aligned}
D_{\mu} J_{B Z}^{\mu} & =\mathrm{J}_{H}^{\perp}-\mathcal{J} \\
\nabla_{\nu} T_{B Z}^{\mu \nu}-\left(J_{B Z}\right)_{\nu} F^{\mu \nu} & =\mathrm{T}_{H}^{\mu \perp}+A^{\mu} \mathcal{J}+\frac{g^{\mu \alpha}}{\sqrt{-g}} \partial_{\nu}\left(\sqrt{-g} \mathcal{T}_{\alpha}^{\nu}\right) .
\end{aligned}
$$

We now want to derive an adiabaticity type equation for Bardeen-Zumino currents using these identities. To this end, consider the combination

$$
\begin{aligned}
\boldsymbol{\beta}_{\mu} & \left(\nabla_{\nu} T_{B Z}^{\mu \nu}-\left(J_{B Z}\right)_{\nu} F^{\mu \nu}-\mathrm{T}_{H}^{\mu \perp}\right)+\left(\Lambda_{\boldsymbol{\beta}}+\boldsymbol{\beta}^{\sigma} A_{\sigma}\right) \cdot\left(D_{\mu} J_{B Z}^{\mu}-\mathrm{J}_{H}^{\perp}\right) \\
& =\frac{\boldsymbol{\beta}^{\mu}}{\sqrt{-g}} \partial_{\nu}\left(\sqrt{-g} \mathcal{T}_{\mu}^{\nu}\right)-\Lambda_{\boldsymbol{\beta}} \cdot \mathcal{J} \\
& =\nabla_{\sigma}\left(\boldsymbol{\beta}^{\nu} \mathcal{T}_{\nu}{ }^{\sigma}\right)-\left(\partial_{\nu} \boldsymbol{\beta}^{\mu}\right) \mathcal{T}_{\mu}{ }^{\nu}-\Lambda_{\boldsymbol{\beta}} \cdot \mathcal{J}
\end{aligned}
$$


If we define the Bardeen-Zumino entropy current as

$$
J_{S, B Z}^{\sigma} \equiv-\boldsymbol{\beta}^{\nu} \mathcal{T}_{\nu}^{\sigma}
$$

we can then write the adiabaticity equation satisfied by Bardeen-Zumino currents as

$$
\begin{aligned}
\nabla_{\sigma} J_{S, B Z}^{\sigma}+\boldsymbol{\beta}_{\mu}( & \left.\nabla_{\nu} T_{B Z}^{\mu \nu}-\left(J_{B Z}\right)_{\nu} F^{\mu \nu}-\mathrm{T}_{H}^{\mu \perp}\right) \\
& +\left(\Lambda_{\boldsymbol{\beta}}+\boldsymbol{\beta}^{\sigma} A_{\sigma}\right) \cdot\left(D_{\mu} J_{B Z}^{\mu}-\mathrm{J}_{H}^{\perp}\right)=-\left(\partial_{\nu} \boldsymbol{\beta}^{\mu}\right) \mathcal{T}_{\mu}^{\nu}-\Lambda_{\boldsymbol{\beta}} \cdot \mathcal{J}
\end{aligned}
$$

The consistent currents are defined as the difference of the covariant currents and the Bardeen-Zumino currents, viz.,

$$
J_{S, \text { cons }}^{\mu} \equiv J_{S}^{\mu}-J_{S, B Z}^{\mu}, \quad T_{\text {cons }}^{m u \nu} \equiv T^{\mu \nu}-T_{B Z}^{\mu \nu}, \quad J_{\text {cons }}^{\mu} \equiv J^{\mu}-J_{B Z}^{\mu} \cdot
$$

By subtracting (A.6) from (2.12), we obtain the adiabaticity equation written in terms of consistent currents and consistent anomalies:

$$
\begin{aligned}
\nabla_{\mu} J_{S, \text { cons }}^{\mu}+\boldsymbol{\beta}_{\mu}\left(\nabla_{\nu} T_{\text {cons }}^{\mu \nu}-\right. & \left.\left(J_{\text {cons }}\right)_{\nu} \cdot F^{\mu \nu}\right) \\
& +\left(\Lambda_{\boldsymbol{\beta}}+\boldsymbol{\beta}^{\lambda} A_{\lambda}\right) \cdot D_{\nu} J^{\nu}=\left(\partial_{\nu} \boldsymbol{\beta}^{\mu}\right) \mathcal{T}_{\mu}{ }^{\nu}+\Lambda_{\boldsymbol{\beta}} \cdot \mathcal{J}
\end{aligned}
$$

This then is the analog of (2.12) in terms of the consistent currents. This expression is useful when we directly want to work with effective actions (without introducing ChernSimons terms).

\section{A.2 The consistent Gibbs current}

If we wish to work directly with the Gibbs current as in section 2.4, then one can introduce the Bardeen-Zumino free energy current given (A.5) via:

$$
\begin{aligned}
\mathcal{G}_{B Z}^{\mu} & \equiv-T\left[J_{S, B Z}^{\mu}+\boldsymbol{\beta}_{\nu} T_{B Z}^{\mu \nu}+\left(\Lambda_{\boldsymbol{\beta}}+\boldsymbol{\beta}^{\sigma} A_{\sigma}\right) \cdot J_{B Z}^{\mu}\right] \\
& =u_{\nu} \mathcal{T}^{\mu \nu}-\left[u_{\nu} T_{B Z}^{\mu \nu}+\mu \cdot J_{B Z}^{\mu}\right] .
\end{aligned}
$$

This Bardeen-Zumino free energy current satisfies the grand canonical counterpart of (A.6)

$$
-\left[\nabla_{\sigma}\left(\frac{\mathcal{G}_{B Z}^{\sigma}}{T}\right)-\frac{\mathcal{G}_{H}^{\perp}}{T}\right]=\frac{1}{2} T_{B Z}^{\mu \nu} \delta_{\mathcal{B}} g_{\mu \nu}+J_{B Z}^{\mu} \cdot \delta_{\mathcal{B}} A_{\mu}-\left(\partial_{\nu} \boldsymbol{\beta}^{\mu}\right) \mathcal{T}_{\mu}^{\nu}-\Lambda_{\boldsymbol{\beta}} \cdot \mathcal{J} .
$$

Subtracting this equation from (2.21), we get

$$
-\nabla_{\sigma}\left(\frac{\mathcal{G}_{\text {cons }}^{\sigma}}{T}\right)=\frac{1}{2} T_{\text {cons }}^{\mu \nu} \delta_{\mathfrak{B}} g_{\mu \nu}+J_{\text {cons }}^{\mu} \cdot \delta_{\mathfrak{B}} A_{\mu}+\left(\partial_{\nu} \boldsymbol{\beta}^{\mu}\right) \mathcal{T}_{\mu}^{\nu}+\Lambda_{\boldsymbol{\beta}} \cdot \mathcal{J}
$$

where we have defined the consistent free energy current

$$
\mathcal{G}_{\text {cons }}^{\sigma} \equiv \mathcal{G}^{\sigma}-\mathcal{G}_{B Z}^{\sigma}
$$

Equation (A.11) plays a useful role in constructing the equilibrium partition function for anomalous hydrodynamics. The current $\mathcal{G}_{\text {cons }}^{\sigma} / T$ is conserved in the hydrostatic limit (unlike $\mathcal{G}^{\sigma} / T$ itself) and hence can be integrated over the base space to give a generating function of hydrostatic correlation functions. 


\section{A.3 Noether construction for free-energy current}

In this subsection, we will show that the free-energy currents for Class A can be obtained by a Noether type construction. To do this, we begin by considering the variation of the transgression form introduced in [12], see section 12

$$
\boldsymbol{V}_{\mathcal{P}} \equiv \frac{\boldsymbol{u}}{2 \boldsymbol{\omega}} \wedge(\mathcal{P}[\boldsymbol{F}, \boldsymbol{R}]-\widehat{\mathcal{P}}[\hat{\boldsymbol{F}}, \hat{\boldsymbol{R}}])
$$

Using the identities

$$
\begin{aligned}
\delta(2 \boldsymbol{\omega}) & =\frac{1}{T} d(T \delta \boldsymbol{u})+T \delta \boldsymbol{u} \wedge \delta_{\mathcal{B}} \boldsymbol{u}+\boldsymbol{u} \wedge(\ldots) \\
\delta \boldsymbol{B} & =D(\delta \boldsymbol{A}+\mu \delta \boldsymbol{u})-\frac{\mu}{T} d(T \delta \boldsymbol{u})+T \delta \boldsymbol{u} \wedge \delta_{\mathcal{B}} \boldsymbol{A}+\boldsymbol{u} \wedge(\ldots) \\
\delta\left(\boldsymbol{B}_{R}\right)^{\mu}{ }_{\nu} & =D\left(\delta \boldsymbol{\Gamma}^{\mu}{ }_{\nu}+T D_{\nu} \boldsymbol{\beta}^{\mu} \delta \boldsymbol{u}\right)-D_{\nu} \boldsymbol{\beta}^{\mu} d(T \delta \boldsymbol{u})+T \delta \boldsymbol{u} \wedge \delta_{\mathcal{B}} \boldsymbol{\Gamma}^{\mu}{ }_{\nu}+\boldsymbol{u} \wedge(\ldots)
\end{aligned}
$$

we can write the variation of $\boldsymbol{V}_{\mathcal{P}}$ in the following form:

$$
\begin{aligned}
\delta \boldsymbol{V}_{\mathcal{P}}=T \delta \boldsymbol{u} \wedge\{ & -\boldsymbol{i}_{\boldsymbol{\beta}} \boldsymbol{V}_{\mathcal{P}}+\delta_{\mathcal{B}} \boldsymbol{u} \wedge \frac{\partial \boldsymbol{V}_{\mathcal{P}}}{\partial(2 \boldsymbol{\omega})}+\delta_{\mathcal{B}} \boldsymbol{A} \wedge \frac{\partial \boldsymbol{V}_{\mathcal{P}}}{\partial \boldsymbol{B}}+\delta_{\mathcal{B}} \boldsymbol{\Gamma}^{\mu}{ }_{\nu} \wedge \frac{\partial \boldsymbol{V}_{\mathcal{P}}}{\partial\left(\boldsymbol{B}_{R}\right)^{\mu}{ }_{\nu}} \\
& +\frac{1}{2} g^{\mu \sigma} \delta_{\mathcal{B}} g_{\nu \sigma} D\left(\frac{\partial \boldsymbol{V}_{\mathcal{P}}}{\partial\left(\boldsymbol{B}_{R}\right)^{\mu}{ }_{\nu}}\right) \\
& +\frac{\mu}{T} \cdot\left[D\left(\frac{\partial \boldsymbol{V}_{\mathcal{P}}}{\partial \boldsymbol{B}}\right)+\frac{\partial \widehat{\mathcal{P}}}{\partial \hat{\boldsymbol{F}}}\right]+\frac{1}{T} \Omega^{\mu}{ }_{\nu}\left[D\left(\frac{\partial \boldsymbol{V}_{\mathcal{P}}}{\partial\left(\boldsymbol{B}_{R}\right)^{\mu}{ }_{\nu}}\right)+\frac{\partial \widehat{\mathcal{P}}}{\partial \hat{\boldsymbol{R}}^{\mu}{ }_{\nu}}\right] \\
& \left.+d\left[\frac{1}{T} \frac{\partial \boldsymbol{V}_{\mathcal{P}}}{\partial(2 \boldsymbol{\omega})}-\frac{\mu}{T} \cdot \frac{\partial \boldsymbol{V}_{\mathcal{P}}}{\partial \boldsymbol{B}}-\frac{1}{T} \Omega^{\mu}{ }_{\nu} \frac{\partial \boldsymbol{V}_{\mathcal{P}}}{\partial\left(\boldsymbol{B}_{R}\right)^{\mu}{ }_{\nu}}-\frac{1}{2} g^{\mu \sigma} \delta_{\mathcal{B}} g_{\nu \sigma} \frac{\partial \boldsymbol{V}_{\mathcal{P}}}{\partial\left(\boldsymbol{B}_{R}\right)^{\mu}{ }_{\nu}}\right]\right\} \\
+ & \delta \boldsymbol{A} \cdot D\left(\frac{\partial \boldsymbol{V}_{\mathcal{P}}}{\partial \boldsymbol{B}}\right)+\delta \boldsymbol{\Gamma}^{\mu}{ }_{\nu} \wedge D\left(\frac{\partial \boldsymbol{V}_{\mathcal{P}}}{\partial\left(\boldsymbol{B}_{R}\right)^{\mu}{ }_{\nu}}\right)-\delta(\mu \boldsymbol{u}) \frac{\partial \hat{\mathcal{P}}}{\partial \hat{\boldsymbol{F}}}-\delta\left(\Omega^{\mu}{ }_{\nu} \boldsymbol{u}\right) \frac{\partial \widehat{\mathcal{P}}}{\partial \hat{\boldsymbol{R}}^{\mu}{ }_{\nu}} \\
+ & d\left\{\delta \boldsymbol{u} \wedge \frac{\partial \boldsymbol{V}_{\mathcal{P}}}{\partial(2 \boldsymbol{\omega})}+\delta \boldsymbol{A} \wedge \frac{\partial \boldsymbol{V}_{\mathcal{P}}}{\partial \boldsymbol{B}}+\delta \boldsymbol{\Gamma}^{\mu}{ }_{\nu} \wedge \frac{\partial \boldsymbol{V}_{\mathcal{P}}}{\partial\left(\boldsymbol{B}_{R}\right)^{\mu}{ }_{\nu}}\right\} .
\end{aligned}
$$

Following the authors of [12], we also introduce a transgression form

$$
\boldsymbol{W}_{\mathrm{CS}} \equiv \frac{\boldsymbol{u}}{2 \boldsymbol{\omega}} \wedge\left(\boldsymbol{I}_{\mathrm{CS}}[\boldsymbol{F}, \boldsymbol{R}]-\boldsymbol{I}_{\mathrm{CS}}[\hat{\boldsymbol{F}}, \hat{\boldsymbol{R}}]\right)
$$

which is related to $\boldsymbol{V}_{\mathcal{P}}$ via the relation $\boldsymbol{V}_{\mathcal{P}}+d \boldsymbol{W}_{\mathrm{CS}}=\boldsymbol{I}_{\mathrm{CS}}[\boldsymbol{F}, \boldsymbol{R}]-\boldsymbol{I}_{\mathrm{CS}}[\hat{\boldsymbol{F}}, \hat{\boldsymbol{R}}]$. Similar manipulations for variation of $\boldsymbol{W}_{\mathrm{CS}}$ yields

$$
\begin{aligned}
\delta \boldsymbol{W}_{\mathrm{CS}}=T \delta \boldsymbol{u} & \wedge\left\{-\boldsymbol{i}_{\boldsymbol{\beta}} \boldsymbol{W}_{\mathrm{CS}}-\Lambda_{\boldsymbol{\beta}} \frac{\partial \boldsymbol{W}_{\mathrm{CS}}}{\partial \boldsymbol{A}}-\partial_{\nu} \boldsymbol{\beta}^{\mu} \frac{\partial \boldsymbol{W}_{\mathrm{CS}}}{\partial \boldsymbol{\Gamma}^{\mu}{ }_{\nu}}\right. \\
& +\delta_{\mathcal{B}} \boldsymbol{u} \wedge \frac{\partial \boldsymbol{W}_{\mathrm{CS}}}{\partial(2 \boldsymbol{\omega})}+\delta_{\mathcal{B}} \boldsymbol{A} \wedge \frac{\partial \boldsymbol{W}_{\mathrm{CS}}}{\partial \boldsymbol{B}} \\
& +\delta_{\mathcal{B}} \boldsymbol{\Gamma}^{\mu}{ }_{\nu} \wedge \frac{\partial \boldsymbol{W}_{\mathrm{CS}}}{\partial\left(\boldsymbol{B}_{R}\right)^{\mu}{ }_{\nu}}+\frac{1}{2} g^{\mu \sigma} \delta_{\mathrm{B}} g_{\nu \sigma} D\left(\frac{\partial \boldsymbol{W}_{\mathrm{CS}}}{\partial\left(\boldsymbol{B}_{R}\right)^{\mu}{ }_{\nu}}\right) \\
& +\frac{\mu}{T} \cdot\left[\frac{\partial \boldsymbol{W}_{\mathrm{CS}}}{\partial \boldsymbol{A}}+D\left(\frac{\partial \boldsymbol{W}_{\mathrm{CS}}}{\partial \boldsymbol{B}}\right)+\frac{\partial \boldsymbol{I}_{\mathrm{CS}}[\hat{\boldsymbol{A}}, \hat{\boldsymbol{\Gamma}}]}{\partial \hat{\boldsymbol{F}}}\right]
\end{aligned}
$$




$$
\begin{aligned}
&+\frac{1}{T} \Omega^{\mu}{ }_{\nu}\left[\frac{\partial \boldsymbol{W}_{\mathrm{CS}}}{\partial \boldsymbol{\Gamma}^{\mu}{ }_{\nu}}+D\left(\frac{\partial \boldsymbol{W}_{\mathrm{CS}}}{\partial\left(\boldsymbol{B}_{R}\right)^{\mu}{ }_{\nu}}\right)+\frac{\partial \boldsymbol{I}_{\mathrm{CS}}[\hat{\boldsymbol{A}}, \hat{\boldsymbol{\Gamma}}]}{\partial \hat{\boldsymbol{R}}^{\mu}{ }_{\nu}}\right] \\
&+\left.d\left[\frac{1}{T} \frac{\partial \boldsymbol{W}_{\mathrm{CS}}}{\partial(2 \boldsymbol{\omega})}-\frac{\mu}{T} \cdot \frac{\partial \boldsymbol{W}_{\mathrm{CS}}}{\partial \boldsymbol{B}}-\frac{1}{T} \Omega^{\mu}{ }_{\nu} \frac{\partial \boldsymbol{W}_{\mathrm{CS}}}{\partial\left(\boldsymbol{B}_{R}\right)^{\mu}{ }_{\nu}}-\frac{1}{2} g^{\mu \sigma} \delta_{{ }_{\mathrm{B}}} g_{\nu \sigma} \frac{\partial \boldsymbol{W}_{\mathrm{CS}}}{\partial\left(\boldsymbol{B}_{R}\right)^{\mu}}\right]\right\} \\
&+\delta \boldsymbol{A} \cdot\left\{\frac{\partial \boldsymbol{W}_{\mathrm{CS}}}{\partial \boldsymbol{A}}+D\left(\frac{\partial \boldsymbol{W}_{\mathrm{CS}}}{\partial \boldsymbol{B}}\right)\right\}+\delta \boldsymbol{\Gamma}^{\mu}{ }_{\nu} \wedge D\left(\frac{\partial \boldsymbol{W}_{\mathrm{CS}}}{\partial\left(\boldsymbol{B}_{R}\right)^{\mu}{ }_{\nu}}\right) \\
&-\delta(\mu \boldsymbol{u}) \frac{\partial \boldsymbol{I}_{\mathrm{CS}}[\hat{\boldsymbol{A}}, \hat{\boldsymbol{\Gamma}}]}{\partial \hat{\boldsymbol{F}}}-\delta\left(\Omega^{\mu}{ }_{\nu} \boldsymbol{u}\right) \frac{\partial \boldsymbol{I}_{\mathrm{CS}}[\hat{\boldsymbol{A}}, \hat{\boldsymbol{\Gamma}}]}{\partial \hat{\boldsymbol{R}}^{\mu}{ }_{\nu}} \\
&+d\left\{\delta \boldsymbol{u} \wedge \frac{\partial \boldsymbol{W}_{\mathrm{CS}}}{\partial(2 \boldsymbol{\omega})}+\delta \boldsymbol{A} \wedge \frac{\partial \boldsymbol{W}_{\mathrm{CS}}}{\partial \boldsymbol{B}}+\delta \boldsymbol{\Gamma}^{\mu}{ }_{\nu} \wedge \frac{\partial \boldsymbol{W}_{\mathrm{CS}}}{\partial\left(\boldsymbol{B}_{R}\right)^{\mu}{ }_{\nu}}\right\}
\end{aligned}
$$

We can then use $\delta \boldsymbol{V}_{\mathcal{P}}+d \delta \boldsymbol{W}_{\mathrm{CS}}=\delta \boldsymbol{I}_{\mathrm{CS}}[\boldsymbol{F}, \boldsymbol{R}]-\delta \boldsymbol{I}_{\mathrm{CS}}[\hat{\boldsymbol{F}}, \hat{\boldsymbol{R}}]$ to get the following identities:

$$
\begin{aligned}
\star \boldsymbol{J}_{\mathcal{P}} & \equiv \frac{\partial \boldsymbol{V}_{\mathcal{P}}}{\partial \boldsymbol{B}}=-\left\{\frac{\partial \boldsymbol{W}_{\mathrm{CS}}}{\partial \boldsymbol{A}}+D\left(\frac{\partial \boldsymbol{W}_{\mathrm{CS}}}{\partial \boldsymbol{B}}\right)\right\}+\frac{\partial \boldsymbol{I}_{\mathrm{CS}}}{\partial \boldsymbol{F}}-\frac{\partial \boldsymbol{I}_{\mathrm{CS}}[\hat{\boldsymbol{A}}, \hat{\boldsymbol{\Gamma}}]}{\partial \hat{\boldsymbol{F}}} \\
D \star \boldsymbol{J}_{\mathcal{P}} & =\frac{\partial \mathcal{P}}{\partial \boldsymbol{F}}-\frac{\partial \widehat{\mathcal{P}}}{\partial \hat{\boldsymbol{F}}} \\
\left(\star \boldsymbol{\Sigma}_{\mathcal{P}}\right)^{\nu}{ }_{\mu} & \equiv 2 \frac{\partial \boldsymbol{V}_{\mathcal{P}}}{\partial\left(\boldsymbol{B}_{R}\right)^{\mu}{ }_{\nu}}=-2\left\{\frac{\partial \boldsymbol{W}_{\mathrm{CS}}}{\partial \boldsymbol{\Gamma}^{\mu}{ }_{\nu}}+D\left(\frac{\partial \boldsymbol{W}_{\mathrm{CS}}}{\partial\left(\boldsymbol{B}_{R}\right)^{\mu}{ }_{\nu}}\right)\right\}+2 \frac{\partial \boldsymbol{I}_{\mathrm{CS}}}{\partial \boldsymbol{R}^{\mu}{ }_{\nu}}-2 \frac{\partial \boldsymbol{I}_{\mathrm{CS}}[\hat{\boldsymbol{A}}, \hat{\boldsymbol{\Gamma}}]}{\partial \hat{\boldsymbol{R}}^{\mu}{ }_{\nu}} \\
\left(D \star \boldsymbol{\Sigma}_{\mathcal{P}}\right)^{\nu}{ }_{\mu} & =2 \frac{\partial \mathcal{P}}{\partial \boldsymbol{R}^{\mu}{ }_{\nu}}-2 \frac{\partial \widehat{\mathcal{P}}^{\mu}}{\partial \hat{\boldsymbol{R}}^{\mu}{ }_{\nu}} .
\end{aligned}
$$

Further, we get an identity of the form

$$
\begin{aligned}
{ }^{{ }_{2 n+1}} \boldsymbol{N}_{H} \equiv & \frac{\mu}{T} \cdot \frac{\partial \mathcal{P}}{\partial \boldsymbol{F}}+\frac{1}{T} \Omega^{\mu}{ }_{\nu} \frac{\partial \mathcal{P}}{\partial \boldsymbol{R}^{\mu}{ }_{\nu}} \\
= & \boldsymbol{i}_{\boldsymbol{\beta}} \boldsymbol{V}_{\mathcal{P}}-\left[\delta_{\mathcal{B}} \boldsymbol{u} \wedge \frac{\partial \boldsymbol{V}_{\mathcal{P}}}{\partial(2 \boldsymbol{\omega})}+\delta_{\mathcal{B}} \boldsymbol{A} \wedge \frac{\partial \boldsymbol{V}_{\mathcal{P}}}{\partial \boldsymbol{B}}+\delta_{\mathcal{B}} \boldsymbol{\Gamma}^{\mu}{ }_{\nu} \wedge \frac{\partial \boldsymbol{V}_{\mathcal{P}}}{\partial\left(\boldsymbol{B}_{R}\right)^{\mu}{ }_{\nu}}\right] \\
& -d\left[\frac{1}{T} \frac{\partial \boldsymbol{V}_{\mathcal{P}}}{\partial(2 \boldsymbol{\omega})}-\frac{\mu}{T} \cdot \frac{\partial \boldsymbol{V}_{\mathcal{P}}}{\partial \boldsymbol{B}}-\frac{1}{T} \Omega^{\mu}{ }_{\nu} \frac{\partial \boldsymbol{V}_{\mathcal{P}}}{\partial\left(\boldsymbol{B}_{R}\right)^{\mu}{ }_{\nu}}-\frac{1}{2} g^{\mu \sigma} \delta_{\mathcal{B}} g_{\nu \sigma} \frac{\partial \boldsymbol{V}_{\mathcal{P}}}{\partial\left(\boldsymbol{B}_{R}\right)^{\mu}{ }_{\nu}}\right] .
\end{aligned}
$$

This is the statement that the bulk free energy current is same as the bulk Noether current corresponding to a bulk Lagrangian $\boldsymbol{V}_{\mathcal{P}}$ up to Komar terms. Taking the normal component and restricting it to the boundary leads to the statement that the covariant free energy current in the boundary, defined via

$$
\star \boldsymbol{N}_{\mathcal{P}, c o v} \equiv-\frac{1}{T} \frac{\partial \boldsymbol{V}_{\mathcal{P}}}{\partial(2 \boldsymbol{\omega})}+\frac{\mu}{T} \cdot \frac{\partial \boldsymbol{V}_{\mathcal{P}}}{\partial \boldsymbol{B}}+\frac{1}{T} \Omega^{\mu}{ }_{\nu} \frac{\partial \boldsymbol{V}_{\mathcal{P}}}{\partial\left(\boldsymbol{B}_{R}\right)^{\mu}{ }_{\nu}}+\frac{1}{2} g^{\mu \sigma} \delta_{\mathcal{B}} g_{\nu \sigma} \frac{\partial \boldsymbol{V}_{\mathcal{P}}}{\partial\left(\boldsymbol{B}_{R}\right)^{\mu}{ }_{\nu}},
$$

satisfies the adiabaticity equation with covariant anomaly.

The last identity we obtain takes the form

$$
\begin{aligned}
\star \boldsymbol{N}_{\mathcal{P}, \text { cons }} & \equiv-\frac{1}{T} \frac{\partial \boldsymbol{V}_{\mathcal{P}}}{\partial(2 \boldsymbol{\omega})}+\frac{\mu}{T} \cdot\left[\frac{\partial \boldsymbol{V}_{\mathcal{P}}}{\partial \boldsymbol{B}}-\frac{\partial \boldsymbol{I}_{\mathrm{CS}}}{\partial \boldsymbol{F}}\right]+\frac{1}{T} \Omega^{\mu}{ }_{\nu}\left[\frac{\partial \boldsymbol{V}_{\mathcal{P}}}{\partial\left(\boldsymbol{B}_{R}\right)^{\mu}{ }_{\nu}}-\frac{\partial \boldsymbol{I}_{\mathrm{CS}}}{\partial \boldsymbol{R}^{\mu}{ }_{\nu}}\right] \\
& =-\boldsymbol{i}_{\boldsymbol{\beta}} \boldsymbol{W}_{\mathrm{CS}}-\Lambda_{\boldsymbol{\beta}} \frac{\partial \boldsymbol{W}_{\mathrm{CS}}}{\partial \boldsymbol{A}}-\partial_{\nu} \boldsymbol{\beta}^{\mu} \frac{\partial \boldsymbol{W}_{\mathrm{CS}}}{\partial \boldsymbol{\Gamma}^{\mu}{ }_{\nu}}
\end{aligned}
$$




$$
\begin{aligned}
& +\delta_{\mathcal{B}} \boldsymbol{u} \wedge \frac{\partial \boldsymbol{W}_{\mathrm{CS}}}{\partial(2 \boldsymbol{\omega})}+\delta_{\mathcal{B}} \boldsymbol{A} \wedge \frac{\partial \boldsymbol{W}_{\mathrm{CS}}}{\partial \boldsymbol{B}}+\delta_{\mathcal{B}} \boldsymbol{\Gamma}^{\mu}{ }_{\nu} \wedge \frac{\partial \boldsymbol{W}_{\mathrm{CS}}}{\partial\left(\boldsymbol{B}_{R}\right)^{\mu}{ }_{\nu}} \\
& +\frac{1}{2} g^{\mu \sigma} \delta_{\mathcal{B}} g_{\nu \sigma} D\left(\frac{\partial \boldsymbol{W}_{\mathrm{CS}}}{\partial\left(\boldsymbol{B}_{R}\right)^{\mu}{ }_{\nu}}\right)+d\left[\frac{1}{T} \frac{\partial \boldsymbol{W}_{\mathrm{CS}}}{\partial(2 \boldsymbol{\omega})}-\frac{\mu}{T} \cdot \frac{\partial \boldsymbol{V}_{\mathcal{P}}}{\partial \boldsymbol{W}_{\mathrm{CS}}}\right]
\end{aligned}
$$

This is the statement that the consistent free energy current in the boundary is same as the boundary Noether current corresponding to a boundary Lagrangian density $\boldsymbol{W}_{\mathrm{CS}}$ up to Komar terms.

\section{B Class ND: from adiabatic to non-dissipative fluids}

As presaged at the end of section 7 , there is a striking similarity between the Class $\mathrm{L}$ Lagrangian solutions to the adiabaticity equation and the effective action formalism for nondissipative fluids which was developed earlier in [45] and explored in [46, 48]. ${ }^{105}$ Intuitively, it is clear that the family of non-dissipative fluids where every on-shell solution to the dynamical equations of motion is constrained to not produce entropy, should be a special case of adiabatic fluids, which are engineered to uplift the statement of entropy conservation off-shell in a suitable manner. The similarities are also striking in the explicit examples discussed in section 8 .

However, at a basic level there is a crucial distinction between the framework of nondissipative fluids and the story we have developed thus far for adiabatic fluids. To wit, the physical fields in the adiabatic fluid formalism are the fluid velocity and the intensive (local) thermodynamic parameters characterizing the fluctuating Gibbs density matrix. On the other hand the non-dissipative fluids use entropy density as a primary variable instead of the temperature. One can nevertheless pass between the two constructions by realizing that temperature and entropy being conjugate variables one can exchange the two by the simple expedient of a Legendre transform.

Aided by this observation, we now consider the Legendre transform of $\mathcal{L}[\mathbf{\Psi}]$ trading the temperature $T$ for its conjugate variable $s$. Part of our basic motivation is of course to make contact with the existing literature on effective actions for hydrodynamics. We will argue upon carrying out this Legendre transform and thence passing to a suitable gauge, we recover the effective action formalism.

In effect we will establish that the family of non-dissipative fluids which we call Class ND are encapsulated within Class L family of adiabatic fluids as presaged in section 1 . This map will in particular make transparent the physical origin of the symmetries employed in the effective action formalism.

\section{B.1 Legendre transformation to an entropic description}

Let us begin by shifting from a description in terms of $\left\{\boldsymbol{\beta}^{\sigma}, \Lambda_{\boldsymbol{\beta}}\right\}$ to a description in terms of a $(d-1)$ form and a $d$-form which we denote as $\left\{\mathbf{S}_{\alpha_{1} \ldots \alpha_{d-1}},\left(\Lambda_{\mathbf{S}}\right)_{\alpha_{1} \ldots \alpha_{d}}\right\}$ respectively. They

\footnotetext{
${ }^{105}$ There are also results in the non-dissipative effective action framework for anomalous transport [20, 30] which we will defer till section 12. As a result the discussion in this section will be restricted to nonanomalous, non-dissipative fluids.
} 
are defined via their Hodge duals:

$$
\begin{aligned}
& { }^{\varepsilon} \mathbf{S}^{\sigma} \equiv \frac{1}{(d-1) !} \varepsilon^{\sigma \alpha_{1} \ldots \alpha_{d-1}} \mathbf{S}_{\alpha_{1} \ldots \alpha_{d-1}}=T s \boldsymbol{\beta}^{\sigma}=s u^{\sigma} \\
& { }^{\varepsilon} \Lambda_{\mathbf{S}} \equiv \frac{1}{d !} \varepsilon^{\alpha_{1} \ldots \alpha_{d}}\left(\Lambda_{\mathbf{S}}\right)_{\alpha_{1} \ldots \alpha_{d}}=T s \Lambda_{\boldsymbol{\beta}}=s\left(\mu-u^{\alpha} A_{\alpha}\right) .
\end{aligned}
$$

These forms $\left\{\mathbf{S}_{\alpha_{1} \ldots \alpha_{d-1}},\left(\Lambda_{\mathbf{S}}\right)_{\alpha_{1} \ldots \alpha_{d}}\right\}$ will play a role analogous to $\left\{\boldsymbol{\beta}^{\sigma}, \Lambda_{\boldsymbol{\beta}}\right\}$ in the Legendre transformed description. To avoid unnecessary clutter, we have adopted above a concise notation for the contraction with $\varepsilon$ tensor which we will use from now on. For any $p$-form $\mathbf{V}_{\alpha_{1} \alpha_{2} \ldots \alpha_{p}}$, we define

$$
{ }^{\varepsilon} \mathbf{V}^{\sigma_{1} \sigma_{2} \ldots \sigma_{\mathbf{d}-\mathbf{p}}} \equiv \frac{1}{p !} \varepsilon^{\sigma_{1} \sigma_{2} \ldots \sigma_{d-p} \alpha_{1} \alpha_{2} \ldots \alpha_{p}} \mathbf{V}_{\alpha_{1} \alpha_{2} \ldots \alpha_{p}}
$$

Let us first attempt to make contact with the variational principle for Lagrangian theories (6.3) by re-expressing them in term of our new variables. To do so we begin with the following identities obtained by varying (B.1):

$$
\begin{aligned}
& T \delta \boldsymbol{\beta}^{\sigma}=\frac{1}{s}{ }^{\varepsilon} \delta \mathbf{S}^{\sigma}-\frac{\boldsymbol{\beta}^{\sigma}}{s \sqrt{-g}} \delta(\sqrt{-g} T s), \\
& T \delta \Lambda_{\boldsymbol{\beta}}=\frac{1}{s}{ }^{\varepsilon} \delta \Lambda_{\mathbf{S}}-\frac{\Lambda_{\boldsymbol{\beta}}}{s \sqrt{-g}} \delta(\sqrt{-g} T s) .
\end{aligned}
$$

From these expressions one can easily check that

$$
\begin{aligned}
T \mathfrak{h}_{\sigma} \delta \boldsymbol{\beta}^{\sigma}+T \mathfrak{n} \cdot\left(\delta \Lambda_{\boldsymbol{\beta}}+A_{\sigma} \delta \boldsymbol{\beta}^{\sigma}\right) & -\frac{1}{\sqrt{-g}} \delta(\sqrt{-g} T s) \\
& =\frac{\mathfrak{h}_{\sigma}}{s}{ }^{\varepsilon} \mathbf{S}^{\sigma}+\frac{\mathfrak{n}}{s} \cdot\left({ }^{\varepsilon} \Lambda_{\mathbf{S}}+A_{\sigma}{ }^{\varepsilon} \mathbf{S}^{\sigma}\right) .
\end{aligned}
$$

In deriving the above we have used the identity $s+\mathfrak{h}_{\sigma} \boldsymbol{\beta}^{\sigma}+\mathfrak{n} \cdot\left(\Lambda_{\boldsymbol{\beta}}+A_{\sigma} \boldsymbol{\beta}^{\sigma}\right)=0$ from (6.6). We can then rewrite (6.3) as

$$
\begin{aligned}
\frac{1}{\sqrt{-g}} \delta(\sqrt{-g}(\mathcal{L}-T s))= & \frac{1}{2} T^{\mu \nu} \delta g_{\mu \nu}+J^{\mu} \cdot \delta A_{\mu}+\nabla_{\mu}\left(\phi \Theta_{\mathrm{PS}}\right)^{\mu} \\
& +\frac{\mathfrak{h}_{\sigma}}{s} \varepsilon \mathbf{S}^{\sigma}+\frac{\mathfrak{n}}{s} \cdot\left({ }^{\varepsilon} \Lambda_{\mathbf{S}}+A_{\sigma}{ }^{\varepsilon} \mathbf{S}^{\sigma}\right)
\end{aligned}
$$

The rewritten variational equation can be interpreted as follows. We think of $\mathcal{L}-T s$ as a functional of the variables $\left\{g_{\mu \nu}, A_{\mu}, \mathbf{S}_{\alpha_{1} \ldots \alpha_{d-1}}, \boldsymbol{\Lambda}_{\mathbf{S} \alpha_{1} \ldots \alpha_{d}}\right\}$ which we can collectively call $\boldsymbol{\Psi}_{S}$ with the subscript denoting passing to the entropic description. To wit, we simply write

$$
\left.\int \sqrt{-g} \mathcal{L}_{S}\left[\mathbf{\Psi}_{S}\right] \equiv \int \sqrt{-g}(\mathcal{L}[\mathbf{\Psi}]-T s)\right|_{\left\{\boldsymbol{\beta}, \Lambda_{\boldsymbol{\beta}}\right\} \mapsto\left\{\mathbf{S}, \boldsymbol{\Lambda}_{\mathbf{S}}\right\}}
$$

Then (B.5) is the defining variational formula for us and gives us the functionals $\left\{\mathfrak{h}_{\sigma}, \mathfrak{n}\right\}$ in this Legendre transformed description. Since entropy density $s$ has taken a 
primary role, it follows that the temperature $T$ is then a derived quantity which can then be computed from

$$
T=-\frac{\delta}{\delta s} \int \sqrt{-g} \mathcal{L}_{S}\left[\mathbf{\Psi}_{S}\right]=-\frac{1}{s^{2}}\left(\mathfrak{h}_{\sigma}{ }^{\varepsilon} \mathbf{S}^{\sigma}+\mathfrak{n} \cdot\left({ }^{\varepsilon} \Lambda_{\mathbf{S}}+A_{\sigma}{ }^{\varepsilon} \mathbf{S}^{\sigma}\right)\right)=-\frac{1}{s}\left(\mu \cdot \mathfrak{n}+u^{\sigma} \mathfrak{h}_{\sigma}\right)
$$

There is no loss of information in the translation between the two descriptions as long as we restrict to non-anomalous fluids. More specifically, much of our analysis in previous sections can be repeated in this Legendre transformed variables. For instance, the Bianchi identities (6.12) can be translated into our new language as

$$
\begin{aligned}
\nabla_{\nu} T^{\mu \nu} & =J_{\nu} \cdot F^{\mu \nu}+\frac{g^{\mu \nu}}{\sqrt{-g}} \delta_{\varepsilon \mathbf{S}}\left(\sqrt{-g} \frac{1}{s} \mathfrak{h}_{\nu}\right)+g^{\mu \nu} \frac{1}{s} \mathfrak{n} \cdot \delta_{\varepsilon \mathbf{S}} A_{\nu}+\nabla^{\mu}(T s) \\
D_{\sigma} J^{\sigma} & =\frac{1}{\sqrt{-g}} \delta_{\varepsilon_{\mathbf{S}}}\left(\sqrt{-g} \frac{1}{s} \mathfrak{n}\right) .
\end{aligned}
$$

where $\delta_{\varepsilon} \mathbf{S}$ denotes Lie derivative along $\left\{{ }^{\varepsilon} \mathbf{S}^{\sigma},{ }^{\varepsilon} \Lambda_{\mathbf{S}}\right\}$. Moreover, the Noether current can be shown in this description to be given by

$$
\begin{aligned}
\mathbf{S}_{\mathrm{N}^{\mu}[X] \equiv} & \xi_{\nu} T^{\mu \nu}+\left(\Lambda+\xi^{\lambda} A_{\lambda}\right) \cdot J^{\mu} \\
& +\frac{\mathfrak{h}_{\nu}}{s}\left(\xi^{\mu \varepsilon} \mathbf{S}^{\nu}-{ }^{\varepsilon} \mathbf{S}^{\mu} \xi^{\nu}\right)+\frac{\mathfrak{n}}{s} \cdot\left(\xi^{\mu}\left({ }^{\varepsilon} \Lambda_{\mathbf{S}}+{ }^{\varepsilon} \mathbf{S}^{\lambda} A_{\lambda}\right)-{ }^{\varepsilon} \mathbf{S}^{\mu}\left(\Lambda+\xi^{\lambda} A_{\lambda}\right)\right)
\end{aligned}
$$

providing the translation of (6.9). As before, (B.8) then imply adiabaticity equation as a corollary.

It is also easy to see that the hydrodynamic equations can be obtained by extremizing $\int \sqrt{-g} \mathcal{L}_{S}\left[\mathbf{\Psi}_{S}\right]$ with respect to arbitrary Lie drags of the forms $\left\{\mathbf{S}_{\nu_{1} \ldots \nu_{d-1}},\left(\Lambda_{\mathbf{S}}\right)_{\nu_{1} \ldots \nu_{d}}\right\}$. However, written in this form, it is not evident that these equations obey the hydrostatic principle. In general, this Legendre transformed description obscures the physics of the hydrostatic state which is better dealt with using $\mathcal{L}$ instead. This makes intuitive physical sense, since the hydrostatic configurations when thought about in terms of the Euclidean background geometry give the temperature a geometric interpretation in terms of the size of the thermal circle, but do not accord a similar preferred status to the entropy density. ${ }^{106}$

\section{B.2 Gauge fixing and the non-dissipative effective action}

Now, we would like to pass to a description in terms of a set of physical dynamical fields $\left\{\phi^{a}, \mathrm{c}\right\}$ such that extremizing $\int \sqrt{-g} \mathcal{L}_{S}\left[\boldsymbol{\Psi}_{S}\right]$ with respect to them gives the correct hydrodynamic equations. Without further ado, based on our preceding analysis of section 7.2 let us introduce a reference manifold $\mathbb{M}$ endowed with reference forms $\left\{\mathbb{S}_{a_{1} \ldots a_{d-1}}[\phi],\left(\Lambda_{\mathbb{S}}\right)_{a_{1} \ldots a_{d}}[\phi]\right\}$ living on it. We then pull-back these reference forms to real

\footnotetext{
${ }^{106}$ The development of the adiabatic fluid formalism was in fact precipitated by attempting to recover the hydrostatic partition function from Class ND.
} 
spacetime using maps $\left\{\phi^{a}, c\right\} .{ }^{107}$ We have

$$
\begin{aligned}
\mathbf{S}_{\nu_{1} \ldots \nu_{d-1}} & =\mathbb{S}_{a_{1} \ldots a_{d-1}} \prod_{i=1}^{d-1} \partial_{\nu_{i}} \phi^{a_{i}} \\
\left(\Lambda_{\mathbf{S}}\right)_{\nu_{1} \ldots \nu_{d}} & =\mathrm{c}\left(\Lambda_{\Phi}\right)_{a_{1} \ldots a_{d}} \mathrm{c}^{-1} \prod_{i=1}^{d} \partial_{\nu_{i}} \phi^{a_{i}}+d\left(\partial_{\left[\nu_{1}\right.} \mathrm{c}\right) \mathrm{c}^{-1} \mathbf{S}_{\left.\nu_{2} \ldots \nu_{d}\right]}
\end{aligned}
$$

or equivalently more compactly as just

$$
\begin{aligned}
{ }^{\varepsilon} \mathbf{S}^{\sigma} & =e_{a}^{\sigma}{ }^{e} \mathbb{S}^{a} \\
{ }^{\varepsilon} \Lambda_{\mathbf{S}} & =\mathrm{c}{ }^{e} \mathbb{S} \mathrm{c}^{-1}+e_{a}^{\sigma} \quad{ }^{e} \mathbb{S}^{a}\left(\partial_{\sigma} \mathrm{c}\right) \mathrm{c}^{-1}
\end{aligned}
$$

where we have defined also an $\varepsilon$-symbol (denoted $\mathbb{e}$ ) associated with the push forward metric g:

$$
\mathbb{e}^{a_{1} \ldots a_{d}} \equiv \varepsilon^{\nu_{1} \ldots \nu_{d}} \prod_{i=1}^{d} \partial_{\nu_{i}} \phi^{a_{i}}
$$

To compute the equations of motion, etc., we obtain first by varying this expression,

$$
\begin{aligned}
{ }^{\varepsilon} \delta \mathbf{S}^{\sigma}= & e_{a}^{\sigma}{ }^{\mathrm{e}} \delta \mathbb{S}^{a}+{ }^{\varepsilon} \delta_{e \delta \phi} \mathbf{S}^{\sigma} \\
= & e_{a}^{\sigma}{ }^{\mathrm{e}} \delta \mathbb{S}-\delta_{\varepsilon} \mathbf{S}\left(e_{a}^{\sigma} \delta \phi^{a}\right)+\mathbf{S}^{\sigma} \nabla_{\lambda}\left(e_{a}^{\lambda} \delta \phi^{a}\right) \\
{ }^{\varepsilon} \Lambda_{\mathbf{S}}= & \mathrm{c}\left({ }^{\circledR} \delta \Lambda_{\mathbb{S}}\right) \mathrm{c}^{-1}+e_{a}^{\sigma}{ }^{\mathbb{e}} \delta \mathbb{S}^{a}\left(\partial_{\sigma} \mathrm{c}\right) \mathrm{c}^{-1} \\
& +\delta_{\varepsilon} \mathbf{S}\left[(\delta \mathrm{c}) \mathrm{c}^{-1}-e_{a}^{\sigma}\left(\partial_{\sigma} \mathrm{c}\right) \mathrm{c}^{-1}\right]+{ }^{\varepsilon} \Lambda_{\mathbf{S}} \nabla_{\lambda}\left(e_{a}^{\lambda} \delta \phi^{a}\right)
\end{aligned}
$$

The derivation follow a very similar pattern as (7.7). Substituting the above expression into (B.5), we finally get

$$
\begin{aligned}
\frac{1}{\sqrt{-g}} \delta\left(\sqrt{-g} \mathcal{L}_{S}\left[\mathbf{\Psi}_{S}\right]\right)= & \frac{1}{2} T^{\mu \nu} \delta g_{\mu \nu}+J^{\mu} \cdot \delta A_{\mu}-\nabla_{\mu} B^{\mu} \\
& +e_{a}^{\sigma}\left[\mathfrak{h}_{\sigma}+\mathfrak{n} \cdot\left(\partial_{\sigma} \mathrm{c} \mathrm{c}^{-1}+A_{\sigma}\right)\right] \frac{1}{s}{ }^{\mathrm{e}} \delta \mathbb{S}^{a}+\mathrm{c}^{-1} \mathfrak{n c} \cdot \frac{1}{s}{ }^{\mathrm{e}} \delta \Lambda_{\mathbb{S}} \\
& -\frac{1}{\sqrt{-g}} \delta_{\varepsilon \mathbf{S}}\left(\sqrt{-g} \frac{\mathfrak{n}}{s}\right) \cdot\left\{(\delta \mathrm{c}) \mathrm{c}^{-1}-e_{a}^{\sigma} \delta \phi^{a}\left(\partial_{\sigma} \mathrm{c} \mathrm{c}^{-1}+A_{\sigma}\right)\right\} \\
& +e_{a}^{\sigma} \delta \phi^{a}\left[\frac{1}{\sqrt{-g}} \delta_{\varepsilon \mathbf{S}}\left(\sqrt{-g} \frac{\mathfrak{h}_{\sigma}}{s}\right)+\frac{\mathfrak{n}}{s} \cdot \delta_{\varepsilon} \mathbf{S} A_{\sigma}+\nabla_{\sigma}(T s)\right]
\end{aligned}
$$

with the boundary term

$$
B^{\mu}=\left(\not \Theta_{\mathrm{PS}}\right)^{\mu}-T s e_{a}^{\mu} \delta \phi^{a}+u^{\mu} \mathfrak{n} \cdot(\delta \mathrm{c}) \mathrm{c}^{-1}-u^{\mu} e_{a}^{\sigma} \delta \phi^{a}\left[\mathfrak{h}_{\sigma}+\mathfrak{n} \cdot\left(\partial_{\sigma} \mathrm{c} \mathrm{c}^{-1}+A_{\sigma}\right)\right]
$$

\footnotetext{
${ }^{107}$ Since we are working with the Legendre transformed system the diffeomorphism and gauge transformation fields $\left\{\phi^{a}, \mathrm{c}\right\}$ are related in a complicated manner to the fields $\{\varphi, \mathrm{c}\}$ we introduced earlier in section 7.3. We prefer to therefore use a different notation (at least stylistically) to emphasize the distinction. As we will see soon the fields introduced in this section are the ones that naturally arise in the non-dissipative fluid effective actions studied in [30, 46, 48].
} 
This then is the analogue of (7.8) in the Legendre transformed description. Thus, extremizing $\int \sqrt{-g} \mathcal{L}_{S}\left[\boldsymbol{\Psi}_{S}\right]$ with respect to $\left\{\phi^{a}, \mathrm{c}\right\}$ variations gives the correct energymomentum and charge conservation equations i.e., (B.8) as required. Furthermore as discussed before we can equivalently work with the variational principle in terms of push forward metric and gauge fields $\left\{\mathfrak{g}_{a b}, \mathbb{A}_{a}\right\}$.

\section{B.3 Static gauge: symmetries of non-dissipative effective actions}

As a final piece of our discussion we now turn to the symmetries in the Legendre transformed variables. We move to the static gauge by using the redundancies in the reference configuration, to facilitate the analysis.

The redundancies to start with, are same as before - we have the diffeomorphisms and gauge transformations on $\mathbb{M}$. Let us explicitly gauge fix these by picking a particular frame for the tensor fields $\left\{\mathbb{S}, \Lambda_{\mathbb{S}}\right\}$. A convenient choice happens to be

$$
\mathbb{S}_{123 \cdots(d-1)}=1, \quad \mathbb{S}_{0 I_{1} I_{2} \cdots I_{(d-2)}}=0, \quad\left(\Lambda_{\mathbb{S}}\right)_{0123 \ldots(d-1)}=0
$$

where as before $I_{k} \in\{1,2, \ldots,(d-1)\}$. Let us now enumerate the residual gauge redundancies which are left unfixed by the conditions above:

$$
\begin{aligned}
\phi^{J} & \mapsto h^{J}\left(\phi^{I}\right), \quad \operatorname{det}\left(\frac{\partial h^{J}}{\partial \phi^{I}}\right)=1 \\
\phi^{0} & \mapsto g\left(\phi^{I}, \phi^{0}\right), \quad \frac{\partial g}{\partial \phi^{0}} \neq 0 \\
\mathrm{c} & \mapsto \mathrm{c} f\left(\phi^{I}\right)
\end{aligned}
$$

Thus, the spatial $\phi$-diffeomorphisms get reduced to the subset of volume preserving diffeomorphisms whereas the analogue of thermal shift gets enhanced to a $\phi^{0}$-dependent shift. In fact, $\phi^{0}$ completely drops out of all hydrodynamic fields altogether in this gauge. ${ }^{108}$ The chemical shift remains unchanged. The first and the third expressions which transform $\left\{\phi^{I}, \mathrm{c}\right\}$ have been described in $[30,48]$ as $\widetilde{\operatorname{Sdiff}_{\mathcal{M}_{\phi, c}}}$, the extended volume preserving diffeomorphism symmetry.

Much of the structure we had in static gauge before Legendre transform survives with small modifications. Since the hydrodynamic fields $u^{\sigma}$ and $\mu$ are unaffected by Legendre transform, they retain their definitions given in (7.31) with the simple replacement $\{\varphi, c\} \mapsto$ $\{\phi, c\}$. We have $(d-1)$ spatial fields $\phi^{I}$ such that $u^{\sigma} \partial_{\sigma} \phi^{I}=0$ and a flavour field c. Using these we define in analogy with the discussion of section 7.5 a distance measure on the spatial geometry of the reference manifold in terms of the matrix $\overline{\mathbb{p}}^{I J} \equiv g^{\mu \nu} \partial_{\mu} \phi^{I} \partial_{\nu} \phi^{J}$. Its inverse then defines a spatial metric $\overline{\mathbb{P}}_{I J}$ as in section 7.5. We can then derive the

\footnotetext{
${ }^{108}$ The reader might worry at this point that our gauge fixing has lost us an equation of motion. This is indeed true and in this sense static gauge is a bit like temporal gauge in electromagnetism where Gauss constraint is lost after gauge fixing. But as we will see in a moment, unlike electromagnetism, adiabaticity equation comes to our rescue and restores the equation that is lost.
} 
expressions for the hydrodynamic fields as

$$
\begin{aligned}
s & =\frac{1}{\sqrt{\overline{\mathbb{P}}}} \\
u^{\sigma} & =\frac{1}{(d-1) !} \varepsilon^{\sigma \alpha_{1} \cdots \alpha_{d-1}} \varepsilon_{I_{1} \cdots I_{d-1}}^{(\overline{\mathrm{P}})} \prod_{i=1}^{d-1} \partial_{\alpha_{i}} \phi^{I_{i}} \\
\mu & =u^{\sigma}\left[\left(\partial_{\sigma} \mathrm{c}\right) \mathrm{c}^{-1}+A_{\sigma}\right]
\end{aligned}
$$

where, as before, $\varepsilon_{I_{1} \cdots I_{d-1}}^{(\overline{\mathrm{p}})}$ is the spatial volume form associated with $\overline{\mathbb{P}}_{I J}$ and $\overline{\mathbb{p}}$ denotes the determinant of $\overline{\mathbb{P}}_{I J}$.

As advertised, the field $\phi^{0}$ completely drops out of all hydrodynamic fields altogether in this gauge. Since there is no $\delta \phi^{0}$ in the variation, thermal shift invariance dictates that all the $e_{a}^{\sigma} \delta \phi^{a}$ factors in (B.14) reduce to $P_{I}^{\sigma} \delta \phi^{I}$. In turn, this means that the absence of $\phi^{0}$ leads to loss of the energy-momentum conservation in the longitudinal direction (along $\left.u^{\mu}\right)$. This however is not as problematic as it seems because of a particular feature of this description: the entropy conservation in this description is automatic. We have

$$
\nabla_{\sigma} J_{S}^{\sigma}=\frac{1}{\sqrt{-g}} \partial_{\sigma}\left(\sqrt{-g} s u^{\sigma}\right)=\frac{1}{\sqrt{-g}} \partial_{\sigma}\left(\frac{\sqrt{-g}}{\sqrt{\overline{\mathbb{P}}}} u^{\sigma}\right)=0
$$

This equation then via adiabaticity equation leads to the energy-momentum conservation along $u^{\mu}$. This then is an additional equation which compensates for the loss of $\phi^{0}$ equation. Effectively we have used the conservation of entropy of the non-dissipative fluids as a dynamical equation of motion.

With these manipulations we have made explicit that the adiabatic fluid formalism along with an appropriate Legendre transform reduces to the standard effective action formalism for hydrodynamics used in $[30,45,46]$ and other references mentioned earlier. In particular, it emphasizes the importance of the symmetries of the latter formalism. The $\widetilde{\operatorname{Sdiff}}_{\mathcal{M}_{\phi, c}}$ symmetry as indicated in [30] is an important part of the framework arising as it does by gauge fixing a covariant formalism involving the background sources and the hydrodynamic fields $\left\{\mathbf{S}, \Lambda_{\mathbf{S}}\right\}$. While we could have perfectly well started with these variables and eschewed the introduction of the $\left\{\boldsymbol{\beta}, \Lambda_{\boldsymbol{\beta}}\right\}$ description used in the preceding sections. However, this formalism for reasons outlined in the preamble to this section suffers when we attempt to describe hydrostatics. Likewise the fact that the entropy current is held rigid in the non-dissipative fluids of Class ND is a substantial obstacle to describing in full generality a Lagrangian solution to adiabatic anomalous hydrodynamics, cf., section 12.2.

\section{Topological currents in odd spacetime dimensions}

On general grounds, one would expect every conserved topological charge on codimensionone spatial slices to be associated with an identically conserved entropy current. In this appendix we provide a general Euler current (with associated charge being the Euler characteristic) and a flavour-charged topological Chern current. 


\section{C.1 Generalized Euler current}

Let $\mathcal{D}$ denote the covariant exterior derivative acting on tensor-valued forms and $u_{\mu}$ be the fluid velocity vector. Then, $u^{\mu} u_{\mu}=-1$ implies that $\mathcal{D} u_{\mu}$ is a transverse vector valued 1-form. Further, we will also need the following transverse anti-symmetric tensor-valued 2-form

$$
P_{\mu}^{\alpha} P_{\nu}^{\beta} \boldsymbol{R}_{\alpha \beta}=\boldsymbol{R}_{\mu \nu}+\boldsymbol{R}_{\mu \alpha} u^{\alpha} u_{\nu}+u_{\mu} u^{\alpha} \boldsymbol{R}_{\alpha \nu}=\boldsymbol{R}_{\mu \nu}+\left(\mathcal{D}^{2} u_{\mu}\right) u_{\nu}-u_{\mu} \mathcal{D}^{2} u_{\nu}
$$

where $\boldsymbol{R}^{\mu}{ }_{\nu}$ is the curvature 2 -form.

Say our fluid is living in $d=3$ spacetime dimensions. Then, we have the following identity [60] due to the transversality properties mentioned above:

$$
\begin{aligned}
\mathcal{D}\left[\varepsilon^{\mu \nu \lambda} u_{\mu}\left(\mathcal{D} u_{\nu} \wedge \mathcal{D} u_{\lambda}-\boldsymbol{R}_{\nu \lambda}\right)\right] \\
\quad=\varepsilon^{\mu \nu \lambda} \mathcal{D} u_{\mu} \wedge \mathcal{D} u_{\nu} \wedge \mathcal{D} u_{\lambda}-\varepsilon^{\mu \nu \lambda} \mathcal{D} u_{\mu} \wedge\left(\boldsymbol{R}_{\nu \lambda}+\left(\mathcal{D}^{2} u_{\nu}\right) u_{\lambda}-u_{\nu} \mathcal{D}^{2} u_{\lambda}\right) \\
\quad=0
\end{aligned}
$$

This implies that we can define an identically conserved current (which we will term the Euler current $^{109}$ ) via

$$
\begin{aligned}
{ }^{\star} \mathrm{J}_{\text {Euler }} & \equiv \frac{1}{2} c_{\text {Euler }} \varepsilon^{\mu \nu \lambda} u_{\mu}\left(\mathcal{D} u_{\nu} \wedge \mathcal{D} u_{\lambda}-\boldsymbol{R}_{\nu \lambda}\right) \\
\mathrm{J}_{\text {Euler }}^{\sigma} & \equiv \frac{1}{2} c_{\text {Euler }} \varepsilon^{\sigma \alpha \beta} \varepsilon^{\mu \nu \lambda} u_{\mu}\left(\nabla_{\alpha} u_{\nu} \nabla_{\beta} u_{\lambda}-\frac{1}{2} R_{\nu \lambda \alpha \beta}\right)
\end{aligned}
$$

which satisfies $\nabla_{\mu} \mathrm{J}$ Euler $=0$. Note that this is a parity-even current appearing in second order in derivative expansion.

Let us now generalize this construction to arbitrary odd spacetime dimensions. ${ }^{110}$ Again we let $\mathcal{D}$ denote the covariant exterior derivative acting on tensor-valued forms. It is uniquely defined by zero-torsion condition $\mathcal{D}\left(d x^{\mu}\right)=0$ and metric compatibility condition $\mathcal{D} g_{\mu \nu}=0$. Let $u_{\mu}$ be the fluid velocity vector. Then, $u^{\mu} u_{\mu}=-1$ implies that $\mathcal{D} u_{\mu}$ is a transverse vector valued 1 -form.

For what follows, it is useful to define a new covariant exterior derivative ${ }^{(\mathfrak{p})} \mathcal{D}$ using the connection 1-form

$$
{ }^{(\mathfrak{p})} \boldsymbol{\Gamma}_{\nu}^{\mu} \equiv \boldsymbol{\Gamma}_{\nu}^{\mu}+\left(\mathcal{D} u^{\mu}\right) u_{\nu}-u^{\mu} \mathcal{D} u_{\nu}
$$

This connection has a torsion ${ }^{(\mathfrak{p})} \mathcal{D}\left(d x^{\mu}\right)=\left(\mathcal{D} u^{\mu}\right) \boldsymbol{u}-u^{\mu} \mathcal{D} \boldsymbol{u}$ but it is still metric compatible ${ }^{(\mathfrak{p})} \mathcal{D} g_{\mu \nu}=0$. In addition, it is also velocity compatible ${ }^{(\mathfrak{p})} \mathcal{D} u^{\mu}=0$, though it is not unique in being velocity compatible. We can work out the curvature 2 -form for this connection as

$$
{ }^{(\mathfrak{p})} \boldsymbol{R}^{\mu}{ }_{\nu}=\boldsymbol{R}^{\mu}{ }_{\nu}-\mathcal{D} u^{\mu} \wedge \mathcal{D} u_{\nu}+\mathcal{D}^{2} u^{\mu} u_{\nu}-u^{\mu} \mathcal{D}^{2} u_{\nu}
$$

\footnotetext{
${ }^{109}$ Sometimes this current is called as the Wen-Zee current, following [59] who realized that 3d Hall currents are often shifted by such a term. The coefficient of this term is hence sometimes called the 'shift'.

${ }^{110}$ During the course of preparation of this paper [61] also constructed the generalization of the Euler current to arbitrary odd dimensions. Our presentation in terms of vector valued one-forms is complementary and equivalent to their explicit construction.
} 
This satisfies ${ }^{(\mathfrak{p})} \mathcal{D}\left({ }^{(\mathfrak{p})} \boldsymbol{R}^{\mu}{ }_{\nu}\right)=0$ and further ${ }^{(\mathfrak{p})} \boldsymbol{R}^{\mu}{ }_{\nu}$ is completely transverse. It then follows that the following form is ${ }^{(\mathfrak{p})} \mathcal{D}$-closed (and is hence $\mathcal{D}$-closed):

$$
{ }^{\star} \mathrm{J}_{\text {Euler }} \equiv-\frac{1}{n !} c_{\text {Euler }} u_{\mu} \varepsilon^{\mu \nu_{1} \nu_{2} \nu_{3} \nu_{4} \ldots \nu_{2 n-1} \nu_{2 n}(\mathfrak{p})} \boldsymbol{R}_{\nu_{1} \nu_{2}}{ }^{(\mathfrak{p})} \boldsymbol{R}_{\nu_{3} \nu_{4}} \ldots{ }^{(\mathfrak{p})} \boldsymbol{R}_{\nu_{2 n-1} \nu_{2 n}}
$$

Here $c_{\mathrm{wz}}$ is some arbitrary numerical constant and we are working in spacetime dimensions $d=2 n+1$. To see how this generalizes the $d=3$ Euler current, we rewrite the above as

$$
\begin{aligned}
{ }_{J_{\text {Euler }} \equiv} \equiv & -\frac{1}{2^{n}} c_{\text {Euler }} u_{\mu} \varepsilon^{\mu \nu_{1} \nu_{2} \ldots \nu_{2 n-1} \nu_{2 n}} \\
& \times\left(\boldsymbol{R}_{\nu_{1} \nu_{2}}-\mathcal{D} u_{\nu_{1}} \wedge \mathcal{D} u_{\nu_{2}}\right) \ldots\left(\boldsymbol{R}_{\nu_{2 n-1} \nu_{2 n}}-\mathcal{D} u_{\nu_{2 n-1}} \wedge \mathcal{D} u_{\nu_{2 n}}\right) \\
\mathrm{J}_{\text {Euler }}^{\sigma} \equiv & -\frac{1}{2^{n}} c_{\text {Euler }} \varepsilon^{\sigma \alpha_{1} \alpha_{2} \ldots \alpha_{2 n-1} \alpha_{2 n}} u_{\mu} \varepsilon^{\mu \nu_{1} \nu_{2} \ldots \nu_{2 n-1} \nu_{2 n}} \\
& \times\left(\frac{1}{2} R_{\nu_{1} \nu_{2} \alpha_{1} \alpha_{2}}-\nabla_{\alpha_{1}} u_{\nu_{1}} \nabla_{\alpha_{2}} u_{\nu_{2}}\right) \\
& \times \ldots\left(\frac{1}{2} R_{\nu_{2 n-1} \nu_{2 n} \alpha_{2 n-1} \alpha_{2 n}}-\nabla_{\alpha_{2 n-1}} u_{\nu_{2 n-1}} \nabla_{\alpha_{2 n}} u_{\nu_{2 n}}\right)
\end{aligned}
$$

This is then a parity-even, identically conserved current with $(d-1)$ derivatives that generalizes the $d=3$ construction of [60] to an arbitrary odd $d$ (see also [61]).

One of the reasons for our interest in the Euler current $J_{\text {Euler }}^{\sigma}$ is that it provides a simple homogeneous solution to the adiabaticity equation. One can simply take the entropy current term to be $\mathrm{J}_{\text {Euler }}^{\sigma}$ and set all other contributions to zero. Thus, given any solution to the adiabaticity equation, we have the freedom to shift the entropy current in odd dimensions by the Euler current (with an arbitrary constant).

\section{C.2 Chern current}

Another identically conserved current for flavour-charged fluids in $d=2 n+1$ dimensions is easily constructed:

$$
\mathrm{J}_{\text {Chern }}^{\sigma}=\frac{1}{2^{n}} c_{\text {Chern }} \varepsilon^{\sigma \alpha_{1} \alpha_{2} \cdots \alpha_{2 n-1} \alpha_{2 n}} F_{\alpha_{1} \alpha_{2}} \cdots F_{\alpha_{2 n-1} \alpha_{2 n}} .
$$

Despite being an exact form (it is the gradient of the Chern-Simon form) as we argue in the main text, it does contribute to the topological degeneracy of states.

It would be interesting to examine if there are other conserved currents that like the Euler current constructed above, include contributions from the background gauge field. We have not been able to find any, but haven't quite proved a no-go theorem either.

\section{Adiabatic hydrodynamics with Weyl invariance}

The hydrodynamics of a CFT has an additional Weyl symmetry over and above the Poincaré and the flavour symmetries. This can be dealt with by treating Weyl symmetry like any other flavouravor symmetry except for a few additional complications that stem from the fact that Weyl symmetry is a spacetime symmetry. In this appendix we give a self-contained construction of the Weyl invariant structures building on earlier work in [56]. 


\section{D.1 Weyl transformation}

Let us begin by adding in a Weyl transformation parameter $\Lambda_{\mathcal{W}}$ to flavour and diffeomorphism $X \equiv\left\{\xi^{\mu}, \Lambda\right\}$. We will denote by $\delta_{x}^{\mathcal{W}}$ the combined flavour, Weyl and diffeomorphisms generated by $\mathcal{X}^{\mathcal{W}} \equiv\left\{\xi^{\mu}, \Lambda, \Lambda_{\mathcal{W}}\right\}$. Thus, we can write

$$
\begin{aligned}
\delta_{x}^{\mathcal{W}} A_{\mu} & =\delta_{x} A_{\mu} \\
\delta_{x}^{\mathcal{W}} g_{\mu \nu} & =\delta_{x} g_{\mu \nu}+2 \Lambda_{\mathcal{W}} g_{\mu \nu} \\
\delta_{x}^{\mathcal{W}} \Gamma_{\lambda \mu}^{\nu} & =\delta_{x} \Gamma_{\lambda \mu}^{\nu}+\delta_{\lambda}^{\nu} \partial_{\mu} \Lambda_{\mathcal{W}}+\delta_{\mu}^{\nu} \partial_{\lambda} \Lambda_{\mathcal{W}}-g_{\lambda \mu} g^{\nu \sigma} \partial_{\sigma} \Lambda_{\mathcal{W}} .
\end{aligned}
$$

A Weyl covariant tensor $Q_{\nu \ldots .}^{\mu \ldots}$ of weight $w$ is a tensor whose Weyl variation is given by

$$
\begin{aligned}
\delta_{x}^{\mathcal{W}} Q_{\nu \ldots}^{\mu \ldots}= & \delta_{x} Q_{\nu \ldots}^{\mu \ldots}-w \Lambda_{\mathcal{W}} Q_{\nu \ldots}^{\mu \ldots} \\
= & {\left[Q_{\nu \ldots}^{\mu \ldots}, \Lambda\right]-w \Lambda_{\mathcal{W}} Q_{\nu \ldots}^{\mu \ldots}+\xi^{\alpha} \partial_{\alpha} Q_{\nu \ldots}^{\mu \ldots}-\left(\partial_{\alpha} \xi^{\mu}\right) Q_{\nu \ldots}^{\alpha \ldots}+\ldots } \\
& +\left(\partial_{\nu} \xi^{\alpha}\right) Q_{\alpha \ldots}^{\mu \ldots}+\ldots
\end{aligned}
$$

The flavour gauge field $A_{\mu}$ has a Weyl weight $w=0$ whereas metric $g_{\mu \nu}$ has a Weyl weight $w=-2$. The hydrodynamic fields $\left\{\boldsymbol{\beta}^{\mu}, \Lambda_{\boldsymbol{\beta}}\right\}$ are invariant under Weyl transformation. ${ }^{111}$ It follows that the velocity $u^{\mu}$, the temperature $T$ and the flavour chemical potential $\mu$ all have $w=1$.

\section{D.2 Weyl connection}

To mimic our construction for flavour symmetries, we extend the sources by adding in a Weyl connection (or a gauge field) $\mathcal{W}_{\mu}$ which transforms as

$$
\delta_{x}^{\mathcal{W}} \mathcal{W}_{\mu}=\delta_{x} \mathcal{W}_{\mu}+\partial_{\mu} \Lambda_{\mathcal{W}}
$$

We can then construct Weyl-invariant Christoffel symbols

$$
\left({ }^{\mathcal{W}} \Gamma\right)^{\mu}{ }_{\nu \lambda} \equiv \Gamma_{\nu \lambda}^{\mu}+g_{\nu \lambda} \mathcal{W}^{\mu}-\delta_{\nu}^{\mu} \mathcal{W}_{\lambda}-\delta_{\lambda}^{\mu} \mathcal{W}_{\nu}
$$

such that

$$
\delta_{x}^{\mathcal{W}}\left({ }^{\mathcal{W}} \Gamma\right)^{\mu}{ }_{\lambda \alpha}=\delta_{x}\left({ }^{\mathcal{W}} \Gamma\right)^{\mu}{ }_{\lambda \alpha}
$$

In turn, this can be used to define a Weyl-covariant derivative [56]

$$
\begin{aligned}
\mathcal{D}_{\lambda}^{\mathcal{W}} Q_{\nu \ldots}^{\mu \ldots} \equiv & D_{\lambda} Q_{\nu \ldots}^{\mu \ldots}+w \mathcal{W}_{\lambda} Q_{\nu \ldots}^{\mu \ldots} \\
& +\left[g_{\lambda \alpha} \mathcal{W}^{\mu}-\delta_{\lambda}^{\mu} \mathcal{W}_{\alpha}-\delta_{\alpha}^{\mu} \mathcal{W}_{\lambda}\right] Q_{\nu \ldots}^{\alpha \ldots}+\ldots \\
& -\left[g_{\lambda \nu} \mathcal{W}^{\alpha}-\delta_{\lambda}^{\alpha} \mathcal{W}_{\nu}-\delta_{\nu}^{\alpha} \mathcal{W}_{\lambda}\right] Q_{\alpha \ldots}^{\mu \ldots}-\ldots
\end{aligned}
$$

\footnotetext{
${ }^{111}$ In hydrodynamics, as elsewhere, a useful thumb rule to determine the Weyl weights is Weyl weight $=$ mass dimension + No. of upper indices - No. of lower indices
} 
where in analogy with flavour covariant derivative, we have added a $w \mathcal{W}_{\lambda}$ term. Further, the additional terms in the definition occur due to the fact that Weyl symmetry in a spacetime symmetry under which Christoffel symbols transform inhomogenously. It is easily checked that these terms serve to correct the Christoffel symbols in $D_{\lambda}$ into Weyl invariant Christoffel symbols. An often useful spacial case is the action on covariant and contravariant vectors

$$
\begin{gathered}
\mathcal{D}_{\lambda}^{\mathcal{W}} V^{\sigma} \equiv D_{\lambda} V^{\sigma}+(w-1) \mathcal{W}_{\lambda} V^{\sigma}+\mathcal{W}^{\sigma} V_{\lambda}-\delta_{\lambda}^{\sigma}(\mathcal{W} . V) \\
\mathcal{D}_{\lambda}^{\mathcal{W}} V_{\sigma} \equiv D_{\lambda} V_{\sigma}+(w+1) \mathcal{W}_{\lambda} V_{\sigma}+\mathcal{W}_{\sigma} V_{\lambda}-g_{\lambda \sigma}(\mathcal{W} \cdot V)
\end{gathered}
$$

and

$$
\begin{aligned}
\mathcal{D}_{\sigma}^{\mathcal{W}} J^{\sigma} & \equiv D_{\sigma} J^{\sigma}+(w-d) \mathcal{W}_{\sigma} J^{\sigma} \\
\mathcal{D}_{\nu}^{\mathcal{W}} T^{\mu \nu} & \equiv D_{\nu} T^{\mu \nu}+(w-d-2) \mathcal{W}_{\nu} T^{\mu \nu}+\mathcal{W}^{\mu} T^{\sigma}{ }_{\sigma}+\mathcal{W}_{\nu}\left(T^{\mu \nu}-T^{\nu \mu}\right)
\end{aligned}
$$

The above Weyl-covariant derivative is metric compatible $\left(\mathcal{D}_{\lambda}^{\mathcal{W}} g_{\mu \nu}=0\right)$ and is torsionless when acting on Weyl-invariant scalar fields. The familiar variational formula for Christoffel symbols

$$
\delta \Gamma_{\nu \lambda}^{\mu}=\frac{1}{2} g^{\mu \alpha}\left(\nabla_{\nu} \delta g_{\lambda \alpha}+\nabla_{\lambda} \delta g_{\nu \alpha}-\nabla_{\alpha} \delta g_{\nu \lambda}\right)
$$

has a Weyl-covariant counterpart

$$
\delta \Gamma^{\mu}{ }_{\nu \lambda}+\delta\left[g_{\nu \lambda} g^{\mu \alpha}\right] \mathcal{W}_{\alpha}=\frac{1}{2} g^{\mu \alpha}\left(\mathcal{D}_{\nu}^{\mathcal{W}} \delta g_{\lambda \alpha}+\mathcal{D}_{\lambda}^{\mathcal{W}} \delta g_{\nu \alpha}-\mathcal{D}_{\alpha}^{\mathcal{W}} \delta g_{\nu \lambda}\right)
$$

This in particular implies that the combination $\delta \Gamma_{\nu \lambda}^{\mu}+\delta\left[g_{\nu \lambda} g^{\mu \alpha}\right] \mathcal{W}_{\alpha}$ is Weyl-invariant ${ }^{112}$ which would prove useful later on.

The curvatures associated with the Weyl-covariant derivative can be defined by the usual procedure of evaluating the commutator between two covariant derivatives on more general fields. For a covariant vector field $V_{\mu}$ of weight $w$, we get

$$
\begin{aligned}
{\left[\mathcal{D}_{\mu}^{\mathcal{W}}, \mathcal{D}_{\nu}^{\mathcal{W}}\right] V_{\lambda} } & =\left[F_{\mu \nu}, V_{\lambda}\right]+w\left({ }^{\mathcal{W}} F\right)_{\mu \nu} V_{\lambda}-\left({ }^{\mathcal{W}} R\right)^{\alpha}{ }_{\lambda \mu \nu} V_{\alpha} \quad \text { with } \\
F_{\mu \nu} & \equiv \nabla_{\mu} A_{\nu}-\nabla_{\nu} A_{\mu}+\left[A_{\mu}, A_{\nu}\right] \\
\left({ }^{\mathcal{W}} F\right)_{\mu \nu} & \equiv \partial_{\mu} \mathcal{W}_{\nu}-\partial_{\nu} \mathcal{W}_{\mu} \\
\left({ }^{\mathcal{W}} R\right)^{\alpha}{ }_{\lambda \mu \nu} & \equiv \partial_{\mu}\left({ }^{\mathcal{W}} \Gamma\right)^{\alpha}{ }_{\lambda \nu}-\partial_{\nu}\left({ }^{\mathcal{W}} \Gamma\right)^{\alpha}{ }_{\lambda \mu}+\left({ }^{\mathcal{W}} \Gamma\right)^{\alpha}{ }_{\beta \mu}\left({ }^{\mathcal{W}} \Gamma\right)^{\beta}{ }_{\lambda \nu}-\left({ }^{\mathcal{W}} \Gamma\right)^{\alpha}{ }_{\beta \nu}\left({ }^{\mathcal{W}} \Gamma\right)^{\beta}{ }_{\lambda \mu}
\end{aligned}
$$

As is evident from their definitions, all these field strengths are Weyl-invariant. A more convenient expression for the Weyl covariant Riemann tensor is given by the formula ${ }^{113}$

$$
\left({ }^{\mathcal{W}} R\right)_{\mu \nu \lambda \sigma}+g_{\mu \nu}\left({ }^{\mathcal{W}} F\right)_{\lambda \sigma}=R_{\mu \nu \lambda \sigma}-4 \delta_{[\mu}^{\alpha} g_{\nu][\lambda} \delta_{\sigma]}^{\beta}\left(\nabla_{\alpha} \mathcal{W}_{\beta}+\mathcal{W}_{\alpha} \mathcal{W}_{\beta}-\frac{\mathcal{W}^{2}}{2} g_{\alpha \beta}\right)
$$

\footnotetext{
${ }^{112} \mathrm{~A}$ quick proof for the Weyl invariance of this combination follows from noting that

$$
\delta\left({ }^{\mathcal{W}} \Gamma\right)^{\mu \lambda}=\delta \Gamma_{\nu \lambda}^{\mu}+\delta\left[g_{\nu \lambda} g^{\mu \alpha}\right] \mathcal{W}_{\alpha}+g_{\nu \lambda} g^{\mu \alpha} \delta \mathcal{W}_{\alpha}-\delta_{\nu}^{\mu} \delta \mathcal{W}_{\lambda}-\delta_{\lambda}^{\mu} \delta \mathcal{W}_{\nu}
$$

and using the statement that $\delta \mathcal{W}_{\alpha}$ being the difference of two Weyl connections, is Weyl-invariant.

${ }^{113}$ Note that our Riemann tensor notion is slightly different from those defined in [56] which is responsible for different signs appearing in our expression.
} 
These curvatures obey Bianchi identities of the form

$$
\begin{aligned}
&\left({ }^{\mathcal{W}} R\right)^{\mu}{ }_{\nu \lambda \sigma}+\left({ }^{\mathcal{W}} R\right)^{\mu}{ }_{\lambda \sigma \nu}+\left({ }^{\mathcal{W}} R\right)^{\mu}{ }_{\sigma \nu \lambda}=0 \\
& \mathcal{D}_{\nu}^{\mathcal{W}}\left({ }^{\mathcal{W}} R\right)^{\alpha \beta}{ }_{\lambda \sigma}+\mathcal{D}_{\lambda}^{\mathcal{W}}\left({ }^{\mathcal{W}} R\right)^{\alpha \beta}{ }_{\sigma \nu}+\mathcal{D}_{\sigma}^{\mathcal{W}}\left({ }^{\mathcal{W}} R\right)^{\alpha \beta}{ }_{\nu \lambda}=0 \\
& \mathcal{D}_{\nu}^{\mathcal{W}}\left({ }^{\mathcal{W}} F\right)_{\lambda \sigma}+\mathcal{D}_{\lambda}^{\mathcal{W}}\left({ }^{\mathcal{W}} F\right)_{\sigma \nu}+\mathcal{D}_{\sigma}^{\mathcal{W}}\left({ }^{\mathcal{W}} F\right)_{\nu \lambda}=0
\end{aligned}
$$

and

$$
\begin{aligned}
& \left({ }^{\mathcal{W}} R\right)_{\mu \nu \lambda \sigma}+\left({ }^{\mathcal{W}} R\right)_{\nu \mu \lambda \sigma}=-2\left({ }^{\mathcal{W}} F\right)_{\lambda \sigma} g_{\mu \nu} \\
& \left({ }^{\mathcal{W}} R\right)_{\mu \nu \lambda \sigma}-\left({ }^{\mathcal{W}} R\right)_{\lambda \sigma \mu \nu}=\left({ }^{\mathcal{W}} F\right)_{\mu \nu} g_{\lambda \sigma}-\left({ }^{\mathcal{W}} F\right)_{\lambda \sigma} g_{\mu \nu}-4\left({ }^{\mathcal{W}} F\right)_{\alpha \beta} \delta_{[\mu}^{\alpha} g_{\nu][\lambda} \delta_{\sigma]}^{\beta}
\end{aligned}
$$

We can use the Weyl covariant Riemann tensor to define the Weyl covariant Ricci tensor, Ricci scalar and Schouten tensor ${ }^{114}$ via

$$
\begin{aligned}
\left({ }^{\mathcal{W}} R\right)_{\mu \nu}+\left({ }^{\mathcal{W}} F\right)_{\mu \nu} & =R_{\mu \nu}+\left((d-2) \delta_{\mu}^{\alpha} \delta_{\nu}^{\beta}+g_{\mu \nu} g^{\alpha \beta}\right)\left(\nabla_{\alpha} \mathcal{W}_{\beta}+\mathcal{W}_{\alpha} \mathcal{W}_{\beta}-\frac{\mathcal{W}^{2}}{2} g_{\alpha \beta}\right) \\
{ }^{\mathcal{W}} R & =R+2(d-1) g^{\alpha \beta}\left(\nabla_{\alpha} \mathcal{W}_{\beta}+\mathcal{W}_{\alpha} \mathcal{W}_{\beta}-\frac{\mathcal{W}^{2}}{2} g_{\alpha \beta}\right) \\
\left({ }^{\mathcal{W}} S\right)_{\mu \nu}+\frac{1}{d-2}\left({ }^{\mathcal{W}} F\right)_{\mu \nu} & =S_{\mu \nu}+\nabla_{\mu} \mathcal{W}_{\nu}+\mathcal{W}_{\mu} \mathcal{W}_{\nu}-\frac{\mathcal{W}^{2}}{2} g_{\mu \nu} .
\end{aligned}
$$

\section{D.3 Covariant form of Weyl transformations}

As in the flavour case, we can rewrite the flavour and diffeomorphism Weyl variations (D.3) and (D.5) in terms of the Weyl-covariant derivative

$$
\begin{aligned}
\delta_{x}^{\mathcal{W}} \mathcal{W}_{\mu} & =\mathcal{D}_{\mu}^{\mathcal{W}}\left(\Lambda_{\mathcal{W}}+\xi^{\alpha} \mathcal{W}_{\alpha}\right)+\xi^{\alpha}\left({ }^{\mathcal{W}} F\right)_{\alpha \mu} \\
\delta_{x}^{\mathcal{W}}\left({ }^{\mathcal{W}} \Gamma\right)^{\mu}{ }_{\nu \lambda} & =\mathcal{D}_{\lambda}^{\mathcal{W}}\left(\mathcal{D}_{\nu}^{\mathcal{W}} \xi^{\mu}\right)+\xi^{\sigma}\left({ }^{\mathcal{W}} R\right)^{\mu}{ }_{\nu \sigma \lambda}
\end{aligned}
$$

where we have assumed $\xi^{\alpha}$ to be a Weyl-invariant vector. The equation for and (D.2) for a Weyl-covariant tensor becomes

$$
\begin{aligned}
\delta_{x}^{\mathcal{W}} Q_{\nu \ldots}^{\mu \ldots}= & {\left[Q_{\nu \ldots}^{\mu \ldots}, \Lambda+\xi^{\alpha} A_{\alpha}\right]-w\left(\Lambda_{\mathcal{W}}+\xi^{\alpha} \mathcal{W}_{\alpha}\right) Q_{\nu \ldots}^{\mu \ldots}+\xi^{\alpha} \mathcal{D}_{\alpha}^{\mathcal{W}} Q_{\nu \ldots}^{\mu \ldots} } \\
& -\left(\mathcal{D}_{\alpha}^{\mathcal{W}} \xi^{\mu}\right) Q_{\nu \ldots}^{\alpha \ldots}+\ldots+\left(\mathcal{D}_{\nu}^{\mathcal{W}} \xi^{\alpha}\right) Q_{\alpha \ldots}^{\mu \ldots}+\ldots
\end{aligned}
$$

In particular, for the metric $g_{\mu \nu}$ we have

$$
\delta_{x}^{\mathcal{W}} g_{\mu \nu}=\mathcal{D}_{\mu}^{\mathcal{W}} \xi_{\nu}+\mathcal{D}_{\nu}^{\mathcal{W}} \xi_{\mu}+2\left(\Lambda_{\mathcal{W}}+\xi^{\alpha} \mathcal{W}_{\alpha}\right) g_{\mu \nu}
$$

We can then write

$$
\delta_{x}^{\mathcal{W}} \Gamma^{\mu}{ }_{\nu \lambda}+\delta_{x}^{\mathcal{W}}\left[g_{\nu \lambda} g^{\mu \alpha}\right] \mathcal{W}_{\alpha}=\frac{1}{2} g^{\mu \alpha}\left[\mathcal{D}_{\nu}^{\mathcal{W}}\left(\delta_{x}^{\mathcal{W}} g_{\lambda \alpha}\right)+\mathcal{D}_{\lambda}^{\mathcal{W}}\left(\delta_{x}^{\mathcal{W}} g_{\nu \alpha}\right)-\mathcal{D}_{\alpha}^{\mathcal{W}}\left(\delta_{x}^{\mathcal{W}} g_{\nu \lambda}\right)\right] .
$$

\footnotetext{
${ }^{114}$ The Schouten tensor in defined as

$$
S_{\mu \nu} \equiv \frac{1}{d-2}\left[R_{\mu \nu}-\frac{1}{2(d-1)} R g_{\mu \nu}\right]
$$

It is often used in defining the Weyl curvature part of Riemann curvature via $C_{\mu \nu \lambda \sigma} \equiv R_{\mu \nu \lambda \sigma}+$ $\delta_{[\mu}^{\alpha} g_{\nu][\lambda} \delta_{\sigma]}^{\beta} S_{\alpha \beta}$. Its significance in Weyl-invariant theories arises from the fact that it acts like a connection for the special conformal transformations.
} 


\section{D.4 Weyl covariance and conservation equations}

In this subsection, we will study the conservation equations in a zero temperature field theory with Weyl invariance. Consider the path integral of this field theory with the background metric and Weyl connection turned on. ${ }^{115}$ We can write the variation of the logarithm of this path integral (up to boundary terms) as

$$
-i \delta \ln Z=\int d^{d} x \sqrt{-g}\left[\frac{1}{2} t^{\alpha \beta} \delta g_{\alpha \beta}+j_{\mathcal{W}}^{\mu} \delta \mathcal{W}_{\mu}+\frac{1}{2}\left(\Sigma_{\mathcal{W}}\right)^{\sigma \mu}{ }_{\nu} \delta\left({ }^{\mathcal{W}} \Gamma\right)^{\nu}{ }_{\mu \sigma}\right]
$$

where we treat $\left\{g_{\alpha \beta}, \mathcal{W}_{\mu},\left({ }^{\mathcal{W}} \Gamma\right)^{\nu}{ }_{\mu \sigma}\right\}$ as independent sources for later convenience. We will call $\left(\Sigma_{\mathcal{W}}\right)^{\sigma \mu}{ }_{\nu}$ as the Weyl spin current. The tensors $\left\{t^{\alpha \beta}, j_{\mathcal{W}}^{\mu}\right\}$ are related to the orbital energy-momentum tensor and the orbital virial current in a way we will make precise below.

Let us now eliminate $\left({ }^{\mathcal{W}} \Gamma\right)^{\nu}{ }_{\mu \sigma}$ variations in favor of variations of the basic sources $\left\{g_{\alpha \beta}, \mathcal{W}_{\mu}\right\}$. First, we use the identity

$$
\begin{aligned}
\frac{1}{2}\left(\Sigma_{\mathcal{W}}\right)^{\sigma \mu}{ }_{\nu} \delta\left({ }^{\mathcal{W}} \Gamma\right)_{\mu \sigma}^{\nu}= & \frac{1}{2}\left(\Sigma_{\mathcal{W}}\right)^{\sigma \mu}{ }_{\nu}\left[\delta \Gamma^{\nu}{ }_{\mu \sigma}+\delta\left(g_{\mu \sigma} g^{\nu \alpha}\right) \mathcal{W}_{\alpha}\right] \\
& +\frac{1}{2} g_{\alpha \beta}\left(\Sigma_{\mathcal{W}}^{\alpha[\beta \sigma]}+\Sigma_{\mathcal{W}}^{\beta[\alpha \sigma]}-\Sigma_{\mathcal{W}}^{\sigma(\alpha \beta)}\right) \delta \mathcal{W}_{\sigma},
\end{aligned}
$$

to write (D.20) in an equivalent form

$$
-i \delta \ln Z=\int d^{d} x \sqrt{-g}\left\{\frac{1}{2} t^{\alpha \beta} \delta g_{\alpha \beta}+J_{\mathcal{W}}^{\mu} \delta \mathcal{W}_{\mu}+\frac{1}{2}\left(\Sigma_{\mathcal{W}}\right)^{\sigma \mu}{ }_{\nu}\left[\delta \Gamma^{\nu}{ }_{\mu \sigma}+\delta\left(g_{\mu \sigma} g^{\nu \alpha}\right) \mathcal{W}_{\alpha}\right]\right\}
$$

where we have defined the total virial current $J_{\mathcal{W}}^{\mu}$ as the sum

$$
J_{\mathcal{W}}^{\sigma} \equiv j_{\mathcal{W}}^{\sigma}+\frac{1}{2} g_{\alpha \beta}\left(\Sigma_{\mathcal{W}}^{\alpha[\beta \sigma]}+\Sigma_{\mathcal{W}}^{\beta[\alpha \sigma]}-\Sigma_{\mathcal{W}}^{\sigma(\alpha \beta)}\right)
$$

Next we integrate by parts using (D.10) and discard the boundary terms to get

$$
-i \delta \ln Z=\int d^{d} x \sqrt{-g}\left\{\frac{1}{2} T_{\mathcal{W}}^{\alpha \beta} \delta g_{\alpha \beta}+J_{\mathcal{W}}^{\mu} \delta \mathcal{W}_{\mu}\right\}
$$

where the Weyl energy-momentum tensor $T_{\mathcal{W}}^{\alpha \beta}$ can be defined by more familiar looking expressions involving the orbital energy-momentum current and Weyl spin current as:

$$
T_{\mathcal{W}}^{\alpha \beta} \equiv t^{\alpha \beta}+\frac{1}{2} \mathcal{D}_{\sigma}^{\mathcal{W}}\left(\Sigma_{\mathcal{W}}^{\alpha[\beta \sigma]}+\Sigma_{\mathcal{W}}^{\beta[\alpha \sigma]}-\Sigma_{\mathcal{W}}^{\sigma(\alpha \beta)}\right) .
$$

We are now ready to derive the conservation equations in the fields theory that follow from Weyl and diffeomorphism symmetries. Assuming there are no Weyl or diffeomorphism anomalies, the path-integral is then invariant under the Weyl and diffeomorphism

\footnotetext{
${ }^{115}$ For simplicity, we will consider the case with no flavour symmetries - the expressions in this subsection can be trivially generalized to account for flavour symmetries if present.
} 
symmetries of the theory. This gives

$$
\begin{aligned}
0= & \int d^{d} x \sqrt{-g}\left\{\frac{1}{2} T_{\mathcal{W}}^{\alpha \beta}\left(\delta_{x}^{\mathcal{W}} g_{\alpha \beta}\right)+J_{\mathcal{W}}^{\mu}\left(\delta_{x}^{\mathcal{W}} \mathcal{W}_{\mu}\right)\right\} \\
= & -\int d^{d} x \sqrt{-g} \xi_{\alpha}\left\{\mathcal{D}_{\beta}^{\mathcal{W}} T_{\mathcal{W}}^{\alpha \beta}-\left(J_{\mathcal{W}}\right)_{\beta}\left({ }^{\mathcal{W}} F\right)^{\alpha \beta}\right\} \\
& -\int d^{d} x \sqrt{-g}\left(\Lambda_{\mathcal{W}}+\xi^{\sigma} \mathcal{W}_{\sigma}\right)\left\{\mathcal{D}_{\beta}^{\mathcal{W}} J_{\mathcal{W}}^{\beta}-g_{\alpha \beta} T_{\mathcal{W}}^{\alpha \beta}\right\}
\end{aligned}
$$

where in the second step, we have integrated by parts and discarded the boundary terms. thus, for a general Weyl-invariant field theory we obtain the conservation equations

$$
\begin{aligned}
\mathcal{D}_{\beta}^{\mathcal{W}} T_{\mathcal{W}}^{\alpha \beta} & =\left(J_{\mathcal{W}}\right)_{\beta}\left({ }^{\mathcal{W}} F\right)^{\alpha \beta} \\
\mathcal{D}_{\beta}^{\mathcal{W}} J_{\mathcal{W}}^{\beta} & =g_{\alpha \beta} T_{\mathcal{W}}^{\alpha \beta}
\end{aligned}
$$

with

$$
\begin{gathered}
T_{\mathcal{W}}^{\alpha \beta} \equiv t^{\alpha \beta}+\frac{1}{2} \mathcal{D}_{\sigma}^{\mathcal{W}}\left(\Sigma_{\mathcal{W}}^{\alpha[\beta \sigma]}+\Sigma_{\mathcal{W}}^{\beta[\alpha \sigma]}-\Sigma_{\mathcal{W}}^{\sigma(\alpha \beta)}\right) \\
J_{\mathcal{W}}^{\sigma} \equiv j_{\mathcal{W}}^{\sigma}+\frac{1}{2} g_{\alpha \beta}\left(\Sigma_{\mathcal{W}}^{\alpha[\beta \sigma]}+\Sigma_{\mathcal{W}}^{\beta[\alpha \sigma]}-\Sigma_{\mathcal{W}}^{\sigma(\alpha \beta)}\right)
\end{gathered}
$$

The energy-momentum conservation equation is reasonably familiar with all the currents now taking their Weyl invariant form. For the Weyl current conservation we see that the stress tensor trace contributes a source term.

\section{D.5 Velocity compatible Weyl connection}

While the above discussion was quite general we now would like to specialize to hydrodynamics, where there is a unique Weyl connection which satisfies the velocity compatibility conditions [56]

$$
u^{\sigma} \mathcal{D}_{\sigma}^{\mathcal{W}} u_{\mu}=0, \quad \mathcal{D}_{\sigma}^{\mathcal{W}} u^{\sigma}=0,
$$

which can equivalently be stated in terms of $\boldsymbol{\beta}^{\mu}$ as

$$
\boldsymbol{\beta}^{\sigma} \mathcal{D}_{\sigma}^{\mathcal{W}} \boldsymbol{\beta}^{\mu}=\boldsymbol{\beta}^{\mu} \mathcal{D}_{\sigma}^{\mathcal{W}} \boldsymbol{\beta}^{\sigma}
$$

Imposing these velocity compatibility conditions gives

$$
\mathcal{W}_{\sigma} \equiv u^{\alpha} \nabla_{\alpha} u_{\sigma}-\frac{\nabla_{\alpha} u^{\alpha}}{d-1} u_{\sigma}=\mathfrak{a}_{\sigma}-\frac{\Theta}{d-1} u_{\sigma}
$$

We can then compute the variation of $\mathcal{W}_{\mu}$ as follows: varying the velocity compatibility conditions, we get

$$
\begin{aligned}
0=\delta\left(u^{\sigma} \mathcal{D}_{\sigma}^{\mathcal{W}} u_{\mu}\right) & =\delta u^{\sigma} \mathcal{D}_{\sigma}^{\mathcal{W}} u_{\mu}+u^{\sigma}\left(\mathcal{D}_{\sigma}^{\mathcal{W}} \delta u_{\mu}-u_{\lambda} \delta \Gamma^{\lambda}{ }_{\mu \sigma}+\delta \mathcal{W}_{\mu} u_{\sigma}-u_{\alpha} \delta\left(g_{\mu \sigma} \mathcal{W}^{\alpha}\right)\right) \\
& =\delta u^{\sigma} \mathcal{D}_{\sigma}^{\mathcal{W}} u_{\mu}+u^{\sigma} \mathcal{D}_{\sigma}^{\mathcal{W}} \delta u_{\mu}-u_{\lambda} u^{\sigma}\left(\delta \Gamma_{\mu \sigma}^{\lambda}+\delta\left[g_{\mu \sigma} g^{\alpha \lambda}\right] \mathcal{W}_{\alpha}\right)-P_{\mu}^{\nu} \delta \mathcal{W}_{\nu} \\
0=\delta\left(u_{\mu} \mathcal{D}_{\sigma}^{\mathcal{W}} u^{\sigma}\right) & =u_{\mu} \mathcal{D}_{\sigma}^{\mathcal{W}} \delta u^{\sigma}+u_{\mu} u^{\sigma}\left(\delta \Gamma^{\lambda}{ }_{\lambda \sigma}+\delta\left[g_{\lambda \sigma} g^{\alpha \lambda}\right] \mathcal{W}_{\alpha}\right)-(d-1) u_{\mu} u^{\nu} \delta \mathcal{W}_{\nu}
\end{aligned}
$$


We can use these equations solve for $\delta \mathcal{W}_{\mu}$ to get

$$
\begin{aligned}
\delta \mathcal{W}_{\mu}=\delta u^{\sigma} \mathcal{D}_{\sigma}^{\mathcal{W}} u_{\mu}+ & u^{\sigma} \mathcal{D}_{\sigma}^{\mathcal{W}} \delta u_{\mu}-\frac{1}{d-1} u_{\mu} \mathcal{D}_{\sigma}^{\mathcal{W}} \delta u^{\sigma} \\
& -u^{\sigma}\left(u_{\lambda} \delta_{\mu}^{\beta}+\frac{1}{d-1} u_{\mu} \delta_{\lambda}^{\beta}\right)\left(\delta \Gamma_{\beta \sigma}^{\lambda}+\delta\left[g_{\beta \sigma} g^{\alpha \lambda}\right] \mathcal{W}_{\alpha}\right)
\end{aligned}
$$

We can then use $\delta u^{\sigma} \mathcal{D}_{\sigma}^{\mathcal{W}} u_{\mu}=T \delta \boldsymbol{\beta}^{\sigma} \mathcal{D}_{\sigma}^{\mathcal{W}} u_{\mu}$ and

$$
\begin{aligned}
u^{\sigma} \mathcal{D}_{\sigma}^{\mathcal{W}} \delta u_{\mu}-\frac{1}{d-1} u_{\mu} \mathcal{D}_{\sigma}^{\mathcal{W}} \delta u^{\sigma}= & \left(u^{\lambda} P_{\mu \sigma}-\frac{1}{d-1} u_{\mu} P_{\sigma}^{\lambda}\right) \mathcal{D}_{\lambda}^{\mathcal{W}}\left(T \delta \boldsymbol{\beta}^{\sigma}\right) \\
& +\frac{1}{2}\left(P_{\mu}^{\alpha} u^{\beta}+P_{\mu}^{\beta} u^{\alpha}-\frac{d}{d-1} u_{\mu} u^{\alpha} u^{\beta}\right)\left(u \cdot \mathcal{D}^{\mathcal{W}}\right) \delta g_{\alpha \beta}
\end{aligned}
$$

to write

$$
\begin{aligned}
\delta \mathcal{W}_{\mu}= & \frac{1}{2}\left(P_{\mu}^{\alpha} u^{\beta}+P_{\mu}^{\beta} u^{\alpha}-\frac{d}{d-1} u_{\mu} u^{\alpha} u^{\beta}\right)\left(u \cdot \mathcal{D}^{\mathcal{W}}\right) \delta g_{\alpha \beta} \\
& -u^{\sigma}\left(u_{\lambda} \delta_{\mu}^{\beta}+\frac{1}{d-1} u_{\mu} \delta_{\lambda}^{\beta}\right)\left(\delta \Gamma_{\beta \sigma}^{\lambda}+\delta\left[g_{\beta \sigma} g^{\alpha \lambda}\right] \mathcal{W}_{\alpha}\right) \\
& +T \delta \boldsymbol{\beta}^{\sigma} \mathcal{D}_{\sigma}^{\mathcal{W}} u_{\mu}+\left(u^{\lambda} P_{\mu \sigma}-\frac{1}{d-1} u_{\mu} P_{\sigma}^{\lambda}\right) \mathcal{D}_{\lambda}^{\mathcal{W}}\left(T \delta \boldsymbol{\beta}^{\sigma}\right)
\end{aligned}
$$

A useful corollary of this result is

$$
\begin{aligned}
J_{\mathcal{W}}^{\mu} \delta \mathcal{W}_{\mu}= & -\frac{1}{2} \delta g_{\alpha \beta}\left(P_{\mu}^{\alpha} u^{\beta}+P_{\mu}^{\beta} u^{\alpha}-\frac{d}{d-1} u_{\mu} u^{\alpha} u^{\beta}\right)\left(u . \mathcal{D}^{\mathcal{W}}\right) J_{\mathcal{W}}^{\mu} \\
& -u^{\sigma}\left(J_{\mathcal{W}}^{\mu} u_{\lambda}+\frac{1}{d-1}\left(u . J_{\mathcal{W}}\right) \delta_{\lambda}^{\mu}\right)\left(\delta \Gamma^{\lambda}{ }_{\mu \sigma}+\delta\left[g_{\mu \sigma} g^{\alpha \lambda}\right] \mathcal{W}_{\alpha}\right) \\
& +T \delta \boldsymbol{\beta}^{\lambda}\left\{J_{\mathcal{W}}^{\mu} \mathcal{D}_{\lambda}^{\mathcal{W}} u_{\mu}-\mathcal{D}_{\sigma}^{\mathcal{W}}\left[J_{\mathcal{W}}^{\mu}\left(u^{\sigma} P_{\mu \lambda}-\frac{1}{d-1} u_{\mu} P_{\lambda}^{\sigma}\right)\right]\right\}+\mathcal{D}_{\sigma}^{\mathcal{W}}(\ldots)
\end{aligned}
$$

\section{D.6 Class L for Weyl covariant fluids}

In this subsection, we will study the Class L for Weyl covariant fluids. In analogy with our previous discussion for a zero temperature field theory, we can write the variation of Lagrangian density (up to boundary terms) as

$$
\int d^{d} x \sqrt{-g}\left[\frac{1}{2} t^{\alpha \beta} \delta g_{\alpha \beta}+J_{\mathcal{W}}^{\mu} \delta \mathcal{W}_{\mu}+\frac{1}{2}\left(\Sigma_{\mathcal{W}}\right)_{\lambda}^{\sigma \mu}\left(\delta \Gamma_{\mu \sigma}^{\lambda}+\delta\left[g_{\mu \sigma} g^{\alpha \lambda}\right] \mathcal{W}_{\alpha}\right)+T \mathfrak{h}_{\lambda}^{\mathcal{W}} \delta \boldsymbol{\beta}^{\lambda}\right]
$$


We can then integrate by parts using (D.36) to get

$$
\begin{aligned}
\frac{1}{2} t^{\alpha \beta} \delta g_{\alpha \beta} & +J_{\mathcal{W}}^{\mu} \delta \mathcal{W}_{\mu}+\frac{1}{2}\left(\Sigma_{\mathcal{W}}\right)_{\lambda}^{\sigma \mu}\left(\delta \Gamma_{\mu \sigma}^{\lambda}+\delta\left[g_{\mu \sigma} g^{\alpha \lambda}\right] \mathcal{W}_{\alpha}\right)+T \mathfrak{h}_{\lambda}^{\mathcal{W}} \delta \boldsymbol{\beta}^{\lambda} \\
=T & \delta \boldsymbol{\beta}^{\lambda}\left\{\mathfrak{h}_{\lambda}^{\mathcal{W}}+J_{\mathcal{W}}^{\mu} \mathcal{D}_{\lambda}^{\mathcal{W}} u_{\mu}-\mathcal{D}_{\sigma}^{\mathcal{W}}\left[J_{\mathcal{W}}^{\mu}\left(u^{\sigma} P_{\mu \lambda}-\frac{1}{d-1} u_{\mu} P_{\lambda}^{\sigma}\right)\right]\right\} \\
& +\frac{1}{2} \delta g_{\alpha \beta}\left\{t^{\alpha \beta}-\left(P_{\mu}^{\alpha} u^{\beta}+P_{\mu}^{\beta} u^{\alpha}-\frac{d}{d-1} u_{\mu} u^{\alpha} u^{\beta}\right)\left(u \cdot \mathcal{D}^{\mathcal{W}}\right) J_{\mathcal{W}}^{\mu}\right\} \\
& +\frac{1}{2}\left[\Sigma_{\mathcal{W} \lambda}^{\sigma \mu}-2 u^{\sigma}\left(J_{\mathcal{W}}^{\mu} u_{\lambda}+\frac{1}{d-1}\left(u . J_{\mathcal{W}}\right) \delta_{\lambda}^{\mu}\right)\right]\left(\delta \Gamma_{\mu \sigma}^{\lambda}+\delta\left[g_{\mu \sigma} g^{\alpha \lambda}\right] \mathcal{W}_{\alpha}\right) \\
& +\mathcal{D}_{\sigma}^{\mathcal{W}}[\ldots]
\end{aligned}
$$

Another integration by parts gives us the fluid energy-momentum tensor $T_{\mathcal{W}}^{\alpha \beta}$

$$
\begin{aligned}
T_{\mathcal{W}}^{\alpha \beta}= & t^{\alpha \beta}-\left(P_{\mu}^{\alpha} u^{\beta}+P_{\mu}^{\beta} u^{\alpha}-\frac{d}{d-1} u_{\mu} u^{\alpha} u^{\beta}\right)\left(u \cdot \mathcal{D}^{\mathcal{W}}\right) J_{\mathcal{W}}^{\mu} \\
& +\frac{1}{2} \mathcal{D}_{\sigma}^{\mathcal{W}}\left(\Sigma^{\alpha[\beta \sigma]}+\Sigma \mathcal{w}^{\beta[\alpha \sigma]}-\Sigma^{\sigma(\alpha \beta)}\right) .
\end{aligned}
$$

with

$$
\Sigma_{\lambda}^{\sigma \mu} \equiv \Sigma_{\mathcal{W} \lambda}^{\sigma \mu}-2 u^{\sigma}\left(J_{\mathcal{W}}^{\mu} u_{\lambda}+\frac{1}{d-1}\left(u . J_{\mathcal{W}}\right) \delta_{\lambda}^{\mu}\right)
$$

along with the adiabatic heat current

$$
\mathfrak{h}_{\lambda}=\mathfrak{h}_{\lambda}^{\mathcal{W}}+J_{\mathcal{W}}^{\mu} \mathcal{D}_{\lambda}^{\mathcal{W}} u_{\mu}-\mathcal{D}_{\sigma}^{\mathcal{W}}\left[J_{\mathcal{W}}^{\mu}\left(u^{\sigma} P_{\mu \lambda}-\frac{1}{d-1} u_{\mu} P_{\lambda}^{\sigma}\right)\right]
$$

Thus, with these virial corrections the Class L for Weyl-covariant fluids reduces to the Class L for the usual fluids with the above energy momentum tensor and adiabatic heat current. 


\section{Part VI}

\section{Computational details}

\section{E Useful variational formulae}

In this appendix we collect various useful variational formulae and some derivations filling in the gaps for various results used in the main text.

\section{E.1 Mapping variations of hydrodynamic fields}

If we denote the hydrodynamic projector by $P_{\alpha \beta} \equiv g_{\alpha \beta}+u_{\alpha} u_{\beta}$, we can derive from (2.1) the following translation between variations of $\left\{\boldsymbol{\beta}^{\mu}, \Lambda_{\boldsymbol{\beta}}\right\}$ and those of the more traditional fields $\left\{u^{\mu}, T, \mu\right\}$ :

$$
\begin{aligned}
\delta u^{\alpha} & =T P_{\beta}^{\alpha} \delta \boldsymbol{\beta}^{\beta}+\frac{1}{2} u^{\alpha} u^{\beta} u^{\rho} \delta g_{\beta \rho} \\
\delta u_{\alpha} & =T P_{\alpha \lambda} \delta \boldsymbol{\beta}^{\lambda}+\frac{1}{2}\left(P_{\alpha}^{\beta} u^{\rho}+P_{\alpha}^{\rho} u^{\beta}-u_{\alpha} u^{\beta} u^{\rho}\right) \delta g_{\beta \rho} \\
\delta T & =T^{2} u_{\alpha} \delta \boldsymbol{\beta}^{\alpha}+\frac{1}{2} T u^{\alpha} u^{\beta} \delta g_{\alpha \beta} \\
\delta \mu & =T\left(\mu u_{\sigma} \delta \boldsymbol{\beta}^{\sigma}+\delta \Lambda_{\boldsymbol{\beta}}+A_{\sigma} \delta \boldsymbol{\beta}^{\sigma}\right)+u^{\sigma} \delta A_{\sigma}+\frac{1}{2} \mu u^{\alpha} u^{\beta} \delta g_{\alpha \beta} .
\end{aligned}
$$

These equations follow from the basic definition of the hydrodynamic fields. Having the explicit expressions at hand comes in handy while deriving various results in the text.

For reference, we also note the variation of the sources along a configuration $\mathcal{B}=$ $\left\{\boldsymbol{\beta}^{\mu}, \Lambda_{\boldsymbol{\beta}}\right\}$ and evaluate them on-shell:

$$
\begin{aligned}
\delta_{\mathcal{B}} g_{\mu \nu} & =2 \nabla_{(\mu} \boldsymbol{\beta}_{\nu)}=\frac{2}{T}\left[\sigma_{\mu \nu}+P_{\mu \nu} \frac{\Theta}{d-1}-\left(\mathfrak{a}_{(\mu}+\nabla_{(\mu} \log T\right) u_{\nu)}\right] \\
& \simeq \frac{2}{T}\left[\sigma_{\mu \nu}+P_{\mu \nu} \frac{\Theta}{d-1}-v_{s}^{2} \Theta u_{\mu} u_{\nu}-\frac{q}{\varepsilon+p} \mathfrak{v}_{(\mu} u_{\nu)}\right]+2^{\text {nd }} \text { order } \\
\delta_{\mathcal{B}} A_{\mu} & =D_{\mu}\left(\Lambda_{\boldsymbol{\beta}}+\boldsymbol{\beta}^{\nu} A_{\nu}\right)+\boldsymbol{\beta}^{\nu} F_{\nu \mu}=u^{\alpha} D_{\alpha}\left(\frac{\mu}{T}\right) u_{\mu}-\frac{1}{T} \mathfrak{v}_{\mu} \\
& \simeq-\frac{1}{T} \frac{d p}{d q} \Theta u_{\mu}-\frac{1}{T} \mathfrak{v}_{\mu}+2^{\text {nd }} \text { order } .
\end{aligned}
$$

\section{E.2 Relating variations of hydrodynamic fields to reference parameterization}

We now turn to the important task of relating the variations of the physical hydrodynamic fields $\left\{\boldsymbol{\beta}^{\mu}, \Lambda_{\boldsymbol{\beta}}\right\}$ to the parameterization in terms of the reference fields (rigid) and physical fluctuating fields introduced in section 7.2. Our goal is to start with the definition (7.6) and derive (7.7). 
Let us begin by varying $\boldsymbol{\beta}^{\mu}$ starting from its definition in the first line of (7.6). An explicit computation gives:

$$
\begin{aligned}
\delta \boldsymbol{\beta}^{\mu} & =\beta^{a}[\varphi] \delta e_{a}^{\mu}+e_{b}^{\mu} \delta \varphi^{a} \frac{\partial}{\partial \varphi^{a}} \beta^{b}[\varphi]+e_{a}^{\mu} \delta \beta^{a}[\varphi] \\
& =-e_{a}^{\mu} \boldsymbol{\beta}^{\nu} \partial_{\nu} \delta \varphi^{a}+e_{b}^{\mu} \delta \varphi^{a} \frac{\partial \beta^{b}}{\partial \varphi^{a}}+e_{a}^{\mu} \delta \beta^{a} \\
& =-\delta_{\mathcal{B}}\left(e_{a}^{\mu} \delta \varphi^{a}\right)+\left[\delta_{\mathcal{B}} e_{a}^{\mu}+e_{b}^{\mu} \frac{\partial \beta^{b}}{\partial \varphi^{a}}\right] \delta \varphi^{a}+e_{a}^{\mu} \delta \beta^{a}
\end{aligned}
$$

To further simplify the expression, consider first the middle term in the above expression, which we argue vanishes.

$$
\begin{aligned}
\delta_{\mathcal{B}} e_{a}^{\mu}+e_{b}^{\mu} \frac{\partial \mathbb{\beta}^{b}}{\partial \varphi^{a}} & =\boldsymbol{\beta}^{\nu} \partial_{\nu} e_{a}^{\mu}-e_{a}^{\nu} \partial_{\nu} \boldsymbol{\beta}^{\mu}+e_{b}^{\mu} \frac{\partial \beta^{b}}{\partial \varphi^{a}} \\
& =\beta^{b} e_{b}^{\nu} \partial_{\nu} e_{a}^{\mu}-\beta^{b} e_{a}^{\nu} \partial_{\nu} e_{b}^{\mu}-e_{a}^{\nu} e_{b}^{\mu} \partial_{\nu} \varphi^{c} \frac{\partial \beta^{b}}{\partial \varphi^{c}}+e_{b}^{\mu} \frac{\partial \beta^{b}}{\partial \varphi^{a}} \\
& =\beta^{b}\left(e_{b}^{\nu} \partial_{\nu} e_{a}^{\mu}-e_{a}^{\nu} \partial_{\nu} e_{b}^{\mu}\right)=0
\end{aligned}
$$

where in the last step we have used the fact that the Lie commutator between two coordinate basis vectors is zero. Thus, we finally obtain

$$
\delta \boldsymbol{\beta}^{\mu}=e_{a}^{\mu} \delta \beta^{a}-\delta_{\mathcal{B}}\left(e_{a}^{\mu} \delta \varphi^{a}\right)
$$

As advertised, the variation of $\varphi^{a}$ enters only as a change along Lie orbit.

We now turn to variation of $\Lambda_{\beta}$ :

$$
\begin{aligned}
\delta \Lambda_{\boldsymbol{\beta}}= & {\left[\delta c c^{-1}, c \Lambda_{\beta} c^{-1}\right]+c \delta \Lambda_{\beta} c^{-1}+\delta \varphi^{a} c \frac{\partial \Lambda_{\beta}}{\partial \varphi^{a}} c^{-1}+\delta \boldsymbol{\beta}^{\sigma}\left(\partial_{\sigma} c\right) c^{-1} } \\
& +\boldsymbol{\beta}^{\sigma} \partial_{\sigma}\left(\delta c c^{-1}\right)+\left[\delta c c^{-1}, \boldsymbol{\beta}^{\sigma}\left(\partial_{\sigma} c\right) c^{-1}\right] \\
= & c \delta \Lambda_{\beta} c^{-1}+\delta \varphi^{a} c \frac{\partial \Lambda_{\beta}}{\partial \varphi^{a}} c^{-1}+e_{a}^{\sigma} \delta \beta^{a}\left(\partial_{\sigma} c\right) c^{-1}-\delta_{\mathcal{B}}\left(e_{a}^{\sigma} \delta \varphi^{a}\right) \partial_{\sigma} c c^{-1} \\
& +\boldsymbol{\beta}^{\sigma} \partial_{\sigma}\left(\delta c c^{-1}\right)+\left[\delta c c^{-1}, \Lambda_{\boldsymbol{\beta}}\right] \\
= & \delta \varphi^{a}\left[e_{a}^{\sigma} \delta_{\mathcal{B}}\left(\partial_{\sigma} c c^{-1}\right)+c \frac{\partial \Lambda_{\beta}}{\partial \varphi^{a}} c^{-1}\right]+c \delta \Lambda_{\beta} c^{-1}+e_{a}^{\sigma} \delta \beta^{a}\left(\partial_{\sigma} c\right) c^{-1} \\
& +\delta_{\mathcal{B}}\left[\delta c c^{-1}-e_{a}^{\sigma} \delta \varphi^{a} \partial_{\sigma} c c^{-1}\right]
\end{aligned}
$$

We now focus on the first term which will end up vanishing after a suitable amount of massaging:

$$
\begin{aligned}
\delta_{\mathcal{B}}\left(\partial_{\sigma} c c^{-1}\right) & =-\partial_{\sigma} \Lambda_{\boldsymbol{\beta}}+\boldsymbol{\beta}^{\alpha} \partial_{\alpha}\left(\partial_{\sigma} c c^{-1}\right)+\partial_{\sigma} \boldsymbol{\beta}^{\alpha}\left(\partial_{\alpha} c c^{-1}\right)+\left[\partial_{\sigma} c c^{-1}, \Lambda_{\boldsymbol{\beta}}\right] \\
& =-\partial_{\sigma}\left(\Lambda_{\boldsymbol{\beta}}-\boldsymbol{\beta}^{\alpha} \partial_{\alpha} c c^{-1}\right)+\left[\partial_{\sigma} c c^{-1}, \Lambda_{\boldsymbol{\beta}}-\boldsymbol{\beta}^{\alpha} \partial_{\alpha} c c^{-1}\right] \\
& =-\partial_{\sigma}\left(c \Lambda_{\beta} c^{-1}\right)+\left[\partial_{\sigma} c c^{-1}, c \Lambda_{\beta} c^{-1}\right] \\
& =-c \partial_{\sigma} \Lambda_{\beta} c^{-1}=-\partial_{\sigma} \varphi^{a} c \frac{\partial \Lambda_{\beta}}{\partial \varphi^{a}} c^{-1} .
\end{aligned}
$$


Using the above result we can write

$$
e_{a}^{\sigma} \delta_{\mathcal{B}}\left(\partial_{\sigma} c c^{-1}\right)+c \frac{\partial \Lambda_{\mathfrak{B}}}{\partial \varphi^{a}} c^{-1}=0
$$

so that in the end

$$
\delta \Lambda_{\boldsymbol{\beta}}=c \delta \Lambda_{\beta} c^{-1}+e_{a}^{\sigma} \delta \beta^{a}\left(\partial_{\sigma} c\right) c^{-1}+\delta_{\mathcal{B}}\left[\delta c c^{-1}-e_{a}^{\sigma} \delta \varphi^{a} \partial_{\sigma} c c^{-1}\right]
$$

Equations (E.5) and (E.9) form the basic map between the variation of the physical fields and those of the reference fields and they are quoted in the text as (7.7).

\section{E.3 Variational rules for anomalous hydrodynamics}

We collect in this appendix various useful formulae for checking the results in section 12.1 and section 12.3. For most of the fields we already know the variations in terms of the hydrodynamic fields and the sources. The new objects whose variations we need in the anomaly discussion are the shadow fields $\hat{\boldsymbol{A}}$ and $\hat{\boldsymbol{\Gamma}}$. These are however conveniently defined for us in terms of the hydrodynamic fields so it is quite simple to see how to write down their variations in terms of our preferred set of fields.

Consider first the flavour shadow connection defined in (12.2); from the basic variations (E.1) it follows immediately that

$$
\begin{aligned}
\delta \hat{A}_{\lambda}=\mu P_{\lambda}^{(\alpha} u^{\beta)} \delta g_{\alpha \beta}+P_{\lambda}^{\alpha} \delta A_{\alpha} \\
\quad+\mu\left(P_{\lambda \alpha}+u_{\lambda} u_{\alpha}\right) T \delta \boldsymbol{\beta}^{\alpha}+u_{\lambda} T\left(\delta \Lambda_{\boldsymbol{\beta}}+A_{\alpha} \delta \boldsymbol{\beta}^{\alpha}\right) .
\end{aligned}
$$

For the mixed anomaly discussion we also need the variation of the spin connection shadow fields. In order to get the variation $\delta \hat{\Gamma}^{\rho}{ }_{\sigma \lambda}$, we first start with the spin chemical potential defined in (12.21) and observe by explicit variation that

$$
\begin{aligned}
\delta \Omega^{\rho}{ }_{\sigma}= & \frac{\delta T}{T}{\Omega^{\rho}}_{\sigma}+T \mathfrak{Q}_{\sigma \kappa}^{\rho \mu} D_{\mu} \delta \boldsymbol{\beta}^{\kappa}+T \boldsymbol{\beta}^{\nu} \mathfrak{Q}_{\sigma \kappa}^{\rho \mu} \delta \Gamma^{\kappa}{ }_{\mu \nu}+T\left(D_{\mu} \boldsymbol{\beta}^{\kappa}\right) \delta \mathfrak{Q}_{\sigma \kappa}^{\rho \mu} \\
= & T{\Omega^{\rho}}_{\sigma} u_{\alpha} \delta \boldsymbol{\beta}^{\alpha}+\frac{1}{2} \Omega^{\rho}{ }_{\sigma} u^{\mu} u^{\nu} \delta g_{\mu \nu}+T \mathfrak{Q}_{\sigma \nu}^{\rho \mu} D_{\mu} \delta \boldsymbol{\beta}^{\nu}+T \boldsymbol{\beta}^{\nu} \mathfrak{Q}_{\sigma \kappa}^{\rho \mu} \delta \Gamma^{\kappa}{ }_{\mu \nu} \\
& -\frac{1}{2} T\left(\delta_{\sigma}^{\mu} D^{\rho} \boldsymbol{\beta}^{\nu}-g^{\rho \mu} \nabla^{\nu} \boldsymbol{\beta}_{\sigma}\right) \delta g_{\mu \nu} .
\end{aligned}
$$

From this we immediately infer

$$
\begin{aligned}
& \delta \hat{\Gamma}^{\rho}{ }_{\sigma \lambda}=\left[\Omega^{\rho}{ }_{\sigma} P_{\lambda}^{(\mu} u^{\nu)}-\frac{1}{2} T u_{\lambda}\left(\delta_{\sigma}^{\mu} \nabla^{\rho} \boldsymbol{\beta}^{\nu}-g^{\rho \mu} \nabla^{\nu} \boldsymbol{\beta}_{\sigma}\right)\right] \delta g_{\mu \nu}+\mathfrak{P}_{\sigma \kappa \lambda}^{\rho \mu \nu} \delta \Gamma^{\kappa}{ }_{\mu \nu} \\
& +\Omega^{\rho}{ }_{\sigma}\left(P_{\lambda \alpha}+u_{\lambda} u_{\alpha}\right) T \delta \boldsymbol{\beta}^{\alpha}+u_{\lambda} T \mathfrak{Q}_{\sigma \kappa}^{\rho \mu} D_{\mu} \delta \boldsymbol{\beta}^{\kappa},
\end{aligned}
$$

where we use the abbreviation $\mathfrak{P}_{\sigma \kappa \lambda}^{\rho \mu \nu}=\delta_{\kappa}^{\rho} \delta_{\sigma}^{\mu} \delta_{\lambda}^{\nu}+\mathfrak{Q}_{\sigma \kappa}^{\rho \mu} u^{\nu} u_{\lambda}$.

\section{F Details of the neutral fluid computation at second order}

In this appendix we work out the Class L theory describing neutral fluids at second order in the gradient expansion. In section 8.1 we have described the basic set-up for this problem. The task at hand is to take the 13 scalar terms given in (8.17) and work out their variations. Once we do that we will be in a position to work out the stress tensor and read off the physical quantities. 


\section{F.1 Variational calculus for the second order scalars}

Let us parameterize the general second order Lagrangian using the basis (8.17) as follows:

$$
\begin{aligned}
\mathcal{L}_{2}= & K_{\sigma}(T) \sigma^{2}+K_{\omega}(T) \omega^{2}+K_{a}(T) \mathfrak{a}^{2}+K_{\Theta}(T) \Theta^{2}+K_{R}(T) R \\
& +K_{t}(T) \nabla_{\mu} T \nabla^{\mu} T+K_{u}(T) \Theta u^{\mu} \nabla_{\mu} T+K_{x}(T) \mathfrak{a}^{\mu} \nabla_{\mu} T+K_{y}(T)\left(u^{\mu} \nabla_{\mu} T\right)^{2} \\
& +f_{a}(T) R_{00}+f_{b}(T) u^{\mu} \nabla_{\mu} \Theta+f_{c}(T) \nabla^{2} T+f_{d}(T) u^{\mu} u^{\nu} \nabla_{\mu} \nabla_{\nu} T
\end{aligned}
$$

We have singled out the last four terms since by a suitable integration by parts they can be eliminated in favour of the nine terms in the first two lines. More specifically we have

$$
\begin{aligned}
f_{a}(T) R_{00}= & -\left[f_{a}^{\prime}(T) \mathfrak{a}^{\mu} \nabla_{\mu} T+f_{a}(T)\left(\sigma^{2}+\omega^{2}+u^{\mu} \nabla_{\mu} \Theta+\frac{\Theta^{2}}{d-1}\right)\right] \\
& +\nabla_{\mu}\left(f_{a}(T) \mathfrak{a}^{\mu}\right) \\
f_{b}(T) u^{\mu} \nabla_{\mu} \Theta= & -\left[f_{b}(T) \Theta^{2}+f_{b}^{\prime}(T) \Theta u^{\mu} \nabla_{\mu} T\right]+\nabla_{\mu}\left(f_{b}(T) \Theta u^{\mu}\right) \\
f_{c}(T) \nabla^{2} T= & -\left[f_{c}^{\prime}(T) \nabla^{\mu} T \nabla_{\mu} T\right]+\nabla_{\mu}\left(f_{c}(T) \nabla^{\mu} T\right) . \\
f_{d}(T) u^{\mu} u^{\nu} \nabla_{\mu} \nabla_{\nu} T= & -\left[f_{d}^{\prime}(T)\left(u^{\mu} \nabla_{\mu} T\right)^{2}+f_{d}(T) \Theta u^{\mu} \nabla_{\mu} T+f_{d}(T) \mathfrak{a}^{\mu} \nabla_{\mu} T\right] \\
& +\nabla_{\mu}\left(f_{d}(T) u^{\mu} u^{\nu} \nabla_{\nu} T\right) .
\end{aligned}
$$

where we dropped the integral signs to keep the expressions compact.

Furthermore, we have at our disposal the field redefinition freedom described in section 7.6. Since we set the first order gradient terms to vanish $\mathcal{L}_{1}=0$, the freedom we have is field redefinition of the ideal fluid Lagrangian (8.2). We claim that by suitable choice of $\delta \varphi^{a}$ we can eliminate all the four terms in the second line of (F.1). Explicitly, under a field redefinition we find as before

$$
\delta \mathcal{L}_{0}[\boldsymbol{\beta}]+\mathcal{L}_{2}[\boldsymbol{\beta}]=p^{\prime}(T)\left(\nabla_{\mu} \log T-v_{s}^{2} \Theta u_{\mu}+\mathfrak{a}_{\mu}\right) e_{a}^{\mu} \delta \varphi^{a}+\mathcal{L}_{2}[\boldsymbol{\beta}]+\cdots
$$

Now what we want to do is to eliminate all terms involving the gradients of temperature. That this is possible is manifest from the equation above, since by choosing appropriate values of $e_{a}^{\mu} \delta \varphi^{a}$ we can set to zero the coefficient functions $\left\{K_{t}(T), K_{u}(T), K_{x}(T), K_{y}(T)\right\}$. However, in doing so we will shift the coefficient functions involving at least one factor of $\Theta$ or $\mathfrak{a}^{\mu}$. It is then easy to see that the combinations

$$
\widetilde{K}_{a}=K_{a}+T^{2} K_{t}-T K_{x}, \quad \widetilde{K}_{\Theta}=K_{\Theta}-T^{2} v_{s}^{4} K_{t}-T v_{s}^{2} K_{u}+T^{2} v_{s}^{4} K_{y} .
$$

are field redefinition invariant. So even if we failed to implement the field redefinitions any transport coefficient not involving these particular combinations would point to an error in the computation.

This means that we only have to compute the variation of the 5 terms in the first line of (F.1). We can do so in a straightforward manner using the basic variational formulae quoted in (E.1). However, in order to demonstrate the efficacy of our field redefintions together with the potential cross-check it offers on the result, we actually vary all the 9 terms in the first two lines of (F.1). 
In what follows we will write the answer eschewing the integrals and factors of $\sqrt{-g}$ though for completeness we will indicate the total derivative pieces we encounter in the process of integrating by parts to facilitate reading off the pre-symplectic potential. In addition to fit the expressions compactly we introduce a shorthand for terms on the l.h.s. viz., $\delta\left(K_{X} X\right) \equiv \frac{1}{\sqrt{-g}} \delta\left(\sqrt{-g} K_{X} X\right)$. For ease of visualization we have also indicated the total derivative terms in a different color; these will be useful later in the computation of the free energy current.

$$
\begin{aligned}
& \delta\left(K_{\sigma} \sigma^{2}\right)=\left(K_{\sigma} \sigma^{2} g^{\mu \nu}+T K_{\sigma}^{\prime} \sigma^{2} u^{\mu} u^{\nu}-4 K_{\sigma} \sigma^{\mu \alpha} \sigma_{\alpha}^{\nu}-4 K_{\sigma} \frac{\Theta}{d-1} \sigma^{\mu \nu}\right. \\
& -4 K_{\sigma} u_{\alpha}\left(u^{\rho} \nabla_{\rho} \sigma^{\alpha(\mu}\right) u^{\nu)}-4 u^{(\nu} \nabla_{\alpha}\left(K_{\sigma} \sigma^{\mu) \alpha}\right) \\
& \left.+2 K_{\sigma} \sigma^{2} u^{\mu} u^{\nu}\right) \frac{1}{2} \delta g_{\mu \nu}-\left(2 K_{\sigma} \sigma^{\alpha \nu} u_{\mu}\right) \delta \Gamma_{\nu \alpha}^{\mu}+\nabla_{\mu}\left(2 K_{\sigma} \sigma^{\mu \nu} \delta u_{\nu}\right) \\
& +\left(T K_{\sigma}^{\prime} \sigma^{2} u_{\alpha}-2 P_{\nu \alpha} \nabla_{\mu}\left(K_{\sigma} \sigma^{\mu \nu}\right)+2 K_{\sigma} a^{\mu} \sigma_{\mu \alpha}\right) T \delta \boldsymbol{\beta}^{\alpha}, \\
& \delta\left(K_{\omega} \omega^{2}\right)=\left(K_{\omega} \omega^{2} g^{\mu \nu}+\left(2 K_{\omega}+T K_{\omega}^{\prime}\right) \omega^{2} u^{\mu} u^{\nu}-4 K_{\omega} \omega^{\mu \alpha} \omega_{\alpha}^{\nu}\right. \\
& +4 K_{\omega} \omega^{(\mu \alpha} u^{\nu)} \mathfrak{a}_{\alpha}+4 \nabla_{\alpha}\left(K_{\omega} \omega^{\alpha(\mu)} u^{\nu)}\right) \frac{1}{2} \delta g_{\mu \nu} \\
& -\nabla_{\mu}\left(2 K_{\omega} \omega^{\mu \nu} \delta u_{\nu}\right) \\
& +\left(T K_{\omega}^{\prime} \omega^{2} u_{\alpha}+2 T P_{\nu \alpha} \nabla_{\mu}\left(K_{\omega} \omega^{\mu \nu}\right)+2 T K_{\omega} \omega_{\alpha}^{\mu} \mathfrak{a}_{\mu}\right) T \delta \boldsymbol{\beta}^{\alpha}, \\
& \delta\left(K_{a} \mathfrak{a}^{2}\right)=\left(K_{a} \mathfrak{a}^{2} g^{\mu \nu}+K_{a}^{\prime} T \mathfrak{a}^{2} u^{\mu} u^{\nu}+2 K_{a} \mathfrak{a}^{\mu} \mathfrak{a}^{\nu}+4 K_{a} \mathfrak{a}^{2} u^{\mu} u^{\nu}\right) \frac{1}{2} \delta g_{\mu \nu} \\
& +\left(2 K_{a} \mathfrak{a}_{\mu} u^{\nu} u^{\alpha}\right) \delta \Gamma_{\nu \alpha}^{\mu}+\nabla_{\mu}\left(2 K_{a} \mathfrak{a}_{\nu} u^{\mu} \delta u^{\nu}\right) \\
& +\left(2 K_{a} \mathfrak{a}_{\mu} P_{\alpha}^{\nu} \nabla_{\nu} u^{\mu}-2 \nabla_{\nu}\left(K_{a} \mathfrak{a}_{\mu} u^{\nu}\right) P_{\alpha}^{\mu}+T K_{a}^{\prime} \mathfrak{a}^{2} u_{\alpha}\right) T \delta \boldsymbol{\beta}^{\alpha}, \\
& \delta\left(K_{\Theta} \Theta^{2}\right)=\left(K_{\Theta} \Theta^{2} g^{\mu \nu}+\left(T K_{\Theta}^{\prime} \Theta^{2}+2 T v_{s}^{2} K_{\Theta}^{\prime} \Theta^{2}-2 K_{\Theta} u^{\alpha} \nabla_{\alpha} \Theta\right) u^{\mu} u^{\nu}\right) \frac{1}{2} \delta g_{\mu \nu} \\
& +\left(2 K_{\Theta} \Theta \delta_{\mu}^{\nu} u^{\alpha}\right) \delta \Gamma_{\nu \alpha}^{\mu}+\nabla_{\mu}\left(2 K_{\Theta} \Theta \delta u^{\mu}\right) \\
& +\left(T K_{\Theta}^{\prime} \Theta^{2} u_{\alpha}-2 P_{\alpha}^{\mu} \nabla_{\mu}\left(\Theta K_{\Theta}\right)\right) T \delta \boldsymbol{\beta}^{\alpha}, \\
& \delta\left(K_{R} R\right)=\left(K_{R} R g^{\mu \nu}+K_{R}^{\prime} T R u^{\mu} u^{\nu}-2 K_{R} R^{\mu \nu}\right. \\
& \left.+2 \nabla^{\mu} \nabla^{\nu} K_{R}-2 g^{\mu \nu} \nabla^{2} K_{R}\right) \frac{1}{2} \delta g_{\mu \nu} \\
& +\nabla_{\mu}\left\{2 \delta g_{\alpha \beta}\left[g^{\alpha \beta} \nabla^{\mu}-g^{\mu \beta} \nabla^{\alpha}\right] K_{R}-\left[g^{\alpha \beta} \nabla^{\mu}-g^{\mu \beta} \nabla^{\alpha}\right]\left(K_{R} \delta g_{\alpha \beta}\right)\right\} \\
& +T^{2} K_{R}^{\prime} R u_{\alpha} \delta \boldsymbol{\beta}^{\alpha},
\end{aligned}
$$




$$
\begin{aligned}
& \delta\left(K_{t} \nabla_{\alpha} T \nabla^{\alpha} T\right)=\left(K_{t}(\nabla T)^{2} g^{\mu \nu}-2 K_{t} \nabla^{\mu} T \nabla^{\nu} T\right. \\
& \left.-T\left(K_{t}^{\prime}(\nabla T)^{2}+2 K_{t} \nabla^{2} T\right) u^{\mu} u^{\nu}\right) \frac{1}{2} \delta g_{\mu \nu}+\nabla_{\mu}\left(2 K_{t} \nabla^{\mu} T \delta T\right) \\
& -\left(K_{t}^{\prime}(\nabla T)^{2}+2 K_{t} \nabla^{2} T\right) T^{2} u_{\alpha} \delta \boldsymbol{\beta}^{\alpha}, \\
& \delta\left(K_{u} \Theta u^{\mu} \nabla_{\mu} T\right)=\left(K_{u} \Theta u^{\alpha} \nabla_{\alpha} T g^{\mu \nu}+\left[K_{u} \Theta u^{\alpha} \nabla_{\alpha} T-T K_{u}\left(\Theta^{2}+u^{\alpha} \nabla_{\alpha} \Theta\right)\right.\right. \\
& \left.\left.-u^{\alpha} \nabla_{\alpha}\left(K_{u} u^{\beta} \nabla_{\beta} T\right)\right] u^{\mu} u^{\nu}\right) \frac{1}{2} \delta g_{\mu \nu} \\
& +\left(P_{\alpha}^{\mu}\left(K_{u} \Theta \nabla_{\mu} T-\nabla_{\mu}\left(K_{u} u^{\mu} \nabla_{\mu} T\right)\right)\right. \\
& \left.-T K_{u}\left(\Theta^{2}+u^{\mu} \nabla_{\mu} \Theta\right) u_{\alpha}\right) T \delta \beta^{\alpha}+K_{u} u^{\beta} \nabla_{\beta} T u^{\nu} \delta_{\mu}^{\alpha} \delta \Gamma_{\alpha \nu}^{\mu} \\
& +\nabla_{\mu}\left(K_{u} \Theta u^{\mu} \delta T+K_{u} u^{\alpha} \nabla_{\alpha} T \delta u^{\mu}\right), \\
& \delta\left(K_{x} \mathfrak{a}^{\mu} \nabla_{\mu} T\right)=\left(K_{x} \mathfrak{a}^{\alpha} \nabla_{\alpha} T g^{\mu \nu}+\left[K_{x} \mathfrak{a}^{\alpha} \nabla_{\alpha} T-T K_{x} \nabla_{\alpha} \mathfrak{a}^{\alpha}\right.\right. \\
& \left.\left.-u^{\alpha} \nabla_{\beta}\left(K_{x} u^{\beta} \nabla_{\alpha} T\right)\right] u^{\mu} u^{\nu}\right) \frac{1}{2} \delta g_{\mu \nu} \\
& +\left(P_{\alpha}^{\mu}\left(K_{x} \nabla_{\mu} u^{\nu} \nabla_{\nu} T-\nabla_{\nu}\left(K_{x} \nabla_{\mu} T u^{\nu}\right)\right)-T K_{x} \nabla_{\mu} \mathfrak{a}^{\mu} u_{\alpha}\right) T \delta \boldsymbol{\beta}^{\alpha} \\
& +K_{x} \nabla_{\mu} T u^{\alpha} u^{\nu} \delta \Gamma_{\alpha \nu}^{\mu}+\nabla_{\mu}\left(K_{x} u^{\mu} \nabla_{\nu} T \delta u^{\nu}+K_{x} \mathfrak{a}^{\mu} \delta T\right), \\
& \delta\left(K_{y}\left(u^{\mu} \nabla_{\mu} T\right)^{2}\right)=\left(K_{y}\left(u^{\alpha} \nabla_{\alpha} T\right)^{2} g^{\mu \nu}+\left[\left(T K_{y}^{\prime}+2 K_{y}\right)\left(u^{\alpha} \nabla_{\alpha} T\right)^{2}\right.\right. \\
& \left.\left.-2 T \nabla_{\alpha}\left(K_{y} u^{\alpha} u^{\beta} \nabla_{\beta} T\right)\right] u^{\mu} u^{\nu}\right) \frac{1}{2} \delta g_{\mu \nu} \\
& +\left(2 K_{y} u^{\beta} \nabla_{\beta} T P_{\alpha}^{\mu} \nabla_{\mu} T-2 T \nabla_{\mu}\left(K_{y} u^{\beta} \nabla_{\beta} T u^{\mu}\right) u_{\alpha}\right. \\
& \left.+T K_{y}^{\prime}\left(u^{\beta} \nabla^{\beta} T\right) u_{\alpha}\right) T \delta \boldsymbol{\beta}^{\alpha}+\nabla_{\mu}\left(2 K_{y} u^{\alpha} \nabla_{\alpha} T u^{\mu} \delta T\right) .
\end{aligned}
$$

In the course of the derivation, we have used for the variation of the curvature term the standard identity

$$
g^{\alpha \beta} \delta R_{\alpha \beta}=\nabla^{\mu}\left[\nabla^{\nu} \delta g_{\mu \nu}-g^{\alpha \beta} \nabla_{\mu} \delta g_{\alpha \beta}\right] .
$$

The stress tensor can be read off from the above expressions as the coefficient of $\frac{1}{2} \delta g_{\mu \nu}$. However, to do so we need to convert $\delta \Gamma_{\nu \alpha}^{\mu}$ variations in some of the terms into $\delta g_{\mu \nu}$ variations. This can be done easily using the identity

$$
\begin{aligned}
X_{\rho}^{\alpha \sigma} \delta \Gamma_{\alpha \sigma}^{\rho}=\nabla_{\rho}\left[X^{\alpha[\beta \rho]}\right. & \left.+X^{\beta[\alpha \rho]}-X^{\rho(\alpha \beta)}\right] \delta g_{\alpha \beta} \\
& -\nabla_{\rho}\left\{\left[X^{\alpha[\beta \rho]}+X^{\beta[\alpha \rho]}-X^{\rho(\alpha \beta)}\right] \delta g_{\alpha \beta}\right\}
\end{aligned}
$$


Using this we compute the combined contribution from (F.5a)-(F.5g) separately to be

$$
\begin{aligned}
& 2\left(K_{\Theta} \Theta\right.\left.\delta_{\mu}^{\nu} u^{\alpha}+K_{a} \mathfrak{a}_{\mu} u^{\nu} u^{\alpha}-K_{\sigma} \sigma^{\alpha \nu} u_{\mu}\right) \delta \Gamma_{\nu \alpha}^{\mu} \\
&=2 \nabla_{\rho}\left(-K_{\Theta} \Theta u^{\rho} g^{\mu \nu}+K_{a}\left(u^{\mu} u^{\nu} \mathfrak{a}^{\rho}-2 \mathfrak{a}^{(\mu} u^{\nu)} u^{\rho}\right)\right. \\
&\left.\quad-K_{\sigma}\left(\sigma^{\mu \nu} u^{\rho}-2 \sigma^{\rho(\mu} u^{\nu)}\right)\right) \frac{1}{2} \delta g_{\mu \nu} \\
&+\nabla_{\mu}\left[K_{a}\left(-u^{\alpha} u^{\beta} \mathfrak{a}^{\mu}+2 \mathfrak{a}^{(\alpha} u^{\beta)} u^{\mu}\right) \delta g_{\alpha \beta}\right] \\
&+\nabla_{\mu}\left[K_{\Theta} \Theta u^{\mu} g^{\alpha \beta} \delta g_{\alpha \beta}+K_{\sigma}\left(\sigma^{\alpha \beta} u^{\mu}-2 \sigma^{\mu(\alpha} u^{\beta)}\right) \delta g_{\alpha \beta}\right] \\
&\left(K_{x} \nabla_{\mu} T u^{\alpha} u^{\nu}+K_{u} u^{\beta} \nabla_{\beta} T \delta_{\mu}^{\alpha} u^{\nu}\right) \delta \Gamma_{\nu \alpha}^{\mu} \\
&=\nabla_{\rho}\left(K_{x}\left(u^{\mu} u^{\nu} \nabla^{\rho} T-2 u^{\rho} u^{(\mu} \nabla^{\nu)} T\right)-K_{u} u^{\beta} \nabla_{\beta} T u^{\rho} g^{\mu \nu}\right) \frac{1}{2} \delta g_{\mu \nu} \\
&+\nabla_{\mu}\left\{\left[-K_{x}\left(u^{\alpha} u^{\beta} \nabla^{\mu} T-2 u^{\mu} u^{(\alpha} \nabla^{\beta)} T\right)+K_{u} u^{\rho} \nabla_{\rho} T u^{\mu} g^{\alpha \beta}\right] \frac{1}{2} \delta g_{\alpha \beta}\right\} .
\end{aligned}
$$

All in all we find the currents for the second order neutral fluid to be:

$$
\begin{aligned}
T_{(2)}^{\mu \nu}= & K_{\sigma} \sigma^{2} g^{\mu \nu}+T K_{\sigma}^{\prime} \sigma^{2} u^{\mu} u^{\nu}-4 K_{\sigma} \sigma^{\mu \alpha} \sigma_{\alpha}^{\nu}-4 K_{\sigma} \frac{\Theta}{d-1} \sigma^{\mu \nu} \\
& -4 K_{\sigma} u_{\alpha}\left(u^{\rho} \nabla_{\rho} \sigma^{\alpha(\mu}\right) u^{\nu)}-4 u^{(\nu} \nabla_{\alpha}\left(K_{\sigma} \sigma^{\mu) \alpha}\right)+2 K_{\sigma} \sigma^{2} u^{\mu} u^{\nu} \\
& +2 \nabla_{\rho}\left(K_{\sigma}\left(\sigma^{\mu \nu} u^{\rho}-2 \sigma^{\rho(\mu} u^{\nu)}\right)\right)+K_{\omega} \omega^{2} g^{\mu \nu}+\left(2 K_{\omega}+T K_{\omega}^{\prime}\right) \omega^{2} u^{\mu} u^{\nu} \\
& -4 K_{\omega} \omega^{\mu \alpha} \omega_{\alpha}^{\nu}+4 K_{\omega} \omega^{(\mu \alpha} u^{\nu)} \mathfrak{a}_{\alpha}+4 \nabla_{\alpha}\left(K_{\omega} \omega^{\alpha(\mu}\right) u^{\nu)}+\widetilde{K}_{a} \mathfrak{a}^{2} g^{\mu \nu} \\
& +\widetilde{K}_{a}^{\prime} T \mathfrak{a}^{2} u^{\mu} u^{\nu}+2 \widetilde{K}_{a} \mathfrak{a}^{\mu} \mathfrak{a}^{\nu}+4 \widetilde{K}_{a} \mathfrak{a}^{2} u^{\mu} u^{\nu}+2 \nabla_{\rho}\left(\widetilde{K}_{a}\left(u^{\mu} u^{\nu} \mathfrak{a}^{\rho}-2 \mathfrak{a}^{(\mu} u^{\nu)} u^{\rho}\right)\right) \\
& +\widetilde{K}_{\Theta} \Theta^{2} g^{\mu \nu}+\left(T \widetilde{K}_{\Theta}^{\prime} \Theta^{2}+2 T v_{s}^{2} \widetilde{K}_{\Theta}^{\prime} \Theta^{2}-2 \widetilde{K}_{\Theta} u^{\alpha} \nabla_{\alpha} \Theta\right) u^{\mu} u^{\nu} \\
& -2 \nabla_{\rho}\left(\widetilde{K}_{\Theta} \Theta u^{\rho} g^{\mu \nu}\right)+K_{R} R g^{\mu \nu}+K_{R}^{\prime} T R u^{\mu} u^{\nu}-2 K_{R} R^{\mu \nu} \\
& +2 \nabla^{\mu} \nabla^{\nu} K_{R}-2 g^{\mu \nu} \nabla^{2} K_{R},
\end{aligned}
$$

while

$$
\begin{aligned}
\mathfrak{h}_{(2)}^{\alpha}= & T K_{\sigma}^{\prime} \sigma^{2} u^{\alpha}-2 P_{\nu}^{\alpha} \nabla_{\mu}\left(K_{\sigma} \sigma^{\mu \nu}\right)+2 K_{\sigma} a^{\mu} \sigma_{\mu}^{\alpha}+T K_{\omega}^{\prime} \omega^{2} u^{\alpha}+2 T P_{\nu}^{\alpha} \nabla_{\mu}\left(K_{\omega} \omega^{\mu \nu}\right) \\
& +2 T K_{\omega} \omega^{\alpha \mu} \mathfrak{a}_{\mu}+2 \widetilde{K}_{a} \mathfrak{a}_{\mu} P^{\nu \alpha} \nabla_{\nu} u^{\mu}-2 \nabla_{\nu}\left(\widetilde{K}_{a} \mathfrak{a}_{\mu} u^{\nu}\right) P^{\mu \alpha}+T \widetilde{K}_{a}^{\prime} \mathfrak{a}^{2} u^{\alpha} \\
& +T \widetilde{K}_{\Theta}^{\prime} \Theta^{2} u^{\alpha}-2 P^{\alpha \mu} \nabla_{\mu}\left(\Theta \widetilde{K}_{\Theta}\right)+T K_{R}^{\prime} R u^{\alpha},
\end{aligned}
$$

where we have written the answer in the field redefinition invariant combination. The 
pre-symplectic potential for this system can be read off from the boundary terms as

$$
\begin{aligned}
\left(\not \Theta_{\mathrm{PS}}\right)_{(2)}^{\mu}= & \left(2 K_{\sigma} \sigma^{\mu \nu}-2 K_{\omega} \omega^{\mu \nu}\right) \delta u_{\nu}+2 K_{a} u^{\mu} \mathfrak{a}_{\nu} \delta u^{\nu}+2 K_{\Theta} \Theta \delta u^{\mu} \\
& +2 \delta g_{\alpha \beta}\left[g^{\alpha \beta} \nabla^{\mu}-g^{\mu \beta} \nabla^{\alpha}\right] K_{R}-\left[g^{\alpha \beta} \nabla^{\mu}-g^{\mu \beta} \nabla^{\alpha}\right]\left(K_{R} \delta g_{\alpha \beta}\right) \\
& +\left(2 K_{t} \nabla^{\mu} T+K_{u} \Theta u^{\mu}+K_{x} \mathfrak{a}^{\mu}+2 K_{y} u^{\mu} u^{\rho} \nabla_{\rho} T\right) \delta T \\
& +u^{\rho} \nabla_{\rho} T\left(K_{u} \delta u^{\mu}\right)+K_{x} u^{\mu} \nabla_{\nu} T \delta u^{\nu} \\
& +K_{a}\left(-u^{\alpha} u^{\beta} \mathfrak{a}^{\mu}+2 \mathfrak{a}^{(\alpha} u^{\beta)} u^{\mu}\right) \delta g_{\alpha \beta} \\
& +K_{\Theta} \Theta u^{\mu} g^{\alpha \beta} \delta g_{\alpha \beta}+K_{\sigma}\left(\sigma^{\alpha \beta} u^{\mu}-2 \sigma^{\mu(\alpha} u^{\beta)}\right) \delta g_{\alpha \beta} \\
& +\left[-K_{x}\left(u^{\alpha} u^{\beta} \nabla^{\mu} T-2 u^{\mu} u^{(\alpha} \nabla^{\beta)} T\right)+K_{u} u^{\rho} \nabla_{\rho} T u^{\mu} g^{\alpha \beta}\right] \frac{1}{2} \delta g_{\alpha \beta}
\end{aligned}
$$

where the first four lines are obtained from the explicit boundary terms in the variations (F.5a)-(F.5i) and the last three lines are obtained from the integration by parts on the variation of the Christoffel symbols (F.8).

\section{F.2 Transport coefficients for neutral fluids}

The expression for the stress tensor as written in (F.9) is rather unilluminating (not to mention formidable), so we need to massage it further to extract some physical information and compare with results in the literature. It is traditional to present the result for the energy momentum tensor in the Landau frame, where the corrections (dissipative or otherwise) to the ideal fluid stress-tensor in the gradient expansion are orthogonal to the fluid velocity field. One can then express the result up to second order [2] $]^{116}$

$$
\begin{aligned}
T_{\mu \nu}= & \epsilon u_{\mu} u_{\nu}+p P_{\mu \nu}-2 \eta \sigma_{\mu \nu}-\zeta P_{\mu \nu} \Theta \\
& +\left[\tau u^{\alpha} \nabla_{\alpha} \sigma_{\langle\mu \nu\rangle}+\kappa_{1} R_{\langle\mu \nu\rangle}+\kappa_{2}\left(F_{R}\right)_{\langle\mu \nu\rangle}+\lambda_{0} \Theta \sigma_{\mu \nu}\right. \\
& \left.\quad+\lambda_{1} \sigma_{\langle\mu}{ }^{\alpha} \sigma_{\alpha \nu\rangle}+\lambda_{2} \sigma_{\langle\mu}{ }^{\alpha} \omega_{\alpha \nu\rangle}+\lambda_{3} \omega_{\langle\mu}{ }^{\alpha} \omega_{\alpha \nu\rangle}+\lambda_{4} \mathfrak{a}_{\langle\mu} \mathfrak{a}_{\nu\rangle}\right] \\
& +P_{\mu \nu}\left[\zeta_{1} u^{\alpha} \nabla_{\alpha} \Theta+\zeta_{2} R+\zeta_{3} R_{00}+\xi_{1} \Theta^{2}+\xi_{2} \sigma^{2}+\xi_{3} \omega^{2}+\xi_{4} \mathfrak{a}^{2}\right] .
\end{aligned}
$$

Most of the fluid dynamical tensors which we are using in the above are given in table 11 and the angle bracket notation indicates projection to the symmetric part transverse to the velocity.

$$
A_{\langle\mu \nu\rangle} \equiv P_{\mu}^{\alpha} P_{\nu}^{\beta}\left(\frac{A_{\alpha \beta}+A_{\beta \alpha}}{2}-\left[\frac{A_{\rho \sigma} P^{\rho \sigma}}{d-1}\right] g_{\alpha \beta}\right) .
$$

In addition we have a few combinations of the curvatures which are defined as

$$
F_{R}^{\mu \nu}=R^{\mu \alpha \nu \beta} u_{\alpha} u_{\beta}, \quad R^{\mu \nu}=R^{\alpha \mu \beta \nu} g_{\alpha \beta}, \quad R_{00}=R^{\mu \nu} u_{\mu} u_{\nu}
$$

with $R_{\alpha \beta \gamma \delta}$ being the Riemann tensor of the background geometry. 


\begin{tabular}{|c|c|c|}
\hline \multicolumn{3}{|c|}{$2^{\text {nd }}$ order neutral fluids: hydrostatic response } \\
\hline Scalars & Vectors & Tensors \\
\hline$\omega^{2}$ & $\omega^{\mu \nu} \mathfrak{a}_{\nu}$ & $\omega^{\alpha<\mu} \omega^{\nu>}{ }_{\alpha}$ \\
$\mathfrak{a}^{2}$ & & $\mathfrak{a}^{<\mu} \mathfrak{a}^{\nu>}$ \\
$R$ & & $R^{<\mu \nu>}$ \\
\hline$R_{\alpha \beta} u^{\alpha} u^{\beta}$ & $P_{\nu}^{\mu} D_{\lambda} \omega^{\nu \lambda}$ & $F_{R}^{<\mu \nu>} \equiv u^{\alpha} u^{\beta} R^{<\mu}{ }_{\alpha}{ }^{\nu>}{ }_{\beta}$ \\
\hline \multicolumn{2}{|c|}{$4 S+4 T=8=5 \mathrm{H}_{F}+3 \mathrm{H}_{S}+0 \mathrm{H}_{V}+0 \mathrm{~A}$} \\
\hline
\end{tabular}

Table 9. The 8 hydrostatic response terms for parity-even neutral fluid at $2^{\text {nd }}$ order in derivative expansion. We have listed the vectors though they do not contribute to frame-invariant transport data. Among the 8 terms, $\mathrm{H}_{F}=5$ combinations are forbidden by hydrostatic principle whereas the remaining $\mathrm{H}_{S}=3$ combinations are generated by using the first 3 scalars in the Lagrangian.

\begin{tabular}{|c|c|c|}
\hline \multicolumn{2}{|c|}{$2^{\text {nd }}$ order neutral fluid: non-hydrostatic transport } \\
\hline Scalars & Vectors & Tensors \\
\hline$\Theta^{2}$ & $\Theta \mathfrak{a}^{\mu}$ & $\sigma^{\alpha<\mu} \sigma^{\nu>}{ }_{\alpha}$ \\
$\sigma^{2}$ & & $\Theta \sigma^{\mu \nu}$ \\
& $\sigma^{\mu \nu} \mathfrak{a}_{\nu}$ & $\sigma^{\alpha<\mu} \omega^{\nu>}{ }_{\alpha}$ \\
\hline$\left(u^{\alpha} D_{\alpha}\right) \Theta$ & $P^{\mu \nu} D_{\nu} \Theta$ & $\left(u^{\alpha} D_{\alpha}\right) \sigma^{\mu \nu}$ \\
& $P_{\nu}^{\mu} D_{\lambda} \sigma^{\nu \lambda}$ & \\
\hline \multicolumn{2}{|c|}{$3 S+4 T=7=2 \overline{\mathrm{H}}_{S}+0 \overline{\mathrm{H}}_{V}+2 \mathrm{~B}+3 \mathrm{D}$} \\
\hline
\end{tabular}

Table 10. The 7 non-hydrostatic transport terms for parity-even neutral fluid at $2^{\text {nd }}$ order in derivative expansion. We have listed the vectors though they do not contribute to frame-invariant transport data. Among the 7 terms, $\overline{\mathrm{H}}_{S}=2$ combinations are generated by inserting the first 2 non-hydrostatic scalars into the Lagrangian. We have $\overline{\mathrm{H}}_{V}=0$ and there are 2 combinations in Class B and 3 combinations in Class D, which are given in table 7 .

The comparison with the expression for the stress tensor (F.12) is however tricky as written for two reasons:

(a) The result (F.12) is given in a basis of independent tensors wherein derivatives of the temperature have been eliminated in favour of those involving the velocity gradients.

(b) It is also presented in the Landau frame, while the stress tensor we compute will naturally be in a frame where the entropy current is $J_{S}^{\mu}=s u^{\mu}$. The latter has been called entropy frame in [46].

\footnotetext{
${ }^{116}$ Note that we have not isolated an explicit factor of $T$ in front of the second order transport coefficients as in [2]. Also some authors (cf., [67]) prefer to make explicit that some second order transport is inherited from first order viscous terms, e.g., it is common to find $\tau=\eta \tau_{\pi}$. We refrain from making such choices to keep expressions manageable.
} 
Using the conservation of the ideal fluid (8.9) we can eliminate temperature gradients and obtain the result for the stress tensor in terms of velocity gradients alone on-shell. Then one can attempt to convert the answer to the Landau frame by an explicit field redefinition. Fortunately, if we set the first order gradient terms to vanish, we can employ a shortcut as discussed in $[46,48]$ which we used in section 8.1. We simply project our result for $T^{\mu \nu}$ onto the invariant tensor and scalar parts using (8.14) and read off the coefficients of the independent tensors used in (F.12). We implement this for each term and use (8.9) at intermediate stages to simplify the computation. When the dust settles we find:

$$
\begin{aligned}
\eta= & \zeta=0 \\
\tau= & -2 T K_{R}^{\prime}-2 K_{\sigma} \\
\kappa_{1}= & -2 K_{R} \quad \kappa_{2}=-2 T K_{R}^{\prime} \\
\lambda_{0}= & \left(2 v_{s}^{2}-\frac{4}{d-1}\right) T K_{R}^{\prime}-2 K_{\sigma}+2 v_{s}^{2} T K_{\sigma}^{\prime} \\
\lambda_{1}= & -2 T K_{R}^{\prime}, \quad \lambda_{2}=4 K_{\sigma}, \quad \lambda_{3}=-2 T K_{R}^{\prime}+4 K_{\omega} \\
\lambda_{4}= & -2 \widetilde{K}_{a}+2 T\left(T K_{R}\right)^{\prime \prime}, \\
\zeta_{1}= & -2 v_{s}^{2} \widetilde{K}_{a}+2 \frac{d-2}{d-1} T K_{R}^{\prime}-2 \widetilde{K}_{\Theta} \\
\zeta_{2}= & \left(\frac{d-3}{d-1}+v_{s}^{2}\right) K_{R}-v_{s}^{2} T K_{R}^{\prime} \\
\zeta_{3}= & -2 v_{s}^{2} \widetilde{K}_{a}+2\left(\frac{d-2}{d-1}+v_{s}^{2}\right) T K_{R}^{\prime}+2\left(v_{s}^{2}-\frac{1}{d-1}\right) K_{R} \\
\xi_{1}= & -\frac{2}{d-1} v_{s}^{2} \widetilde{K}_{a}+2 v_{s}^{2} T\left(v_{s}^{2} T K_{R}^{\prime}\right)^{\prime}+2\left(\frac{d-2}{d-1}+v_{s}^{2}\right)\left(\frac{1}{d-1}-v_{s}^{2}\right) T K_{R}^{\prime} \\
& -\left[\left(1+v_{s}^{2}\right) \widetilde{K}_{\Theta}-v_{s}^{2} T \widetilde{K}_{\Theta}^{\prime}\right] \\
\xi_{2}= & -2 v_{s}^{2} \widetilde{K}_{a}+2\left(\frac{d-2}{d-1}+v_{s}^{2}\right) T K_{R}^{\prime}+\left(1-v_{s}^{2}\right) K_{\sigma}-v_{s}^{2} T K_{\sigma}^{\prime} \\
\xi_{3}= & -2 v_{s}^{2} \widetilde{K}_{a}+2\left(\frac{d-2}{d-1}+v_{s}^{2}\right) T K_{R}^{\prime}+\left(\frac{d-5}{d-1}+3 v_{s}^{2}\right) K_{\omega}-v_{s}^{2} T K_{\omega}^{\prime} \\
\xi_{4}= & {\left[\left(\frac{d-3}{d-1}+v_{s}^{2}\right) \widetilde{K}_{a}+v_{s}^{2} T \widetilde{K}_{a}^{\prime}\right]-2\left(\frac{d-2}{d-1}+v_{s}^{2}\right) T\left(T K_{R}^{\prime}\right)^{\prime} }
\end{aligned}
$$

These are the physically interesting results for the transport coefficients written in terms of the field redefinition invariant combinations of the coefficient functions $K_{\sigma}(T)$, $K_{\omega}(T), \widetilde{K}_{a}(T), \widetilde{K}_{\Theta}(T)$ and $K_{R}(T)$. There are several interesting relations that these hydrodynamic data obey: for one because 15 a-priori independent data are expressed in terms of 5 functions, we expect to see 10 relations between them (which can be obtained 
by eliminating the $K_{i}$ ). More explicitly, we obtain the first 5 relations by substituting

$$
\begin{aligned}
K_{R} & =-\frac{1}{2} \kappa_{1} \\
K_{\omega} & =\frac{1}{4}\left[\lambda_{3}-T \kappa_{1}^{\prime}\right] \\
\widetilde{K}_{a} & =-\frac{1}{2}\left[\lambda_{4}+T\left(T \kappa_{1}\right)^{\prime \prime}\right]
\end{aligned}
$$

into the expressions for $\left\{\kappa_{2}, \zeta_{2}, \zeta_{3}, \xi_{3}, \xi_{4}\right\}$. These 5 relations are the ones that appear in the hydrostatic partition function analysis [3]. In addition, if we substitute

$$
\begin{aligned}
K_{\sigma} & =\frac{1}{2}\left[T \kappa_{1}^{\prime}-\tau\right] \\
\widetilde{K}_{\Theta} & =-\frac{1}{2}\left[\zeta_{1}+\frac{d-2}{d-1} T \kappa_{1}^{\prime}-v_{s}^{2}\left(\lambda_{4}+T\left(T \kappa_{1}\right)^{\prime \prime}\right)\right]
\end{aligned}
$$

into the expressions for $\left\{\lambda_{0}, \lambda_{1}, \lambda_{2}, \xi_{1}, \xi_{2}\right\}$ we get 5 additional relations which cannot be obtained from hydrostatic analysis. Among these 5 relations into the 2 relations for $\left\{\lambda_{0}-\right.$ $\left.\xi_{2}, \lambda_{2}\right\}$ which remove the two Class B transport coefficients whereas the 3 relations for $\left\{\lambda_{0}+\xi_{2}, \lambda_{1}, \xi_{1}\right\}$ remove the three Class D transport coefficients. Further, we can clearly see that the two relations we highlighted for the Weyl invariant fluid in (8.26) continue to hold even for the general neutral fluid:

$$
\tau=\lambda_{1}-\frac{1}{2} \lambda_{2}, \quad \lambda_{1}=\kappa_{2} .
$$

These relations serve to project out one Class B transport coefficient and one Class D transport coefficient respectively.

\section{F.3 Entropy current for the neutral fluid}

The entropy current can be easily computed by variation of the Lagrangian with respect to the temperature. The quickest way is to use the identity (6.6) in terms of the adiabatic heat and charge currents. Using the result obtained in (F.10) for the adiabatic heat current it is trivial to compute the second order corrections to the entropy current. We find a simple expression:

$$
J_{S,(2)}^{\mu}=\left(K_{\sigma}^{\prime} \sigma^{2}+K_{\omega}^{\prime} \omega^{2}+\widetilde{K}_{a}^{\prime} \mathfrak{a}^{2}+\widetilde{K}_{\Theta}^{\prime} \Theta^{2}+K_{R}^{\prime} R^{2}\right) u^{\mu} .
$$

which of course fits with the explicit $T$ dependence of $\mathcal{L}_{2}$.

It is instructive to examine the Noether current in Class L for this system, which encodes the non-canonical part of the entropy current (upto Komar terms). To achieve this we need the pre-symplectic potential which has been computed in (F.11). One then computes:

$$
\begin{aligned}
\mathrm{N}_{(2)}^{\mu}[\mathcal{B}] & =\left[\boldsymbol{\beta}^{\mu} \mathcal{L}-\left(\phi_{\mathcal{B}} \Theta_{\mathrm{PS}}\right)^{\mu}\right]_{(2)} \\
& =\frac{K_{\sigma}}{T}\left(\sigma^{2} u^{\mu}-2 \sigma^{\mu \nu} T \delta_{\mathcal{B}} u_{\nu}-T\left(\sigma^{\alpha \beta} u^{\mu}-2 \sigma^{\mu(\alpha} u^{\beta)}\right) \delta_{\mathfrak{B}} g_{\alpha \beta}\right)
\end{aligned}
$$




$$
\begin{aligned}
& +\frac{K_{\omega}}{T}\left(\omega^{2} u^{\mu}+2 \omega^{\mu \nu} T \delta_{\mathfrak{B}} u_{\nu}\right) \\
& +\frac{K_{a}}{T}\left[u^{\mu}\left(\mathfrak{a}^{2}-2 \mathfrak{a}_{\nu} T \delta_{\mathcal{B}} u^{\nu}\right)+T\left(u^{\alpha} u^{\beta} \mathfrak{a}^{\mu}-2 \mathfrak{a}^{(\alpha} u^{\beta)} u^{\mu}\right) \delta_{\mathfrak{B}} g_{\alpha \beta}\right] \\
& +\frac{K_{\Theta}}{T}\left(\Theta^{2} u^{\mu}-2 \Theta T \delta_{\mathcal{B}} u^{\mu}-T \Theta u^{\mu} g^{\alpha \beta} \delta_{\mathcal{B}} g_{\alpha \beta}\right) \\
& +\frac{K_{R}}{T} R u^{\mu}-2 \delta_{\mathfrak{B}} g_{\alpha \beta}\left[g^{\alpha \beta} \nabla^{\mu}-g^{\mu \beta} \nabla^{\alpha}\right] K_{R}+\left[g^{\alpha \beta} \nabla^{\mu}-g^{\mu \beta} \nabla^{\alpha}\right]\left(K_{R} \delta_{\mathcal{B}} g_{\alpha \beta}\right) \\
& +\frac{K_{t}}{T}\left(\nabla_{\alpha} T \nabla^{\alpha} T u^{\mu}-2 T \nabla^{\mu} T \delta_{\mathcal{B}} T\right) \\
& +\frac{K_{u}}{T}\left(\Theta u^{\alpha} \nabla_{\alpha} T u^{\mu}-T \Theta u^{\mu} \delta_{\mathcal{B}} T-T u^{\alpha} \nabla_{\alpha} T \delta_{\mathcal{B}} u^{\mu}\right. \\
& \left.+\frac{1}{2} T u^{\rho} \nabla_{\rho} T u^{\mu} g^{\alpha \beta} \delta_{\mathfrak{B}} g_{\alpha \beta}\right) \\
& +\frac{K_{x}}{T}\left(\mathfrak{a}^{\alpha} \nabla_{\alpha} T u^{\mu}-T \mathfrak{a}^{\mu} \delta_{\mathcal{B}} T-T u^{\mu} \nabla_{\nu} T \delta_{\mathcal{B}} u^{\nu}\right. \\
& \left.\quad+T\left(\frac{1}{2} u^{\alpha} u^{\beta} \nabla^{\mu} T-u^{\mu} u^{(\alpha} \nabla^{\beta)} T\right) \delta_{\mathfrak{B}} g_{\alpha \beta}\right) \\
& +\frac{K_{y}}{T}\left(\left(u^{\alpha} \nabla_{\alpha} T\right)^{2} u^{\mu}-2 T u^{\mu} u^{\rho} \nabla_{\rho} T \delta_{\mathcal{B}} T\right)
\end{aligned}
$$

Using the on-shell expressions:

$$
\begin{aligned}
\delta_{\mathcal{B}} u^{\mu} & \simeq-\Theta v_{s}^{2} \boldsymbol{\beta}^{\mu}, \quad \delta_{\mathcal{B}} u_{\mu} \simeq \Theta v_{s}^{2} \boldsymbol{\beta}_{\mu}, \quad \delta_{\mathcal{B}} T \simeq-\Theta v_{s}^{2}, \\
\delta_{\mathcal{B}} g_{\alpha \beta} & =\frac{2}{T}\left(\sigma_{\alpha \beta}+P_{\alpha \beta} \frac{\Theta}{d-1}-\Theta v_{s}^{2} u_{\alpha} u_{\beta}\right)
\end{aligned}
$$

we can simplify the above to

$$
\begin{aligned}
\mathrm{N}_{(2)}^{\mu}[\mathcal{B}]= & -\frac{K_{\sigma}}{T} \sigma^{2} u^{\mu}+\frac{K_{\omega}}{T} \omega^{2} u^{\mu}+\frac{K_{a}}{T}\left(\mathfrak{a}^{2} u^{\mu}-2 v_{s}^{2} \Theta \mathfrak{a}^{\mu}\right)-\frac{K_{\Theta}}{T} \Theta^{2} u^{\mu} \\
& +\frac{K_{R}}{T} R u^{\mu}-2 \delta_{\mathfrak{B}} g_{\alpha \beta}\left[g^{\alpha \beta} \nabla^{\mu}-g^{\mu \beta} \nabla^{\alpha}\right] K_{R}+\left[g^{\alpha \beta} \nabla^{\mu}-g^{\mu \beta} \nabla^{\alpha}\right]\left(K_{R} \delta_{\mathfrak{B}} g_{\alpha \beta}\right) \\
& +T K_{t}\left(\mathfrak{a}^{2} u^{\mu}+\Theta^{2} v_{s}^{4} u^{\mu}-2 \Theta v_{s}^{2} \mathfrak{a}^{\mu}\right)+K_{u} \Theta^{2} v_{s}^{2} u^{\mu} \\
& +K_{x}\left(-\mathfrak{a}^{2} u^{\mu}+2 \Theta v_{s}^{2} \mathfrak{a}^{\mu}\right)-T K_{y} \Theta^{2} v_{s}^{4} u^{\mu} \\
= & -\frac{K_{\sigma}}{T} \sigma^{2} u^{\mu}+\frac{K_{\omega}}{T} \omega^{2} u^{\mu}+\frac{\widetilde{K}_{a}}{T}\left(\mathfrak{a}^{2} u^{\mu}-2 v_{s}^{2} \Theta \mathfrak{a}^{\mu}\right)-\frac{\widetilde{K}_{\Theta}}{T} \Theta^{2} u^{\mu} \\
& +\frac{K_{R}}{T}\left(R u^{\mu}+2 \nabla^{\mu}\left(\Theta\left(1+v_{s}^{2}\right)\right)-2 \nabla_{\alpha}\left(\sigma^{\alpha \mu}+P^{\alpha \mu} \frac{\Theta}{d-1}-\Theta v_{s}^{2} u^{\alpha} u^{\mu}\right)\right) \\
& -2 \frac{\left(T K_{R}\right)^{\prime}}{T}\left(\Theta^{2} v_{s}^{2} u^{\mu}-\Theta \mathfrak{a}^{\mu}\left(\frac{d-2}{d-1}+v_{s}^{2}\right)+\sigma^{\mu \alpha} \mathfrak{a}_{\alpha}\right)
\end{aligned}
$$

It is useful to note that the free energy current is field redefinition invariant as can be explicitly seen from the fact that the terms combine into the combinations suggested in (F.4). 


\section{G The hydrostatic entropy current}

In section 4 and section 6 we constructed an entropy current starting from a basic variational principle, which we used, for example, in deriving (F.22). As we have mentioned in the course of our discussions, a very impressive analysis of the entropy current arising from hydrostatics was described in [36, 37]. We revisit that argument in our language providing a simple translation to the considerations of these papers to the current discussion.

\section{G.1 The entropy analysis of Bhattacharyya}

Consider a system in hydrostatic equilibrium as described in section 4, for which we can write down an equilibrium partition function. To understand the structure of the entropy current, we will need to depart from hydrostatics, which we shall do in the gradient expansion, by introducing time dependence as described in [36, 37]. A useful proxy for the time dependence is the operator $\delta_{\mathfrak{B}}$, since in equilibrium $\left.\delta_{\mathfrak{B}}\right|_{\text {Hydrostatics }}=\delta_{\mathscr{K}}$ annihilates the background sources. Thus introducing linear time dependence is tantamount to working at linear order in variations captured by $\delta_{\mathfrak{B}}$.

With this understanding, let us state the various results obtained in [36] in order:

- The second law of thermodynamics implies that every equilibrium configurations is associated with a partition function.

- The leading $\mathcal{O}\left(\delta_{\mathcal{B}}\right)$ terms in the entropy current are determined by this partition function.

- Demanding on-shell conservation of this entropy current to linear order in $\delta_{\mathcal{B}}$ is same as demanding that energy-momentum and charge currents be derived by varying the associated partition function with respect to background sources. This gives all the equality type constraints.

- The divergence of entropy current at quadratic order in $\delta_{\mathfrak{B}}$ expansion leads to inequality type constraints.

- Terms in the entropy current at higher order in the $\delta_{\mathfrak{B}}$ expansion can then be arranged as to give a non-negative definite quadratic form for the total entropy production $\Delta$. They do not produce any new constraints.

The crucial step here is, of course, the construction of an entropy current from the hydrostatic partition function. Paraphrasing our discussion in section 4.2, we can describe this construction as follows:

1. We begin with the thermodynamic formula for entropy

$$
\text { Total entropy }=\frac{\partial}{\partial T} \int_{\Sigma_{E}}\left[\boldsymbol{\beta}^{\sigma} \mathcal{L}\right]_{\text {Hydrostatic }} d^{d-1} S_{\sigma} .
$$

which can then be rewritten up to boundary terms as

$$
\text { Total entropy }=\int_{\Sigma_{E}}\left[-\boldsymbol{\beta}_{\lambda} T^{\sigma \lambda}-\left(\Lambda_{\boldsymbol{\beta}}+\boldsymbol{\beta}^{\lambda} A_{\lambda}\right) \cdot J^{\sigma}+\boldsymbol{\beta}^{\sigma} \mathcal{L}\right]_{\text {Hydrostatic }} d^{d-1} S_{\sigma} .
$$


2. Next we compute the time derivative of this entropy. Whenever the hydrostatic equality type constraints are satisfied, the time derivative of the first two terms in (G.2) gives a boundary term which is just the total free energy current. In particular, we can read off the spatial component of the free energy current from this boundary term.

3. In turn, this gives an expression for the hydrostatic contribution to the entropy current $\left(J_{S}^{\mu}\right)_{\text {Hydrostatic }}$.

Instead of working in the microcanonial ensemble, we can directly move to canonical ensemble in the first step, and focus on free energy current instead of entropy current. With this change in ensemble, the above set of arguments is then equivalent to the algorithm we described in section 4.2 .

\section{G.2 Comparison with the charged fluid analysis of Bhattacharyya}

Armed with this understanding we can compare the analysis of [37] for parity-even charged fluids with the arguments presented in section 14.3.

1. One begins by counting the total number of transport coefficients in frame invariant language. This gives 16 Scalars +17 Vectors +18 Tensors $=51$ a-priori different transport coefficients.

2. Class A: remove Class A using the particular combination from anomaly-induced transport theory. In this example, there is nothing to remove, which gives $\mathrm{A}=0$.

3. Classes $\left\{\mathrm{H}_{S}, \mathrm{H}_{V}, \mathrm{H}_{F}\right\}$ (or the 3-fold fate of non-anomaly induced hydrostatic transport coefficients): focus on the remaining terms that survive hydrostatics; these amount to 9 Scalars +6 vectors +9 Tensors $=24$ a-priori hydrostatic transport coefficients. Thus, we are leaving out 7 Scalars +11 vectors +9 Tensors $=27$ transport coefficients that do not survive hydrostatic limit. By looking at the partition function we see that, of these 24,7 come from $\mathrm{H}_{S}$ terms. This gives $\mathrm{H}_{S}=7, \mathrm{H}_{V}=0$ as obtained in [37]. Thus we have $\mathrm{H}_{F}=24-(7+0)=17$.

The first half of [37] (and appendix A therein) is devoted to showing that one can complete these to 7 solutions of adiabaticity equation. This involves constructing the entropy current etc... By this point, we understand how to do this very well covariantly, so we can just skip ahead and construct a covariant entropy current as outlined in the main text. Henceforth, we have to only worry about the 27 nonhydrostatic terms.

4. Class B: next we examine $\frac{1}{2} T^{\mu \nu} \delta_{\mathfrak{B}} g_{\mu \nu}+J^{\mu} \cdot \delta_{\mathfrak{B}} A_{\mu}$ for these non-hydrostatic terms. This is contained in eqs. (5.6) and (5.7) and appendix B of [37]. Before we proceed, let us note that the last two terms of $(5.7)_{[37]}$ with $T_{8}$ and $T_{9}$ respectively are identically zero - the tensor term summation should stop with $T_{7}$. With this small amendment we have

$$
\begin{aligned}
& 2 \text { Scalars }+3 \text { Vectors }+2 \text { Tensors }=7 \text { terms in eq. }(5.6)_{[37]} \\
& 5 \text { Scalars }+0 \text { vectors }+4 \text { tensors }=9 \text { terms in eq. }(5.7)_{[37]}
\end{aligned}
$$


Comparing this against the total count of 7 Scalars +11 Vectors +9 Tensors for non-hydrostatic terms, we conclude that 0 Scalars +8 Vectors +3 Tensors $=11$ terms go away at this step. This gives us 11 terms in Class B in agreement with our counting. We remove these and thence focus on the 16 terms that are left.

5. Class $\overline{\mathrm{H}}_{S}$ : at this point, [37] argues that 5 out of the 16 terms that survived this far, can be absorbed as total derivatives into the entropy current. We know independently that this is the correct counting based on $\overline{\mathrm{H}}_{S}=5$, following from the procedure described below equation (14.7). At this point we are left with 11 terms.

6. Class D: all of the 11 terms are now dissipative. Moreover, their contribution to $\Delta$ can be explicitly assembled schematically into the combinations

$$
T \Delta=2 \eta\left(\sigma+\delta_{\mathcal{B}}^{2} \mathcal{O}_{1}\right)^{2}+\zeta\left(\Theta+\delta_{\mathcal{B}}^{2} \mathcal{O}_{2}\right)^{2}+\sigma_{\mathrm{Ohm}}\left(\mathfrak{v}+\delta_{\mathcal{B}}^{2} \mathcal{O}_{3}\right)^{2}+\mathcal{O}\left(\partial^{4}\right)
$$

for some operators $\mathcal{O}_{i}$, which can be obtained from the explicit construction in [37] if necessary. We see that this works directly by using the differential operators at our disposal.

This completes then a cross-check of our results with the analysis of [37].

\section{H Bianchi identities for anomalous hydrodynamics}

In this appendix we derive Bianchi identities and on-shell constraints for anomalous hydrodynamics. This fills in the details and complements the discussion in section 12 .

\section{H.1 Bianchi identities from anomalous part of effective action}

Our goal here is to evaluate eq. (12.22) which we reproduce for convenience:

$$
\begin{aligned}
\delta \boldsymbol{V}_{\mathcal{P}}[\boldsymbol{A}, \boldsymbol{\Gamma}, \hat{\boldsymbol{A}}, \hat{\boldsymbol{\Gamma}}]= & \delta \boldsymbol{A} \wedge \cdot^{\star 2 n+1} \mathbf{J}_{H}-\delta \hat{\boldsymbol{A}} \wedge \cdot{ }^{\star 2 n+1} \hat{\mathbf{J}}_{H} \\
& +\frac{1}{2} \delta \boldsymbol{\Gamma}^{a}{ }_{b} \wedge{ }^{{ }^{2 n+1}} \boldsymbol{\Sigma}_{H}{ }^{b}{ }_{a}-\frac{1}{2} \delta \hat{\boldsymbol{\Gamma}}^{a}{ }_{b} \wedge{ }^{{ }^{2 n+1}} \hat{\boldsymbol{\Sigma}}_{H}{ }^{b}{ }_{a} \\
& +d\left\{\delta \boldsymbol{A} \wedge \cdot \star \boldsymbol{J}_{\mathcal{P}}+\frac{1}{2} \delta \boldsymbol{\Gamma}^{\alpha}{ }_{\beta} \wedge \star \boldsymbol{\Sigma}_{\mathcal{P}}{ }^{\beta}{ }_{\alpha}+\delta \boldsymbol{u} \wedge \star \boldsymbol{q}_{\mathcal{P}}\right\},
\end{aligned}
$$

In order to further evaluate this expression, we need variations of various objects. We begin by first using the relations derived in appendix E.3. In particular substituting (E.10) and (E.12) into (12.22) we find that the variation of the transgression form takes the following form:

$$
\begin{aligned}
\delta \int_{\mathcal{M}_{d+1}} & \boldsymbol{V}_{\mathcal{P}}[\boldsymbol{A}, \boldsymbol{\Gamma} ; \hat{\boldsymbol{A}}, \hat{\boldsymbol{\Gamma}}] \\
= & f_{\mathcal{M}}\left[\left(\mathrm{J}_{H}^{m}-P_{n}^{m} \hat{\mathrm{J}}_{H}^{n}\right) \cdot \delta A_{m}+\frac{1}{2}\left(\Sigma_{H}{ }^{m s}{ }_{r}-\mathfrak{P}_{p r n}^{q s m} \hat{\Sigma}_{H}{ }^{n p}{ }_{q}\right) \delta \Gamma^{r}{ }_{s m}\right] \\
- & f_{\mathcal{M}}\left[\left(\mu \cdot \hat{\mathrm{J}}_{H}^{q}+\frac{1}{2} \Omega^{r}{ }_{s} \hat{\Sigma}_{H}{ }^{q s}{ }_{r}\right) P_{q}^{(m} u^{n)}\right. \\
& \left.-\frac{1}{4} T u_{q} \hat{\Sigma}_{H}{ }^{q s}{ }_{r}\left(\delta_{s}^{m} D^{r} \boldsymbol{\beta}^{n}-g^{r m} D^{n} \boldsymbol{\beta}_{s}\right)\right] \delta g_{m n}
\end{aligned}
$$




$$
\begin{aligned}
& -f_{\mathcal{M}}\left(\mu \cdot \hat{\mathrm{J}}_{H}^{q}+\frac{1}{2} \Omega^{r}{ }_{s} \hat{\Sigma}_{H}{ }^{q s}{ }_{r}\right)\left(P_{q m}+u_{q} u_{m}\right) T \delta \boldsymbol{\beta}^{m} \\
& -f_{\mathcal{M}} T u_{m}\left[\hat{\mathrm{J}}_{H}^{m} \cdot\left(\delta \Lambda_{\boldsymbol{\beta}}+A_{n} \delta \boldsymbol{\beta}^{n}\right)+\frac{1}{2} \mathfrak{Q}_{p r}^{q s} \hat{\Sigma}_{H}{ }^{m p}{ }_{q} D_{s} \delta \boldsymbol{\beta}^{r}\right] \\
& +\int_{\mathcal{M}} \sqrt{-g}\left[J_{\mathcal{P}}^{\alpha} \cdot \delta A_{\alpha}+\frac{1}{2} \Sigma_{\mathcal{P}}{ }^{\alpha \sigma}{ }_{\rho} \delta \Gamma^{\rho}{ }_{\sigma \alpha}+q_{\mathcal{P}}^{(\alpha} u^{\beta)} \delta g_{\alpha \beta}+\left(q_{\mathcal{P}}\right)_{\sigma} T \delta \boldsymbol{\beta}^{\sigma}\right]
\end{aligned}
$$

where we have introduced a new projector $\mathfrak{P}_{\sigma \kappa \lambda}^{\rho \mu \nu}=\delta_{\kappa}^{\rho} \delta_{\sigma}^{\mu} \delta_{\lambda}^{\nu}+\mathfrak{Q}_{\sigma \kappa}^{\rho \mu} u^{\nu} u_{\lambda}$ to keep the expression compact.

The variational formula needs to be massaged further to bring it into an amenable form from which we can read off the bulk and boundary currents. For one the variation of the Christoffel symbols need to be converted to metric variations. For another the bulk term involving $D_{s} \delta \boldsymbol{\beta}^{r}$ should be integrated by parts and will thus contribute some boundary terms. Both of these features arise from the gravitational contribution. Indeed setting the spin connection terms to zero we see that (H.2) reduces to (12.14).

Firstly, the variation of the Christoffel symbols can be converted into a variation of the metric by observing the identity

$$
\int_{\mathcal{M}} \sqrt{-g} \frac{1}{2} \Sigma_{\mathcal{P}}^{\alpha \sigma}{ }_{\rho} \delta \Gamma^{\rho}{ }_{\sigma \alpha}=\int_{\mathcal{M}} \sqrt{-g} \frac{1}{2} D_{\rho}\left[\Sigma_{\mathcal{P}}^{\alpha[\beta \rho]}+\Sigma_{\mathcal{P}}^{\beta[\alpha \rho]}-\Sigma_{\mathcal{P}}^{\rho(\alpha \beta)}\right] \frac{1}{2} \delta g_{\alpha \beta},
$$

We use this expression on the boundary $\mathcal{M}$ to simplify the term in the last line of (H.2). In the intermediate step we have discarded a total derivative term using the fact that $\partial \mathcal{M}=0$. For the bulk term however we have to do a bit more work since now the boundary contributions from total derivative terms cannot be ignored. These can however be accounted for by recalling that our coordinatization of the bulk spacetime $\mathcal{M}_{d+1}$ was such that the normal direction to $\mathcal{M}$ was denoted as $\perp$. Putting this together we find from the bulk term involving the Christoffel symbol variation

$$
\begin{aligned}
f_{\mathcal{M}} & \frac{1}{2}\left(\Sigma_{H}^{m s}{ }_{r}-\mathfrak{P}_{p r n}^{q s m} \hat{\Sigma}_{H}^{n p}{ }_{q}\right) \delta \Gamma^{r}{ }_{s m} \\
= & f_{\mathcal{M}}\left\{\frac{1}{2} D_{k}\left[\Sigma_{H}^{m[n k]}+\Sigma_{H}^{n[m k]}-\Sigma_{H}^{k(m n)}\right] \frac{1}{2} \delta g_{m n}\right. \\
& \left.\quad-\frac{1}{2} D_{k}\left[\left(\mathfrak{P}_{p r s}^{q[n m} g^{k] r}+\mathfrak{P}_{p r s}^{q[m n} g^{k] r}-\mathfrak{P}_{p r s}^{q(m k} g^{n) r}\right) g_{q l} \hat{\Sigma}_{H}^{s p l}\right] \frac{1}{2} \delta g_{m n}\right\} \\
& -\int_{\mathcal{M}} \sqrt{-g} \frac{1}{2}\left(\mathfrak{P}_{\rho \gamma \lambda}^{\eta(\alpha \perp} g^{\beta) \gamma} g_{\eta \kappa} \hat{\Sigma}_{H}^{\lambda \rho \kappa}\right) \frac{1}{2} \delta g_{\alpha \beta} \\
= & f_{\mathcal{M}} \frac{1}{2} D_{p}\left[\left(\Sigma_{H}^{m[n p]}+\Sigma_{H}^{n[m p]}-\Sigma_{H}^{p(m n)}\right)-\left(P_{q}^{m} \hat{\Sigma}_{H}^{q[n p]}+P_{q}^{n} \hat{\Sigma}_{H}^{q[m p]}-\hat{\Sigma}_{H}^{p(m n)}\right)\right] \frac{1}{2} \delta g_{m n} \\
& -\int_{d} \sqrt{-g} \frac{1}{2} \hat{\Sigma}_{H}^{\perp(\mu \nu)} \frac{1}{2} \delta g_{\mu \nu},
\end{aligned}
$$

where extrinsic boundary terms of the form $\Sigma_{H}^{\alpha \perp \beta}+\Sigma_{H}^{\beta \perp \alpha}$ have been set to zero and in the second step we used the following identities to implement some simplifications:

$$
\begin{aligned}
& \mathfrak{P}_{p r s}^{q[n m} g^{k] r} g_{q l}=\delta_{p}^{[n} \delta_{l}^{k]} \delta_{s}^{m}+\frac{1}{2}\left(\delta_{p}^{[n} \delta_{l}^{k]}-\delta_{l}^{[n} \delta_{p}^{k]}\right) u^{m} u_{s}=\delta_{p}^{[n} \delta_{l}^{k]} P_{s}^{m}, \\
& \mathfrak{P}_{p r s}^{q(m k} g^{n) r} g_{q l}=\delta_{p}^{(m} \delta_{l}^{n)} \delta_{s}^{l} k+\frac{1}{2}\left(\delta_{p}^{(m} \delta_{l}^{n)}-\delta_{l}^{(m} \delta_{p}^{n)}\right) u^{k} u_{s}=\delta_{p}^{(m} \delta_{l}^{n)} \delta_{s}^{k}
\end{aligned}
$$


Finally, performing an integration by parts also in the third line of eq. (H.2), we obtain the final simplified variational formula of interest

$$
\begin{aligned}
& \delta \int_{\mathcal{M}_{d+1}} \boldsymbol{V}_{\mathcal{P}}[\boldsymbol{A}, \boldsymbol{\Gamma} ; \hat{\boldsymbol{A}}, \hat{\boldsymbol{\Gamma}}] \\
& =f_{\mathcal{M}}\left(J_{(d+1)}^{m} \cdot \delta A_{m}+\frac{1}{2} \delta g_{m n} T_{(d+1)}^{m n}+T \mathfrak{h}_{m}^{(d+1)} \delta \boldsymbol{\beta}^{m}+T \mathfrak{n}_{(d+1)} \cdot\left(\delta \Lambda_{\boldsymbol{\beta}}+A_{a} \delta \boldsymbol{\beta}^{a}\right)\right) \\
& \quad+\int_{\mathcal{M}} \sqrt{-g}\left(\left(J^{\alpha}\right)_{\mathrm{A}} \cdot \delta A_{\alpha}+\frac{1}{2} \delta g_{\alpha \beta}\left(T^{\alpha \beta}\right)_{\mathrm{A}}+\left(q_{\mathcal{P}}\right)_{\sigma} T \delta \boldsymbol{\beta}^{\sigma}\right)
\end{aligned}
$$

where the bulk currents now take the form

$$
\begin{aligned}
T_{(d+1)}^{m n}= & \frac{1}{2} T u_{p} \hat{\Sigma}_{H}^{p s}{ }_{r}\left(\delta_{s}^{m} D^{r} \boldsymbol{\beta}^{n}-g^{r m} D^{n} \boldsymbol{\beta}_{s}\right)-\left(\mu \cdot \hat{\mathrm{J}}_{H}^{p}+\frac{1}{2} \Omega^{r}{ }_{s} \hat{\Sigma}_{H}{ }^{p s}{ }_{r}\right)\left(P_{p}^{m} u^{n}+P_{p}^{n} u^{m}\right) \\
& +\frac{1}{2} D_{p}\left[\left(\Sigma_{H}^{m[n p]}+\Sigma_{H}^{n[m p]}-\Sigma_{H}^{p(m n)}\right)-\left(P_{q}^{m} \hat{\Sigma}_{H}^{q[n p]}+P_{q}^{n} \hat{\Sigma}_{H}^{q[m p]}-\hat{\Sigma}_{H}^{p(m n)}\right]\right) \\
J_{(d+1)}^{m}= & \mathrm{J}_{H}^{m}-P_{n}^{m} \hat{\mathrm{J}}_{H}^{n} \\
\mathfrak{h}_{m}^{(d+1)}= & -\left(\mu \cdot \hat{\mathrm{J}}_{H}^{p}+\frac{1}{2} \Omega^{r}{ }_{s} \hat{\Sigma}_{H}{ }^{p s}{ }_{r}\right)\left(P_{p m}+u_{p} u_{m}\right)+\frac{1}{2 T} D_{s}\left(T u_{r} \mathfrak{Q}_{p m}^{q s} \hat{\Sigma}_{H}{ }^{r p}{ }_{q}\right) \\
\mathfrak{n}_{(d+1)}= & -u_{m} \hat{\mathrm{J}}_{H}^{m}
\end{aligned}
$$

and boundary currents turn out to be

$$
\begin{aligned}
\left(T^{\alpha \beta}\right)_{\mathrm{A}} & =q_{\mathcal{P}}^{\alpha} u^{\beta}+q_{\mathcal{P}}^{\beta} u^{\alpha}+\frac{1}{2} D_{\rho}\left(\Sigma_{\mathcal{P}}^{\alpha[\beta \rho]}+\Sigma_{\mathcal{P}}^{\beta[\alpha \rho]}-\Sigma_{\mathcal{P}}^{\rho(\alpha \beta)}\right)-\frac{1}{2} \hat{\Sigma}_{H}^{\perp(\alpha \beta)}, \\
\left(J^{\alpha}\right)_{\mathrm{A}} & =J_{\mathcal{P}}^{\alpha}
\end{aligned}
$$

We have written this expression, allowing a-priori for a contribution to the stress tensor involving the shadow Hall current $\frac{1}{2} \hat{\Sigma}_{H}^{\perp(\alpha \beta)}$. In the main text, (12.28), the stress tensor is quoted without this term. This is due to the fact that $\hat{\Sigma}_{H}^{\perp(\alpha \beta)}=0$ always holds for our choice of spin chemical potential. To prove this, observe that the connection $\hat{\boldsymbol{\Gamma}}^{\mu}{ }_{\nu}$ is metric compatible due to our spin chemical potential being anti-symmetric:

$$
\widehat{\nabla}_{\sigma} g_{\mu \nu}=-u_{\sigma}\left(\Omega_{\mu \nu}+\Omega_{\nu \mu}\right)=0
$$

From metric compatibility it follows immediately that the associated curvature tensor $\hat{\boldsymbol{R}}^{\nu}{ }_{\mu}$ is anti-symmetric. From the definition $(12.23)$ one can see that $\left(\hat{\boldsymbol{\Sigma}}_{H}\right)^{\mu}{ }_{\nu}$ inherits this antisymmetry. In what follows we will therefore often set

$$
\hat{\Sigma}_{H}^{\perp(\alpha \beta)}=0 .
$$

Equation (H.6) is our master equation for the Lagrangian variation. Our main interest is not in a generic variation, but rather in the variations engendered by diffeomorphisms and gauge transformations of the fields on $\mathcal{M}_{d+1}$, which is what is needed to derive the 
Bianchi identities. Using the general formula (6.8) for the bulk integral, we find for the particular case where $\delta=\delta_{x}$ is a gauge transformation and diffeomorphism: ${ }^{117}$

$$
\begin{aligned}
\delta_{x} \int_{\mathcal{M}_{d+1}} \boldsymbol{V}_{\mathcal{P}}[\boldsymbol{A}, \boldsymbol{\Gamma} \hat{\boldsymbol{A}}, \hat{\boldsymbol{\Gamma}}] \\
=f_{\mathcal{M}} \xi_{m}\left\{-D_{n}\left(T_{(d+1)}^{m n}\right)+F_{n}^{m} \cdot\left(\mathrm{J}_{H}^{n}-P_{p}^{n} \hat{\mathrm{J}}_{H}^{p}\right)\right. \\
\left.\quad+g^{m n} T \mathfrak{n}_{(d+1)} \cdot \delta_{\mathcal{B}} A_{n}+\frac{g^{m n}}{\sqrt{-g_{d+1}}} \delta_{\mathcal{B}}\left[\sqrt{-g_{d+1}} T \mathfrak{h}_{m}^{(d+1)}\right]\right\} \\
\quad-f_{\mathcal{M}}\left(\Lambda+\xi^{n} A_{n}\right) \cdot\left\{D_{m}\left(J_{(d+1)}^{m}\right)-\frac{1}{\sqrt{-g_{d+1}}} \delta_{\mathcal{B}}\left[\sqrt{-g_{d+1}} T \mathfrak{n}_{(d+1)}\right]\right\} \\
\quad+\int_{\mathcal{M}} \sqrt{-g} \xi_{\alpha}\left(\frac{1}{2} D_{\gamma}\left(\Sigma_{H}^{\perp[\alpha \gamma]}-\hat{\Sigma}_{H}^{\perp[\alpha \gamma]}\right)-\left(\mu \cdot \hat{\mathrm{J}}_{H}^{\perp}+\frac{1}{2} \Omega^{\nu}{ }_{\mu} \hat{\Sigma}_{H}{ }^{\perp \mu}{ }_{\nu}\right) u^{\alpha}\right) \\
\quad+\int_{\mathcal{M}} \sqrt{-g}\left(\Lambda+\xi^{\alpha} A_{\alpha}\right) \cdot\left(\mathrm{J}_{H}^{\perp}-\hat{\mathrm{J}}_{H}^{\perp}\right) \\
\quad+\int_{\mathcal{M}} \sqrt{-g}\left(\left(J^{\alpha}\right)_{\mathrm{A}} \cdot \delta_{x} A_{\alpha}+\left(T^{\alpha \beta}\right)_{\mathrm{A}} \frac{1}{2} \delta_{x} g_{\alpha \beta}+\left(q_{\mathcal{P}}\right)_{\sigma} T \delta_{x} \boldsymbol{\beta}^{\sigma}\right)
\end{aligned}
$$

In writing the above expression we have done some integration by parts mostly to remove the derivatives of the diffeomorphism field $\xi^{m}$.

We can now directly read off the Bianchi identities for the bulk theory: these are simply given by the first two lines of (H.11). They satisfy the expected form of the equations (6.12) as derived earlier from general considerations. Indeed in so far as the bulk theory is concerned, we have a gapped topological system which obeys bulk diffeomorphism and gauge invariance and so we should have a-priori expected to see this work out as stated. Note that upon setting the spin currents to zero we recover the flavour Bianachi identities as indicated in section 12.1 .

Once the bulk Bianchi identities are satisfied for arbitrary bulk $X$ we see that the variation of the anomalous Lagrangian is purely a boundary term. This has both the physics of the hydrodynamic system of interest as well as the anomaly inflow term that enable us to write down the expressions for the covariant currents. We have one final manipulation to do to bring this into a canonical form. Expressing the variations $\delta_{x} g_{\alpha \beta}$ and $\delta_{x} A_{\alpha}$ in terms of the gauge transformation fields $X$ and performing yet another integration by parts we finally convert (H.11) into

$$
\begin{aligned}
\delta_{x} & \int_{\mathcal{M}_{d+1}} \boldsymbol{V}_{\mathcal{P}}[\boldsymbol{A}, \boldsymbol{\Gamma} ; \hat{\boldsymbol{A}}, \hat{\boldsymbol{\Gamma}}] \\
= & \int_{\mathcal{M}} \sqrt{-g} \xi_{\alpha}\left(\frac{1}{2} D_{\gamma}\left(\Sigma_{H}^{\perp[\alpha \gamma]}-\hat{\Sigma}_{H}^{\perp[\alpha \gamma]}\right)-\left(\mu \cdot \hat{\mathrm{J}}_{H}^{\perp}+\frac{1}{2} \Omega^{\nu}{ }_{\mu} \hat{\Sigma}_{H}{ }^{\perp \mu}{ }_{\nu}\right) u^{\alpha}\right. \\
& \left.\quad-D_{\beta}\left(T^{\alpha \beta}\right)_{\mathrm{A}}+\left(J^{\beta}\right)_{\mathrm{A}} \cdot F^{\alpha}{ }_{\beta}+\frac{g^{\alpha \sigma}}{\sqrt{-g}} \delta_{\mathcal{B}}\left[\sqrt{-g} T\left(q_{\mathcal{P}}\right)_{\sigma}\right]\right) \\
& +\int_{\mathcal{M}} \sqrt{-g}\left(\Lambda+\xi^{\alpha} A_{\alpha}\right) \cdot\left(-D_{\alpha}\left(J^{\alpha}\right)_{\mathrm{A}}+\mathrm{J}_{H}^{\perp}-\hat{\mathrm{J}}_{H}^{\perp}\right)
\end{aligned}
$$

\footnotetext{
${ }^{117}$ As in all of our discussion of anomalies, the fields $X=\left\{\xi^{m}, \Lambda\right\}$ are taken to live on $\mathcal{M}_{d+1}$.
} 
We are now in a position to read off the boundary Bianchi identities which are obeyed by our anomalous fluid. We find that these take the form (picking out coefficients of the arbitrary $\xi_{\alpha}$ and $\left(\lambda+\xi^{\alpha} A_{\alpha}\right)$ from the above expression)

$$
\begin{aligned}
D_{\beta}\left(T^{\alpha \beta}\right)_{\mathrm{A}}=\left(J^{\beta}\right)_{\mathrm{A}} & \cdot F_{\beta}^{\alpha}+\frac{g^{\alpha \sigma}}{\sqrt{-g}} \delta_{\mathcal{B}}\left[\sqrt{-g} T\left(q_{\mathcal{P}}\right)_{\sigma}\right] \\
& +\frac{1}{2} D_{\gamma}\left(\Sigma_{H}^{\perp[\alpha \gamma]}-\hat{\Sigma}_{H}^{\perp[\alpha \gamma]}\right)-\left(\mu \cdot \hat{\mathrm{J}}_{H}^{\perp}+\frac{1}{2} \Omega^{\nu}{ }_{\mu} \hat{\Sigma}_{H}{ }^{\perp \mu}{ }_{\nu}\right) u^{\alpha}
\end{aligned}
$$

and

$$
D_{\alpha}\left(J^{\alpha}\right)_{\mathrm{A}}=\mathrm{J}_{H}^{\perp}-\hat{\mathrm{J}}_{H}^{\perp} .
$$

The terms on the r.h.s. of the expressions of (H.13) and (H.14) with the one $\perp$ component of the Hall currents are due to bulk inflow.

\section{H.2 On-shell constraints from the full Lagrangian}

For reference, we quote both the bulk and the boundary on-shell constraints that are obtained in section 12.4 by extremizing the full effective Lagrangian $\mathcal{L}_{\text {eff }}[\boldsymbol{\Psi}]=d \mathcal{L}_{\mathrm{n}-\mathrm{a}}[\boldsymbol{\Psi}]+$ $\boldsymbol{V}_{\mathcal{P}}[\boldsymbol{A}, \boldsymbol{\Gamma}, \hat{\boldsymbol{A}}, \hat{\boldsymbol{\Gamma}}]$ with respect to the pullback fields. For the bulk theory, this yields the following on-shell constraints:

$$
\begin{aligned}
g^{m n} T \mathfrak{n}_{(d+1)} \cdot \delta_{\mathcal{B}} A_{n}+\frac{g^{m n}}{\sqrt{-g_{d+1}}} \delta_{\mathcal{B}}\left[\sqrt{-g_{d+1}} \mathfrak{h}_{m}^{(d+1)}\right] & \simeq 0, \\
\frac{1}{\sqrt{-g_{d+1}}} \delta_{\mathcal{B}}\left[\sqrt{-g_{d+1}} T \mathfrak{n}_{(d+1)}\right] & \simeq 0 .
\end{aligned}
$$

The boundary on-shell constraints, on the other hand, are given by

$$
\begin{aligned}
\frac{g^{\mu \nu}}{\sqrt{-g}} \delta_{\mathcal{B}}\left(\sqrt{-g} T\left[\left(\mathfrak{h}_{\nu}\right)_{\mathrm{n}-\mathrm{a}}+\left(q_{\mathcal{P}}\right)_{\nu}\right]\right)+g^{\mu \nu} T \mathfrak{n}_{\mathrm{n}-\mathrm{a}} \cdot \delta_{\mathcal{B}} A_{\nu} & \simeq 0, \\
\frac{1}{\sqrt{-g}} \delta_{\mathcal{B}}\left(\sqrt{-g} T \mathfrak{n}_{\mathrm{n}-\mathrm{a}}\right) & \simeq 0 .
\end{aligned}
$$

Combining these on-shell constraints with the anomalous Bianchi identities we obtain the equations of motion. In the bulk, we find from (H.11),

$$
D_{n}\left(T_{(d+1)}^{m n}\right)=F_{n}^{m} \cdot\left(J_{H}^{n}-P_{p}^{n} \hat{\mathrm{J}}_{H}^{p}\right), \quad D_{m}\left(J_{(d+1)}^{m}\right)=0 .
$$

Similarly, in the boundary theory, we obtain

$$
\begin{aligned}
D_{\beta}\left(T_{\mathrm{n}-\mathrm{a}}^{\alpha \beta}+\left(T^{\alpha \beta}\right)_{\mathrm{A}}\right) \simeq & \left(J_{\mathrm{n}-\mathrm{a}}^{\sigma}+\left(J^{\sigma}\right)_{\mathrm{A}}\right) \cdot F^{\alpha}{ }_{\sigma}+\frac{1}{2} D_{\gamma}\left(\Sigma_{H}^{\perp[\alpha \gamma]}-\hat{\Sigma}_{H}^{\perp[\alpha \gamma]}\right) \\
& -\left(\mu \cdot \hat{\mathrm{J}}_{H}^{\perp}+\frac{1}{2} \Omega^{\nu}{ }_{\mu} \hat{\Sigma}_{H}{ }^{\perp \mu}{ }_{\nu}\right) u^{\alpha}, \\
D_{\sigma}\left(J_{\mathrm{n}-\mathrm{a}}^{\sigma}+\left(J^{\sigma}\right)_{\mathrm{A}}\right) \simeq & \mathrm{J}_{H}^{\perp}-\hat{\mathrm{J}}_{H}^{\perp} .
\end{aligned}
$$

We note that we could have arrived at these results on a slightly easier path. As discussed in general in section 7.4, equations of motion are easier to obtain on the reference 
manifold where the relevant part of the constrained variation is already built in. Concretely, we could have started from (H.6) written on the reference manifold $\mathbb{M}$ by replacing all currents by boldface currents and changing indices from Greek to Latin. Then directly performing the variation (7.20) would give

$$
\begin{aligned}
& -\S \int_{\mathbb{M}_{d+1}} \boldsymbol{V}_{\mathcal{P}}\left[\mathbb{A}_{P}, \mathbb{T}_{M N}^{P} ; \hat{\mathbb{A}}_{P}, \hat{\mathbb{T}}_{M N}^{P}\right] \\
& =f_{\mathbb{M}_{d+1}}\left(\mathbb{J}_{(d+1)}^{M} \cdot \delta \mathbb{A}_{M}+\frac{1}{2} \delta \mathfrak{g}_{M N} \mathbb{T}_{(d+1)}^{M N}\right)+\int_{\mathbb{M}} \sqrt{-\mathfrak{g}}\left(\left(\mathbb{J}^{a}\right)_{\mathrm{A}} \cdot \delta \mathbb{A}_{a}+\frac{1}{2} \delta \mathfrak{g}_{a b}\left(\mathbb{T}^{a b}\right)_{\mathrm{A}}\right) \\
& =f_{\mathbb{M}_{d+1}} \delta \varphi_{M}\left[-\mathbb{D}_{N}\left(\mathbb{T}_{(d+1)}^{M N}+\mathbb{F}^{M} \cdot\left(\mathbb{J}_{H}^{N}-P_{P}^{N} \hat{\mathbb{J}}_{H}^{P}\right)\right)\right] \\
& -f_{\mathbb{M}_{d+1}}\left(-c^{-1} \delta c+\mathbb{A}_{M} \delta \varphi^{M}\right) \cdot \mathbb{D}_{M}\left(\mathbb{J}_{(d+1)}^{M}\right) \\
& +\int_{\mathbb{M}} \sqrt{-g} \delta \varphi_{a}\left[\frac{1}{2} \mathbb{D}_{c}\left(\mathbb{\Sigma}_{H}^{\perp[a c]}-\hat{\mathbb{\Sigma}}_{H}^{\perp[a c]}\right)-\left(\mu \cdot \hat{\mathbb{J}}_{H}^{\perp}+\frac{1}{2} \Omega^{b}{ }_{c} \hat{\mathbb{\Sigma}}_{H}{ }^{\perp c} b\right) \mathbb{u}^{a}\right] \\
& +\int_{\mathbb{M}} \sqrt{-\mathfrak{g}}\left(-c^{-1} \delta c+\mathbb{A}_{b} \delta \varphi^{c}\right) \cdot\left(\mathbb{J}_{H}^{\perp}-\hat{\mathbb{J}}_{H}^{\perp}\right) \\
& +\int_{\mathbb{M}} \sqrt{-\mathfrak{g}}\left[\delta \varphi_{a}\left(-\mathbb{D}_{b}\left(\mathbb{T}^{a b}\right)_{\mathrm{A}}+\left(\mathbb{J}^{b}\right)_{\mathrm{A}} \cdot \mathbb{F}^{a}{ }_{b}\right)-\left(-c^{-1} \delta c+\mathbb{A}_{b} \delta \varphi^{c}\right) \mathbb{D}_{a}\left(\mathbb{J}^{a}\right)_{\mathrm{A}}\right] .
\end{aligned}
$$

From this we can immediately read off the anomalous part of the equations of motion (H.17) and (H.18). While working on the reference manifold is thus manifestly easier, we still gain computational insight from doing the full analysis from a point of view of physical $\mathcal{M}$.

\section{H.3 Bianchi identities of anomalous Schwinger-Keldysh action}

We will now give some details of the derivation of anomalous Schwinger-Keldysh currents as outlined in section 13.3. Since we double also the sources, we want to avoid complications that arise from an analysis on two different manifolds and work instead on the unambiguously defined reference manifold as described in section 13. For simplicity we can work in the hydrodynamic limit from the beginning, i.e., we only keep track of terms linear in difference fields. Let us start by computing the variation of the influence functional adapting the results form [30]. We have

$$
\begin{aligned}
\delta S_{I F}= & \int_{\mathbb{M}_{d+1}}\left\{\delta \hat{\boldsymbol{A}}\left[\Psi_{\mathrm{R}}\right] \wedge^{\star_{2 n+1}} \hat{\mathbf{J}}_{H}\left[\Psi_{\mathrm{R}}\right]-\delta \hat{\boldsymbol{A}}\left[\Psi_{\mathrm{L}}\right] \wedge^{\star^{2 n+1}} \hat{\mathbf{J}}_{H}\left[\Psi_{\mathrm{L}}\right]\right. \\
& \left.+\delta \hat{\boldsymbol{\Gamma}}\left[\Psi_{\mathrm{R}}\right]^{M}{ }_{N} \wedge^{{ }^{2 n+1}} \hat{\boldsymbol{\Sigma}}_{H}\left[\Psi_{\mathrm{R}}\right]^{N}{ }_{M}-\delta \hat{\boldsymbol{\Gamma}}\left[\Psi_{\mathrm{L}}\right]^{M}{ }_{N} \wedge^{\star^{2 n+1}} \hat{\boldsymbol{\Sigma}}_{H}\left[\Psi_{\mathrm{L}}\right]^{N}{ }_{M}\right\} \\
& +\int_{\mathbb{M}}\left\{\text { Boundary terms }\left[\Psi_{\mathrm{R}}, \Psi_{\mathrm{L}}\right] \approx \mathcal{O}\left(\left(\Psi_{\mathrm{R}}-\Psi_{\mathrm{L}}\right)^{2}\right)\right\}
\end{aligned}
$$

Isolating the variations of difference fields makes the linearization in difference fields manifest and we can for ease of notation immediately take the hydrodynamic limit of all the remaining quantities involved. Using the variation rules (E.10), (E.12) and integration 
by parts, the variation of the influence functional $S_{I F}$, (H.20), with respect to the difference fields takes the form ${ }^{118}$

$$
\left.\delta S_{I F}\right|_{\text {hydro }}=f_{\mathbb{M}_{d+1}}\left(\left(\mathbb{T}_{(d+1)}^{M N}\right)_{I F} \frac{1}{2} \delta \overline{\mathfrak{g}}_{M N}+\left(\mathbb{J}_{(d+1)}^{M}\right)_{I F} \cdot \delta \overline{\mathbb{A}}_{M}\right),
$$

where hydro denotes the limit where all the expressions that are not variations of difference fields are evaluated at $\varphi_{\mathrm{R}}^{a}(x)=\varphi_{\mathrm{L}}^{a}(x) \equiv \varphi^{a}(x)$ and $c_{\mathrm{R}}(x)=c_{\mathrm{L}}(x) \equiv c(x)$ and we find the following bulk currents generated by the influence functional:

$$
\begin{aligned}
\left(\mathbb{T}_{(d+1)}^{M N}\right)_{I F}= & 2\left(\mu \cdot \hat{\mathbb{J}}_{H}^{P}+\frac{1}{2} \mathbb{\Omega}^{T}{ }_{S} \hat{\mathbb{Z}}_{H}{ }^{P S}{ }_{T}\right) P_{P}^{(M} \mathbb{u}^{N)} \\
& +\frac{1}{2} \mathbb{D}_{P}\left(P_{Q}^{M} \hat{\mathbb{\Sigma}}_{H}^{Q[N P]}+P_{Q}^{N} \hat{\mathbb{\Sigma}}_{H}^{Q[M P]}-\hat{\mathbb{\Sigma}}_{H}^{P(M N)}\right) \\
& -\frac{1}{2} \mathbb{T} \mathfrak{u}_{Q} \hat{\mathbb{Z}}_{H}{ }^{Q S}{ }_{T}\left(\delta_{S}^{M} \mathbb{D}^{T} \beta^{N}-g^{T M} \mathbb{D}^{N} \beta_{S}\right) \\
\left(\mathbb{J}_{(d+1)}^{M}\right)_{I F}= & P_{N}^{M} \hat{\mathbb{J}}_{H}^{N}
\end{aligned}
$$

We now consider the variation of the left and right anomalous terms in $S_{\text {tot }}$. These are completely analogous to our earlier discussion in section 12.3 except that we have two sets of contributions to keep track of. Sticking to the hydrodynamic limit, we find that one copy each of the $\mathrm{R}$ and $\mathrm{L}$ anomalous variation (H.6) combine in the hydrodynamic limit to give ${ }^{119}$

$$
\begin{aligned}
& \left.\delta\left(\int_{\mathbb{M}_{d+1}} \boldsymbol{V}_{\mathcal{P}}\left[\mathbb{\Psi}_{\mathrm{R}}\right]-\boldsymbol{V}_{\mathcal{P}}\left[\mathbb{\Psi}_{\mathrm{L}}\right]\right)\right|_{\text {hydro }} \\
& \quad=f_{\mathbb{M}_{d+1}}\left(\mathbb{T}_{(d+1)}^{M N} \delta \overline{\mathfrak{g}}_{M N}+\mathbb{J}_{(d+1)}^{M} \delta \overline{\mathbb{A}}_{M}\right)+\int_{\mathbb{M}} \sqrt{-\breve{g}}\left(\left(\mathbb{T}^{a b}\right)_{\mathrm{A}} \frac{1}{2} \delta \overline{\mathfrak{g}}_{a b}+\left(\mathbb{J}^{a}\right)_{\mathrm{A}} \cdot \delta \overline{\mathbb{A}}_{a}\right) .
\end{aligned}
$$

The currents showing up in the above equation are the ones we have already recorded in (H.7) and (12.28) (just on the reference manifold). As expected, to linear order in the variations as required for the hydrodynamic limit the contributions simply combine to give the common current times the difference of the $\mathrm{R}$ and $\mathrm{L}$ sources.

We can now put everything together to find the bulk and boundary Bianchi identities and the dynamical equations of motion. Firstly, combining eqs. (H.21) and (H.23), we

\footnotetext{
${ }^{118}$ We adhere to our conventions stated earlier: boundary reference manifold indices are from the earlier part of the lowercase Latin alphabet, while the bulk reference manifold is denoted as $\mathbb{M}_{d+1}$ and indexed by letters from the second half of the uppercase Latin alphabet.

${ }^{119}$ The currents written in (H.23) strictly speaking live on the reference manifold. However, we have refrained from introducing further notation to distinguish them from their physical counterparts; they are distinguish by their indices which indicate their origins. They are of course pushed-forward along the diffeomorphism and gauge transformation fields like any other tensor field defined on $\mathbb{M}$.
} 
obtain for the variation of the entire anomaly part of the action

$$
\begin{aligned}
\left.\delta S_{\text {anom }}\right|_{\text {hydro }} \equiv & \left.\delta\left(S_{I F}+\int_{\mathcal{M}_{d+1}}\left(\boldsymbol{V}_{\mathcal{P}}\left[\Psi_{\mathrm{R}}\right]-\boldsymbol{V}_{\mathcal{P}}\left[\Psi_{\mathrm{L}}\right]\right)\right)\right|_{\text {hydro }} \\
= & f_{\mathbb{M}_{d+1}}\left[\frac{1}{2} \mathbb{D}_{P}\left(\mathbb{Z}_{H}^{M[N P]}+\mathbb{\Sigma}_{H}^{N[M P]}-\mathbb{\Sigma}_{H}^{P(M N)}\right) \frac{1}{2} \delta \overline{\mathfrak{g}}_{M N}+\mathbb{J}_{H}^{M} \cdot \delta \overline{\mathbb{A}}_{M}\right] \\
& +\int_{\mathbb{M}} \sqrt{-\breve{g}}\left\{\left[\frac{1}{2} \mathbb{D}_{c}\left(\mathbb{\Sigma}_{\mathcal{P}}^{a[b c]}+\mathbb{Z}_{\mathcal{P}}^{b[a c]}-\mathbb{\Sigma}_{\mathcal{P}}^{c(a b)}\right)+2 \mathbb{q}_{\mathcal{P}}^{\left(a, u^{b}\right)}\right] \frac{1}{2} \delta \overline{\mathfrak{g}}_{a b}+\mathbb{J}_{\mathcal{P}}^{a} \cdot \delta \overline{\mathbb{A}}_{a}\right\} .
\end{aligned}
$$

From this one can readily compute the equations of motion (see section 13.3).

\section{Class $\mathrm{L}_{\mathrm{T}}$ details}

This appendix collects some intermediate steps of the computations relevant for the Class $\mathrm{L}_{\mathrm{T}}$ discussions of section 15. In section I.1 we check that our diffeomorphism, flavour gauge, and $U(1)_{\mathrm{T}}$ transformations form an algebra. section I.2 fills in some intermediate steps involved in the derivation of the $U(1)_{\mathrm{T}}$ Bianchi identity.

\section{I.1 Consistency of $U(1)_{\mathrm{T}}$ transformations}

We would like to check that the diffeomorphism, flavour gauge and $U(1)_{\mathrm{T}}$ transformations given in (15.3), (15.4) and (15.5) form a Lie algebra. In particular, we would like to ensure that the Wess-Zumino consistency conditions are satisfied. This in particular requires that the commutator of two transformations parameterized by $X_{1}=\left\{\bar{\xi}_{1}^{\mu}, \bar{\Lambda}_{1}, \bar{\Lambda}^{(\mathrm{T})}{ }_{1}\right\}$ and $X_{2}=\left\{\bar{\xi}_{2}^{\mu}, \bar{\Lambda}_{2}, \bar{\Lambda}^{(\mathrm{T})}{ }_{2}\right\}$, is itself given by a diffeomorphism, flavour and $U(1)_{\mathrm{T}}$ transformation with parameters $X_{3}=\left\{\bar{\xi}_{3}^{\mu}, \bar{\Lambda}_{3}, \bar{\Lambda}^{(\mathrm{T})}{ }_{3}\right\}$. Note that we work directly with in the untwisted formalism; the conclusions will of course be unchanged should we switch to the twisted transformation variables parameterizing individual elements of the Class $\mathrm{L}_{\mathrm{T}}$ symmetries.

We will proceed systematically analyzing the commutator of two successive transformations. A-priori the transformation of the basic hydrodynamic fields $\boldsymbol{\Psi}$ will fix the dependence of $\left\{\bar{\xi}_{3}^{\mu}, \bar{\Lambda}_{3}\right\}$ on $X_{1}$ and $X_{2}$. However, since the $U(1)_{\mathrm{T}}$ transformation mixes with diffeomorphisms, it also follows that the parameter $\bar{\Lambda}^{(T)} 3$ is already constrained. Consistency of our transformations requires that the partner sources and $A^{(\top)}$ transform by the now determined values of $X_{3}$. Ensuring that this is upheld will form the main check of the analysis below.

Let us start with the transformation for the background metric $g_{\mu \nu}$. From (15.3) we find

$$
\left[\delta x_{1}, \delta x_{2}\right] g_{\mu \nu}=\left[£_{\bar{\xi}_{1}}, £_{\bar{\xi}_{2}}\right] g_{\mu \nu} \equiv £_{\bar{\xi}_{3}} g_{\mu \nu}
$$

with

$$
\begin{aligned}
\bar{\xi}_{3}^{\sigma} & =\left[\bar{\xi}_{1}, \bar{\xi}_{2}\right]^{\sigma} \\
\xi_{3}^{\sigma} & =\left[\xi_{1}, \xi_{2}\right]^{\sigma}+\left(\boldsymbol{\lambda}_{2}^{(\mathrm{T})} £_{\xi_{1}} \boldsymbol{\beta}^{\sigma}-\boldsymbol{\lambda}_{1}^{(\mathrm{T})} £_{\xi_{2}} \boldsymbol{\beta}^{\sigma}\right)+x \boldsymbol{\beta}^{\sigma} \\
\boldsymbol{\lambda}_{3}^{(\mathrm{T})} & =£_{\xi_{1}} \boldsymbol{\lambda}_{2}^{(\mathrm{T})}-£_{\xi_{2}} \boldsymbol{\lambda}_{1}^{(\mathrm{T})}+\boldsymbol{\lambda}_{1}^{(\mathrm{T})} £_{\boldsymbol{\beta}} \boldsymbol{\lambda}_{2}^{(\mathrm{T})}-\boldsymbol{\lambda}_{2}^{(\mathrm{T})} £_{\boldsymbol{\beta}} \boldsymbol{\lambda}_{1}^{(\mathrm{T})}-x
\end{aligned}
$$


where we define

$$
\boldsymbol{\lambda}_{k}^{(\mathrm{T})} \equiv \bar{\Lambda}_{k}^{(\mathrm{T})}+\bar{\xi}_{k}^{\sigma} \mathrm{A}_{\sigma}^{(\mathrm{T})}=\Lambda_{k}^{(\mathrm{T})}+\xi_{k}^{\sigma} \mathrm{A}_{\sigma}^{(\mathrm{T})}
$$

to keep the expressions compact. Further, $x$ is an arbitrary scalar parameterizing a family of relations that are all consistent with $\bar{\xi}_{3}^{\sigma}=\xi_{3}^{\sigma}+\boldsymbol{\lambda}_{3}^{(\mathrm{T})} \boldsymbol{\beta}^{\sigma}$. The value of $x$ will have to be determined later on to ensure consistency of the formalism.

The parameter $\bar{\xi}_{3}$ encodes the effective diffeomorphism in the untwisted variables. Once we have ascertained this it is clear that the $\boldsymbol{\beta}$ transformation in (15.3) is consistent with (I.2) since $\boldsymbol{\beta}^{\mu}$ itself is Lie transported along $\mathcal{X}$.

Let us then turn to the flavour gauge transformations, taking the commutator of the gauge transformations we have using (I.2)

$$
\begin{aligned}
{\left[\delta_{x_{1}}, \delta x_{2}\right] A_{\mu}=} & £_{\bar{\xi}_{1}}\left(£_{\bar{\xi}_{2}} A_{\mu}+\left[A_{\mu}, \bar{\Lambda}_{2}\right]+\partial_{\mu} \bar{\Lambda}_{2}\right)+\left[£_{\bar{\xi}_{2}} A_{\mu}+\left[A_{\mu}, \bar{\Lambda}_{2}\right]+\partial_{\mu} \bar{\Lambda}_{2}, \bar{\Lambda}_{1}\right] \\
& -£_{\bar{\xi}_{2}}\left(£_{\bar{\xi}_{1}} A_{\mu}+\left[A_{\mu}, \bar{\Lambda}_{1}\right]+\partial_{\mu} \bar{\Lambda}_{1}\right)-\left[£_{\bar{\xi}_{1}} A_{\mu}+\left[A_{\mu}, \bar{\Lambda}_{1}\right]+\partial_{\mu} \bar{\Lambda}_{1}, \bar{\Lambda}_{2}\right] \\
= & £_{\bar{\xi}_{3}} A_{\mu}+£_{\bar{\xi}_{1}} D_{\mu} \bar{\Lambda}_{2}-£_{\bar{\xi}_{2}} D_{\mu} \bar{\Lambda}_{1}+\left[A_{\mu},\left[\bar{\Lambda}_{2}, \bar{\Lambda}_{1}\right]\right] \\
& +\left[£_{\bar{\xi}_{2}} A_{\mu}+\partial_{\mu} \bar{\Lambda}_{2}, \bar{\Lambda}_{1}\right]-\left[£_{\bar{\xi}_{1}} A_{\mu}+\partial_{\mu} \bar{\Lambda}_{1}, \bar{\Lambda}_{2}\right] \\
= & £_{\bar{\xi}_{3}} A_{\mu}+D_{\mu} \bar{\Lambda}_{3}
\end{aligned}
$$

where we defined $\bar{\Lambda}_{3} \equiv \Lambda_{3}+\boldsymbol{\lambda}_{3}^{(\mathrm{T})} \Lambda_{\boldsymbol{\beta}}$ with

$$
\begin{aligned}
\bar{\Lambda}_{3}=£_{\bar{\xi}_{1}} & \bar{\Lambda}_{2}-£_{\bar{\xi}_{2}} \bar{\Lambda}_{1}-\left[\bar{\Lambda}_{1}, \bar{\Lambda}_{2}\right] \\
\Lambda_{3}=\boldsymbol{\lambda}_{2}^{(\mathrm{T})} & \left(£_{\xi_{1}} \Lambda_{\boldsymbol{\beta}}+\left[\Lambda_{\boldsymbol{\beta}}, \Lambda_{1}\right]\right)-\boldsymbol{\lambda}_{1}^{(\mathrm{T})}\left(£_{\xi_{2}} \Lambda_{\boldsymbol{\beta}}+\left[\Lambda_{\boldsymbol{\beta}}, \Lambda_{2}\right]\right) \\
& +£_{\bar{\xi}_{1}} \Lambda_{2}-£_{\bar{\xi}_{2}} \Lambda_{1}-\left[\Lambda_{1}, \Lambda_{2}\right]-\boldsymbol{\lambda}_{1}^{(\mathrm{T})} \boldsymbol{\lambda}_{2}^{(\mathrm{T})}\left[\Lambda_{\boldsymbol{\beta}}, \Lambda_{\boldsymbol{\beta}}\right]+x \Lambda_{\boldsymbol{\beta}}
\end{aligned}
$$

Having ensured that the flavour gauge field transformation works, it is clear that the transformation of $\Lambda_{\boldsymbol{\beta}}$ will also follow along similar lines.

We can now fix the free parameter $x$ by demanding consistency of the $U(1)_{\text {T }}$ gauge field transformation. To wit, $\mathrm{A}^{(\mathrm{T})}{ }_{\mu}$ transforms like an abelian gauge field, so it works in a way similar to the flavour field $A_{\mu}$ :

$$
\begin{aligned}
{\left[\delta_{x_{1}}, \delta_{x_{2}}\right] A^{(\mathrm{T})}{ }_{\mu} } & =£_{\bar{\xi}_{3}} A^{(\mathrm{T})}{ }_{\mu}+£_{\bar{\xi}_{1}} \partial_{\mu} \bar{\Lambda}^{(\mathrm{T})}{ }_{2}-£_{\bar{\xi}_{2}} \partial_{\mu} \bar{\Lambda}^{(\mathrm{T})}{ }_{1} \\
& =£_{\bar{\xi}_{3}} A^{(\mathrm{T})}{ }_{\mu}+\partial_{\mu} \bar{\Lambda}^{(\mathrm{T})}{ }_{3}
\end{aligned}
$$

with transformation parameters

$$
\begin{aligned}
& \bar{\Lambda}^{(\mathrm{T})}{ }_{3}=£_{\bar{\xi}_{1}} \bar{\Lambda}^{(\mathrm{T})}{ }_{2}-£_{\bar{\xi}_{2}} \bar{\Lambda}^{(\mathrm{T})}{ }_{1}, \\
& \Lambda^{(\mathrm{T})}{ }_{3}=\boldsymbol{\lambda}_{2}^{(\mathrm{T})} £_{\xi_{1}} \Lambda_{\boldsymbol{\beta}}^{(\mathrm{T})}-\boldsymbol{\lambda}_{1}^{(\mathrm{T})} £_{\xi_{2}} \Lambda_{\boldsymbol{\beta}}^{(\mathrm{T})}+£_{\bar{\xi}_{1}} \Lambda^{(\mathrm{T})}{ }_{2}-£_{\bar{\xi}_{2}} \Lambda^{(\mathrm{T})}{ }_{1}-x\left(\boldsymbol{\beta}^{\nu} \mathrm{A}^{(\mathrm{T})}{ }_{\nu}\right),
\end{aligned}
$$

such that consistently $\bar{\Lambda}^{(\mathrm{T})}{ }_{3}=\Lambda^{(\mathrm{T})}{ }_{3}-\boldsymbol{\lambda}_{3}^{(\mathrm{T})}\left(\boldsymbol{\beta}^{\nu} \mathrm{A}^{(\mathrm{T})}{ }_{\nu}\right)$. From demanding the relation (I.3) to hold, we can fix the parameter $x$. Indeed, using the definitions (I.2) and (I.11), the relation $\lambda_{3}^{(\mathrm{T})}=\bar{\Lambda}_{3}^{(\mathrm{T})}+\bar{\xi}_{3}^{\sigma} \mathrm{A}_{\sigma}^{(\mathrm{T})}$ only holds if

$$
x=\bar{\xi}_{1}^{\mu} \bar{\xi}_{2}^{\nu} \mathrm{F}^{(\mathrm{T})}{ }_{\mu \nu}
$$


Let us now consider the sources $\left\{\tilde{g}_{\mu \nu}, \tilde{A}_{\mu}\right\}$. For the partner metric source $\tilde{g}_{\mu \nu}$ we find:

$$
\begin{aligned}
{\left[\delta x_{1}, \delta x_{2}\right] \tilde{g}_{\mu \nu}=} & £_{\bar{\xi}_{1}}\left(£_{\bar{\xi}_{2}} \tilde{g}_{\mu \nu}+\bar{\Lambda}^{(\mathrm{T})}{ }_{2} \delta_{\mathcal{B}} g_{\mu \nu}\right)+\bar{\Lambda}^{(\mathrm{T})}{ }_{1} \delta x_{2}\left(\delta_{\mathfrak{B}} g_{\mu \nu}\right) \\
& -£_{\bar{\xi}_{2}}\left(£_{\bar{\xi}_{1}} \tilde{g}_{\mu \nu}+\bar{\Lambda}^{(\mathrm{T})}{ }_{1} \delta_{\mathcal{B}} g_{\mu \nu}\right)-\bar{\Lambda}^{(\mathrm{T})}{ }_{2} \delta_{x_{1}}\left(\delta_{\mathfrak{B}} g_{\mu \nu}\right) \\
= & £_{\bar{\xi}_{3}} \tilde{g}_{\mu \nu}+\bar{\Lambda}^{(\mathrm{T})}{ }_{3} \delta_{\mathcal{B}} g_{\mu \nu}
\end{aligned}
$$

where we used

$$
\delta_{x_{1}}\left(\delta_{\mathcal{B}} g_{\mu \nu}\right)=\delta_{\left(\delta_{1} \mathcal{B}\right)} g_{\mu \nu}+\delta_{\mathcal{B}}\left(\delta_{x_{1}} g_{\mu \nu}\right)=£_{\left[\bar{\xi}_{1}, \boldsymbol{\beta}\right]} g_{\mu \nu}+£_{\boldsymbol{\beta}}\left(£_{\bar{\xi}_{1}} g_{\mu \nu}\right)=£_{\bar{\xi}_{1}}\left(\delta_{\mathcal{B}} g_{\mu \nu}\right)
$$

and similarly for $\delta_{x_{2}}\left(\delta_{\mathfrak{B}} g_{\mu \nu}\right)$. Note that the successive application of transformations in (I.13) introduces an inhomogeneous term (the last terms in the first and second line) which must be evaluated on the new configuration of fields and sources.

The commutator of two transformations of $\tilde{A}_{\mu}$ works in a similar fashion:

$$
\begin{aligned}
{\left[\delta x_{1}, \delta x_{2}\right] \tilde{A}_{\mu}=} & £_{\bar{\xi}_{1}}\left(£_{\bar{\xi}_{2}} \tilde{A}_{\mu}+\partial_{\mu} \bar{\Lambda}_{2}+\left[A_{\mu}, \bar{\Lambda}_{2}\right]+\bar{\Lambda}^{(\mathrm{T})}{ }_{2} \delta_{\mathcal{B}} A_{\mu}\right) \\
& +\left[£_{\bar{\xi}_{2}} \tilde{A}_{\mu}+\partial_{\mu} \bar{\Lambda}_{2}+\left[A_{\mu}, \bar{\Lambda}_{2}\right]+\bar{\Lambda}^{(\mathrm{T})}{ }_{2} \delta_{\mathcal{B}} A_{\mu}, \bar{\Lambda}_{1}\right]+\bar{\Lambda}^{(\mathrm{T})}{ }_{1} \delta_{x_{2}}\left(\delta_{\mathcal{B}} A_{\mu}\right) \\
& -£_{\bar{\xi}_{2}}\left(£_{\bar{\xi}_{1}} \tilde{A}_{\mu}+\partial_{\mu} \bar{\Lambda}_{1}+\left[A_{\mu}, \bar{\Lambda}_{1}\right]+\bar{\Lambda}^{(\mathrm{T})}{ }_{1} \delta_{\mathcal{B}} A_{\mu}\right) \\
& -\left[£_{\bar{\xi}_{1}} \tilde{A}_{\mu}+\partial_{\mu} \bar{\Lambda}_{1}+\left[A_{\mu}, \bar{\Lambda}_{1}\right]+\bar{\Lambda}^{(\mathrm{T})}{ }_{1} \delta_{\mathcal{B}} A_{\mu}, \bar{\Lambda}_{2}\right]-\bar{\Lambda}^{(\mathrm{T})}{ }_{2} \delta_{x_{1}}\left(\delta_{\mathcal{B}} A_{\mu}\right) \\
= & £_{\bar{\xi}_{3}} \tilde{A}_{\mu}+D_{\mu} \bar{\Lambda}_{3}+\bar{\Lambda}^{(\mathrm{T})}{ }_{3} \delta_{\mathcal{B}} A_{\mu},
\end{aligned}
$$

using $\delta_{x_{1}}\left(\delta_{\mathfrak{B}} A_{\mu}\right)=£_{\bar{\xi}_{1}}\left(\delta_{\mathfrak{B}} A_{\mu}\right)+\left[\delta_{\mathfrak{B}} A_{\mu}, \bar{\Lambda}_{1}\right]$ and similarly for $\delta_{x_{2}}\left(\delta_{\mathfrak{B}} A_{\mu}\right)$.

To summarize, the above computations show that the Class $\mathrm{L}_{\mathrm{T}}$ transformation rules (15.3), (15.4) and (15.5) form a Lie algebra where the commutator of two transformations $X_{1}=\left\{\bar{\xi}_{1}^{\mu}, \bar{\Lambda}_{1}, \bar{\Lambda}^{(\mathrm{T})}{ }_{1}\right\}$ and $X_{2}=\left\{\bar{\xi}_{2}^{\mu}, \bar{\Lambda}_{2}, \bar{\Lambda}^{(\mathrm{T})}{ }_{2}\right\}$ is another diffeomorphism/gauge/U(1) transformation with parameters

$$
\bar{\xi}_{3}^{\sigma}=\left[\bar{\xi}_{1}, \bar{\xi}_{2}\right]^{\sigma}, \quad \bar{\Lambda}_{3}=£_{\bar{\xi}_{1}} \bar{\Lambda}_{2}-£_{\bar{\xi}_{2}} \bar{\Lambda}_{1}-\left[\bar{\Lambda}_{1}, \bar{\Lambda}_{2}\right], \quad \bar{\Lambda}_{3}^{(\mathrm{T})}{ }_{3}=£_{\bar{\xi}_{1}} \bar{\Lambda}^{(\mathrm{T})}{ }_{2}-£_{\bar{\xi}_{2}} \bar{\Lambda}^{(\mathrm{T})}{ }_{1} .
$$

\section{I.2 Deriving Class $\mathrm{L}_{\mathrm{T}}$ Bianchi identities}

We now outline some of the computations relevant to obtain Bianchi identities eqs. (15.15)(15.19) from the master Lagrangian $\mathcal{L}_{\mathrm{T}}\left[\boldsymbol{\Psi}_{\mathrm{T}}\right]$. The steps are straightforward, but rendered somewhat complex by the sheer number of fields in $\boldsymbol{\Psi}_{\mathrm{T}}$. We start with the computation of a full diffeomorphism, flavour, and $U(1)_{\mathrm{T}}$ transformation on $\mathcal{L}_{\mathrm{T}}$ using the untwisted transformation parameters introduced in section 15.2:

$$
\begin{aligned}
\frac{1}{\sqrt{-g}} \delta_{x}\left(\sqrt{-g} \mathcal{L}_{\mathrm{T}}\right)-\nabla_{\mu}\left(\phi_{x} \Theta_{\mathrm{PS}}^{\top}\right)^{\mu} \\
=\frac{1}{2} T_{\mathrm{L}}^{\mu \nu} \delta_{x} g_{\mu \nu}+J_{\mathrm{L}}^{\mu} \cdot \delta_{x} A_{\mu}+T \mathfrak{h}_{\sigma} \delta_{x} \boldsymbol{\beta}^{\sigma}+T \mathfrak{n} \cdot\left(\delta_{x} \Lambda_{\boldsymbol{\beta}}+A_{\sigma} \delta_{x} \boldsymbol{\beta}^{\sigma}\right) \\
\quad+\frac{1}{2} T_{\mathrm{L}^{c}}^{\mu \nu} \delta_{x} \tilde{g}_{\mu \nu}+J_{\mathrm{L}^{c}}^{\mu} \cdot \delta_{x} \tilde{A}_{\mu}+J_{\mathrm{T}}^{\sigma} \delta_{x} \mathrm{~A}_{\sigma}^{(\mathrm{T})}+T \mathfrak{n}_{\mathrm{T}}\left(\delta_{x} \Lambda_{\boldsymbol{\beta}}^{(\mathrm{T})}+\mathrm{A}_{\sigma}^{(\mathrm{T})} \delta_{x} \boldsymbol{\beta}^{\sigma}\right)
\end{aligned}
$$




$$
\begin{aligned}
& =\bar{\xi}^{\sigma}\left\{-D_{\mu}\left(T_{\mathrm{L}+\mathrm{L}^{c}}\right)_{\sigma}^{\mu}+J_{\mathrm{L}+\mathrm{L}^{c}}^{\nu} \cdot F_{\sigma \nu}+J_{\mathrm{T}}^{\nu} \mathrm{F}_{\sigma \nu}^{(\mathrm{T})}\right. \\
& +\frac{1}{\sqrt{-g}} \delta_{\mathcal{B}}\left(\sqrt{-g} T \mathfrak{h}_{\sigma}\right)+T \mathfrak{n} \cdot \delta_{\mathcal{B}} A_{\sigma}+T \mathfrak{n}_{\mathrm{T}} \cdot \delta_{\mathcal{B}} A_{\sigma}^{(T)}+D_{\nu}\left(g_{\sigma \mu}^{\prime} T_{\mathrm{L}^{c}}^{\mu \nu}\right) \\
& \left.-\frac{1}{2} T_{\mathrm{L}^{c}}^{\mu \nu} D_{\sigma} g_{\mu \nu}^{\prime}-J_{\mathrm{L}^{c}}^{\nu} \cdot F_{\sigma \nu}^{\prime}-\mathrm{A}_{\sigma}^{(\mathrm{T})}\left(\frac{1}{2} T_{\mathrm{L}^{c}}^{\mu \nu} \delta_{\mathfrak{B}} g_{\mu \nu}+J_{\mathrm{L}^{c}}^{\mu} \cdot \delta_{\mathcal{B}} A_{\mu}\right)\right\} \\
& -\left(\bar{\Lambda}+\bar{\xi}^{\nu} A_{\nu}\right) \cdot\left\{D_{\mu} J_{\mathrm{L}+\mathrm{L}^{c}}^{\mu}-\frac{1}{\sqrt{-g}} \delta_{\mathcal{B}}(\sqrt{-g} T \mathfrak{n})\right\} \\
& +\left(\bar{\Lambda}+\bar{\xi}^{\nu} A_{\nu}^{\prime}\right) \cdot\left[D_{\mu} J_{\mathrm{L}^{c}}^{\mu}-\left[\tilde{A}_{\mu}, J_{\mathrm{L}^{c}}^{\mu}\right]\right] \\
& -\left(\bar{\Lambda}^{(\mathrm{T})}+\bar{\xi}^{\sigma} \mathrm{A}_{\sigma}^{(\mathrm{T})}\right)\left\{D_{\mu} J_{\mathrm{T}}^{\mu}-\frac{1}{2} T_{\mathrm{L}^{c}}^{\mu \nu} \delta_{\mathfrak{B}} g_{\mu \nu}-J_{\mathrm{L}^{c}}^{\mu} \cdot \delta_{\mathcal{B}} A_{\mu}-\frac{1}{\sqrt{-g}} \delta_{\mathcal{B}}\left(\sqrt{-g} T \mathfrak{n}_{\mathrm{T}}\right)\right\} \\
& +D_{\mu}\left(\bar{\xi}_{\nu} T_{\mathrm{L}+\mathrm{L}^{c}}^{\mu \nu}+\left(\bar{\Lambda}+\bar{\xi}^{\nu} A_{\nu}\right) \cdot J_{\mathrm{L}+\mathrm{L}^{c}}^{\mu}+\left(\bar{\Lambda}^{(\mathrm{T})}+\bar{\xi}^{\nu} \mathrm{A}_{\nu}^{(\mathrm{T})}\right) J_{\mathrm{T}}^{\mu}\right. \\
& -\boldsymbol{\beta}^{\mu}\left\{T \mathfrak{h}_{\sigma} \bar{\xi}^{\sigma}+T \mathfrak{n} \cdot\left(\bar{\Lambda}+\bar{\xi}^{\nu} A_{\nu}\right)+T \mathfrak{n}_{\mathrm{T}}\left(\bar{\Lambda}^{(\mathrm{T})}+\bar{\xi}^{\nu} \mathrm{A}_{\nu}^{(\mathrm{T})}\right)\right\} \\
& \left.-\bar{\xi}_{\nu} g_{\alpha \rho}^{\prime} g^{\rho \mu} T_{\mathrm{L}^{c}}^{\alpha \nu}-\left(\bar{\Lambda}+\bar{\xi}^{\nu} A_{\nu}^{\prime}\right) \cdot J_{\mathrm{L}^{c}}^{\mu}\right) .
\end{aligned}
$$

In deriving this we have used the transformation rules given in eqs. (15.3), (15.4) and (15.5) and integrated terms by parts where necessary. Furthermore, we used $\delta_{\mathcal{B}} \mathrm{A}^{(\mathrm{T})}{ }_{\mu}=\boldsymbol{\beta}^{\nu} \mathrm{F}^{(\mathrm{T})}{ }_{\nu \mu}$.

From this one can read off the diffeomorphism and flavour Bianchi identities:

- The diffeomorphism identity (15.15) can be read off as the coefficient of $\bar{\xi}^{\sigma}$. This is clear since switching off $\Lambda^{(\mathrm{T})}$ implies that $\bar{\xi}^{\sigma} \rightarrow \xi^{\sigma}$ and indeed the coefficient of the latter is the desired term. The answer then if the set of terms in the curly braces of the first three lines and in addition, the contribution from the fifth line. This is because we have an isolated $\bar{\xi}^{\sigma} \tilde{A}_{\sigma}$ term, which is not the flavour invariant combination and should be accounted for in the diffeomorphism transformations. ${ }^{120}$

- The flavour Bianchi identity (15.16) is given by the coefficient of $\left(\bar{\Lambda}+\bar{\xi}^{\nu} A_{\nu}\right)$. This gets contributions from the fourth and fifth lines respectively.

We can read off another interesting identity from the coefficient of $\left(\bar{\Lambda}^{(\mathrm{T})}+\bar{\xi}^{\sigma} \mathrm{A}^{(\mathrm{T})}{ }_{\sigma}\right)$ in (I.17):

$$
D_{\mu} J_{\top}^{\mu}=\frac{1}{2} T_{\mathrm{L}^{c}}^{\mu \nu} \delta_{\mathcal{B}} g_{\mu \nu}+J_{\mathrm{L}^{c}}^{\mu} \cdot \delta_{\mathcal{B}} A_{\mu}+\frac{1}{\sqrt{-g}} \delta_{\mathcal{B}}\left(\sqrt{-g} T \mathfrak{n}_{\mathrm{T}}\right)
$$

This is just the grand canonical adiabaticity equation for $\left\{T_{\mathrm{L}^{c}}^{\mu \nu}, J_{\mathrm{L}^{c}}^{\mu}\right\}$, where the free energy current is identified with $J_{\top}^{\mu}-T \mathfrak{n}_{\mathrm{T}} \boldsymbol{\beta}^{\mu}$. One might at this point think that we are done with deriving the three Bianchi identities following from the diffeomorphism, flavour, and $U(1)_{T}$ transformations. However, owing to the twisted character of the $U(1)_{\mathrm{T}}$ symmetry, (I.18)

\footnotetext{
${ }^{120}$ This term and contributions involving $\tilde{g}_{\mu \nu}$ or $g_{\mu \nu}^{\prime}$ as we have written owe their origin to the tensorial properties of $\left\{\tilde{g}_{\mu \nu}, \tilde{A}_{\mu}\right\}$ which matters when we perform diffeomorphisms.
} 
is not the actual $U(1)_{\mathrm{T}}$ Bianchi identity, but rather a combination of it with the other Bianchi identities. To extract the actual $U(1)_{\mathrm{T}}$ Bianchi identity, we need to shift back to the original transformation parameters $\left\{\xi^{\mu}, \Lambda, \Lambda^{(\mathrm{T})}\right\}$. We now turn to this exercise.

As mentioned in the discussion above, upon shifting to the original transformation parameters $\left\{\xi^{\mu}, \Lambda, \Lambda^{(\mathrm{T})}\right\}$ the diffeomorphism and flavour Bianchi identities do not change. However, to get the actual $U(1)_{\mathrm{T}}$ Bianchi identity, we have to do this shift and then read off the coefficient of $\left(\Lambda^{(\mathrm{T})}+\xi^{\sigma} \mathrm{A}^{(\mathrm{T})}{ }_{\sigma}\right)$. To do this, it is convenient to define the $U(1)_{\mathrm{T}}$ Noether current as in (15.18).

We can then use this expression to simplify (I.17). Let us temporarily group together the terms in the r.h.s. of this expression into the total derivative and non-derivative terms

$$
\frac{1}{\sqrt{-g}} \delta_{x}\left(\sqrt{-g} \mathcal{L}_{\mathrm{T}}\right)-\nabla_{\mu}\left(\phi_{x} \Theta_{\mathrm{PS}}^{\mathrm{T}}\right)^{\mu} \equiv \mathfrak{O}\left[\bar{\xi}^{\mu}, \bar{\Lambda}, \Lambda_{\boldsymbol{\beta}}^{(\mathrm{T})}\right]+D_{\mu}\left(\mathfrak{T}^{\mu}\left[\bar{\xi}^{\mu}, \bar{\Lambda}, \Lambda_{\boldsymbol{\beta}}^{(\mathrm{T})}\right]\right)
$$

We can now use (15.18) to simplify the two contributions separately. Firstly we have A straightforward substitution leads to a simplification of $\mathfrak{T}^{\mu}\left[\bar{\xi}^{\mu}, \bar{\Lambda}, \Lambda_{\boldsymbol{\beta}}^{(\mathrm{T})}\right]$ to

$$
\begin{aligned}
\mathfrak{T}^{\mu}\left[\xi^{\mu}, \Lambda, \Lambda_{\boldsymbol{\beta}}\right]= & \xi_{\nu} T_{\mathrm{L}+\mathrm{L}^{c}}^{\mu \nu}+\left(\Lambda+\xi^{\nu} A_{\nu}\right) \cdot J_{\mathrm{L}+\mathrm{L}^{c}}^{\mu}+\left(\Lambda^{(\mathrm{T})}+\xi^{\nu} \mathrm{A}^{(\mathrm{T})}{ }_{\nu}\right) \mathrm{N}_{\mathrm{T}}^{\mu} \\
& -\boldsymbol{\beta}^{\mu}\left\{T \mathfrak{h}_{\sigma} \xi^{\sigma}+T \mathfrak{n} \cdot\left(\Lambda+\xi^{\nu} A_{\nu}\right)\right\}-\xi_{\nu} g_{\alpha \rho}^{\prime} g^{\rho \mu} T_{\mathrm{L}^{c}}^{\alpha \nu}-\left(\Lambda+\xi^{\nu} A_{\nu}^{\prime}\right) \cdot J_{\mathrm{L}^{c}}^{\mu}
\end{aligned}
$$

Further, we can simplify the non-total derivative pieces combined into $\mathfrak{O}$ by using the easily verified identity

$$
\begin{aligned}
\mathfrak{O}\left[\boldsymbol{\beta}, \Lambda, \Lambda_{\boldsymbol{\beta}}^{(\mathrm{T})}\right]= & -D_{\mu}\left(\mathrm{N}_{\mathrm{T}}^{\mu}+\left(\Lambda_{\boldsymbol{\beta}}^{(\mathrm{T})}+\boldsymbol{\beta}^{\sigma} \mathrm{A}_{\sigma}^{(\mathrm{T})}-1\right)\left(J_{\mathrm{T}}^{\mu}-T \mathfrak{n}_{\mathrm{T}} \boldsymbol{\beta}^{\mu}\right)\right) \\
& +\frac{1}{2} T_{\mathrm{L}+\mathrm{L}^{c}}^{\mu \nu} \delta_{\mathcal{B}} g_{\mu \nu}+J_{\mathrm{L}+\mathrm{L}^{c}}^{\mu} \cdot \delta_{\mathcal{B}} A_{\mu}+\frac{1}{2} T_{\mathrm{L}^{c}}^{\mu \nu} \delta_{\mathcal{B}} \tilde{g}_{\mu \nu}+J_{\mathrm{L}^{c}}^{\mu} \cdot \delta_{\mathcal{B}} \tilde{A}_{\mu} \\
& +J_{\mathrm{T}}^{\mu} \delta_{\mathcal{B}} \mathrm{A}^{(\mathrm{T})}{ }_{\mu}+\left(\Lambda_{\boldsymbol{\beta}}^{(\mathrm{T})}+\boldsymbol{\beta}^{\sigma} \mathrm{A}_{\sigma}^{(\mathrm{T})}\right)\left(D_{\mu} J_{\mathrm{T}}^{\mu}-\frac{1}{2} T_{\mathrm{L}^{c}}^{\mu \nu} \delta_{\mathcal{B}} g_{\mu \nu}-J_{\mathrm{L}^{c}}^{\mu} \cdot \delta_{\mathcal{B}} A_{\mu}\right. \\
& \left.-\frac{1}{\sqrt{-g}} \delta_{\mathcal{B}}\left(\sqrt{-g} T \mathfrak{n}_{\mathrm{T}}\right)\right)
\end{aligned}
$$

upon defining the Noether current

$$
\begin{aligned}
\mathrm{N}_{\mathrm{T}}^{\mu} \equiv-\frac{\mathcal{G}_{\mathrm{T}}^{\mu}}{T} \equiv & J_{\mathrm{T}}^{\mu}+\boldsymbol{\beta}_{\nu} T_{\mathrm{L}+\mathrm{L}^{c}}^{\mu \nu}+\left(\Lambda_{\boldsymbol{\beta}}+\boldsymbol{\beta}^{\nu} A_{\nu}\right) \cdot J_{\mathrm{L}+\mathrm{L}^{c}}^{\mu}-\left\{\mathfrak{h}_{\sigma} \boldsymbol{\beta}^{\sigma}+\mathfrak{n} \cdot\left(\Lambda_{\boldsymbol{\beta}}+\boldsymbol{\beta}^{\nu} A_{\nu}\right)+\mathfrak{n}_{\mathrm{T}}\right\} u^{\mu} \\
& -\boldsymbol{\beta}_{\nu} g_{\alpha \beta}^{\prime} g^{\mu \alpha} T_{\mathrm{L}^{c}}^{\beta \nu}-\left(\Lambda_{\boldsymbol{\beta}}+\boldsymbol{\beta}^{\nu} A_{\nu}^{\prime}\right) \cdot J_{\mathrm{L}^{c}}^{\mu}
\end{aligned}
$$

Plugging this into the full variation (15.17) we find

$$
\begin{aligned}
\frac{1}{\sqrt{-g}} \delta_{x}\left(\sqrt{-g} \mathcal{L}_{\mathrm{T}}\right)-\nabla_{\mu}\left(\phi_{x} \Theta_{\mathrm{PS}}^{\mathrm{T}}\right)^{\mu} & \\
=\xi^{\sigma}\{- & D_{\mu}\left(T_{\mathrm{L}+\mathrm{L}^{c}}\right)_{\sigma}^{\mu}+J_{\mathrm{L}+\mathrm{L}^{c}}^{\nu} \cdot F_{\sigma \nu}+J_{\mathrm{T}}^{\nu} \mathrm{F}_{\sigma \nu}^{(\mathrm{T})} \\
& \quad+\frac{1}{\sqrt{-g}} \delta_{\mathcal{B}}\left(\sqrt{-g} T \mathfrak{h}_{\sigma}\right)+T \mathfrak{n} \cdot \delta_{\mathcal{B}} A_{\sigma}+T \mathfrak{n}_{\mathrm{T}} \cdot \delta_{\mathcal{B}} A_{\sigma}^{(\mathrm{T})}+D_{\nu}\left(g_{\sigma \mu}^{\prime} T_{\mathrm{L}^{c}}^{\mu \nu}\right)
\end{aligned}
$$




$$
\begin{aligned}
& \left.-\frac{1}{2} T_{\mathrm{L}^{c}}^{\mu \nu} D_{\sigma} g_{\mu \nu}^{\prime}-J_{\mathrm{L}^{c}}^{\nu} \cdot F_{\sigma \nu}^{\prime}-\mathrm{A}_{\sigma}^{(\mathrm{T})}\left(\frac{1}{2} T_{\mathrm{L}^{c}}^{\mu \nu} \delta_{\mathcal{B}} g_{\mu \nu}+J_{\mathrm{L}^{c}}^{\mu} \cdot \delta_{\mathcal{B}} A_{\mu}\right)\right\} \\
& -\left(\Lambda+\xi^{\nu} A_{\nu}\right) \cdot\left\{D_{\mu} J_{\mathrm{L}+\mathrm{L}^{c}}^{\mu}-\frac{1}{\sqrt{-g}} \delta_{\mathfrak{B}}(\sqrt{-g} T \mathfrak{n})\right\} \\
& +\left(\Lambda+\xi^{\nu} A_{\nu}^{\prime}\right) \cdot\left[D_{\mu} J_{\mathrm{L}^{c}}^{\mu}-\left[\tilde{A}_{\mu}, J_{\mathrm{L}^{c}}^{\mu}\right]\right] \\
& +\left(\Lambda^{(\mathrm{T})}+\xi^{\sigma} \mathrm{A}_{\sigma}^{(\mathrm{T})}\right)\left\{-D_{\mu}\left(\mathrm{N}_{\mathrm{T}}^{\mu}+\left(\Lambda_{\boldsymbol{\beta}}^{(\mathrm{T})}+\boldsymbol{\beta}^{\sigma} \mathrm{A}_{\sigma}^{(\mathrm{T})}-1\right)\left(J_{\mathrm{T}}^{\mu}-T \mathfrak{n}_{\mathrm{T}} \boldsymbol{\beta}^{\mu}\right)\right)\right. \\
& +\frac{1}{2} T_{\mathrm{L}+\mathrm{L}^{c}}^{\mu \nu} \delta_{\mathfrak{B}} g_{\mu \nu}+J_{\mathrm{L}+\mathrm{L}^{c}}^{\mu} \cdot \delta_{\mathfrak{B}} A_{\mu}+\frac{1}{2} T_{\mathrm{L}^{c}}^{\mu \nu} \delta_{\mathfrak{B}} \tilde{g}_{\mu \nu}+J_{\mathrm{L}^{c}}^{\mu} \cdot \delta_{\mathfrak{B}} \tilde{A}_{\mu}+J_{\top}^{\mu} \delta_{\mathfrak{B}} \mathrm{A}^{(\mathrm{T})} \mu \\
& \left.+\left(\Lambda_{\boldsymbol{\beta}}^{(\mathrm{T})}+\boldsymbol{\beta}^{\sigma} \mathrm{A}_{\sigma}^{(\mathrm{T})}\right)\left(D_{\mu} J_{\top}^{\mu}-\frac{1}{2} T_{\mathrm{L}^{c}}^{\mu \nu} \delta_{\mathfrak{B}} g_{\mu \nu}-J_{\mathrm{L}^{c}}^{\mu} \cdot \delta_{\mathcal{B}} A_{\mu}-\frac{1}{\sqrt{-g}} \delta_{\mathcal{B}}\left(\sqrt{-g} T \mathfrak{n}_{\mathrm{T}}\right)\right)\right\} \\
& +D_{\mu}\left(\mathfrak{T}^{\mu}\left[\xi, \Lambda, \Lambda^{(\mathrm{T})}\right]-\boldsymbol{\beta}^{\mu} T \mathfrak{n}_{\mathrm{T}}\left(\Lambda^{(\mathrm{T})}+\xi^{\nu} \mathrm{A}^{(\mathrm{T})}{ }_{\nu}\right)\right)
\end{aligned}
$$

\begin{tabular}{|c|c|c|c|}
\hline Symbol & Definition & Symbol & Definition \\
\hline \multicolumn{4}{|c|}{ Basic hydrodynamical variables $\boldsymbol{\Psi}$ on physical spacetime $\mathcal{M}$} \\
\hline$g_{\mu \nu}$ & Background metric & $A_{\mu}$ & Background gauge field \\
\hline $\boldsymbol{\beta}^{\mu}$ & Thermal vector $=\frac{1}{T} u^{\mu}$ & $\Lambda_{\beta}$ & Thermal twist $=\frac{\mu}{T}-\boldsymbol{\beta}^{\nu} A_{\nu}$ \\
\hline \multicolumn{4}{|c|}{ Hydrostatic variables $\mathcal{K}$} \\
\hline$K^{\mu}$ & Hydrostatic thermal vector & $\Lambda_{K}$ & Hydrostatic thermal twist \\
\hline \multicolumn{4}{|c|}{ Hydrodynamic tensors at first order in gradients } \\
\hline$\sigma^{\mu \nu}$ & Shear tensor $=P^{\mu \alpha} P^{\nu \beta}\left(\nabla_{(\alpha} u_{\beta)}-\frac{\Theta}{d-1} P_{\alpha \beta}\right)$ & $\Theta$ & Expansion $=\nabla_{\mu} u^{\mu}$ \\
\hline$\omega^{\mu \nu}$ & Vorticity tensor $=P^{\mu \alpha} P^{\nu \beta} \nabla_{[\alpha} u_{\beta]}$ & $\mathfrak{a}^{\mu}$ & Acceleration $=u^{\nu} \nabla_{\nu} u^{\mu}$ \\
\hline $\mathfrak{v}^{\mu}$ & Potential gradient $=E^{\mu}-T P^{\mu \nu} \nabla_{\nu}\left(\frac{\mu}{T}\right)$ & $E^{\mu}$ & Electric field $=F^{\mu \nu} u_{\nu}$ \\
\hline$B^{\mu \nu}$ & Magnetic field $=P^{\mu \alpha} P^{\nu \beta} F_{\alpha \beta}$ & & \\
\hline \multicolumn{4}{|c|}{ Currents $\mathcal{C}_{\mathcal{H}}[\mathbf{\Psi}]$} \\
\hline$T^{\mu \nu}$ & Energy-momentum tensor & $J^{\mu}$ & Charge current \\
\hline$J_{S}^{\mu}$ & Fluid entropy current & $\mathcal{G}^{\mu}$ & Gibbs free energy current (2.18) \\
\hline \multicolumn{4}{|c|}{ Further physical currents } \\
\hline $\mathfrak{h}_{\sigma}$ & Adiabatic heat current $(6.3)$ & $\mathfrak{n}$ & Adiabatic charge density (6.3) \\
\hline$\left(\phi \Theta_{\mathrm{PS}}\right)^{\mu}$ & Presymplectic potential $(6.3)$ & $\mathcal{K}^{\mu \nu}$ & Komar charge $(6.21)$ \\
\hline $\mathrm{N}^{\mu}$ & Noether current (6.9) & & \\
\hline
\end{tabular}

The $U(1)_{\mathrm{T}}$ Bianchi identity can now be read off as the coefficient of $\left(\Lambda^{(\mathrm{T})}+\xi^{\sigma} \mathrm{A}^{(\mathrm{T})}{ }_{\sigma}\right)$ in the three lines preceding the total derivative term. We have chosen to leave the latter in terms of the intermediate quantities defined earlier, to avoid unnecessary repetition. After setting $\left(\Lambda^{(\mathrm{T})}+\xi^{\sigma} A^{(\mathrm{T})}{ }_{\sigma}\right)=1$, the $U(1)_{\mathrm{T}}$ Bianchi identity takes the form (15.17).

\section{J Notation and conventions}

Table 11. Basic fields, sources and hydrodynamic quantities on physical spacetime manifold $\mathcal{M}$. 


\begin{tabular}{|c|c|c|c|}
\hline Symbol & Definition & Symbol & Definition \\
\hline \multicolumn{4}{|c|}{ Basic hydrodynamical variables $\Psi$ on reference spacetime $\mathbb{M}$} \\
\hline $9 a b$ & Background metric (7.12) & $\mathbb{A}_{a}$ & Background gauge field (7.12) \\
\hline$\beta^{a}$ & Thermal vector $=\frac{1}{\mathbb{T}} \mathrm{u}^{a}$ & $\Lambda_{\beta}$ & Thermal twist $=\frac{\mu}{\mathbb{T}}-\beta^{a} \mathbb{A}_{a}$ \\
\hline \multicolumn{4}{|c|}{ Derived hydrodynamical variables on reference spacetime $\mathbb{M}$} \\
\hline $\mathbb{T}$ & Temperature & $\mu$ & Chemical potential \\
\hline $\mathrm{u}^{a}$ & Velocity & & \\
\hline \multicolumn{4}{|c|}{ Transition functions from $\mathcal{M}$ to $\mathbb{M}$} \\
\hline$\varphi^{a}$ & Diffeomorphism field (7.6) & $c$ & Gauge transformation (7.6) \\
\hline \multicolumn{4}{|c|}{ Currents (7.19) } \\
\hline $\mathbb{T}^{a b}$ & Energy-momentum tensor & $\mathbb{J}^{a}$ & Charge current \\
\hline $\mathbb{J}_{S}^{a}$ & Fluid entropy current & $\mathbb{h}_{a}$ & Adiabatic heat current \\
\hline $\mathrm{m}$ & Adiabatic charge density & & \\
\hline
\end{tabular}

Table 12. Basic sources, fields and currents on reference manifold $\mathbb{M}$.

\begin{tabular}{|c|c|c|c|}
\hline Symbol & Definition & Symbol & Definition \\
\hline \multicolumn{4}{|c|}{ Variational symbols and derivatives } \\
\hline$\delta$ & Unconstrained variation & $\delta_{x}$ & Generic diffeo/gauge transf. (6.7) \\
\hline$\delta_{\mathcal{B}}$ & Diffeo/gauge trf. w.r.t. $\mathcal{B}=\left\{\boldsymbol{\beta}^{\mu}, \Lambda_{\boldsymbol{\beta}}\right\}$ (2.22) & $\delta_{\mathbb{B}}$ & Diffeo/gauge trf. on $\mathbb{M}$ w.r.t. $\mathbb{B}=\left\{\mathbb{\beta}^{a}, \Lambda_{\beta}\right\}$ \\
\hline$\delta_{\varphi}$ & Lie drag on $\mathbb{M}$ along $\left\{\delta \varphi^{a},-c^{-1} \delta c\right\}$ & $\delta$ & Constrained variation along Lie orbits (7.2) \\
\hline$D_{\alpha}$ & Gauge covariant derivative (2.8) & $£_{\xi}$ & Lie derivative along $\xi^{\mu}$ \\
\hline $\mathcal{D}_{\lambda}^{\mathcal{W}}$ & Weyl covariant derivative (D.6) & $\delta_{x}^{\mathcal{W}}$ & $\delta_{x}+$ Weyl transformation (D.1) \\
\hline \multicolumn{4}{|c|}{ Indices } \\
\hline $\begin{array}{r}\alpha, \beta, \mu, \nu, \ldots \\
m, n, p, \ldots\end{array}$ & $\begin{array}{l}\text { Physical manifold } \mathcal{M} \\
\text { Physical bulk manifold } \mathcal{M}_{d+1}\end{array}$ & $\begin{array}{r}a, b, c, \ldots \\
{ }_{M, N}, P, \ldots \\
\end{array}$ & $\begin{array}{l}\text { Reference manifold } \mathbb{M} \\
\text { Reference bulk manifold } \mathrm{M}_{d+1}\end{array}$ \\
\hline
\end{tabular}

Table 13. Variational symbols, derivatives, index conventions. 


\begin{tabular}{|c|c|c|c|}
\hline Symbol & Definition & Symbol & Definition \\
\hline \multicolumn{4}{|c|}{ Shadow connections } \\
\hline $\begin{array}{c}\hat{\Gamma}_{\nu \rho}^{\mu} \\
\Omega_{\nu}^{\mu}{ }_{\nu}\end{array}$ & $\begin{array}{l}\text { Shadow spin connection }=\Gamma_{\nu \rho}^{\mu}+\Omega^{\mu}{ }_{\nu} u_{\rho} \\
\text { Spin potential }=\frac{1}{2} T\left(D_{\nu} \boldsymbol{\beta}^{\mu}-D^{\mu} \boldsymbol{\beta}_{\nu}\right)\end{array}$ & $\hat{A}^{\mu}$ & Shadow gauge field $=A^{\mu}+\mu u^{\mu}$ \\
\hline \multicolumn{4}{|c|}{ Anomaly induced currents } \\
\hline$\Sigma_{H}^{a b}{ }_{c}$ & Bulk Hall spin current (12.23) & $\mathrm{J}_{H}^{a}$ & Bulk Hall charge current (12.23) \\
\hline $\mathrm{T}_{H}^{\mu \perp}$ & Covariant Lorentz anomaly $=\frac{1}{2} D_{\nu} \Sigma_{H}^{\perp[\mu \nu]}$ & $\mathrm{J}_{H}^{\perp}$ & Covariant flavour anomaly \\
\hline$J_{\mathcal{P}}^{\mu}$ & Anomalous flavour current (12.25) & $\Sigma_{\mathcal{P}}$ & Anomalous spin current (12.25) \\
\hline$q_{\mathcal{P}}^{\mu}$ & Mixed anomalous current (12.25) & $\left(J_{S}^{\alpha}\right)_{\mathrm{A}}$ & Anomalous entropy current (12.30) \\
\hline$\left(T^{\alpha \beta}\right)_{\mathrm{A}}$ & Anomalous boundary stress tensor (12.28) & $\left(J^{\alpha}\right)_{\mathrm{A}}$ & Anomalous boundary current (12.28) \\
\hline$T_{(d+1)}^{m n}$ & Anomalous bulk stress tensor (H.6) & $J_{(d+1)}^{m}$ & Anomalous bulk current (H.6) \\
\hline $\mathfrak{h}_{m}^{(d+1)}$ & Anomalous bulk heat current (H.6) & $\mathfrak{n}_{(d+1)}$ & Anomalous bulk charge density (H.6) \\
\hline \multicolumn{4}{|c|}{ Basic fields as differential forms } \\
\hline$u$ & Velocity 1-form $=u_{\mu} d x^{\mu}$ & $\iota_{\boldsymbol{u}} \boldsymbol{X}^{(2)}$ & Velocity contraction $=u^{\mu} X_{\mu \nu}^{(2)} d x^{\nu}$ \\
\hline$a$ & Acceleration 1-form $=\mathfrak{a}_{\mu} d x^{\mu}$ & $2 \omega$ & Vorticity 2-form $=d \boldsymbol{u}+\boldsymbol{u} \wedge \boldsymbol{a}$ \\
\hline$A$ & Gauge field 1-form $=A_{\mu} d x^{\mu}$ & $\Gamma_{\nu}^{\mu}$ & Connection 1-form $=\Gamma_{\nu \rho}^{\mu} d x^{\rho}$ \\
\hline$\hat{A}$ & Shadow gauge field $=\boldsymbol{A}+\mu \boldsymbol{u}$ & $\hat{\Gamma}_{\nu}^{\mu}$ & Shadow connection $=\boldsymbol{\Gamma}_{\nu}^{\mu}+\Omega^{\mu}{ }_{\nu} \boldsymbol{u}$ \\
\hline $\boldsymbol{F}$ & Field strength $=d \boldsymbol{A}+\boldsymbol{A} \wedge \boldsymbol{A}$ & $\boldsymbol{R}_{\nu}^{\mu}$ & Curvature $=d \boldsymbol{\Gamma}_{\nu}^{\mu}+\boldsymbol{\Gamma}_{\rho}^{\mu} \wedge \boldsymbol{\Gamma}_{\nu}^{\rho}$ \\
\hline$E$ & Electric field $=-\iota_{\boldsymbol{u}} \boldsymbol{F}$ & $\left(\boldsymbol{E}_{R}\right)_{\nu}^{\mu}$ & Electric curvature $=-\iota_{\boldsymbol{u}} \boldsymbol{R}_{\nu}^{\mu}$ \\
\hline$B$ & Magnetic field $=\boldsymbol{F}-\boldsymbol{u} \wedge \boldsymbol{E}$ & $\left(\boldsymbol{B}_{R}\right)_{\nu}^{\mu}$ & Magnetic curvature $=\boldsymbol{R}^{\mu}{ }_{\nu}-\boldsymbol{u} \wedge\left(\boldsymbol{E}_{R}\right)^{\mu}{ }_{\nu}$ \\
\hline$A^{(T)}$ & $U(1)_{\mathrm{T}}$ gauge field 1-form & $\mathrm{F}^{(\mathrm{T})}$ & $U(1)_{\mathrm{T}}$ field strength 2-form $=d \mathrm{~A}^{(\mathrm{T})}$ \\
\hline \multicolumn{4}{|c|}{ Abbreviations } \\
\hline $\mathfrak{Q}_{\nu \beta}^{\mu \alpha}$ & Antisymmetrizer $=\frac{1}{2}\left(\delta_{\beta}^{\mu} \delta_{\nu}^{\alpha}-g^{\mu \alpha} g_{\nu \beta}\right)$ & $\mathfrak{P}_{\sigma \kappa \lambda}^{\rho \mu \nu}$ & Projector $=\delta_{\kappa}^{\rho} \delta_{\sigma}^{\mu} \delta_{\lambda}^{\nu}+\mathfrak{Q}_{\sigma \kappa}^{\rho \mu} u^{\nu} u_{\lambda}$ \\
\hline$f_{\mathcal{M}}$ & Bulk integral $=\int_{\mathcal{M}_{d+1}} \sqrt{-g_{d+1}}$ & $\mathrm{R}, \mathrm{L}$ & Shortcuts for right and left SK copies \\
\hline
\end{tabular}

Table 14. Class A and Schwinger-Keldysh (SK) quantities on physical spacetime $\mathcal{M}$.

\begin{tabular}{|c|c|c|c|}
\hline Symbol & Definition & Symbol & Definition \\
\hline \multicolumn{4}{|c|}{ Shadow connections } \\
\hline $\begin{array}{r}\hat{\mathbb{T}}^{a}{ }_{b c} \\
\mathbb{\Omega}^{a}{ }_{b}\end{array}$ & $\begin{array}{l}\text { Shadow spin connection }=\mathbb{V}^{a}{ }_{b c}+\Omega^{a} b_{b} \mathbb{u}_{c} \\
\text { Spin potential }=\frac{1}{2} \mathbb{T}\left(\mathbb{D}_{b} \mathbb{\beta}^{a}-\mathbb{D}^{a} \beta_{b}\right)\end{array}$ & $\hat{\mathbb{A}}^{a}$ & Shadow gauge field $=\mathbb{A}^{a}+\mu \pi^{a}$ \\
\hline \multicolumn{4}{|c|}{ Anomaly induced currents } \\
\hline $\mathbb{\Sigma}_{H}^{M N}{ }_{P}$ & Bulk Hall spin current & $\mathbb{J}_{H}^{M}$ & Bulk Hall charge current \\
\hline $\mathbb{J}_{\mathcal{P}}^{a}$ & Anomalous flavour current (13.17) & $\mathbb{\Sigma}_{\mathcal{P}}$ & Anomalous spin current (13.17) \\
\hline $\mathbb{q}_{\mathcal{P}}^{a}$ & Mixed anomalous current current (13.17) & $\left(\mathbb{J}_{S}^{a}\right)_{\mathrm{A}}$ & Anomalous entropy current \\
\hline$\left(\mathbb{T}^{a b}\right)_{\mathrm{A}}$ & Anomalous boundary stress tensor & $\left(\mathbb{J}^{a}\right)_{\mathrm{A}}$ & Anomalous boundary current \\
\hline $\mathbb{T}_{(d+1)}^{M N}$ & Anomalous bulk stress tensor & $\mathbb{J}_{(d+1)}^{M}$ & Anomalous bulk current \\
\hline $\mathbb{T}_{\text {hydro }}^{a b}$ & Hydrodynamic SK stress tensor (13.12) & $\mathbb{J}_{\text {hydro }}^{a}$ & Hydrodynamic SK charge current (13.12) \\
\hline$\left(\mathbb{T}_{(d+1)}^{M N}\right)_{I F}$ & Cross contour stress tensor (H.21) & $\left(\mathbb{J}_{(d+1)}^{M}\right)_{I F}$ & Cross contour charge current (H.21) \\
\hline \multicolumn{4}{|c|}{ Abbreviations } \\
\hline $\mathfrak{s}$ & Ratio of measures $=\sqrt{-\mathscr{g}_{\mathrm{L}}} / \sqrt{-\mathfrak{g}_{\mathrm{R}}}$ & $\mathfrak{s}_{\mathrm{R}, \mathrm{L}}$ & Ratio of measures $=\sqrt{-\mathscr{g}_{\mathrm{R}, \mathrm{L}}} / \sqrt{-\breve{g}}$ \\
\hline $\bar{\psi}$ & Difference fields $=\Downarrow_{R}-\Downarrow_{L}$ & $\breve{\Downarrow}$ & Common fields $=\frac{1}{2}\left(\Psi_{R}+\Psi_{L}\right)$ \\
\hline$f_{\mathbb{M}_{d+1}}$ & Bulk integral $=\int_{\mathbb{M}_{d+1}} \sqrt{-\mathbb{g}_{d+1}}$ & & \\
\hline
\end{tabular}

Table 15. Class A and Schwinger-Keldysh (SK) quantities on reference manifold M. 


\begin{tabular}{|c|c|c|c|}
\hline Symbol & Definition & Symbol & Definition \\
\hline \multicolumn{4}{|c|}{ Basic fields } \\
\hline$\tilde{g}_{\mu \nu}$ & Partner metric & $\tilde{A}_{\mu}$ & Partner gauge field \\
\hline$g_{\mu \nu}^{\prime}$ & Shifted partner metric $=g_{\mu \nu}-\tilde{g}_{\mu \nu}$ & $A_{\mu}^{\prime}$ & Shifted partner gauge field $=A_{\mu}-\tilde{A}_{\mu}$ \\
\hline$g_{\mu \nu}^{\mathrm{L}}$ & SK difference $=g_{\mu \nu}^{\prime}-\boldsymbol{\beta}_{\mu} \mathrm{A}^{(\mathrm{T})}{ }_{\nu}-\boldsymbol{\beta}_{\nu} \mathrm{A}^{(\mathrm{T})}{ }_{\mu}$ & $A_{\mu}^{\mathrm{L}}$ & SK difference $=A_{\mu}^{\prime}-\left(\Lambda_{\boldsymbol{\beta}}+\boldsymbol{\beta}^{\alpha} A_{\alpha}\right) \mathrm{A}^{(\mathrm{T})}{ }_{\mu}$ \\
\hline$A^{(T)}{ }_{\mu}$ & $U(1)_{\mathrm{T}} \mathrm{KMS}$ gauge field & $\Lambda_{\beta}^{(\mathrm{T})}$ & $U(1)_{\mathrm{T}}$ holonomy field \\
\hline$\Psi_{\mathrm{T}}$ & $=\left\{\boldsymbol{\beta}^{\mu}, \Lambda_{\boldsymbol{\beta}}, g_{\mu \nu}, A_{\mu}, \tilde{g}_{\mu \nu}, \tilde{A}_{\mu}, \mathrm{A}^{(\mathrm{T})}{ }_{\mu}, \Lambda_{\boldsymbol{\beta}}^{(\mathrm{T})}\right\}$ & & \\
\hline \multicolumn{4}{|c|}{ Currents } \\
\hline$T_{\mathrm{L}}^{\mu \nu}$ & Stress tensor associated to $g_{\mu \nu}$ & $J_{\mathrm{L}}^{\mu}$ & Charge current associated to $A_{\mu}$ \\
\hline$T_{\mathrm{L}^{c}}^{\mu \nu}$ & Stress tensor associated to $\tilde{g}_{\mu \nu}$ & $J_{\mathrm{L}^{c}}^{\mu}$ & Charge current associated to $\tilde{A}_{\mu}$ \\
\hline$J_{T}^{\mu}$ & $U(1)_{\mathrm{T}}$ KMS current & $\mathfrak{n}_{\mathrm{T}}$ & Adiabatic $U(1)_{\mathrm{T}}$ charge density \\
\hline $\mathrm{N}_{\mathrm{T}}^{\sigma}$ & Class $\mathrm{L}_{\mathrm{T}}$ Noether current (15.18) & $\mathcal{G}_{\top}^{\sigma}$ & Class $\mathrm{L}_{\mathrm{T}}$ free energy current $=-T \mathrm{~N}_{\mathrm{T}}^{\sigma}$ \\
\hline$\left(J_{S}\right)_{\mathrm{T}}^{\mu}$ & Class $\mathrm{L}_{\mathrm{T}}$ entropy current (16.5) & & \\
\hline
\end{tabular}

Table 16. Class $\mathrm{L}_{\mathrm{T}}$ quantities.

\begin{tabular}{|c|l|c|l|}
\hline Symbol & Definition & Symbol & Definition \\
\hline \multicolumn{3}{|c|}{ Hydrodynamical fields for Legendre transform to entropic description } \\
\hline $\mathbf{S}_{\alpha_{1} \cdots \alpha_{d-1}}$ & $(d-1)$-form entropy current dual to $T s \boldsymbol{\beta}^{\mu}$ & ${ }^{e} \mathbb{S}^{a}$ & Dual of $\mathbf{S}_{\alpha_{1} \cdots \alpha_{d-1}}$ on M (B.10) \\
$\left(\Lambda_{\mathbf{S}}\right)_{\alpha_{1} \ldots \alpha_{d}}$ & $d$-form gauge parameter dual to $T s \Lambda_{\boldsymbol{\beta}}$ & ${ }^{e} \Lambda_{\mathbb{S}}$ & Dual of $\left(\Lambda_{\mathbf{S}}\right)_{\alpha_{1} \ldots \alpha_{d}}$ on M (B.10) \\
\hline
\end{tabular}

Table 17. Class ND fields.

Open Access. This article is distributed under the terms of the Creative Commons Attribution License (CC-BY 4.0), which permits any use, distribution and reproduction in any medium, provided the original author(s) and source are credited.

\section{References}

[1] L.D. Landau and E.M. Lifshitz, Fluid mechanics, Course of theoretical physics volume 6, Butterworth-Heinemann (1987).

[2] S. Bhattacharyya, Constraints on the second order transport coefficients of an uncharged fluid, JHEP 07 (2012) 104 [arXiv:1201.4654] [INSPIRE].

[3] N. Banerjee et al., Constraints on fluid dynamics from equilibrium partition functions, JHEP 09 (2012) 046 [arXiv:1203.3544] [INSPIRE].

[4] K. Jensen et al., Towards hydrodynamics without an entropy current, Phys. Rev. Lett. 109 (2012) 101601 [arXiv:1203.3556] [INSPIRE].

[5] J. Erdmenger, M. Haack, M. Kaminski and A. Yarom, Fluid dynamics of R-charged black holes, JHEP 01 (2009) 055 [arXiv: 0809.2488] [INSPIRE].

[6] N. Banerjee et al., Hydrodynamics from charged black branes, JHEP 01 (2011) 094 [arXiv:0809.2596] [INSPIRE].

[7] D.T. Son and P. Surowka, Hydrodynamics with triangle anomalies, Phys. Rev. Lett. 103 (2009) 191601 [arXiv:0906 .5044] [INSPIRE]. 
[8] R. Loganayagam, Anomaly induced transport in arbitrary dimensions, arXiv:1106.0277 [INSPIRE].

[9] S. Jain and T. Sharma, Anomalous charged fluids in $1+1 D$ from equilibrium partition function, JHEP 01 (2013) 039 [arXiv:1203.5308] [INSPIRE].

[10] N. Banerjee, S. Dutta, S. Jain, R. Loganayagam and T. Sharma, Constraints on anomalous fluid in arbitrary dimensions, JHEP 03 (2013) 048 [arXiv: 1206.6499] [INSPIRE].

[11] K. Jensen, R. Loganayagam and A. Yarom, Thermodynamics, gravitational anomalies and cones, JHEP 02 (2013) 088 [arXiv:1207.5824] [INSPIRE].

[12] K. Jensen, R. Loganayagam and A. Yarom, Anomaly inflow and thermal equilibrium, JHEP 05 (2014) 134 [arXiv: 1310.7024] [INSPIRE].

[13] K. Jensen, R. Loganayagam and A. Yarom, Chern-Simons terms from thermal circles and anomalies, JHEP 05 (2014) 110 [arXiv:1311.2935] [INSPIRE].

[14] M. Torabian and H.-U. Yee, Holographic nonlinear hydrodynamics from AdS/CFT with multiple/non-Abelian symmetries, JHEP 08 (2009) 020 [arXiv: 0903.4894] [INSPIRE].

[15] D.E. Kharzeev and H.J. Warringa, Chiral magnetic conductivity, Phys. Rev. D 80 (2009) 034028 [arXiv: 0907.5007] [INSPIRE].

[16] K. Jensen, Chiral anomalies and AdS/CMT in two dimensions, JHEP 01 (2011) 109 [arXiv: 1012.4831] [INSPIRE].

[17] I. Amado, K. Landsteiner and F. Pena-Benitez, Anomalous transport coefficients from Kubo formulas in Holography, JHEP 05 (2011) 081 [arXiv: 1102.4577] [INSPIRE].

[18] K. Landsteiner, E. Megias and F. Pena-Benitez, Gravitational anomaly and transport, Phys. Rev. Lett. 107 (2011) 021601 [arXiv: 1103.5006] [INSPIRE].

[19] D.E. Kharzeev and H.-U. Yee, Anomalies and time reversal invariance in relativistic hydrodynamics: the second order and higher dimensional formulations, Phys. Rev. D 84 (2011) 045025 [arXiv: 1105.6360] [INSPIRE].

[20] S. Dubovsky, L. Hui and A. Nicolis, Effective field theory for hydrodynamics: Wess-Zumino term and anomalies in two spacetime dimensions, Phys. Rev. D 89 (2014) 045016 [arXiv:1107.0732] [INSPIRE].

[21] R. Loganayagam and P. Surowka, Anomaly/transport in an ideal Weyl gas, JHEP 04 (2012) 097 [arXiv: 1201.2812] [INSPIRE].

[22] Y. Neiman and Y. Oz, Relativistic hydrodynamics with general anomalous charges, JHEP 03 (2011) 023 [arXiv: 1011.5107] [INSPIRE].

[23] K. Jensen, Triangle anomalies, thermodynamics and hydrodynamics, Phys. Rev. D 85 (2012) 125017 [arXiv:1203.3599] [INSPIRE].

[24] V.P. Nair, R. Ray and S. Roy, Fluids, anomalies and the chiral magnetic effect: a group-theoretic formulation, Phys. Rev. D 86 (2012) 025012 [arXiv:1112.4022] [INSPIRE].

[25] M. Valle, Hydrodynamics in $1+1$ dimensions with gravitational anomalies, JHEP 08 (2012) 113 [arXiv:1206.1538] [INSPIRE].

[26] K. Landsteiner, E. Megias and F. Pena-Benitez, Anomalous transport from Kubo formulae, Lect. Notes Phys. 871 (2013) 433 [arXiv:1207.5808] [INSPIRE]. 
[27] R. Banerjee, Exact results in two dimensional chiral hydrodynamics with gravitational anomalies, Eur. Phys. J. C 74 (2014) 2824 [arXiv:1303.5593] [INSPIRE].

[28] E. Megias and F. Pena-Benitez, Holographic gravitational anomaly in first and second order hydrodynamics, JHEP 05 (2013) 115 [arXiv: 1304.5529] [INSPIRE].

[29] R. Banerjee, P. Chakraborty, S. Dey, B.R. Majhi and A.K. Mitra, Two dimensional hydrodynamics with gauge and gravitational anomalies, Phys. Rev. D 89 (2014) 104013 [arXiv:1307.1313] [INSPIRE].

[30] F.M. Haehl, R. Loganayagam and M. Rangamani, Effective actions for anomalous hydrodynamics, JHEP 03 (2014) 034 [arXiv: 1312.0610] [INSPIRE].

[31] R. Banerjee and S. Dey, Constitutive relations and response parameters in two dimensional hydrodynamics with gauge and gravitational anomalies, Phys. Lett. B 733 (2014) 198 [arXiv:1403.7357] [INSPIRE].

[32] A. Jimenez-Alba and L. Melgar, Anomalous transport in holographic chiral superfluids via Kubo formulae, JHEP 10 (2014) 120 [arXiv:1404.2434] [INSPIRE].

[33] H.-U. Yee, Chiral magnetic and vortical effects in higher dimensions at weak coupling, Phys. Rev. D 90 (2014) 065021 [arXiv: 1406.3584] [INSPIRE].

[34] G.M. Monteiro, A.G. Abanov and V.P. Nair, Hydrodynamics with gauge anomaly: variational principle and Hamiltonian formulation, arXiv:1410.4833 [INSPIRE].

[35] R. Banerjee, S. Dey and B.R. Majhi, Entropy current in two dimensional anomalous hydrodynamics and $a$ bound on the sum of the response parameters, arXiv:1412.5878 [INSPIRE].

[36] S. Bhattacharyya, Entropy current and equilibrium partition function in fluid dynamics, JHEP 08 (2014) 165 [arXiv:1312.0220] [INSPIRE].

[37] S. Bhattacharyya, Entropy current from partition function: one example, JHEP 07 (2014) 139 [arXiv: 1403.7639] [INSPIRE].

[38] L. Onsager, Reciprocal relations in irreversible processes. I, Phys. Rev. 37 (1931) 405.

[39] L. Onsager, Reciprocal relations in irreversible processes. II, Phys. Rev. 38 (1931) 2265.

[40] G. Policastro, D.T. Son and A.O. Starinets, The shear viscosity of strongly coupled $N=4$ supersymmetric Yang-Mills plasma, Phys. Rev. Lett. 87 (2001) 081601 [hep-th/0104066] [INSPIRE].

[41] S. Bhattacharyya, V.E. Hubeny, S. Minwalla and M. Rangamani, Nonlinear fluid dynamics from gravity, JHEP 02 (2008) 045 [arXiv:0712.2456] [INSPIRE].

[42] A.H. Taub, General relativistic variational principle for perfect fluids, Phys. Rev. 94 (1954) 1468 [INSPIRE].

[43] B. Carter, Elastic perturbation theory in general relativity and a variation principle for a rotating solid star, Commun. Math. Phys. 30 (1973) 261.

[44] S. Dubovsky, T. Gregoire, A. Nicolis and R. Rattazzi, Null energy condition and superluminal propagation, JHEP 03 (2006) 025 [hep-th/0512260] [INSPIRE].

[45] S. Dubovsky, L. Hui, A. Nicolis and D.T. Son, Effective field theory for hydrodynamics: thermodynamics and the derivative expansion, Phys. Rev. D 85 (2012) 085029 [arXiv:1107.0731] [INSPIRE]. 
[46] J. Bhattacharya, S. Bhattacharyya and M. Rangamani, Non-dissipative hydrodynamics: effective actions versus entropy current, JHEP 02 (2013) 153 [arXiv:1211.1020] [INSPIRE].

[47] A. Nicolis and D.T. Son, Hall viscosity from effective field theory, arXiv:1103.2137 [INSPIRE].

[48] F.M. Haehl and M. Rangamani, Comments on Hall transport from effective actions, JHEP 10 (2013) 074 [arXiv: 1305.6968] [INSPIRE].

[49] M. Geracie and D.T. Son, Effective field theory for fluids: hall viscosity from a Wess-Zumino-Witten term, JHEP 11 (2014) 004 [arXiv: 1402.1146] [INSPIRE].

[50] R.P. Feynman and F.L.J. Vernon, The theory of a general quantum system interacting with a linear dissipative system, Annals Phys. 24 (1963) 118 [INSPIRE].

[51] I.-S. Liu, Method of Lagrange multipliers for exploitation of the entropy principle, Archive Ration. Mech. Anal. 46 (1972) 131.

[52] V.E. Hubeny, S. Minwalla and M. Rangamani, The fluid/gravity correspondence, arXiv: 1107.5780 [INSPIRE].

[53] M.A. York and G.D. Moore, Second order hydrodynamic coefficients from kinetic theory, Phys. Rev. D 79 (2009) 054011 [arXiv:0811.0729] [InSPIRE].

[54] F.M. Haehl, R. Loganayagam and M. Rangamani, The eightfold way to dissipation, arXiv:1412.1090 [INSPIRE].

[55] F. M. Haehl, R. Loganayagam and M. Rangamani, Adiabaticity and KMS gauge invariance, work in progress.

[56] R. Loganayagam, Entropy current in conformal hydrodynamics, JHEP 05 (2008) 087 [arXiv:0801.3701] [INSPIRE].

[57] M. Rangamani, Gravity and hydrodynamics: lectures on the fluid-gravity correspondence, Class. Quant. Grav. 26 (2009) 224003 [arXiv:0905.4352] [INSPIRE].

[58] N. Read, Non-Abelian adiabatic statistics and Hall viscosity in quantum Hall states and $p(x)+i p(y)$ paired superfluids, Phys. Rev. B 79 (2009) 045308 [arXiv:0805.2507] [INSPIRE].

[59] X.G. Wen and A. Zee, Shift and spin vector: new topological quantum numbers for the Hall fluids, Phys. Rev. Lett. 69 (1992) 953 [INSPIRE].

[60] S. Golkar, M.M. Roberts and D.T. Son, Effective field theory of relativistic quantum Hall systems, JHEP 12 (2014) 138 [arXiv:1403.4279] [INSPIRE].

[61] S. Golkar, M.M. Roberts and D.T. Son, The Euler current and relativistic parity odd transport, arXiv: 1407.7540 [INSPIRE].

[62] R. Bertlmann, Anomalies in quantum field theory, International series of monographs on physics volume 91, Oxford University Press, Oxford U.K. (1996).

[63] J.A. Harvey, TASI 2003 lectures on anomalies, hep-th/0509097 [INSPIRE].

[64] S. Bhattacharyya et al., Local fluid dynamical entropy from gravity, JHEP 06 (2008) 055 [arXiv:0803.2526] [INSPIRE].

[65] S. Bhattacharyya, R. Loganayagam, I. Mandal, S. Minwalla and A. Sharma, Conformal nonlinear fluid dynamics from gravity in arbitrary dimensions, JHEP 12 (2008) 116 [arXiv: 0809.4272] [INSPIRE]. 
[66] S. Dubovsky, private communication.

[67] R. Baier, P. Romatschke, D.T. Son, A.O. Starinets and M.A. Stephanov, Relativistic viscous hydrodynamics, conformal invariance and holography, JHEP 04 (2008) 100 [arXiv:0712.2451] [INSPIRE].

[68] K. Jensen et al., Parity-violating hydrodynamics in $2+1$ dimensions, JHEP 05 (2012) 102 [arXiv:1112.4498] [INSPIRE].

[69] L.V. Delacrétaz, A. Nicolis, R. Penco and R.A. Rosen, Wess-Zumino terms for relativistic fluids, superfluids, solids and supersolids, Phys. Rev. Lett. 114 (2015) 091601 [arXiv: 1403.6509] [INSPIRE].

[70] E. Shaverin and A. Yarom, Universality of second order transport in Gauss-Bonnet gravity, JHEP 04 (2013) 013 [arXiv:1211.1979] [INSPIRE].

[71] A. Yarom, private communication.

[72] S. Grozdanov and A.O. Starinets, On the universal identity in second order hydrodynamics, JHEP 03 (2015) 007 [arXiv:1412.5685] [InSPIRE].

[73] V. Iyer and R.M. Wald, Some properties of Noether charge and a proposal for dynamical black hole entropy, Phys. Rev. D 50 (1994) 846 [gr-qc/9403028] [InSPIRE].

[74] R. Loganayagam, Anomalies and the helicity of the thermal state, JHEP 11 (2013) 205 [arXiv: 1211.3850] [INSPIRE].

[75] T. Azeyanagi, R. Loganayagam, G.S. Ng and M.J. Rodriguez, Holographic thermal helicity, JHEP 08 (2014) 040 [arXiv:1311.2940] [InSPIRE].

[76] L. Di Pietro and Z. Komargodski, Cardy formulae for SUSY theories in $d=4$ and $d=6$, JHEP 12 (2014) 031 [arXiv:1407.6061] [INSPIRE].

[77] C.G. Callan Jr. and J.A. Harvey, Anomalies and Fermion zero modes on strings and domain walls, Nucl. Phys. B 250 (1985) 427 [InSPIRE].

[78] K. Jensen, R. Loganayagam and A. Yarom, to appear.

[79] J.S. Schwinger, Brownian motion of a quantum oscillator, J. Math. Phys. 2 (1961) 407 [INSPIRE].

[80] L.V. Keldysh, Diagram technique for nonequilibrium processes, Zh. Eksp. Teor. Fiz. 47 (1964) 1515 [INSPIRE].

[81] K.-c. Chou, Z.-b. Su, B.-l. Hao and L. Yu, Equilibrium and nonequilibrium Formalisms Made Unified, Phys. Rept. 118 (1985) 1 [INSPIRE].

[82] A. Adams, N. Arkani-Hamed, S. Dubovsky, A. Nicolis and R. Rattazzi, Causality, analyticity and an IR obstruction to UV completion, JHEP 10 (2006) 014 [hep-th/0602178] [INSPIRE].

[83] P. Kovtun, G.D. Moore and P. Romatschke, Towards an effective action for relativistic dissipative hydrodynamics, JHEP 07 (2014) 123 [arXiv: 1405.3967] [INSPIRE].

[84] S. Grozdanov and J. Polonyi, Viscosity and dissipative hydrodynamics from effective field theory, arXiv: 1305.3670 [INSPIRE].

[85] S. Grozdanov and J. Polonyi, Dynamics of the electric current in an ideal electron gas: a sound mode inside the quasi-particles, arXiv:1501.06620 [INSPIRE]. 
[86] S. Bhattacharyya, J.R. David and S. Thakur, Second order transport from anomalies, JHEP 01 (2014) 010 [arXiv: 1305.0340] [INSPIRE].

[87] P. Kovtun, D.T. Son and A.O. Starinets, Viscosity in strongly interacting quantum field theories from black hole physics, Phys. Rev. Lett. 94 (2005) 111601 [hep-th/0405231] [INSPIRE].

[88] G. Plewa and M. Spalinski, On the gravity dual of strongly coupled charged plasma, JHEP 05 (2013) 002 [arXiv: 1212.2344] [INSPIRE].

[89] M. Haack and A. Yarom, Universality of second order transport coefficients from the gauge-string duality, Nucl. Phys. B 813 (2009) 140 [arXiv:0811.1794] [INSPIRE].

[90] D. Nickel and D.T. Son, Deconstructing holographic liquids, New J. Phys. 13 (2011) 075010 [arXiv: 1009.3094] [INSPIRE].

[91] P.B. Arnold, G.D. Moore and L.G. Yaffe, Transport coefficients in high temperature gauge theories. 1. Leading log results, JHEP 11 (2000) 001 [hep-ph/0010177] [INSPIRE].

[92] P.B. Arnold, G.D. Moore and L.G. Yaffe, Transport coefficients in high temperature gauge theories. 2. Beyond leading log, JHEP 05 (2003) 051 [hep-ph/0302165] [INSPIRE].

[93] G.D. Moore and K.A. Sohrabi, Thermodynamical second-order hydrodynamic coefficients, JHEP 11 (2012) 148 [arXiv:1210.3340] [INSPIRE].

[94] J.L. Manes and M. Valle, Parity violating gravitational response and anomalous constitutive relations, JHEP 01 (2013) 008 [arXiv:1211.0876] [INSPIRE].

[95] J.L. Mañes and M. Valle, Parity odd equilibrium partition function in $2+1$ dimensions, JHEP 11 (2013) 178 [arXiv:1310.2113] [INSPIRE].

[96] E. Megias and M. Valle, Second-order partition function of a non-interacting chiral fluid in $3+1$ dimensions, JHEP 11 (2014) 005 [arXiv: 1408.0165] [INSPIRE].

[97] A. Kamenev, Field theory of non-equilibrium systems, Cambridge University Press, Cambridge U.K. (2011).

[98] V. Iyer, Lagrangian perfect fluids and black hole mechanics, Phys. Rev. D 55 (1997) 3411 [gr-qc/9610025] [INSPIRE].

[99] O. Saremi and D.T. Son, Hall viscosity from gauge/gravity duality, JHEP 04 (2012) 091 [arXiv: 1103.4851] [INSPIRE].

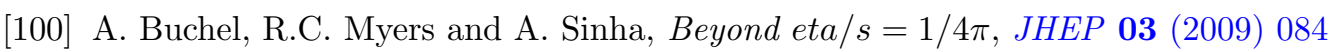
[arXiv: 0812.2521] [INSPIRE].

[101] S. Cremonini, The shear viscosity to entropy ratio: a status report, Mod. Phys. Lett. B 25 (2011) 1867 [arXiv: 1108.0677] [INSPIRE].

[102] I. Kanitscheider and K. Skenderis, Universal hydrodynamics of non-conformal branes, JHEP 04 (2009) 062 [arXiv:0901.1487] [InSPIRE].

[103] J.R. David, M. Mahato and S.R. Wadia, Hydrodynamics from the D1-brane, JHEP 04 (2009) 042 [arXiv: 0901.2013] [InSPIRE].

[104] J.R. David, M. Mahato, S. Thakur and S.R. Wadia, Hydrodynamics of R-charged D1-branes, JHEP 01 (2011) 014 [arXiv: 1008.4350] [INSPIRE].

[105] F. Bigazzi and A.L. Cotrone, An elementary stringy estimate of transport coefficients of large temperature QCD, JHEP 08 (2010) 128 [arXiv:1006.4634] [INSPIRE]. 
[106] Y. Tachikawa, Black hole entropy in the presence of Chern-Simons terms, Class. Quant. Grav. 24 (2007) 737 [hep-th/0611141] [INSPIRE].

[107] T. Azeyanagi, R. Loganayagam and G.S. Ng, Anomalies, Chern-Simons terms and black hole entropy, to appear.

[108] N. Iqbal and H. Liu, Universality of the hydrodynamic limit in AdS/CFT and the membrane paradigm, Phys. Rev. D 79 (2009) 025023 [arXiv:0809.3808] [INSPIRE].

[109] J. Bhattacharya, S. Bhattacharyya, S. Minwalla and A. Yarom, A theory of first order dissipative superfluid dynamics, JHEP 05 (2014) 147 [arXiv:1105.3733] [INSPIRE].

[110] J. Sonner and B. Withers, A gravity derivation of the Tisza-Landau model in AdS/CFT, Phys. Rev. D 82 (2010) 026001 [arXiv: 1004.2707] [InSPIRE].

[111] C.P. Herzog, N. Lisker, P. Surowka and A. Yarom, Transport in holographic superfluids, JHEP 08 (2011) 052 [arXiv:1101.3330] [InSPIRE].

[112] J. Bhattacharya, S. Bhattacharyya and S. Minwalla, Dissipative superfluid dynamics from gravity, JHEP 04 (2011) 125 [arXiv:1101.3332] [INSPIRE].

[113] P.C. Hohenberg and B.I. Halperin, Theory of dynamic critical phenomena, Rev. Mod. Phys. 49 (1977) 435 [INSPIRE]. 